\title{
Mechanistic Investigations of Metal- Metal Cooperativity in Dinickel Complexes and Iron/Cobalt Prussian Blue Analogues
}

\author{
DISSERTATION \\ zur Erlangung des mathemisch-naturwissenschaftlichen Doktorgrades \\ „Doctor rerum naturalium“ \\ der Georg-August-Universität zu Göttingen \\ im Promotionsprogramm der Georg-August-University School of Science (GAUSS)
}

vorgelegt von

Hendrik Stevens

aus

Berlin

Göttingen 2020 



\section{Betreuungsausschuss}

Prof. Dr. Franc Meyer

Institut für Anorganische Chemie, Georg-August-Universität Göttingen

Prof. Dr. Sven Schneider

Institut für Anorganische Chemie, Georg-August-Universität Göttingen

\section{Prüfungskomitee}

\section{Referent: Prof. Dr. Franc Meyer}

Institut für Anorganische Chemie, Georg-August-Universität Göttingen

Korreferent: Prof. Dr. Sven Schneider

Institut für Anorganische Chemie, Georg-August-Universität Göttingen

Prof. Dr. Dirk Schwarzer

Max-Planck-Institut für Biophysikalische Chemie, Göttingen

Prof. Dr. Dietmar Stalke

Institut für Anorganische Chemie, Georg-August-Universität Göttingen

Prof. Dr. Lutz Ackermann

Institut für Organische und Biomolekulare Chemie, Georg-August-Universität Göttingen

Dr. Christian Sindlinger

Institut für Anorganische Chemie, Georg-August-Universität Göttingen 


\section{Danksagung}

Gerne möchte ich an dieser Stelle mehreren Menschen für ihre außerordentliche Unterstützung danken. Denn ohne sie wäre es mir nicht möglich gewesen, diese Dissertation anzufertigen und zu einem Abschluss zu bringen.

Zunächst möchte ich mich bei Prof. Dr. Franc Meyer bedanken, der mir die wissenschaftliche Freiheit und die Möglichkeiten an die Hand gab, für mich interessante Themen selbständig zu erforschen. Zusätzlich möchte ich mich bei ihm für seinen Optimismus und unsere konstruktiven Diskussionen bedanken, ohne die das vorliegende Dissertationsprojekt an so manchen Stellen womöglich schon gescheitert wäre.

Weiterer Dank richtet sich an Prof. Dr. Sven Schneider, der durch Jahresgespräche meinen Arbeitsprozess förderte und Ideen zu weiteren Experimenten anregte.

In besonderem Maße möchte ich auch Thomas Kothe danken. Aus den gemeinsamen Unterredungen zum Thema resultierten stets fruchtbare Experimente und kreative Impulse, die diese Dissertation thematisch zu der Form gebracht haben, in der sie nun hier vorliegt.

Zusätzlich möchte ich bei den KollegInnen und KollaborateurInnen bedanken, die diese Themen durch Messungen fundiert haben: Prof. Dr. Dirk Schwazer und Dr. Jennifer Ahrens für die Pump-Probe Messungen; Dr. Sebastian Dechert für XRD Messungen und Kristallstrukturverfeinerungen; Dr. Serhiy Demeshko für magnetische Messungen; Dr. Serhiy Demeshko, Dr. Eleonora Ferretti und Dr. Joanne Wong für Mößbauer Messungen; Dr. Michael John und Ralf Schöne für die Hilfe bei komplexeren NMR-Messungen; Andreas Schwarz und Jörg Teichgräber für die Unterstützung beim Erwerb von Chemikalien und Equipment sowie der Analytikabteilung für Elementaranalysen.

Ich möchte mich außerdem bei Dr. Claudia Stückl und Britta Müller bedanken für die Hilfe beim Überwinden von diversen bürokratischen Hürden.

Da die meiste Zeit im Labor verbracht wurde, gilt mein Dank zudem meinen bisherigen und jetzigen LaborkollegInnen für die immerfort erheiternd bereichernde Arbeitsatmosphäre, dazu gehören: Dr. Mike Schütze, Claudia Schremmer, Thomas Kothe, Giuseppe Lococciolo und Dr. Allyssa Massie.

In diesem Zusammenhang möchte ich mich natürlich bei allen Mitgliedern des AK Meyer bedanken und besonders bei Dr. Sandeep Gupta, Dr. Shao-An Hua, Dr. Joanne Wong, Anna Kölpin, Sheida Rajabi, Massimiliano Morganti, Jerome Spyra, Martin Dzemski, Kevin Lüken und Roland Schulz. Auch ehemaligen Mitgliedern spreche ich hiermit meinen Dank aus, dazu zählen Dr. Eleonora Ferretti, Dr. Munmun Ghosh und Dr. Christopher Inman.

Für das Korrekturlesen dieser Dissertation danke ich Dr. Alexander Paesch, Dr. Allyssa Massie, Dr. Joanne Wong, Dr. Christopher Inman und Thomas Kothe. 
Für die mußestündlichen Aktivitäten außerhalb des Labors möchte mich sehr bedanken bei Thomas Kothe, Dr. Alexander Paesch, Josh Abbenseth, Sarah Bete, Jan Gerkens und vielen anderen. Im Besonderen danke ich Christina Piljavec, der es scheinbar möglich war, mich in dieser intensiven Zeit des Schreibens auszuhalten.

Schließlich bedanke ich mich bei meinen Eltern, Martina und Günther Stevens ebenso wie bei meinen Großmüttern, Christine Stevens und Ulrita Teschendorff. 


\section{Publications Derived from this Work}

Parts of this work have already been published and will be reprinted in this dissertation.

- H. Stevens, P.-C. Duan, S. Dechert, F. Meyer, J. Am. Chem. Soc., 2020, 142, 67176728. DOI: $10.1021 /$ jacs.0c00758. The rights for reprint have been granted by the American Chemical Society (Copyright (C) 2020). 


\section{List of Contents}

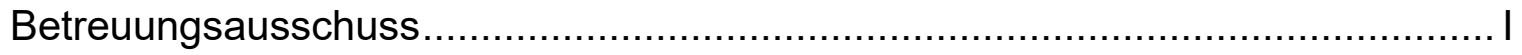

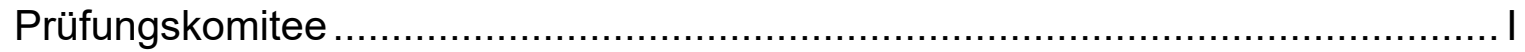

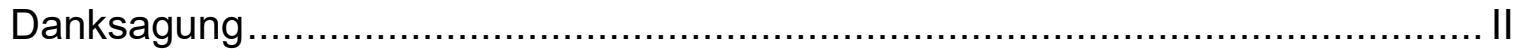

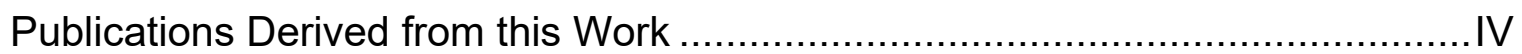

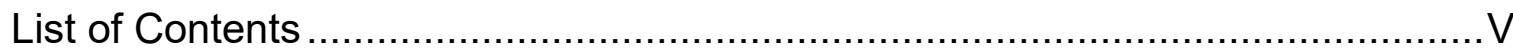

1. Investigations on a Reversible $\mathrm{C}-\mathrm{H}$ Activation by a $\mathrm{Ni}(\mathrm{I}) \mathrm{Ni}(\mathrm{I})$ Intermediate Bearing a Preorganized Ligand Scaffold ....................................................... 1

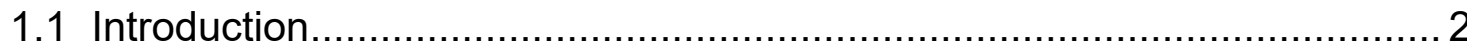

1.1.1 Small Molecule Activation by Mononuclear Ni(I) Systems Bearing $\beta$ Diketiminato Ligands .................................................................. 3

1.1.2 Oxidative Addition of $\mathrm{H}_{2}$ and $\mathrm{C}-\mathrm{H}$ Bonds towards Nickel Hydride Complexes.

1.1.3 Development of Bimetallic Nickel $\beta$-Diketiminato Complexes Through a Preorganized Ligand Scaffold ............................................................ 9

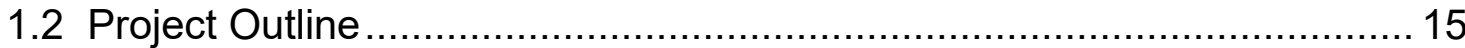

1.3 Synthesis of Pyrazole-Bridged NacNac Ligand $\mathbf{H}_{3} L^{\mathrm{Ph}}$ Bearing $m$-Terphenyl

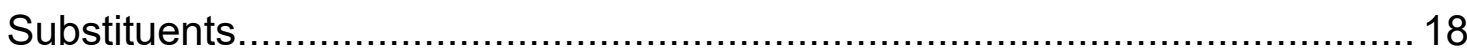

1.4 Mechanistic Study of the Reversible C-H Activation of the Bimetallic Nickel

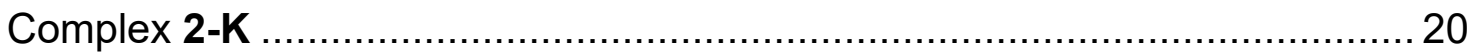

1.4.1 Synthesis of $\mathrm{L}^{\mathrm{Ph}} \mathrm{Ni}_{2}(\mu-\mathrm{Br})(\mathbf{1})$ and Product of the Hydrolysis (1-OH) 20

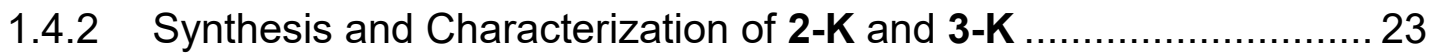

1.4.3 Mechanistic Insight into the Reversible C-H Activation through $\mathrm{H}_{2}$

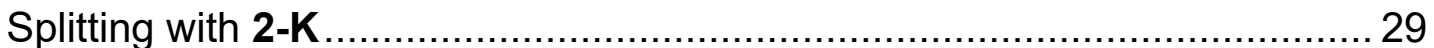

1.4.4 Trapping of the $\mathrm{Ni}(\mathrm{I}) \mathrm{Ni}(\mathrm{I})$ Intermediate by the Reaction with Benzaldehyde as a Two-electron Acceptor............................................... 33

1.5 Influence of the Alkali Metal Cation on the C-H Activation........................... 37

1.5.1 Exchange of Potassium with Sodium: C-H Activated Species 2-Na

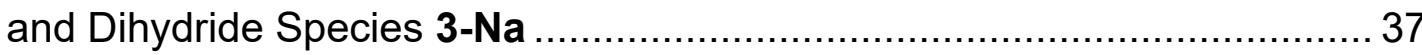

1.5.2 Investigations of the Interconversion between $\mathrm{C}-\mathrm{H}$ Activated Species 2-Na and Dihydride 3-Na ............................................................. 41

1.5.3 Mechanistic Investigation on the Twofold C-H Activated 5-Na ........ 45

1.5.4 Competition Experiments for the Binding of $\mathrm{Na}^{+}$and $\mathrm{K}^{+}$to the $\mathrm{C}-\mathrm{H}$ Activated Species 2-Na and 2-K in Solution ............................................ 49

1.6 Reactivity of C-H Activated Species 2-K Towards Small Molecules......... 52 
1.6.1 Investigations on the Activation of Carbon Oxides with $\mathrm{C}-\mathrm{H}$ Activated Species 2-K

1.6.2 Investigations on One-Electron Reductions of $\mathrm{O}_{2}$ and $\mathrm{N}_{2}$ with $\mathrm{C}-\mathrm{H}$

Activated species 2-K

1.7 Summary and Outlook

2. Mechanistic Studies on the CTIST of Di- and Tetranuclear Fe/Co Prussian Blue Analogues using Time-Resolved Spectroscopy

2.1 Introduction

2.1.1 From 3D-Networks to Linear and Square Prussian Blue Analogues70

2.1.2 Pump-Probe Spectroscopy on Fe/Co PBAs .75

2.2 Project Outline .78

2.3 Synthesis and Characterization of the Tetranuclear Fe/Co Prussian Blue Analogue $\left[\mathrm{Fe}_{2} \mathrm{CO}_{2}\right]$

2.4 Dinuclear Fe/Co PBAs Containing Polypyrazolyl Lutidine Ligands

2.4.1 Synthesis of the Polypyrazolyl Lutidine Ligands $\mathbf{p z} \mathbf{z}_{\mathbf{4}}$ Lut .85

2.4.2 Synthesis of Dinuclear Fe/Co PBAs Containing Lutidine-Based

Pentadentate Ligands $\mathrm{pz}^{\mathrm{R}} \mathbf{4} \mathrm{Lut}$.

2.4.3 Electronic Structure Characterization of [tp ${ }^{R}-F e / p z^{R}{ }_{4}$ Lut-Co] 92

2.5 Dinuclear PBAs Bearing Different Pentadentate Ligand Systems Bearing Pyrazole or Amine Moieties .96

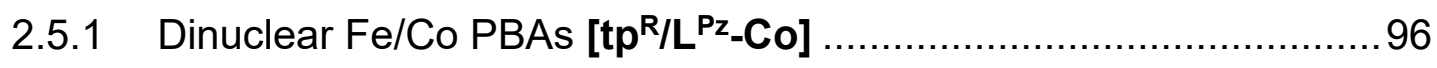

2.5.2 Linear PBAs with pentadentate N4Py Capping Ligand .98

2.6 Ultrafast Time-Resolved Spectroscopy of the Photo-Induced Electron Transfer in Di- and Tetranuclear Fe/Co PBAs 102

2.6.1 Femtosecond UV/vis-Pump UV/vis-Probe Spectroscopy on

[tp*-Fe/pz* ${ }_{4}$ Lut-Co] and [Fe2 $\left.\mathrm{Co}_{2}\right]$ 103

2.6.2 Femtosecond UV/vis-Pump Mid-IR-Probe Spectroscopy on [tp*-Fe/pz* ${ }_{4}$ Lut-Co] and $\left[\mathrm{Fe}_{2} \mathrm{Co}_{2}\right]$ 105

2.7 Summary and Outlook ............................................................ 108

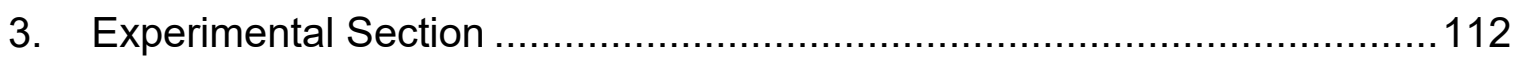

3.1 General Considerations ............................................................. 112

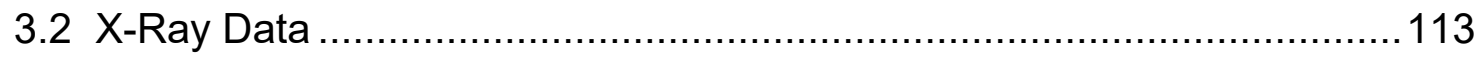

3.3 Investigations on a Reversible C-H Activation Through a $\mathrm{Ni}(\mathrm{I}) \mathrm{Ni}(\mathrm{I})$ Intermediate and Investigations Towards Small Molecule Activation.............115

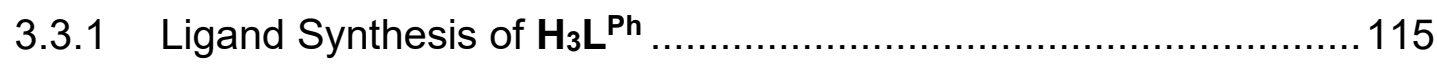

3.3.2 Synthesis of Nickel(II) Complexes .....................................118 


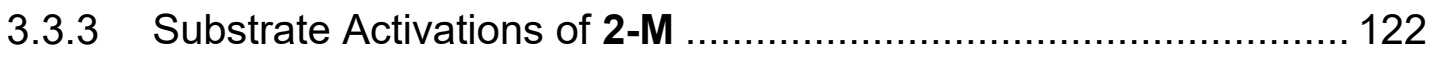

3.4 Mechanistic Studies on the CTIST of Di- and Tetranuclear Co/Fe Prussian Blue Analogues using Time-Resolved Spectroscopy ................................. 125

3.4.1 Physical Measurements ............................................... 125

3.4.2 Laser Systems ........................................................ 125

3.4.3 Magnetic Measurements ................................................. 126

3.4.4 Ligand Syntheses.............................................................. 126

3.4.5 General Procedure for the Synthesis of Dinuclear Co/Fe PBAs

Containing Hydrotrispyrazolylborate and Tetrapyrazolyl Lutidine ligands .. 128

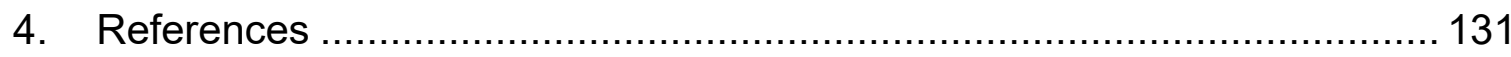

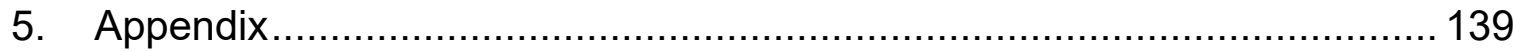

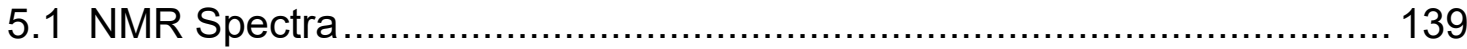

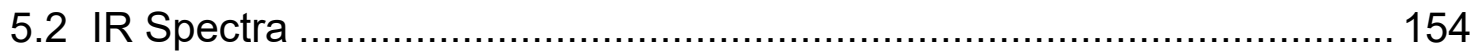

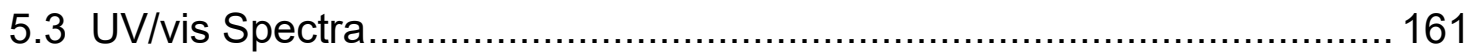

5.4 Magnetic Susceptibility Measurements ...................................... 163

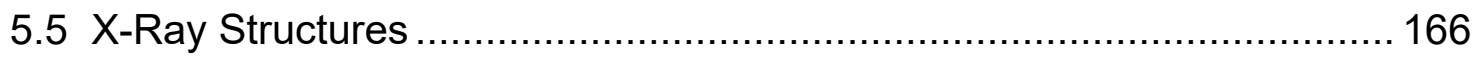





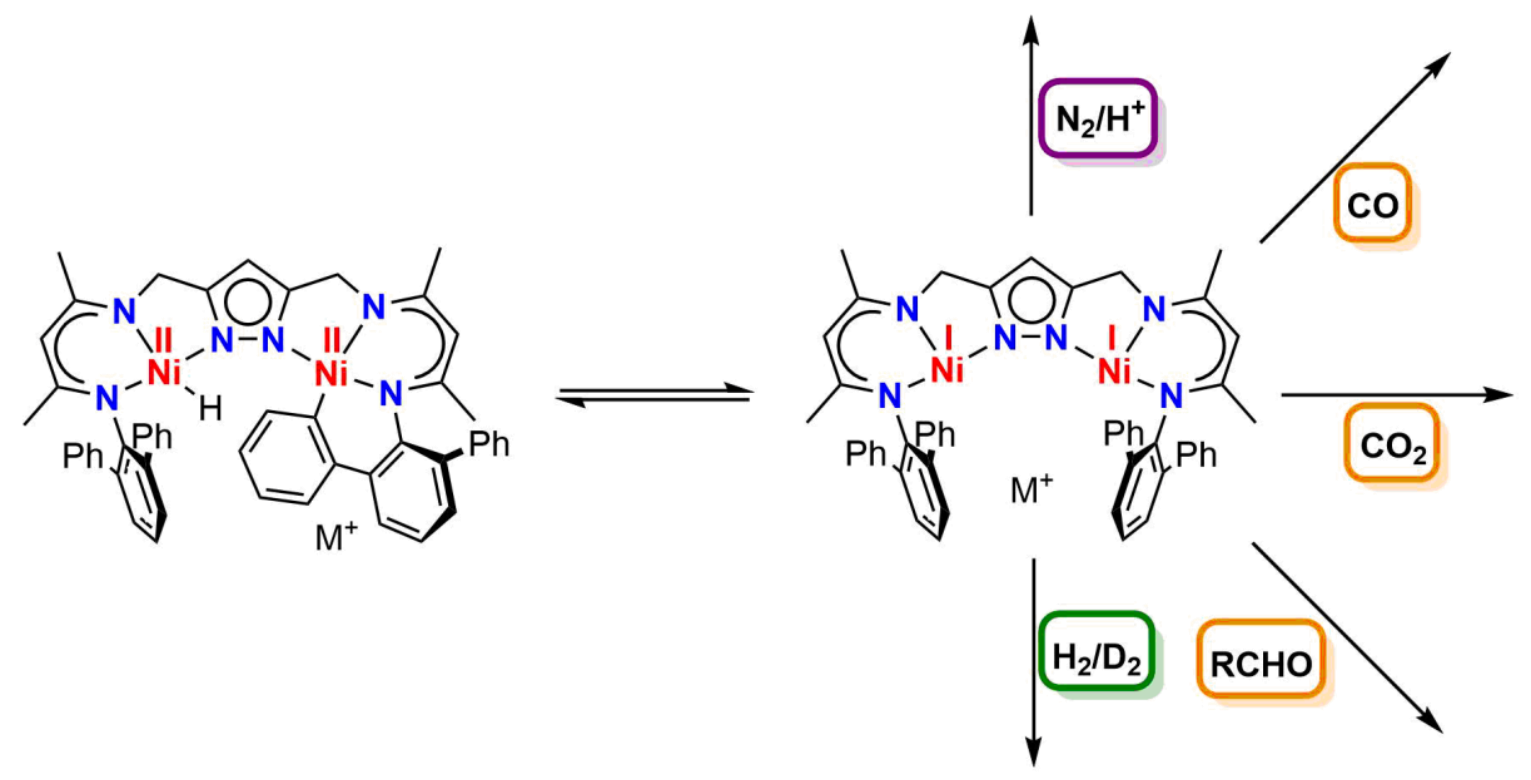

1. Investigations on a Reversible C-H Activation by a $\mathrm{Ni}(\mathrm{I}) \mathrm{Ni}(\mathrm{I})$ Intermediate Bearing a Preorganized Ligand Scaffold 


\subsection{Introduction}

Small molecule activation by transition metal complexes is an essential part of industrial processes, due to their mostly overall energy- and cost-efficient applications. The transformation of such molecules, for example $\mathrm{N}_{2}, \mathrm{CO}$ or $\mathrm{CO}_{2}$, often require the development of suitable systems towards stabilization of low-valent metal centers or of reactive ligands such as hydrides. These systems then provide a coordination site and consecutive reaction to activate and fix the substrates. ${ }^{1}$

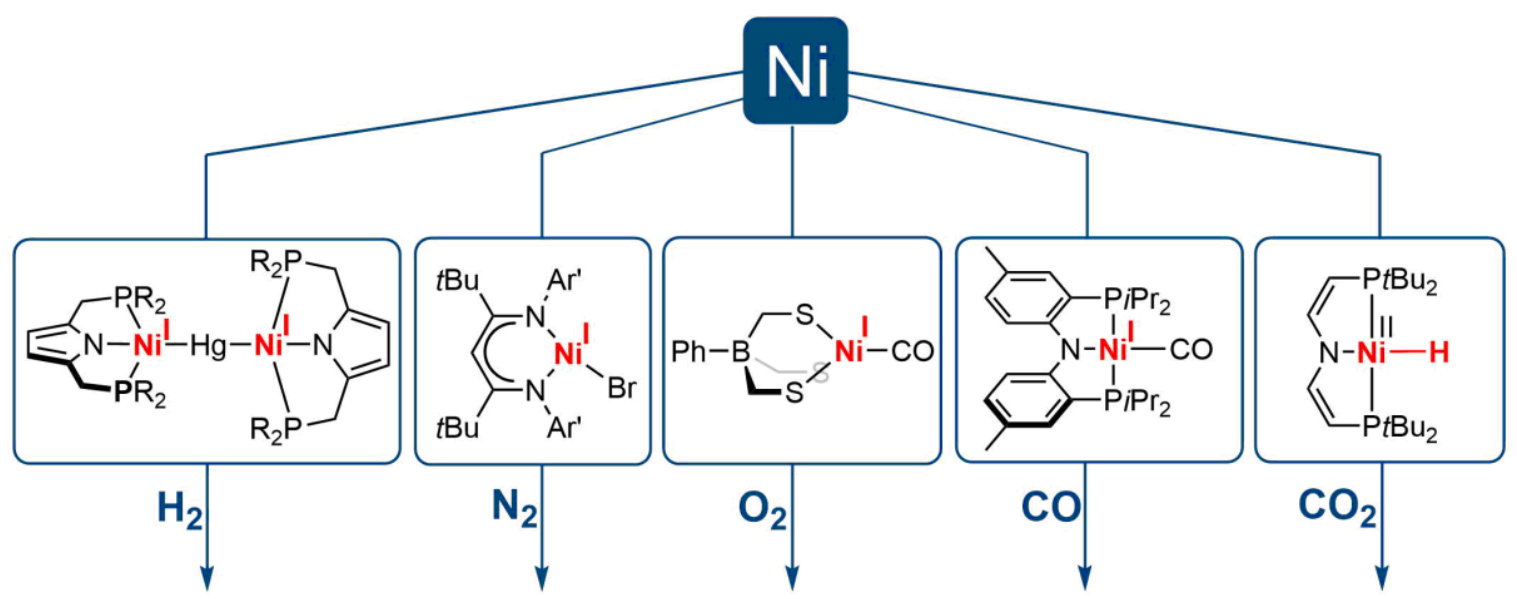

Scheme 1.1 Exemplary $\mathrm{Ni}(\mathrm{I})$ or nickel-hydride complexes showing activation of the highlighted small molecules. ${ }^{2-6}$

Multiple first-row transition metals, such as iron and nickel, have been employed for such reactions. In particular, nickel complexes showed interesting results on the activation of small molecules based on the stabilization of $\mathrm{Ni}(\mathrm{I})$ and $\mathrm{Ni}(0)$ species as strong one- or twoelectron reducing agents. ${ }^{1,7-9}$ Additionally, the possibility of developing Ni(II) hydride complexes allow other reactivities in from of two-electron reduction coupled with protonation on certain small molecules. ${ }^{10}$ This versatility of nickel is presented in the activation of a variety of small molecules based on multiple different coordination compounds with different kinds of ligands (examples in Scheme 1.1). Further stabilization of the reactive center is gained through masking of the $\mathrm{Ni}(\mathrm{I})$ ion utilizing reactive ligands, for example aryl moieties, which are able to interact reversibly. ${ }^{11}$ Thorough investigations on such $\mathrm{Ni}$ (II) synthons is the key to understand the mechanistic pathways for small molecule activation. Additionally, such "masquerade" opens up to a variety of other transformations dependent on the reaction conditions and based on the coordinated ligands. Such a system shall be presented in this work through the employment of a reactive phenyl moiety on the ligand system and metal-metal cooperativity ${ }^{12}$, which results in a masked $\mathrm{Ni}(\mathrm{I}) \mathrm{Ni}(\mathrm{I})$ system. 


\subsubsection{Small Molecule Activation by Mononuclear Ni(I) Systems Bearing $\beta$ - Diketiminato Ligands}

A closer look at the examples in Scheme 1.1 presents the $N$-aryl $\beta$-diketiminato ligands $(\mathrm{NacNac})$ in the activation of small molecules. ${ }^{1,8}$ The Schiff bases (Scheme 1.2), derived from acetylacetone $(a c a c)$, show remarkable properties, due to their stabilization of multiple other low-valent metal centers besides nickel, such as $\mathrm{Fe}^{\mathrm{I}}, \mathrm{Mg}^{\mathrm{I}}$ et cetera. ${ }^{13}$

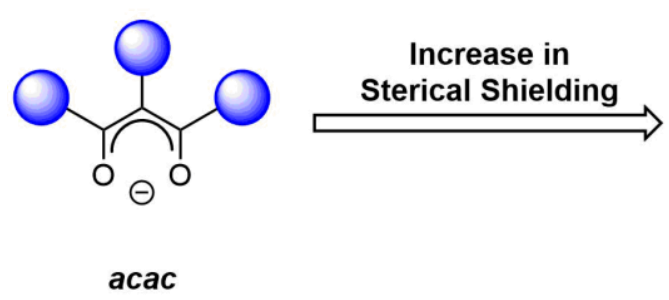

Scheme 1.2 N-aryl $\beta$-diketiminato ligands NacNac derived from acetylacetone (acac) by condensation reaction.

These properties are based on the steric shielding NacNac ligands are providing through substituents on the aryl moieties. This incorporation of bulky alkyl or aryl groups results in kinetic stabilization of reactive species and therefore provide an improvement over the oxygen derivative. This is typically realized through the substitution of the ortho-positions (Scheme 1.2) The bulkier the substituents the more orthogonal orientation of the aryl groups relative to the NacNac backbone is found. Therefore, with bulkier ligands, causing less $\pi$-orbital overlap, the electronic influence of the aryl moieties is negligible. Substitution of the $\alpha$ - and $\beta$-carbons (Scheme 1.2) has an impact on the electronic structure of the anionic ligand, but modifications to these positions are rather synthetically challenging. ${ }^{14}$ All in all, the kinetic inhibition through bulky aryl substituents is the main cause of the stabilization of reactive species, which results in a high interest in NacNac bearing coordination compounds. ${ }^{1,13,15-18}$

The employment of NacNac for the stabilization of nickel compounds has resulted in a broad range of small molecule activation. Due to the planarity and therefore support of a trigonal, square planar or tetrahedral coordination sphere the stabilization of $\mathrm{Ni}(\mathrm{I})$ and $\mathrm{Ni}(\mathrm{II})$ is possible. The geometry around the nickel center is dependent on the bulkiness of the aryl substituents. ${ }^{16}$ The first example of such a system was presented by the group of Holland in 2002 by employing a NacNac with tert-butyl and 2,6-di-(iso-propyl)phenyl (dipp) substituents (Scheme 1.3, top). ${ }^{19}$ Here, the attempt of methylation of a nickel chlorido precursor resulted in the reduction to a $\mathrm{Ni}(\mathrm{I})$-thf complex through the elimination of ethane. Shortly after, a similar system of Warren et al. was published. ${ }^{20}$ Here a $\mathrm{Ni}$ (II)lutidine alkyl system undergoes reductive elimination to the $\mathrm{Ni}(\mathrm{I})$ complex. Further reactivity with $\mathrm{NO}$ resulted in the $\mathrm{Ni}(\mathrm{I})-\mathrm{NO}$ complex. Only small structural changes were observed on the NO ligand, which indicates no electron transfer to the ligand (Scheme 1.3, bottom). 

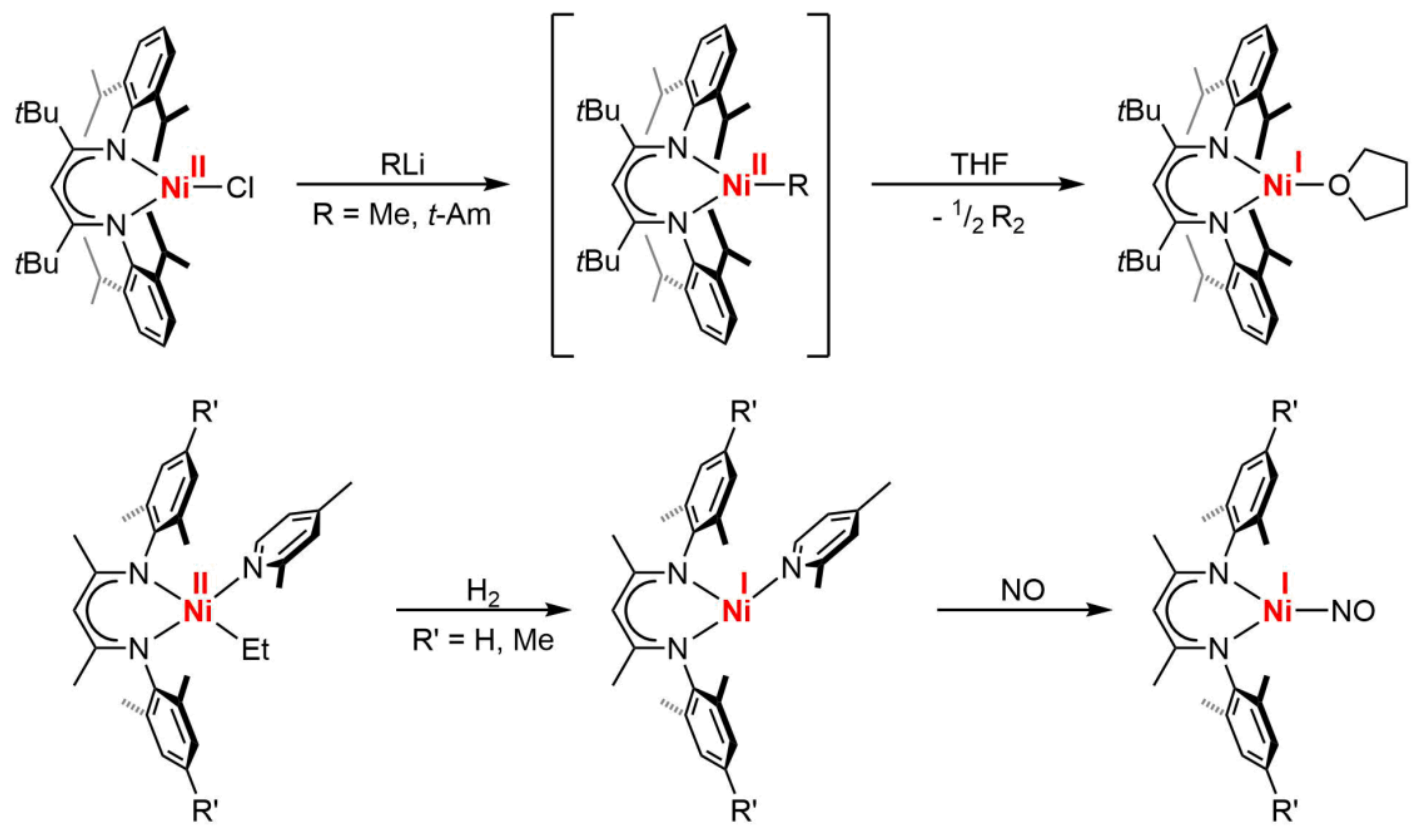

Scheme 1.3 First examples of $\mathrm{Ni}(\mathrm{I})$ systems bearing $\mathrm{N}$-aryl $\beta$-diketiminato ligands; top: by Holland et al. in 2002 through reduction of $\mathrm{Ni}(\mathrm{II})$ chlorido complex with organolithium or Grignard reagents ${ }^{19}$; bottom: by Warren et al. in 2003 through reduction of a $\mathrm{Ni}(\mathrm{II})$ alkyl complex with dihydrogen and reaction with $\mathrm{NO}^{20}$

In contrast to the genuine $\mathrm{Ni}(\mathrm{I})$ systems presented, the group of Stephan published a rather remarkable dinickel complex in 2005 , which indirectly showed Ni(I) reactivity. ${ }^{11}$ Through utilization of an electron accepting ligand, here toluene, the group isolated a $\mathrm{Ni}(\mathrm{II}) \mathrm{Ni}$ (II) dimer bridged by a Birch-like reduced toluene ligand (Scheme 1.4).

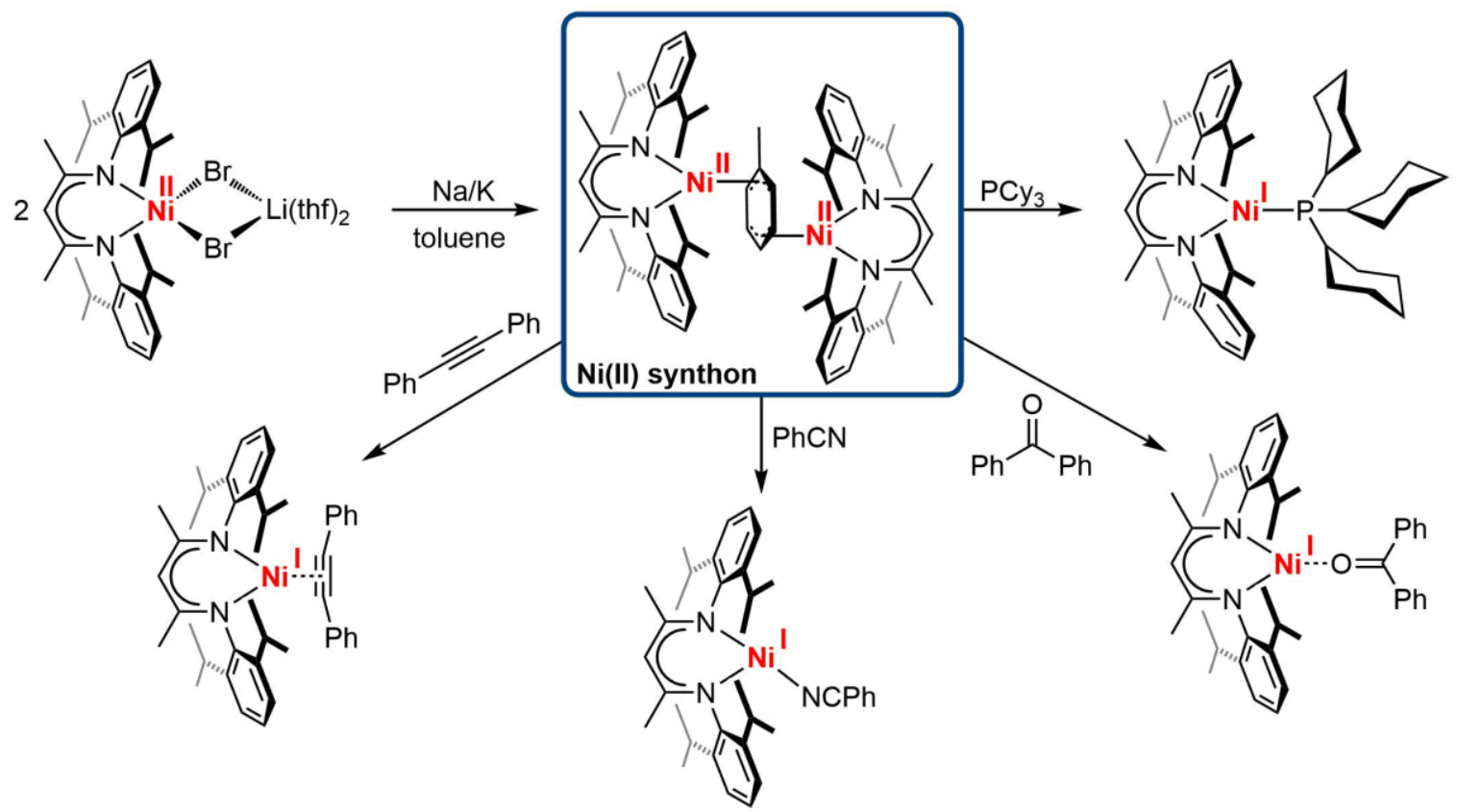

Scheme 1.4 Synthesis of a dimeric Ni(II) synthon bearing a NacNac and a bridged Birch-like reduced toluene ligands by Stephan et al. and reactivity with different substrates. ${ }^{11}$ 
Although the diamagnetic species was characterized by ${ }^{1} \mathrm{H}-\mathrm{NMR}$ spectroscopy and shows distinct signals, reactions with different substrates resulted in monomeric $\mathrm{Ni}(\mathrm{I})$ complexes. Instead of one-electron reduction of the substrates, adducts bound to the $\mathrm{Ni}(\mathrm{I})$ center are observed in the cases of $\mathrm{PCy}_{3}, \mathrm{PhCN}$, diphenylacetylene and benzophenone (Scheme 1.4). This behavior is caused by the equilibrium between the Ni(II) dimer and $\mathrm{Ni}(\mathrm{I})$ monomer in solution, which was shown by EPR spectroscopy. Therefore, the Ni(II) synthon is treated as a toluene-masked $\mathrm{Ni}(\mathrm{I})$ precursor, being diamagnetic in nature, but resulting in paramagnetic adducts with substrates. This first example of such a "masquerade" for $\mathrm{Ni}(\mathrm{I})$ complexes allows characterization through NMR spectroscopy, despite of its $\mathrm{Ni}(\mathrm{I})$ reactivity. ${ }^{1}$

This methodology was then optimized and expanded for another NacNac nickel complex by Driess et al. using 2,6-diethylphenyl substituents. ${ }^{1}$ Furthermore, the group investigated the reactivity of the masked complex A (Scheme 1.5) towards small substrates, such as $\mathrm{N}_{2} \mathrm{O}, \mathrm{O}_{2}, \mathrm{~S}_{8}$ and $\mathrm{P}_{4}$. As an example, dioxygen activation should be discussed. Reaction of A with an excess of dioxygen resulted in the isolation of a side-on Ni(II) superoxido complex. The singly-reduced dioxygen species A-SO reacted with another equivalent of A or an oxophilic compound to the A-OH through hydrogen-atom abstraction (HA) (Scheme 1.5). This behavior indicated an O-atom transfer ability of A-SO to a nickel oxido system (brackets in Scheme 1.5), which undergoes dimerization and HA to A-OH. Remarkably, two different geometries, square planar and tetrahedral, are observed for the two nickel centers of A-OH. Similar reactivity to A-OH was found, when A was oxidized with $\mathrm{N}_{2} \mathrm{O}$ as an oxygen-atom supplier. Substrate scope oxygen atom-transfer was tested on oxygenation reactions, which mostly resulted in oxygen-atom transfer, consistent with the previous investigations. ${ }^{21}$

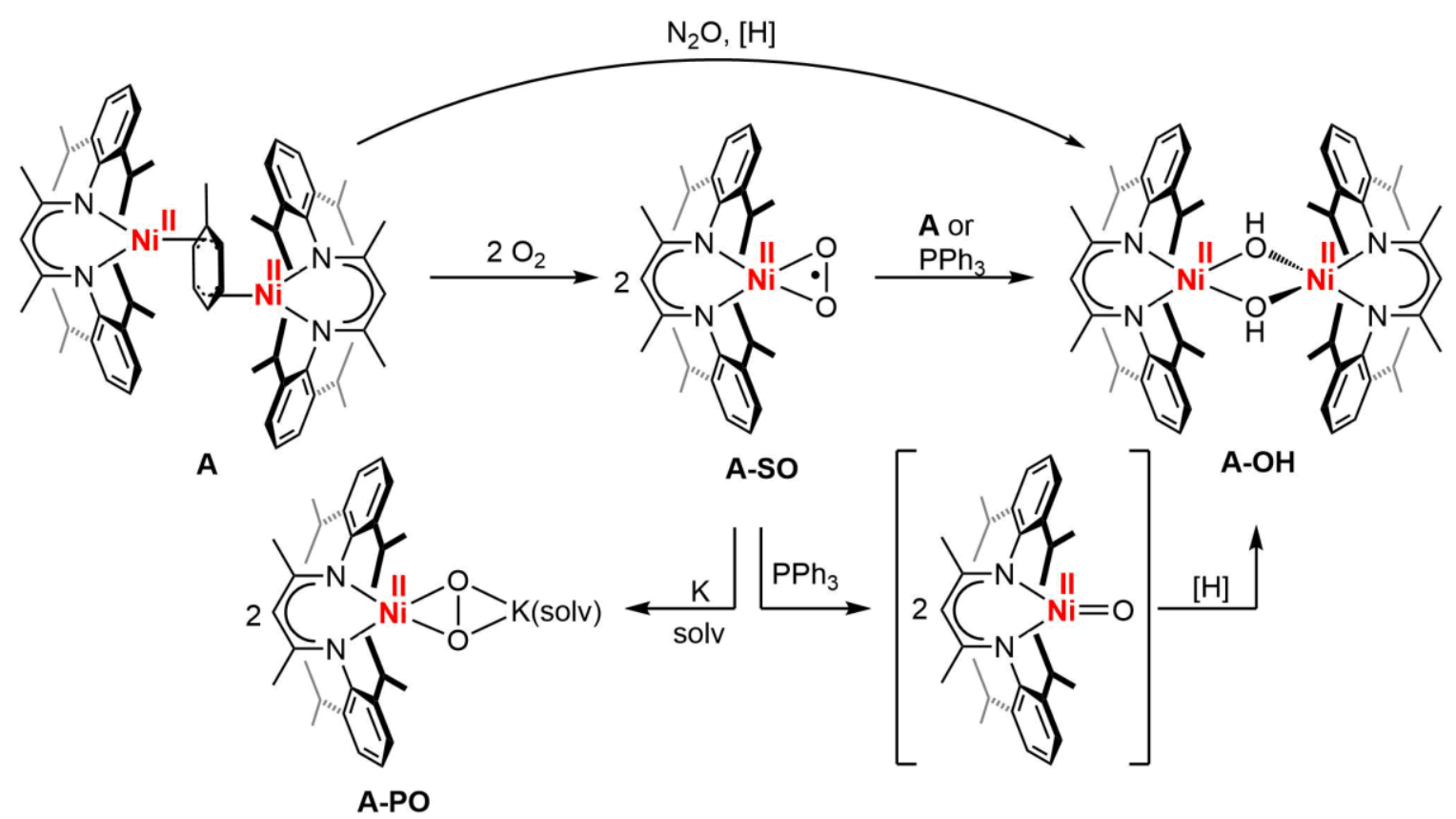

Scheme 1.5 Oxygen activation of the masked $\mathrm{Ni}(\mathrm{l})$ system $\mathbf{A}$ to a nickel superoxido complex A-SO by Driess et al. and consecutive reactive through oxygen-atom transfer and hydrogen-atom transfer to $\mathbf{A}-\mathbf{O H}$ or reduction to the peroxido species $\mathbf{A}-\mathbf{P O} \mathbf{.}^{21}$ 
Reduction of the superoxido complex A-SO with potassium metal, resulted in the isolation of the side-on peroxido complex A-PO (Scheme 1.5). ${ }^{22}$ Compared to A-SO, A-PO did not show reactivity towards substrates and therefore shows a higher stability than the starting material. Instead, heterobimetallic systems indirectly with zinc ${ }^{22}$ and directly with iron ${ }^{23}$ were synthesized starting from the superoxido complex A-SO. Both peroxido complexes were unstable and reacted further to the bridged Ni-Zn bis-hydroxido complex or through intramolecular $\mathrm{C}-\mathrm{H}$ activation of one of the iso-propyl moieties to the bridged Ni-Fe alkoxido-hydroxido system.

In addition to the reduction of oxygen, multiple other small molecules activations $\left(\mathrm{H}_{2}{ }^{24}\right.$, $\mathrm{N}_{2}{ }^{24}, \mathrm{CO}^{25,26}$ and $\mathrm{CO}_{2}{ }^{27}$ ) were presented and show the importance of such systems for future small molecule activation investigations. Interestingly, most of the activations resulted in the creation of dimeric structures to stabilize the reactive products. Furthermore, twoelectron reductions have been observed for multiple activated species employing NacNacbased $\mathrm{Ni}(\mathrm{I})$ complexes. Therefore, the idea arose of implementing a ligand scaffold, which preorganizes two metal centers through two independent coordination pockets.

\subsubsection{Oxidative Addition of $\mathrm{H}_{2}$ and $\mathrm{C}-\mathrm{H}$ Bonds towards Nickel Hydride Complexes}

The storage of electrons and protons in form of metal hydride units allows for selective transformations of substrates. A biological example for this kind of storage is seen in the FeMo cofactor of the metalloenzyme nitrogenase. Here, the reducing equivalents are stored in Fe-H units, which are used for the subsequent reduction of nitrogen upon elimination of $\mathrm{H}_{2}$ (Scheme 1.6, left). ${ }^{28}$
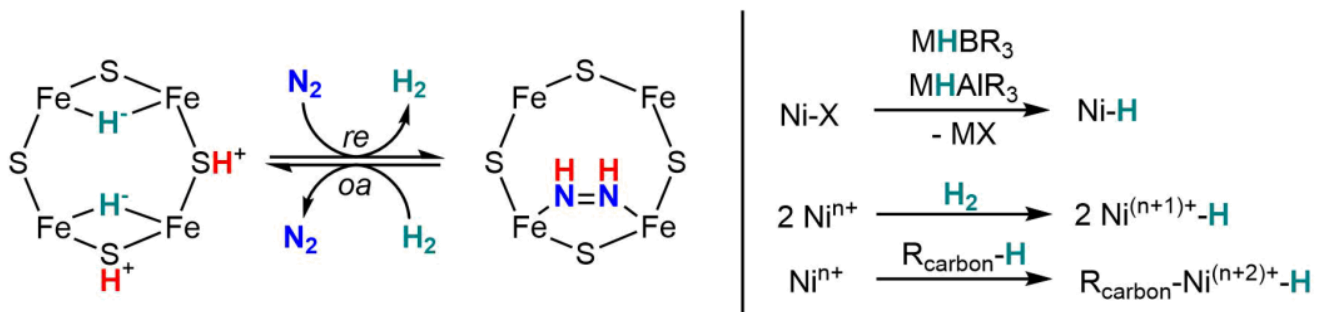

Scheme 1.6 Left: Scheme for the reductive elimination ( $r e$ ) and oxidative-addition (oa) mechanism of the FeMo cofactor of nitrogenase; ${ }^{28}$ right: access to nickel hydride complexes through hydride donor reagents or oxidative addition of substrates. ${ }^{10}$

The storage of reducing equivalents in metal hydride bonds received significant attention with various metals in particular nickel. The resulting nickel-hydride complexes can be used variously in transformations of substrates for example reductions. ${ }^{10}$ The synthetic strategies to obtain nickel hydride complexes is rather diverse, in which hydride donors 
such as borohydrides or aluminiumhydrides are often used. However oxidative addition of dihydrogen or $\mathrm{C}-\mathrm{H}$ bonds have also been reported (Scheme 1.6, right). ${ }^{10}$

The oxidative addition of $\mathrm{H}_{2}$ with low-valent nickel center to obtain the hydride complexes is has been well documented. One of the reason for this is the high interest in model complexes for the [NiFe] hydrogenase activity. ${ }^{29,30}$ These model systems mainly correspond to the oxidative addition on $\mathrm{Ni}(0)$ centers or heterolytic splitting on $\mathrm{Ni}$ (II) ions. For the addition of $\mathrm{H}_{2}$ to $\mathrm{Ni}(\mathrm{I})$ complexes only a few systems are found in the literature. ${ }^{2,24,31,32}$ Such a system bearing a $\beta$-diketiminato ligand was used by the group of Limberg. Utilization of low-valent $\mathrm{Ni}(\mathrm{I})$ systems $\mathbf{B}$ and $\mathbf{B}-\mathbf{B r}$ readily undergo oxidative addition under $\mathrm{H}_{2}$ atmosphere to form the dihydride-bridged species $\mathbf{B}-\mathbf{H}_{2}$ (Scheme 1.7). ${ }^{24,33}$ Although, the two tetrahedral $h s-\mathrm{Ni}(\mathrm{II})$ hydride dimer $\mathbf{B}-\mathbf{H}_{2}$ are antiferromagnetically coupled, characterization through NMR spectroscopy was not obtained. The weak ligation of the bridged dihydride allows reductive elimination and $\mathbf{B}-$ $\mathbf{H}_{2}$ forms the dinitrogen bridged $\mathrm{Ni}(\mathrm{I}) \mathrm{Ni}(\mathrm{I})$ complex $\mathbf{B}-\mathbf{N}_{\mathbf{2}}$ under $\mathrm{N}_{2}$ atmosphere. Instead of a reduction of dinitrogen, the coordination of dinitrogen to the two Ni(I) centers is observed. The reverse reaction to $\mathbf{B}-\mathbf{H}_{2}$ occurs rapidly under $\mathrm{H}_{2}$ atmosphere. Therefore, similar to the system developed by Stephan et al., B-H $\mathbf{H}_{2}$ reacts as a masked $\mathrm{Ni}(\mathrm{I})$ synthon upon elimination of $\mathrm{H}_{2}$ and thus a storage of reduction equivalents similar to the FeMo cofactor can be attributed. Further investigation on $\mathbf{B}-\mathbf{N}_{\mathbf{2}}$ with bulkier NacNac backbone substituents, resulted in stepwise reduction of the dinitrogen ligand to a single-reduced and twice-reduced moiety, also observed for the FeMo cofactor. ${ }^{3,25}$

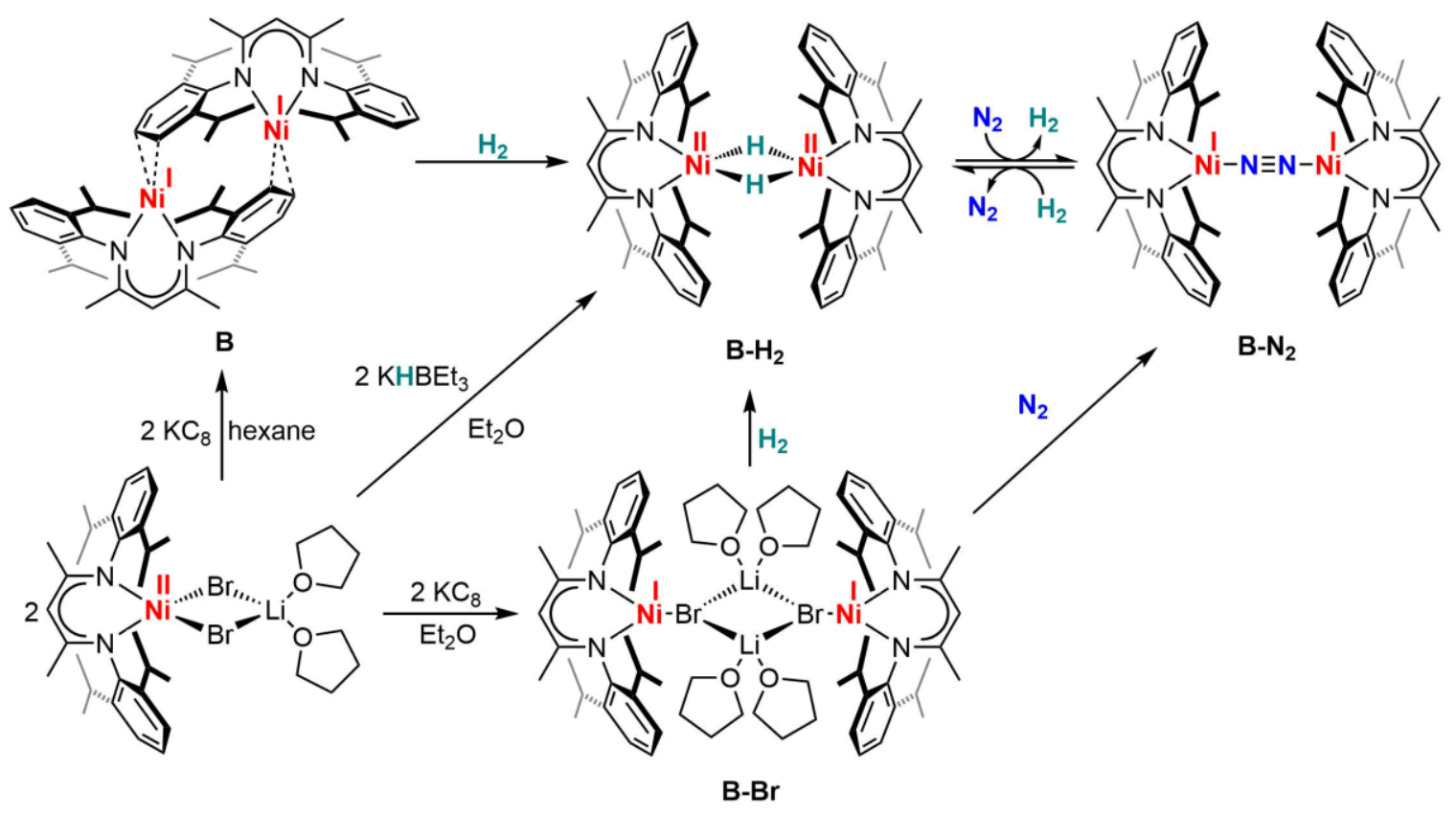

Scheme 1.7 Oxidative addition of $\mathrm{H}_{2}$ on the low-valent $\mathrm{Ni}(\mathrm{I})$ complexes $\mathbf{B}$ and $\mathbf{B}-\mathbf{B r}$ to the bridged dihydride species $\mathbf{B}-\mathbf{H}_{2}$; further ligand exchange of $\mathbf{B}-\mathbf{B r}$ and $\mathbf{B}-\mathbf{H}_{2}$ to form $\left[\mathrm{LNi}(I)\left(\mu-\mathrm{N}_{2}\right) \mathrm{Ni}(\mathrm{I}) \mathrm{L}\right] \mathbf{B}-\mathbf{N}_{\mathbf{2}}$ under $\mathrm{N}_{2}$ atmosphere. ${ }^{24,33}$

In addition to oxidative addition of dihydrogen for nickel hydride complexes, the usage of substrates with activated $\mathrm{C}-\mathrm{H}$ bonds proved to be a reliable source for the reactive species. 
While for the addition of dihydrogen to both $\mathrm{Ni}(0)$ and $\mathrm{Ni}(\mathrm{I})$ complexes are well, the majority in $\mathrm{C}-\mathrm{H}$ functionalization is found to be $\mathrm{Ni}(0)$ complexes. ${ }^{34-41} \mathrm{Nickel}(\mathrm{I})$ complexes undergoing $\mathrm{C}-\mathrm{H}$ activation have been postulated but rarely evidenced. ${ }^{42-46}$

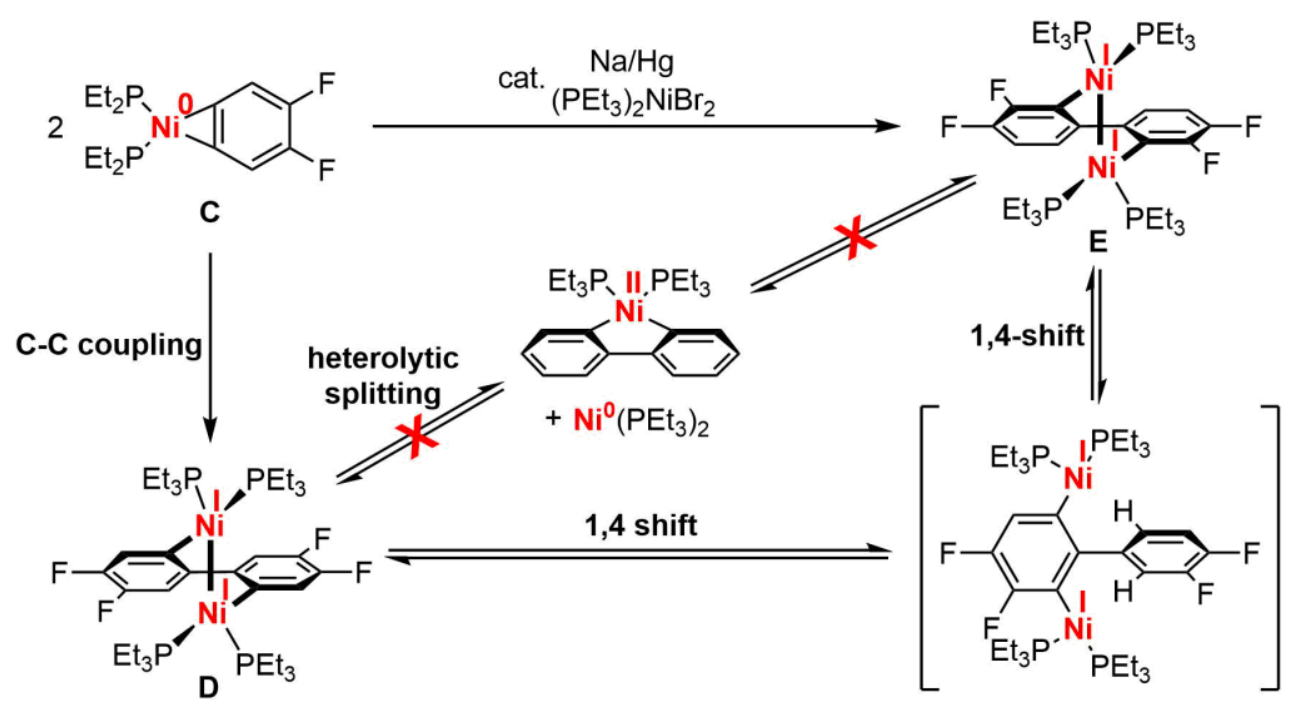

Scheme $1.8 \mathrm{Ni}(0)$-catalyzed isomerization of an aryne complex $\mathrm{C}$ to a $\mathrm{Ni}(\mathrm{I}) \mathrm{Ni}(\mathrm{l})$ biarylyl complex by Johnson et al. with hypothesized mechanism. ${ }^{42,43}$

The nickel(0) difluoroaryne complex $\mathbf{C}$ by Johnson et al. reacts upon the addition of a catalytic amount of $\left(\mathrm{PEt}_{3}\right)_{2} \mathrm{NiBr}_{2}$ and sodium amalgam to form the biarylyl $\mathrm{Ni}(\mathrm{I}) \mathrm{Ni}(\mathrm{I})$ complex E (Scheme 1.8). ${ }^{42}$ Based on NMR studies and isolation of certain intermediates, it was theorized a mechanism going through Lewis-acid catalyzed C-C coupling to afford $\mathbf{D}$ and isomerization through two 1,4-shifts to form $\mathbf{E} .{ }^{43}$ Based on reactivity studies of different intermediates the isomerization does not appear to go through the heterolytic cleavage of the Ni-Ni bond to the mononuclear biarylyl Ni(II) complex and $\mathrm{Ni}(0)\left(\mathrm{PEt}_{3}\right)_{2}$. Rather a C-H activation of $\mathbf{D}$ to the shown intermediate (in brackets) occurs, which immediately undergoes 1,4-shift again. This unique behavior of a $\mathrm{Ni}(\mathrm{I})$ center undergoing $\mathrm{C}-\mathrm{H}$ activation was not observed until then and therefore Johnson et al. gave the first evidence for such a reactivity.

Investigations by the group of Chirik on the $\alpha$-diimine dinickel(II) bridged-dihydride complex $\mathbf{F}$ resulted in multiple catalytic applications, ${ }^{45,47,48}$ for example C-H functionalization in form of hydrogen isotope exchange (HIE). ${ }^{49}$ For HIE (Scheme 1.9) reactivity studies found that the monomeric $\mathrm{Ni}(\mathrm{I})$ complex $\mathbf{F}$ ' appeared to be the active catalyst. To increase the concentration of the monomeric nickel(I) hydride species and therefore catalytic activity in HIE, substitution of the dipp moieties to more sterically demanding substituents was pursued. The utilization of isopinocampheyl as substituents in ${ }^{i p c} \mathrm{~L}$ (Scheme 1.9) resulted in monomeric structures for the nickel(I) halogen complexes. Although the resulting hydride complex $\mathbf{G}$ appeared to be a nickel(II) dimer in the solid state and in benzene solution, solution-based measurements in THF showed a significant higher presence of the nickel(I) monomer $\mathbf{G}^{\prime}$ than of the previously reported $\mathbf{F}$. Additionally, $\mathbf{G}$ proved to be a superior catalyst for hydrogen isotope exchange compared 
to $\mathbf{F}$, due to the higher concentration of the monomer. While a detection of monomeric nickel(I) center for $\mathbf{F}$ was only possible upon addition of pyridine, due to the more thermodynamically favored dimer, detection of $\mathbf{G}$ ' gave additional evidence for a nickel(I) based catalysis. $^{46}$

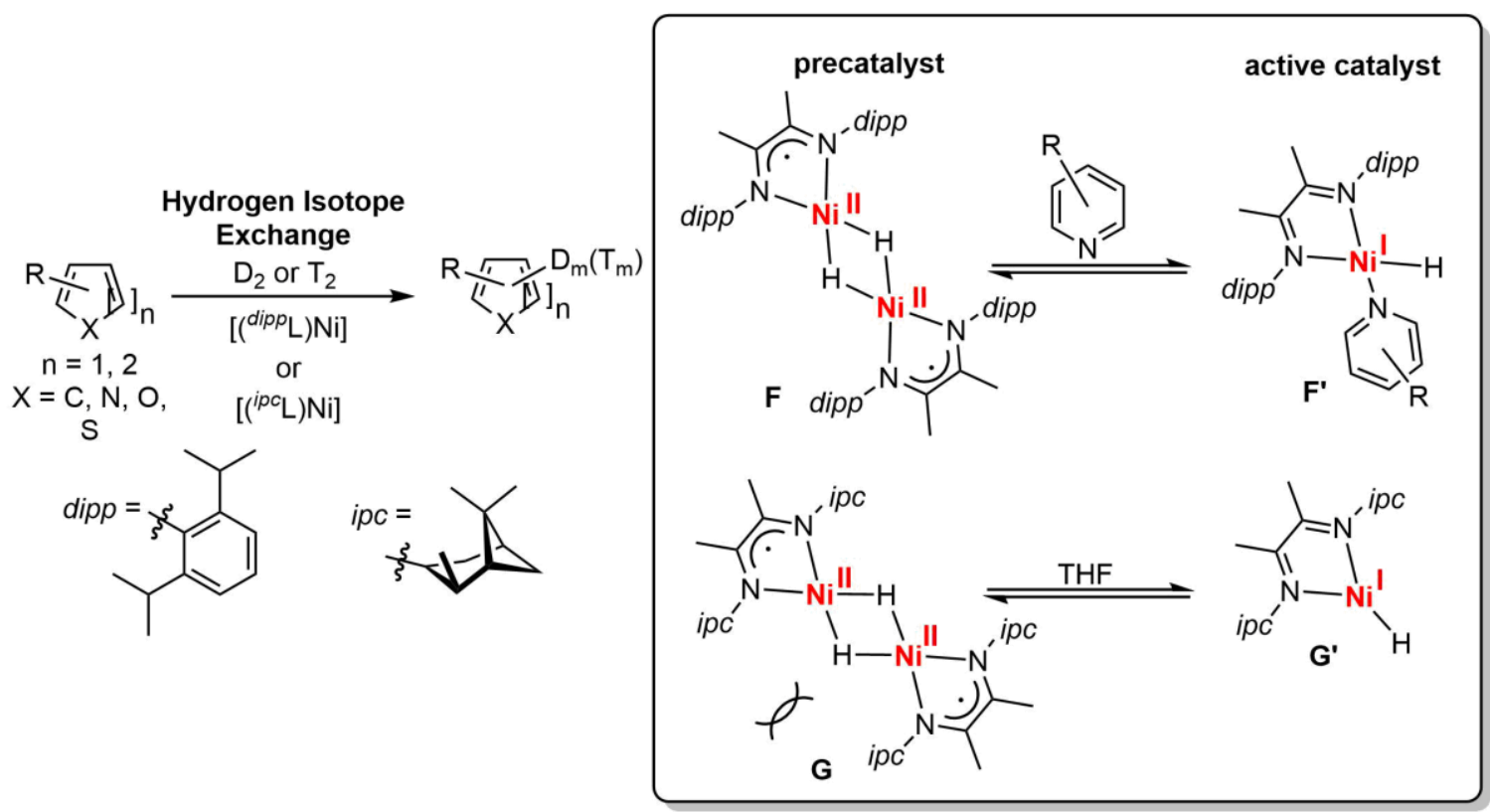

Scheme 1.9 Nickel(I) based catalysts bearing a-diimine ligands by Chirik et al. for HIE. ${ }^{45,46}$

For both presented systems the possibility of C-H activation utilizing nickel(I) was evidenced but an isolation of the direct oxidative addition product has not been observed, due to high reactivity of the nickel(III) intermediate. Therefore, a bimetallic system supporting $\mathrm{Ni}(\mathrm{I}) \mathrm{Ni}(\mathrm{I})$ could be essential for the isolation of an intermediate. Additionally, a preorganized ligand scaffold helps facilitating a close proximity of the two metal centers, splitting the two-electron onto both nickel ions.

\subsubsection{Development of Bimetallic Nickel $\beta$-Diketiminato Complexes Through a Preorganized Ligand Scaffold}

As mentioned in the previous section, the strong-field NacNac ligand is capable of supporting a nickel(I) oxidation state, resulting in interesting properties towards the activation of small molecules and substrates with $\mathrm{C}\left(\mathrm{sp}^{2}\right)-\mathrm{H}$ bonds. Most of the presented reactivities result in bridging of mononuclear nickel complexes and two-electron reduction of the substrate. The stabilization by two metal ions is therefore needed to support the reactive species and divide the needed redox equivalents onto both metal centers. Beginning in 2005, research has been presented on the development of ligands which 
support bimetallic complexes through two $N$-aryl $\beta$-diketiminato coordination pockets bridged by a linker.

The incorporation of ethylene and cyclohexylene bridges for two NacNac pockets as spacers by Hulztsch et al. began the development of this class of supporting ligands. The ligands were used for coordination of lanthanide complexes and structural dependence on the choice of the linker was studied. ${ }^{50}$ In 2008 , the group of Harder started implementing $p$-phenylene, $m$-phenylene and $m$-pyridylene linkers. In particular, the m-pyridylene linked $\mathrm{NacNac}$ ligand can be viewed as the first example of a linker bearing another coordination site. Reactivity of complexes of calcium and zinc towards epoxide and $\mathrm{CO}_{2}$ polymerization resulted in higher activity of the bimetallic systems, indicating a positive effect of the preorganized ligand scaffold..$^{51}$

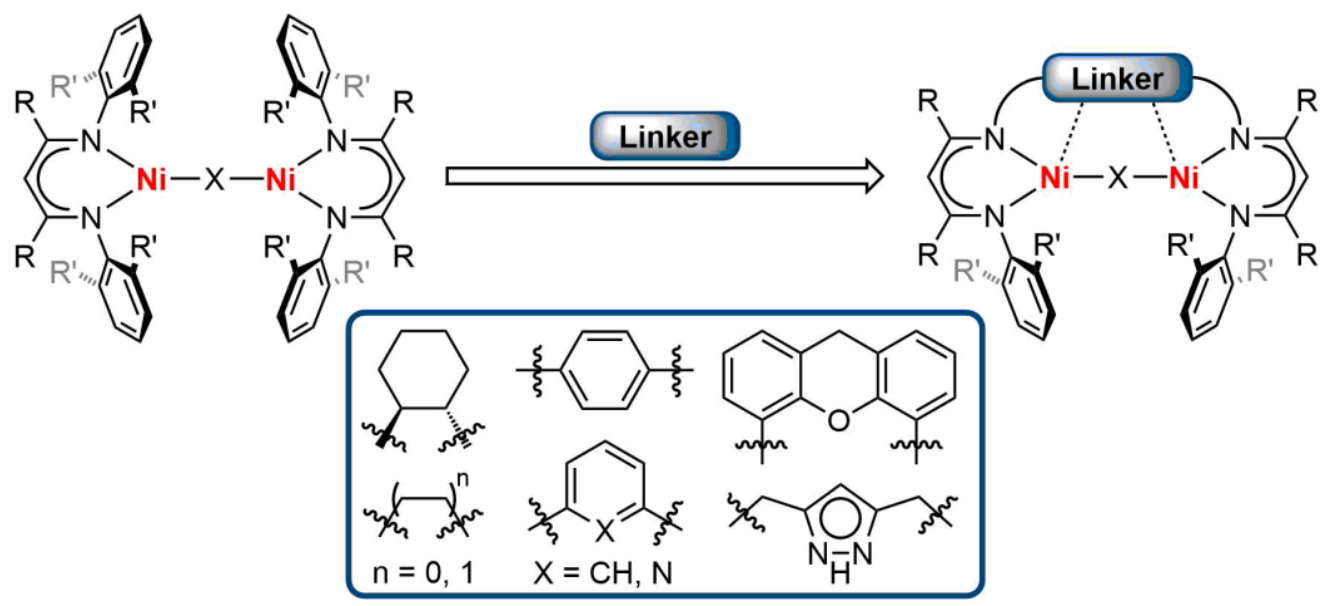

Scheme 1.10 Preorganization of two NacNac ligands through the introduction of a linker supporting bimetallic coordination, examples of the linker are presented in the square. ${ }^{32,50-53}$

Previously, the group of Hulztsch and Limberg used a xanthene-bridged ligand system for the similar reactivity towards the same kind of polymerization. Based on the observed lower reactivity, steric hinderance of the ligand system was reasoned. ${ }^{52}$ A second publication by Harder et al. showed the possibility of the smallest possible linker or rather the absence of it through direct N-N connection. Although this example did not show promising results in the polymerization experiments, demonstration of the direct dimerization of two NacNac pockets has to be mentioned (all examples are presented in Scheme 1.10)..$^{53}$

Although these examples show the development of such ligands with coordinating or noncoordinating linkers, investigation on nickel complexes was not a focus until 2013. The first bimetallic system utilizing nickel was presented by the group of Limberg, employing the $m$-pyridylen-linked scaffold developed by Harder et al. ${ }^{51}$ for a bivalent $\mathrm{Ni}-\mathrm{H}$ and $\mathrm{Ni}(\mathrm{I})$ system. The idea behind such a bimetallic system was the mimicking of intermediates in the $[\mathrm{NiFe}]$ hydrogenase mechanism. Due to the monodentate nature of the pyridylen-linker, I-Br with two different bromido coordination modes was observed (Scheme 1.11). Subsequent reaction with KHBEt3 resulted in a bivalent $\mathrm{Ni}-\mathrm{H}$ and $\mathrm{Ni}(\mathrm{I})$ complex $\mathbf{C}$ in a 
square planar and trigonal planar environment, respectively. A hypothesized mechanism implied an $\mathrm{Ni}(\mathrm{II})-(\mu-\mathrm{H})-\mathrm{Ni}(\mathrm{II})-\mathrm{H}$ intermediate, which undergoes elimination of an $\mathrm{H}$-atom to give dihydrogen and $\mathbf{C}$. The bivalent system $\mathbf{J}$ was further characterized by $\mathrm{X}$-ray absorption spectroscopy to evaluate the similarity to the metalloenzyme. ${ }^{54}$ In addition, to further resemble the [NiFe] hydrogenase reactivity a thiolate ligand was incorporated (ISEt in Scheme 1.11). Upon the same reaction as for I-Br with $\mathrm{KHBEt}_{3}$, a SEt-bridged $\mathrm{Ni}(\mathrm{I}) \mathrm{Ni}(\mathrm{I})$ product J-SEt was isolated (Scheme 1.11, bottom). The intermediate $\mathrm{Ni}(\mathrm{II})-(\mu-\mathrm{SEt})-\mathrm{Ni}(\mathrm{II})-\mathrm{H}$ of the reaction undergoes heterolytic cleavage, protonation of one nitrogen of the NacNac ligand and electron transfer to the Ni(II) ion. The reversibility of the process was induced by protons in form of lutidinium triflate and results in the triflate complex I-OTf with substitution of the bridged bromido ion with a triflate anion. The reactivity of the bimetallic nickel system $\mathbf{I - B r}$ already shows the interesting pathways through metal-metal cooperativity in combination with the nature of the pyridylen-linker, due to the fact that the mononuclear derivatives undergo other transformations.
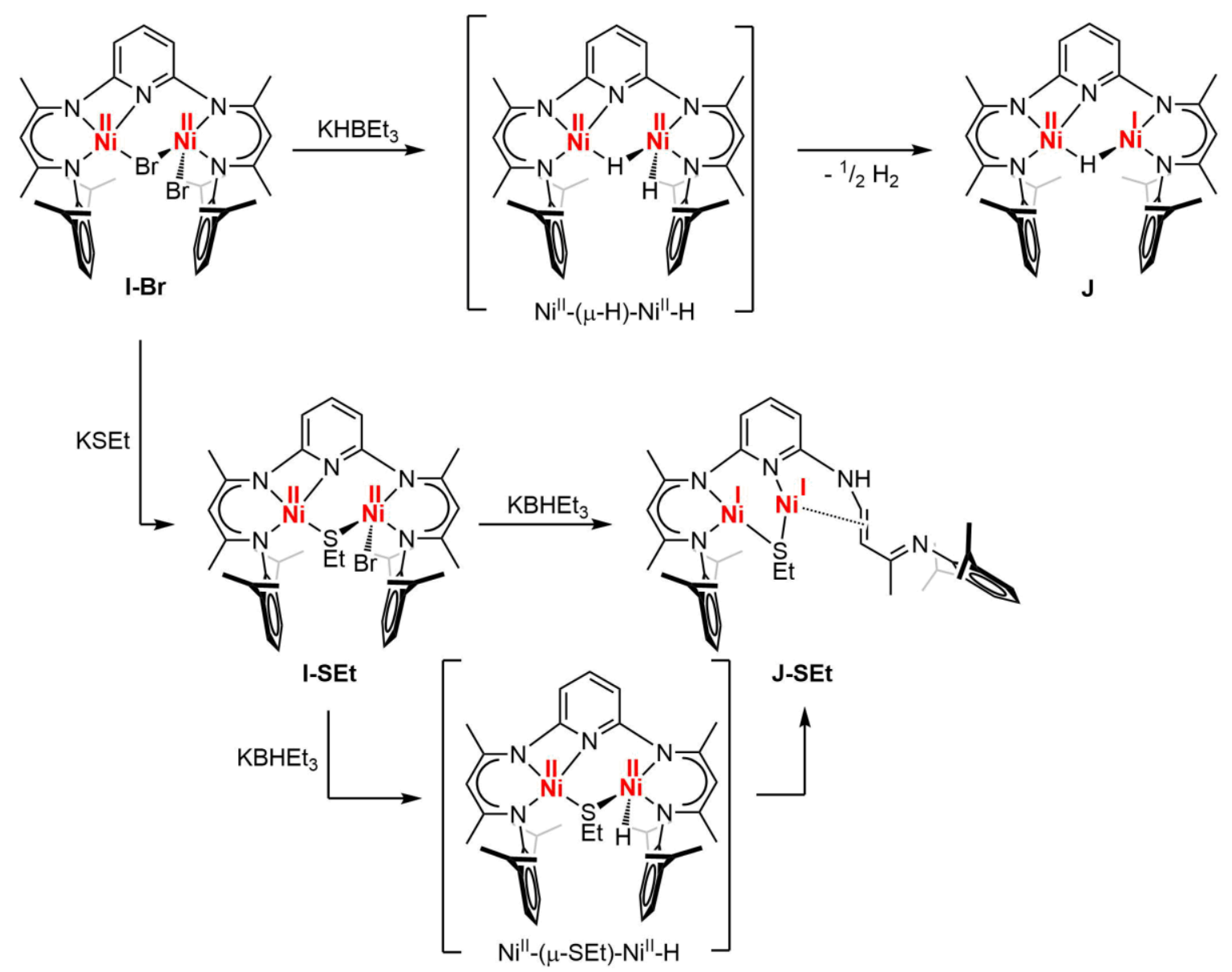

Scheme 1.11 Bimetallic nickel system by Limberg et al. for bio-inspired modelling of intermediates in the [NiFe] hydrogenase mechanism; monodentate nature of the pyridylen-linker results in asymmetric behavior of the two nickel ions. ${ }^{55}$

The interesting properties of the Harder and Limberg system drove Manz and Meyer to the development of newer bimetallic nickel systems. ${ }^{56}$ Due to the advanced expertise in pyrazole bridged systems in the Meyer group, the NacNac variant $\mathbf{L}^{i \operatorname{Pr}}$ was synthesized and 
investigated (Scheme 1.12). The ability of the pyrazole-bridge to offer two coordination sites for two metals changes the reactivity relative to the earlier discussed system I-Br. Therefore, reaction of the $\mathrm{C}_{2 \mathrm{v}}$ symmetric precursor $1^{i \mathrm{Pr}}$ with KHBEt 3 resulted in the dihydride complex $3^{i \operatorname{Pr}}$ (Scheme 1.12), instead of a Ni-H/Ni(I) system as observed with C. ${ }^{32}$ Although the compound $3^{i \mathbf{P r}}$ appears as a dihydride system, reaction with $\mathrm{D}_{2}$ resulted in the pairwise exchange to $\mathbf{3}^{i \mathbf{P r}}-\mathbf{D}_{2}$. This reactivity indicates a $\mathrm{Ni}(\mathrm{I}) \mathrm{Ni}(\mathrm{I})$ system as an intermediate similar to complex $\mathbf{B}-\mathbf{H}_{2}$ without a preorganized ligand scaffold (Section 1.1.2, Scheme 1.7). In particular, the reactivity towards $\mathrm{H}_{2}$ elimination was found to be dependent on the alkali metal and abstraction of the cation resulted in immediate decay of the compound to a $\mathrm{Ni}(\mathrm{I}) \mathrm{Ni}(\mathrm{I})$ species shown by magnetic susceptibility measurements (Scheme 1.12, bottom left). Further, addition of phenylacetylene resulted in release of $\mathrm{H}_{2}$ and two-electron reduction of the alkyne in $\mathbf{3}^{\mathbf{i P r}}$-Sty (Scheme 1.12, bottom right). Similar to the toluene-bridged system by Stephan et al. ${ }^{11}$, a masked bimetallic Ni(I) species is postulated, utilizing the stability of a dihydride system and reactivity of a $\mathrm{Ni}(\mathrm{I}) \mathrm{Ni}(\mathrm{I})$ complex. More recent experiments also show that the $\mathrm{Ni}(\mathrm{I}) \mathrm{Ni}(\mathrm{I})$ complex is stable and can be selectively synthesized through two-electron reduction of the precursor $\mathbf{1}^{i \mathbf{P r}}$ with strong reducing agents. ${ }^{57}$ This reactivity of two-fold reduction of substrates upon elimination of $\mathrm{H}_{2}$ is similar to the FeMo cofactor

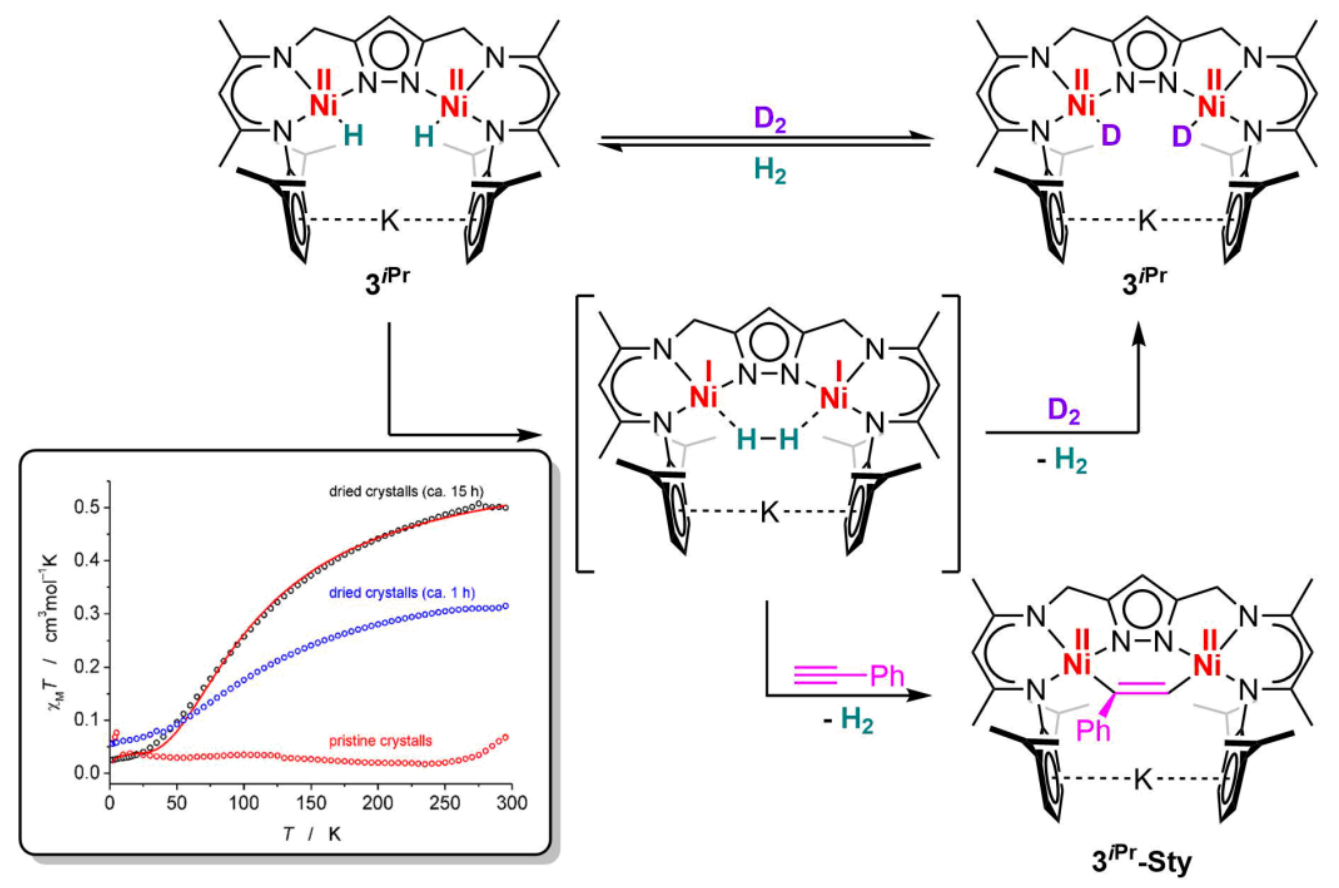

Scheme 1.12 Bimetallic nickel dihydride system $3^{\text {iPr }-K}$ by the group of Meyer; reaction with $\mathrm{D}_{2}$ results in pairwise exchange through a $\mathrm{Ni}(\mathrm{I}) \mathrm{Ni}(\mathrm{I})$ intermediate, shown by trapping experiments and magnetic susceptibility measurements. The rights for reprint have been granted by the American Chemical Society (Copyright @ 2017).

The "masquerade" of a two-electron reservoir allowed the focus on further substrate activation, in particular small molecules. Some examples will be presented to show the versatility of the system and impact of the metal-metal cooperativity. Similar to the mononuclear system by Driess et al. (Scheme 1.5), reaction with an excess of oxygen 
resulted in a superoxido species $\mathbf{3}^{\text {iPr}}$-SO. Instead of a side-on binding mode, an end-on bridged superoxido ligand is observed (Scheme 1.13, top). Addition of a stoichiometric amount of dioxygen resulted in the expected peroxido species $3^{i \mathbf{P r}}$-PO, which was also accessible through reduction of the superoxido system $3^{i \text { Pr }}-\mathbf{S O} .^{58}$

For the reaction of $\mathbf{3}^{i \mathrm{Pr}}$ towards $\mathrm{NO}$ a different kind of reactivity is observed. Through reduction, two molecules of NO are coupled to hyponitrite, which is found in between the two nickel ions in $3^{i \mathbf{P r}}-\mathbf{H N}$. Protonation of the hyponitrite results in elimination of nitrous oxide and isolation of the hydroxido-bridged complex $\mathbf{1}^{\mathbf{i P r}} \mathbf{- O H}$ (Scheme 1.13, middle). ${ }^{59}$ Such a two-electron reduction was also observed for the substrate nitrosobenzene. While for the mononuclear system single-reduction is observed ${ }^{60}$, the reaction of $\mathbf{3}^{\mathbf{i P r}}$ with nitrosobenzene ends up in a twice-reduced substrate. The species $3^{i \mathbf{P r}}-\mathbf{N B}$ can be oneelectron oxidized to $3^{i \mathbf{P r}}-\mathbf{N B}^{+}$or protonated to $3^{i \mathbf{P r}}-\mathbf{N B H}$, and the nitrosobenzene derived ligand is still stabilized between the two nickel ions (Scheme 1.13, bottom). Redox transformation between $3^{i \mathbf{P r}}-\mathbf{N B H}$ and $\mathbf{3}^{i \mathbf{P r}}-\mathbf{N B}^{+}$through hydrogen atom abstraction allowed investigation on the BDFE of the N-H bond. ${ }^{61}$ Those twice reduced species have also been extended to sulfur, resulting in sulfido bridged complexes. ${ }^{57}$ All in all, the twoelectron reservoir of the bimetallic system $3^{i \mathrm{Pr}}$ is rather unique and the similarity in reactivity to the FeMo cofactor (Scheme 1.6) is rather interesting for other substrate activations.
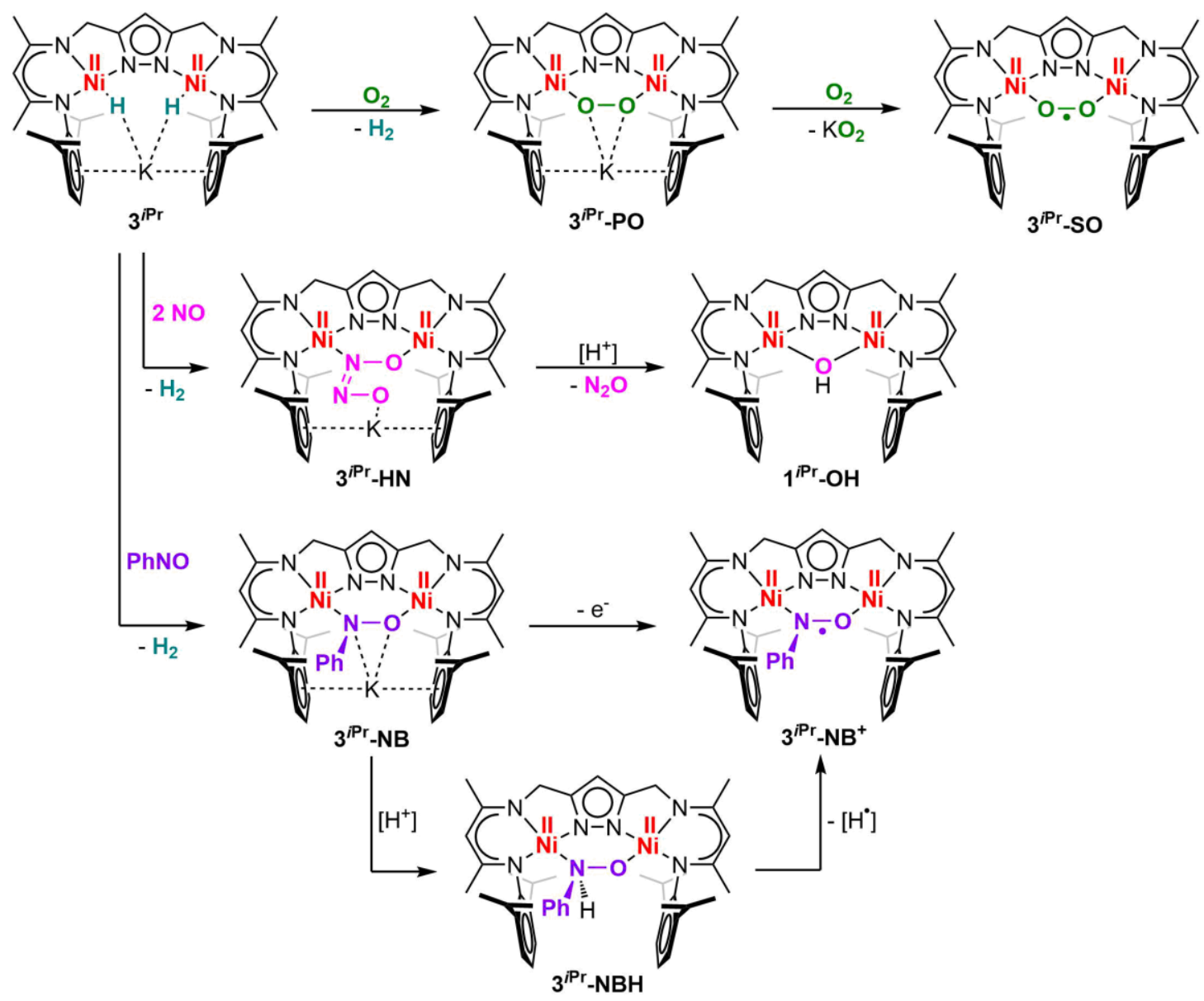
Scheme 1.13 Two-electron reductions of $3^{\text {iPr }}$ with oxygen, ${ }^{58}$ nitrous oxide ${ }^{59}$ and nitrosobenzene, ${ }^{61}$ showing the versatility of the dihydride system $3^{\text {iPr }}$.

Although two-electron reductions are the most prominent found for this system, in a unique case a single-electron reduction is observed (Scheme 1.14). In particular, the $\mathrm{N}_{2}$ activation of $3^{i \mathbf{P r}}$ is not observed under the shown conditions of two-electron reduction, but in presence of protons formation of 1.5 equiv. $\mathrm{H}_{2}$ and the $\mathrm{Ni}$ (II)Ni(II) species $3^{i \mathrm{Pr}_{-}} \mathbf{N}_{2}$ with a singly reduced $\mathrm{N}_{2}{ }^{-}$substrate is observed. This kind of activation has only been observed for dimeric $\mathrm{Ni}(\mathrm{I})$ species through reduction of the $\mathrm{Ni}(\mathrm{I})-\mathrm{N}_{2}-\mathrm{Ni}(\mathrm{I})$ complex. ${ }^{3}$ Further redox transformations allowed the isolation of multiple steps of the alternating nitrogen fixation pathway. ${ }^{56} 3^{i \mathrm{Pr}}-\mathbf{N}_{2}$ can also be used as a starting synthon for other one electron reductions. In particular, the reaction of $\mathbf{3}^{i \mathbf{P r}}-\mathbf{N}_{2}$ with $\mathrm{CO}$ resulted in the first example of a singlyreduced $\mathrm{CO}$ species $\mathbf{3}^{\mathbf{i P r}}-\mathbf{C O} .^{57}$ Again, a two-electron reduction is not observed for $\mathrm{CO}$ based on $3^{\mathrm{iPr}}$, which indicates the importance of the $3^{i \mathbf{P r}}-\mathbf{N}_{2}$ as a starting material.
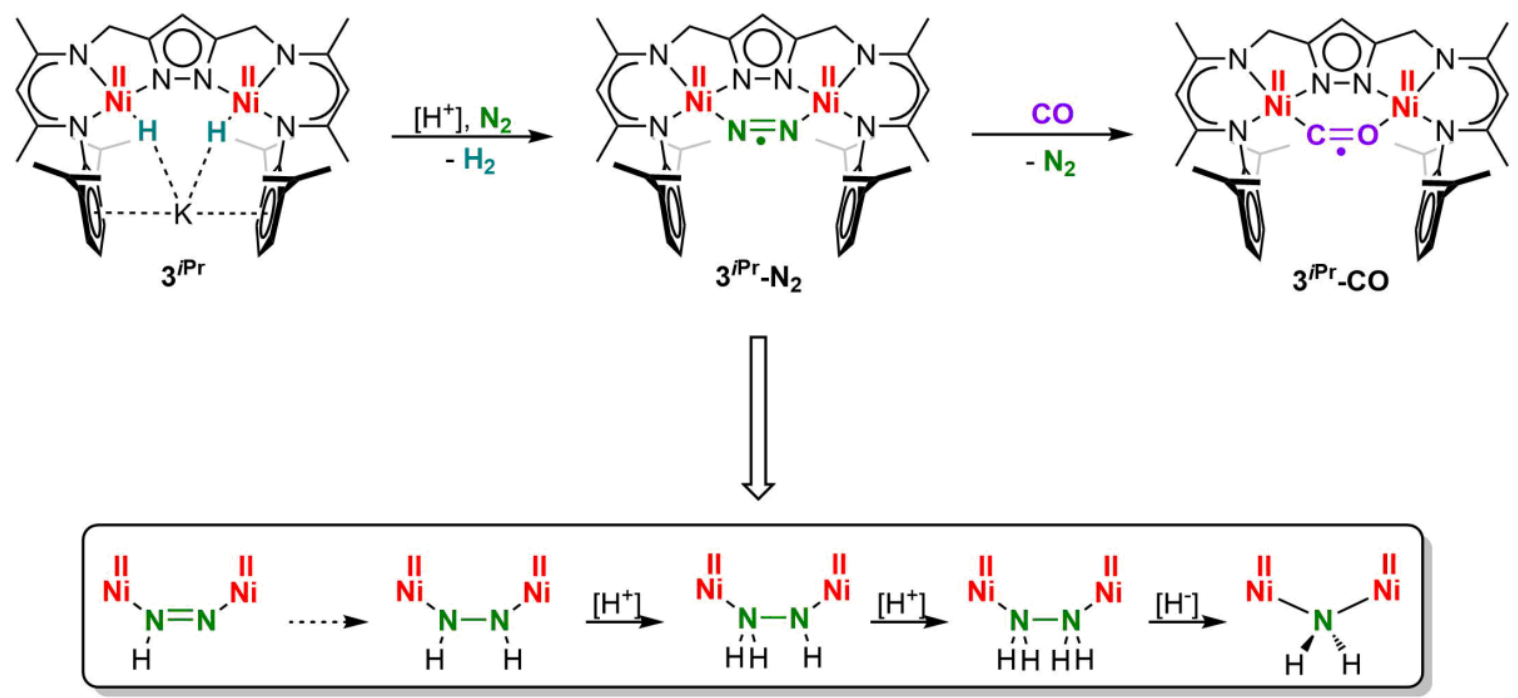

Scheme 1.14 Single-electron reduction of dinitrogen and carbon monoxide; rectangle: nitrogen activation allows isolation of multiple species of the "alternating" nitrogen fixation pathway. ${ }^{56,57,62}$

The presented results on bimetallic systems with a preorganized ligand scaffold bearing two NacNac donors show the remarkable properties such system can provide. From one to two electrons in small molecule activation, the spectrum of substrates is therefore limitless and even advances the mononuclear systems. Additionally, the masking of the reactive species through toluene (Scheme 1.4) or dihydrogen (Scheme 1.12), in a Ni(II)Ni(II) synthon allows trivial isolation and handling of the system. 


\subsection{Project Outline}

The reactivity of $\mathrm{Ni}(\mathrm{I})$ systems (Section 1.1.1) is based on the low reduction potential and therefore provides a one-electron reservoir for the reduction of small molecules. ${ }^{8,9}$ Bimetallic $\mathrm{Ni}(\mathrm{I}) \mathrm{Ni}(\mathrm{I})$ systems (Section 1.1.3) show a similar behavior but mediate dominantly two-electron transfers, which is not observed often for 3d-metals. Additionally, the metal-metal cooperativity allows unique reactivity, because of the stabilization through two metal ions. As presented by Manz and Duan et al., these systems can also be masked as dihydride systems. Although $\mathbf{3}^{\mathbf{i P r}}$ (Scheme 1.15, left) already demonstrates a range of small molecule activation, ${ }^{32,58}$ Duan investigated the modification of the aryl moieties, changing from dipp to $m$-terphenyl substituents (Scheme 1.15, right). ${ }^{57}$ The $m$-terphenyl moieties provide steric modification compared to the dipp substituents, but an expansion of the $\pi$-system might result in different electronic properties of the NacNac subunit.

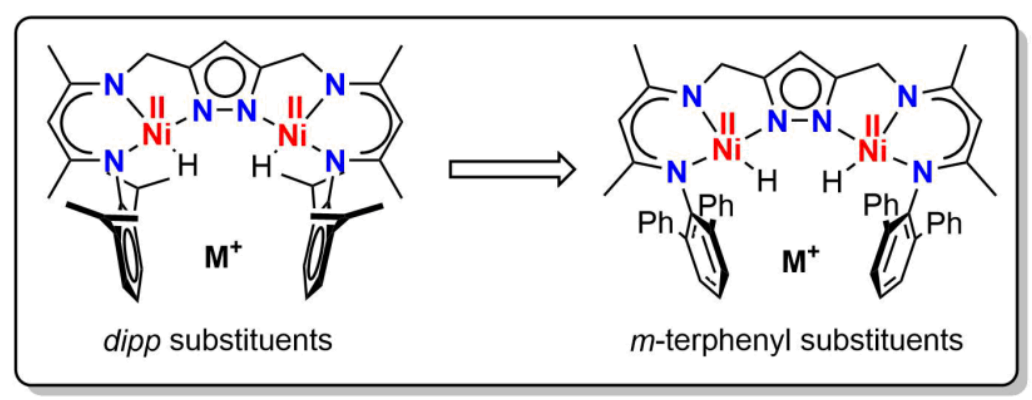

Scheme 1.15 System $3^{\text {iPr }}$ modified through $m$-terphenyl substitution of the dipp moieties. ${ }^{32,57}$

Preliminary results have shown that an incorporation of $m$-terphenyl substituents resulted in an $\mathrm{C}\left(\mathrm{sp}^{2}\right)-\mathrm{H}$ activation of the ligand, instead of the dihydride species 3-K (Scheme 1.16). While utilization of $\mathrm{KHBEt}_{3}$ resulted in the isolation of the $\mathrm{C}-\mathrm{H}$ activated species 2-K, treating the nickel precursor $\mathbf{1}$ with the sodium analogue gave a mixture of 2-Na and 3-Na. Additionally, the $\mathrm{C}-\mathrm{H}$ activation is reversible by addition of dihydrogen, resulting in an interconversion to the pure dihydride species 3-M. ${ }^{57}$

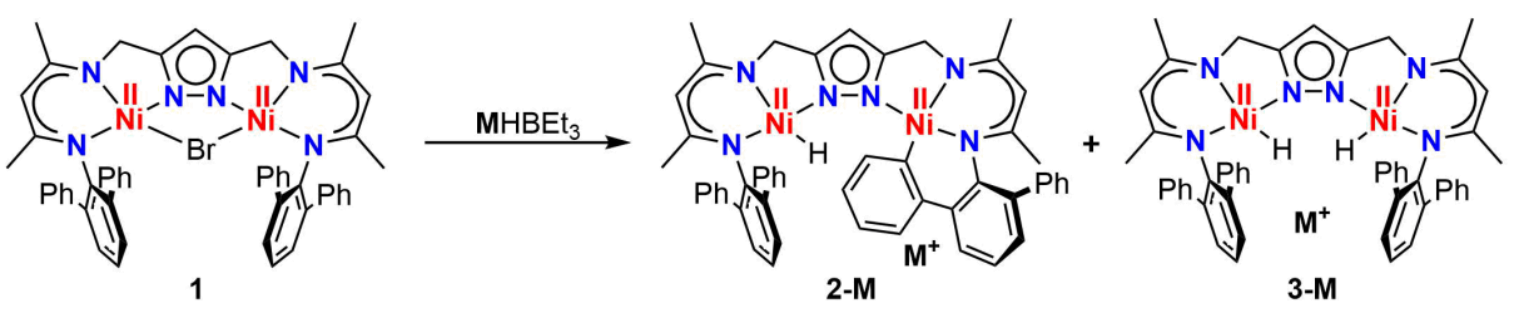

Scheme 1.16 Mixture of $\mathrm{C}-\mathrm{H}$ activated product 2-M and dihydride species 3-M dependent on the alkali metal $\mathbf{M}^{+}=\mathrm{K}$ or $\mathrm{Na}^{57}$

Due to a lack of mechanistic investigations, our understanding of $\mathrm{H}_{2}$-activation by the $\mathrm{C}-\mathrm{H}$ activated and dihydride species is poor. Further to this, understanding of the selective 
conversion to the $\mathrm{C}-\mathrm{H}$ activated species 2-Na is also lacking. These areas will be the starting point for this chapter. Firstly, an optimization of the ligand synthesis, shortly described in Scheme 1.17, will be investigated. While the established synthesis of Manz and Duan works reliably, the overall yield is rather low. ${ }^{58}$

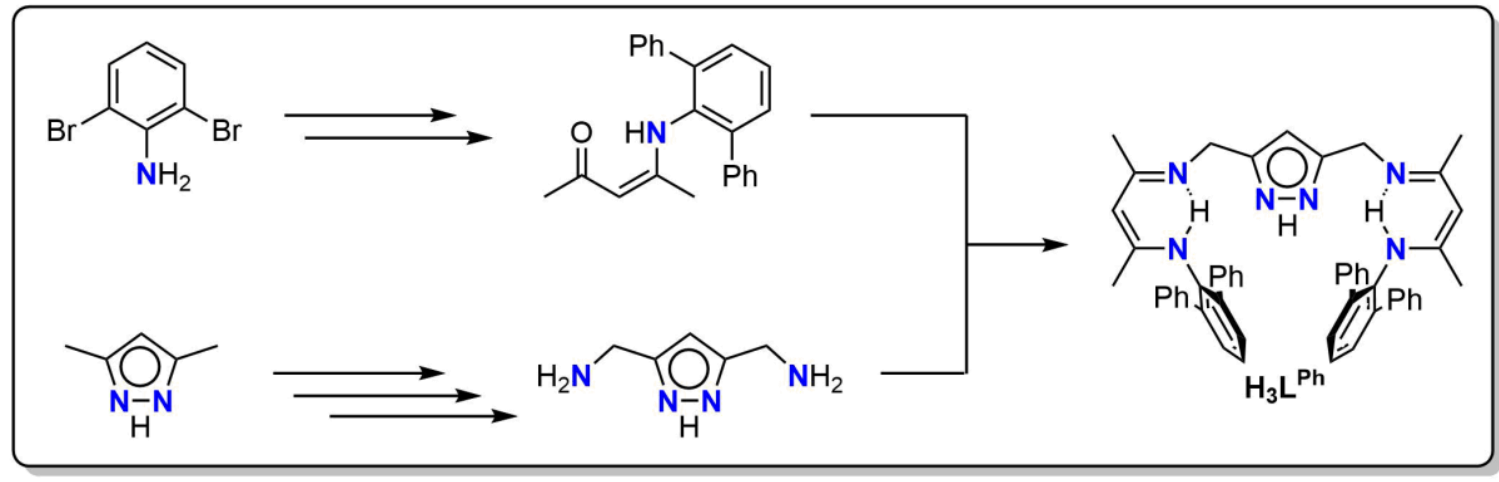

Scheme 1.17 Schematic overview of the synthetic route for pyrazole bridged NacNac ligand with m-terphenyl moieties $\mathrm{H}_{3} \mathrm{~L}^{\mathrm{Ph}}$.

The main aspect of the upcoming work will focus on the investigation of the reversible activation of the $\mathrm{C}-\mathrm{H}$ moiety. Particularly, the mechanism of how dihydrogen is incorporated is of interest. Hitherto, a Ni-C bond insertion (Scheme 1.18, right) was assumed by P.-Ch. Duan, however for the other ligand system $3^{i \mathrm{Pr}}$ a reductive elimination through a $\mathrm{Ni}(\mathrm{I}) \mathrm{Ni}(\mathrm{I})$ intermediate was observed (Scheme 1.18, left).

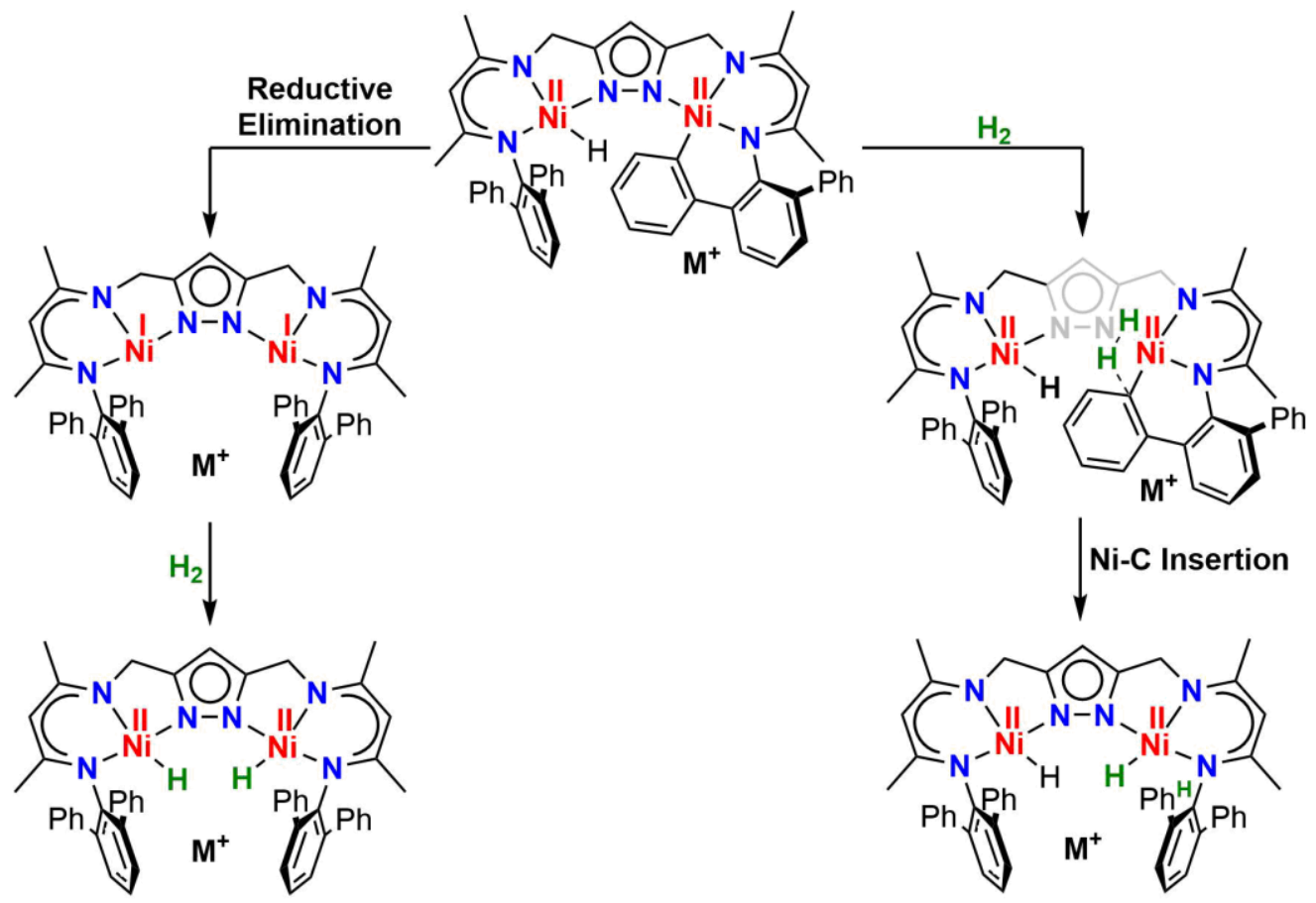

Scheme 1.18 Possible pathways for $\mathrm{H}_{2}$ activation based on earlier findings of reductive elimination for $\mathbf{L}^{\text {iPr }}$ (left) and heterolytic splitting for $\mathbf{L}^{\mathrm{Ph}}$ (right). ${ }^{32,57}$ 
In this context, the influence of the alkali metal is investigated because with sodium the dihydride species 3-Na was isolated along with the C-H activated species 2-Na. Due to the isolation of a mixture, a selective synthesis route will be developed towards isolation of the C-H activated species $\mathbf{2}-\mathbf{N a} .^{57}$

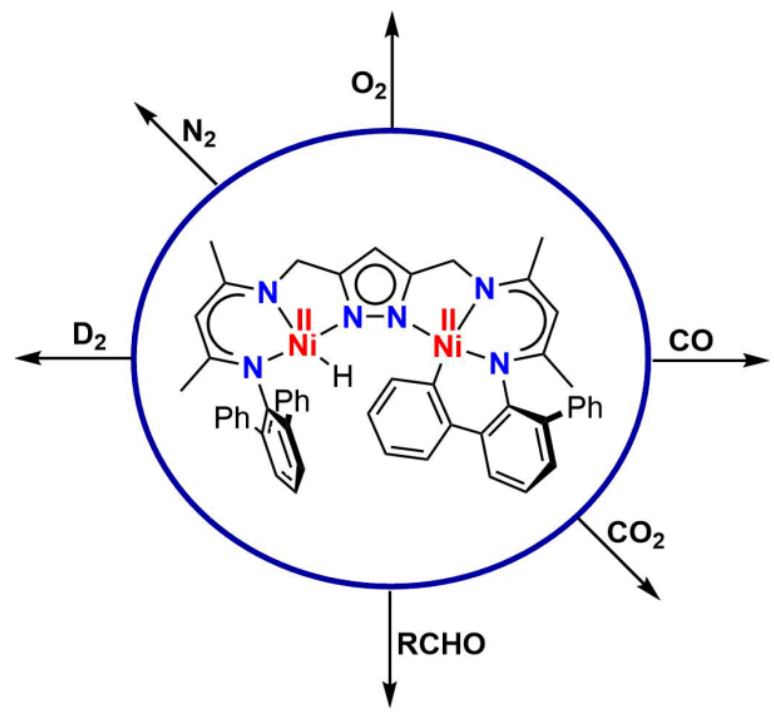

Scheme 1.19 Small molecule activation based on mono-tucked-in 2-M.

Based on the information gained through the investigation of the $\mathrm{C}-\mathrm{H}$ activation, the reactivity towards small molecules should be investigated (Scheme 1.19). The choice of the small molecules is based on the findings for the $\mathrm{L}^{i \mathrm{Pr}}$ system. Other small molecules (e.g. $\mathrm{N}_{2}, \mathrm{CO}, \mathrm{CO}_{2}$ ), for which no direct reactivity with $3^{i P r}$ was observed, will also be addressed. 


\subsection{Synthesis of Pyrazole-Bridged NacNac Ligand $\mathrm{H}_{3} \mathrm{~L}^{\mathrm{Ph}}$ Bearing $m$-Terphenyl Substituents}

While the synthetic strategy for the ligands $\mathbf{H}_{3} \mathbf{L}^{i \mathbf{P r}}$ and $\mathbf{H}_{3} \mathbf{L}^{\mathbf{P h}}$ is overall similar, the commercial availability of 2,6-diphenylaniline is the limiting factor. Therefore, one more step, a Suzuki-Miyaura-coupling, based on 2,6-dibromoaniline was added. ${ }^{63}$ The synthesis route is presented in Scheme 1.20. The overall yield for the longest linear sequence is 35 $\%$ based on 3,5-pyrazole dicarbamide (I), which was provided by A. Schwarz through literature procedures. ${ }^{32}$ Instead of the Suzuki-Miyaura-coupling presented in Duan's thesis with the atom inefficient $\mathrm{Pd}\left(\mathrm{PPh}_{3}\right)_{4},{ }^{57}$ the use of a different procedure by Lachmanová et al. ${ }^{63}$ utilizing $\mathrm{Pd}(\mathrm{OAc})_{2}$ as the catalyst was used. The final product $\mathbf{H}_{3} \mathbf{L}^{\mathbf{P h}}$ was characterized by multiple methods (Appendix).
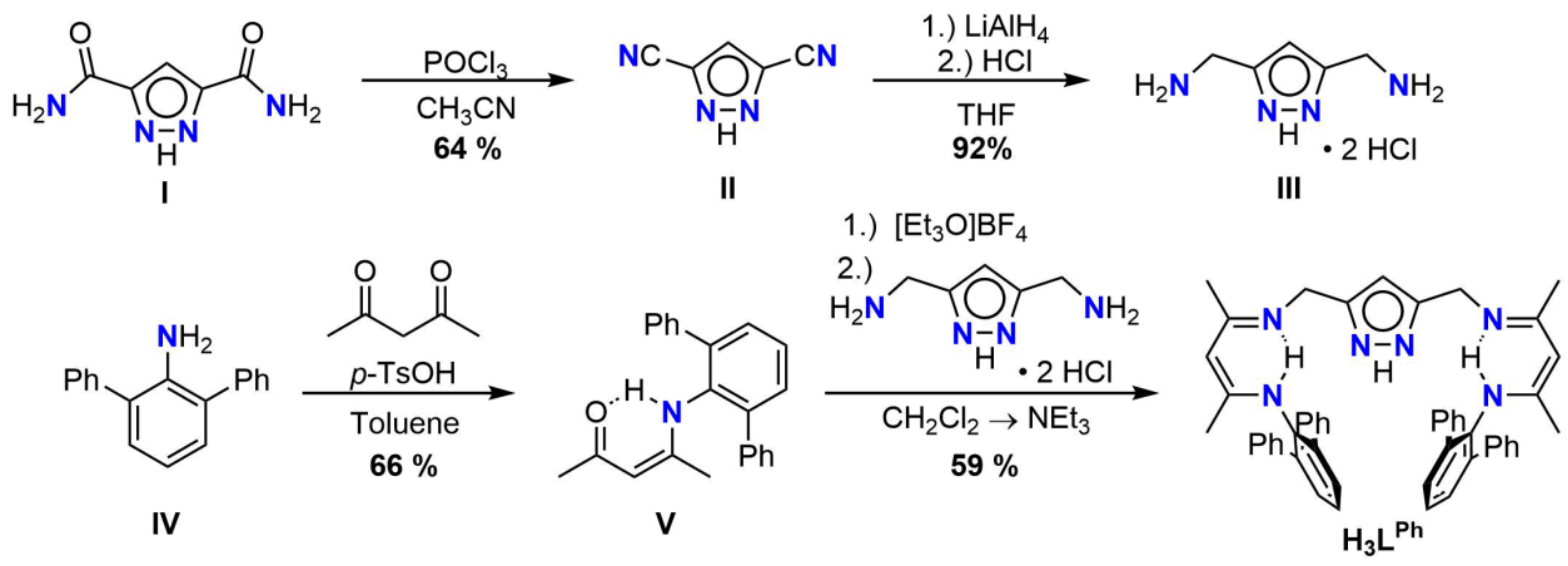

Scheme 1.20 Detailed synthesis route for $\mathrm{H}_{3} \mathrm{~L}^{\mathrm{Ph}}$ based on P.-Ch. Duan's work ${ }^{57}$; dehydration with $\mathrm{POCl}_{3}$ to nitrile II and reduction with $\mathrm{LiAlH}_{4}$ to III; Suzuki coupling product IV condensation reaction with acac to the one armed Nacac $\mathbf{V}$ and second condensation reaction with III utilizing ethyl derivative of the Meerwein salt to the final ligand $\mathrm{H}_{3} \mathbf{L}^{\mathrm{Ph}}$.

Further optimizations were also successful and resulted in a drastic increase of the overall yield. Firstly, the dehydration reaction of $\mathbf{I}$ was carried out with a stepwise addition of $\mathrm{POCl}_{3}$ at $0^{\circ} \mathrm{C}$ (Experimental Section for details) and resulted in a two-fold improvement of the yield. Secondly, the isolation of III was done by protonation with hydrochloric acid, giving III as the hydrochloride in higher purity and yield. The final step was optimized by using a simplified workup procedure, which involved a toluene extraction and ethanol wash instead of the unreliable crystallization. Some of these compounds serendipitously crystallized and were studied via single crystal X-ray diffraction studies.

The resulting molecular structures of $\mathbf{V}$ and $\mathbf{H}_{3} \mathbf{L}^{\mathbf{P h}}$ are shown in Figure 1.1. The solid structure of $\mathbf{V}$ shows that two molecules are connected through an intermolecular $\mathrm{N} \cdots \mathrm{O}$ hydrogen bridge ${ }^{64}$ with a distance of 2.9893(12) $\AA$, which also indicates the enol-form of the imine group (insert in Figure 1.1). This hydrogen bridge is not found any more in the structure of $\mathbf{H}_{3} \mathbf{L}^{\mathbf{P h}}$, but rather two molecules interact through a $\mathrm{N}_{\mathrm{pz} 1} \cdots \mathrm{N}_{\mathrm{pz} 2}$ hydrogen bridge with a length of 2.7919(42) A (insert in Figure 1.1). This type of pyrazole based hydrogen 
bridge has also been observed for other pyrazolyl substituted systems. ${ }^{65,66}$ Additionally, the enol form is found on the pyrazole substituted nitrogen side, due to corresponding smaller $\mathrm{C}-\mathrm{C}$ and longer C-N bond lengths of the NacNac subunit (see detailed bond lengths in the Appendix.)
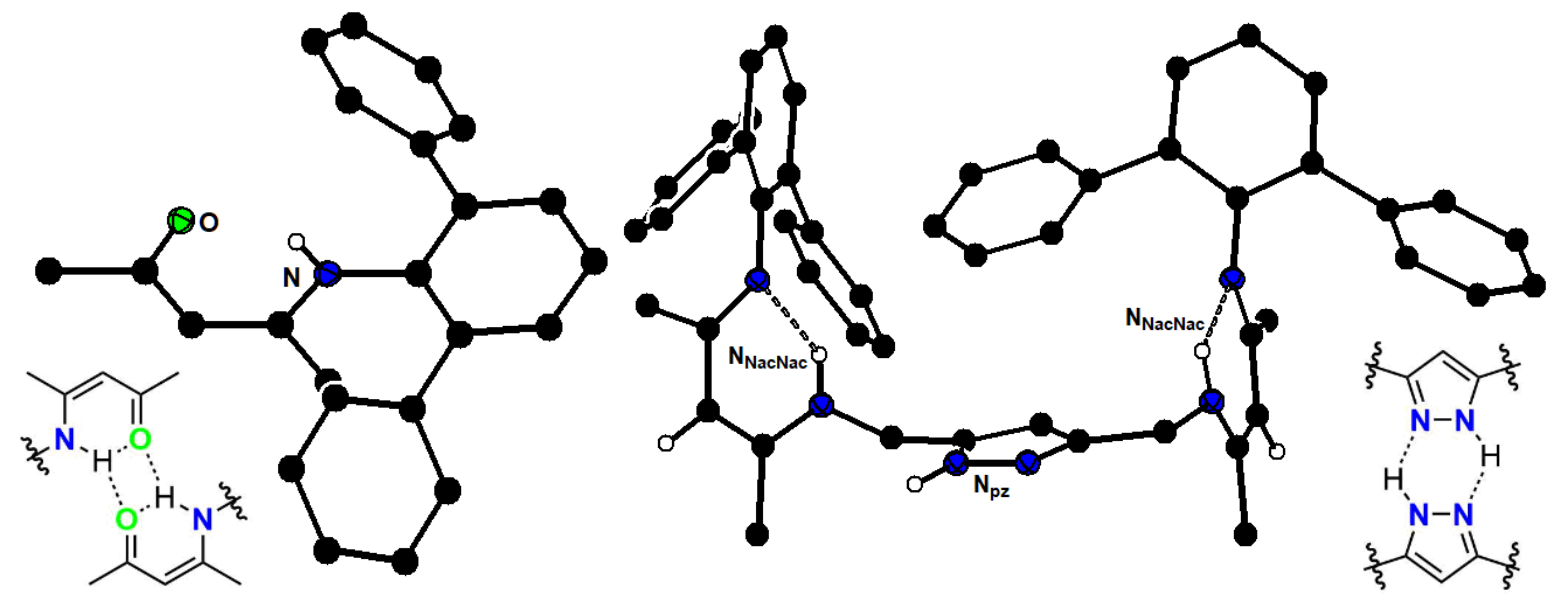

Figure 1.1 Molecular structure of sidearm $\mathbf{V}$ (left) and ligand $\mathrm{H}_{3} \mathrm{~L}^{\mathrm{Ph}}$ (right); most of the hydrogen atoms are omitted for clarity; schematic representations of the intermolecular interactions found in the solid state structure.

The successful optimization of the yield and purity of the ligand $\mathbf{H}_{3} \mathbf{L}^{\mathbf{P h}}$ gives access to larger quantities of this ligand, which is a precursor to compound $\mathbf{1}, \mathrm{LPhNi}_{2}(\mu-\mathrm{Br})$. 


\subsection{Mechanistic Study of the Reversible C-H Activation of the Bimetallic Nickel Complex 2-K}

Based on 1, the hydride complexes with different alkali metal cations will be synthesized. The characterization and the mechanism of the $\mathrm{H}_{2}$ activation will be discussed.

\subsubsection{Synthesis of $\mathrm{L}^{\mathrm{Ph}} \mathrm{Ni}_{2}(\mu-\mathrm{Br})(\mathbf{1})$ and Product of the Hydrolysis (1-OH)}

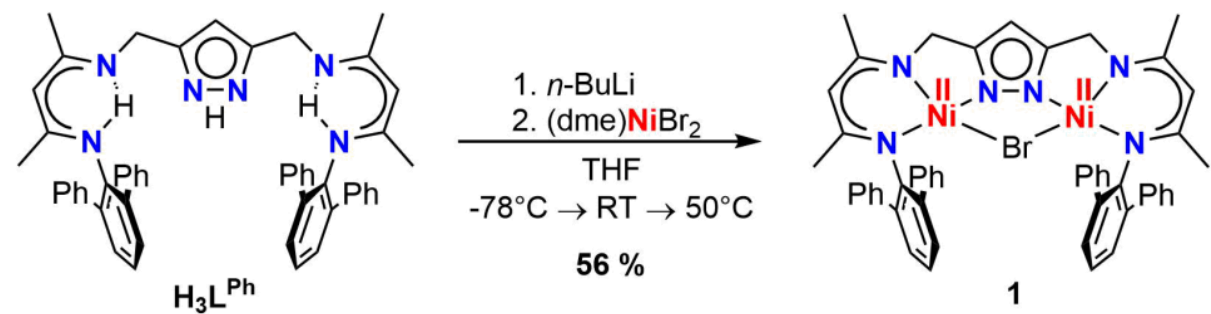

Scheme 1.21 Synthesis of [ $\left.\mathrm{LPhNi}_{2}(\mu-\mathrm{Br})\right]$; deprotonation of $\mathrm{H}_{3} \mathrm{~L}^{\mathrm{Ph}}$ with $n$ butyl lithium and consecutive addition of (dme) $\mathrm{NiBr}_{2}$ in $\mathrm{THF}$.

Similar to the iso-propyl derivative $\mathbf{1}^{\mathbf{i P r}}$, the bromido-bridged dinickel(II) complex $\mathbf{1}$ was synthesized. ${ }^{57}$ The deprotonation of $\mathbf{H}_{\mathbf{3}} \mathbf{L}^{\mathbf{P h}}$ in THF with $n$-butyl lithium resulted in a dark red solution to which (dme) $\mathrm{NiBr}_{2}$ was added. The precipitation and subsequent washing gave the product $\left[\mathrm{L}^{\mathrm{Ph}^{2}} \mathrm{Ni}_{2}(\mu-\mathrm{Br})\right] 1$ with a yield of $56 \%$ (Scheme 1.21 ).

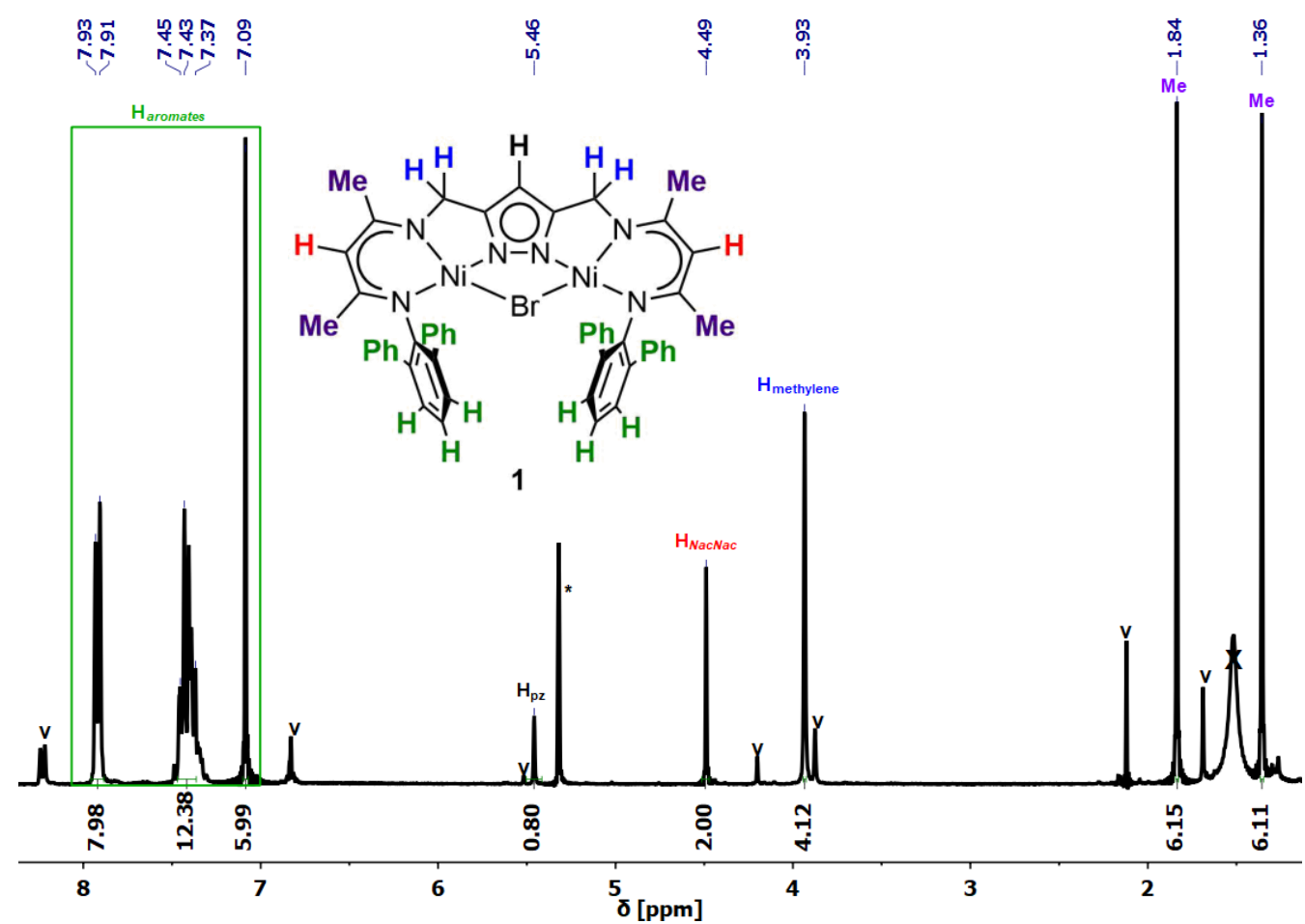

Figure 1.2 ${ }^{1} \mathrm{H}-\mathrm{NMR}$ spectrum of bromido bridged dinickel complex 1 in $\mathrm{CD}_{2} \mathrm{Cl}_{2}$ ( $^{*}$ ); 1 $\mathrm{OH}$ signals are marked with a $\mathbf{V}$; residual water in $\mathrm{CD}_{2} \mathrm{Cl}_{2}$ is marked with $\mathbf{X}$. 
Although a small impurity ( $\sim 9 \%$ ) of the hydrolysis product (1-OH) was still found, further purification resulted in a drastic decrease of yield. Therefore $\mathbf{1}$ was used as a precursor for the following chemical transformations without further purification. This compound was previously characterized by Duan, ${ }^{57}$ but certain data will be discussed shortly. The ${ }^{1} \mathrm{H}-$ NMR spectrum of $\mathbf{1}$ is presented in Figure 1.2 and is consistent with the data shown by Duan. The exclusive chemical shift area of the pyrazole signal $(\sim 5-6 \mathrm{ppm})$ allows to distinguish between different pyrazole-based products. Next to the proton signal of the pyrazole of $1\left(\delta_{4-\mathrm{Hpz}}=5.46 \mathrm{ppm}\right)$ proton signal of $\mathbf{1 - O H}$ is found at $5.52 \mathrm{ppm}$. The remaining proton signals show the $\mathrm{C}_{2 \mathrm{v}}$ symmetry of the molecule in solution. This is especially indicative based on the singlets for the methylene (3.93 ppm) and NacNac backbone (4.49 $\mathrm{ppm})$ proton resonances.

For later experiments the electrochemical behavior of 1 was investigated. For this a further purified sample of $\mathbf{1}$ was used, due to the sensitivity of cyclic voltammetry towards impurities (Appendix). The cyclic voltammograms of the two observed reductions are presented in Figure 1.3. The low solubility of $\mathbf{1}$ in THF resulted in a low current of the measurement and explains the high signal-to-noise ratio observed. The first reduction is observed at $\mathrm{E}_{1 / 2}=-2.25 \mathrm{~V}$ and shows a quasi-reversible behavior with a dependency on the scan rate $\left(\Delta \mathrm{E}_{1000 \mathrm{mV} / \mathrm{s}}=0.206 \mathrm{~V}\right.$ with $\mathrm{I}_{\mathrm{p}, \mathrm{c}} / \mathrm{I}_{\mathrm{p}, \mathrm{a}}=0.79$ and $\Delta \mathrm{E}_{50 \mathrm{mV} / \mathrm{s}}=0.102 \mathrm{~V}$ with $\mathrm{I}_{\mathrm{p}, \mathrm{c}} / \mathrm{I}_{\mathrm{p}, \mathrm{a}}=$ $0.90)$. The lowest reversibility is observed for fast scan rates and vice versa. This redox behavior is consistent with an $\mathrm{E}_{\mathrm{r}} \mathrm{C}_{\mathrm{r}}$ mechanism, which has a reaction rate of the equilibrium in the domain of the scan rate. ${ }^{67}$ The electrochemical properties change drastically upon a second irreversible reduction at $\mathrm{E}_{\mathrm{p}, \mathrm{c}}=-2.85 \mathrm{~V}$. The first reduction becomes irreversible and two oxidation process are observed at $\mathrm{E}_{\mathrm{p}, \mathrm{a} 1}=-2.13 \mathrm{~V}$ and $\mathrm{E}_{\mathrm{p}, \mathrm{a} 2}=-0.84 \mathrm{~V}$ with a scan rate of $1000 \mathrm{mV} / \mathrm{s}$, indicating a chemical reaction faster than the time scale of the measurement.

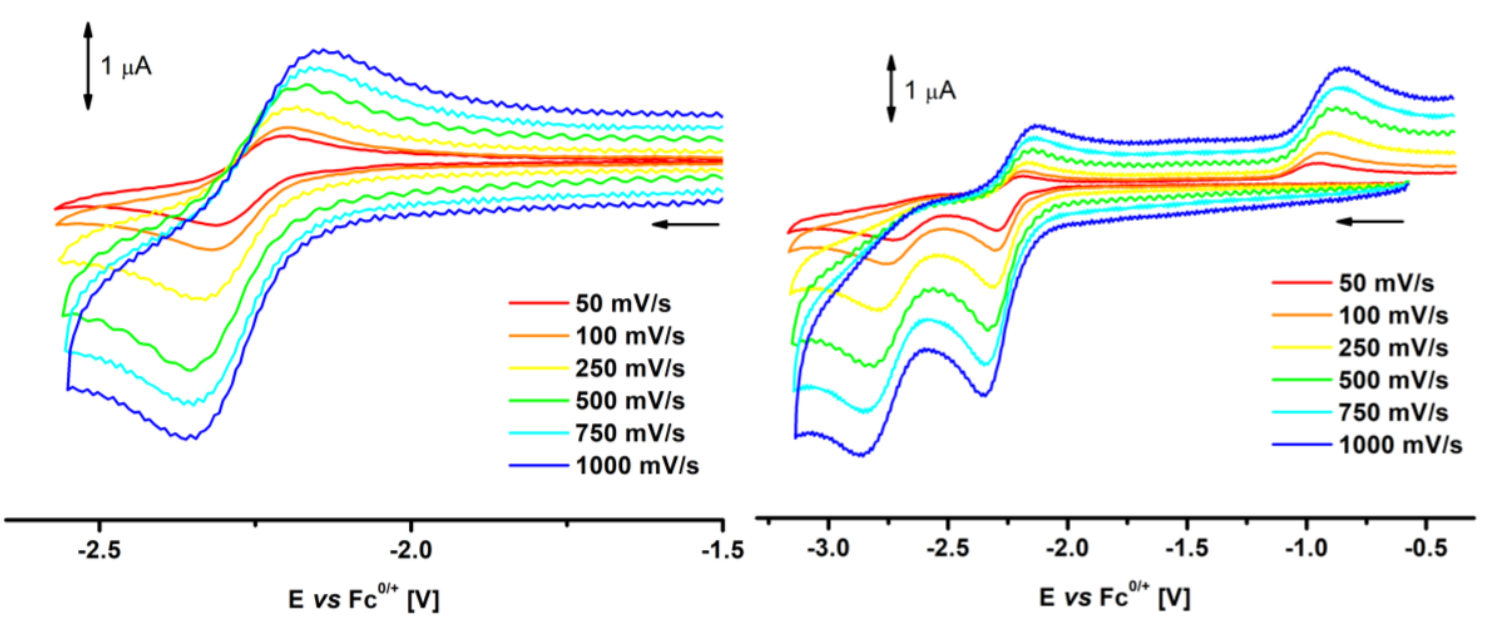

Figure 1.3 $\mathrm{CVs}$ of 1 in THF at room temperature at different scan rates with $\mathrm{NBu}_{4} \mathrm{PF}_{6}(0.1 \mathrm{M})$ as

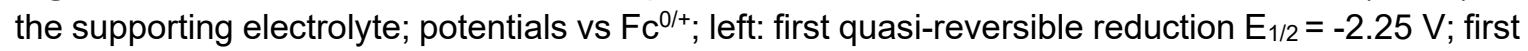
and second irreversible reductions $E_{p, c 1}=-2.34 \vee$ and $E_{p, c 2}=-2.85 \vee$ with two new oxidation processes at $E_{p, a 1}=-2.13 \mathrm{~V}$ and $E_{p, a 2}=-0.84 V$ at a scan rate of $1000 \mathrm{mV} / \mathrm{s}$. 
A possible EEC mechanism ${ }^{67}$ is presented in Scheme 1.22 and is based the results of the chemical reduction of $1 .{ }^{57}$ The first metal-based reduction results in the formation of a Tshaped Ni(I) ion, similar for other NacNac nickel complexes. ${ }^{11,24}$ Due to the Ni(II) ion present, the bromide is still coordinated to the complex and consequently a quasi-reversible behavior is observed. The scan rate dependency of the first reduction indicates that no bromide ion abstraction is observed. In contrast to this, an abstraction of the bromide ion is reasonable upon second reduction, due to the changes in potential for both back oxidations and insignificant dependency on the scan-rate. Further reaction to the $\mathrm{C}-\mathrm{H}$ activated species 2-K was observed, when $\mathbf{1}$ was treated with a strong reducing agent (vide infra). In case of the iso-propyl derivative $\mathbf{1}^{i \mathbf{P r}}$ the resulting $\mathrm{Ni}(\mathrm{I}) \mathrm{Ni}(\mathrm{I})$ species was isolated after chemical reduction with potassium graphite $\left(\mathrm{KC}_{8}\right)$ (Scheme 1.29 in Section 1.4.3). ${ }^{57}$ This phenomenon will be thoroughly discussed during the mechanistic investigation presented later on.

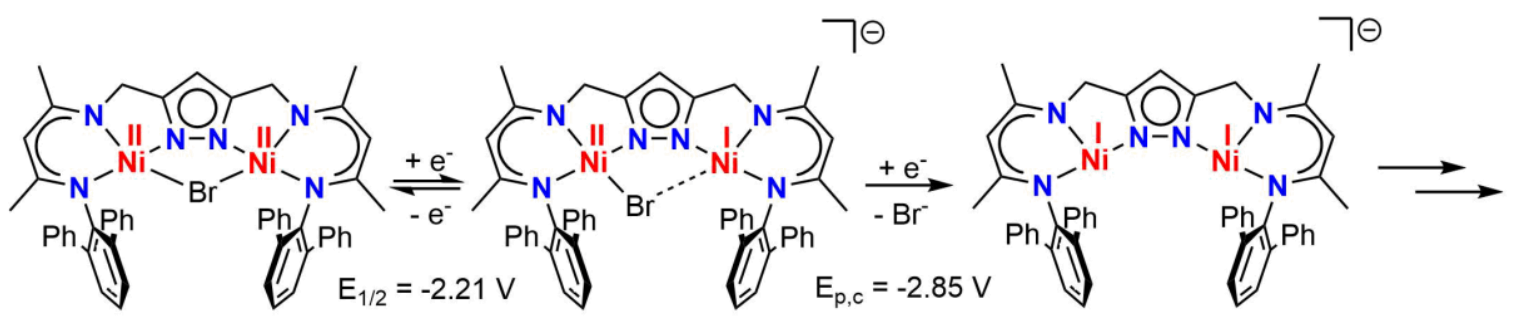

Scheme 1.22 Proposed metal-based EEC mechanism of the redox properties of 1 based on behavior of literature known NacNac nickel complexes. ${ }^{11,24}$

Next to the characterization of 1 the hydrolysis product $\mathrm{L}^{\mathrm{Ph}} \mathrm{Ni}_{2}(\mu-\mathrm{OH}) \mathbf{1 - O H}$ will be discussed. 1-OH is selectively synthesized by reaction of potassium hydroxide with $\mathbf{1}$ (Scheme 1.23) or by hydrolysis of the later discussed hydride complexes. ${ }^{56}$

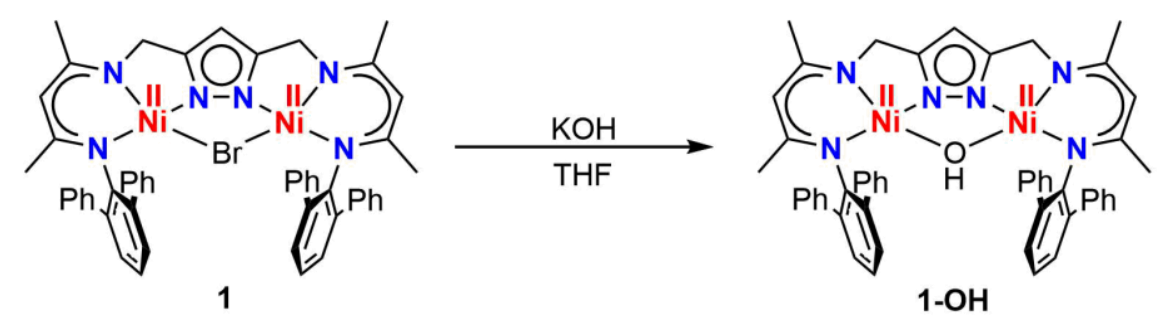

Scheme 1.23 Selective synthesis of 1-OH through anion exchange with potassium hydroxide in THF.

Green crystals of 1-OH were isolated by slow evaporation of a saturated dichloromethane solution. The hydroxido bridged complex was fully characterized by Duan. ${ }^{57}$ The ${ }^{1} \mathrm{H}-\mathrm{NMR}$ spectrum of 1-OH in THF-d8 (Figure 1.4) shows, that the complex features $\mathrm{C}_{2 \mathrm{v}}$-symmetry like the precursor 1 . The characteristic signals are the pyrazole signal at $5.42 \mathrm{ppm}$ and the proton signal of the hydroxide group at $-6.06 \mathrm{ppm}$. The hydroxido bridge is also observed in the IR spectrum giving rise to a band at $3605 \mathrm{~cm}^{-1}$ for the $\mathrm{O}-\mathrm{H}$ stretching frequency. 


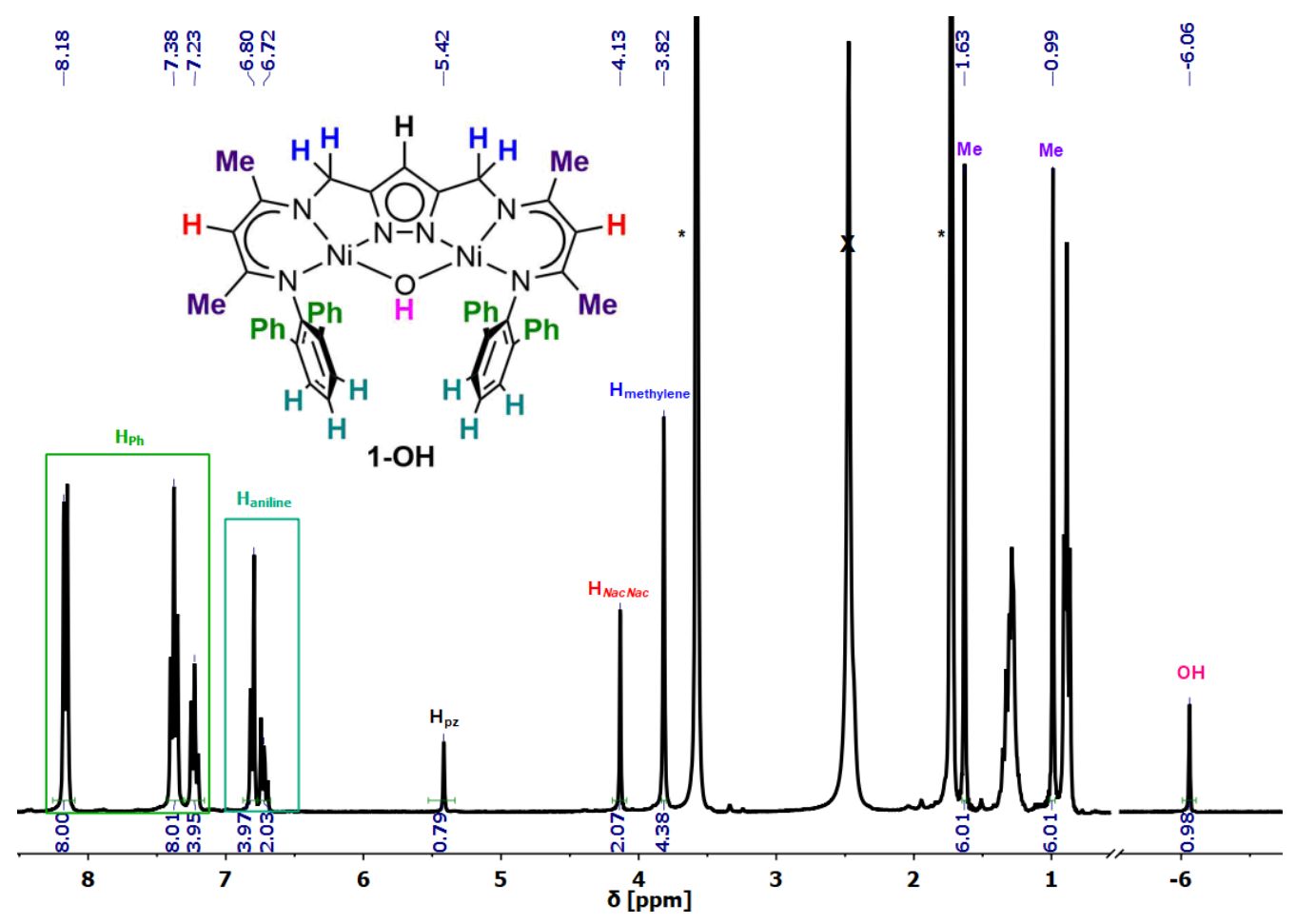

Figure $1.4{ }^{1} \mathrm{H}-\mathrm{NMR}$ spectrum of $1-\mathrm{OH}$ in $\mathrm{THF}^{-\mathrm{d}_{8}}\left({ }^{*}\right)$. Residual water in THF- $\mathrm{d}_{8}$ is marked with $\mathbf{X}$.

This compound was thoroughly characterized to simplify analysis of subsequent reactions as it is a common decomposition product in this work. After synthesis of $\mathbf{1}$, the next chapters will focus on the hydride complexes (2-M and 3-M) and the effect of the alkali metal $(\mathrm{M}=\mathrm{K}$ or $\mathrm{Na})$ on reactivity.

\subsubsection{Synthesis and Characterization of $2-\mathrm{K}$ and $3-\mathrm{K}$}

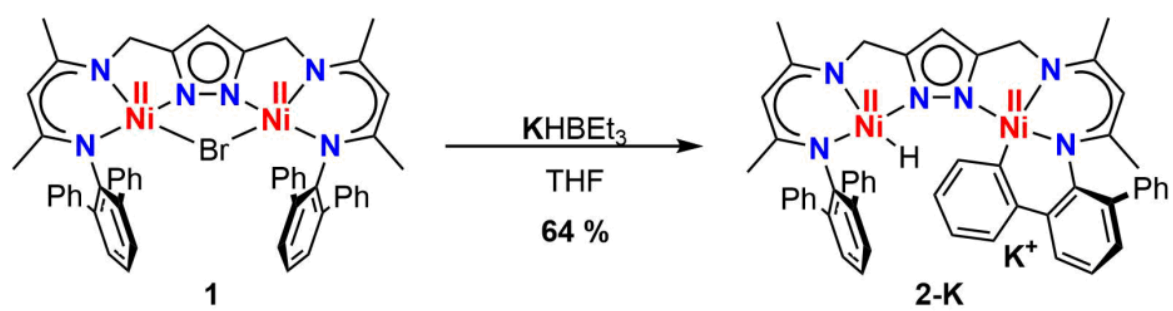

Scheme 1.24 Synthesis of the $\mathrm{C}-\mathrm{H}$ activated nickel hydride complex $\mathrm{K}\left[\mathrm{L}^{\mathrm{Ph}} \mathrm{Ni}_{2}\left(\mathrm{H}, \eta^{1}\right.\right.$-phenyl) $]$ 2-K.

As already mentioned, the reaction of 1 with KHBEt 3 resulted in the isolation of a different product, rather than the expected dihydride species $\mathbf{3}-\mathbf{K}$ as observed for $\mathbf{3}^{\mathbf{i P r}}$. The $\mathrm{C}-\mathrm{H}$ activated species $\mathbf{2}-\mathbf{K}$ was isolated after washing the crude product with hexane and crystallization through vapor diffusion of pentane into a saturated THF solution (Scheme 
1.24). ${ }^{57}$ The $\mathrm{C}-\mathrm{H}$ activation was confirmed via multiple characterization methods. ${ }^{1} \mathrm{H}-\mathrm{NMR}$ spectroscopy is especially useful because of the characteristic signals for the hydride and phenyl moiety and the reduction of the symmetry (from $\mathrm{C}_{2 \mathrm{v}}$ to $\mathrm{C}_{1}$ ). The ${ }^{1} \mathrm{H}-\mathrm{NMR}$ spectrum of $\mathbf{2}-\mathbf{K}$ is presented in Figure 1.5.

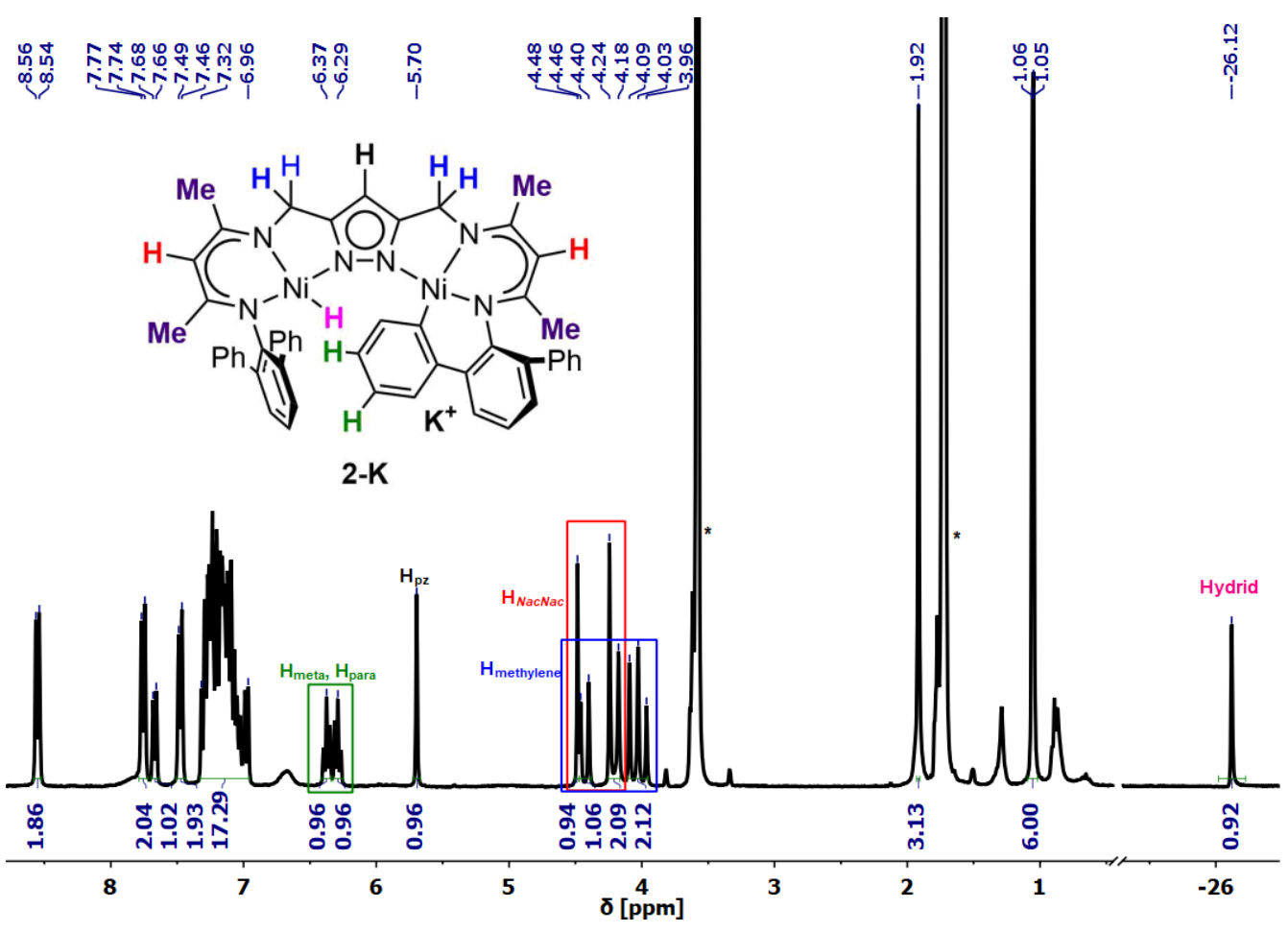

Figure $1.5{ }^{1} \mathrm{H}-\mathrm{NMR}$ spectrum of 2-K in THF-d $\left(^{*}\right)$; important signals for characteristic protons, such as pyrazole and hydride, are highlighted.

The intramolecular coordination of the phenyl moiety on one of the two nickel ions results in the loss of the $\mathrm{C}_{2}$-axis and the vertical mirror plane $\sigma_{\mathrm{v}}$ of the molecule. This is observed through the diastereotopic splitting of the methylene signals $\left(\mathbf{H}_{\text {methylene }}\right)$ and the NacNac backbone signals $\left(\mathbf{H}_{\mathrm{NacNac}}\right)$, which therefore provide useful information on the symmetry of this molecule. Additionally, the hydride signal (-26.12 ppm) is observed to be high-field shifted compared to the signal in $3^{i \operatorname{Pr}}$ by $\sim 2$ ppm $(-24.17 \mathrm{ppm})^{32}$ This is in agreement with typical chemical shifts for nickel hydride species $\left(\delta_{\text {Hydride }}=-5--38 \mathrm{ppm}\right) .{ }^{10}$ Finally, the protons meta- and para-oriented to the carbon on the coordinated nickel $\left(\mathbf{H}_{\mathbf{m}}, \mathbf{H}_{\mathbf{p}}\right)$ are assigned to low field shifted triplett signals of the aromatic protons. Those indicators will be used later on to indicate symmetry changes and phenyl coordination.

The molecular structure of $\mathbf{2 - K}$ determined by $\mathbf{X}$-ray diffraction was already discussed by Duan and therefore will only be presented briefly. ${ }^{57}$ It is displayed in Figure 1.6 and shows the intramolecular $\mathrm{C}-\mathrm{H}$ bond activation of the phenyl moiety. This coordination results in a distortion of the square planar environment around the nickel center. This geometry is in contrast to the other nickel center, which features lesser distortion $\left(\angle\left(\mathrm{N}_{\text {trans }}-\mathrm{Ni}-\mathrm{N}_{\mathrm{pz}}\right)=\right.$ $177.22(15)^{\circ}$ vs. $\left.167.15(14)^{\circ}\right)$ The Ni-C bond distance $(1.902(4) \AA)$ is consistent with other phenyl coordinated nickel complexes $(\mathrm{d}(\mathrm{Ni}-\mathrm{CPh})=1.8503(16)-1.902(4) \AA)^{68-70}$. On the other nickel ion, the $\mathrm{Ni}-\mathrm{H}$ bond length has to be treated carefully based of the inherent 
ambiguities of $\mathrm{H}$ atom locations in XRD analyses and therefore will not be discussed. Two molecules are bridged through a potassium cation, resulting in a chain-like assembly in solid state (Appendix). Due to the phenyl coordination and the correlated twist of the phenyl sidearm the potassium cannot coordinate between the two $\pi$-systems as observed for the iso-propyl derivative.

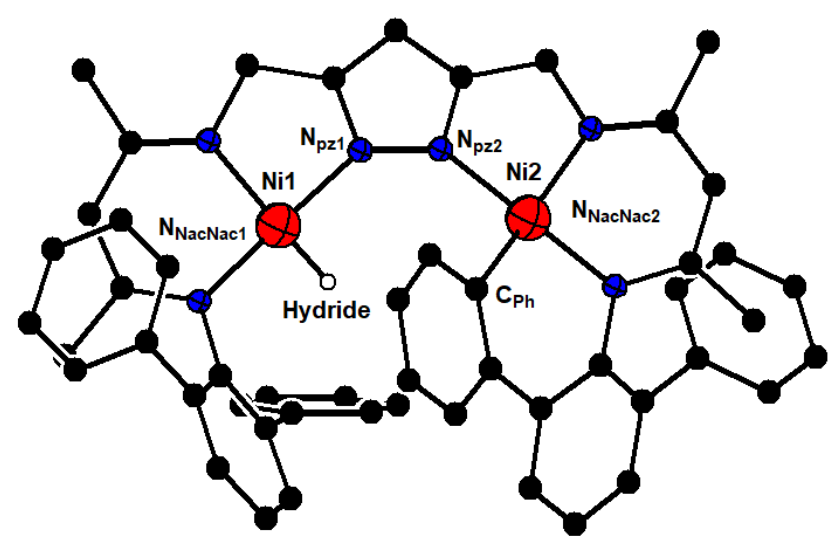

Figure 1.6 Molecular structure of $\mathrm{C}-\mathrm{H}$ activated species 2-K, protons and potassium cation are omitted for clarity. ${ }^{57}$

Several attempts with different techniques (nujol, solution and ATR) to observe a Ni-H stretching frequency in the IR spectrum were unsuccessful. The ATR-IR spectrum is presented in the Appendix, but it must be noted that no Ni-H vibration was observed in all of the IR measuring methods. The absence of a band at $\sim 3500 \mathrm{~cm}^{-1}$ shows that the hydrolysis product 1-OH is not present and hydrolysis is not the reason for the missing of the Ni-H band.

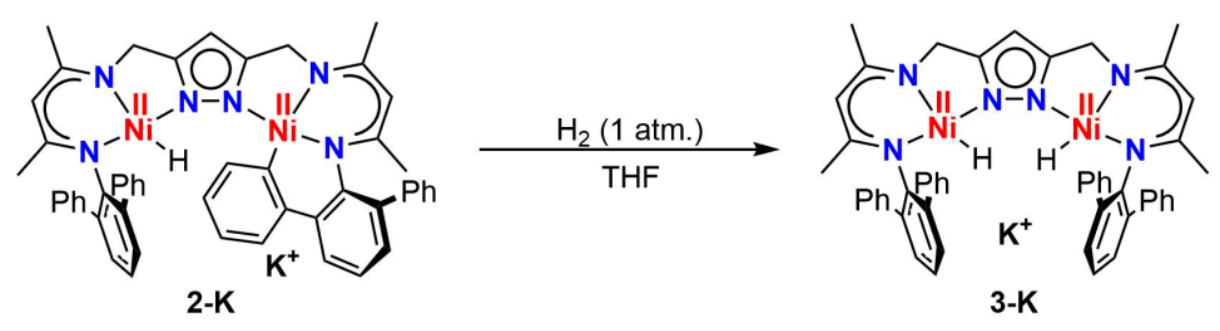

Scheme 1.25 Reversible $\mathrm{C}-\mathrm{H}$ activation of 2-K with dihydrogen to dihydride 3-K.

It was previously observed that $\mathbf{3}-\mathbf{K}$ was generated upon treatment of $\mathbf{2}-\mathbf{K}$ with dihydrogen, which suggests the reversibility of the $\mathrm{C}-\mathrm{H}$ activation (top, Figure 1.7). This was indicated via ${ }^{1} \mathrm{H}-\mathrm{NMR}$ spectroscopy. The shifted hydride signal $(-26.12 \mathrm{ppm} \rightarrow-23.80 \mathrm{ppm})$, absence of the aromatic triplets and the convergence of the methylene and NacNac signals indicated a full conversion to the dihydride species 3-K (Scheme 1.25), due to the increase in symmetry compared to the asymmetric $\mathbf{2 - K}$. Additional studies also showed that treating 
2-K with lower partial pressures of $\mathrm{H}_{2}\left(10 \%\right.$ with $\left.\mathrm{N}_{2}\right)$ also resulted in the dihydride species, although over a longer period of time.

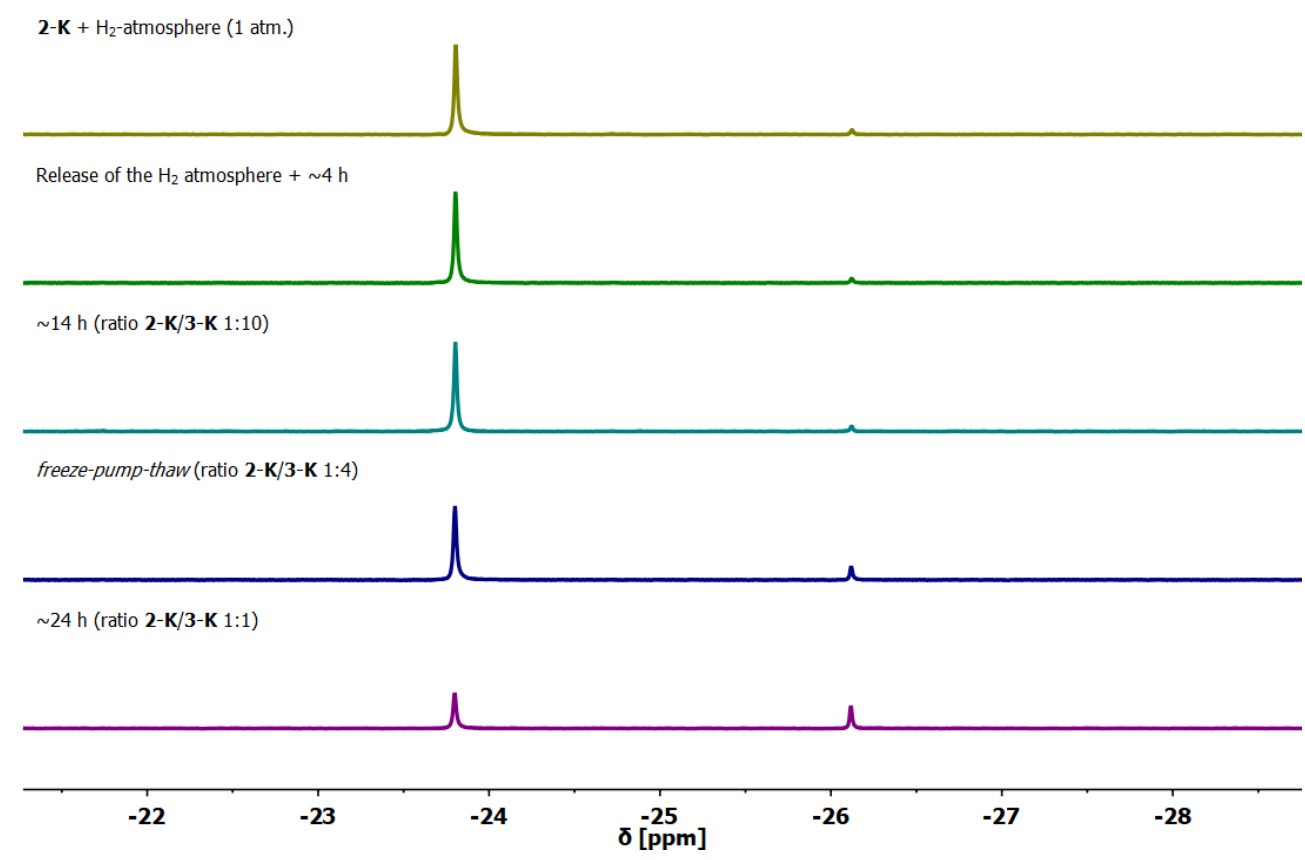

Figure $1.7{ }^{1} \mathrm{H}-\mathrm{NMR}$ spectra in THF-d 8 of $3-\mathrm{K}$ over time to evaluate the stability of 3-K $\left(\delta_{\text {Hydride }}=-23.80 \mathrm{ppm}\right)$ to undergo $\mathrm{C}-\mathrm{H}$ activation to $2-\mathrm{K}\left(\delta_{\text {Hydride }}=-26.12 \mathrm{ppm}\right)$ through exchange of atmosphere to dinitrogen atmosphere and freeze-pump-thaw procedure.

Upon removal of dihydrogen through multiple pump-freeze-thaw processes 3-K reacts back to $\mathbf{2 - K}$, as observed through ${ }^{1} \mathrm{H}-\mathrm{NMR}$ spectroscopy over two days (Figure 1.7). Only after multiple freeze-pump-thaw procedures, $\mathbf{2 - K}$ was fully recovered. Still a stability of the species over a longer period of time was not observed, making isolation of the species rather challenging. Addition of [2.2.2]-cryptand aided the crystallinity and resulted in the isolation of crystalline material (vide infra).

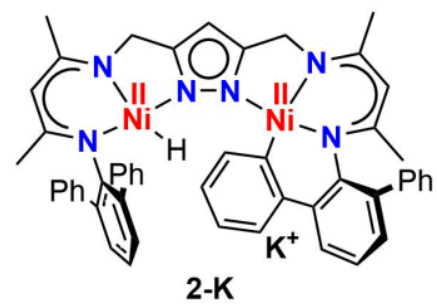

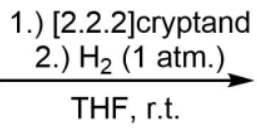

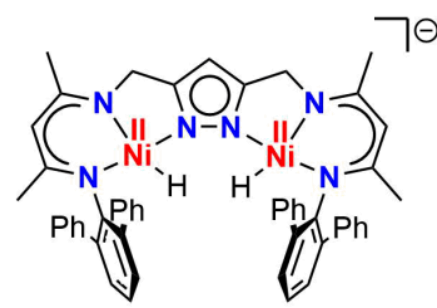

3-K(crypt)

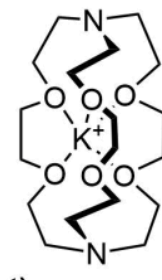

Scheme 1.26 Isolation of 3-K(crypt) via abstraction of the potassium cation using [2.2.2]cryptand under $\mathrm{H}_{2}$ atmosphere.

While the stability of $\mathbf{3}-\mathbf{K}$ was problematic for isolation through crystallization, the utilization of cryptand, for abstraction of the potassium cation resulted in immediate crystallization of 3-K(crypt) out of THF under $\mathrm{H}_{2}$-atmosphere (Scheme 1.26). 


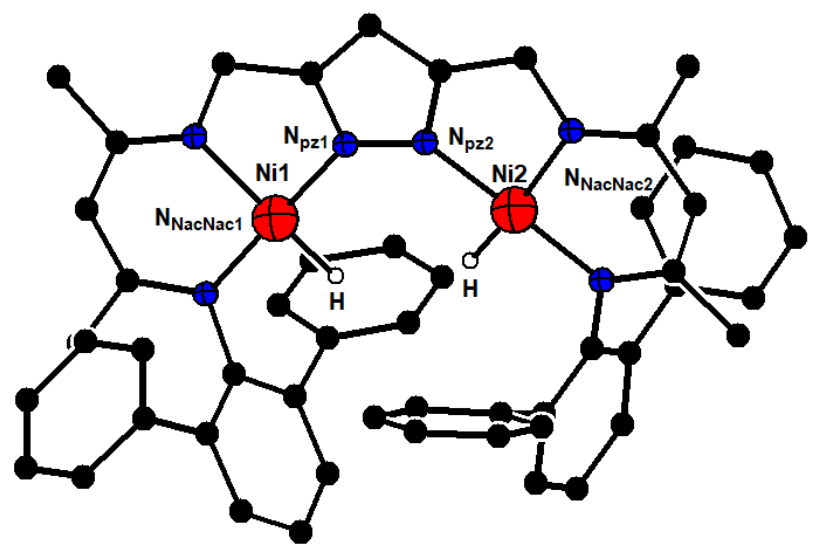

Figure 1.8 Molecular structure of 3-K(crypt); most hydrogens, apart from hydrides, and THF molecules are omitted for clarity.

Table 1.1 Selected bond lengths and angles of 2-K, 3-K(crypt) and for reference $3^{\text {iPr-K}_{-} \text {(DB18C6) }}{ }^{32}$.

\begin{tabular}{|c|c|c|c|}
\hline $\mathrm{d}[\AA \AA \AA] / \angle\left[^{\circ}\right]$ & $2-K^{57}$ & 3-K(crypt) & $3^{\mathrm{iPr}-K(D B 18 C 6)^{[a]}}$ \\
\hline \multirow{2}{*}{$\mathrm{Ni}-\mathrm{N}_{\mathrm{NacNac1}}$} & $1.879(3)$ & $1.8709(16)$ & \multirow{4}{*}{$\begin{array}{l}1.8729(17) \\
1.9201(17)\end{array}$} \\
\hline & $1.915(3)$ & $1.9154(17)$ & \\
\hline \multirow{2}{*}{$\mathrm{Ni}-\mathrm{N}_{\mathrm{NacNac2}}$} & $1.877(4)$ & $1.8645(17)$ & \\
\hline & $1.936(4)$ & $1.9179(17)$ & \\
\hline $\mathrm{Ni}-\mathrm{N}_{\mathrm{pz} 1}$ & 1.910(3) & $1.8651(16)$ & \multirow{2}{*}{$1.8855(17)$} \\
\hline $\mathrm{Ni}-\mathrm{N}_{\mathrm{pz2}}$ & $1.933(3)$ & $1.8672(17)$ & \\
\hline \multirow{2}{*}{$\mathrm{Ni}-\mathrm{H}$} & \multirow{2}{*}{$1.291(3)$} & $1.39(2)$ & $1.44(3)$ \\
\hline & & $1.42(2)$ & $1.46(3)$ \\
\hline \multirow{2}{*}{$\mathrm{Ni} \cdots \mathrm{Ni}$} & \multirow{2}{*}{$4.266(8)$} & \multirow{2}{*}{$4.0478(8)$} & $4.0636(7)$ \\
\hline & & & $4.1152(7)$ \\
\hline \multirow{2}{*}{$\mathbf{N}_{\text {trans }}-\mathrm{Ni}-\mathrm{N}_{\mathrm{pz}}$} & $167.15(14)$ & $177.67(7)$ & $177.22(8)$ \\
\hline & $177.22(15)$ & $178.71(7)$ & $177.25(8)$ \\
\hline
\end{tabular}

${ }^{\text {[a] }}$ Crystal structure shows a vertical mirror plane through the molecule.

Therefore, analysis by single crystal X-ray diffraction was possible and resulted in the molecular structure of 3-K(crypt) presented in Figure 1.8. The dinickel dihydride core is similar to that of $\mathbf{3}^{\mathbf{i P r}}-\mathbf{K}(\mathbf{D B 1 8 C 6})$ where DB18C6 is dibenzo-18-crown-6-ether. ${ }^{32}$ Only slight distortions from the square planar environment $\left(\angle\left(\mathrm{N}_{\text {trans }}-\mathrm{Ni}-\mathrm{N}_{\mathrm{pz}}=177.67(7)\right.\right.$ and $\left.178.71(7)^{\circ}\right)$ are observed. This geometry is different compared to the more-distorted nickel ion of 2-K $\left(\angle\left(\mathrm{N}_{\text {trans }}-\mathrm{Ni}-\mathrm{N}_{\mathrm{pz}}=167.15(14)^{\circ}\right)\right.$ bearing the phenyl moiety. These differences are also found in the nickel-nickel distance $(\mathrm{d}(\mathrm{Ni} \cdots \mathrm{Ni})=4.0478(8) \AA)$, which is observed to be elongated in the case of $2-\mathrm{K}(\mathrm{d}(\mathrm{Ni} \cdots \mathrm{Ni})=4.266(8) \AA)$. Nevertheless, the Ni-N bond lengths of 3-K(crypt) are in good agreement with $\mathbf{2}-\mathbf{K}$, e.g. d $\left(\mathrm{Ni}-\mathrm{N}_{\mathrm{NaCNa}}\right)=1.8645(17)-$ 
1.9179(17) vs 1.877(4) - 1.936(4) $\AA$ for 2-K. No drastic differences in the molecular structures between $\mathbf{3}-\mathbf{K}(\mathbf{c r y p t})$ and $\mathbf{3}^{\mathbf{i P r}}-\mathbf{K}(\mathrm{DB} 18 \mathrm{C6})$ are observed. This is mostly, due to the orthogonal orientation of the phenyl moieties of the $m$-terphenyl substituents, which inhibits $\pi$-orbital overlapping and therefore electronic structure differences. When the crystals of 3-K(crypt) were redissolved without $\mathrm{H}_{2}$-atmosphere, degradation to 2-K(crypt) was observed. This behavior is consistent with the observations for the iso-propyl system, due to facile elimination of dihydrogen upon abstraction of the potassium cation by the cryptand or crown ether. ${ }^{32}$

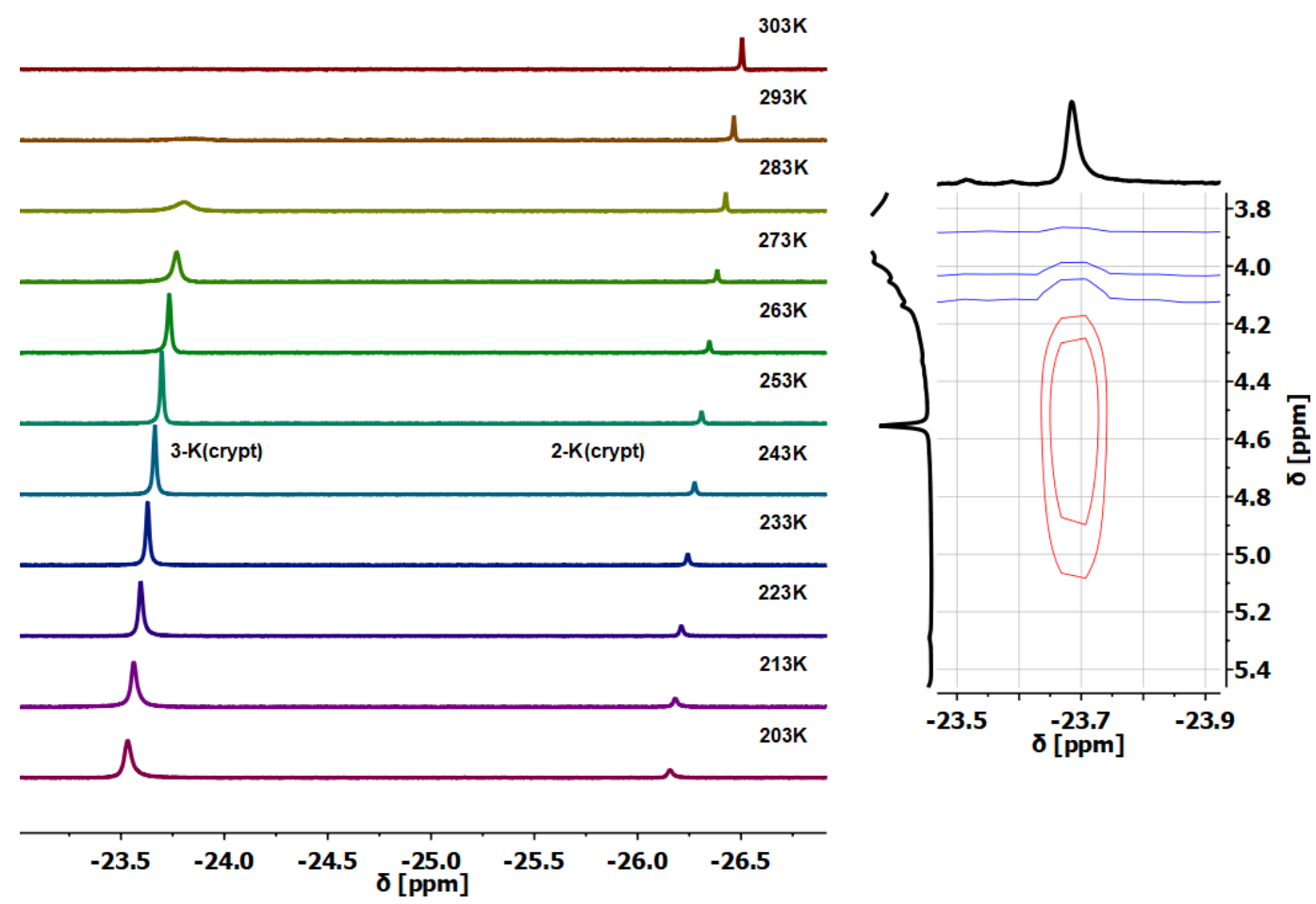

Figure 1.9 Left: Temperature dependent ${ }^{1} \mathrm{H}-\mathrm{NMR}$ spectra of 3-K(crypt) in THF-d8 under $\mathrm{H}_{2}$ atmosphere (1 atm.) from -90 to $30^{\circ} \mathrm{C}$, only the $\mathrm{Ni}-\mathrm{H}$ region is shown; right: part of the EXSY-NMR spectrum of 3-K(crypt) at $-30^{\circ} \mathrm{C}$ showing exchange of 3-K(crypt) with free dihydrogen.

Similarities of 3-K(crypt) to the iso-propyl variant $3^{i \mathbf{P r}}$-K(DB18C6) are also observed by in situ ${ }^{1} \mathrm{H}$-NMR measurements of 3-K(crypt) in THF-d8 under $\mathrm{H}_{2}$-atmosphere. At room temperature a broadening of the dihydride $(-23.66 \mathrm{ppm})$, free dihydrogen $(4.56 \mathrm{ppm})$ and pyrazole signal $(5.30 \mathrm{ppm})$ is observed. This dynamic between the proton signals was further investigated by temperature dependent ${ }^{1} \mathrm{H}-\mathrm{NMR}$ spectroscopy from $-90^{\circ} \mathrm{C}$ to $+30^{\circ} \mathrm{C}$ (Figure 1.9, left). Upon cooling, the broad signals coalesce with a maximum sharpness at $\mathrm{T}=-30^{\circ} \mathrm{C}$ and allows visibility of $\mathbf{3}-\mathbf{K}(\mathbf{c r y p t})$. Below $-30^{\circ} \mathrm{C}$ the signals appear to become broader again, most probably due to molecular tumbling. Further investigations utilizing EXSY-NMR measurements at $-30^{\circ} \mathrm{C}$ showed an exchange between the signal for solvated $\mathrm{H}_{2}$ and the hydride signal of 3-K(crypt) (Figure 1.9, right). This exchange has also been observed for $3^{i P r}{ }^{32}$ Interestingly, such broadening of the pyrazole, dihydrogen and hydride signal was only observed, when abstraction of the potassium was forced through crown 
ether or cryptand addition, indicating a faster exchange on the NMR timescale for the "naked" anion 3-.

Activation of $\mathrm{H}_{2}$ by $\mathbf{2}-\mathbf{K}$ is of interest due to the different mechanism by which this reactivity occurs in comparison to $\mathbf{3}-\mathbf{K}$. Therefore, the next section will focus on the pathway of the reversible $\mathrm{C}-\mathrm{H}$ activation to give insights into potential reactivity towards other small molecules.

\subsubsection{Mechanistic Insight into the Reversible $\mathrm{C}-\mathrm{H}$ Activation through $\mathrm{H}_{2}$ Splitting with 2-K}

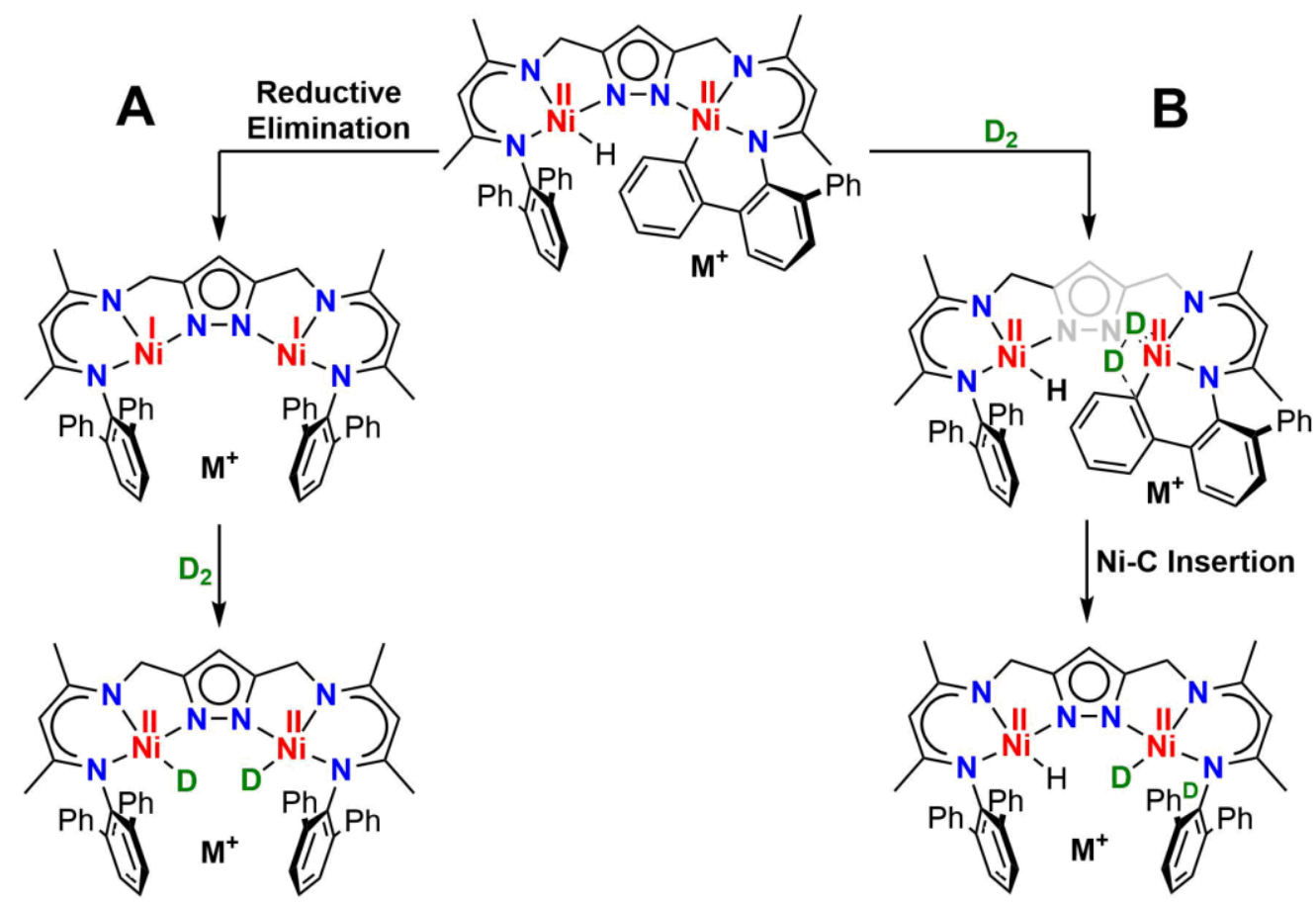

Scheme 1.27 Two mechanistic pathways for $D_{2}$-activation with 2-K; mechanism $A$ : reductive elimination ; mechanism $\mathrm{B}$ : direct reaction of dihydrogen with the $\mathrm{Ni}-\mathrm{C}$ bond.

In Chapter 1.1, two mechanisms were proposed for the activation of $\mathrm{H}_{2}$ by $\mathbf{2}-\mathbf{K}$. Mechanism $\mathrm{A}$ in Scheme 1.27 involves the intramolecular reductive elimination via formation of the $\mathrm{C}-\mathrm{H}$ bond to give a $\mathrm{Ni}(\mathrm{I}) \mathrm{Ni}(\mathrm{I})$ intermediate, which then reacts with dihydrogen. Alternatively, postulated is the direct reaction of $\mathrm{H}_{2}$ with the Ni-C bond, here shown in Scheme 1.27 as mechanism B, which then regenerates the phenyl moiety and hydride on the nickel ion. Previous studies assumed reaction pathway B as the dominant mechanism in the reactivity of $\mathbf{2 - K}$ with $\mathrm{D}_{2}$, due to the presence of a broad signal in the typical region for aromatic protons in the ${ }^{2} \mathrm{H}-\mathrm{NMR}$ spectrum. ${ }^{57}$ In combination with a an absence of the hydride signal in the $1 \mathrm{H}-\mathrm{NMR}$ spectrum meant further evaluation to be necessary. 
Considering mechanism B, one nickel-hydride unit is left unchanged, so that it is found in the product of the reaction, even when using deuterium instead of hydrogen. This reactivity means that a hydride signal is still found in the ${ }^{1} \mathrm{H}-\mathrm{NMR}$ spectrum, while a deuteride signal is found in the ${ }^{2} \mathrm{H}-\mathrm{NMR}$. Additionally, the incorporation of deuterium into the phenyl moiety might be observed, resulting in a signal in the aromatic proton region of the ${ }^{2} \mathrm{H}-$ NMR spectrum of 3-K-HD (Scheme 1.27, Mechanism B). Contrarily, mechanism A involves both nickel centers, therefore a hydride signal in the ${ }^{1} \mathrm{H}-\mathrm{NMR}$ spectrum is not expected. The $\mathrm{Ni}(\mathrm{I}) \mathrm{Ni}(\mathrm{I})$ system reacts with $\mathrm{D}_{2}$ to a dideuteride species $\mathbf{3}-\mathbf{K}-\mathbf{D}_{\mathbf{2}}$, which would be observed in the ${ }^{2} \mathrm{H}-\mathrm{NMR}$ spectrum by a deuteride signal around $-24 \mathrm{ppm}$ (Scheme 1.27, Mechanism A).
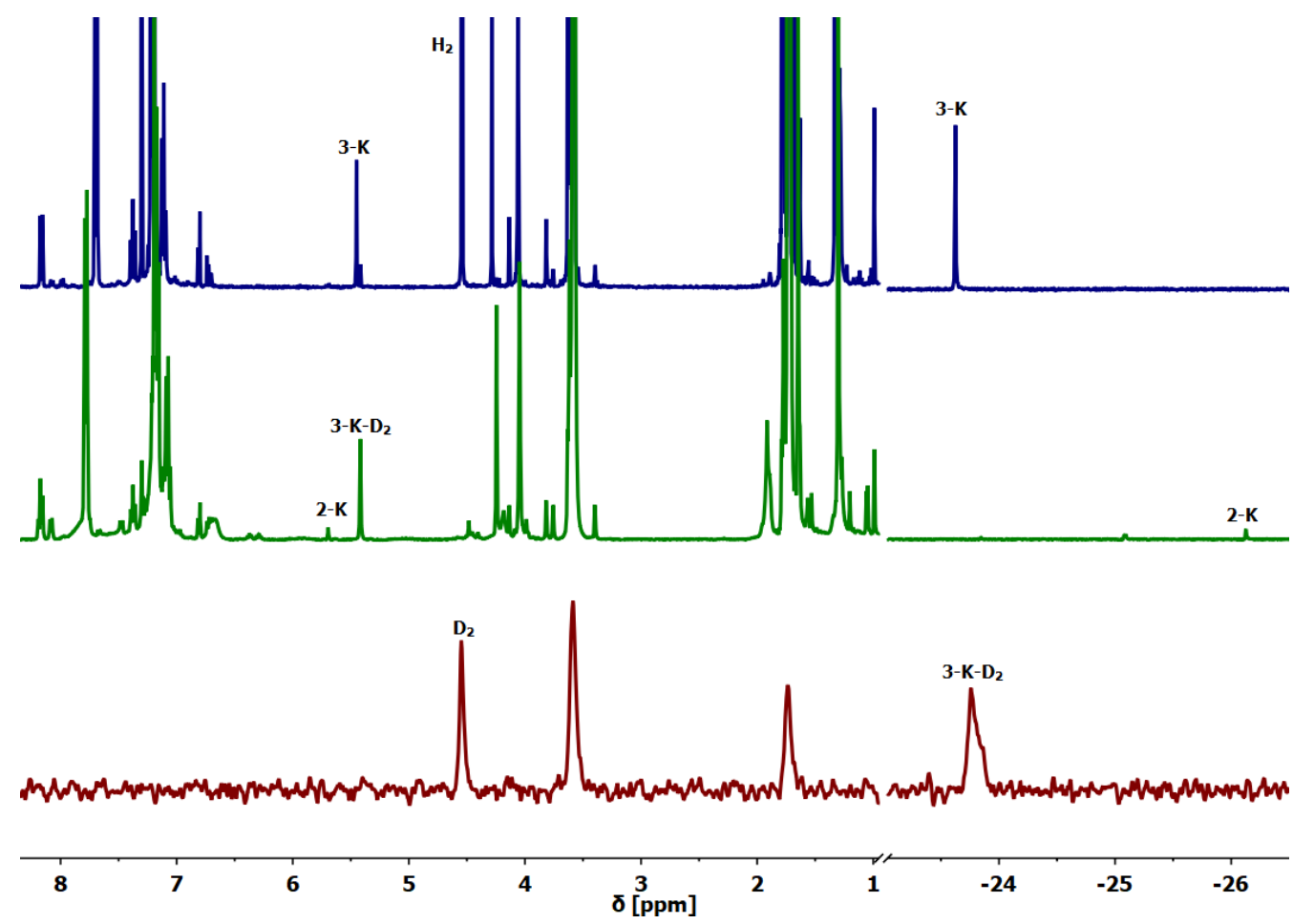

Figure 1.10 Top: ${ }^{1} \mathrm{H}-\mathrm{NMR}$ spectrum of $3-\mathrm{K}$ in THF-d8; middle: ${ }^{1} \mathrm{H}-\mathrm{NMR}$ spectrum of 2-K under $\mathrm{D}_{2}$ (1 atm.) in THF-d8; bottom: ${ }^{2} \mathrm{H}-\mathrm{NMR}$ of 2-K under $\mathrm{D}_{2}$ (1 atm.) in THF-h8; The pyrazole signal next to $3-\mathrm{K}$ corresponds to $1-\mathrm{OH}$, because of traces of water in the $\mathrm{H}_{2}$ and $\mathrm{D}_{2}$ gas.

Therefore, the C-H activated species $\mathbf{2}-\mathbf{K}$ was reacted with $\mathrm{D}_{2}$ in THF-d8 and THF to investigate the outcome of the reaction. The NMR spectra of the resulting products under $\mathrm{D}_{2}$-atmosphere are shown in Figure 1.10. The ${ }^{1} \mathrm{H}$-NMR spectrum under $\mathrm{D}_{2}$ (Figure 1.10, middle) shows a species with a pyrazole signal at $5.42 \mathrm{ppm}$ as the main product, due to the two singlets for the methylene and NacNac backbone signals it is assumed to show $\mathrm{C}_{2 v^{-}}$ symmetry. Apart from the missing dihydride signal at $-23.62 \mathrm{ppm}$, the spectrum is consistent with spectrum for the dihydride species 3-K. The absent hydride signal in the ${ }^{1} \mathrm{H}-\mathrm{NMR}$ spectrum is observed in the ${ }^{2} \mathrm{H}-\mathrm{NMR}$ spectrum at $-23.76 \mathrm{ppm}$, indicating a dideuteride species 3-K-D $\mathbf{2}$. Additionally, no other signal apart from $\mathrm{D}_{2}$ and $\mathrm{THF}$ is found in the ${ }^{2} \mathrm{H}-\mathrm{NMR}$ spectrum, in particular no signal is observed in the aromatic proton region. An incorporation of deuterium into the phenyl moieties did not occur. Even after longer 
reaction times $(>2 \mathrm{~d})$, no signal of an aromatic proton in the ${ }^{2} \mathrm{H}-\mathrm{NMR}$ spectrum was observed. Therefore, a reductive elimination process according to mechanism A (Scheme 1.27) is likely.

EXSY-NMR spectroscopy was utilized to investigate an equilibrium between the proposed $\mathrm{Ni}(\mathrm{I}) \mathrm{Ni}(\mathrm{I})$ species and 2-K. This study works by looking for an exchange reaction of specific protons and the resulting dynamic between the two protons. Due to the expected equilibrium between the $\mathrm{Ni}(\mathrm{I}) \mathrm{Ni}(\mathrm{I})$ species and $\mathbf{2}-\mathbf{K}$ and therefore the exchange of the seven other ortho-protons with the hydride the exchange between the hydride signal and an aromatic proton signal should be investigated (Scheme 1.28).
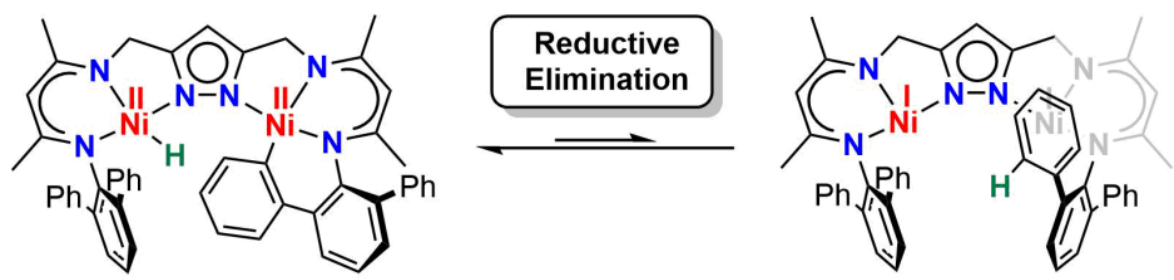

Scheme 1.28 Schematic presentation of the proton exchange of seven possible aromatic ortho-protons with hydride during reductive elimination of $\mathrm{C}-\mathrm{H}$ activated 2-K.

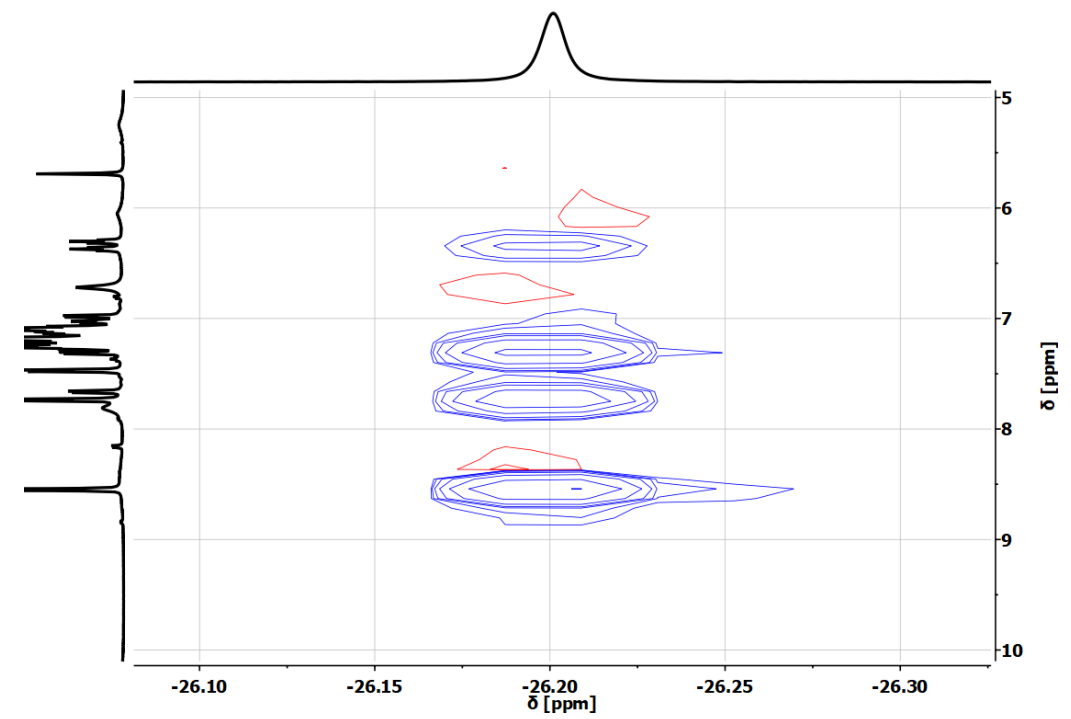

Figure 1.11 Correlation EXSY-NMR measurement of 2-K at $50^{\circ} \mathrm{C}$ in THF-d8; signals do not show high dependency on the mixing time, therefore assumption for NOESY correlation.

The measurements were carried out at different temperatures and mixing times up to $0.5 \mathrm{~s}$ to look for a correlation between the hydride and an aromatic proton signal. Apart from the NOESY correlation, due to the proximity of the hydride to the phenyl moiety coordinated on the other nickel center, no cross peaks were found (Figure 1.11).

The signals shown did not have a dependency on the mixing time even at the longest possible mixing time of $0.5 \mathrm{~s}$. The NOESY correlation also shows the intramolecular interaction of the hydride signal with the two triplets for the already discussed meta-protons 
$\left(\mathrm{H}_{\text {meta }}\right)$. Additionally, mixture of dihydride $\mathbf{3}-\mathbf{K}$ and $\mathbf{2}-\mathbf{K}$ was measured. Here, only correlation between the dihydrogen and the dihydride were observed, as expected for the exchange of the two. This behavior will be discussed in detail later (vide infra).

The EXSY-NMR measurement showed that the equilibrium cannot be observed on the timescale of the measurement, but the $\mathrm{D}_{2}$-experiments already showed evidence for a possible $\mathrm{Ni}(\mathrm{I}) \mathrm{Ni}(\mathrm{I})$ intermediate. The readily elimination of $\mathrm{H}_{2}$ in $\mathbf{3}^{\text {iPr }}$ allows following assumption on the pathway of $\mathbf{1}$ to $\mathbf{2}-\mathbf{K}$. Here, a hypothesis is that reaction of $\mathbf{1}$ with $\mathrm{KHBEt}_{3}$ results in 3-K. This species undergoes $\mathrm{H}_{2}$-loss through the same mechanism as postulated for $3^{i \mathrm{Pr}}$ to the $\mathrm{Ni}(\mathrm{I}) \mathrm{Ni}(\mathrm{I})$ intermediate. The $\mathrm{Ni}(\mathrm{I}) \mathrm{Ni}(\mathrm{I})$ reacts further in the case of $\mathbf{3}-\mathbf{K}$ via C-H activation to $\mathbf{2 - K}$. Therefore, an isolation of $\mathbf{2}-\mathbf{K}$ should be possible by reduction of the bromide 1 to the $\mathrm{Ni}(\mathrm{I}) \mathrm{Ni}(\mathrm{I})$ intermediate. A similar experiment for this system should then go directly to $\mathbf{2}-\mathbf{K}$ and gives evidence for the reductive elimination pathway (Scheme 1.29). A possible reduction is consistent with the CV data obtained for $\mathbf{1}$, in which a second irreversible reduction is observed $\left(\mathrm{E}_{\mathrm{p}, \mathrm{c}}=-2.85 \mathrm{~V}\right)$.

When 1 was treated with 2.2 equivalent of $\mathrm{KC}_{8}$ in $\mathrm{THF}$, the green suspension of 1 turned slowly into a red solution over a time of 6 hours. After evaporation of the solvent and multiple washings with hexane, the crude product was characterized by ${ }^{1} \mathrm{H}-\mathrm{NMR}$ spectroscopy and compared to 2-K (Appendix). The ${ }^{1} \mathrm{H}-\mathrm{NMR}$ spectrum of the reaction of $\mathbf{1}$ with $\mathrm{KC}_{8}$ indicates significant formation of $\mathbf{2}-\mathbf{K}$.

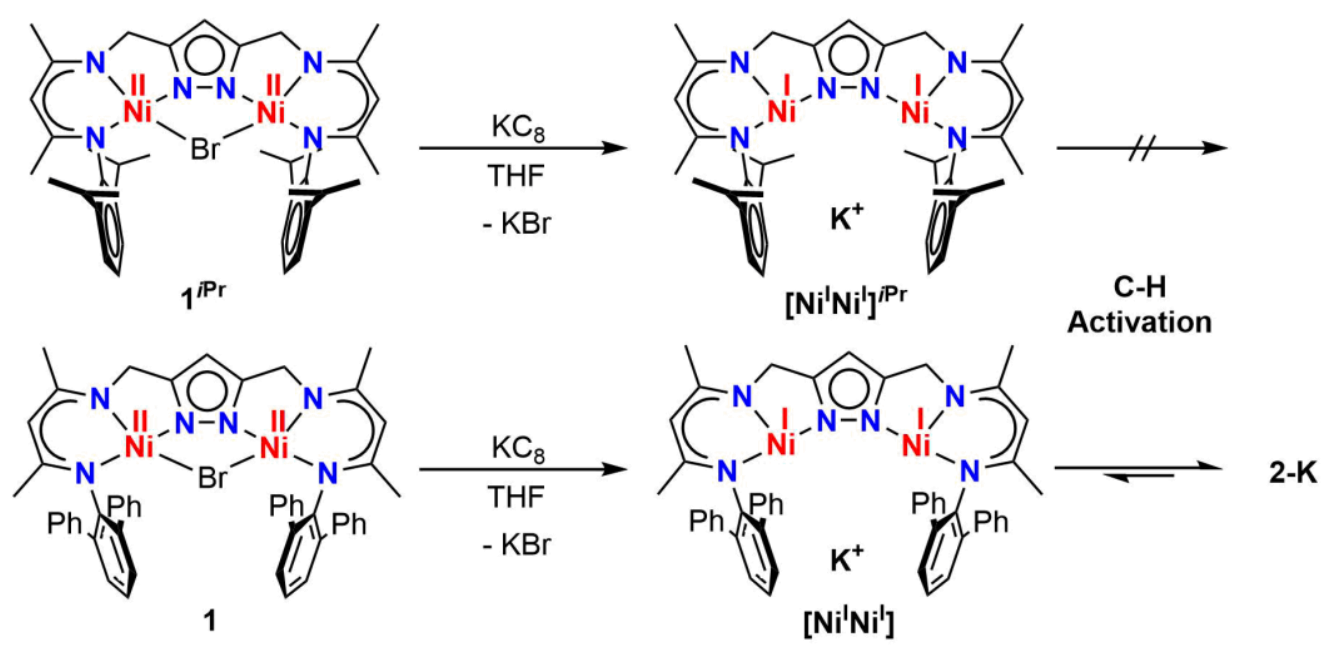

Scheme 1.29 Two-electron reduction of bromide precursor of $\mathbf{1}^{\mathrm{iPr}}$ (top) and $\mathrm{L}^{\mathrm{Ph}}$ system

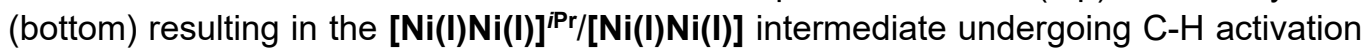
only for the $\mathrm{L}^{\mathrm{Ph}}$ system. ${ }^{57}$

The ${ }^{1} \mathrm{H}-\mathrm{NMR}$ of the reduction product is consistent with the one for $\mathbf{2}-\mathbf{K}$. This allows also a different and more efficient synthesis route to the mono hydride because of the heterogenous reactivity of $\mathrm{KC}_{8}$ and trivial separation of the by-products. Additionally, the assumption can be made, that the $\mathrm{Ni}(\mathrm{I}) \mathrm{Ni}(\mathrm{I})$ species plays an important role as an intermediate for the production of the $\mathrm{C}-\mathrm{H}$ activated species $\mathbf{2}-\mathbf{K}$, although the equilibrium is mostly on the side of the $\mathrm{C}-\mathrm{H}$ activation. Therefore, the direct isolation of the $\mathrm{Ni}(\mathrm{I}) \mathrm{Ni}(\mathrm{I})$ 
species is rather challenging and other means of trapping by reactions with substrates were attempted.

\subsubsection{Trapping of the $\mathrm{Ni}(\mathrm{I}) \mathrm{Ni}(\mathrm{I})$ Intermediate by the Reaction with Benzaldehyde as a Two-electron Acceptor}

Based on the deuterium labeling and reduction experiments described in the last section, the trapping of the $\mathrm{Ni}(\mathrm{I}) \mathrm{Ni}(\mathrm{I})$ intermediate by reaction with an electron acceptor is feasible. The use of phenylacetylene or nitroso benzene, similar to the $\mathbf{L}^{i \mathbf{P r}}$ system $^{32,61}$, resulted mostly in hydrolysis or decay of $\mathbf{2 - K}$. Therefore, different reagents for the two-electron reduction were attempted. Based on the work of $\mathrm{T}$. Kothe using $\left[\mathrm{L}^{i \mathrm{Pr}}(\mathrm{Ni}-\mathrm{H})_{2}\right] \mathrm{K}$, the reactivity of 2-K with benzaldehyde was attempted (Scheme 1.30).
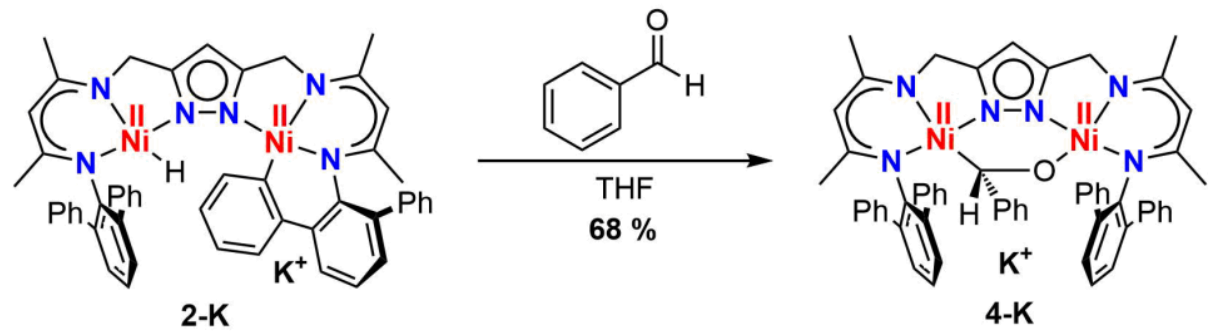

Scheme 1.30 Two-electron reduction of benzaldehyde with 2-K with in THF.

When 2-K was reacted with benzaldehyde in THF the deep red color of $\mathbf{2}-\mathbf{K}$ changed to dark brown. Crystallization through vapor diffusion of pentane, resulted in dark brown crystals suitable for XRD analyses in a $68 \%$ yield. The same reactivity to 4-Na was also observed, when the sodium analogue 2-Na was used as the starting material. The molecular structure of 4-K shows the successful incorporation of the aldehyde into the bimetallic pocket (Figure 1.12). Additionally, the compound was characterized by NMR, IR and UV/vis spectroscopy though characterization was hampered due to low solubility of 4-K in common solvents. Selected bond lengths and angles with $\mathbf{2 - K}$ as reference are presented in Table 1.2. Both nickel centers surrounded in a distorted square planar environment similar to the starting material, due to the interaction with the aldehyde being out of plane. On the side of the aldehyde $\mathrm{N}_{\text {trans-Ni-Oald }}$ is $168.50(8)^{\circ}$, which shows the degree of distortion. A similar situation is found on the other side of the aldehyde coordination with an angle of $168.43(9)^{\circ}$. This is less pronounced on the NacNac ligand itself with angles closer to $180^{\circ}$ found $\left(\angle\left(\mathrm{N}_{\text {trans }}-\mathrm{Ni}-\mathrm{N}_{\mathrm{pz}}=172.90(8)\right.\right.$ and $\left.178.36(8)^{\circ}\right)$.

Rather more interesting are the structural changes of the bridging aldehyde, coordinated between the two nickel centers. The $\mathrm{C}-\mathrm{O}$ bond length of the aldehyde is significantly elongated (1.388(3) $\AA$ ) versus the free terephtalaldehyde ${ }^{71}\left(\mathrm{~d}\left(\mathrm{C}_{\text {ald }}-\mathrm{O}\right)=1.204(2) \AA\right)$. The bond length of benzyl alcohol $\left(\mathrm{d}\left(\mathrm{C}_{\text {alk }}-\mathrm{O}\right)=1.424(6) \AA\right)^{72}$ is closer to the observed species, 
indicating a two-electron reduced aldehyde. The assumption of a reduced species observed is also consistent with the contraction of the C-C-O angle $\left(114.58(19)^{\circ}\right)$, compared to the nearly $120^{\circ}$ angle of terephatalaldehyde ${ }^{71}\left(124.23(13)^{\circ}\right)$. Finally, the charge of the 4-K is compensated by a potassium, indicating a doubly negative charged benzyl alcoholate molecule. The alcoholate substrate is in agreement with the data from IR spectrum of 4-K, not showing a typical CO stretching band around $1702 \mathrm{~cm}^{-1}$ for free benzaldehyde. ${ }^{73}$ The stretching frequency of the reduced species is overlapping with other ligand vibrations. To exclude a hydride reactivity though, the proton on the aldehyde molecule must be originating from the benzaldehyde used and not from the hydride of $\mathbf{2}-\mathbf{K}$.

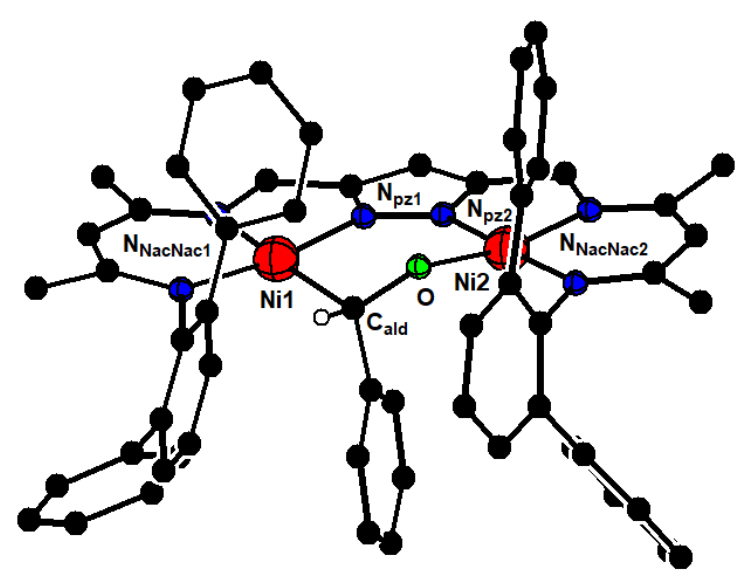

Figure 1.12 Molecular structure of anionic part of 4-K. The potassium cation, solvent molecules and hydrogens, apart from the aldehyde, are omitted for clarity.
Table 1.2 Selected bond lengths and angles of $\mathbf{2}-\mathbf{K}^{57}$ and $\mathbf{4 - K}$ at $133 \mathrm{~K}$.

\begin{tabular}{|c|c|c|}
\hline $\mathrm{d}[\AA \AA] / \angle\left[^{\circ}\right]$ & $2-K$ & $4-K$ \\
\hline \multirow{2}{*}{$\mathrm{Ni}-\mathrm{N}_{\mathrm{NacNac1}}$} & $1.879(3)$ & $1.9336(18)$ \\
\hline & $1.915(3)$ & $1.9709(18)$ \\
\hline \multirow{2}{*}{$\mathrm{Ni}-\mathrm{N}_{\mathrm{NacNac2}}$} & $1.877(4)$ & $1.8813(18)$ \\
\hline & $1.936(4)$ & $1.9088(18)$ \\
\hline $\mathrm{Ni}-\mathrm{N}_{\mathrm{pz} 1}$ & $1.910(3)$ & $1.8919(18)$ \\
\hline $\mathrm{Ni}-\mathrm{N}_{\mathrm{pz2}}$ & $1.933(3)$ & $1.8526(18)$ \\
\hline $\mathrm{Ni}-\mathrm{C}$ & $1.902(4)$ & $2.015(2)$ \\
\hline Ni-O & - & $1.8299(16)$ \\
\hline $\mathrm{Ni} \cdots \mathrm{Ni}$ & $4.2658(8)$ & $3.9594(4)$ \\
\hline $\mathrm{C}_{\text {ald }}-\mathrm{O}$ & - & $1.388(3)$ \\
\hline $\mathrm{C}_{\mathrm{Ph}}-\mathrm{C}_{\text {ald }}-\mathrm{O}$ & - & $114.58(19)$ \\
\hline
\end{tabular}

The ${ }^{1} \mathrm{H}-\mathrm{NMR}$ spectrum of $\mathbf{4 - K}$ is shown in Figure 1.13 and is consistent with a $\mathrm{C}_{1}$ symmetrical molecule. Similar to $\mathbf{2 - K}$, the splitting of methylene $\left(\mathrm{H}_{\text {methylene: }} 4.13\right.$ and $3.68 \mathrm{ppm}$ ) and NacNac backbone signals ( $\mathrm{H}_{\mathrm{NacNac}}$ : 4.61 and $\left.4.36 \mathrm{ppm}\right)$ is observed. This is expected based on the crystal structure, due to the bridging coordination of the aldehyde, resulting in the phenyl positioning of the aldehyde vertical to the ligand plane. Interestingly, the methylene signal at $3.68 \mathrm{ppm}$ shows no diastereotopy and is observed as a singlet. Interpretation of the whole spectra was rather challenging, due to the overlapping of multiple signals in the aromatic region of the ${ }^{1} \mathrm{H}-\mathrm{NMR}$ and ${ }^{13} \mathrm{C}-\mathrm{NMR}$ spectra. In this regard, application of 2D spectra did not proof successful, because of the same reason. Concerning the bridging ligand, the proton of the aldehyde is found at $2.03 \mathrm{ppm}$ significantly high field shifted compared to typical chemical shifts for aldehydes of $\sim 10-11 \mathrm{ppm}$. The corresponding ${ }^{13} \mathrm{C}$-NMR signal is observed at $86.70 \mathrm{ppm}$, as expected, high field shifted compared to typical aldehydes signal mostly found around $190-200 \mathrm{ppm}$. All these 
observations clearly give evidence for a two-electron reduced species. The chemical shifts are consistent with the elongation of the $\mathrm{C}-\mathrm{O}$ bond in the molecular structure of 4-K.

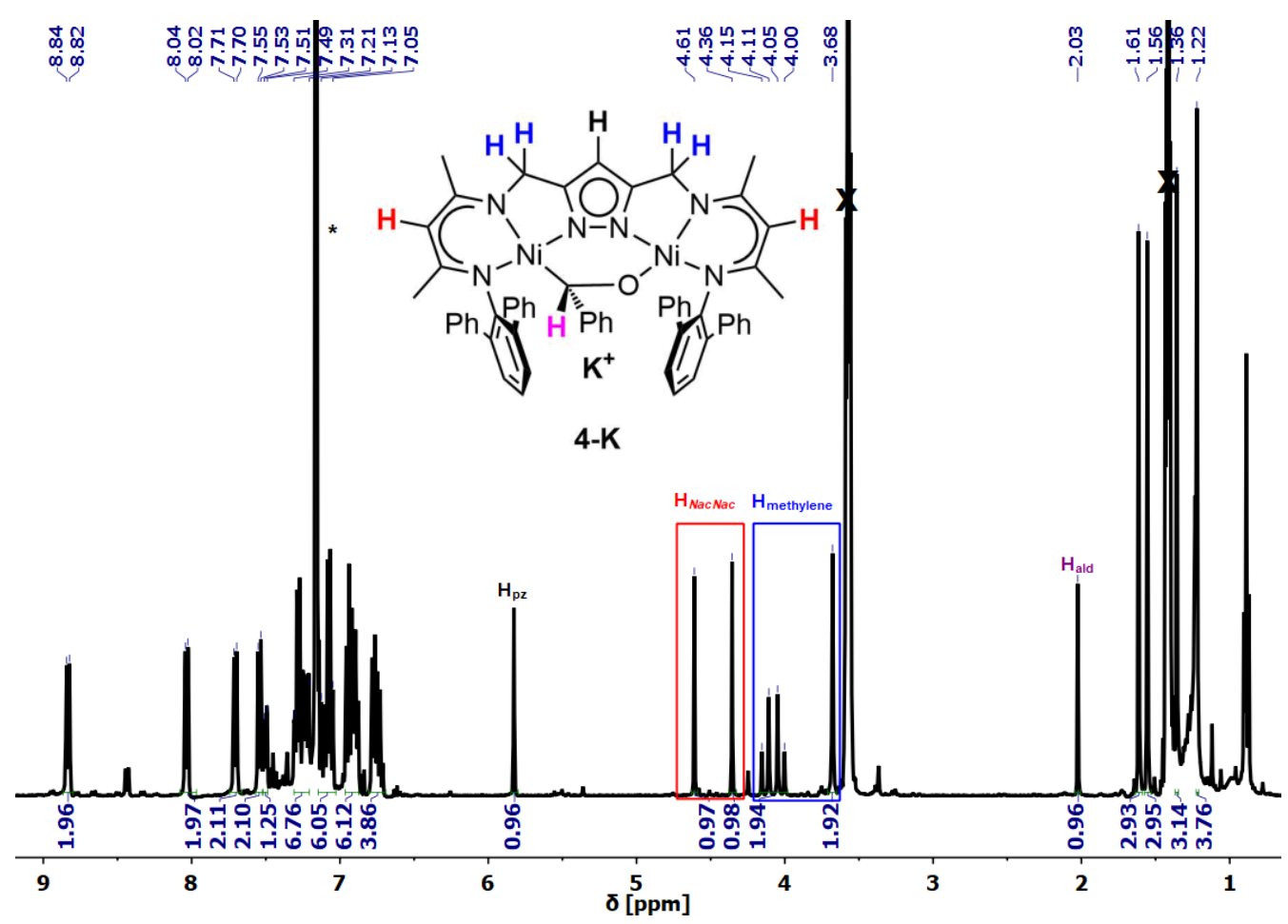

Figure $1.13{ }^{1} \mathrm{H}-\mathrm{NMR}$ spectrum of 4-K in benzene- $\mathrm{d}_{6}\left({ }^{*}\right)$; specific protons are marked in colors; THF impurity due to crystallization is marked with $\mathbf{X}$.

In contrast to $\mathrm{K}\left[\mathrm{L}^{i \mathrm{Pr}^{2}} \mathrm{Ni}_{2}\left(\mu-\mathrm{CH}_{2} \mathrm{CHPh}\right)\right] 3^{\text {iPr }}$-Sty (Scheme 1.12 ), the phenyl signals of the benzaldehyde do not show a significant low-field shift and dynamic behavior. ${ }^{32}$ The origin of the remaining proton on the aldehyde was investigated through deuteration experiments. For this $d_{1}$-benzaldehyde was synthesized according to the literature. ${ }^{74}$ Reaction of it with 2-K resulted in the formation of 4-K-D and commutant 1-OH. This outcome is due to the reasonable water content of the aldehyde after workup. Further purification through distillation or molecular sieves did not result in a lower water content. The resulting ${ }^{2} \mathrm{H}-$ NMR spectrum (Appendix) revealed the presence of $d_{1}$-benzaldehyde ${ }^{74}(9.12 \mathrm{ppm})$ signal and assumed $d_{1}$-benzyl alcohol ${ }^{75}(4.60 \mathrm{ppm})$. The later one would be expected as the hydrolysis byproduct of 4-K-D.

In summary, 2-K shows a reactivity towards benzaldehyde, resulting in the incorporation of the doubly reduced dianionic species similar to doubly deprotonated benzyl alcohol. This two-electron reduction was shown by XRD analysis, NMR and IR spectroscopy. The crystal structure shows an overall negatively charged complex compensated by a potassium cation. The aldehyde carbon appears to be $\mathrm{sp}^{3}$-hydridized and the bond length to the oxygen is close to that of free benzyl alcohol. The high-field shift of the aldehyde proton signal is consistent with a reduced species. Additionally, the CO stretching frequency in the IR spectrum is not observed in the region for the aldehyde and therefore overlaid by other 
bands in the lower energy region. To the best of our knowledge, such reactivity has not been observed for nickel hydride systems or bimetallic nickel systems. Similar complexes have only been observed for mononuclear $\mathrm{Ni}(0)$ aldehyde adducts with strongly donating ligands resulting in a strong back bonding and reduction of the aldehyde. ${ }^{76-83}$

Overall the results are in agreement with the mechanistic study of the dihydrogen reactivity of the previous chapter and support the remarkable hypothesis that $\mathbf{2 - K}$ is a "masked" $\mathrm{Ni}(\mathrm{I}) \mathrm{Ni}(\mathrm{I})$ system with a reversible intramolecular $\mathrm{C}-\mathrm{H}$ activation, which can be used as a two-electron reservoir. The equilibrium between $\mathbf{2}-\mathbf{K}$ and the $\mathrm{Ni}(\mathrm{I}) \mathrm{Ni}(\mathrm{I})$ intermediate lies far on the side of $\mathbf{2 - K}$, so only trapping experiments with reactive molecules, such as dihydrogen/deuterium or aldehyde, gave evidence for this interesting phenomenon (Scheme 1.31). Similar reactivity for nickel hydride complexes undergoing reductive elimination of a phenyl substituent has not been observed in the literature ${ }^{10}$ and shows the interesting properties of bimetallic systems utilizing metal-metal cooperativity resulting in unique reactivity. In the upcoming chapters the effect of the Lewis acidity of the alkali metals will be discussed.

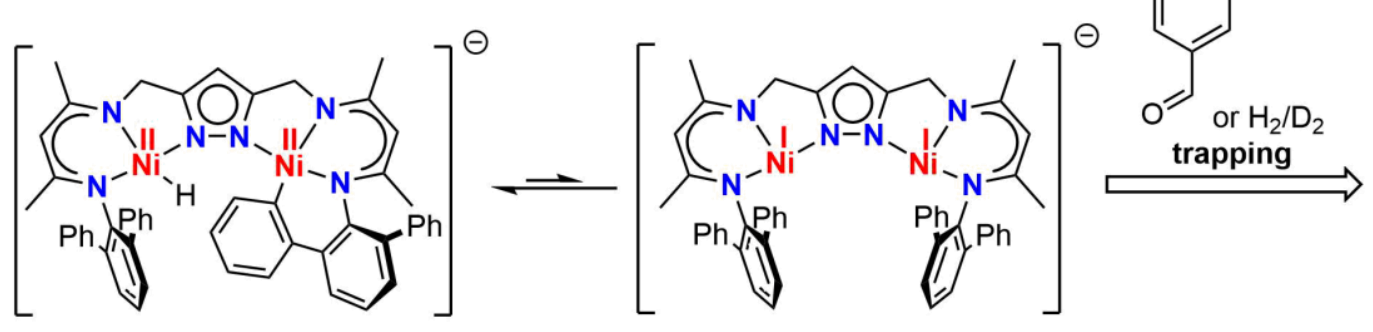

Scheme 1.31 Equilibrium of 2-K and [ $\mathrm{Ni}(\mathrm{I}) \mathrm{Ni}(\mathrm{I})]$, only observable through trapping / reactivity of the instable $[\mathrm{Ni}(\mathrm{I}) \mathrm{Ni}(\mathrm{I})]$ species with reactive electron acceptors, e.g. $\mathrm{H}_{2} / \mathrm{D}_{2}$ or benzaldehyde. 


\subsection{Influence of the Alkali Metal Cation on the C-H Activation}

As discussed in the previous chapter, the species 2-K has remarkable properties, due to the reversibility of the $\mathrm{C}-\mathrm{H}$ activation via to a $\mathrm{Ni}(\mathrm{I}) \mathrm{Ni}(\mathrm{I})$ species and possible two-electron reduction of substrates such as $\mathrm{H}_{2} / \mathrm{D}_{2}$ or benzaldehyde. The dihydride species 3-K, only observable under $\mathrm{H}_{2}$-atmosphere, has a low stability in the absence of dihydrogen and reacts back to the $\mathrm{C}-\mathrm{H}$ activated species $\mathbf{2}-\mathrm{K}$ through release of dihydrogen. Further studies of Duan showed that exchanging the potassium cation to sodium resulted in a higher stability of the dihydride species, which allowed isolation of a mixture of both after reaction. This chapter focuses on the effect of the alkali metal by investigating the properties of the sodium salts 2-Na and 3-Na.

\subsubsection{Exchange of Potassium with Sodium: $\mathrm{C}-\mathrm{H}$ Activated Species 2-Na and Dihydride Species 3-Na}

Like mentioned above, the reaction of 1 with $\mathrm{KHBEt}_{3}$ allows the isolation of the monotucked-in species 2-K, which can only be transformed in the dihydride species utilizing dihydrogen. In contrast, when $\mathbf{1}$ is reacted with $\mathrm{NaHBEt}_{3}$ a mixture of both species 2-Na and 3-Na is isolated (Scheme 1.32). ${ }^{57}$

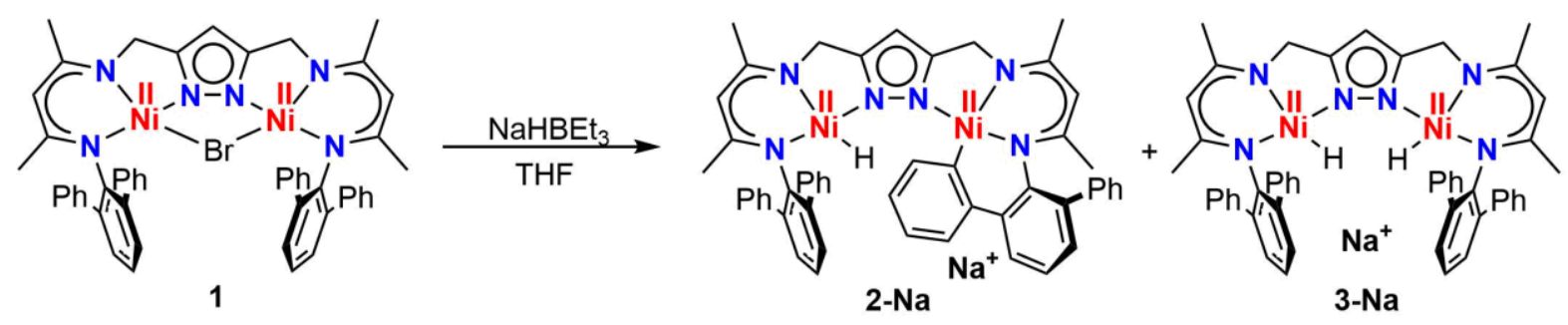

Scheme 1.32 Reaction of the bromide precursor 1 with $\mathrm{NaHBEt}_{3}$, resulting in a mixture of $\mathrm{C}-\mathrm{H}$ activated 2-Na and dihydride 3-Na.

No correlation of the ratio $\mathbf{2 - N a / 3 - N a}$ to the reaction conditions was found, indicating that the change of ratio is mostly dependent on the crystallization behavior. An influence of the stoichiometry of $\mathrm{NaHBEt}_{3}$ relative to 1 was not observed and always resulted in a 1:1 mixture before workup as shown by ${ }^{1} \mathrm{H}-\mathrm{NMR}$ spectroscopy. This outcome also indicates again the crystallization condition, due to the ratios different from 1:1 after workup. Therefore, no selective synthesis for 2-Na could be developed prior to this work. 3-Na was isolated through reaction of the mixture with dihydrogen and subsequent crystallization. A mixture of 2-Na and 3-Na or the isolated compounds were characterized through NMR, IR, UV/vis spectroscopy and XRD analysis, but for later reference and comparison the NMR data and the XRD analysis done by Dr. P.-Ch. Duan will be discussed shortly. The ${ }^{1} \mathrm{H}-\mathrm{NMR}$ spectra of a typical mixture of 2-Na and 3-Na and the separated species are presented in Figure 1.14. 


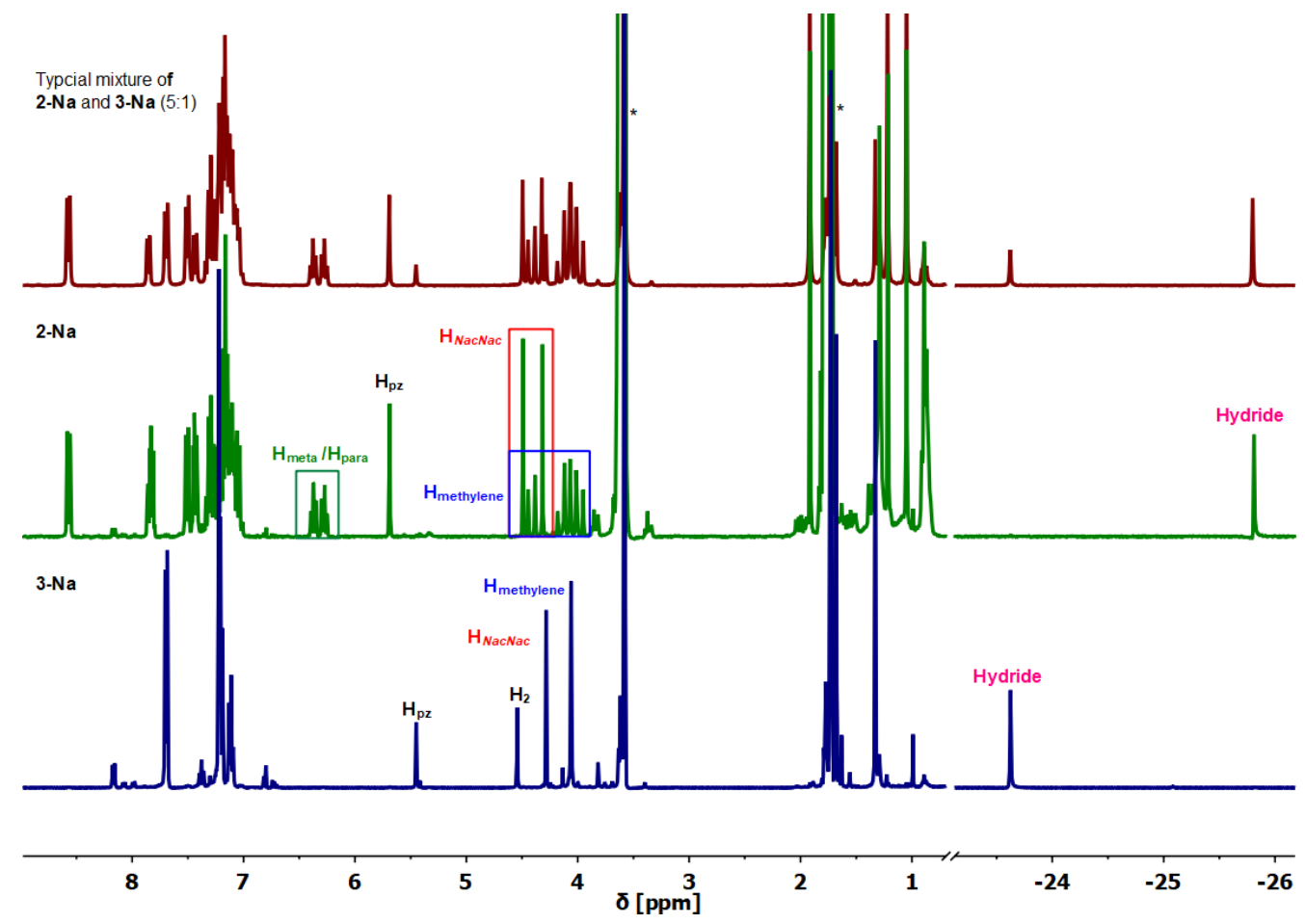

Figure $1.14{ }^{1} \mathrm{H}-\mathrm{NMR}$ spectra in $\mathrm{THF}-\mathrm{d}_{8}\left(^{*}\right)$ : top: mixture of 2-Na and 3-Na $(5: 1$ based on the ratio of the pyrazole signal); middle: isolated 2-Na; bottom: $3-\mathrm{Na}$ under $\mathrm{H}_{2}-$ atmosphere.

The overall ${ }^{1} \mathrm{H}-\mathrm{NMR}$ spectrum of $\mathbf{2 - N a}$ appears similar to $\mathbf{2 - K}$, because of the lack of twofold symmetry. In contrast, the ${ }^{1} \mathrm{H}-\mathrm{NMR}$ spectrum of 3-Na still shows $\mathrm{C}_{2 \mathrm{v}}$ symmetry. This assignment is based on the equivalent methylene and $\mathrm{NacNac}$ backbone protons resulting in two singlets each, due to the $\mathrm{C}_{2}$ rotation axes and the mirror plane $\sigma_{\mathrm{v}}$. Although the spectra look similar to the potassium analogue, small differences in proton shifts can be identified, due to the difference in Lewis acidity of the two alkali metals. This is mostly found for the signals where an interaction with the alkali metal is most probable in solution. Therefore, the hydride signal of $\mathbf{2 - N a}$ is found with a shift of $\Delta \delta_{\text {hydride }}=0.30 \mathrm{ppm}$ compared to $\mathbf{2 - K}$. The difference is even smaller for the changes of the dihydride species $\mathbf{3 - N a}$ and $3-K\left(\Delta \delta_{\text {hydride }}=+0.20 \mathrm{ppm}\right)$. The splitting of the triplets for the meta and para protons of the coordinated phenyl ring in $\mathbf{2 - N a}$ and $\mathbf{2 - K}$ are found to be separated more for the potassium analogue, resulting in a chemical shift separation of $\Delta \delta=+0.08 \mathrm{ppm}$ for 2 -Na and $\Delta \delta=+0.10 \mathrm{ppm}$ for $\mathbf{2}-\mathbf{K}$. Additionally, the triplet signal at $6.37 \mathrm{ppm}$ is not changing with differing the cation. An explanation for that behavior might be the interaction of the potassium with the $\pi$-system. This is found in the crystal structure of $\mathbf{2 - K}$ and is likely still observed in solution. Due to the presence of both complexes 2-Na and 3-Na, sequential crystallization for XRD analysis of both species was possible.

The two structures are shown in Figure 1.15 and a table with selective bond lengths and angles, together with those of $\mathbf{2 - K}$ as a reference will be presented later on (Table 1.3). While the dihydride complex 3-Na presents two nearly square planar environments, a distortion of one nickel center is found for 2-Na due to the phenyl coordination. This similarity to the potassium analogue $\mathbf{2}-\mathbf{K}$ is also shown trough bond angle. The angle of the 
$\mathrm{N}_{\text {NacNac }}-\mathrm{Ni}-\mathrm{N}_{\text {trans }}$ is observed at nearly the optimal $180^{\circ}$ for $\mathbf{3}-\mathrm{Na}\left(175.91(11)^{\circ}\right.$ and $178.19(11)^{\circ}$ in comparison to $2-\mathbf{N a}\left(164.96(7)^{\circ}\right.$ and $\left.176.70(8)\right)$. This is also consistent with the earlier discussed dihydride 3-K(crypt), in which distortion is less pronounced. As expected from 2-K, the biggest distortion is found on the phenyl coordinated nickel center, in which the phenyl group is lifted out of the $\mathrm{N}_{\mathrm{NacNac}}-\mathrm{Ni}-\mathrm{N}_{\mathrm{NacNac}}$ plane by $26.06^{\circ}$, even more pronounced than for $\mathbf{2}-\mathbf{K}\left(21.45^{\circ}\right)$. This difference is also shown in the Ni-Ni distance being even longer for 2-Na than for 2-K, 4.3451(5) and 4.2658(8) $\AA$, respectively.
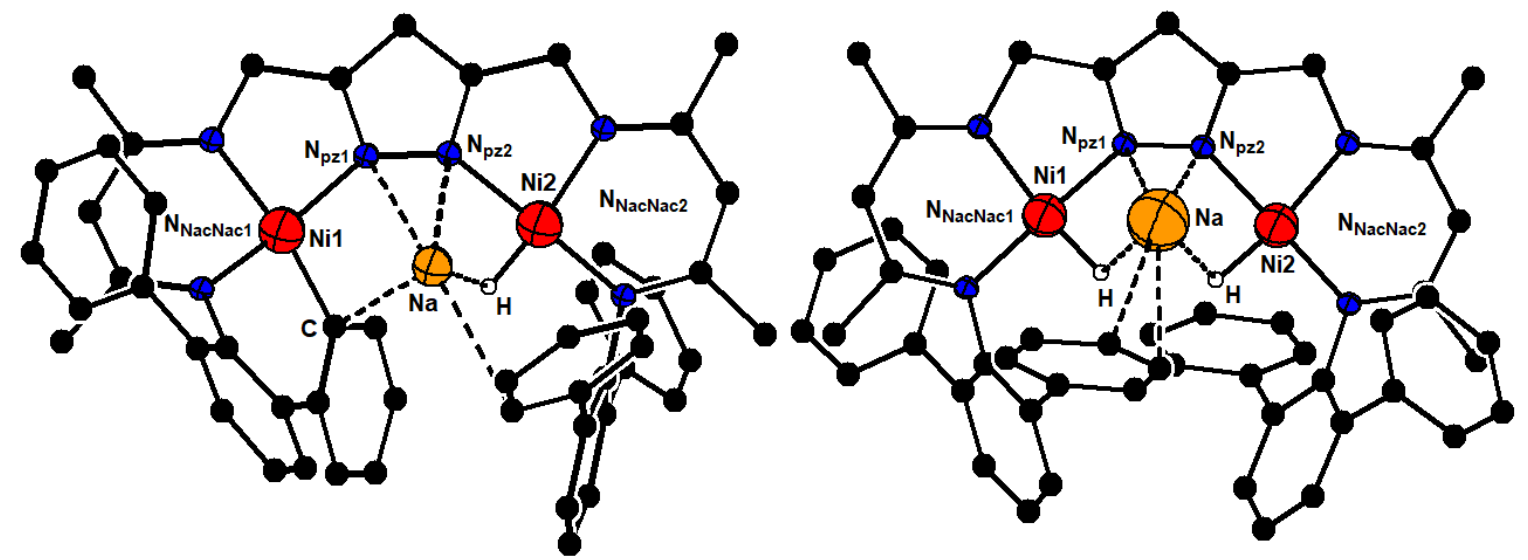

Figure 1.15 Molecular structures of 2-Na (left) and 3-Na (right); hydrogen atoms and the solvent molecules are omitted for clarity. ${ }^{57}$

Table 1.3 Selected bond lengths and angles of $\mathrm{C}-\mathrm{H}$ activated $2-\mathrm{K}^{57}, \mathbf{2 -}-\mathrm{Na}^{57}$ and dihydride species $3-\mathrm{Na}^{57}$ and 3-K(crypt).

\begin{tabular}{|c|c|c|c|c|}
\hline $\mathrm{d}[\AA ̊] / \angle\left[^{\circ}\right]$ & 2-Na & $2-K$ & $3-\mathrm{Na}$ & 3-K(crypt) \\
\hline \multirow{2}{*}{ Ni1-N $N_{\text {NacNac1 }}$} & $1.8810(15)$ & $1.879(3)$ & $1.859(3)$ & $1.8709(16)$ \\
\hline & $1.9178(15)$ & $1.915(3)$ & $1.914(2)$ & $1.9154(17)$ \\
\hline \multirow{2}{*}{ Ni2-N $\mathrm{N}_{\mathrm{NacNac2}}$} & $1.8818(16)$ & $1.877(4)$ & $1.873(2)$ & $1.8645(17)$ \\
\hline & $1.9105(15)$ & $1.936(4)$ & $1.903(3)$ & $1.9179(17)$ \\
\hline Ni1-N ${ }_{p z 1}$ & $1.9250(15)$ & $1.910(3)$ & $1.863(3)$ & $1.8651(16)$ \\
\hline $\mathrm{Ni2}-\mathrm{N}_{\mathrm{pz2}}$ & $1.9392(16)$ & $1.933(3)$ & $1.873(2)$ & $1.8672(17)$ \\
\hline $\mathrm{Ni}-\mathrm{C}$ & $1.900(18)$ & $1.902(4)$ & - & - \\
\hline \multirow{2}{*}{$\mathrm{Ni}-\mathrm{H}$} & \multirow{2}{*}{$1.34(3)$} & \multirow{2}{*}{$1.29(3)$} & $1.40(3)$ & $1.39(2)$ \\
\hline & & & $1.46(3)$ & $1.42(2)$ \\
\hline $\mathrm{Ni} \cdots \mathrm{Ni}$ & $4.3451(5)$ & $4.2658(8)$ & $4.0566(6)$ & $4.0478(8)$ \\
\hline $\mathrm{N}_{\text {trans }}-\mathrm{Ni}-\mathrm{C}$ & $153.94(8)$ & $158.55(17)$ & - & - \\
\hline \multirow{2}{*}{$\mathrm{N}_{\text {trans }}-\mathrm{Ni}-\mathrm{N}_{\mathrm{pz}}$} & $164.96(7)$ & $167.15(14)$ & $175.91(11)$ & $177.67(7)$ \\
\hline & $176.70(8)$ & $177.22(15)$ & $178.19(11)$ & $178.71(7)$ \\
\hline
\end{tabular}


Concerning the bond lengths only small differences can be observed. As already mentioned, the $\mathrm{Ni}-\mathrm{H}$ bond lengths is prone upon misleading due to the low resolution of the hydrogen atom in XRD analysis and therefore will not be discussed. For the Ni-C bond of 2-Na and 2-K the difference is small with 1.900(18) and 1.902(4) $\AA$, respectively. Interestingly, the interaction with the cation is as expected based on the ${ }^{1} \mathrm{H}-\mathrm{NMR}$ data. While for 2-K an interaction with the ortho and meta carbons of the coordinated ring is observed, for 2-Na this interaction is only found for the ortho carbon, due to the smaller size of the sodium cation. Additionally, instead of the chain-like structure for $\mathbf{2}-\mathbf{K}$, the interaction with one molecule of THF is found in the solid-state structure of 2-Na.

Through the discussed interaction of the alkali metal with the complex, changes of the properties are expected. Interestingly, these influence results in the higher stability of the species 3-Na compared to 3-K, which allowed isolation of 3-Na for characterization purposes. To investigate the stability, 3-Na was treated through freeze-pump-thaw procedure to evaluate the $\mathrm{C}-\mathrm{H}$ activation to $2-\mathrm{Na}$ by ${ }^{1} \mathrm{H}-\mathrm{NMR}$ spectroscopy (Figure 1.16).

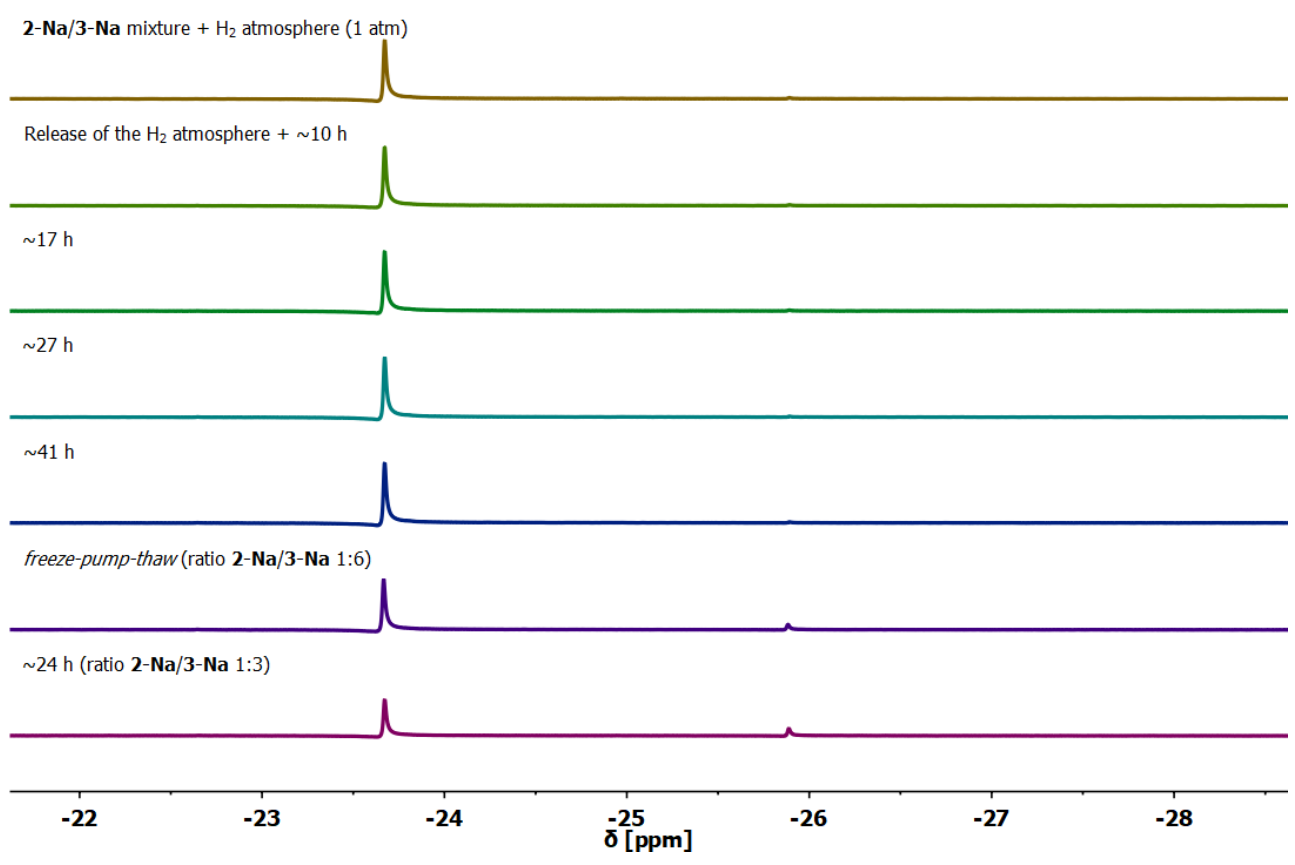

Figure 1.16 ${ }^{1} \mathrm{H}-\mathrm{NMR}$ spectra in THF-d8 of 3-Na over time to evaluate the stability of $3-\mathrm{Na}\left(\delta_{\text {Hydride }}=-23.63 \mathrm{ppm}\right.$ ) to undergo $\mathrm{C}-\mathrm{H}$ activation to $2-\mathrm{Na}$ ( $\delta_{\text {Hydride }}$ $=-25.81 \mathrm{ppm}$ ) through exchange of atmosphere by opening in a dinitrogen atmosphere and freeze-pump-thaw procedure.

The changes over time before applying a freeze-pump-thaw procedure show the stability of the complex in dihydrogen saturated solution. Even after freeze-pump-thaw 3-Na was observed in a ratio of $6: 1$ and after 24 hours $3: 1$. This stability is also the reason, why a complete transformation to 2-Na was not observed, but more degradation to the hydrolysis product 1-OH and a later discussed new species 5-Na (vide infra) was found over a longer period of time. A selective way for the transformation to $\mathbf{2 - N a}$ is therefore missing, which is why, one of the main focus of this work concentrated on the understanding of the 
interconversion between 2-Na and 3-Na. These results of the investigation will be discussed in the next section.

\subsubsection{Investigations of the Interconversion between $\mathrm{C}-\mathrm{H}$ Activated Species 2-Na and Dihydride 3-Na}

Although 2-K is isolable right after workup of the reaction of $\mathbf{1}$ with the corresponding superhydride, 2-Na is found in a mixture with the dihydride species 3-Na. As discussed in the previous section, this mixture is rather difficult to fully transform into the $\mathrm{C}-\mathrm{H}$ activated species 2-Na, but the dihydride 3-Na can be produced by addition of dihydrogen (Scheme 1.33). A thorough understanding of the interconversion of those two compounds is therefore relevant to evaluate future reactivity differences in comparison to the potassium analogue. Because of these reasons, a selective isolation to 2-Na was sought.

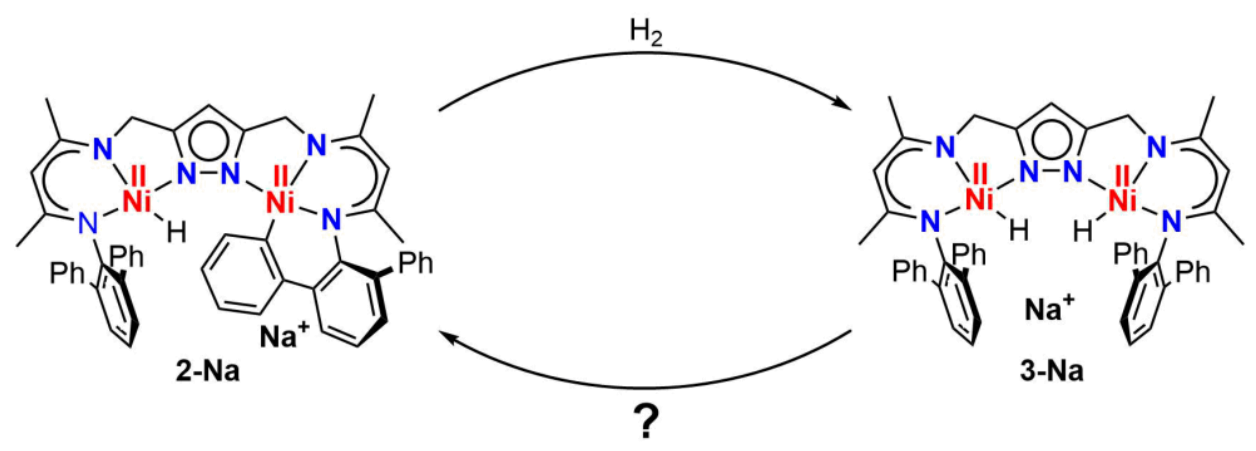

Scheme 1.33 Interconversion of 2-Na and 3-Na; with a missing condition for the transformation to the $\mathrm{C}-\mathrm{H}$ activated species 2-Na.

The already known reaction of 2-Na with dihydrogen was monitored by UV/vis spectroscopy, due to individual bands for both species (Figure 1.17). The fast reactivity with dihydrogen did not allow kinetic interpretation of the transformation, because of the fast reaction with dihydrogen relative to the preparation of the experiment. For reference purposes, the UV/vis spectra of the sample after reaction with aerial moisture is shown to evaluate any hydrolysis during the application of dihydrogen.

The characteristic band of 2-Na at $540 \mathrm{~nm}$ is lost during the reaction with dihydrogen. Additionally, the mostly MLCT and $\pi \rightarrow \pi^{*}$ transition containing bands found at $393 \mathrm{~nm}$ are shifted to lower wavelength for 3-Na and increase in intensity, the latter can be attributed to the symmetry change. A shoulder at roughly $420 \mathrm{~nm}$ can be observed for 3-Na, which most may also be found in 2-Na but is overlaid by the broadness of the band at $393 \mathrm{~nm}$. The only similarity between the two spectra is a shoulder at $340 \mathrm{~nm}$, which is found for both species. The UV/vis spectrum changed when the sample was then opened to moisture in the air. Two new bands at $581 \mathrm{~nm}$ and $442 \mathrm{~nm}$ are observed that originate from 1-OH. 
The absence of those bands in the previous spectrum clearly shows that no significant hydrolysis has occurred after the application of a dihydrogen atmosphere. The very sharp band at $377 \mathrm{~nm}$ is higher in intensity but nevertheless looks similar to the band at $393 \mathrm{~nm}$ for 3-Na. All three species therefore show characteristic bands, which allows to distinguish those compounds for future investigations.

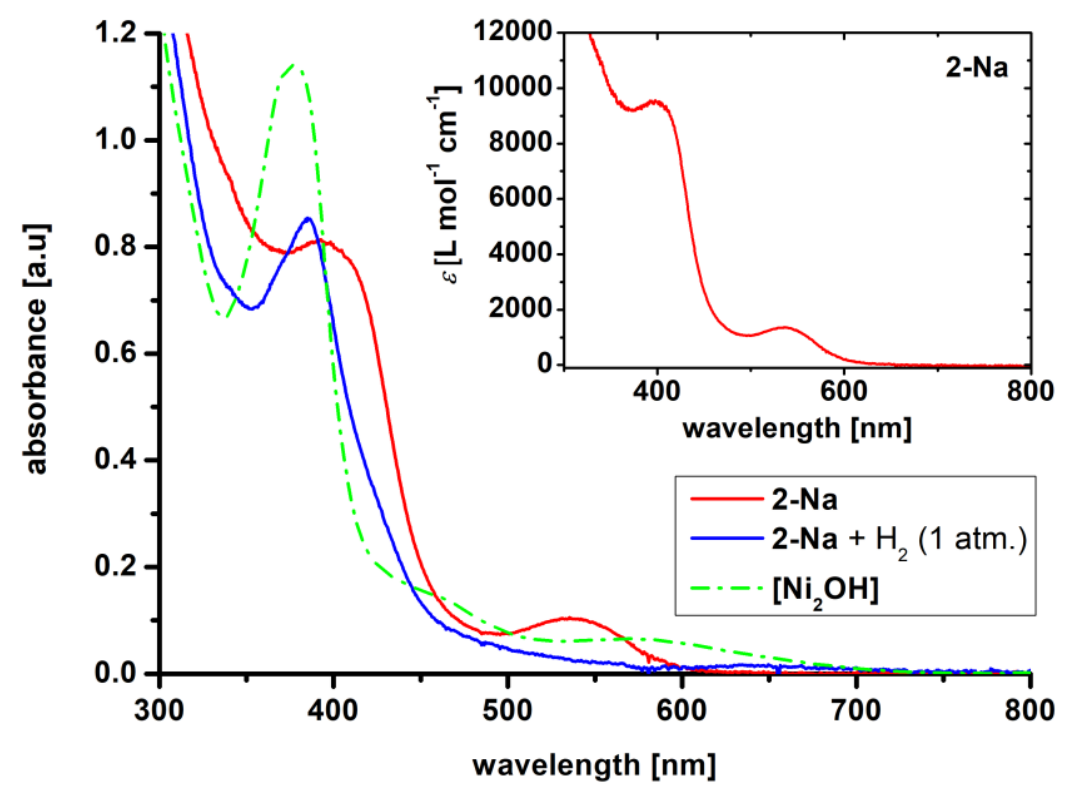

Figure $1.17 \mathrm{UV} /$ vis spectra in THF; red: $\mathrm{C}-\mathrm{H}$ activated species 2-Na; blue: 2-Na under a $\mathrm{H}_{2}$ atmosphere $(1 \mathrm{~atm}$.) resulting in dihydride species 3-Na; green dotted: sample opened to moisture for hydrolysis product $1-\mathrm{OH}$ as a reference; inset: UV/vis spectrum of 2-Na with extinction coefficient.

Based on the result that 2-K can be synthesized based on $\mathbf{1}$ utilizing a strong reducing agent, such as $\mathrm{KC}_{8}$ (Section 1.4.3), a similar reactivity was expected utilizing a sodium based reducing agents. Reducing agents with a suitable reduction potential ${ }^{84}$, such as sodium amalgam, sodium dispersion on $\mathrm{NaCl}$ and sodium naphtalide (short: $\mathrm{Na}[\mathrm{Naph}]$ ), were attempted (Scheme 1.34).

Sodium amalgam $\left(\mathrm{E}^{0}=-2.36 \mathrm{~V}\right)^{84}$ does not show a suitable reduction potential and therefore led to reisolation of the bromide precursor $\mathbf{1}$. However, based on the CV of $\mathbf{1}$ (Figure 1.3) the second reduction, which is assumed to lead to the $\mathrm{Ni}(\mathrm{I}) \mathrm{Ni}(\mathrm{I})$ species, is much lower $\left(\mathrm{E}_{\mathrm{p}, \mathrm{c}}=-2.85 \mathrm{~V}\right)$. When using the sodium dispersion on sodium chloride $\left(\mathrm{E}^{0}=\right.$ $-3.04 \mathrm{~V})^{84}$ a reaction was observed. The characterization through ${ }^{1} \mathrm{H}-\mathrm{NMR}$ spectroscopy of the crude product showed two major species, one of them being 2-Na. The chloride analogue of 1 was identified through ${ }^{1} \mathrm{H}-\mathrm{NMR}$ spectroscopy as a degradation product (Scheme 1.34, middle). This behavior can be expected due to the moderate solubility of sodium chloride in THF and therefore incorporation into the complex, similar to other anions such as triflate (Section 1.6.2). ${ }^{57}$ As an alternative reductant sodium naphalide $(\mathrm{Na}[\mathrm{Naph}])$ was employed. Addition of two equivalents of $\mathrm{Na}[\mathrm{Naph}]$ resulted in the isolation of the $\mathrm{C}-\mathrm{H}$ activated species 2-Na with a yield of $96 \%$. It has to be noted, that the 
use of a significant excess of sodium naphtalide resulted in the formation of side products and therefore strict stoichiometry had to be maintained. Also, for a dearth of sodium naphtalide, for example one equivalent, only partial conversion to 2-Na was observed. The discovery of a selective synthesis of 2-Na allowed further studies on the C-H activated species, but did not give information on the interconversion of the two hydride species 2Na and 3-Na.

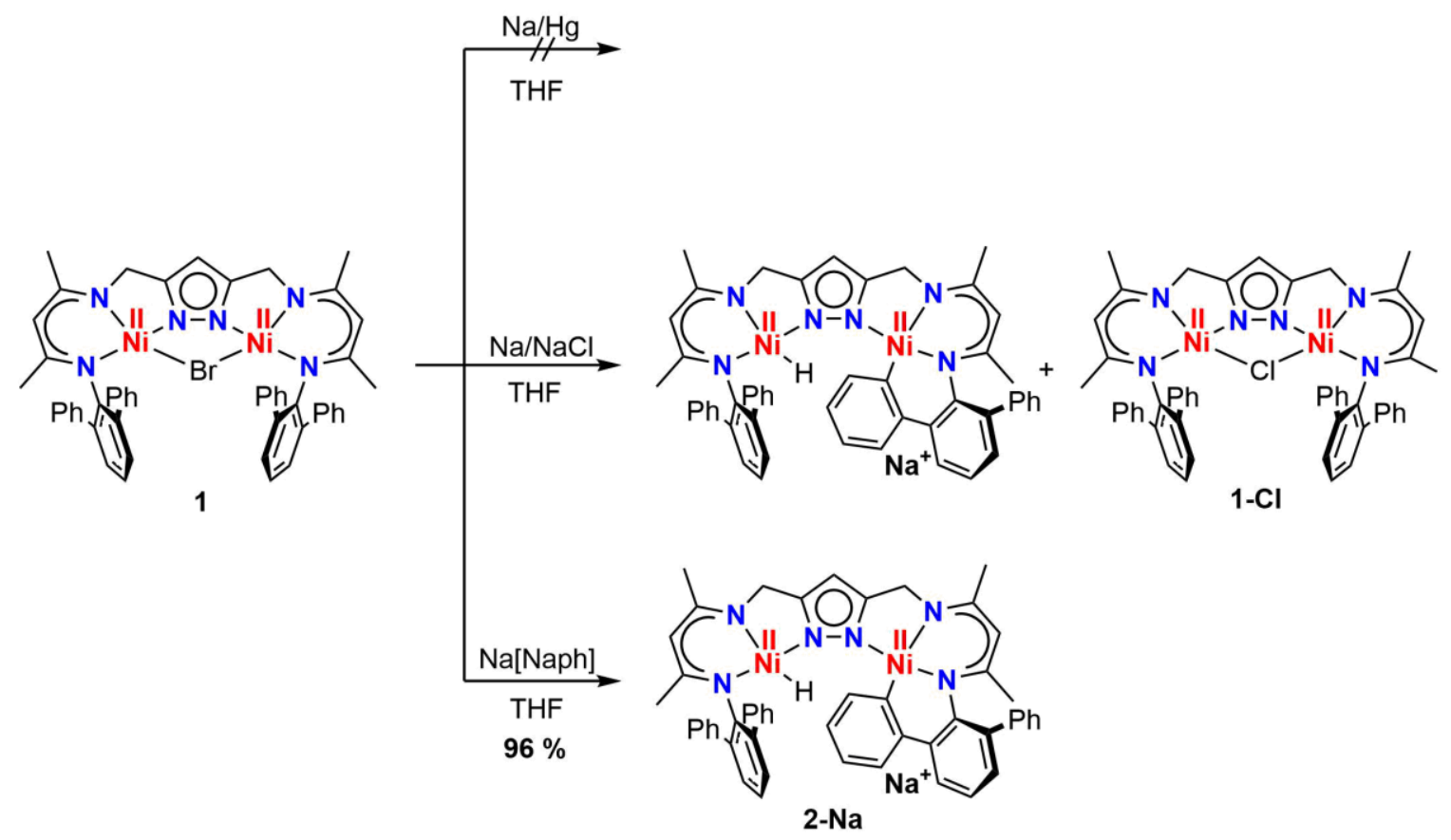

Scheme 1.34 Reduction of bromide precursor 1 with various strong reducing agents.

Based on $\mathrm{H}_{2}$ release as a potential thermodynamic driving force for the interconversion of 3-Na to 2-Na, increase of temperature was tested. Therefore, a mixture of 2-Na and 3-Na was warmed up to $60^{\circ} \mathrm{C}$ in an NMR-experiment and the changes in ratio between 2-Na and 3-Na over time were monitored. The resulting spectra are shown in Figure 1.18 over a time period of 2 days after applying a freeze-pump-thaw procedure. Although the ratio between the two species did not change, the overall quantity of both decreased and a new species featuring the pyrazole signal at $5.66 \mathrm{ppm}$, two new triplet signals at 5.91 and $6.25 \mathrm{ppm}$ and no corresponding hydride signal was observed. Additionally, only a singlet at $4.19 \mathrm{ppm}$ for the NacNac backbone protons was found, but the diastereotopy of the methylene protons (doublets at 4.26 and $4.08 \mathrm{ppm}$ ) was still present. The assignment indicates that the new product 5-Na likely has $\mathrm{C}_{2}$-symmetry. Because of the similarity of the triplets for the aromatic protons of 5-Na to those of 2-Na, just being more separated and increased in relative intensity, indicated a $\mathrm{C}-\mathrm{H}$ activation. The relative integrals of the triplets correspond to two protons each, which emphasized, that a second $\mathrm{C}-\mathrm{H}$ activation is present. This hypothesis is supported by the molecular structure of the new species of 5-Na (vide infra). Treating 2-Na solely under the same reaction conditions resulted in short reaction times, which suggests $\mathbf{2}-\mathbf{N a}$ as the precursor for the second $\mathrm{C}-\mathrm{H}$ activation. 
Although the transformation to the new species 5-Na was shown, the full interconversion between 2-Na and 3-Na was still not accomplished. In contrast, freeze-pump-thaw experiments at room temperature resulted in the mentioned hydrolysis product 1-OH next to a small amount of 5-Na without high changes of the ratio 2-Na/3-Na. In summary, no conditions were found to promote $\mathrm{C}-\mathrm{H}$ activation and fully convert 3-Na into 2-Na, but the selective synthesis via reduction of $\mathbf{1}$ with sodium naphtalide allows $\mathbf{2}-\mathbf{N a}$ to be the starting point for future investigations. Additionally, through thermal degradation of a mixture of 2-Na a doubly activated species 5-Na was observed and isolated, for which the reductive elimination mechanism cannot be operative. Therefore, 5-Na should provide different properties compared to $\mathbf{2}-\mathbf{N a}$ based on the incapability of a forming $\mathrm{Ni}(\mathrm{I}) \mathrm{Ni}(\mathrm{I})$ intermediate.

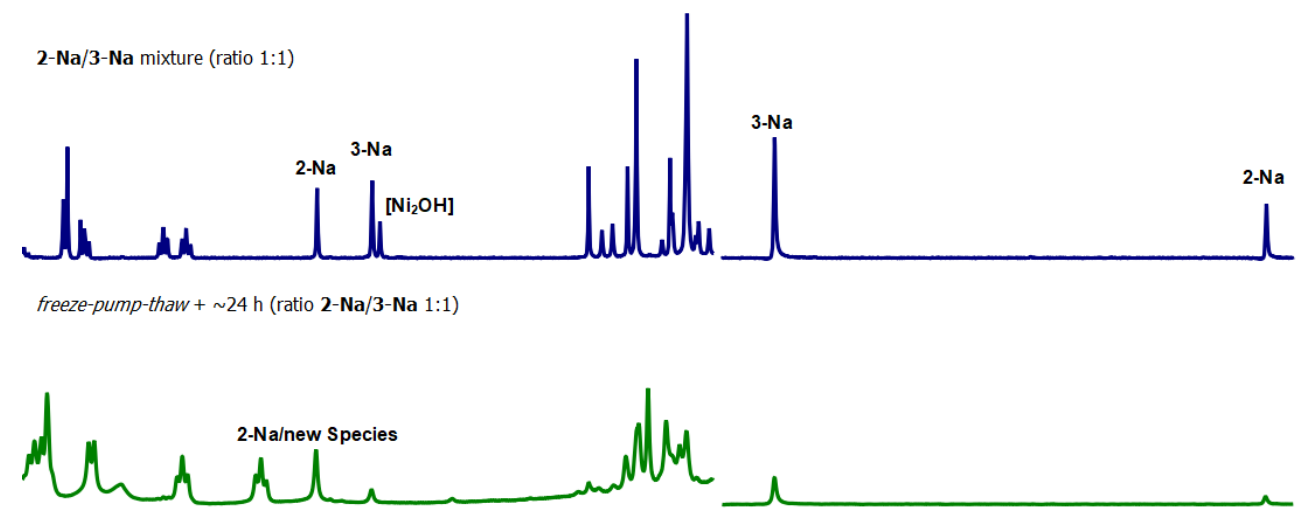

$48 \mathrm{~h}$ at $60^{\circ} \mathrm{C}$

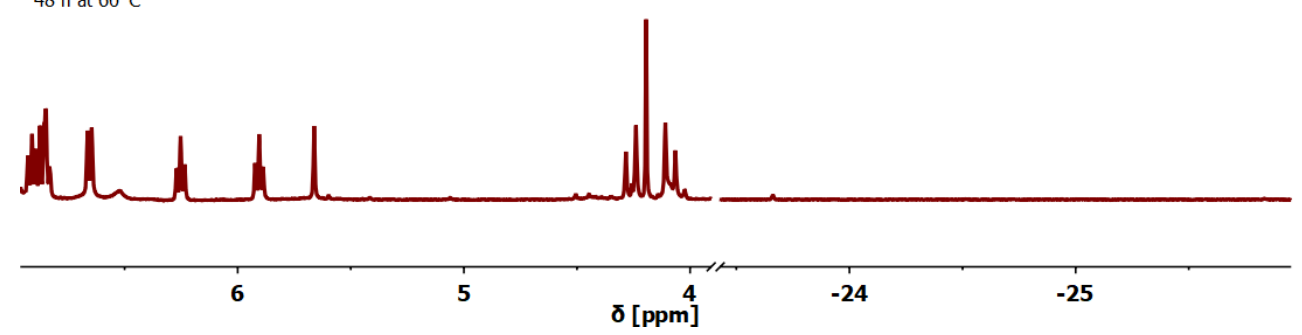

Figure 1.18 Monitoring of the interconversion of 2-Na and 3-Na in THF-d8 at $60^{\circ} \mathrm{C}$ over time; top: starting mixture of 2-Na / 3-Na 1:1; middle: $24 \mathrm{~h}$ after freeze-pumpthaw; bottom: $48 \mathrm{~h}$; 


\subsubsection{Mechanistic Investigation on the Twofold C-H Activated 5-Na}

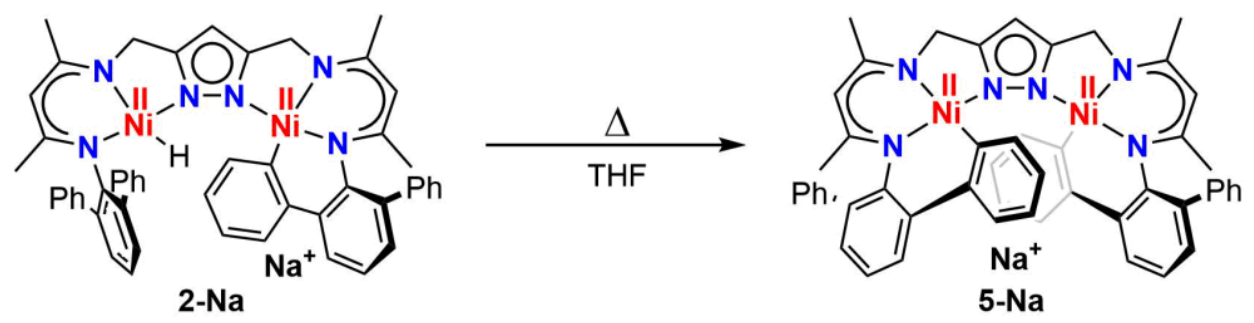

Scheme 1.35 Thermal transformation of $2-\mathrm{Na}$ into the doubly $\mathrm{C}-\mathrm{H}$ activated species 5-Na.

As already mentioned in the last chapter, the second $\mathrm{C}-\mathrm{H}$ activation to $\mathbf{5 - N a}$ is thermally promoted through heating of $\mathbf{2 - N a}$ at $60^{\circ} \mathrm{C}$ for several days (Scheme 1.35). After full transformation, monitored by ${ }^{1} \mathrm{H}-\mathrm{NMR}$ spectroscopy, 5-Na was crystallized similar to the other complexes utilizing vapor diffusion of pentane into the THF solution of 5-Na. 5-Na was then fully characterized by spectroscopic methods and XRD.

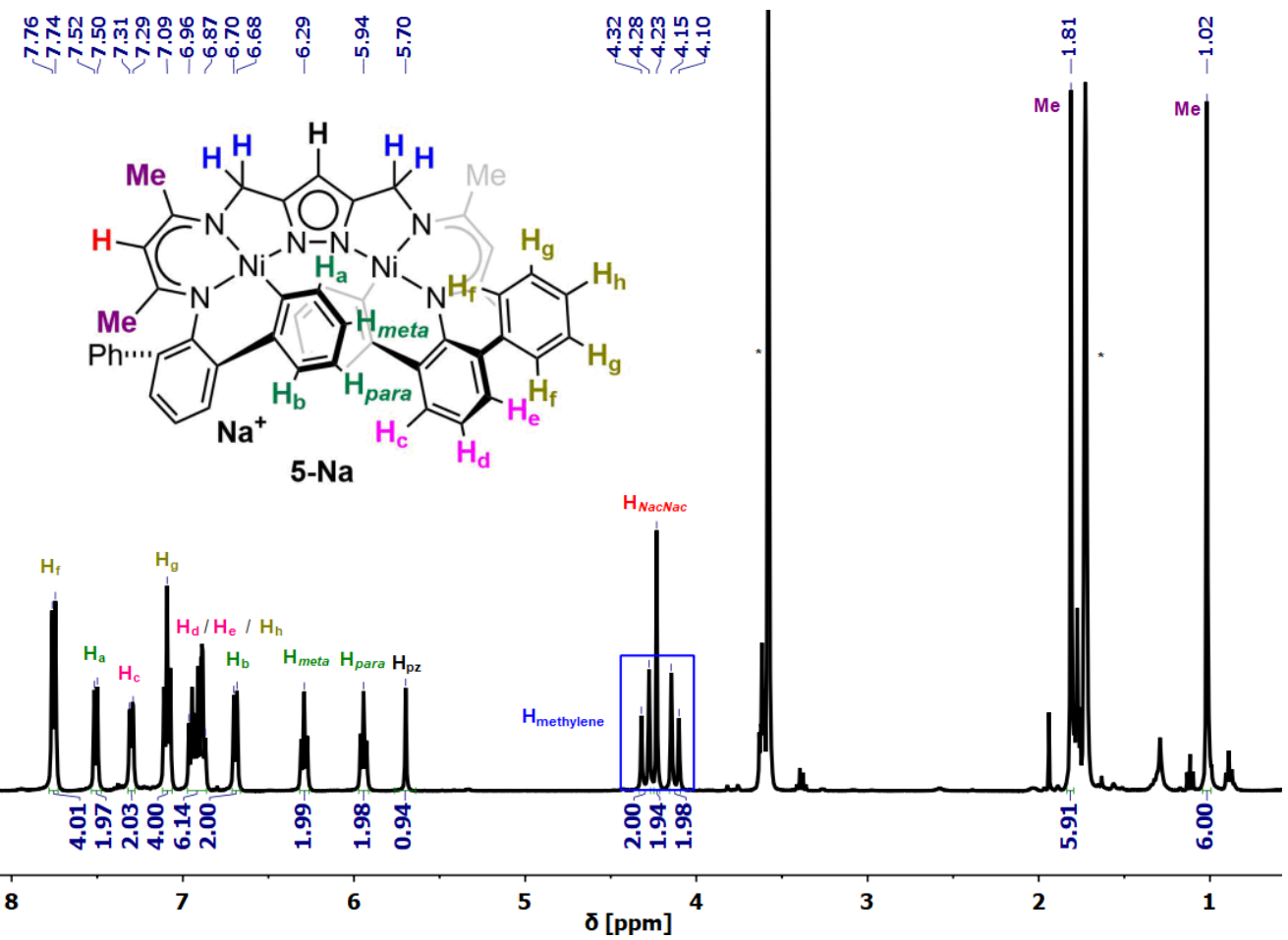

Figure $1.19{ }^{1} \mathrm{H}-\mathrm{NMR}$ spectrum of doubly $\mathrm{C}-\mathrm{H}$ activated $5-\mathrm{Na}$ in THF-ds $\left(^{*}\right)$ with full signal interpretation.

The ${ }^{1} \mathrm{H}-\mathrm{NMR}$ spectrum of $\mathrm{C}_{2}$-symmetric 5-Na (Figure 1.19) was already shortly discussed in the previous chapter, but small impurities of 2-Na, 3-Na and 1-OH did not allow full interpretation. The chemical shift difference of the meta and para protons (green, Figure 1.19 ) is with $\Delta \delta=+0.35 \mathrm{ppm}$ larger than for $\mathbf{2}-\mathbf{K}$ or $\mathbf{2 - N a}$, meaning a drastic change in the chemical environment for the protons. Interestingly, similar to the other $\mathrm{C}-\mathrm{H}$ activated 
species only the para-proton (6.29 ppm for 2-Na $v s .5 .90 \mathrm{ppm}$ for 5-Na) exhibits a drastic change of the chemical shift, while the chemical shift of the meta-proton only changes

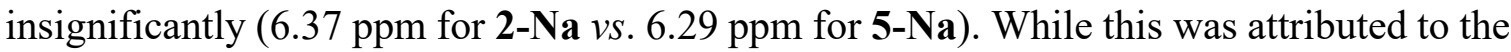
different interaction of potassium and sodium in $\mathbf{2}-\mathbf{K}$ and $\mathbf{2}-\mathbf{N a}$, for $\mathbf{5}-\mathbf{N a}$ the crystal structure showed no interaction of the alkali metal with the coordinating phenyl groups in solid state (vide infra). The most low-field shifted signal of the aromatic protons belongs to the ortho protons of the free phenyl moieties $\left(\mathrm{H}_{\mathrm{f}}\right.$ in Figure 1.19), while the first signal found for the coordinated phenyl ring is found at $7.51 \mathrm{ppm}\left(\mathrm{H}_{\mathrm{a}}\right.$ in Figure 1.19). The equivalency of both sides of the non-coordinating phenyl moiety indicates a free rotation in solution. Overall, apart from the $\mathrm{H}_{\text {meta }}$ and $\mathrm{H}_{\text {para }}$ signals, no drastic difference is observed for the chemical shifts of the remaining aromatic protons. All protons signals are correlated with the corresponding ${ }^{13} \mathrm{C}-\mathrm{NMR}$ signal (Appendix) using COSY, HSQC and HMBC NMR spectroscopy

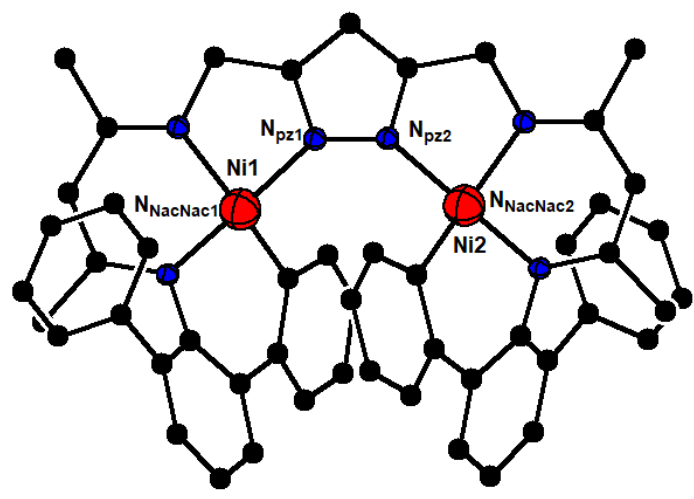

Figure 1.20 Molecular structure of doubly $\mathrm{C}-\mathrm{H}$ activated species $\mathbf{5 - N a ; ~ s o l v e n t ~ m o l e c u l e s , ~}$ cation and protons are omitted for clarity.

Table 1.4 Selected bond lengths and angles of 2-Na and 5-Na.

\begin{tabular}{|c|c|c|}
\hline $\mathrm{d}[\AA \AA \AA] / \angle\left[^{\circ}\right]$ & $2-\mathrm{Na}$ & $5-\mathrm{Na}$ \\
\hline $\mathbf{N i 1}-\mathbf{N}_{\text {NacNac1 }}$ & $\begin{array}{l}1.8810(15) \\
1.9178(15)\end{array}$ & $\begin{array}{l}1.8646(15) \\
1.9205(16)\end{array}$ \\
\hline $\mathbf{N i 2}-\mathbf{N}_{\mathrm{NacNac} 2}$ & $\begin{array}{l}1.8818(16) \\
1.9105(15)\end{array}$ & $\begin{array}{l}1.8630(18) \\
1.9310(18)\end{array}$ \\
\hline $\mathbf{N i 1}-\mathbf{N}_{\mathrm{pz} 1}$ & $1.9250(15)$ & $1.9046(16)$ \\
\hline $\mathbf{N i 2}-\mathbf{N}_{\mathrm{pz2}}$ & $1.9392(16)$ & $1.8963(18)$ \\
\hline Ni-C & $1.900(18)$ & $\begin{array}{r}1.8854(18) \\
1.900(2)\end{array}$ \\
\hline $\mathbf{N i} \cdot \cdot \mathbf{N i}$ & $4.3451(5)$ & $4.1840(8)$ \\
\hline $\mathbf{N}_{\text {trans }}-\mathrm{Ni}-\mathrm{C}$ & $153.94(8)$ & $\begin{array}{l}153.14(7) \\
153.50(8)\end{array}$ \\
\hline $\mathbf{N}_{\text {trans }}-\mathbf{N i}-\mathbf{N}_{p z}$ & $\begin{array}{l}164.96(7) \\
176.70(8)\end{array}$ & $\begin{array}{l}166.14(7) \\
166.55(6)\end{array}$ \\
\hline
\end{tabular}

The molecular structure of 5-Na is shown in Figure 1.20. Multiple crystallization attempts of 5-Na still resulted in a disorder of the sodium cation; this is why location of $\mathrm{Na}^{+}$relative to the anion is not discussed. Selected bond lengths and angles in comparison with the starting material 2-Na are shown in Table 1.4. The geometries around both nickel ions is greatly distorted. While for 2-Na the angle $\angle\left(\mathrm{N}_{\text {trans }}-\mathrm{Ni}-\mathrm{N}_{\mathrm{pz}}\right)=176.70(8)^{\circ}$ is still close to the expected $180^{\circ}$ for square planar coordination, now both metal centers are shifted out of the ligand plane to accommodate the phenyl coordination $\left(\angle\left(\mathrm{N}_{\text {trans }}-\mathrm{Ni}-\mathrm{N}_{\mathrm{pz}}\right)=166.14(8)\right.$ and $\left.166.55(6)^{\circ}\right)$. This results in nearly the same angle $\mathrm{N}_{\text {trans }}-\mathrm{Ni}-\mathrm{C}$ of around $153^{\circ}$ for both nickel ions. The similarities to one side of $\mathbf{2 - N a}$ can be also found in the nickel-carbon bond lengths, showing the same length around $1.90 \AA$, with a small deviation for one of the two nickel centers in 5-Na $(\mathrm{d}(\mathrm{Ni}-\mathrm{C})=1.8854(18) \AA)$. Interestingly, the remaining bond lengths $\left(\mathrm{Ni}-\mathrm{N}_{\mathrm{Nac}}\right.$ and $\mathrm{Ni}-\mathrm{N}_{\mathrm{pz}}$ ) and the $\mathrm{Ni}-\mathrm{Ni}$ distance shorten upon the second $\mathrm{C}-\mathrm{H}$ activation. The UV/vis spectrum of 5-Na (Appendix) shows also a band around $550 \mathrm{~nm}$, consistent with 
the UV/vis spectrum of 2-Na. When 2-K in THF was treated under the same conditions, the conversion to 5-K appeared slower compared to 5-Na. After abstraction of the alkali metal by trapping with dibenzo-18-crown-6-ether (DB18C6) no reaction of 2-Na(DB18C6) was observed (both in Appendix). This behavior shows that the alkali metal has a significant influence on the reactivity of the complexes and therefore on their capability of C-H activation or $\mathrm{H}_{2}$-activation.

Due to the missing Ni-H moiety in 5-Na a conversion back to the other complexes 2-Na and 3-Na has to proceed via a different mechanism. The reversibility of the second $\mathrm{C}-\mathrm{H}$ activation was studied through application of dihydrogen on 5-Na and monitored by ${ }^{1} \mathrm{H}$ NMR spectroscopy over time (Scheme 1.36 and Figure 1.21).

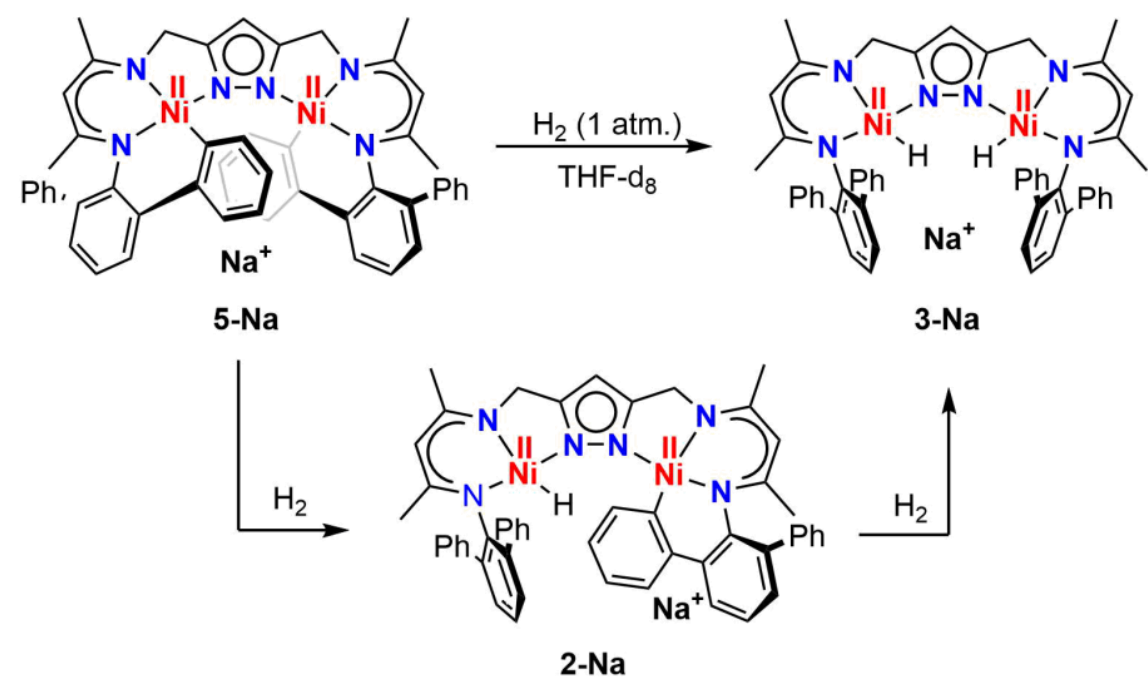

Scheme 1.36 Reaction of 5-Na with dihydrogen for investigation of the reversibility of the second $\mathrm{C}-\mathrm{H}$ activation.

A direct conversion to 3-Na is observed based on the appearance of the hydride signal at $\delta$ Hydride $=-23.63 \mathrm{ppm}$. Only a minimal amount of $\mathbf{2 - N a}$ is found $\left(\delta_{\text {Hydride }}=-25.81 \mathrm{ppm}\right)$, which indicates a two-step reaction to 3-Na with 2-Na as an intermediate. The first reaction step appears to be slower, followed by the immediate transformation of 2-Na to 3-Na. After several days no full conversion was observed, but instead the conversion remained at $41 \%$ when the solution was heated. Application of higher pressure (3 atm.) resulted in $67 \%$ conversion (Appendix). The back reaction from 3-Na to 5-Na is promoted by temperature, therefore an equilibrium is expected to be the reason for the incomplete transformation.

The slow transformation of 5-Na to 2-Na suggests a different reaction mechanism compared to the earlier discussed reductive elimination pathway (Scheme 1.27 in Section 1.4.3, left). Several deuterium experiments did not result in any deuterium signals in the ${ }^{2} \mathrm{H}-\mathrm{NMR}$ spectroscopy after 2 weeks of reaction time (Appendix). Interestingly, the reactivity of $\mathbf{2 - K}$ towards a second $\mathrm{C}-\mathrm{H}$ activation appears to be lower and therefore resulted in even longer reaction times to $\mathbf{5 - K}$. This behavior is in contrast to the first $\mathrm{C}-\mathrm{H}$ activation of $\mathbf{3}-\mathbf{K}$ to $\mathbf{2 - K}$, which readily is observed on absence of dihydrogen. This is why, future work should be focused on deuteration experiments and reactivity of 5-Na or 5-K, 
which can provide information of the nature of the complex. The time frame of this $\mathrm{PhD}$ work did not allow to investigate 5-Na further.
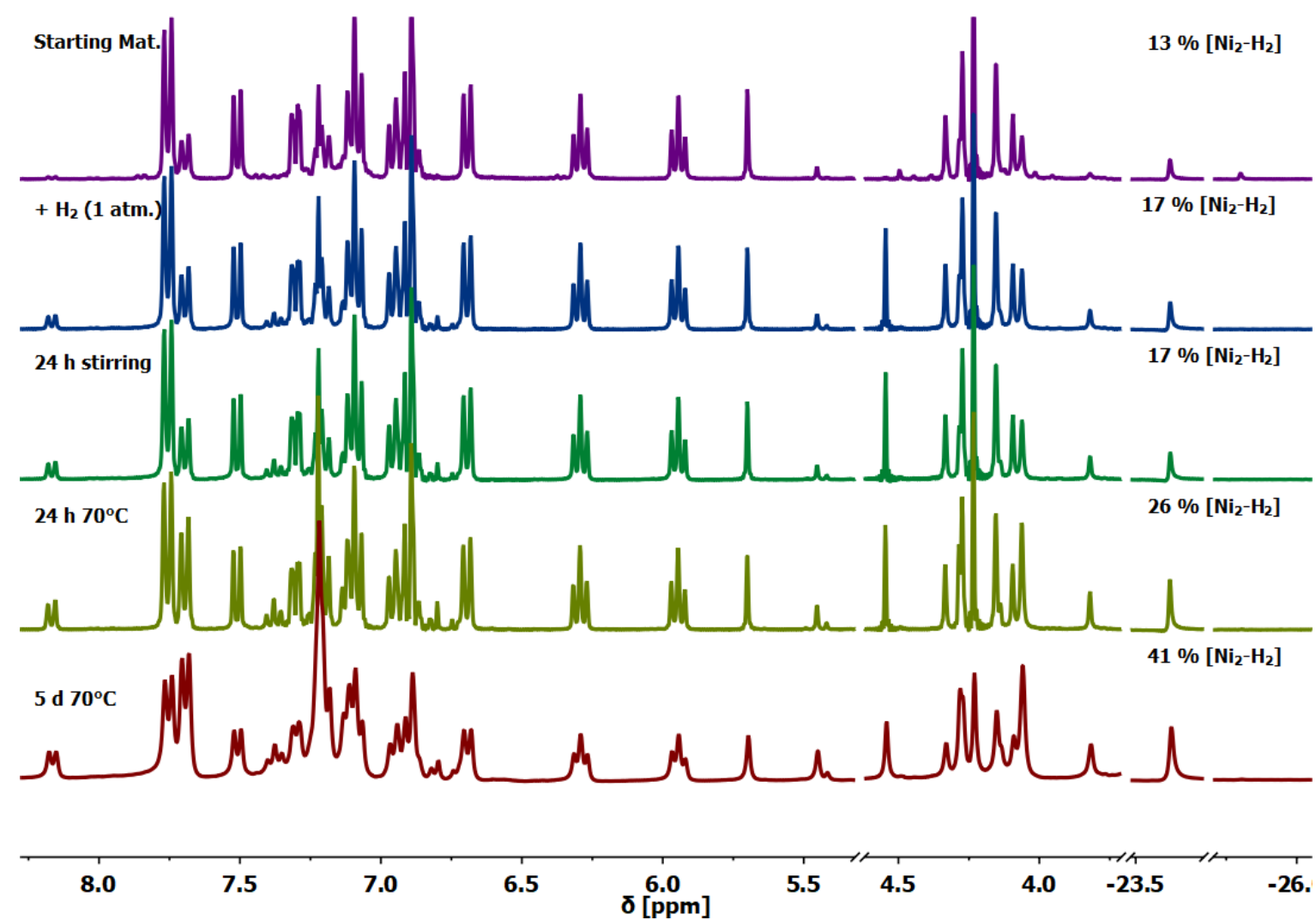

Figure 1.21 ${ }^{1} \mathrm{H}-\mathrm{NMR}$ spectra of $5-\mathrm{Na}$ in THF- $\mathrm{d}_{8}$ and under $\mathrm{H}_{2}$ atmosphere (1 atm.) over time; amount of 3-Na was investigated by the corresponding pyrazole proton signals of both species. 


\subsubsection{Competition Experiments for the Binding of $\mathrm{Na}^{+}$and $\mathrm{K}^{+}$to the $\mathrm{C}-\mathrm{H}$ Activated Species 2-Na and 2-K in Solution}

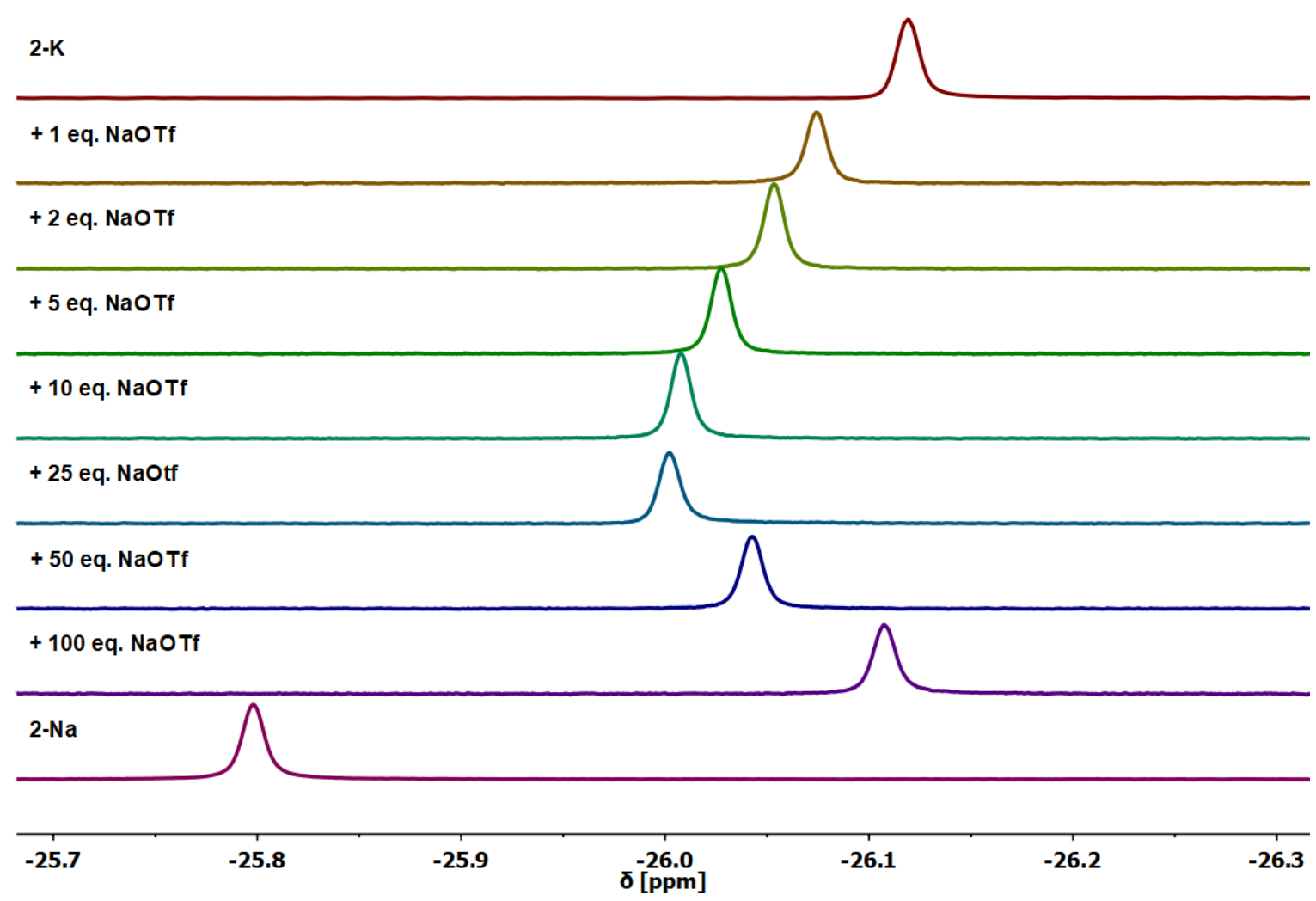

Figure $1.22{ }^{1} \mathrm{H}-\mathrm{NMR}$ spectra of the addition of $1-100$ eq. of sodium triflate to a THF-d8 solution of 2-K in the area of the hydride signal; ${ }^{1} \mathrm{H}-\mathrm{NMR}$ spectra of $2-\mathrm{Na}\left(\delta_{\text {Hydride }}=-25.81 \mathrm{ppm}\right)$ and $2-\mathrm{K}\left(\delta_{\text {Hydride }}\right.$ $-26.12 \mathrm{ppm}$ ) in THF-d8 are presented as references.

The different behavior of hydride complexes 2-Na and 2-K suggests a strong dependence on the alkali metal ion and their Lewis acidity. The results in reactivity of the dihydride complexes 3-Na and 3-K to transform via $\mathrm{C}-\mathrm{H}$ activation to the species $\mathbf{2}-\mathbf{N a}$ and $\mathbf{2}-\mathbf{K}$ was already shown in the previous sections. In the ${ }^{1} \mathrm{H}-\mathrm{NMR}$ spectra of the two species 2-Na and 2-K, the signals of the corresponding hydride of are found at -25.81 and $-26.12 \mathrm{ppm}$, respectively. This behavior could be attributed due to the different grade of interaction of the alkali metal also found in the solid-state structure of both complexes. To assess the relative affinity of anion $3^{-}$to $\mathrm{Na}^{+}$and $\mathrm{K}^{+}$titration experiments were performed and monitored by ${ }^{1} \mathrm{H}-\mathrm{NMR}$ spectroscopy. First, different amounts of sodium triflate were added to a THF-d 8 solution of $\mathbf{2}-\mathbf{K}$ and the ${ }^{1} \mathrm{H}-\mathrm{NMR}$ chemical shifts of the hydride signal were evaluated (Figure 1.22).

The addition of up to 25 equivalents of NaOTf resulted in a downfield shift of the hydride signal from -26.12 to $-26.00 \mathrm{ppm}$. Interestingly, further addition of sodium cations reversed the trend and showed a high field shift of the hydride signal back to $-26.11 \mathrm{ppm}$ after addition of 100 eq. of sodium triflate. The reversed trend suggests that additional $\mathrm{Na}^{+}$ becomes dominant at higher $\mathrm{Na}^{+}$concentrations. This assumption is consistent with the same trend for the pyrazole backbone signal. Here, the same chemical shifts are observed 
for 2-Na and 3-K (5.70 ppm) but a clear shift to higher field is observed upon addition of $>10$ eq. of sodium triflate ( $5.55 \mathrm{ppm}$ after addition of 100 eq. of NaOTf). Addition of further equivalents was not possible, due to limited solubility of sodium triflate in THF. This unexpected behavior is also observed in the area of the pyrazole signal and the two aryl protons $\mathrm{H}_{\text {meta }}$ and $\mathrm{H}_{\text {para }}$ (Figure 1.23). An interaction with multiple sodium cations is reasonable.

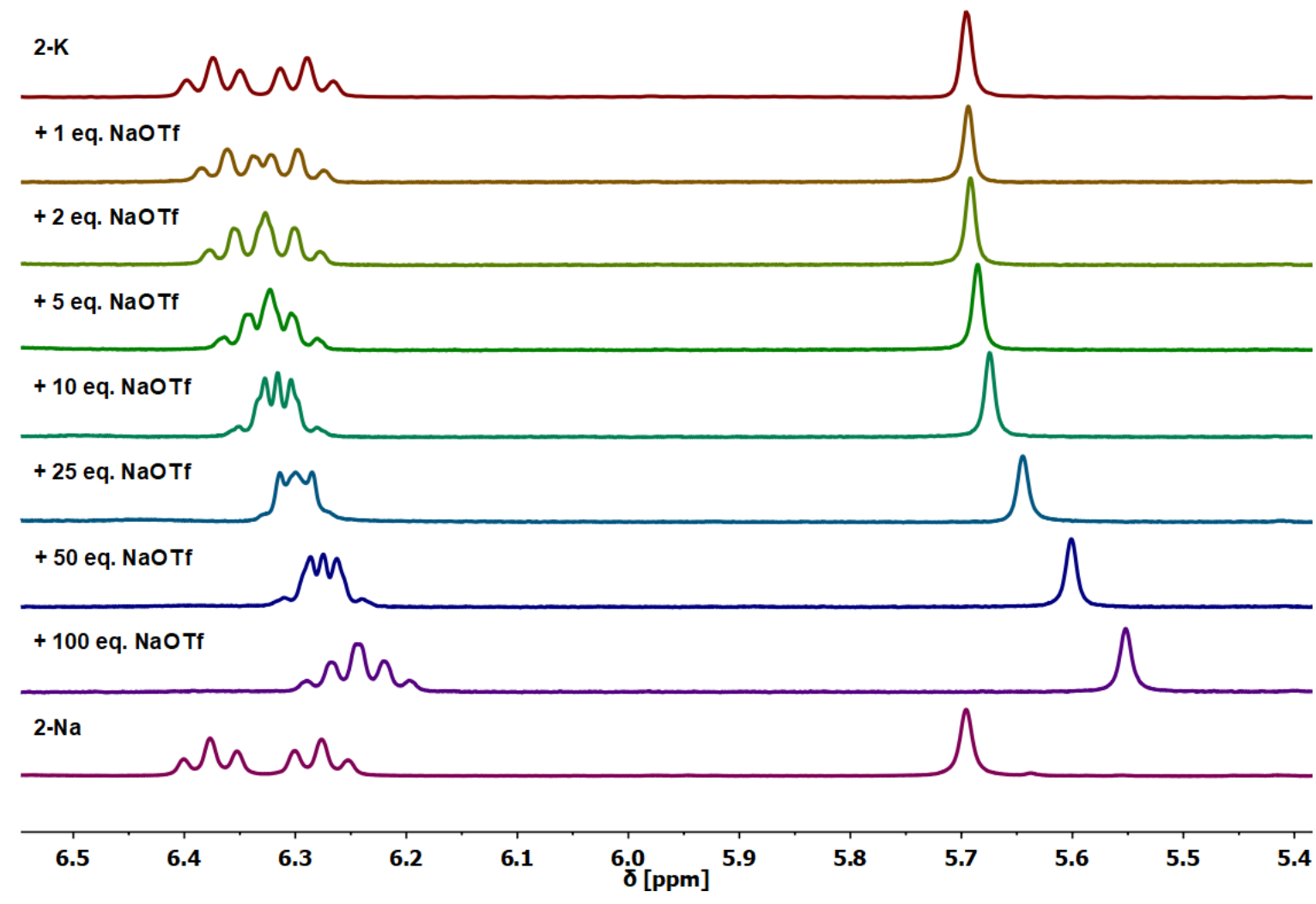

Figure $1.23{ }^{1} \mathrm{H}-\mathrm{NMR}$ spectra of the addition of 1 - 100 eq. of sodium triflate to a THF-d 8 solution of 2-K in the area pyrazole and aryl signals; ${ }^{1} \mathrm{H}-\mathrm{NMR}$ spectra of $\mathbf{2}-\mathbf{N a}$ and $\mathbf{2 - K}$ in THF- $\mathrm{d}_{8}$ are presented as references.

The pyrazole and aryl proton signals experience a high field shift through the addition of sodium triflate. This excess of sodium also results in the convergence of the signals for the meta and para protons of the coordinated phenyl moiety until 25 equivalents of NaOTf. Addition of more sodium cations results in separation of the two signals. All those changes of the aryl and pyrazole proton signals upon addition of NaOTf are not found for the sodium analogue 2-Na, indicative for a different interaction. Overall a hypothetical conversion, based on Equation 1.1 from $\mathbf{2}-\mathbf{K}$ to $\mathbf{2}-\mathbf{N a}$ is found at a maximum of $39 \%$. This calculation has to be taken with a grain of salt, because of the different kind of interaction with a higher excess of sodium triflate ( $>25$ eq.), which has a converse effect.

Equation $1.1 \quad$ Conversion $(2-N a)=\frac{\delta_{\text {hydride }}(\text { measured })-\delta_{\text {hydride }}(2-\boldsymbol{K})}{\delta_{\text {hydride }}(\mathbf{2}-\mathbf{N a})-\delta_{\text {hydride }}(2-\boldsymbol{K})}$ 
Equation 1.2

$$
\text { Conversion }(\mathbf{2}-\boldsymbol{K})=\frac{\delta_{\text {hydride }}(\mathbf{2}-\mathbf{N a})-\delta_{\text {hydride }}(\text { measured })}{\delta_{\text {hydride }}(\mathbf{2}-\mathbf{N a})-\delta_{\text {hydride }}(\mathbf{2}-\boldsymbol{K})}
$$

For comparison, the vice versa experiment, addition of KOTf to 2-Na, was conducted (Figure 1.24). Already addition of one equivalent of potassium triflate resulted in a drastic high field shift of the hydride signal. Calculation of the conversion based on Equation 1.2 indicates a nearly full transformation (93\%) to $\mathbf{2}-\mathbf{K}$. Due to limited solubility in THF no further addition ( $>10$ eq.) of KOTf was possible. The other signals, such as the pyrazole and aryl protons $\left(\mathrm{H}_{\text {meta }} / \mathrm{H}_{\text {para }}\right)$, are not shifted as significant as observed for the other experiment. The chemical shifts at 1 eq. of additional $\mathrm{Na}^{+}$or $\mathrm{K}^{+}$are comparable due to the same cation ratio in both experiments. The small error between $-26.08 \mathrm{ppm}$ for the addition of NaOTf to 2-K versus -26.09 ppm for the addition of KOTf to 2-Na can be attributed to the weighing error of the small amounts needed for both measurements. In contrast to the hydride signal of 2-Na and 2-K, the affinity is based on the overall interaction of the alkali metal with the complex. Nevertheless, the higher affinity of 2-M for potassium was shown through competitivity experiments of $\mathbf{2}-\mathbf{N a}$ and $\mathbf{2}-\mathbf{K}$, which indicates additional interactions of the potassium cation apart from the hydride. A quantitative evaluation is hampered by the different solubility of KOTf and NaOTf and the dominant behavior of additional sodium ions for $>10$ eq. of NaOTf.

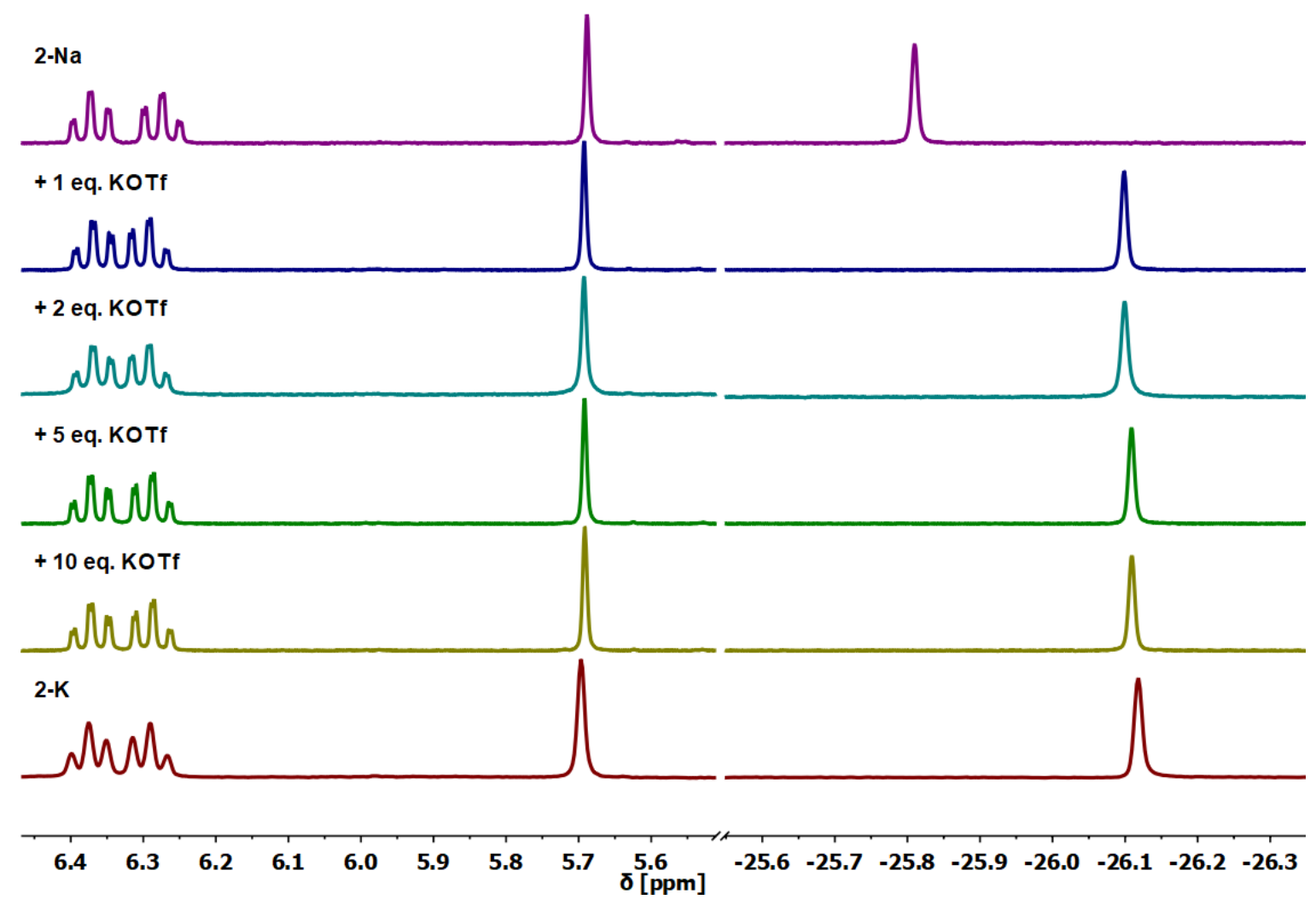

Figure 1.24 ${ }^{1} \mathrm{H}-\mathrm{NMR}$ spectra of the addition of $1-10$ eq. of potassium triflate to a THF-d8 solution of 2-K; ${ }^{1} \mathrm{H}-\mathrm{NMR}$ spectra of $\mathbf{2 - N a}$ and $\mathbf{2 - K}$ in THF-d8 are presented as references. 


\subsection{Reactivity of C-H Activated Species 2-K Towards Small Molecules}

As shown in the previous sections, the $\mathrm{C}-\mathrm{H}$ activated species 2-Na and 2-K provide reactivity as a $\mathrm{Ni}(\mathrm{I}) \mathrm{Ni}(\mathrm{I})$ reagent and therefore allow two-electron reductions of substrates, such as $\mathrm{H}_{2} / \mathrm{D}_{2}$ and benzaldehyde (Section 1.4.3). This behavior of 2-M is similar to the original dihydride complex $\mathbf{3}^{\mathbf{i P r}}$ bearing iso-propyl moieties but is less pronounced due to lower thermodynamic driving force. Nevertheless, investigations towards small molecule activation may be of particular interest, because of the potential differences in reactivity. Additionally, the more pronounced presence of the monohydride can also result in contrasting outcomes compared to the iso-propyl system.

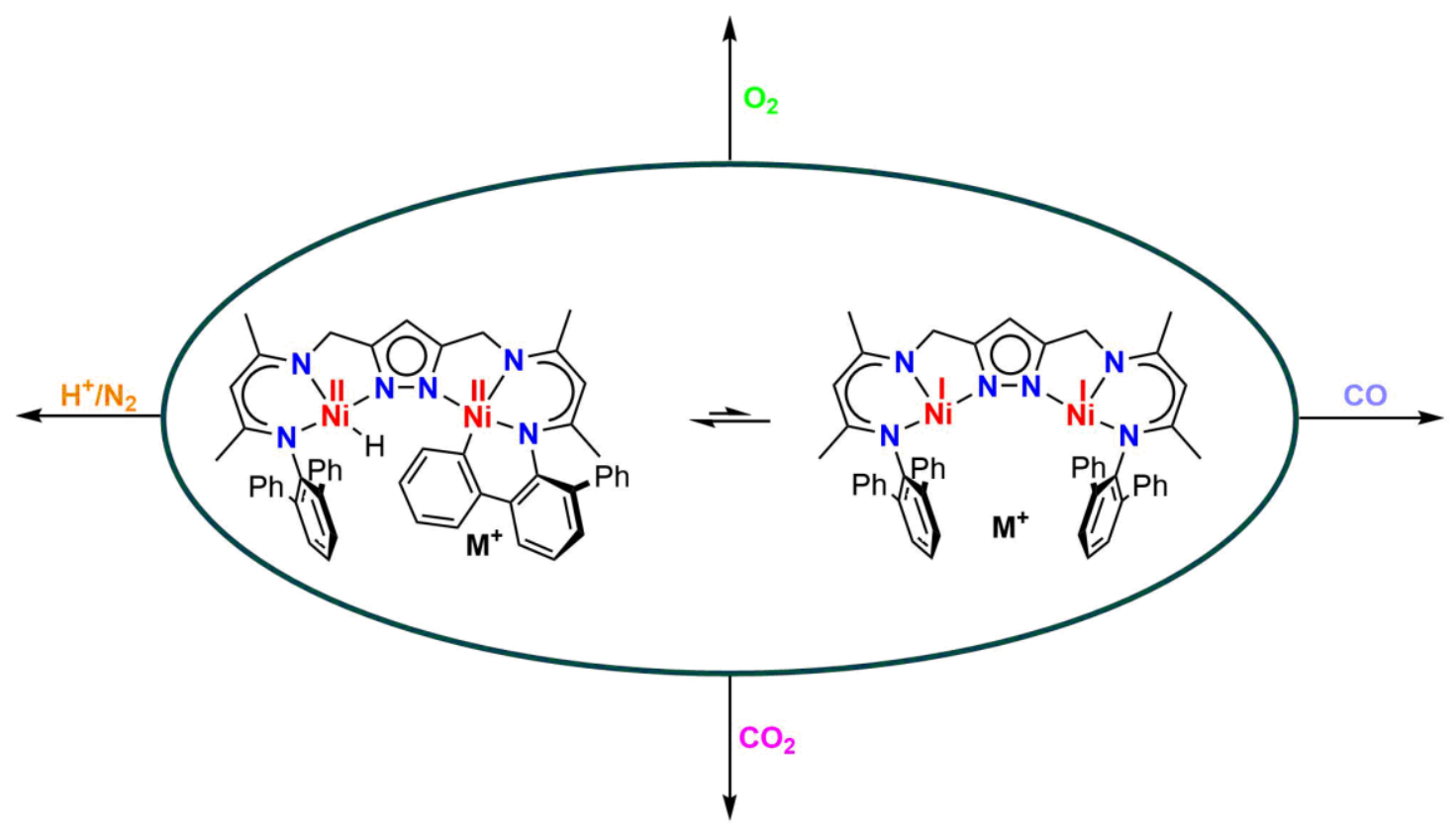

Scheme 1.37 Carried out investigations on Small Molecule Activation with $\mathrm{C}-\mathrm{H}$ activated 2-M.

Most of the chosen substrates, such as oxygen, nitrogen and carbon-oxygen substrates, were based on already investigated reactions of $3^{i \mathbf{P r}}$ for comparison (Scheme 1.37). ${ }^{56-58}$ It has to be noted that the upcoming sections are preliminary results, due to the time restrictions of the dissertation. Nevertheless, those investigations will be discussed because of the mechanistic implications started earlier in this chapter. 


\subsubsection{Investigations on the Activation of Carbon Oxides with $\mathrm{C}-\mathrm{H}$ Activated Species 2-K}

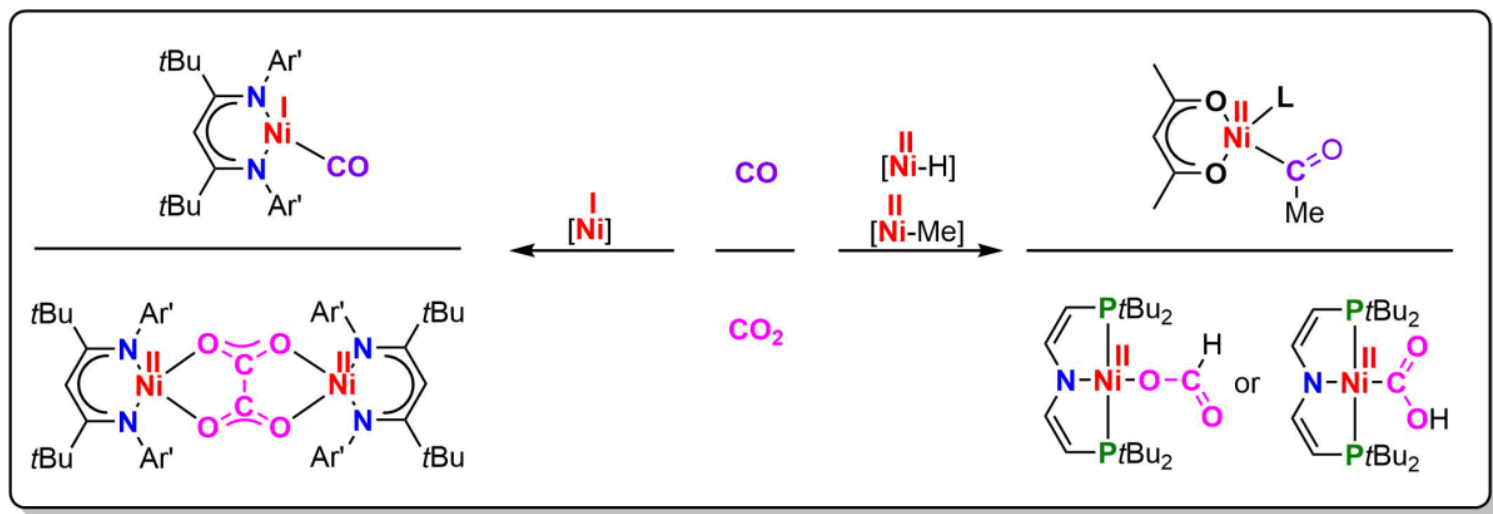

Scheme 1.38 Different reactivities of carbon oxides with $\mathrm{Ni}-\mathrm{H}, \mathrm{Ni}-\mathrm{Me}$ or $\mathrm{Ni}(\mathrm{I})$ systems; left: reactivity of $\mathrm{Ni}(\mathrm{I})$ systems towards $\mathrm{CO}$ and $\mathrm{CO}_{2}$ by Limberg et al. ${ }^{25,27}$; top right: $\mathrm{Ni}-\mathrm{C}$ insertion of $\mathrm{CO}$ by Klein et al. ${ }^{85}$; bottom right: Reverse water-gas shift reaction and formic acid formation of Schneider et al. ${ }^{6}$.

The two-electron reduction reactivity of $\mathbf{2 - K}$ towards benzaldehyde was shown as an trapping experiment the $\mathrm{Ni}(\mathrm{I}) \mathrm{Ni}(\mathrm{I})$ intermediate in Section 1.4.4, which already presented the possibility of such reactions with unsaturated substrates having $\mathrm{C}-\mathrm{O}$ double bonds. In this section, the small carbon oxides, such as $\mathrm{CO}$ and $\mathrm{CO}_{2}$, will be investigated. The possibility of reaction with a $\mathrm{Ni}(\mathrm{I})$ center, as well as with Ni-hydride or Nickel-methyl systems was intriguing. The reactivity of a Ni(I)-NacNac complex with $\mathrm{CO}$ results in the simple coordination of the CO molecule and stabilization of the Ni(I) entity (Scheme 1.38, top left). ${ }^{25}$ The same system reacts with $\mathrm{CO}_{2}$ under a $\mathrm{C}-\mathrm{C}$ coupling to a dimer containing a bridging oxalate dianion (Scheme 1.38, bottom left). ${ }^{27}$ In contrast to the $\mathrm{Ni}(\mathrm{I})$ reactivity, $\mathrm{Ni}-\mathrm{C}$ insertion takes place in alkyl nickel compounds resulting in a Ni-acetyl complex (Scheme 1.38, top right) ${ }^{85}$ and a Nickel-hydride system by Schneider et al. reacts with carbon dioxide to produce formic acid or metallacarboxylates dependent on the reaction conditions (Scheme 1.38, bottom right). ${ }^{6}$ Due to the diversity of $\mathbf{2 - K}$ featuring all characteristics of the presented species, three different kinds of reactivity are possible. Investigations will therefore give more insight in the versatility of $\mathbf{2}-\mathbf{K}$ towards small molecule activation.

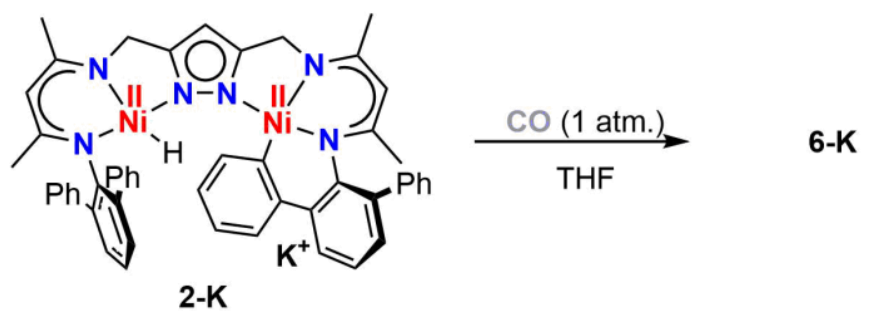

Scheme 1.39 Reaction of 2-K with carbon monoxide (1 atm.) in THF to one major product 6-K 
Upon addition of carbon monoxide, the solution of $\mathbf{2}-\mathbf{K}$ changes over a reaction time of 2 hours to a light red color (Scheme 1.39). For the iso-propyl system no reactivity was observed, when dihydride $3^{i \operatorname{Pr}}$ was treated with carbon monoxide, although it reacts readily with benzaldehyde to products similar to $\mathbf{4 - K}$ and $\mathbf{4 - N a}{ }^{57}$ The ${ }^{1} \mathrm{H}-\mathrm{NMR}$ spectrum of $\mathbf{6}-\mathbf{K}$ is presented in Figure 1.25 and shows significant changes compared to the starting material $\mathbf{2 - K}$. The pyrazole signal of $\mathbf{6 - K}$ is observed at $5.66 \mathrm{ppm}$, slightly shifted compared to the signal of $2-K\left(\delta_{\mathrm{pz}}=5.70 \mathrm{ppm}\right)$. A similar symmetry of the product to the starting material is found based on the splitting of the NacNac proton signals ( $\delta=4.55$ and $4.18 \mathrm{ppm})$ and the diastereotopy of one set of the methylene protons $(\delta=4.46$ and $4.15 \mathrm{ppm})$. The other signal of the methylene protons $(\delta=4.34 \mathrm{ppm})$ shows a coupling to the drastically lowfield shifted triplet at $10.62 \mathrm{ppm}$, consistent with the ${ }^{1} \mathrm{H}-{ }^{13} \mathrm{C}$ HMBC correlation (Figure 1.26, left). Additionally, no correlation to a carbon was found for the triplet signal at 10.62 ppm in the ${ }^{1} \mathrm{H}_{-}{ }^{13} \mathrm{C}$ HSQC Experiment.
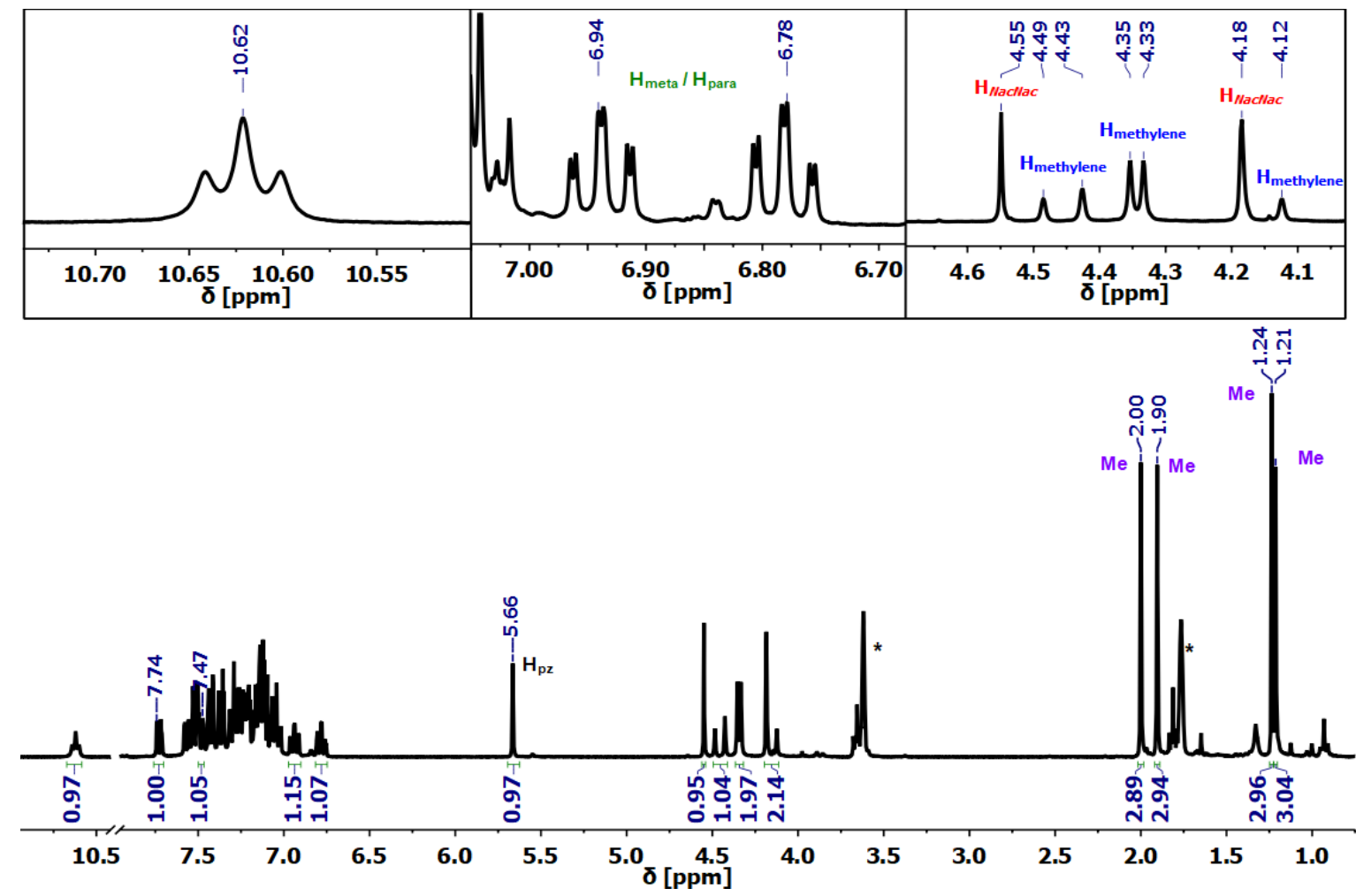

Figure $1.25{ }^{1} \mathrm{H}-\mathrm{NMR}$ in THF-d $\left.8{ }^{*}\right)$ of the reaction 2-K with $\mathrm{CO}(1 \mathrm{~atm}$.) to a product $6-\mathrm{K}$; insets of the triplet (10.62 ppm), $\mathrm{H}_{\text {meta }}$ and $\mathrm{H}_{\text {para }}(6.94$ and $6.78 \mathrm{ppm})$ and methylene/NacNac signals (4.60 $4.10 \mathrm{ppm})$ are shown for clarification.

Moreover, a coordination of the phenyl group is still present, due to the presence of the characteristic $\mathrm{H}_{\text {meta }}$ and $\mathrm{H}_{\text {para }}$ protons $(\delta=6.94$ and $6.78 \mathrm{ppm})$. These signals are observed low-field shifted compared to the starting material. A coupling of those two signals with two doublets $\left(\delta_{\mathrm{H}}=7.73\right.$ and $\left.7.49 \mathrm{ppm}\right)$ also in the aryl proton region and with two quaternary carbons $\left(\delta_{\mathrm{C}}=158.47\right.$ and $\left.139.90 \mathrm{ppm}\right)$ in the aryl carbon region is consistent with the $\mathrm{C}$-H activated phenyl moiety (Figure 1.26 , right). ${ }^{13} \mathrm{CO}$ experiments (both ${ }^{13} \mathrm{C}$ - 
NMR spectra are presented in the Appendix) resulted in a main signal at $192.75 \mathrm{ppm}$, which indicates a coordinated $\mathrm{CO}$ molecule because of the small difference to free carbon monoxide $\left(\delta_{\mathrm{C}}=185.30 \mathrm{ppm}\right)$. To further elucidate the outcome of the reaction, the product was studied by IR spectroscopy. While ATR-IR does not show a band at the expected region of coordinated CO $\left(1900-2100 \mathrm{~cm}^{-1}\right)$, a small broad band $\left(\tilde{v}=1963 \mathrm{~cm}^{-1}\right)$ is observed in the IR spectrum utilizing nujol (both in Appendix). Any signs for an insertion of the CO into the $\mathrm{Ni}-\mathrm{H}$ or $\mathrm{Ni}-\mathrm{C}$ bond were considered further, this is why those reactivities were turned down. Several attempts of crystallization only resulted in the formation of microcrystalline powder. Also, exchange or rather abstraction of the cation through crown ethers/cryptand did not result in single crystal formation. ESI-MS measurements did not show the expected molecular ion peak.
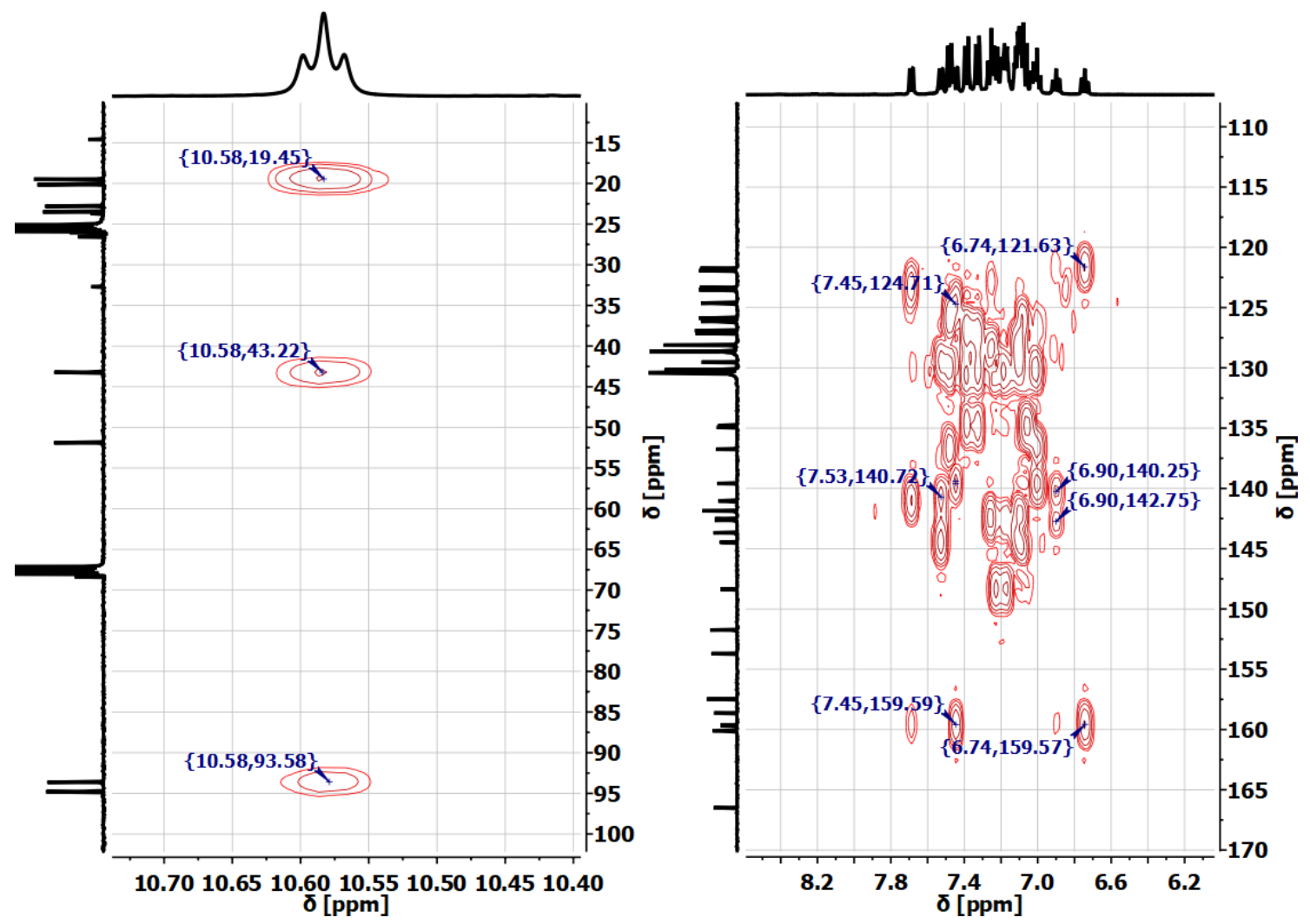

Figure 1.26 ${ }^{1} \mathrm{H}-{ }^{13} \mathrm{C}$ HMBC experiment of 6-K in THF-d8 at room temperature; left: coupling of the low-field shifted triplet signal; right: coupling of $\mathrm{H}_{\text {meta }}$ and $\mathrm{H}_{\text {para }}$ with other aromatic protons.

Based on all the presented observations for 6-K,it is assumed that the Ni-H moiety undergoes reductive $\mathrm{N}-\mathrm{H}$ elimination induced by coordination of carbon monoxide (Scheme 1.40). Therefore, a protonation of the NacNac nitrogen is observed and no changes of the $\mathrm{C}-\mathrm{H}$ activated phenyl is found. Due to the broadness of the band found in the IR spectra of 6-K, both products, shown in Scheme 1.40, could be the outcome of the reaction. The mechanism of the reaction is still unknown and due to time limitations was not investigated further. The reductive elimination or heterolytic cleavage is consistent with the monometallic and bimetallic system of Schneider or Limberg et al., respectively, who observe such reactivity in their respective systems upon coordination of carbon monoxide 
or reduction of a thiolate bridge dinickel complex B-SEt (Scheme 1.11, Section 1.1.3). ${ }^{55,86}$ In the case of Schneider et al. this behavior has been observed upon radiation and is found to be common for the photochemistry of metal-hydrides. ${ }^{87}$ Rather the NacNac ligand is still coordinated through the nitrogen or the double bond, similar to C-SEt (Scheme 1.11, Section 1.1.3), is still unknown. No indication for an E-configuration has been found via NMR spectroscopy. Nevertheless, this reaction outcome indicates a hydride reactivity, which has not been observed for similar systems. ${ }^{10}$ In contrast to $3^{\mathbf{P P r}}$, which did not show reactivity towards carbon monoxide, the present Ni-hydride dictates the outcome due to disfavored reaction of the $\mathrm{Ni}(\mathrm{I}) \mathrm{Ni}(\mathrm{I})$ state. Another example of typical hydride reactivity ${ }^{10}$ for $\mathbf{2}-\mathbf{K}$ will be presented in a later section.

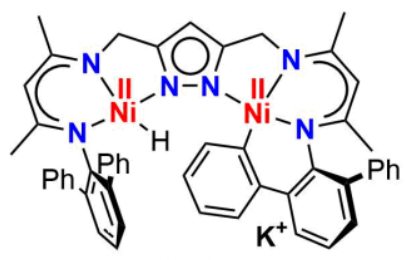

2-K

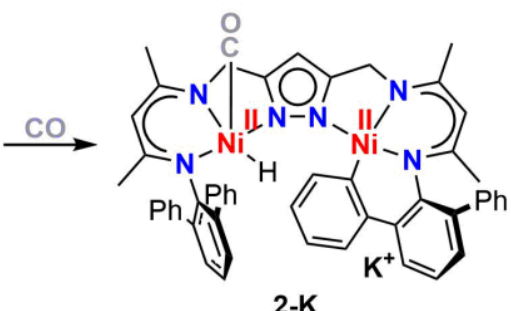

2-K

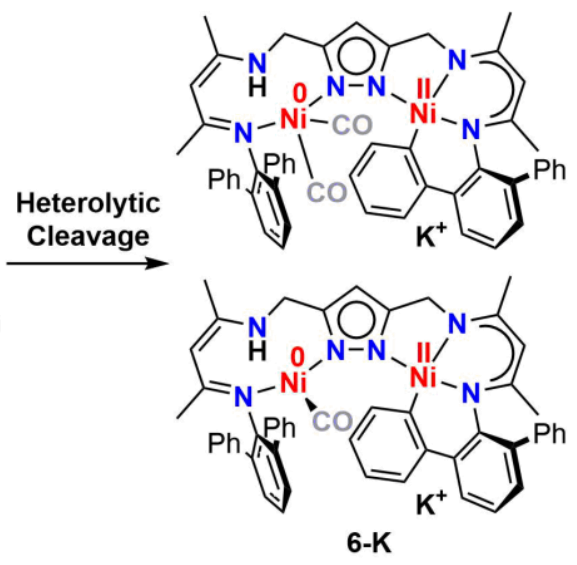

Scheme 1.40 Hypothesized reaction pathway to $6-\mathrm{K}$, heterolytic cleavage of the $\mathrm{Ni}-\mathrm{H}$ unit and protonation of one NacNac nitrogen based on characterization methods mentioned in the text.

In contrast to the $\mathrm{CO}$ reactivity, interpretation of the results for carbon dioxide is rather challenging, due to isolation of a mixture after the reaction. Therefore, only preliminary results on the subject will be presented. When $\mathbf{2 - K}$ was treated with carbon dioxide, the ${ }^{1} \mathrm{H}$ NMR spectrum of the crude product indicates a high amount of the symmetrical hydrolysis product 1-OH next to an asymmetrical species 7-K in a ratio of 1:1 (Scheme 1.41 and Figure 1.27, top). The vast amount of hydrolysis was correlated to the challenging process of drying carbon dioxide, due to the presence of a high amount of water.

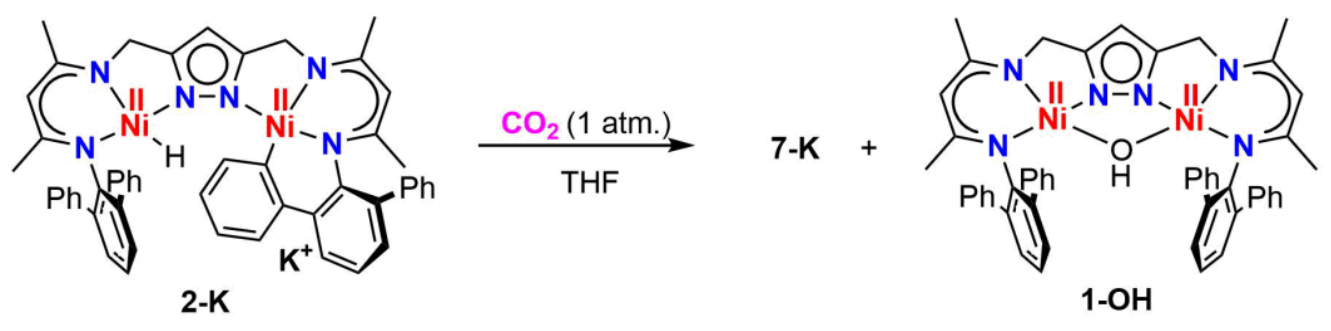

Scheme 1.41 Reaction of $\mathrm{C}-\mathrm{H}$ activated species 2-K with carbon dioxide in THF to unknown species $7-\mathrm{K}$ and hydrolysis product $1-\mathrm{OH}$.

The assumption of asymmetry in 7-K is based on the methylene (3.92 and $3.53 \mathrm{ppm}$ ) and NacNac (4.11 and $3.87 \mathrm{ppm}$ ) protons, which are observed to be diastereotopic or split, 
respectively. This assignment is consistent with the methyl signals (1.61, 1.08 and $0.95 \mathrm{ppm}$ ) splitting and the low-field shifted doublet of a doublet of two phenyl moieties $(8.56 \mathrm{ppm})$. All those observations are indicative for $\mathrm{C}_{1}$ symmetry of the complex $\mathbf{7 - K}$.

The ${ }^{13} \mathrm{C}$-NMR spectrum of the crude product (Figure 1.27, bottom) did not show a lowfield shifted signal at 200-300 ppm, which would be indicative for a $\mathrm{CO}_{2}$ insertion into the $\mathrm{Ni}-\mathrm{H}$ or $\mathrm{Ni}-\mathrm{C}$ bond.

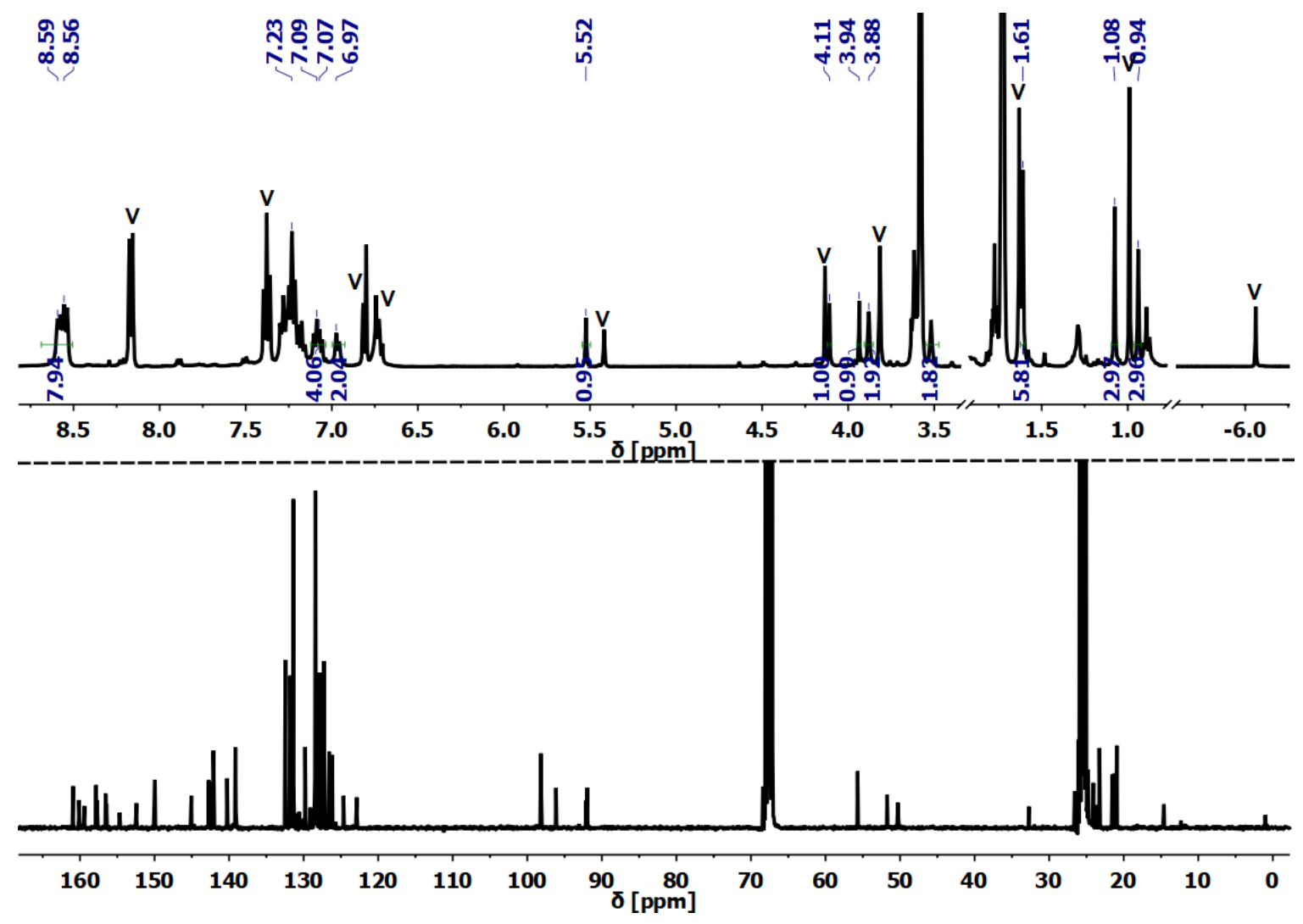

Figure 1.27 Top: ${ }^{1} \mathrm{H}-\mathrm{NMR}$ spectrum of the reaction of 2-K with $\mathrm{CO}_{2}(1 \mathrm{~atm}$.) in THF-d8 at rt, assignment is only for the characteristic proton signals of species $7-\mathbf{K}$ and $\mathbf{V}$ marks the proton signals for $1-\mathrm{OH}$; bottom: ${ }^{13} \mathrm{C}-\mathrm{NMR}$ of the reaction of $\mathbf{2}-\mathrm{K}$ with $\mathrm{CO}_{2}(1 \mathrm{~atm}$.) in THF-d8 at rt.

Further analysis through head-space analysis and ${ }^{13} \mathrm{CO}_{2}$ were not finished in the time of the $\mathrm{PhD}$. A reasonable guess would be the reduction of $\mathrm{CO}_{2}$ to $\mathrm{CO}$ and $\mathrm{H}_{2} \mathrm{O}$, which has to be confirmed with the mentioned experiments.

\subsubsection{Investigations on One-Electron Reductions of $\mathrm{O}_{2}$ and $\mathrm{N}_{2}$ with $\mathrm{C}-\mathrm{H}$ Activated species 2-K}

While in the previous section the reaction of unsaturated carbon oxides with $\mathbf{2}-\mathbf{K}$ were investigated, this section focuses around the reaction of $\mathbf{2 - K}$ with $\mathrm{O}_{2}$ and $\mathrm{N}_{2}$. Previous 
investigations for the iso-propyl system $3^{i \mathrm{Pr}}$ showed the reactivity for both substrates, resulting in one- or two-electron reductions of the diatomic molecules. ${ }^{56-58}$ The reactivity of $3^{i \mathrm{Pr}}$ towards dioxygen to the peroxido and superoxido species was already presented in the introduction (Section 1.1.3) and therefore will not be discussed in detail again. In contrast to the oxygen activation, the pathway for the nitrogen reactivity is induced by protonation and results in the one-electron reduction of the coordinated $\mathrm{N}_{2}$ ligand. ${ }^{56} \mathrm{~A}$ twoelectron reduction is not observed and makes the reactivity and the product rather interesting compared to other $\mathrm{Ni}(\mathrm{I}) \mathrm{Ni}(\mathrm{I})$ bimetallic systems. Also, proton-induced $\mathrm{N}_{2}$ activation is rather unknown. Both reactivities of the $3^{i P r}$ system are presented in Scheme 1.42 .
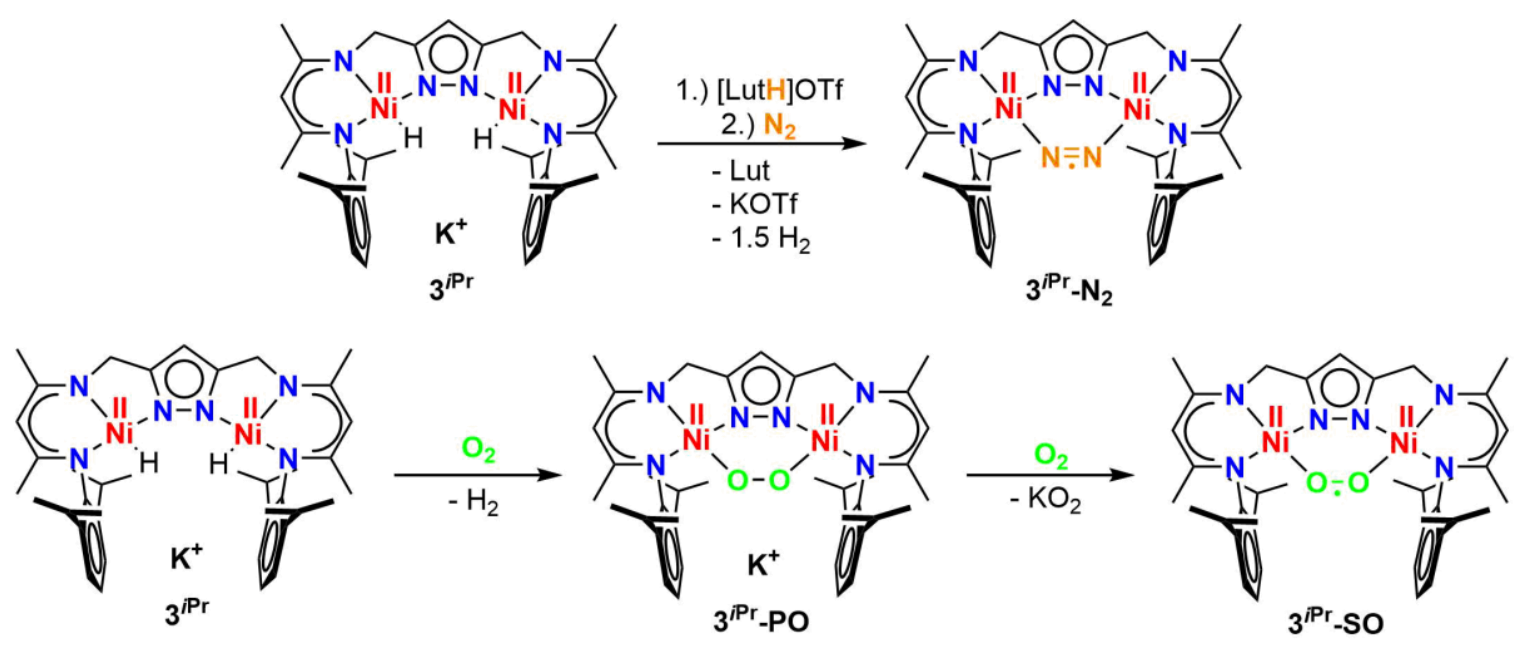

Scheme 1.42 Reactivity of the iso-propyl system $3^{\text {iPr }}$ towards dioxygen and dinitrogen; top: dinitrogen activation is induced by protonation in dinitrogen atmosphere; bottom: dioxygen readily reacts with the dihydride system to the peroxido and with excess of $\mathrm{O}_{2}$ to the superoxido.

Based on the remarkable activation properties of $3^{i \mathbf{P r}}$, similar studies have been employed for the $\mathrm{C}-\mathrm{H}$ activated species $\mathbf{2 - K}$, due to the similar $\mathrm{Ni}(\mathrm{I}) \mathrm{Ni}(\mathrm{I})$ reactivity discussed in earlier parts of this work (Chapter 1.4).

The reactivity of $\mathbf{2}-\mathbf{K}$ towards oxygen was investigated multiple times. No paramagnetic species but rather hydrolysis was observed when oxygen was added at room temperature in excess. For the iso-propyl system $3^{i \mathbf{P r}}$, these conditions resulted in the isolation of the superoxide species $3^{i \mathbf{P r}}$-SO. Also, addition of oxygen to $2-\mathbf{K}$ at lower temperatures $\left(-78^{\circ} \mathrm{C}\right)$ only resulted in the hydrolysis product $\mathbf{1 - O H}$, even when the sample was monitored through ${ }^{1} \mathrm{H}-\mathrm{NMR}$ spectroscopy. Based on those results and focus on other projects, further work on this investigation were not attempted. Therefore, reactivity towards dioxygen cannot be shown, but should not be excluded due to the fact that insufficient drying of dioxygen or instability of the peroxido complex could be the reason for the decay to the hydroxido complex 1-OH. 


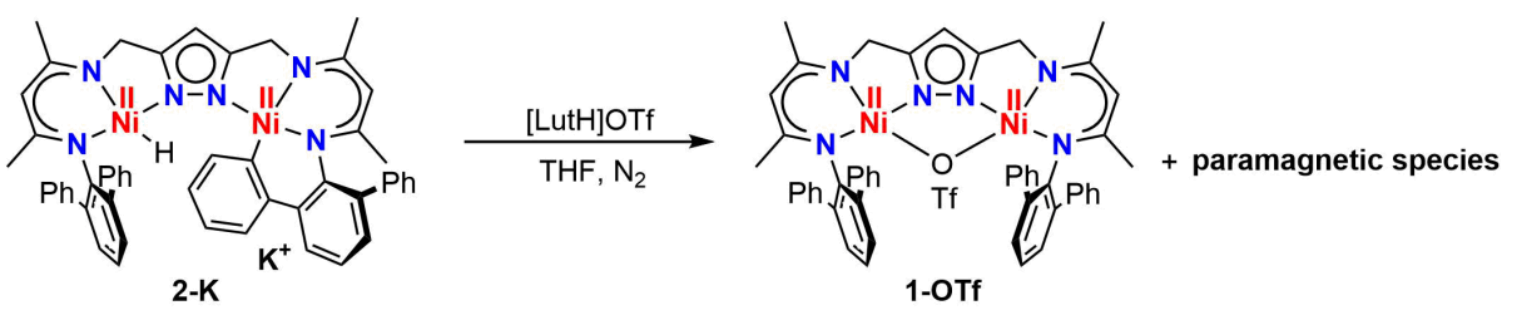

Scheme 1.43 Protonation of 2-K results in triflate complex 1-OTf.

The protonation of $\mathbf{2}-\mathbf{K}$ under $\mathrm{N}_{2}$-atmosphere with one equivalent of 2,6-lutidinium triflate results in a dark red solution. Crystallization attempts gave a red microcrystalline solid, which was analyzed by NMR spectroscopy. Compared to the paramagnetic species $3^{i \mathbf{P r}_{-}} \mathbf{N}_{\mathbf{2}}$ (Scheme 1.42, top), the ${ }^{1} \mathrm{H}-\mathrm{NMR}$ spectrum of the crude product appears to be rather a mixture of a diamagnetic and paramagnetic species (Appendix). Although the ${ }^{19} \mathrm{~F}-\mathrm{NMR}$ spectrum of the species shows only one discrete signal (-79.37 ppm) the ${ }^{1} \mathrm{H}-\mathrm{NMR}$ spectrum presents broadened signals. Previous investigations resulted in the isolation of a triflate complex 1-OTf (Scheme 1.43), as a degradation product. While the reason for the broadening of the signals was not discovered, based on the chemical shift of the fluorine signal 1-OTf is part of the mixture. Similar degradation was also observed for the isopropyl system, resulting $\mathbf{1}^{\text {iPr}}$-OTf. The origin of the broadened signals was not identified.

The degradation was correlated to the coordinating anion triflate, which is why other synthesis routes to the dinitrogen complex $3^{i \mathbf{P r}}-\mathbf{N}_{2}$ were investigated. One alternative is the application of hydride abstractors, such as triphenylcarbenium hexafluorophosphate ([trityl] $\mathrm{PF}_{6}$ ). Because of the presence of a non-coordinating anion, the degradation of the product is less likely.

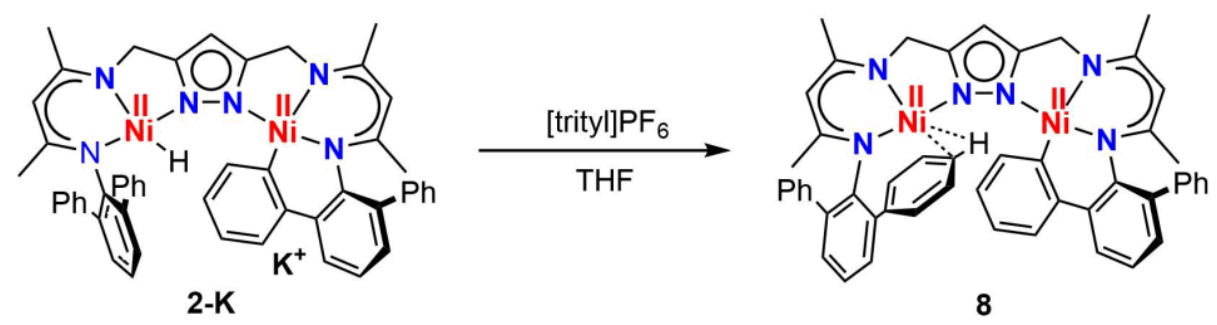

Scheme 1.44 Hydride abstraction of 2-K with [trityl]PF 6 allowing isolation of 8 with a Ni-CH agostic bond.

Treatment of 2-K with [trityl] $\mathrm{PF}_{6}$ in THF results in a dark brown solution. Crystallization via vapor diffusion of pentane gave a small amount of dark blue crystals $(<1 \%)$, which were suitable for XRD analysis. The molecular structure of $\mathbf{8}$ is shown in Figure 1.28. Instead of a $\mathrm{N}_{2}$-reduction, the hydride is abstracted while the coordinated phenyl moiety remains present. The agostic interaction of the free phenyl group stabilizes the unsaturated $\mathrm{Ni}(\mathrm{II})$ center originating from the $\mathrm{Ni}-\mathrm{H}$ unit (Scheme 1.44).

The overall structure of $\mathbf{8}$ is similar to $\mathbf{2}-\mathbf{K}$. Both geometries around the nickel ions are fairly distorted due to the interaction with the corresponding phenyl moiety. But the bond lengths of 2-K and $\mathbf{8}$ with the $\mathbf{L}^{\mathbf{P h}}$ are consistent with each other. The same is found for the 
$\mathrm{C}-\mathrm{H}$ activated phenyl group (Figure 1.28). The bond lengths around both nickel centers are consistent with $\mathrm{Ni}(\mathrm{II})$ ions. Therefore, a $\mathrm{Ni}(\mathrm{I})$ species in the case of the agostic bond is less probable. The nickel-nickel distance is slightly elongated compared to the starting material $2-\mathrm{K}$, due to the agostic interaction and the resulting steric repulsion of the two phenyl moieties in $\mathbf{8}$. This steric hinderance is also observed in the coordination angles of the $\mathrm{C}-\mathrm{H}$ activated phenyl group. The nickel center, which is further lifted out-of-plane compared to the starting material 2-K $\left(\angle\left(\mathrm{N}_{\text {trans }}-\mathrm{Ni}-\mathrm{CPh}_{\mathrm{Ph}}\right)=154.82(13)\right.$ vs. $\left.158.55(17)^{\circ}\right)$. The agostic interaction shows a Ni-H distance of 1.7224(5) $\AA$ and an angle Ni-H-C angle of $113.52(21)^{\circ}$. Those attributes are consistent with agostic bonds of organometallic compounds $\left(\mathrm{d}(\mathrm{M}-\mathrm{H})=1.8-2.3 \AA\right.$ and $\left.\angle(\mathrm{M}-\mathrm{H}-\mathrm{C})=90-140^{\circ}\right){ }^{88}$

Table 1.5 Selected bond lengths and angles of $\mathbf{2 - K}$ and $\mathbf{8}$.

\begin{tabular}{|c|c|c|}
\hline $\mathrm{d}[\AA \AA \AA] / \angle\left[^{\circ}\right]$ & $2-K$ & 8 \\
\hline \multirow{2}{*}{$\mathbf{N i 1}-\mathbf{N}_{\mathrm{NacNac1}}$} & $1.879(3)$ & $1.852(3)$ \\
\hline & $1.915(3)$ & $1.917(3)$ \\
\hline \multirow{2}{*}{ Ni2-N $\mathbf{N a c N a c 2}_{2}$} & $1.877(4)$ & $1.845(3)$ \\
\hline & $1.936(4)$ & $1.860(3)$ \\
\hline $\mathbf{N i 1}-\mathbf{N}_{\mathrm{pz} 1}$ & $1.910(3)$ & $1.879(3)$ \\
\hline $\mathrm{Ni2}-\mathrm{N}_{\mathrm{pz2}}$ & $1.933(3)$ & $1.878(3)$ \\
\hline $\mathrm{Ni}-\mathrm{C}_{\mathrm{Ph}}$ & $1.902(4)$ & $1.899(3)$ \\
\hline $\mathrm{Ni} \cdot \mathrm{C}_{\mathrm{agos}}$ & - & $2.275(3)$ \\
\hline $\mathrm{Ni} \cdot \cdots \mathrm{Ni}$ & $4.266(8)$ & $4.4034(6)$ \\
\hline \multirow{2}{*}{$\mathbf{N}_{\text {trans }}-\mathbf{N i}-\mathbf{N}_{\mathrm{pz}}$} & $167.15(14)$ & $165.27(12)$ \\
\hline & $177.22(15)$ & $171.55(12)$ \\
\hline $\mathbf{N}_{\text {trans }}-\mathrm{Ni}-\mathrm{C}_{\mathrm{Ph}}$ & $158.55(17)$ & $154.82(13)$ \\
\hline $\begin{array}{l}\text { N }_{\text {NacNac-Ni- }}-\mathrm{Ni} \\
\mathrm{C}_{\text {agos }}\end{array}$ & - & $144.41(13)$ \\
\hline
\end{tabular}

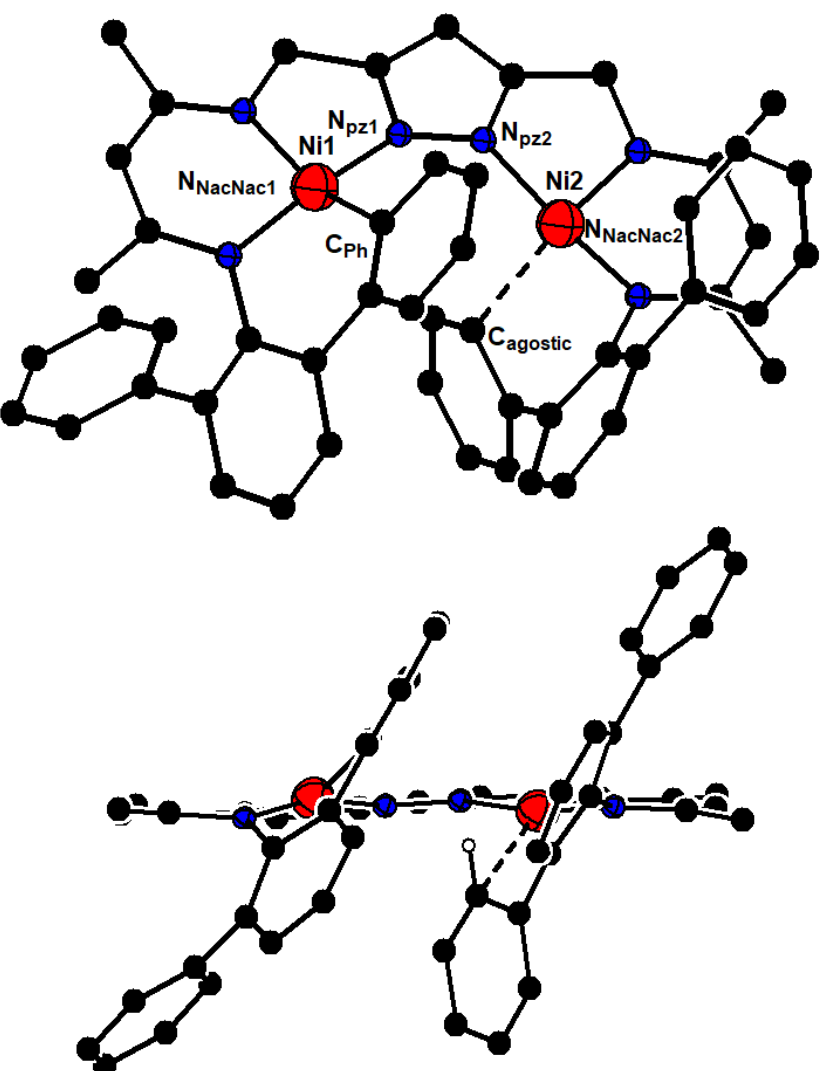

Figure $\mathbf{1 . 2 8}$ Two perspectives of the molecular structure of $\mathbf{8}$, hydrogen atoms and solvent molecules are omitted for clarity.

Further characterization of $\mathbf{8}$ was attempted, but due to the low crystalline yield and sideproducts of the reaction, quantitative purification was challenging. Further investigations via NMR and IR spectroscopy are needed to confirm the nature of the interaction to be agostic or simply a $\pi$-interaction 


\subsection{Summary and Outlook}

Through this work, nickel complexes from hydride to organometallic systems bearing the ligand system $\mathbf{H}_{3} \mathbf{L}^{\mathbf{P h}}$ were presented. $\mathbf{H}_{3} \mathbf{L}^{\mathbf{P h}}$ was derived from the established iso-propyl system $\mathbf{H}_{3} \mathbf{L}^{i \mathbf{P r}}$ by utilization of $m$-terphenyl substituents (Scheme 1.45). The synthesis route was developed by Duan. ${ }^{57}$ However, in this work, optimization of the reaction conditions and work-up resulted in an increase of the overall yield to $35 \%$. This allowed larger scale synthesis with minimal effort in work-up for access to $\mathbf{H}_{\mathbf{3}} \mathbf{L}^{\mathbf{P h}}$.

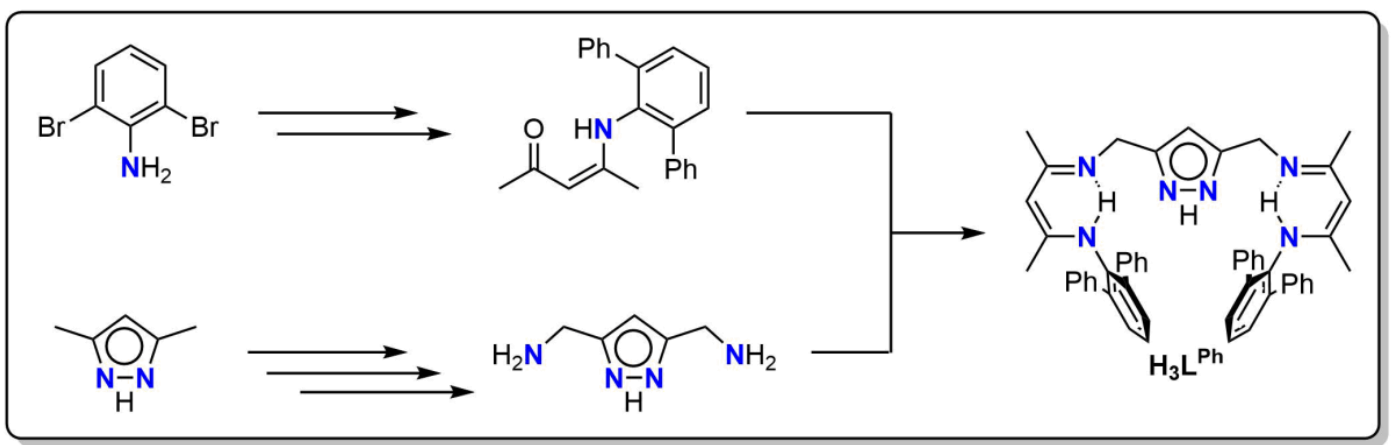

Scheme 1.45 Synthesis route for the diphenyl aniline derived ligand $\mathrm{H}_{3} \mathrm{~L}^{\mathrm{Ph}}$.

Using the ligand $\mathbf{H}_{\mathbf{3}} \mathbf{L}^{\mathbf{P h}}$, the bromido bridged dinickel complex $\mathbf{1}$ was synthesized. Additional characterization of the redox behavior of 1 showed two reductions, likely metalbased. The first reduction $\left(\mathrm{E}_{1 / 2}=-2.32 \mathrm{~V} v s . \mathrm{Fc} / \mathrm{Fc}^{+}\right)$appears to be quasi-reversible, but upon second reduction $\left(\mathrm{E}_{\mathrm{p}, \mathrm{c}}=-2.85 \mathrm{~V} v \mathrm{~s} . \mathrm{Fc} / \mathrm{Fc}^{+}\right)$both processes become irreversible (Figure 1.29). An EEC mechanism was assumed, due to the electrochemical behavior and similarity to $\mathbf{1}^{i \mathbf{P r}}$.

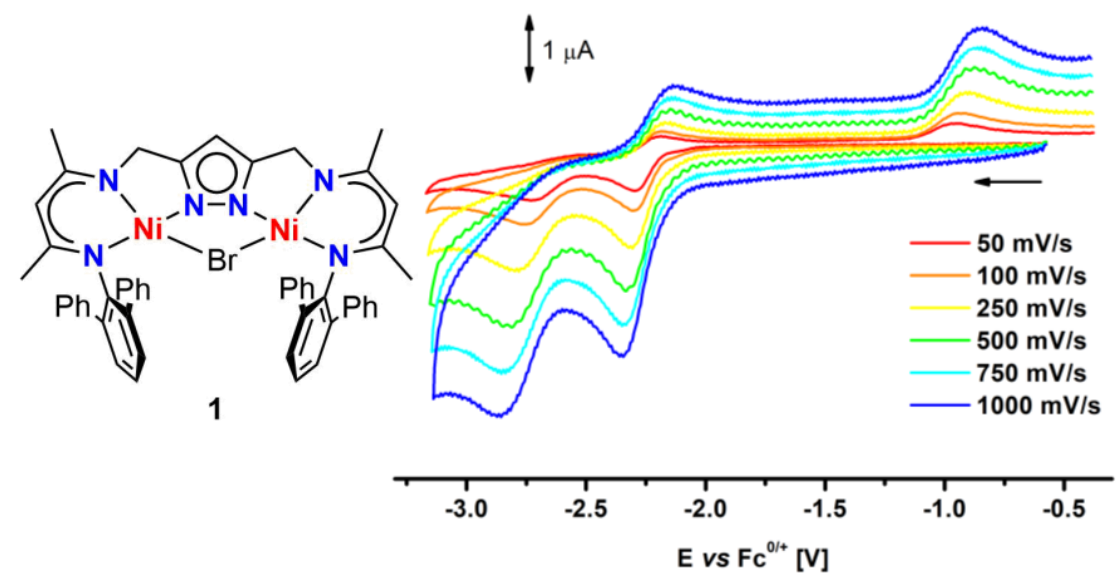

Figure 1.29 Bromide precursor 1 and the cyclic voltammogram showing the two reductions of 1 .

Identification of the redox behavior of $\mathbf{1}$ allowed development of an alternative synthesis procedure to 2-K and 2-Na. Although the established synthetic route by Duan through the 
addition of superhydride (Scheme 1.46, top), resulted in $\mathbf{2}-\mathbf{K}$ in moderate crystal yield, utilization of a strong reducing agent, such as $\mathrm{KC}_{8}$, gave improved conditions for the synthesis (Scheme 1.46, bottom) resulting in higher yield and purity. This behavior itself suggested a $\mathrm{Ni}(\mathrm{I}) \mathrm{Ni}(\mathrm{I})$ complex as an intermediate for the $\mathrm{C}-\mathrm{H}$ activation due to the absence of a hydride source.

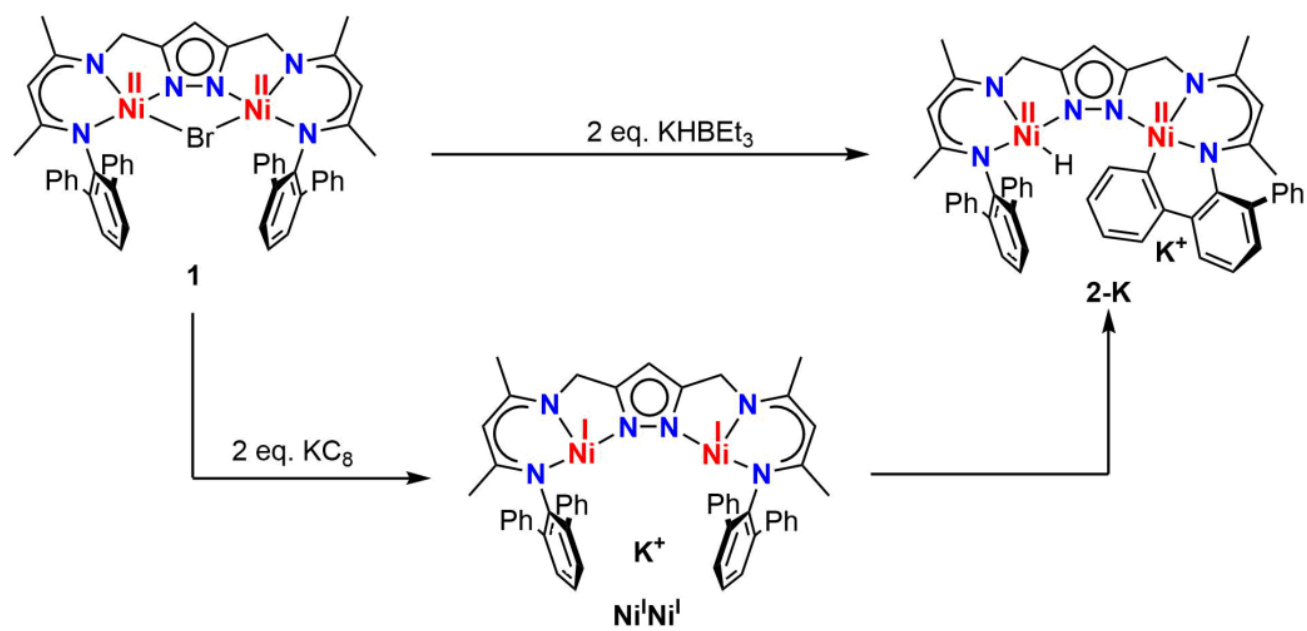

Scheme 1.46 Synthesis routes to the $\mathrm{C}-\mathrm{H}$ activated bimetallic Ni complex 2-K through potassium triethyl borohydride or reduction with potassium graphite.

The reversibility of the $\mathrm{C}-\mathrm{H}$ activation is observed when $\mathbf{2}-\mathbf{K}$ is reacted with dihydrogen to the dihydride species 3-K. 3-K is fairly instable without the dihydrogen atmosphere and reacts back to $\mathbf{2 - K}$ over a period of time. Based on those findings, the mechanism of the reversible $\mathrm{C}-\mathrm{H}$ activation or $\mathrm{H}_{2}$ splitting required further investigations. $\mathrm{D}_{2}$-experiments showed an incorporation of deuterium in 3-K-D $\mathbf{D}_{2}$. No hydride or deuterium on the phenyl groups was found through ${ }^{1} \mathrm{H}-\mathrm{NMR}$ or ${ }^{2} \mathrm{H}-\mathrm{NMR}$ spectroscopy, respectively. Additionally, trapping experiments with $\mathbf{2 - K}$ and an electrophile, such as benzaldehyde, showed twoelectron reduction of the substrate between the two nickel centers in 4-K (Scheme 1.47).

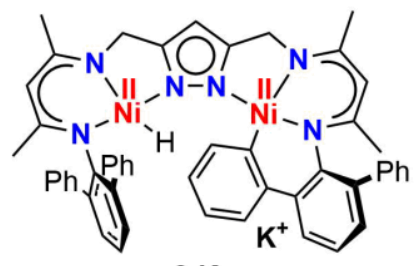

2-K
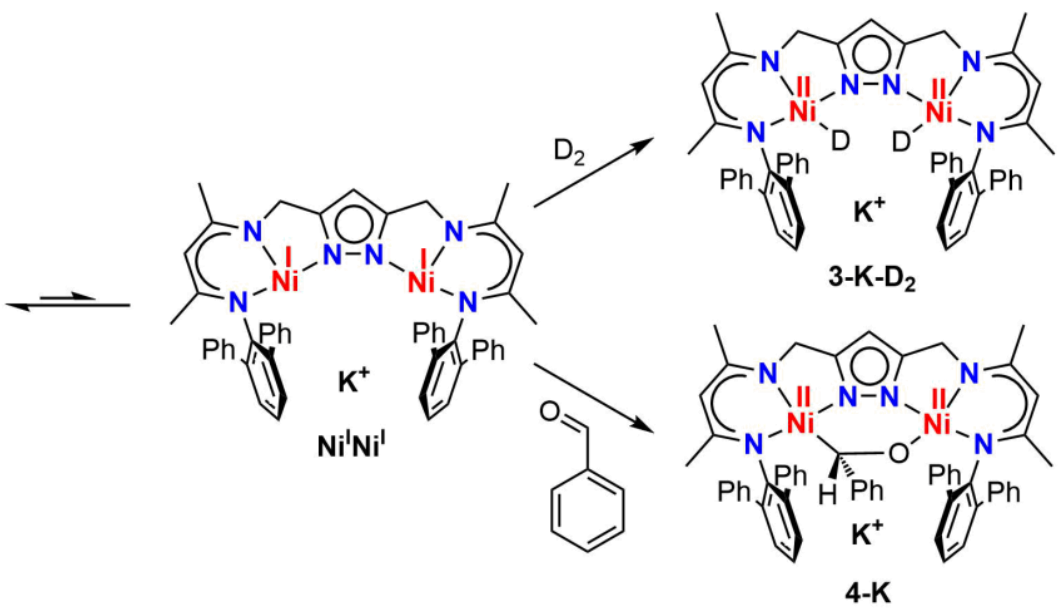

Scheme 1.47 C-H activated 2-K as a masked $\mathrm{Ni}(\mathrm{I}) \mathrm{Ni}(\mathrm{I})$ complex shown by reactivity with $\mathrm{D}_{2}$ or $\mathrm{PhCHO}$. 
Therefore, an equilibrium with a $\mathrm{Ni}(\mathrm{I}) \mathrm{Ni}(\mathrm{I})$ species through reductive elimination was proposed, which is consistent with the alternative synthesis route for 2-K (Scheme 1.46, bottom). This equilibrium of $\mathbf{2}-\mathbf{K}$ is similar to $3^{i \mathbf{P r}}$, but less pronounced due to the lesser thermodynamic driving force of the reductive elimination. This type of reversible $\mathrm{C}-\mathrm{H}$ activation on $\mathrm{Ni}(\mathrm{I})$ centers is the first example to the best of our knowledge and allows $\mathrm{Ni}(\mathrm{I}) \mathrm{Ni}(\mathrm{I})$ reactivity without the sensitivity of the strongly reducing character. Such a "masquerade" has only been known for reducible substrates, such as toluene or dihydrogen. 11,32
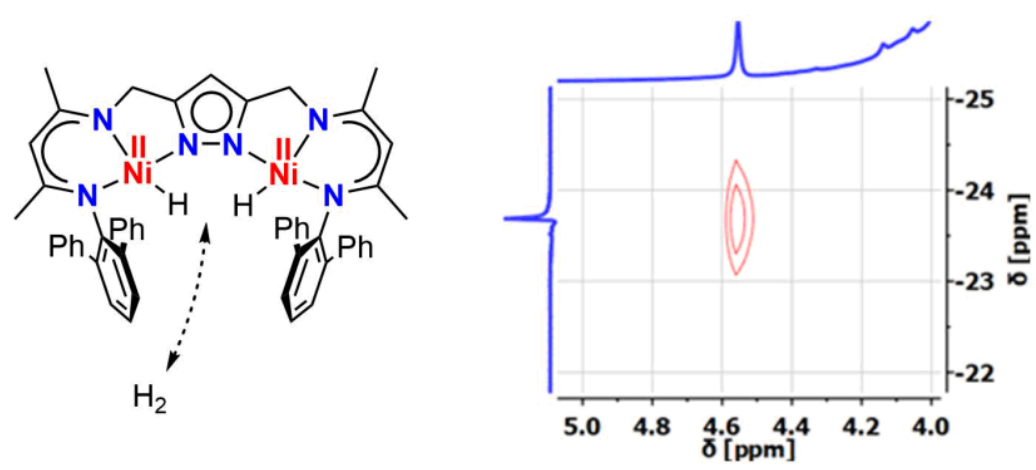

Figure 1.30 Exchange with 3-K(crypt) with free dihydrogen shown through EXSY-NMR measurements (right).

The instability of the dihydride species 3-K did not allow for its isolation, but after abstraction of the potassium with cryptand, the rapid crystallization of 3-K(crypt) out of THF gave crystals suitable for X-ray diffraction. In solution, 3-K(crypt) under $\mathrm{H}_{2}$ atmosphere shows a dynamic equilibrium between the hydrides and free dihydrogen, which is shown by EXSY-NMR measurements (Figure 1.30). This exchange reaction is consistent with the observations for $\mathbf{3}^{\mathbf{i P r}}$ but is only observed for $\mathbf{3}-\mathbf{K}$ when the potassium was complexed by crown ethers or cryptands.

Exchange of the alkali metal to the lighter homologue sodium results in a mixture of the hydride species 2-Na and 3-Na. Although the ratio between the two species cannot be changed in favor of the $\mathrm{C}-\mathrm{H}$ activated species 2-Na, reaction with dihydrogen resulted in the isolation and characterization of solely the dihydride species 3-Na (Scheme 1.48, left). The dihydride species 3-Na shows a higher stability without $\mathrm{H}_{2}$-atmosphere than the potassium analogue, but also degrades over time. The full conversion to 2-Na was not observed, which motivated the search for alternative synthesis routes. Based on the findings for $\mathbf{2 - K}$, the reduction pathway of $\mathbf{1}$ employing sodium naphtalide resulted in the isolation of the C-H activated species 2-Na in nearly quantitative yield (Scheme 1.48, middle).

At higher temperatures the second $\mathrm{C}-\mathrm{H}$ activation to the species 5-Na was observed (Scheme 1.48, right). 5-Na was fully characterized by XRD analysis, NMR, IR and UV/vis spectroscopy. The transformation to $\mathbf{5 - M}$ is found to be dependent on the alkali metal; potassium showed longer reaction times to $\mathbf{5 - K}$ and abstraction of the cation resulted in no reaction at all. Reaction of 5-Na with dihydrogen resulted in a slow reaction to 3-Na with only a small amount of the intermediate $\mathbf{2 - N a}$, indicative for a fast second reaction. The 
absence of a hydride and therefore disfavored reductive elimination pathway explains the slow reaction. Deuterium experiments were unsuccessful, due to the long reaction time and decay of the starting material during heating.

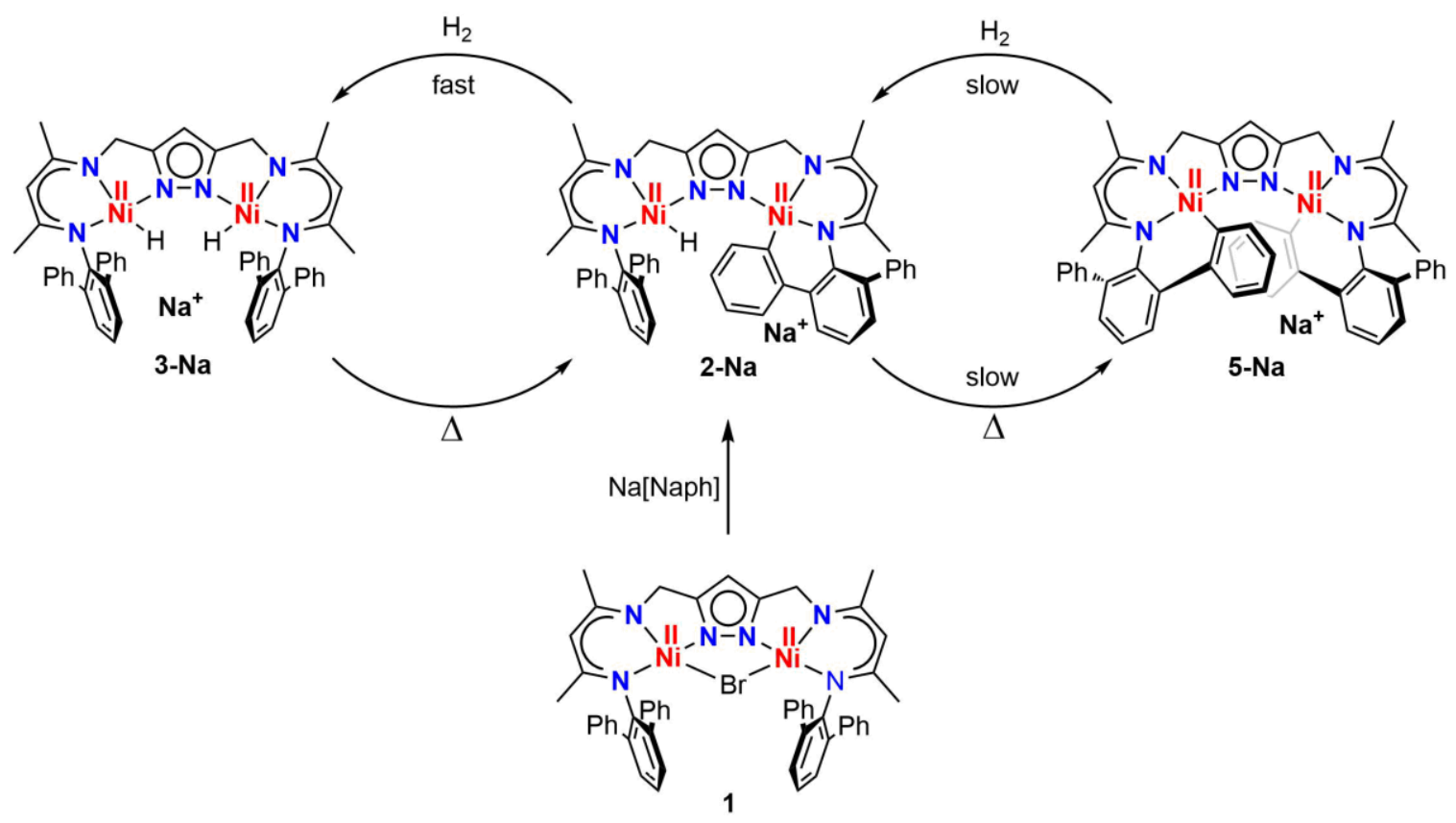

Scheme 1.48 Overall reactivity scheme of the hydride and aryl complexes 2-Na, 3-Na and 5-Na and selective synthesis procedure to the $\mathrm{C}-\mathrm{H}$ activated species 2-Na via reduction of 1 .

The $\mathrm{Ni}(\mathrm{I}) \mathrm{Ni}(\mathrm{I})$ reactivity of $\mathbf{2}-\mathbf{K}$ was already investigated via trapping experiments, which is why, the activation of small molecules was tested in the last part of this project. For these studies, multiple bi- or triatomic substrates were investigated, some of them known from the investigations of the $i$ so-propyl derivative $3^{i \mathrm{Pr}}$.

Based on the previous investigation on $3^{i \mathrm{Pr}}$, carbon monoxide reacted unexpectedly with 2-K through a heterolytic cleavage of the $\mathrm{Ni}-\mathrm{H}$ unit induced by coordination of carbon monoxide to the asymmetrical 6-K (Scheme 1.49). 6-K was characterized by NMR, IR spectroscopy and labeling experiments. The Ni-Ph unit is still observed in $\mathbf{6}-\mathbf{K}$, shown by ${ }^{1} \mathrm{H}$ - and ${ }^{13} \mathrm{C}-\mathrm{NMR}$ spectra. Although crystallization of the compound could not be achieved, full characterization through other measurements was possible, which resulted in the hypothesis for the outcome of the reaction. In addition to carbon monoxide the activation of triatomic carbon dioxide was investigated. Preliminary experiments support the assumption that an insertion into the $\mathrm{Ni}-\mathrm{H}$ or $\mathrm{Ni}-\mathrm{C}$ bond is not observed, rather reduction of $\mathrm{CO}_{2}$ to $\mathrm{CO}$ and $\mathrm{H}_{2} \mathrm{O}$ is found (Scheme 1.49). Only the presence of 1-OH and an unknown species 7-K is found after reaction with $\mathrm{CO}_{2}$.

The last discussed investigation revolved around the activation of dinitrogen through protonation/hydride abstraction and one-electron reduction of the substrate. Here, when lutidinium triflate was used, the coordination potential of the anion resulted in the isolation of a triflate complex 1-OTf (Scheme 1.49), which was shown by ${ }^{1} \mathrm{H}$ - and ${ }^{19} \mathrm{~F}-\mathrm{NMR}$ spectroscopy. Much more interesting was the employment of a hydride abstractor, such as 
[trityl] $\mathrm{PF}_{6}$, which resulted in elimination of a hydride resulting in a "naked" $\mathrm{Ni}(\mathrm{II})$ center with an agostic bond to a free phenyl moiety in $\mathbf{8}$ (Scheme 1.49). The coordinated phenyl group was still observed intact, which suggested hydride abstraction from $\mathbf{2}-\mathbf{K}$ without further reactivity. In contrast to $\mathbf{3}^{\mathbf{i P r}}$ this compound $\mathbf{8}$ did not react further with dinitrogen due to the absence of a second $\mathrm{Ni}-\mathrm{H}$ unit. Therefore, the reactivity of $\mathbf{2}-\mathbf{K}$ towards hydride abstractors has implications for the mechanistic pathway proposed for the nitrogen activation by $3^{i \mathbf{P r}}$ to give $3^{i \mathbf{P r}}-\mathbf{N}_{2}$.

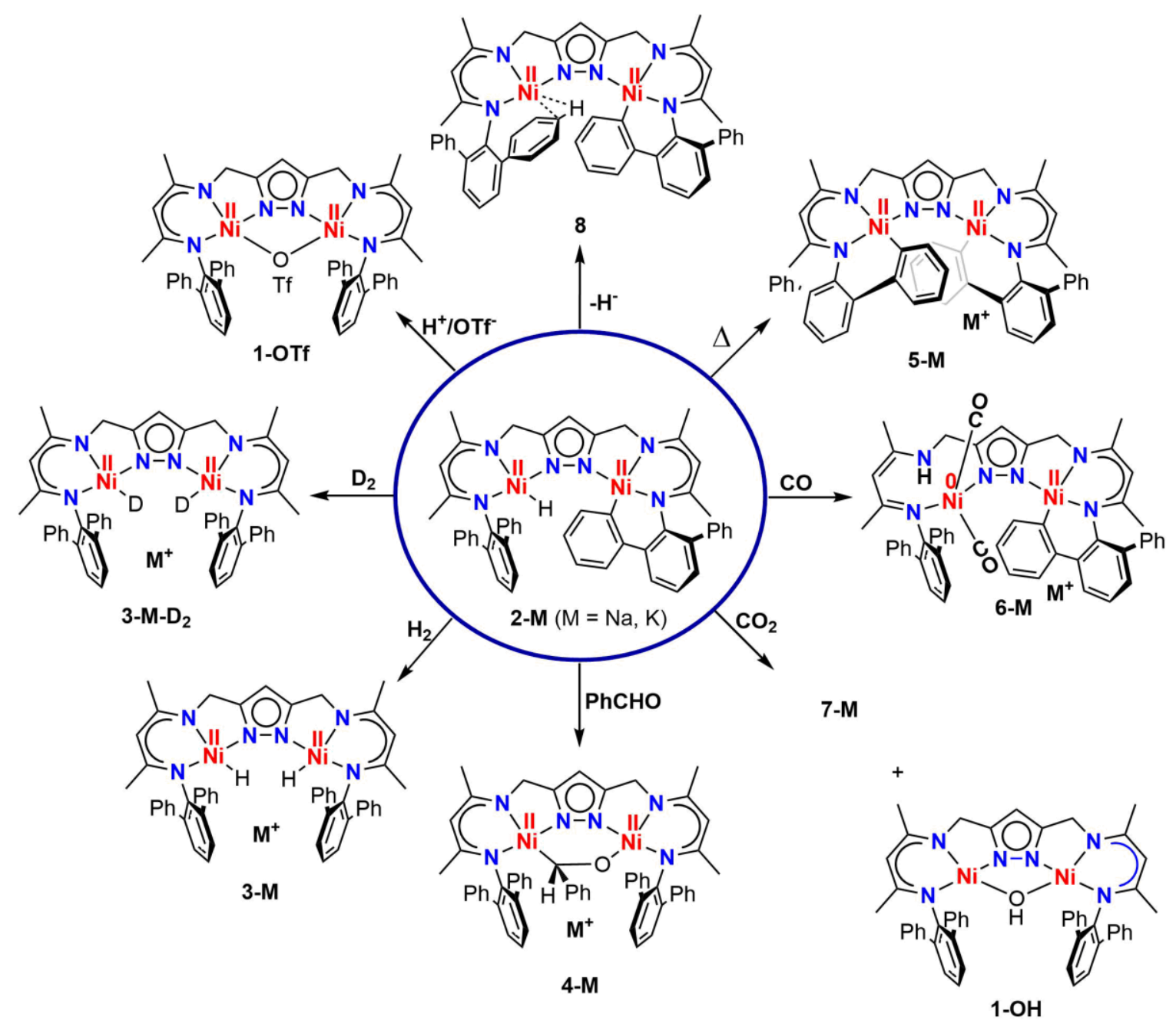

Scheme 1.49 Overview of the reactivity of 2-M $(M=N a, K)$ towards small molecule substrates.

All in all, 2-M $(\mathrm{M}=\mathrm{Na}, \mathrm{K})$ provides an interesting bimetallic system with unique reactivity due to the metal-metal cooperativity of the two nickel centers (Scheme 1.49). 2-M can show manifold reactivity that reflect its character as a masked $\mathrm{Ni}(\mathrm{I}) \mathrm{Ni}(\mathrm{I})$ compound, $\mathrm{Ni}-\mathrm{H}$ or a $\mathrm{Ni}$-Ph system, which can undergo two-electron substrate reduction and hydride dependent on the substrate and the conditions of the reaction. Such a combination of reactivity is novel for nickel systems and therefore provides an advancement in the versatility of such complexes. 


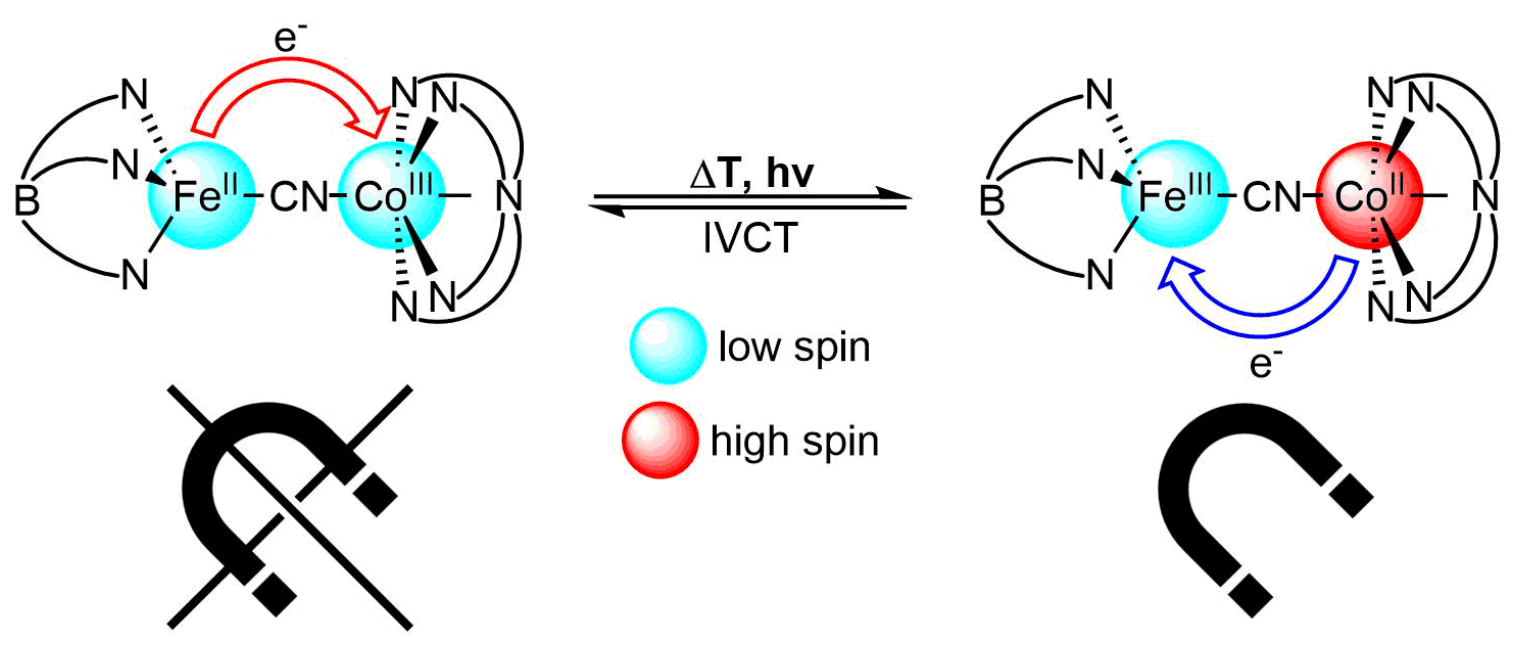

2. Mechanistic Studies on the CTIST of Di- and Tetranuclear Fe/Co Prussian Blue Analogues using Time-Resolved Spectroscopy
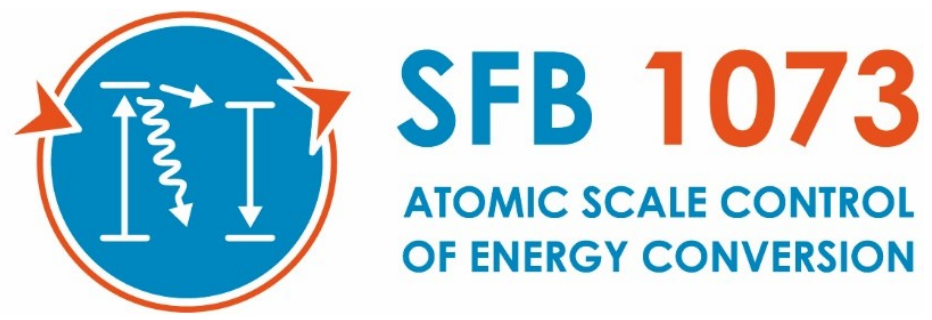


\subsection{Introduction}

The development of new information storage devices is one of the fields of research gaining increased attention due to the need for faster, longer-lasting and lower-energy computational systems. Construction of bits with increasing amount of information while decreasing the amount of physical space is a major goal. Although the potential size of the storage units can be reduced to millimeter or nanometer scale through bulk materials, a further decrease in dimensions would be preferred. ${ }^{89}$ Therefore, molecule based memory devices have gained significant interest, for example spin crossover ${ }^{90}$ or single molecule magnet materials. ${ }^{91,92}$ Those bits on a molecular level, which contain the binary information, need to be interchangeable between two states " 1 " and " 0 " through an external stimulus, such as light, temperature or pressure.
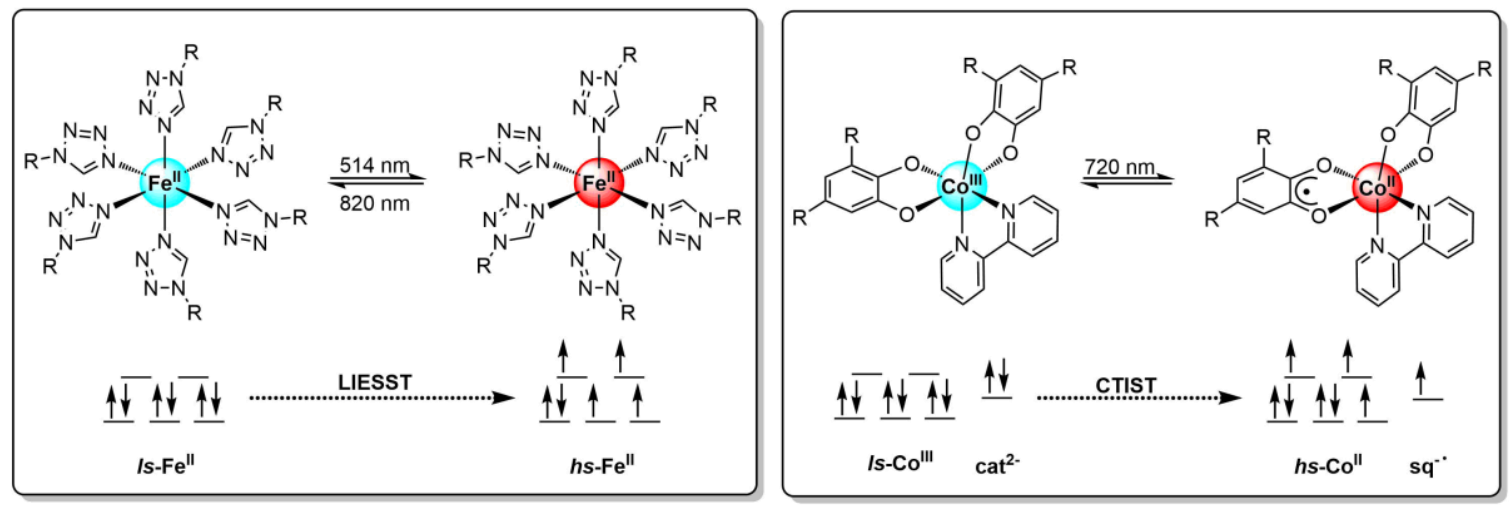

Scheme 2.1 Examples for LIESST and CTIST in metal complexes; left: LIESST in [Fe(ptz)6](BF 4$)_{2}$ by Hauser et al. ${ }^{93}$; right: metal-to-ligand CTIST in [Ph${ }_{2}$ bpyCo(3,5-dtbcat)(3,5-dtbsq)] by Hendrickson et al. ${ }^{94}$.

While the latter two stimuli are harder to employ for solid state materials because of indirect effects of the material (thermal expansion) or problematic realization of the stimuli (exceptionally high pressures), photo-driven changes in molecular systems have already been shown through Light Induced Excited Spin State Trapping (LIESST) or ChargeTransfer Induced Spin Trapping (CTIST) effects. In contrast to LIESST, which uses a spin transition by an indirect excitation pathway found dominantly in $\mathrm{Fe}(\mathrm{II})$ spin crossover complexes $^{90,95-97}$, CTIST compounds inhibit a coupling of the spin transition with a metalto-metal or metal-to-ligand charge transfer ${ }^{98,99}$ For both effects chosen examples are shown in Scheme 2.1. [ $\left.\mathrm{Fe}(\mathrm{ptz})_{6}\right]\left(\mathrm{BF}_{4}\right)_{2}$ is the first example of a system exhibiting a LIESST. This system reported by Hauser et al. exhibits the spin transition $l s-\mathrm{Fe}(\mathrm{II}) \rightarrow h s-\mathrm{Fe}(\mathrm{II})$ upon irradiation with blue light at $20 \mathrm{~K}$ (Scheme 2.1, left). ${ }^{93}$ An example for a CTIST reported by Hendrickson et al. is shown in the right part of Scheme 2.1. It is driven by a Co(III)catechol $\rightarrow \mathrm{Co}(\mathrm{II})$-semiquinone metal-to-ligand charge transfer. Instead of a metal-toligand charge transfer, Prussian Blue Analogues (PBAs) utilize a metal-to-metal charge transfer to facilitate a magnetic switching via CTIST. ${ }^{100}$ Additionally, PBAs allow certain 
benefits, due to their chemical composition, which will be discussed in detail in the next Section. 


\subsubsection{From 3D-Networks to Linear and Square Prussian Blue Analogues}

The pigment Prussian Blue $\mathrm{Fe}_{4}\left[\mathrm{Fe}(\mathrm{CN})_{6}\right]_{3} \cdot 14 \mathrm{H}_{2} \mathrm{O}$ (Figure 2.1) was widely used as a coloring agent because of its distinct blue color. The characteristic color is based on a metalto-metal electron transfer (MMCT) between the two bivalent iron centers resulting in an absorption band at $700 \mathrm{~nm}$.

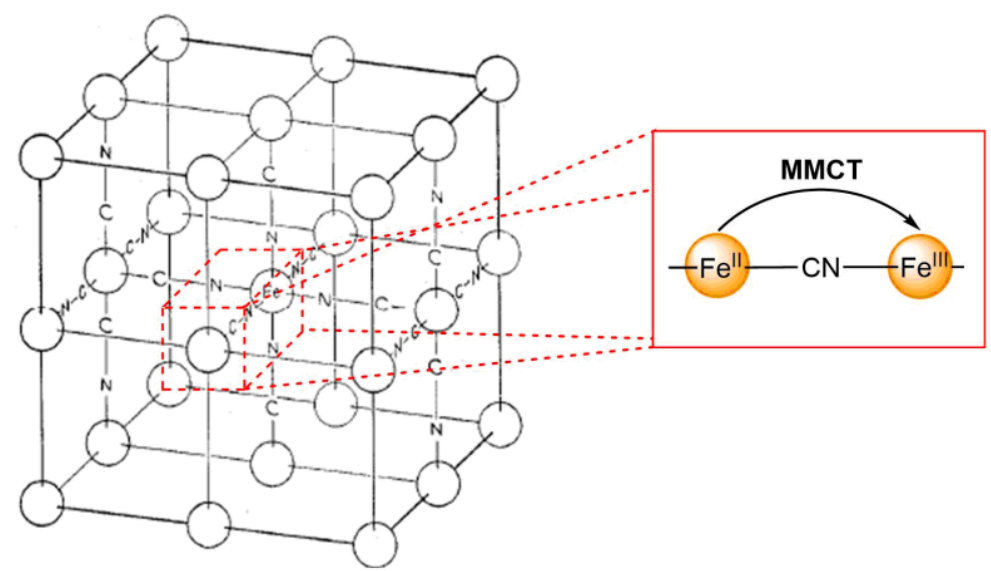

Figure 2.1 Illustration of the crystal lattice of Prussian Blue $\mathrm{Fe}(\mathrm{III})_{4}\left[\mathrm{Fe}(\mathrm{II})(\mathrm{CN})_{6}\right]_{3} \cdot 14 \mathrm{H}_{2} \mathrm{O}$ with schematic MMCT between the two mixed-valent iron centres. ${ }^{101}$ The rights for reprint have been granted by the American Chemical Society (Copyright $\odot$ 1962).

While the $\mathrm{Fe}(\mathrm{II}) \rightarrow \mathrm{Fe}$ (III) Inter-Valence-Charge transfer (IVCT) in Prussian Blue only results in the deep blue color, Hashimoto et al. realized in 1996 that exchanging one of the iron centers with a $\mathrm{Co}(\mathrm{II})$ ion in $\mathrm{K}_{0.2} \mathrm{Co}_{1.4}\left[\mathrm{Fe}(\mathrm{CN})_{6}\right] \cdot 6.9 \mathrm{H}_{2} \mathrm{O}$, couples the $\mathrm{Fe}(\mathrm{II}) \rightarrow \mathrm{Co}(\mathrm{III})$ electron transfer with the spin transition $l_{s}-\mathrm{Co}(\mathrm{III}) \rightarrow h s-\mathrm{Co}(\mathrm{II})$, affording the first $\mathrm{Co} / \mathrm{Fe}$ PBA material exhibiting a CTIST. ${ }^{102}$ The additional spin transition of Fe/Co PBAs gives rise to magnetic and optic bistability resulting in a diamagnetic state $(S=0)$ and a paramagnetic state $(S=1$ or 2$)$, dependent on the magnetic coupling (Scheme 2.2).

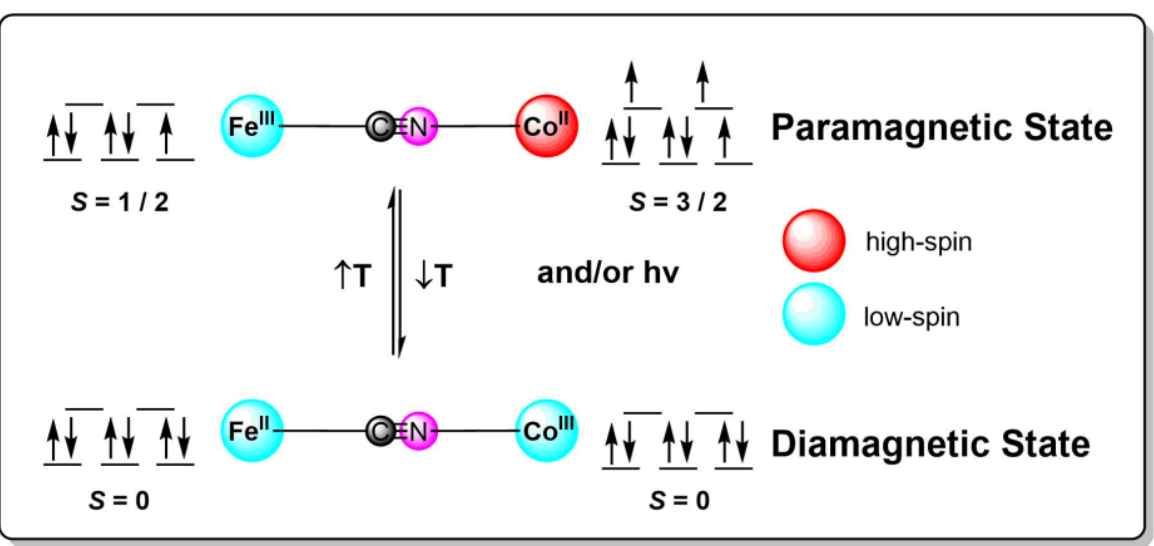

Scheme 2.2 Thermal and photo driven CTIST in Fe/Co Prussian Blue Analogues. ${ }^{100}$ Published by The Royal Society of Chemistry (Copyright $\odot$ 2016). 
This transition of the material showed high dependencies on the kind and amount of alkali metals in the crystal lattice. For example $\mathrm{Co}_{3}\left[\mathrm{Fe}(\mathrm{CN})_{6}\right]_{2}$ did not show a thermal or photodriven CTIST. ${ }^{103}$

Based on multiple investigations, this is due to different occupations of water molecules and alkali metal ions in the PBA lattice. Those defects change the ligand-field splitting of the cobalt ions through weaker ligand strength of the alkali metal compared to water. ${ }^{104,105}$ This effect was also confirmed by the group of Verdaguer ${ }^{106}$ by electronic potential changes. ${ }^{106}$ Although a variety of systems with different ratio of alkali metals or water have been synthesized, any reliable development of the materials towards magnetic switching is problematic due to the inhomogeneous distribution of those defects. ${ }^{100}$ The unreliable synthesis and insolubility in common solvents limits characterization methods to solid state analyses of mostly amorphous material. Therefore, investigations on the mechanism of the CTIST of those materials appears to be difficult.
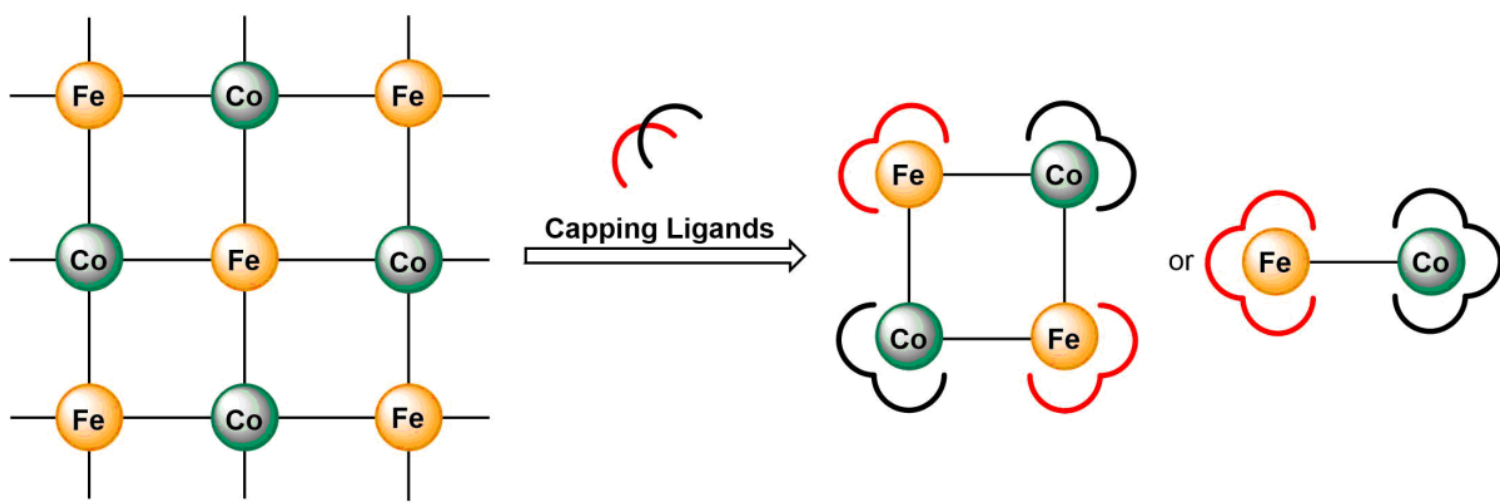

Scheme 2.3 Simplified utilization of capping ligands for di- and tetranuclear Fe/Co PBAs.

These problems with the 3D network materials resulted in the employment of organic capping ligands, predictably lowering the dimensions of the Fe/Co PBA through blocking the coordination spheres of the iron or cobalt ions (Scheme 2.3). Therefore, through denticity of the capping ligands different sizes of the molecular assembly can be produced. Additionally, fine-tuning of the electronic structure by the capping ligands is also possible. ${ }^{100}$ A definitive advantage for the molecular Fe/Co PBAs is the possibility of using solution-based characterization methods, such as UV/vis spectroscopy to further elucidate the light-induced switching of the two magnetic state. ${ }^{107,108}$ During the time first publications for the materials were presented, also the first molecular system gained interest. Although those complexes were the first molecular examples, none of them showed a CTIST in solid state or solution. For example, the group of Martinez introduced cyclam-based Fe/Co PBAs ${ }^{109-111}$, which were characterized in solution, but a CTIST effect was not observed.

The first molecular examples showing such behavior were presented in 2004 and 2005 by Berlinguette et al., utilizing tetramethyl phenanthroline (tmphen) as the capping ligand, 
reducing the 3D network to trigonal bipyramidal structures (Figure 2.2). ${ }^{112,113}$ The species, shown in Figure 2.2, is going through $\left[\mathrm{Co}(\mathrm{II}) \mathrm{Co}(\mathrm{III})_{2} \mathrm{Fe}(\mathrm{II})_{2}\right] \rightarrow$ $\left[\mathrm{Co}(\mathrm{II})_{2} \mathrm{Co}(\mathrm{III}) \mathrm{Fe}(\mathrm{II}) \mathrm{Fe}(\mathrm{III})\right] \rightarrow\left[\mathrm{Co}(\mathrm{II})_{3} \mathrm{Fe}(\mathrm{III})_{2}\right]$ transitions with increasing temperature. This thermally driven CTIST was confirmed by XRD analysis, ${ }^{57} \mathrm{Fe}$ Mößbauer spectroscopy and magnetic susceptibility measurements. Although the photo driven CTIST was not shown in the publication of 2004, studies in collaboration with the group of Clérac presented the photochemical excitation at $10 \mathrm{~K}$ of the low-temperature phase $\left[\mathrm{Co}(\mathrm{II}) \mathrm{Co}(\mathrm{III})_{2} \mathrm{Fe}(\mathrm{II})_{2}\right]$ with white light to the high temperature phase $\left[\mathrm{Co}(\mathrm{II})_{3} \mathrm{Fe}(\mathrm{III})_{2}\right] .{ }^{114}$
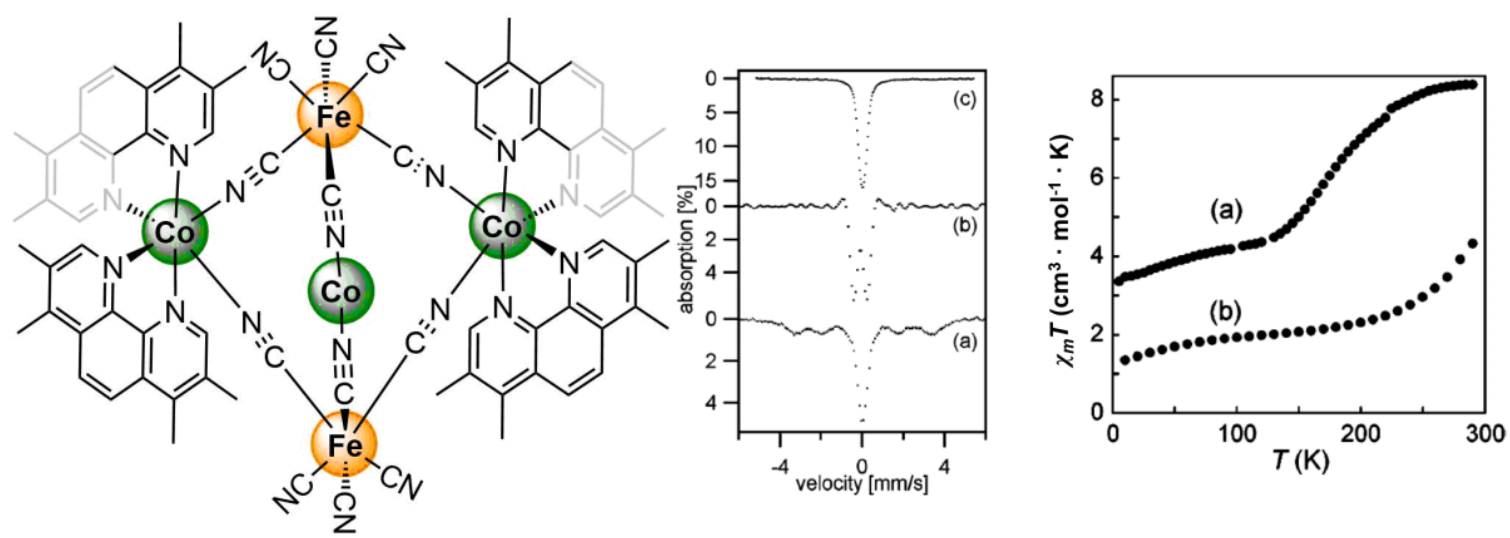

Figure 2.2 First molecular Fe/Co PBA system $\left[(\text { tmphen })_{2} \mathrm{Co}\right]_{3}\left[\mathrm{Fe}(\mathrm{CN})_{6}\right]_{2}$ with a thermal and photo driven CTIST from Berlinguette et. al. (tmphen ligands are omitted for the last cobalt for clarity);

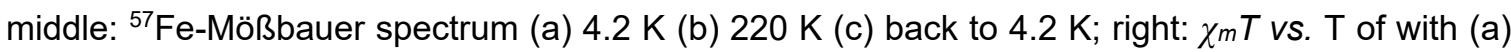
and without (b) solvent in the crystal lattice. ${ }^{112}$ The rights for reprint have been granted by The American Chemical Society (Copyright @ 2004).

The first example of a complex completely resembling a smaller unit of the bulk material was reported by Berlinguette et al. by $\left[(\mathrm{pztp}) \mathrm{Fe}(\mathrm{III})(\mathrm{CN})_{3}\right]_{4}\left[\mathrm{Co}(\mathrm{II})\left\{(\mathrm{pz})_{3} \mathrm{CCH}_{2} \mathrm{OH}\right\}\right]_{4}{ }^{4+} .{ }^{115}$ In the octanuclear cubane assembly the metal centers were capped utilizing two different pyrazole based tridentate scorpionate ligands. In contrast to the complex presented earlier by Berlinguette, the cubane system showed a direct thermally driven transition at around $250 \mathrm{~K}$ between the diamagnetic state $[l s-\mathrm{Fe}(\mathrm{II}) / l s-\mathrm{Co}(\mathrm{III})]$ and the paramagnetic state $[l s$ $\mathrm{Fe}(\mathrm{III}) / h s-\mathrm{Co}(\mathrm{II})]$, confirming the presence of magnetic switching. The same transformation was evidenced by an increase in magnetic susceptibility when the sample was irradiated with white light at $30 \mathrm{~K}$.

Since the first development of such molecular based systems, multiple other systems have been discovered with the most publications on square-like assemblies. ${ }^{100}$ One of such is the systems reported by Oshio et al. (Figure 2.3). Here, presence of a thermally driven CTIST was correlated to the use of specific substituents on the capping ligands. While the combination of tp and di-tert-butyl bipyridine (dtbbpy) as capping ligands showed a diamagnetic state as the magnetic ground state over the whole temperature range (blue dots in Figure 2.3, right), for the tris-(dimethyl pyrazolyl) borate (tp*) and bipyridine (bpy) the paramagnetic state was found (red dots in Figure 2.3, right). The mixture of both substituted ligands tp* and dtbbpy allowed observation of a two-step thermal driven CTIST 
with switching temperatures of $275 \mathrm{~K}$ and $310 \mathrm{~K}$ (green dots in Figure 2.3, right). ${ }^{116}$ The high switching temperature allowed the observation of the thermally driven CTIST for the first time in solution via UV/vis and IR spectroscopy next to solid state studies using ${ }^{57} \mathrm{Fe}$ Mößbauer spectroscopy, XRD and magnetic susceptibility measurements (Figure 2.7 and Figure 2.8 in Chapter 2.3). Interestingly, further work of Oshio et al. showed that the partial switching in the solid state is not fully switched on one edge of the square, but rather in a $50 / 50$ ratio of diamagnetic and paramagnetic molecules in the crystal lattice at $300 \mathrm{~K} .{ }^{117,118}$

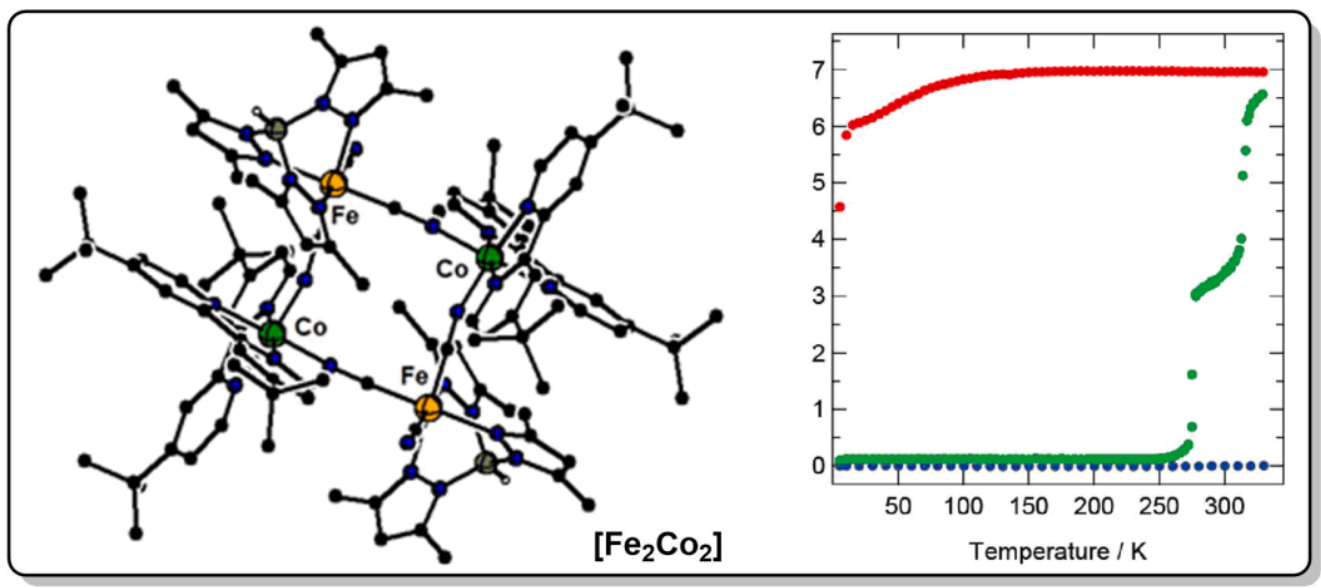

Figure 2.3 Left: Molecular structure of tetranuclear $\mathrm{Fe} / \mathrm{Co}$ PBA $\left[\left(\mathrm{tpFe}(\mathrm{CN})_{3}\right)_{2}\left(\mathrm{Co}(\mathrm{dtbbpy})_{2}\right] \mathrm{PF}_{6}\left[\mathrm{Fe}_{2} \mathrm{Co}_{2}\right]\right.$ from Oshio et al..;16 right: $\chi_{m} T$ vs. $T$ measurements of the three tetranuclear PBAs with different magnetic behavior dependent on the substitution of the capping ligands, red: paramagnetic $\left[\left(\mathrm{tp}^{*} \mathrm{Fe}(\mathrm{CN})_{3}\right)_{2}\left(\mathrm{Co}(\mathrm{bpy})_{2}\right]\right.$, blue: diamagnetic $\left[\left(\operatorname{tpFe}(\mathrm{CN})_{3}\right)_{2}\left(\mathrm{Co}(\mathrm{dtbbpy})_{2}\right]\right.$, green: $\left[\mathrm{Fe}_{2} \mathrm{Co}_{2}\right]$ with thermally driven CTIST. The rights for reprint have been granted by The American Chemical Society (Copyright @ 2011).

The ultimate miniaturization was realized by saturating the coordination sphere of the cobalt atom with the pentadentate ligand 2,6-bis(1,1-bis(2-pyridyl)ethyl)pyridine (PY5Me 2 , resulting in dinuclear systems. The first example by Clérac, utilizing 2,6bis(benzimidazole-2-yl)pyridine (bbp) for the iron ion, showed a $l s-\mathrm{Co}$ (II) $\rightarrow h s$-Co(II) spin-crossover (SCO) at $220 \mathrm{~K}$. However, through protonation of the bbp ligand at room temperature an electron transfer was facilitated, which results in the diamagnetic species (Figure 2.4, left). ${ }^{119}$ This proton dependent magnetic switching was investigated via the characteristic bands for the diamagnetic and paramagnetic species in the UV/vis spectrum. The second system [FeCo], using tp instead of bbp, showed a thermally induced transition at $165 \mathrm{~K}$ in the solid state. Irradiation with white light showed again that this system can also be photo-driven and affords a meta stable state at $5 \mathrm{~K}$ (Figure 2.4, right). In the most recent study on [FeCo], the magnetic switching was shown on the atomic scale via X-Ray absorption spectroscopy (XAS) and X-ray magnetic circular dichroism (XMCD) in the solid-state. As expected, no structural changes between the thermally induced paramagnetic state at $300 \mathrm{~K}$ and light induced paramagnetic state at $5 \mathrm{~K}$ were observed. ${ }^{120}$ For tuning of the electronic structure for [FeCo], suitable ligands were found by comparing the redox behavior of the individual mononuclear complexes $\left[(\mathrm{tp}) \mathrm{Fe}(\mathrm{CN})_{3}\right]^{-}$and $\left[\left(\mathrm{PY} 5 \mathrm{Me}_{2}\right) \mathrm{Co}\left(\mathrm{CH}_{3} \mathrm{CN}\right)\right]^{2+}$. While the correlation between redox behavior and magnetic 
switching has been investigated for Fe/Co PBA materials by Verdaguer ${ }^{106}$, the correlation for molecular system with the properties of the individual mononuclear components is rather untouched. Therefore, the system of Clérac provides a possible "recipe" for future development of dinuclear systems.
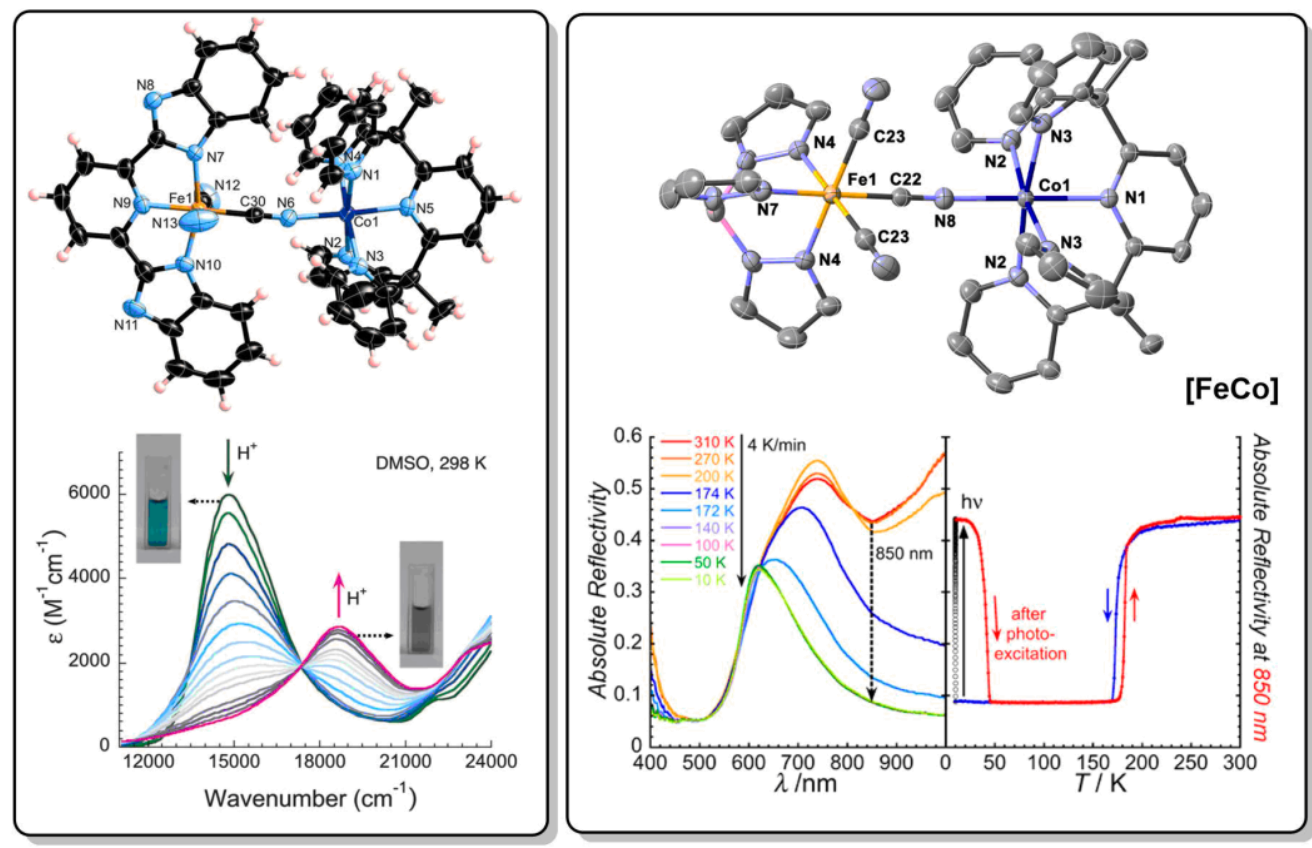

Figure 2.4 Structures of dinuclear Fe/Co PBAs demonstrating a spin transition through external stimuli; left: [(bbp) $\left.\mathrm{Fe}(\mathrm{CN})_{3} \mathrm{Co}\left(\mathrm{PY}_{5} \mathrm{Me}_{2}\right)\right]$ with a protonation induced electron transfer shown through UV/vis spectroscopy in DMSO solution; ${ }^{119}$ right: $\left[\mathrm{tpFe}(\mathrm{CN})_{3} \mathrm{Co}\left(\mathrm{PY}_{5} \mathrm{Me}_{2}\right)\right]^{+}([\mathrm{FeCo}])$ with thermal and photo driven CTIST illustrated by solid state UV/vis spectroscopy and SQUID measurements. ${ }^{121}$ The rights for reprint have been granted by The Royal Chemical Society (Copyright @ 2013 ). and The American Chemical Society (Copyright @ 2014).

Although a variety of PBAs demonstrating CTIST were developed and some of them were shown in this introduction, the mechanism of the CTIST is still not well understood. One challenge is the time dependent measurement of such electron transfer reactions, due to their very fast nature. A method capable of observing such fast transformations is ultrafast transient absorption spectroscopy. To the best of our knowledge, the only publication regarding Fe/Co PBAs, which utilized transient absorption spectroscopy on the nanosecond and femtosecond timescale, was presented in 2005 on dinuclear systems in the solidstate. $^{122}$ The importance of this method for the understanding of the CTIST mechanism $\mathrm{Fe} / \mathrm{Co} \mathrm{PBAs}$ will be discussed in the next section. 


\subsubsection{Pump-Probe Spectroscopy on Fe/Co PBAs}

Light-harvesting processes, such as photosynthesis, are not well understood, because of the short lifetimes (mostly $100 \mathrm{fs}$ ) of the intermediates and therefore challenging for stationary characterization methods. ${ }^{123}$ This is also a problem for investigation of the mechanism of LIESST and CTIST processes due to fast intersystem crossing. ${ }^{124}$ One particular approach to investigating such excitation mechanisms is ultrafast transient absorption spectroscopy. The schematic setup for such a pump-probe spectroscopy is shown in Scheme 2.4.

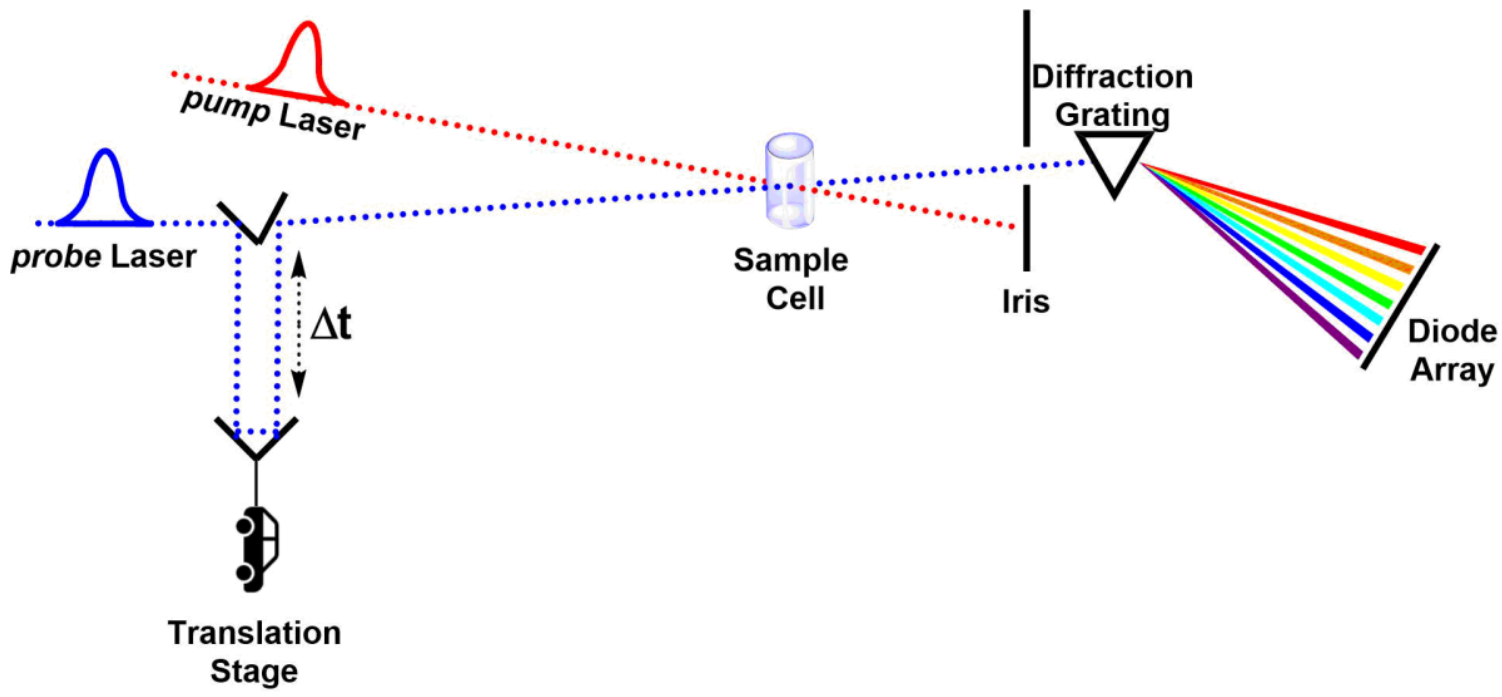

Scheme 2.4 Schematic setup of ultrafast transient absorption spectroscopy utilizing a pump laser and a probe laser time shifted through a translation stage. ${ }^{123}$
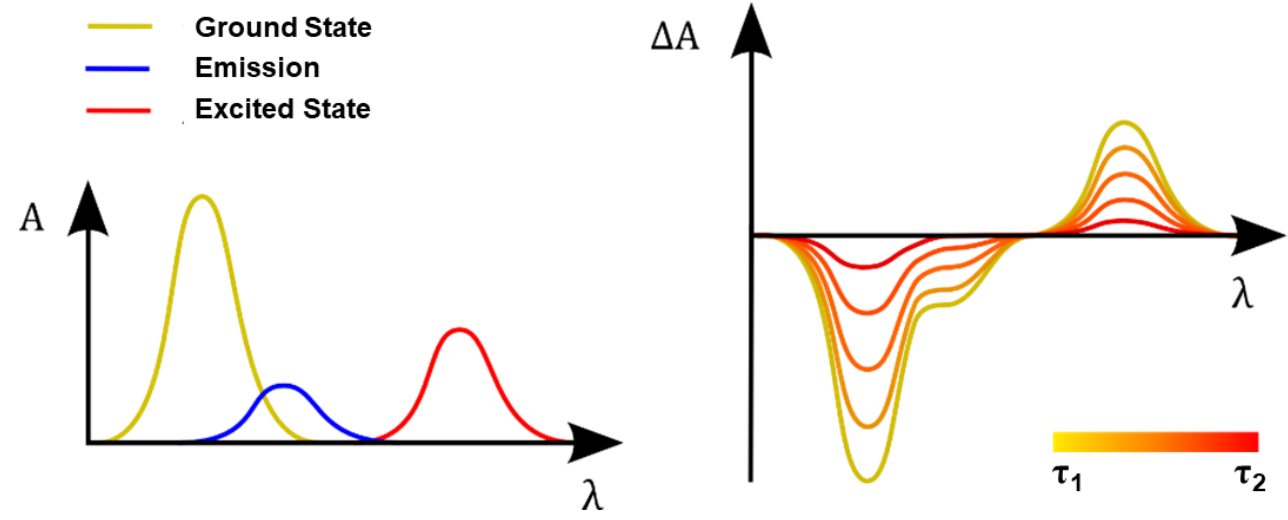

Figure 2.5 Left: Spectral contributions to the overall pump-probe spectrum; right: schematic pump-probe spectrum between two time delays $\tau_{1}$ and $\tau_{2 .}{ }^{125}$ Copyright $\odot 2018$ Sierke Verlag.

Through an excitation pulse (pump laser in Scheme 2.4) at a certain wavelength, a fraction of the molecules in the sample are promoted to an excited state. Simultaneously, a weak white light probe laser (probe laser in Scheme 2.4) irradiates the sample through a delay line, consisting of a precisely moveable translation stage. The delay $\tau$ in relation to the 
pump pulse results in a time dependent probing of the sample. The possibility of small changes in distance through the translation stage in combination of the fast pulses result in a time resolution of lower than $100 \mathrm{fs}$, consequently an examination on the intermediates formed upon photooxidation may be possible. In contrast to typical spectroscopy (UV/vis, IR and so on), a difference in optical density $\Delta \mathrm{A}$ or $\triangle \mathrm{mOD}$ between the excited state and the ground state spectra at a certain delay $\tau$ is obtained. The resulting spectra can then be analyzed by global analysis to extract lifetimes and the number of intermediates. A typical pump-probe spectrum is shown in Figure 2.5 (right).

Coupling with various types of spectroscopy, including Extended X-Ray absorption (EXAFS), UV/vis and IR spectroscopy, is feasible and allows the observation of structural and electronical changes. ${ }^{122,124,126}$ For this, all contributions from the various processes must be considered to fully understand the spectral changes observed. The first observed process is the bleaching of the ground state. Due to the excitation of a partial number of molecules in the sample, the number of molecules in the ground state decreases, which results in a negative absorption difference at the expected wavelength of the ground state spectrum. A second contribution may be observed in the $\Delta \mathrm{A}$ arising from the stimulated emission. These Stoke shifted bands are observed in combination with the ground state bleach, but the shift may be extremely small dependent on the system, therefore overlapping with the ground state bleach. Because of that process the probing laser is chosen to be weaker to not interfere drastically with the population of the excited state. The third process arises from the absorption of the excited state itself, resulting in a positive $\Delta \mathrm{A}$. After excitation of the molecules, chemical reactions may also occur on the timescale of the measurement. ${ }^{123}$ All those contributions must be taken into account, when interpreting the resulting spectrum in order to determine a reaction pathway. All in all, ultrafast transient absorption spectroscopy can be used as a powerful tool to investigate those rapid fast processes.

One useful application of transient absorption spectroscopy is the investigation of the CTIST for Fe/Co PBAs, which has been started by the group of Bernhard. ${ }^{122}$ For this, cyano-bridged dinuclear mixed-valent complexes Fe-Co and Ru-Co containing pentadentate ligands were investigated via UV/vis transient absorption spectroscopy (Figure 2.6). Variable temperature magnetic susceptibility measurements of the Fe-Co

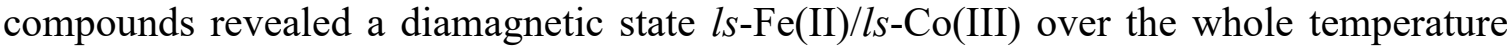
range, which allowed an excitation at 400 and $550 \mathrm{~nm} \cdot{ }^{127,128}$ Two different relaxation pathways of the excited states were obtained using different laser pulses (fs or ns) at the same excitation wavelengths and temperature. While for the femtosecond pulse a fairly short-lived excited state was observed, which decays back to the ground state via reverse electron donation through a hot vibrational ground state $\left(\tau_{1}=0.75 \mathrm{ps} ; \tau_{2}=28 \mathrm{ps}\right)$, the longer nanosecond pulse results in a long-lived species. This difference of lifetime is due to the nature of the nanosecond excitation, which results in a higher population of the paramagnetic state $l s-\mathrm{Fe}(\mathrm{III}) / h s-\mathrm{Co}(\mathrm{II})$. Therefore, a long-lived excited state is observed $\left(\tau_{1}=10.4 \mathrm{~ns}\right)$. The transition back to the ground state is spin-forbidden and the system relaxes back via intersystem crossing (ISC). Interestingly, this could indicate that a CTIST 
of this species is only possible with a longer exposure time for excitation of the diamagnetic state. The hypothesized pathway is shown in Figure 2.6 (right).
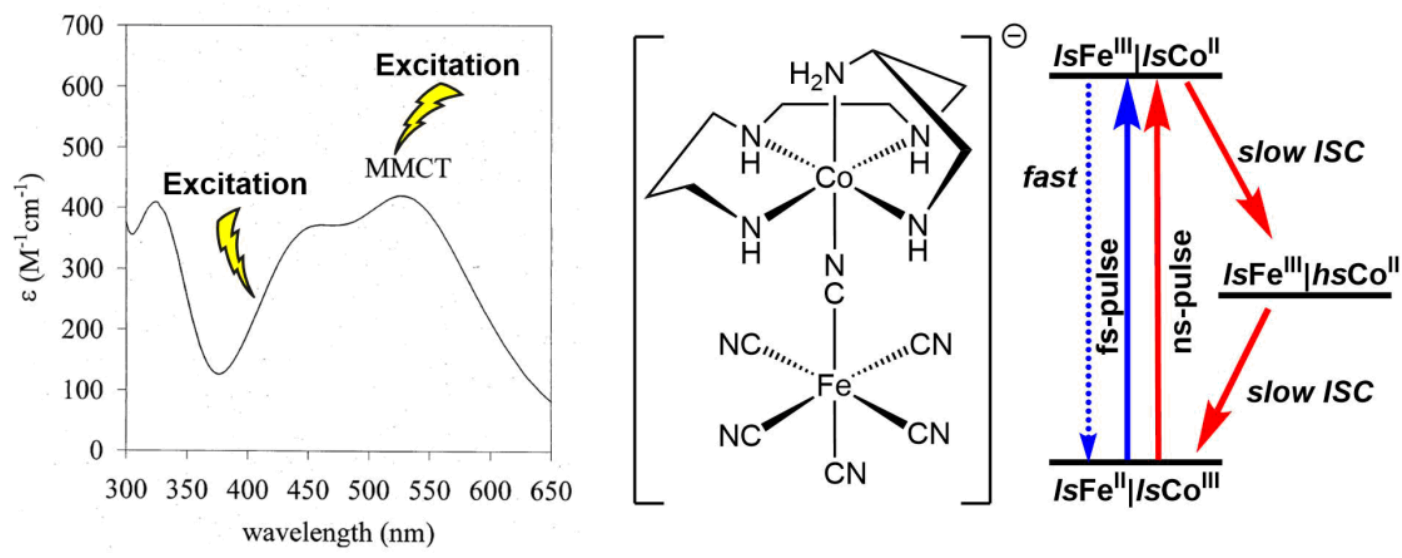

Figure 2.6 Left: Stationary UV/vis spectrum of dinuclear Fe/Co PBA by Bernhard et al. ${ }^{127}$ (middle); right: different excitation pathways of Fe/Co PBA dependent on the lifetime of the laser pulse. ${ }^{122}$ The rights for reprint have been granted by The American Chemical Society (Copyright $@ 2000$ and 2005).

Some questions still remain, for example whether the same pathway is to be expected for all dinuclear Fe/Co PBAs, or whether the pulse dependency is only found for the specific system presented? Is this behavior also dependent on the dominant ground state being the diamagnetic state or is a corresponding pathway also observed for the paramagnetic state? Additionally, IR spectroscopy could be utilized to interpret structural changes associated with the CTIST process with the help of the cyanide stretching frequency. Those questions should be investigated through utilization of an established Fe/Co PBA as a benchmark system, complemented by the development of new literature unknown dinuclear $\mathrm{Fe} / \mathrm{Co}$ PBAs to evaluate the information gained through transient absorption spectroscopy. 


\subsection{Project Outline}

As mentioned in the introduction, the photo driven CTIST in Fe/Co PBAs provides a possible application of such system as bits in information storage devices through their light induced transition between a magnetic and a nonmagnetic state. The mechanism behind the light-harvesting process is still rather unclear, but preliminary investigations have been conducted using ultrafast transient absorption spectroscopy. The upcoming work should therefore provide further information to elucidate the mechanism.

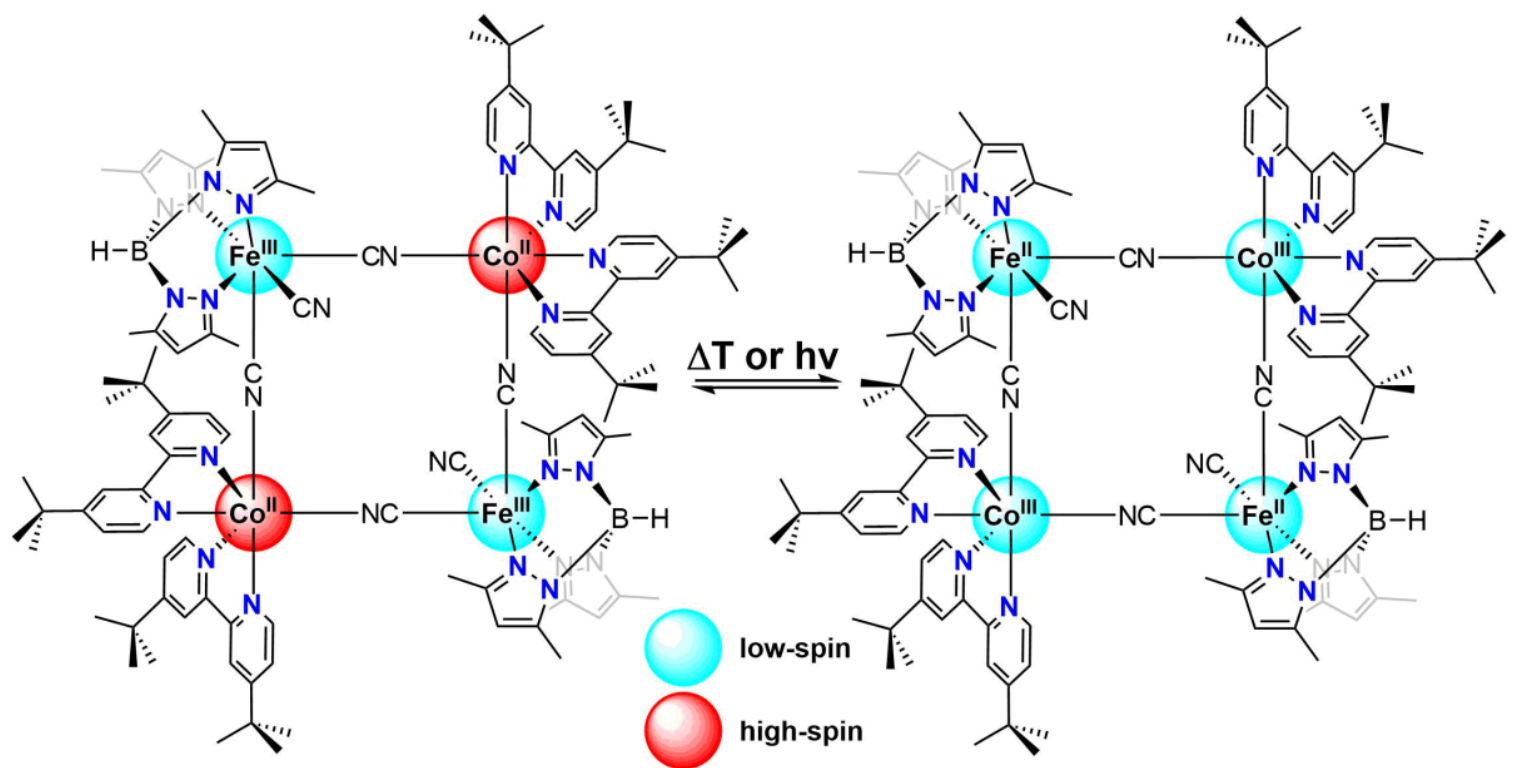

Scheme 2.5 Interconversion of the anionic part of $\left[\mathrm{Fe}_{2} \mathrm{Co}_{2}\right]$ at $\mathrm{T}_{1 / 2}=275 \mathrm{~K}$ between paramagnetic state $\left[(/ s-\mathrm{Fe}(\mathrm{III}))_{2} /(h s-\mathrm{Co}(\mathrm{II}))_{2}\right]$ and diamagnetic state $\left[(/ s-\mathrm{Fe}(\mathrm{III}))_{2} /(/ s-\mathrm{Co}(\mathrm{III}))_{2}\right] .{ }^{116}$

In the first part of this thesis, the tetranuclear Fe/Co PBA [ $\left.\mathbf{F e}_{2} \mathbf{C o}_{2}\right]$ from Oshio et al. ${ }^{116}$ with a high switching temperature of 275 and $310 \mathrm{~K}$ should be synthesized and characterized (Scheme 2.5). The oxidation state sensitive cyanide stretching frequencies can be used to observe intermediates during the excitation via UV/vis-pump IR-probe spectroscopy. Additionally, the high switching temperature of $\left[\mathbf{F e}_{2} \mathbf{C o}_{2}\right]$ allows the investigation of both spin states as the ground state in solution. This investigation should elucidate differences in the CTIST upon excitation of the paramagnetic state $\left[\left(l_{s}\right.\right.$ $\left.\mathrm{Fe}(\mathrm{III}))_{2} /(h s-\mathrm{Co}(\mathrm{II}))_{2}\right]$ or the diamagnetic state $\left[\left(l_{s}-\mathrm{Fe}(\mathrm{III})\right)_{2} /(l s-\mathrm{Co}(\mathrm{III}))_{2}\right]$. The information gained should be further on used to evaluate the electronic transition of literature unknown $\mathrm{Fe} / \mathrm{Co}$ PBAs.

Pentadentate polypyridine ligands were already presented for dinuclear Fe/Co PBAs, such as [FeCo], to tune the electronic structure of the cobalt ion in favor of a thermal and photo driven CTIST. The application of pyrazole, amine or imidazole donors instead is rather unknown for this field of dinuclear assemblies, despite of the broad availability of these Ndonor groups. The $\mathrm{N}_{5}$-ligands (Scheme 2.6) should be utilized as capping ligands and the corresponding dinuclear Fe/Co PBAs should be synthesized. 
<smiles>c1cc(C(n2cccn2)n2cccn2)nc(C(n2cccn2)n2cccn2)c1</smiles>

$\mathrm{pz}_{4}$ Lut<smiles>Cc1ccn(C(c2cccc(C(n3ccc(C)n3)n3ccc(C)n3)n2)n2ccc(C)n2)n1</smiles>

$\mathrm{pz}^{\mathrm{Me}}{ }_{4} \mathrm{Lut}$<smiles>Cc1cc(C)n(C(c2cccc(C(n3nc(C)cc3C)n3nc(C)cc3C)n2)n2nc(C)cc2C)n1</smiles>

pz* ${ }_{4}$ Lut<smiles>CC(c1cccc(C(C)(n2cccn2)n2cccn2)n1)(n1cccn1)n1cccn1</smiles>

$\mathrm{pz}_{4}$ depy

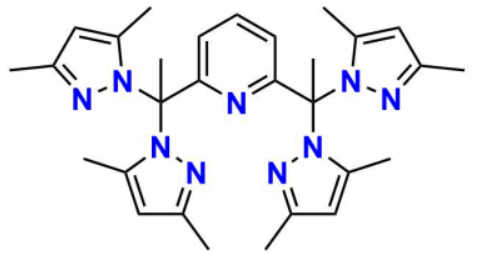

$\mathrm{pz}_{4}{ }^{*}$ depy<smiles>Cc1cc(C(F)(F)F)nn1C(c1cccc(C(n2nc(C(F)(F)F)cc2C(F)(F)F)n2nc(C(F)(F)F)cc2C(F)(F)F)n1)n1nc(C(F)(F)F)cc1C(F)(F)F</smiles><smiles>CC(c1cc[nH]n1)(c1cc[nH]n1)c1cccc(C(C)(c2cc[nH]n2)c2cc[nH]n2)n1</smiles><smiles>c1ccc(CN(Cc2ccccn2)C(c2ccccn2)c2ccccn2)nc1</smiles>

N4Py

Scheme 2.6 Pentadentate $\mathrm{N}_{5}$-ligands for the synthesis of dinuclear Fe/Co PBAs for the main-

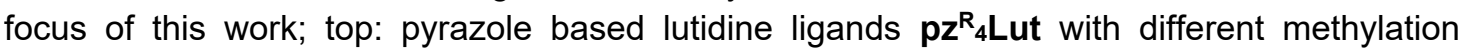
patterns; bottom left: $\mathrm{Pz}_{4} \mathrm{PyOMe} 2\left(\mathbf{L}^{\mathrm{pz}}\right) ;{ }^{129}$ bottom right: pentadentate derivative of the wellknown tripodal tpa ligand system developed by Feringa et al.

Similar to the literature known dinuclear system [FeCo], the synthesis of the new complexes should utilize the iron cyanide precursor bearing scorpionate ligands $\mathbf{t p}, \mathbf{t p}^{\mathbf{M e}}$ and $\mathbf{t p} *$ to also vary the electronic structure of the iron center (Scheme 2.7).

The resulting dinuclear Fe/Co PBAs should then be thoroughly characterized to investigate the ground state electronic structure at different temperatures. Ideally, a thermal induced switching between the magnetic and nonmagnetic state, similar to $\left[\mathbf{F e}_{2} \mathbf{C o}_{2}\right]$, would be observed. Afterwards, the excitation pathways of $\left[\mathbf{F e}_{2} \mathbf{C o}_{2}\right]$ and the dinuclear $\mathrm{Fe} / \mathrm{Co} \mathrm{PBAs}$ should be investigated via femtosecond transient absorption spectroscopy, applying UV/vis and IR probe lasers. The structural and electronical changes observed should give new information on the nature of this light-harvesting process and benefit future development in this field. 


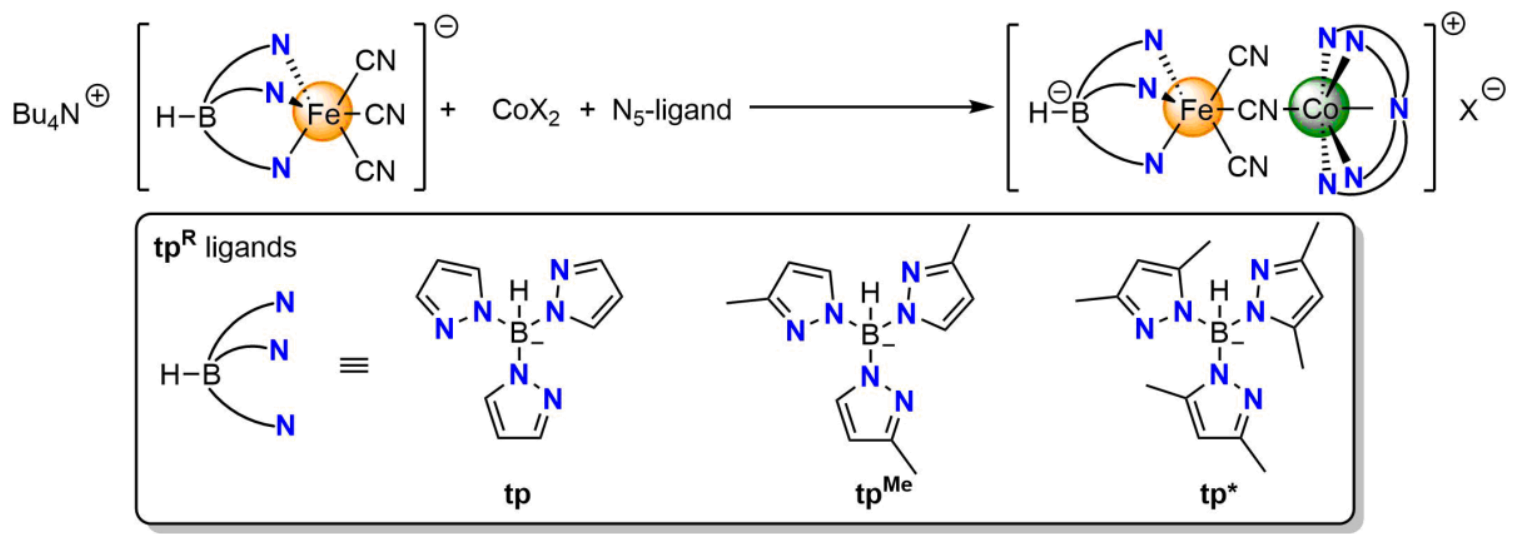

Scheme 2.7 Proposed synthesis route for the dinuclear Fe/Co PBAs consisting of pentadentate

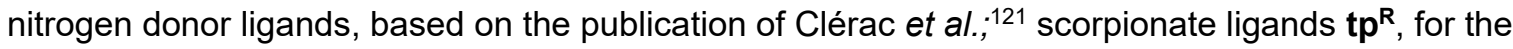
capping of the iron ion. 


\subsection{Synthesis and Characterization of the Tetranuclear $\mathrm{Fe} / \mathrm{Co}$ Prussian Blue Analogue $\left[\mathrm{Fe}_{2} \mathrm{CO}_{2}\right]$}

The synthesis of $\left[\mathrm{Fe}_{2} \mathbf{C o}_{2}\right]$ was carried out as described in the literature ${ }^{116}$, producing the cobalt(II)-dtbbpy precursor in situ and reacting with $\mathrm{Bu} 4 \mathrm{~N}\left(\mathrm{tp}^{*} \mathrm{Fe}(\mathrm{CN})_{3}\right]$. The simplified procedure is shown in Scheme 2.8. After anion exchange and recrystallization, $\left[\mathrm{Fe}_{2} \mathbf{C o}_{2}\right]$ was obtained as dark red crystals. The crystals of $\left[\mathbf{F e}_{2} \mathbf{C o}_{2}\right]$ were analyzed by X-ray diffraction (XRD) measurements, IR and UV/vis spectroscopy, which confirmed the successful synthesis.

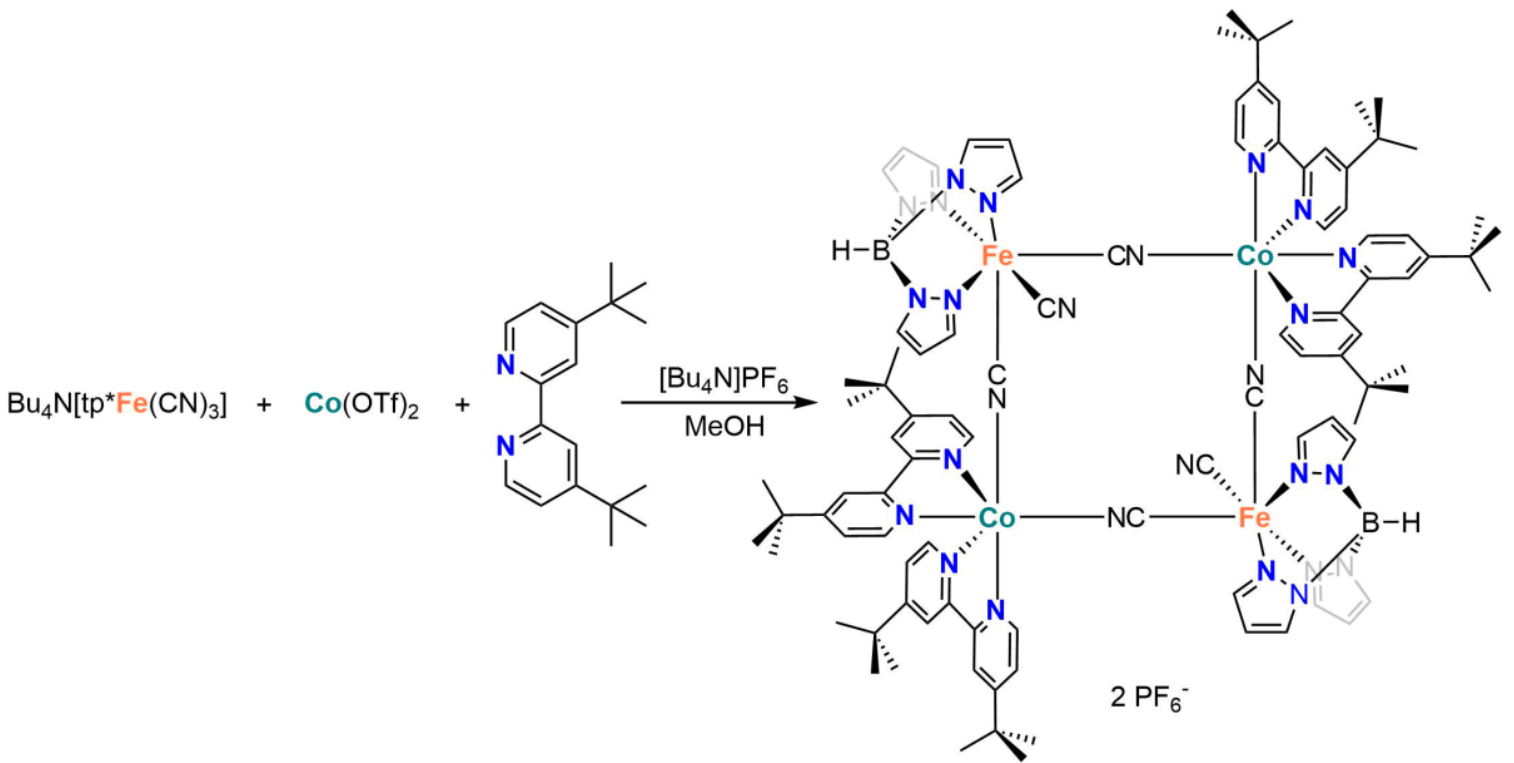

Scheme 2.8 Synthesis of tetranuclear PBA $\left[\mathrm{Fe}_{2} \mathrm{Co}_{2}\right]$ based of the literature procedure; in situ production of (dtbbpy $)_{2} \mathrm{Co}(\mathrm{OTf})_{2}$ and reaction with $\mathrm{Bu} 4 \mathrm{~N}\left[\mathrm{tp}{ }^{*} \mathrm{Fe}(\mathrm{CN})_{3}\right]$ followed by salt metathesis.

The molecular structure of $\left[\mathbf{F e}_{2} \mathbf{C o}_{2}\right]$ at $133 \mathrm{~K}$ confirms the tetranuclear assembly. Compared to the literature, diethyl ether and water are incorporated into the crystal structure instead of methanol, but this does not affect the subsequent solution-based measurements (vide infra). Overall the bond lengths and angles are consistent with the parameters reported in the literature, even though, the unit cell is not the same.

In the solution FT-IR spectrum of $\left[\mathbf{F e}_{2} \mathbf{C o}_{2}\right]$ at room temperature the bands for the C-N and $\mathrm{B}-\mathrm{H}$ stretching vibrations bonds are found at the published frequencies for the paramagnetic state $\left[(l s-\mathrm{Fe}(\mathrm{III}) / h s-\mathrm{Co}(\mathrm{II}))_{2}\right]$ (Table 2.2 in Section 2.4.2). Upon cooling to $246 \mathrm{~K}$ the bands shift to the ones of the diamagnetic state [( $\left.l s-\mathrm{Fe}(\mathrm{II}) / l s-\mathrm{Co}(\mathrm{III}))_{2}\right]$ (Figure 2.7). Interestingly, at roughly $23.4^{\circ} \mathrm{C}$ a small amount of the diamagnetic state is observed, which must be considered for the pump-probe spectroscopy later on. 


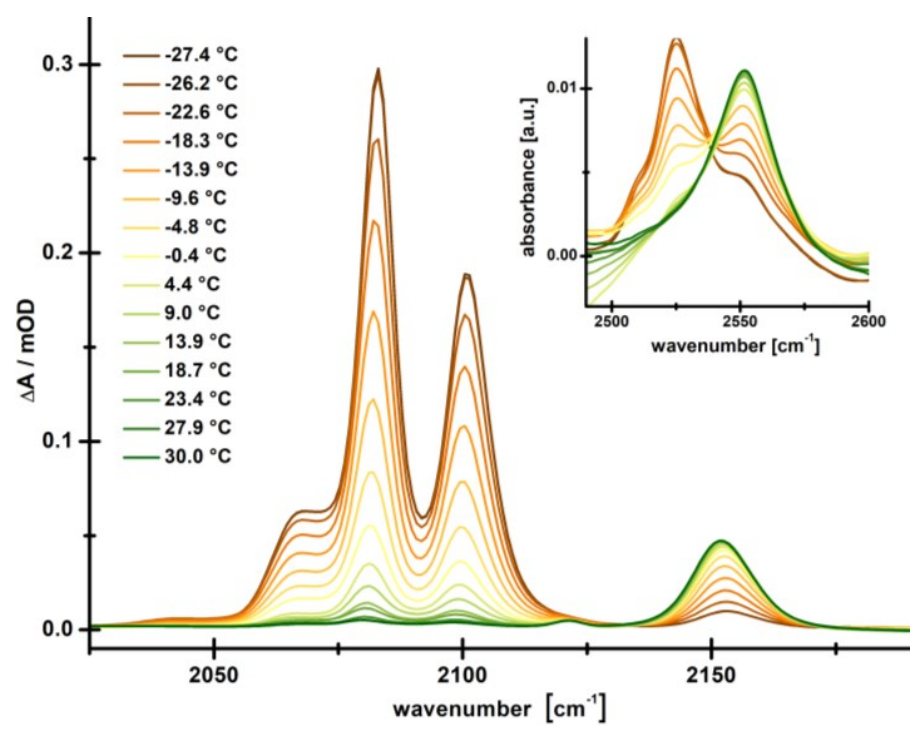

Figure 2.7 Temperature dependent FT-IR spectrum of $\left[\mathrm{Fe}_{2} \mathrm{Co}_{2}\right]$ in acetonitrile, carried out by Dr. Ahrens, MPI.

The same thermal changes were also observed in the UV/vis spectrum upon cooling. The $\mathrm{Co}(\mathrm{II}) \rightarrow \mathrm{Fe}$ (III) IVCT band of the paramagnetic state at $400 \mathrm{~nm}$ vanishes and give rise to a broad band at $800 \mathrm{~nm}$ for the $\mathrm{Fe}(\mathrm{II}) \rightarrow \mathrm{Co}$ (III) IVCT of the diamagnetic state (Figure 2.8, left). While screening for a suitable solvent for the femtosecond transient absorption spectroscopy, other solvents than acetonitrile were used and a dependency of the switching temperature on the solvent used was observed. The results of this investigation are shown in Figure 2.8 (right). For more nonpolar derivatives of acetonitrile, such as propio- and butyronitrile, lower switching temperatures were observed.
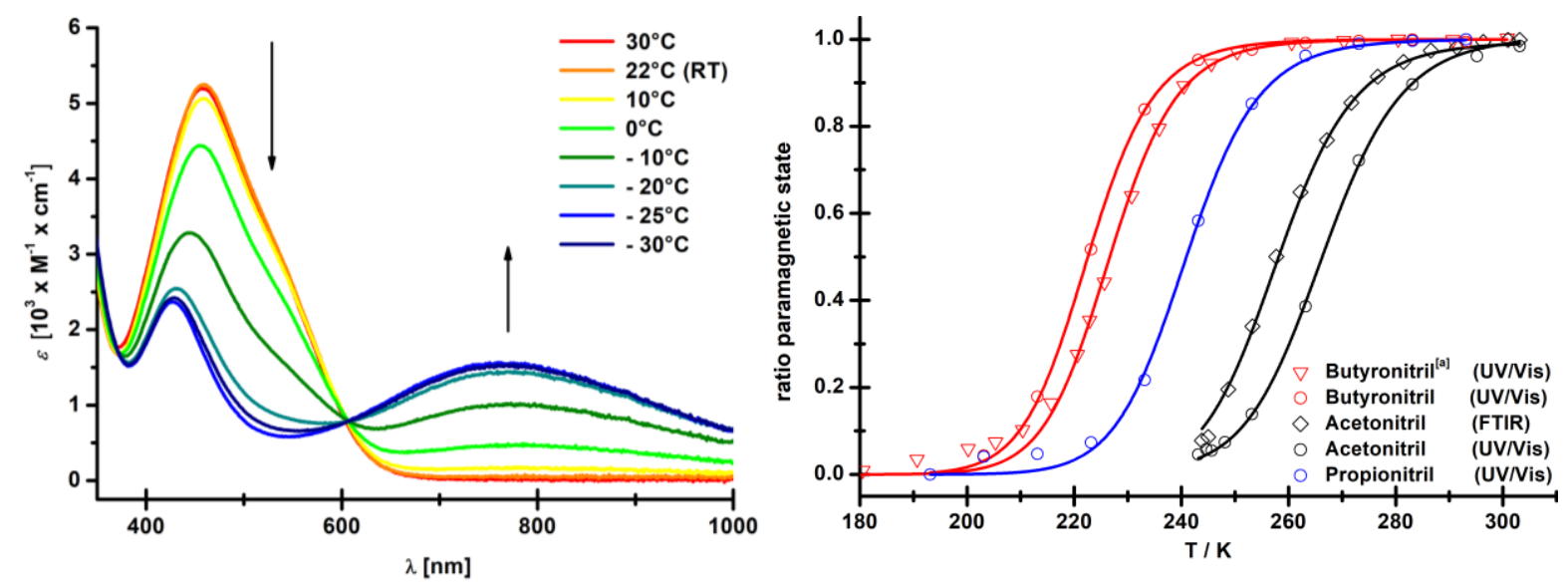

Figure 2.8 Left: temperature dependent UV/vis spectrum of $\left[\mathrm{Fe}_{2} \mathrm{Co}_{2}\right]$ in acetonitrile; right: temperature and solvent dependent ratio of the paramagnetic state of $\left[\mathrm{Fe}_{2} \mathrm{Co}_{2}\right]$ with $[a]$ taken from the literature

The discussed measurements illustrate the ideal properties of $\left[\mathbf{F e}_{2} \mathbf{C o}_{2}\right]$ as a suitable benchmark system for investigating the CTIST via transient absorption spectroscopy. The 
C-N and B-H stretching frequencies will be used to distinguish between dia- and paramagnetic state. In addition, the UV/vis spectrum of $\left[\mathbf{F e}_{2} \mathbf{C o}_{2}\right]$ in the diamagnetic state shows a characteristic IVCT band at $800 \mathrm{~nm}$, which allows selective excitation. Due to the high switching temperatures of $\left[\mathbf{F e}_{2} \mathbf{C o}_{2}\right]$ the photo physics of both states could be investigated individually. In contrast to the tetranuclear assembly of the benchmark system, the next section will focus around the development of dinuclear Fe/Co PBAs to further investigate the photophysical properties of the most simple and miniaturized $\mathrm{Fe} / \mathrm{Co}$ units. 


\subsection{Dinuclear Fe/Co PBAs Containing Polypyrazolyl Lutidine Ligands}

The pentadentate polypyrazolyl lutidine ligands (Scheme 2.9), derived from tetrakis(2-pyridyl)- $m$-lutidine PY5, have been used in multiple fields of research, for example PCET studies with a cobalt-hydroxide complex ${ }^{130}$ and tandem Suzuki coupling / transfer hydrogenation catalysis with $\mathrm{Pd} / \mathrm{Ru}$ heterobimetallic complexes. ${ }^{131}$ Despite of their availability, pentadentate pyrazole ligands have not been used for $\mathrm{Fe} / \mathrm{Co}$ PBAs until now. An investigation on the electronic structure changes induced by employing a fivemembered ring with two nitrogen atoms is therefore still untouched. Additionally, the ligands are easily synthesized based on 2,6-pyridinecarbaldehyde through a cobalt catalyzed Peterson rearrangement reaction. Modifications of the pyrazole moieties are possible. due to the commercial availability of the corresponding substituted pyrazole derivatives.<smiles>[R16]c1cccc(C([R])(c2ccccn2)c2cccc(C([R])(c3ccccn3)c3ccccn3)n2)n1</smiles>
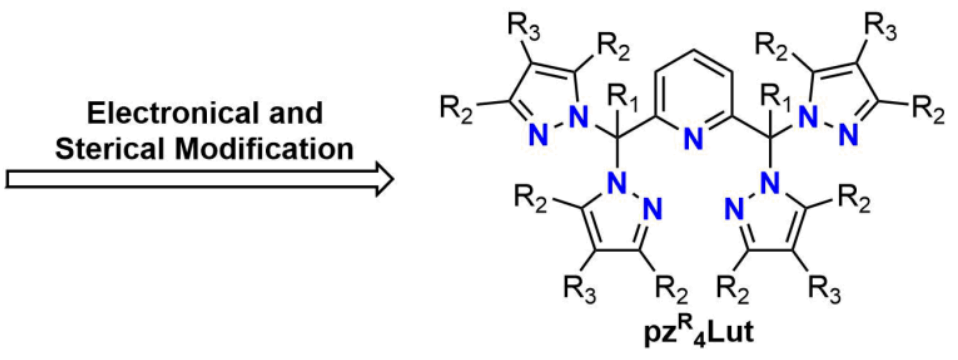

Scheme 2.9 Modification of 2,6-bis((bis-2-pyridyl)methane)pyridine (PY5R $)$ by incorporation of pyrazole instead of pyridine sidearms, resulting in $\alpha, \alpha, \alpha^{\prime}, \alpha^{\prime}-$ tetra(pyrazolyl)lutidine $\left(\mathbf{p z}^{\mathbf{R}}{ }_{4} \mathrm{Lut}\right)$ with variable $\mathrm{R}$ groups for electronic and steric tuning. 


\subsubsection{Synthesis of the Polypyrazolyl Lutidine Ligands $\mathbf{p z}^{\mathbf{R}_{\mathbf{4}}} \mathbf{L u t}$}<smiles>Cc1cccc(C)n1</smiles><smiles>COc1cccc(Cl)c1Cl</smiles><smiles>[R][R]([R])c1cc([R])n(S(=O)n2nc([R])cc2[R])n1</smiles><smiles>CC(=O)C1CCCCC1</smiles><smiles>[R]c1ccn(C(c2cccc(C(n3nc([R])cc3[R])n3nc([R])cc3[R])n2)n2nc([R])cc2[R])n1</smiles>

Scheme 2.10 Synthesis of $\mathbf{p z}_{4}$ Lut and $\mathbf{p z}^{*}{ }_{4}$ Lut based on 2,6-lutidine (VI) through oxidation with $\mathrm{SeO}_{2}$ to the dialdehyde (VII) and cobalt-catalyzed Peterson rearrangement with di-pyrazolyl sulfone (VIII-R).

$\mathbf{p z}_{4} \mathbf{L u t}$ is synthesized through a cobalt-catalyzed Peterson rearrangement reaction of 2,6pyridine carbaldehyde VII with di-pyrazolyl sulfone VIII-H (Scheme 2.10). ${ }^{132}$ The 2,6pyridine carbaldehyde VII is easily available through an oxidation of 2,6-lutidine VI with selenium dioxide. ${ }^{133}$ Afterwards the di-pyrazolyl sulfone VIII-R is produced in situ by deprotonation of the corresponding pyrazole with sodium hydride and reaction with thionyl chloride afterwards. As shown in Scheme 2.10, $\mathbf{p z} \mathbf{z}_{\mathbf{4}} \mathbf{L u t}$ and $\mathbf{p z}^{*}{ }_{4} \mathbf{L u t}$ were synthesized according to literature procedure in moderate yields and characterization via NMR spectroscopy and ESI mass spectrometry are consistent with the literature. ${ }^{134,135}$
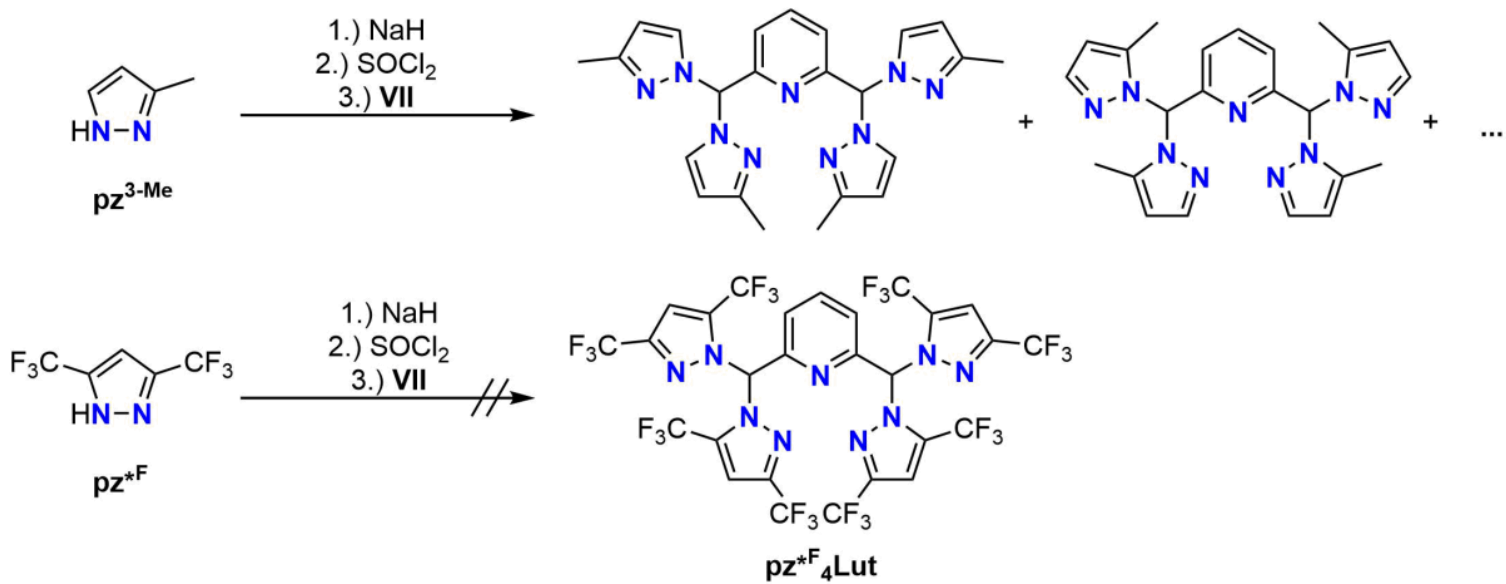

Scheme 2.11 Proposed synthesis of $\mathrm{pz}^{\mathrm{Me}}{ }_{4} \mathrm{Lut}$ and $\mathrm{pz}^{\mathrm{*}}{ }_{4} \mathrm{Lut}$; top: for $\mathrm{pz}^{\mathrm{Me}}{ }_{4} \mathrm{Lut}$ regioselectivity was not observed; bottom: $\mathbf{p} \mathbf{z}^{* \mathrm{~F}}$ showed no reactivity towards the coupling reaction.

When asymmetric 3-methylpyrazole $\mathbf{p z}^{\mathbf{M e}}$ was used, regio selectivity of the reaction was not observed, confirmed by ${ }^{1} \mathrm{H}-\mathrm{NMR}$ spectroscopy and mass spectrometry (ESI-MS). In the ${ }^{1} \mathrm{H}-\mathrm{NMR}$ spectrum of the product after workup, multiple signals for the methyl and pyrazole protons were observed, but only one molecular peak was found in the ESI-MS. 
The ${ }^{1} \mathrm{H}-\mathrm{NMR}$ spectrum and mass spectrum are found in the Appendix. The reaction of the deprotonated pyrazole with thionyl chloride in a non-regioselective manner could be the reason for the number of different isomers (Scheme 2.11, top). Also, for the electron-poor 3,5-bis(trifluoromethyl)pyrazole $\mathbf{p} \mathbf{z}^{* \mathbf{F}}$ the resulting pentadentate ligand was not isolated. Due to the electron withdrawing substituents compared to the non-fluorinated $\mathbf{p z} \mathbf{z}^{*}$ moiety, a mixture of partial and fully substituted ligand was observed, which were not separable. Longer reactions times did not result in the full substitution (Scheme 2.11, bottom). Therefore, the ligands $\mathbf{p z}^{\mathrm{Me}}{ }_{4} \mathbf{L u t}$ and $\mathbf{p z}^{* \mathrm{~F}}{ }_{4} \mathbf{L u t}$ were not obtained in the time frame of the dissertation.
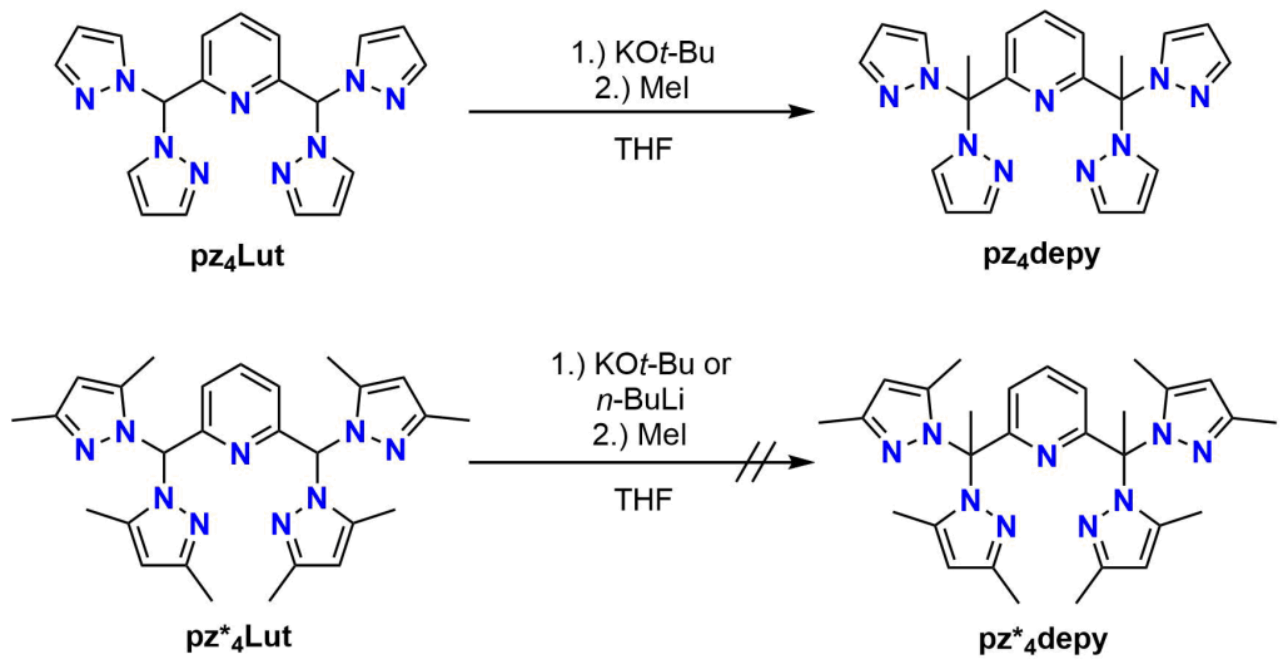

Scheme 2.12 Methylation attempts for the picolinic position of $p z_{4} L u t$ and $\mathbf{p z}^{*}{ }_{4}$ Lut; top: $\mathbf{p z} \mathbf{z}_{4} \mathbf{d e p y}$ was synthesized by literature procedure; bottom: steric hinderance resulted in the incomplete methylation of $\mathbf{p z}^{*}{ }_{4} \mathrm{Lut}$.

In addition to the substitution of the pyrazole sidearms, methylation of the picolinic position for the synthesized ligands was also feasible. A similar modification is also seen in PY5Me for $[\mathbf{F e C o}] .{ }^{121}$ For $\mathbf{p z}_{4} \mathbf{L u t}$ the methylation of the picolinic positions was already shown by the group of Berlinguette. ${ }^{130}$ Here, $\mathbf{p z}_{4} \mathbf{L} \mathbf{u t}$ was first deprotonated by $n$-BuLi or $\mathrm{KO} t$-Bu and then reacted with an excess of methyl iodide to obtain pz4depy (Scheme 2.12, top). Deprotonation with $\mathrm{KO} t$-Bu was also attempted with $\mathbf{p z}^{*}{ }_{4} \mathbf{L u t}$, but no reaction was observed after addition of methyl iodide and workup. The same outcome was observed when $n$-BuLi was used for deprotonation, hence it appears that the steric hindrance likely is the reason why no substitution was observed.

Three ligands $\mathbf{p z} \mathbf{4}$ Lut, $\mathbf{p z}^{*}{ }_{4}$ Lut and $\mathbf{p z _ { 4 }}$ depy were obtained (Scheme 2.13). The characterization data of the ligands is consistent with the literature. With $\mathbf{p z} \mathbf{z}_{4} \mathbf{L u t}, \mathbf{p z} \mathbf{z}_{\mathbf{4}} \mathbf{d e p y}$ and $\mathbf{p z}^{*}{ }_{4}$ Lut in hand, the synthesis of the corresponding dinuclear PBAs using these pentadentate ligands will be discussed and their magnetic properties characterized. 

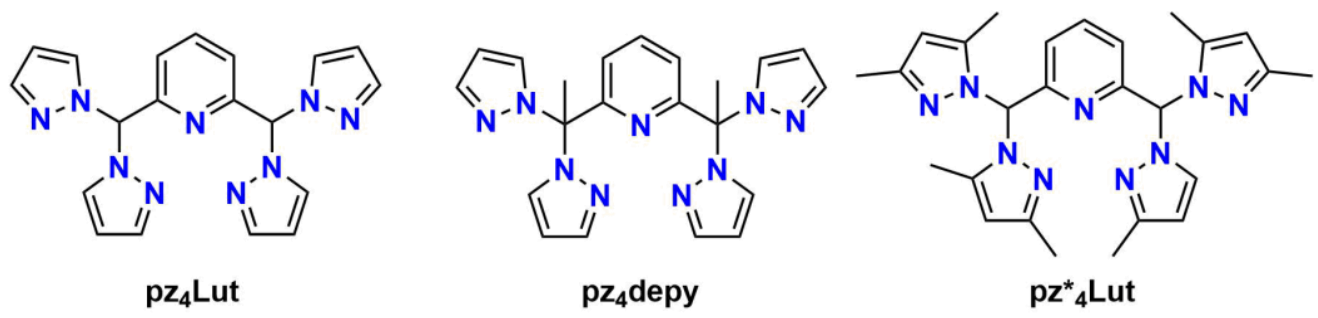

Scheme 2.13 Overview of the synthesized polypyrazole lutidine based ligands $\mathbf{p z}^{\mathbf{R}}{ }_{4} \mathbf{P y}$ for dinuclear PBAs utilizing different methylation patterns: pyrazole sidearms or picolinic positions.

\subsubsection{Synthesis of Dinuclear Fe/Co PBAs Containing Lutidine-Based Pentadentate Ligands $\mathrm{pz}^{\mathrm{R}}{ }_{4} \mathrm{Lut}$}

As $\mathbf{p z} \mathbf{R}_{\mathbf{4}}$ Lut serve as capping ligands for the cobalt ion, the capping ligand for the iron ion were chosen to be hydrotrispyrazolyl borate ligands $\mathbf{t p}^{\mathbf{R}}$ for comparison reasons to [ $\left.\mathbf{F e}_{2} \mathbf{C o}_{2}\right]$ and $\left[\mathbf{F e C o}\right.$. The different substitutions $\mathbf{t p}, \mathbf{t p}^{\mathrm{Me}}$ and $\mathbf{t} \mathbf{p} *$ were synthesized according to the literature and used for the synthesis of the corresponding iron cyanido precursor $\left[\mathbf{t p}^{\mathbf{R}}\right.$-Fe]. ${ }^{121,136-138}$

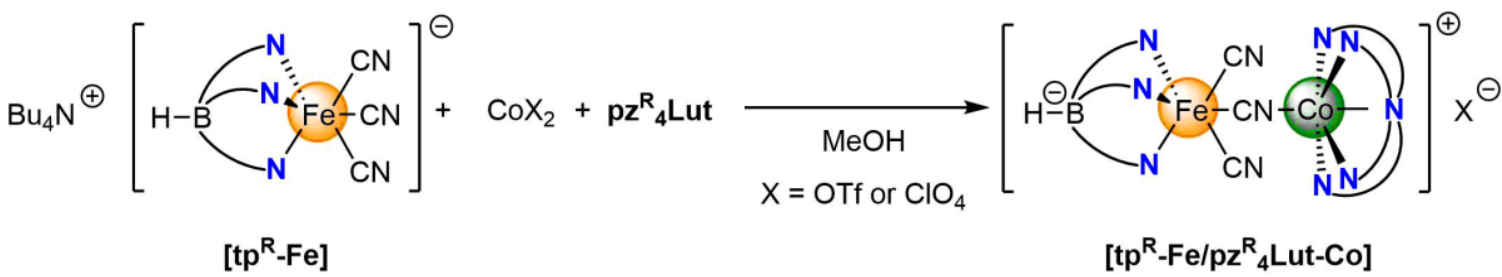

Scheme 2.14 General synthesis procedure of the dinuclear PBAs [tp ${ }^{\mathrm{R}}-\mathrm{Fe} / \mathrm{pz}^{\mathrm{R}}{ }_{4} \mathrm{Lut}-\mathrm{Co}$ ] based on the iron cyanido precursor $\left[\mathbf{t p}^{\mathrm{R}}-\mathrm{Fe}\right]$ and the pentadentate ligands $\mathbf{p z}^{\mathrm{R}}{ }_{4} \mathrm{Lut}$.

Based on the literature procedure by Nihei et al. the synthesis of the dinuclear system was carried out (Scheme 2.14). ${ }^{116}$ Addition of a solution of $\left[\mathbf{t p}^{\mathbf{R}}-\mathbf{F e}\right]$ in methanol to $\mathrm{Co}(\mathrm{OTf})_{2} / \mathrm{Co}\left(\mathrm{ClO}_{4}\right)_{2}$ and $\mathbf{~} \mathbf{~ z}^{\mathbf{R}}{ }_{4}$ Lut in methanol, resulted for most ligand combinations in a dark red solution, which was set up for vapor diffusion with diethyl ether. In cases of [tp-Fe/pz 4 Lut-Co] and [tp ${ }^{\mathrm{Me}}-\mathbf{F e} / \mathbf{p z}_{4}$ Lut-Co] precipitation afforded an orange solid despite different anions used. Afterwards, the solid was only soluble in DMSO and different solvents for inducing crystal growth resulted in a microcrystalline product.

After crystallization, six out of the nine ligand combinations gave dinuclear Fe/Co PBAs with a yield of $62-81 \%$. Those compounds were characterized by XRD analyses, IR and UV/vis spectroscopy and mass spectrometry, which will be discussed to elucidate the magnetic state of the dinuclear Fe/Co PBAs. Because of the similarity of the molecular structures only the one of the perchlorate salt of $\left[\mathbf{t p} *-\mathbf{F e} / \mathbf{p z}{ }^{*} \mathbf{L u t}-\mathbf{C o}\right]$ is shown in Figure 
2.9, but selected bond lengths and angles of all crystal structures and also of [FeCo] in the diamagnetic and paramagnetic state for comparison are shown in Table 2.1. Figures of the molecular structures of the other $\left[\mathbf{t p}^{\mathbf{R}}-\mathbf{F e} / \mathbf{p z}^{\mathbf{R}}{ }_{4} \mathbf{L u t}-\mathbf{C o}\right]$ complexes are provided in the Appendix.

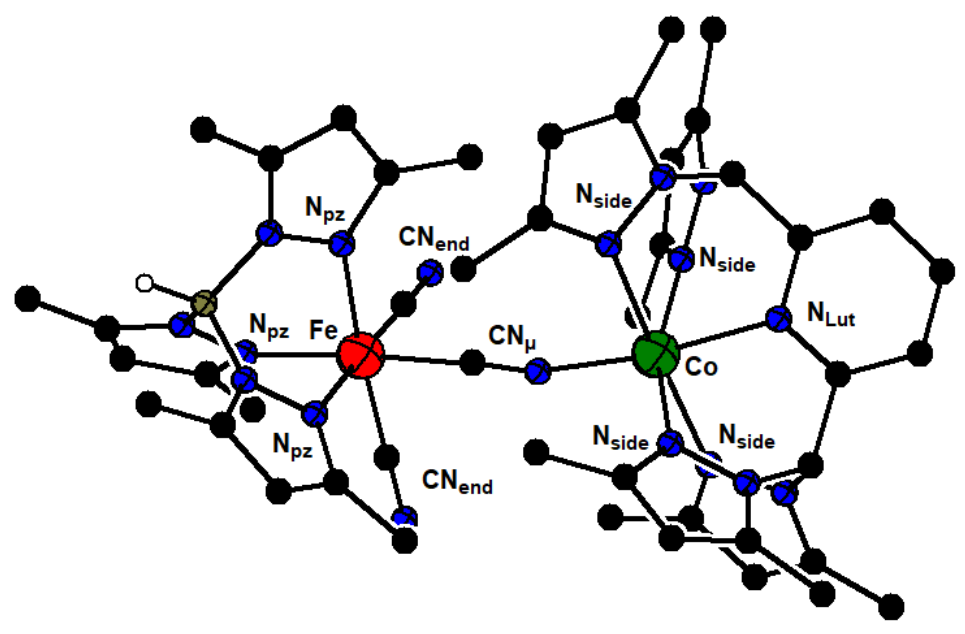

Figure 2.9 Molecular structure of the [tp*-Fe/pz* $\left.{ }_{4} \mathrm{Lut}-\mathrm{Co}\right]$; hydrogen atoms, perchlorate anion and solvent molecules are omitted for clarity.

For $[\mathbf{F e C o}]$, the structural transition from $h s-\mathrm{Co}(\mathrm{II})$ to $l s-\mathrm{Co}(\mathrm{III})$, induced by the thermal CTIST, is observed when comparing the structures of [FeCo] at $180 \mathrm{~K}$ for the paramagnetic state, and $90 \mathrm{~K}$ for the diamagnetic state. All cobalt-ligand bonds exhibit a shortening with the biggest difference for the Co-NC bond by nearly $0.08 \AA$. In contrast, the changes in the iron-ligand bons are not as pronounced. Additionally, the bridging $\mathrm{C}-\mathrm{N}$ bond marginally changes from 1.148 to $1.159 \AA$, comparably insignificant in comparison to the cobalt-ligand bond length changes. Interestingly, the Co-N-C bond angle as well as the N-C-Fe angle, show no significant changes.

As already mentioned, the molecular structure of the other $\left[\mathbf{t p}^{\mathbf{R}}-\mathbf{F e} / \mathbf{p z}^{\mathbf{R}}{ }_{\mathbf{4}}\right.$ Lut-Co] look rather similar, but comparing them in detail, marginal differences in the bond lengths and angles were observed. The $\mathrm{C}-\mathrm{N}$ bond lengths of the bridging cyanide ions do not differ significantly $(1.139 \rightarrow \sim 1.15 \AA)$. In addition, the terminal cyanide groups display similar differences, varying from 1.144 to $1.163 \AA$. Small differences were observed for the metalbased bond distances, e.g. Co-N $\mathrm{N}_{\text {Lut }} 2.1152$ to $2.1878 \AA$. In contrast, bigger differences were observed for the C-N-Co bond angles with $156.94^{\circ}$ of [tp*-Fe/pz 4 Lut-Co] significantly differing from the ideal $180^{\circ}$. While most of the bond lengths and angles vary from PBA to PBA, a correlation between methylation and the resulting structures cannot be made. When comparing the structures to [FeCo] at $180 \mathrm{~K}$, the assumption can be made that the dinuclear PBAs with polypyrazolyl lutidine ligands are in the paramagnetic state, meaning $[l s-\mathrm{Fe}(\mathrm{III}) / h s-\mathrm{Co}(\mathrm{II})]$ at $133 \mathrm{~K}$. The Co-Nlut bond, which can be found in all shown PBAs, is in good agreement with the bond length of [FeCo] at $180 \mathrm{~K}$, while the difference to the structure at $90 \mathrm{~K}$ can be up to $0.14 \AA$ for [tp*-Fe/pz $\mathbf{p d e p y - C o ]}$. For [tp-Fe/pz $\mathbf{t e p y - C o ]}$ two different modifications of the crystal structure are found (Appendix). 
Table 2.1 Selected bond lengths and angles of the linear PBAs with polypyrazole lutidine ligands $\mathbf{p z}^{\mathrm{R}}{ }_{4} \mathrm{Py}$ at $133 \mathrm{~K}$ and $\left[\mathrm{FeCo}\right.$ ] of Clérac et al. ${ }^{121}$ at $90 \mathrm{~K}$ and $180 \mathrm{~K}$.

\begin{tabular}{|c|c|c|c|c|c|c|c|c|}
\hline \multirow{2}{*}{$\begin{array}{c}\mathrm{d}[\AA \AA] \\
\angle\left[^{\circ}\right]\end{array}$} & \multicolumn{2}{|c|}{ tp-Fe } & \multirow{2}{*}{$\begin{array}{c}\mathrm{tp}^{\mathrm{Me}}{ }_{-\mathrm{Fe}} \\
\mathrm{pz}^{*}{ }_{4} \mathrm{Lut}- \\
\text { Co }\end{array}$} & \multicolumn{3}{|c|}{ tp*-Fe } & \multicolumn{2}{|c|}{ [FeCo] } \\
\hline & $\begin{array}{c}\mathrm{pz}_{4} \text { depy- } \\
\text { Co }\end{array}$ & $\begin{array}{c}\text { pz }{ }_{4} \text { Lut- } \\
\text { Co }\end{array}$ & & $\begin{array}{l}\mathrm{pz}_{4} \text { Lut- } \\
\text { Co }\end{array}$ & $\begin{array}{c}\mathrm{pz}_{4} \text { depy- } \\
\text { Co }\end{array}$ & $\begin{array}{c}\mathrm{pz}^{*}{ }_{4} \text { Lut- } \\
\text { Co }\end{array}$ & $90 \mathrm{~K}$ & $180 \mathrm{~K}$ \\
\hline$C-N_{\mu}$ & 1.139 & 1.148 & 1.154 & 1.153 & 1.154 & 1.153 & 1.159 & 1.148 \\
\hline $\mathrm{C}-\mathrm{N}_{\text {end }}$ & $\begin{array}{l}1.147 \\
1.148\end{array}$ & $\begin{array}{l}1.144 \\
1.153\end{array}$ & $\begin{array}{l}1.146 \\
1.163\end{array}$ & $\begin{array}{l}1.146 \\
1.152\end{array}$ & $\begin{array}{l}1.147 \\
1.153\end{array}$ & $\begin{array}{l}1.153 \\
1.157\end{array}$ & 1.148 & 1.147 \\
\hline Co-N Lut & 2.154 & 2.148 & 2.137 & 2.1527 & 2.1878 & 2.1152 & 2.047 & 2.111 \\
\hline Co- $\mathrm{N}_{\text {side }}$ & $\begin{array}{r}2.083- \\
2.105\end{array}$ & $\begin{array}{r}2.200- \\
2.269\end{array}$ & $\begin{array}{r}2.188- \\
2.282\end{array}$ & $\begin{array}{r}2.1280- \\
2.1463\end{array}$ & $\begin{array}{r}2.0876- \\
2.1095\end{array}$ & $\begin{array}{r}2.219- \\
2.245\end{array}$ & $\begin{array}{l}2.080 \\
2.081\end{array}$ & $\begin{array}{l}2.137 \\
2.146\end{array}$ \\
\hline Co-NC & 2.056 & 2.067 & 2.040 & 2.0482 & 2.0559 & 2.032 & 1.968 & 2.047 \\
\hline $\mathrm{Fe}-\mathrm{N}_{\mathrm{pz}}$ & $\begin{array}{r}1.957- \\
1.974\end{array}$ & $\begin{array}{r}1.972- \\
1.984\end{array}$ & $\begin{array}{r}1.996- \\
2.011\end{array}$ & $\begin{array}{r}1.9912- \\
2.0129\end{array}$ & $\begin{array}{r}2.0017- \\
2.0186\end{array}$ & $\begin{array}{r}1.996- \\
2.004\end{array}$ & $\begin{array}{l}1.933 \\
1.933\end{array}$ & $\begin{array}{l}1.974 \\
1.987\end{array}$ \\
\hline $\mathrm{Fe}-\mathrm{C}_{\mu}$ & 1.915 & 1.935 & 1.919 & 1.9141 & 1.9187 & 1.909 & 1.875 & 1.891 \\
\hline Fe- $C_{\text {end }}$ & $\begin{array}{l}1.922 \\
1.937\end{array}$ & $\begin{array}{l}1.923 \\
1.924\end{array}$ & $\begin{array}{l}1.928 \\
1.929\end{array}$ & $\begin{array}{l}1.9266 \\
1.9307\end{array}$ & $\begin{array}{l}1.9311 \\
1.9367\end{array}$ & $\begin{array}{l}1.913 \\
1.916\end{array}$ & 1.942 & 1.931 \\
\hline $\mathrm{Co}-\mathrm{N}-\mathrm{C}$ & 159.9 & 177.9 & 172.9 & 156.94 & 174.73 & 165.5 & 171.9 & 171.0 \\
\hline Fe-C-N & 174.4 & 177.8 & 177.7 & 179.00 & 177.05 & 177.2 & 178.3 & 177.4 \\
\hline
\end{tabular}

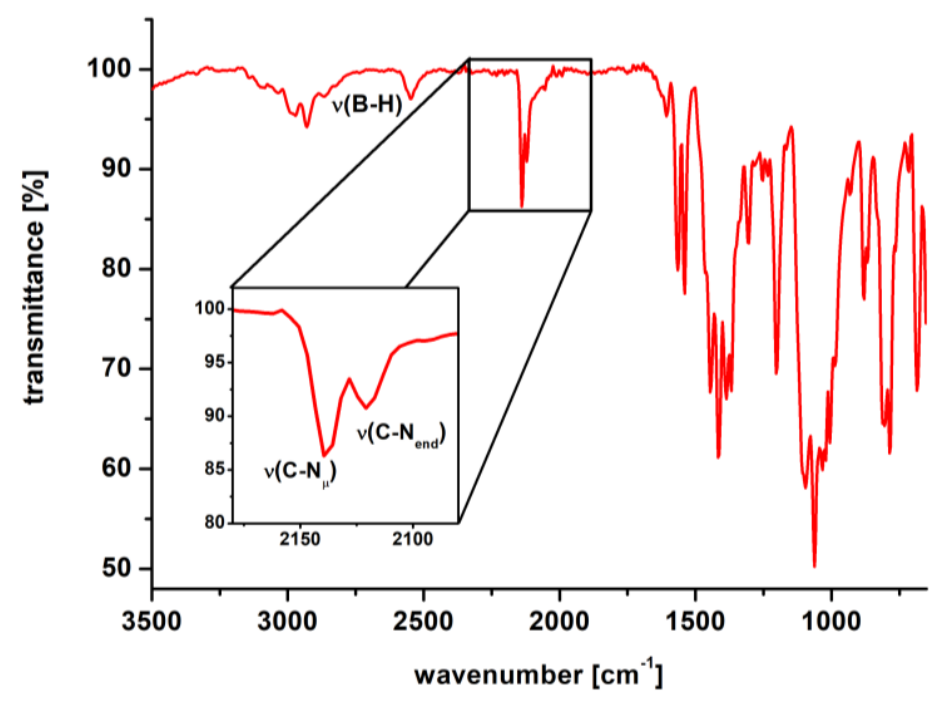

Figure 2.10 ATR-IR (diamond) spectrum of [tp*-Fe/pz* ${ }_{4}$ Lut-Co] at room temperature with inset for distinguishing bridging and terminal cyanide ligands.

Apart from X-ray diffraction analyses, as already mentioned, IR spectroscopy provides information on the magnetic state based on the stretching vibration frequencies of the $\mathrm{C}-\mathrm{N}$ and B-H bonds. Due to the characteristic B-H and C-N stretching vibrations, they can be used for probing electronic structure changes in PBAs. ${ }^{100}$ As shown for $\left[\mathbf{F e}_{2} \mathbf{C o}_{2}\right]$ (Chapter 2.3), the stretching frequencies of borohydride and terminal or bridging cyanide highly 
depend on the magnetic state and therefore can be used here to characterize the magnetic state of the synthesized complexes. As all complexes show similar infrared spectra, only the spectrum of [tp*-Fe/pz* $\left.{ }_{4} \mathbf{L u t}-\mathbf{C o}\right]$ is shown in Figure 2.10, but representative stretching frequencies of all complexes along with those of [FeCo] and $\left[\mathrm{Fe}_{2} \mathrm{Co}_{2}\right]$ for comparison are presented in Table 2.2.

Table 2.2 Selected stretching frequencies for $\mathrm{tp}^{\mathrm{R}} / \mathrm{pz}^{\mathrm{R}}{ }_{4} \mathrm{Py}$, [FeCo ${ }^{121}$ and $\left[\mathrm{Fe}_{2} \mathrm{CO}_{2}\right]^{116}$ at room temperature based on the solid-state IR spectra.

\begin{tabular}{|c|c|c|c|c|}
\hline \multicolumn{2}{|c|}{ Vibrations $\tilde{0}\left[\mathrm{~cm}^{-1}\right]$} & $\mathrm{v}(\mathrm{BH})$ & $v\left(C_{\mu}\right)$ & $v\left(C_{\text {end }}\right)$ \\
\hline \multirow{2}{*}{ Tp-Fe } & $\mathrm{pz}_{4} \mathrm{depy}-\mathrm{Co}$ & 2512 & 2160,2149 & 2136,2123 \\
\hline & pz* ${ }_{4}$ Lut-Co & 2492 & 2150 & 2120 \\
\hline $\mathrm{tp}^{\mathrm{Me}}-\mathrm{Fe}$ & pz ${ }_{4}$ Lut-Co & 2519 & 2139 & 2122 \\
\hline \multirow{3}{*}{ tp*-Fe } & $\mathrm{pz}_{4}$ Lut-Co & 2538 & 2134 & 2115 \\
\hline & pz ${ }_{4}$ depy-Co & 2546 & 2139 & 2119 \\
\hline & pz ${ }_{4}$ Lut-Co & 2547 & 2138 & 2120 \\
\hline [FeCo] & para. & 2512 & 2156 & 2122 \\
\hline \multirow{2}{*}[\mathrm{Fe}_{2}\mathrm{CO}_{2}]{} & para. $^{[\mathrm{a}]}$ & 2545 & 2152 & 2127 \\
\hline & dia..$^{[b]}$ & - & 2098,2077 & 2070 \\
\hline
\end{tabular}

[a] at $335 \mathrm{~K} ;[\mathrm{b}]$ at $220 \mathrm{~K}$

While the frequencies of the synthesized complexes differ by up to $50 \mathrm{~cm}^{-1}$ for the $\mathrm{B}-\mathrm{H}$ vibration and up to $26 \mathrm{~cm}^{-1}$ for the $\mathrm{C}-\mathrm{N}$ vibration, this does not correlate to different electronic states and is probably due to different solvent molecules in the crystal lattice. This can be shown by analysis of the C-N vibrations of [tp-Fe/pzadepy-Co], where two bands are observed. This is due to diethyl ether and different amounts of methanol molecules in the crystal lattice resulting also in two molecules in the asymmetric unit cell, splitting up the cyanide stretching frequencies. This is consistent with the Mößbauer data, where two iron species are distinguished (vide infra). The $\mathrm{C}-\mathrm{N}$ and $\mathrm{B}-\mathrm{H}$ stretching frequencies of $\left[\mathbf{t p}^{\mathrm{R}}-\mathbf{F e} / \mathbf{p z}^{\mathrm{R}}{ }_{4} \mathbf{L u t}-\mathbf{C o}\right]$ in comparison to the ones of [FeCo] and $\left[\mathrm{Fe}_{2} \mathbf{C o}_{2}\right]$ (Table 2.2) support that the synthesized dinuclear systems are in the paramagnetic state $[l s-\mathrm{Fe}(\mathrm{III}) / h s-\mathrm{Co}(\mathrm{II})]$ at room temperature.

In addition to XRD analyses and IR spectroscopy, the compounds where further characterized by ESI mass spectrometry to determine the stability of the dinuclear structure in solution. An exemplary spectrum is shown in Figure 2.11 Although solubility of [tp-Fe/pz 4 Lut-Co] was an issue for purification, the ESI-MS spectrum of the crude product shows the mass peak for the cationic part $\left[\operatorname{tpFe}(\mathrm{CN})_{3} \mathrm{Co}\left(\mathbf{p z z}_{4} \mathbf{L u t}\right)\right]^{+}\left([\mathrm{M}-\mathrm{OTf}]^{+}=777.14\right.$ $\mathrm{m} / \mathrm{z}$ ), which is why [tp-Fe/pz4 $\mathbf{L u t}-\mathbf{C o}$ ] was used further for further studies. Fragmentations were also observed, for example $\mathbf{p z}^{\mathbf{R}}{ }_{4} \mathbf{L u t}$ and $\left[\mathrm{pz}^{\mathrm{R}}{ }_{3} \mathrm{Lut}\right]^{+}$for some complexes. No higher 
order assemblies, such as square or cubane structures, were observed in the mass spectra of the synthesized compounds.

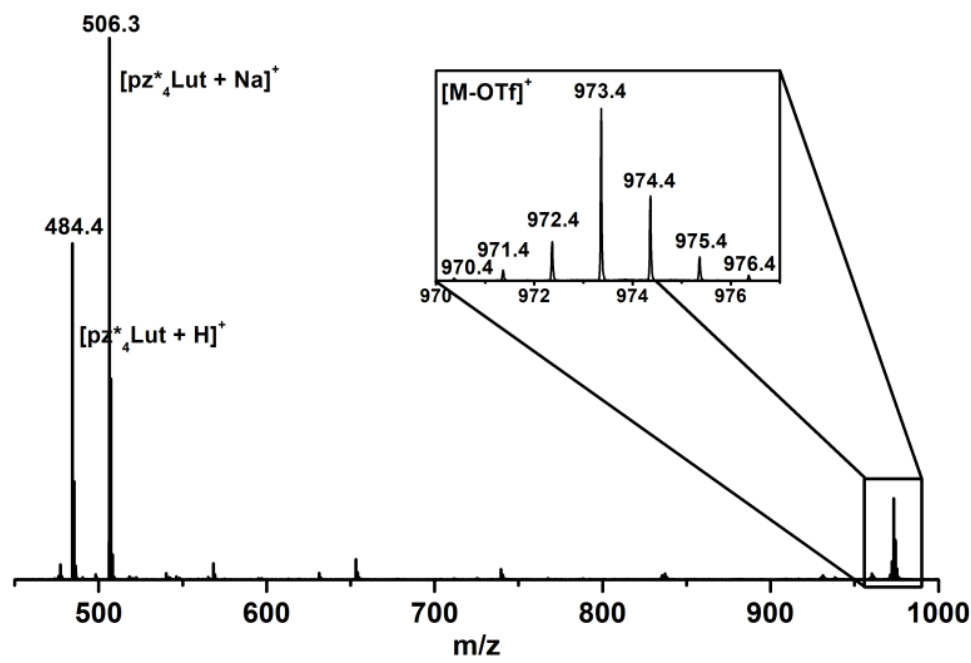

Figure 2.11 Exemplary ESI-MS(+)-spectrum of [tp*-Fe/pz* ${ }_{4}$ Lut-Co] in acetonitrile.

In summary, six linear Fe/Co PBAs containing polypyrazolyl lutidine ligands $\mathbf{p z}^{\mathbf{R}}{ }_{4} \mathbf{L}$ ut have been successfully synthesized and the molecular structures were characterized by ESI-MS, IR spectroscopy and XRD analyses. Although the complexes show different methylation patterns on both ligands, the bond lengths around the metal ions differ only slightly and no correlation between the substitution pattern and the metric parameters of the molecular structures could be derived. Comparison of the molecular structures of $\left[\mathbf{t p}^{\mathbf{R}}-\mathbf{F e} / \mathbf{p z}^{\mathbf{R}}{ }_{4} \mathbf{L u t}-\mathbf{C o}\right]$ to the ones of $[\mathbf{F e C o}]$ at $90 \mathrm{~K}$ and $180 \mathrm{~K}$, the resemblance of all structures to the paramagnetic state is dominant, and thus the paramagnetic $[l s-\mathrm{Fe}(\mathrm{III}) / h s-\mathrm{Co}(\mathrm{II})]$ state is the preferred ground state at $133 \mathrm{~K}$. Additionally, the stretching vibrations of the cyanide ligands of all synthesized complexes show frequencies $\left(2134-2156 \mathrm{~cm}^{-1}\right.$ for $v\left(\mathrm{CN}_{\mu}\right)$ and $2115-2127 \mathrm{~cm}^{-1}$ for $\left.v\left(\mathrm{CN}_{\mathrm{end}}\right)\right)$ similar to [FeCo] at room temperature $\left(2152 \mathrm{~cm}^{-1}\right.$ for $\nu\left(\mathrm{CN}_{\mu}\right)$ and $2127 \mathrm{~cm}^{-1}$ for $\left.\nu\left(\mathrm{CN}_{\mathrm{end}}\right)\right)$, giving evidence for the paramagnetic $[l s-\mathrm{Fe}(\mathrm{III}) / h s-\mathrm{Co}(\mathrm{II})]$ state from $133 \mathrm{~K}-298 \mathrm{~K}$. To further discuss the electronic and magnetic structure over the whole temperature range, further measurements have been done, which will be discussed in the next section. 


\subsubsection{Electronic Structure Characterization of $\left[\mathrm{tp}^{\mathrm{R}}-\mathrm{Fe} / \mathrm{pz}^{\mathrm{R}}{ }_{4} \mathrm{Lut}-\mathrm{Co}\right]$}

To further elucidate the electronic structure the dinuclear PBAs $\left[\mathbf{t p}^{\mathbf{R}}-\mathbf{F e} / \mathbf{p} \mathbf{z}^{\mathbf{R}} \mathbf{4} \mathbf{L u t}-\mathbf{C o}\right]$ UV/vis absorption, Mößbauer and magnetic measurements have been conducted. Since the $\mathrm{UV} / \mathrm{vis}$ spectra of the synthesized Fe/Co PBAs all displayed similar features, only the variable temperature UV/vis spectra of [tp*-Fe/pz* $\left.{ }_{4} \mathbf{L u t}-\mathbf{C o}\right]$ is shown in Figure 2.12. All the other spectra are provided in the Appendix.

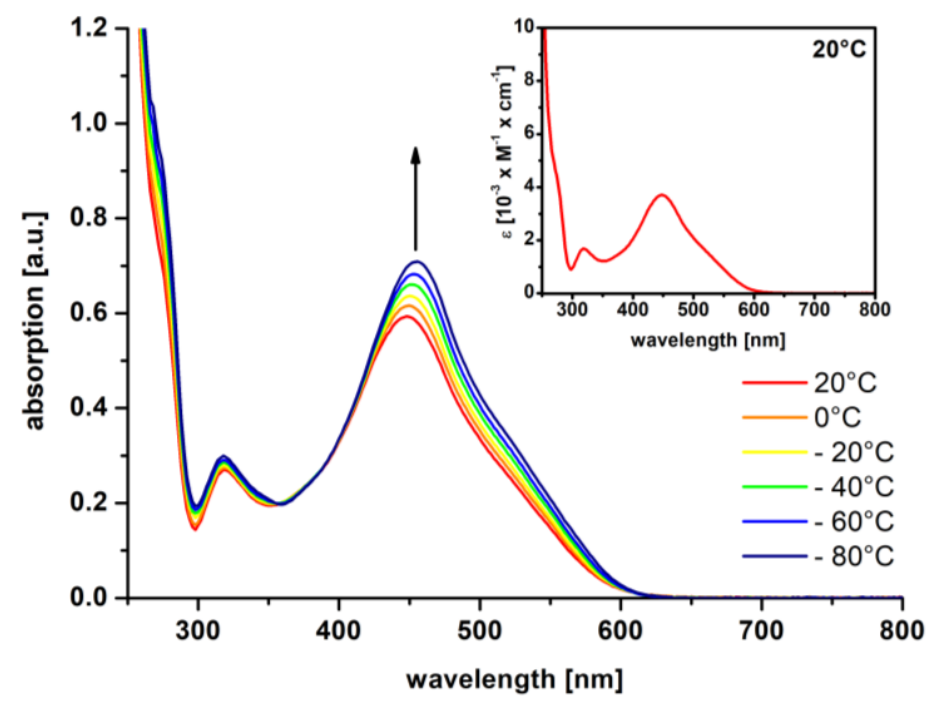

Figure 2.12 Variable temperature UV/vis spectrum of [tp*-Fe/pz ${ }^{*}{ }_{4}$ Lut-Co] in propionitrile; inset: UV/vis spectrum at $20^{\circ} \mathrm{C}$ with extinction coefficient.

Around 400 and $500 \mathrm{~nm}$, the MLCT and LMCT bands of the Co and Fe chromophores are observed. Moreover, the shoulder at $500-550 \mathrm{~nm}$ is assigned to the $\mathrm{Co}$ (II) $\rightarrow \mathrm{Fe}$ (III) IVCT transition, which is also found in other $\mathrm{Fe} / \mathrm{Co} \mathrm{PBAs}$ at room temperature. ${ }^{116,121}$ Interestingly, the band at $\sim 300 \mathrm{~nm}$ cannot be found for all complexes and is overlapped either by the broad MLCT or LMCT bands. A missing band above $650 \mathrm{~nm}$ indicates that there is no $\mathrm{Fe}(\mathrm{II}) \rightarrow \mathrm{Co}$ (III) IVCT and therefore no diamagnetic species at room temperature, solidifying the results of the IR data. When the sample was cooled down the spectra do not change drastically. The small increase of the intensity is due to the Boltzmann distribution of the ground state at lower temperature found for all presented compounds. A thermal driven CTIST in the temperature range of $133 \mathrm{~K}-298 \mathrm{~K}$ is not observed.

While assumptions on the oxidation and spin states can be made based on the molecular structure and electronic transitions, ${ }^{57} \mathrm{Fe}$ Mößbauer spectroscopy can be a tool to precisely assign the electronic state of the iron ion. For this, the samples were measured as a solid at $80 \mathrm{~K}$. The extracted data is tabulated in Table 2.3 and the spectrum of [tp*-Fe/pz* ${ }_{4}$ Lut-Co] is shown in Figure 2.13 (left). All complexes show an isomer shift between $\delta=0.02-0.11$ $\mathrm{mm} \cdot \mathrm{s}^{-1}$ and quadrupole splitting $\left|\Delta E_{Q}\right|$ between 1.16 and $1.74 \mathrm{~mm} \cdot \mathrm{s}^{-1}$. In comparison to 
$\left[\mathrm{Fe}_{2} \mathrm{Co}_{2}\right]$, the low isomer shift can be assigned rather to the paramagnetic state $\left(0.00 \mathrm{~mm} \cdot \mathrm{s}^{-1}\right.$ for $\left.\left[\mathrm{Fe}_{2} \mathbf{C o}_{2}\right]\right)$ rather than to the diamagnetic state $\left(0.22 \mathrm{~mm} \cdot \mathrm{s}^{-1}\right.$ for $\left.\left[\mathbf{F e}_{2} \mathbf{C o}_{2}\right]\right)$. Differentiating between $l s$ - $\mathrm{Fe}(\mathrm{II})$ and $l s$-Fe(III) through the isomer shift is rather challenging, but the quadrupole splitting is different for the electronic states of the iron center. Due to the symmetric $l s-\mathrm{d}^{6}$ configuration of $l s-\mathrm{Fe}(\mathrm{II})$, a smaller quadrupole splitting is expected. This is consistent with the data for $\left[\mathbf{F e}_{2} \mathbf{C o}_{2}\right]$. The high quadrupole splitting for the synthesized complexes $\left(\left|\Delta E_{Q}\right|=1.74 \mathrm{~mm} \cdot \mathrm{s}^{-1}\right.$ for [tp-Fe/pz_depy-Co] $)$ indicates the less symmetrical $\mathrm{d}^{7}$ configuration of $l s-\mathrm{Fe}(\mathrm{III})$. The two iron species for [tp-Fe/pz 4 depy-Co] (Figure 2.13, right) could be attributed to the two modifications of the crystal structure and two slightly different Fe environments (vide supra).
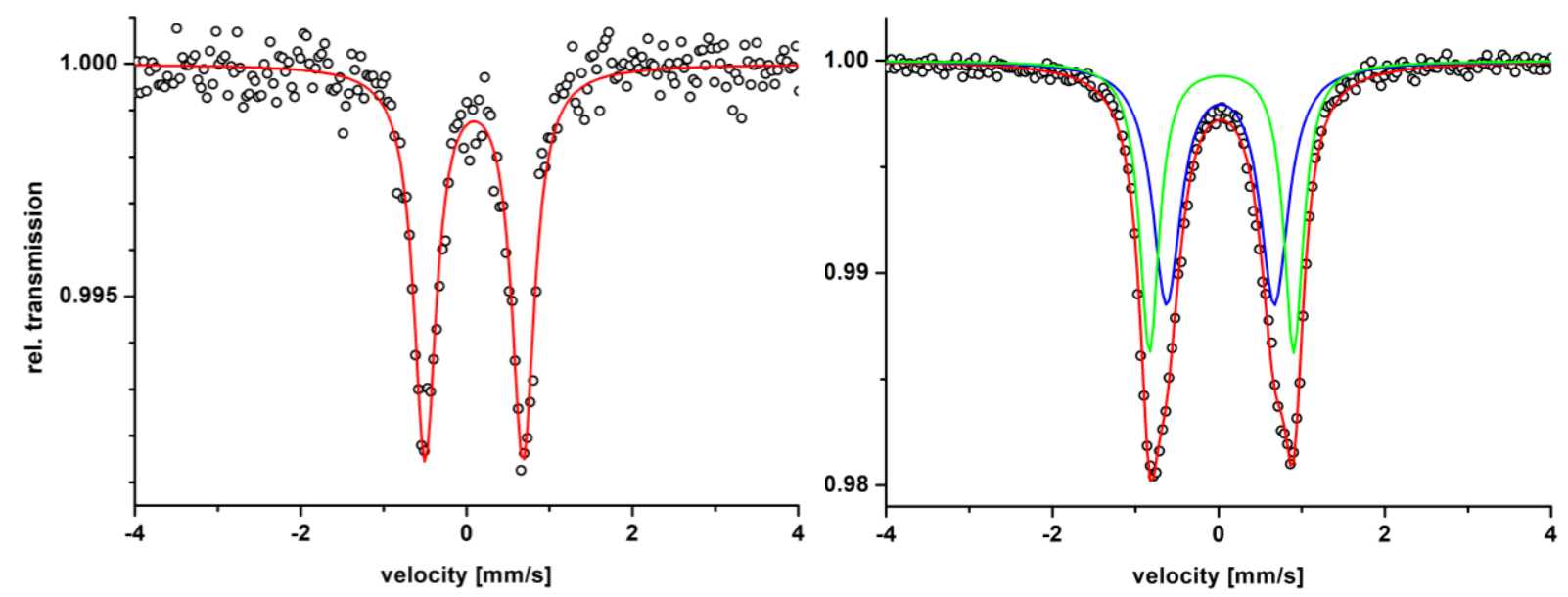

Figure 2.13 Solid-state ${ }^{57} \mathrm{Fe}$ Mößbauer spectra of [tp*/pz ${ }_{4}^{*} \mathrm{Lut}$ (left) and [tp/pz 4 depy] (right) at $80 \mathrm{~K}$; dots show the experimental data; red, green and blue lines show the simulated fit.

Table $2.3{ }^{57} \mathrm{Fe}$ Mößbauer isomer shift $(\delta)$ and quadrupole splitting $\left(\left|\Delta \mathrm{E}_{\mathrm{Q}}\right|\right)$ for the presented PBAs at $80 \mathrm{~K}$ from the spectra; for comparison the data of $\left[\mathrm{Co}_{2} \mathrm{Fe}_{2}\right]$ is included.

\begin{tabular}{|c|c|c|c|}
\hline \multicolumn{2}{|c|}{ Fe/Co PBAs } & $\delta\left[\mathrm{mm} \cdot \mathrm{s}^{-1}\right]$ & $\left|\Delta E_{Q}\right|\left[\mathrm{mm} \cdot \mathrm{s}^{-1}\right]$ \\
\hline \multirow{2}{*}{ tp-Fe } & pz $z_{4}$ depy-Co & $0.02 / 0.08$ & $1.30 / 1.74$ \\
\hline & pz ${ }_{4}$ Lut-Co & 0.05 & 1.16 \\
\hline tp $\mathrm{Me}_{-\mathrm{Fe}}$ & pz ${ }_{4}{ }_{4}$-ut-Co & 0.08 & 1.58 \\
\hline \multirow{3}{*}{ tp*-Fe } & pzaLut-Co & 0.08 & 1.48 \\
\hline & $\mathrm{pz}_{4}$ depy-Co & 0.11 & 1.33 \\
\hline & pz ${ }_{4}{ }_{4}$ Lut-Co & 0.09 & 1.18 \\
\hline \multirow{2}{*}[\mathrm{Fe}_{2}\mathrm{CO}_{2}]{} & para. ${ }^{[\mathrm{a}]}$ & 0.00 & 0.91 \\
\hline & dia. ${ }^{[b]}$ & 0.22 & 0.43 \\
\hline
\end{tabular}

[a] recorded at $330 \mathrm{~K}$; [b] recorded at $20 \mathrm{~K}$

To investigate the magnetic state of the synthesized complexes over the whole temperature range variable temperature magnetic susceptibility measurements were carried out. The 
data $\chi_{m} T$ vs. $\mathrm{T}$ for $\left[\mathbf{t p} * \mathbf{F e} / \mathbf{p z}{ }_{4}{ }_{4} \mathbf{L u t}-\mathbf{C o}\right]$ is presented (Figure 2.14) and fitted with the following spin Hamiltonian.

$$
\begin{aligned}
& \widehat{H}=-2 J \hat{S}_{F e} \hat{S}_{C o}+g \mu_{B} \vec{B} \vec{S}_{F e}+\mu_{B}\left(S_{x(C o)} g_{x(C o)} B_{x}+S_{y(C o)} g_{y(C o)} B_{y}+\right. \\
& \left.S_{z(C o)} g_{z(C o)} B_{z}\right)+D_{C o}\left[\hat{S}_{z(C o)}^{2}-\frac{1}{3} S_{C o}\left(S_{C o}+1\right)\right]
\end{aligned}
$$

At $300 \mathrm{~K}$ the value for $\chi_{m} T$ of $3.7 \mathrm{~cm}^{3} \cdot \mathrm{K} \cdot \mathrm{mol}^{-1}$ is assigned to the sum of the spin-only value of $h s$-Co(II) $(S=3 / 2)$ and $l s-\mathrm{Fe}(\mathrm{III})(S=1 / 2)$. At $2 \mathrm{~K}$ the value of $\chi_{m} T$ is at $1.4 \mathrm{~cm}^{3} \cdot \mathrm{K} \cdot \mathrm{mol}^{-1}$, which is attributed to a zero-field splitting of the $h s-\mathrm{Co}$ (II) ion with $D=-125 \mathrm{~cm}^{-1}$. The smaller slope of the graph between $20-90 \mathrm{~K}$ is due to a weak antiferromagnetic coupling of the two metal centers with a coupling constant of $J=-1.3 \mathrm{~cm}^{-1}$. Since the magnetic data are similar for the other five complexes, their magnetic properties will not be discussed in detail. The remaining magnetic susceptibility graphs and extracted data are reported in the Appendix.

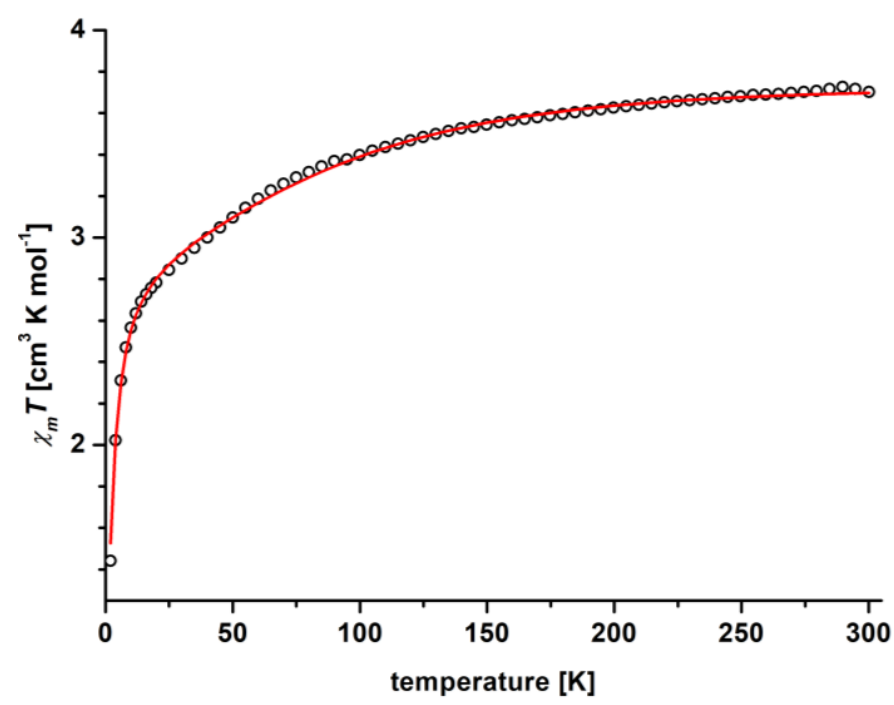

Figure $2.14 \chi_{m} T$ vs. T for crystalline [tp*-Fe/pz* ${ }_{4}$ Lut-Co]; Dots represent experimental data and the solid red line represent fits to the data (see text).

In conclusion, the electronic state of all six synthesized dinuclear Fe/Co PBAs has been well characterized by UV/vis, ${ }^{57} \mathrm{Fe}$ Mößbauer spectroscopy and magnetic susceptibility measurement. The variable temperature UV/vis spectra do not show significant changes but rather show the characteristic bands for the paramagnetic state in comparison with literature-known systems $[\mathbf{F e C o}]$ and $\left[\mathrm{Fe}_{2} \mathbf{C o}_{2}\right]$. The presence of the paramagnetic state $[l s$ $\mathrm{Fe}(\mathrm{III}) / h s-\mathrm{Co}(\mathrm{II})]$ is supported by the Mößbauer data corresponding to a $l s-\mathrm{Fe}(\mathrm{III})$ with a low isomer shift and a high quadrupole splitting at $80 \mathrm{~K}$. Finally, magnetic susceptibility measurements show the paramagnetic state over the measured temperature range of 2 $300 \mathrm{~K}$. Additionally, based on the fitting of the data a weak antiferromagnetic coupling of the two metal centers and zero-field-splitting of the $h s-\mathrm{Co}$ (II) ion is observed. While this makes excitation of the diamagnetic state for transient absorption spectroscopy impossible, an excitation at the $\mathrm{Co}(\mathrm{II}) \rightarrow \mathrm{Fe}(\mathrm{III})$ IVCT at $550 \mathrm{~nm}$ will be investigated. Investigations 
on the photophysical properties via ultrafast pump probe spectroscopy of the paramagnetic state will be presented later. 


\subsection{Dinuclear PBAs Bearing Different Pentadentate Ligand Systems Bearing Pyrazole or Amine Moieties}

Due to the absence of a thermally driven CTIST in the dinuclear PBAs $\left[\mathbf{t p}^{\mathbf{R}}-\mathbf{F e} / \mathbf{p z}^{\mathbf{R}}{ }_{4}\right.$ Lut-Co], other linear PBA systems utilizing different N-donor capping ligands for the modulation of the electronic structure were investigated (Scheme 2.15).
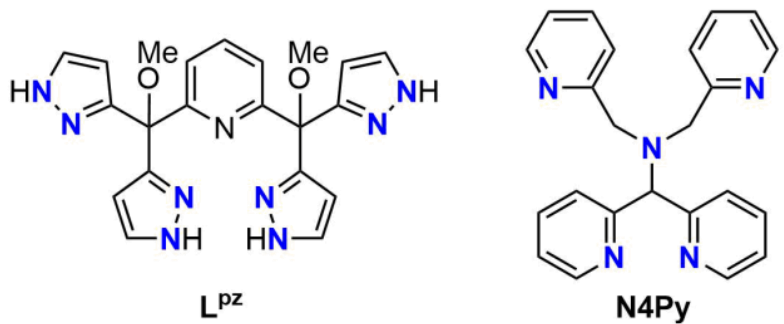

N4Py

Scheme 2.15 $N_{5}$-Ligands $L^{p z}$ from the Siewert et al. ${ }^{129}$ and N4Py reported by Feringa et al. ${ }^{139}$

\subsubsection{Dinuclear Fe/Co PBAs [tp $\left.{ }^{\mathrm{R}} / \mathrm{L}^{\mathrm{Pz}}-\mathrm{Co}\right]$}

The $N$-connected pyrazole lutidine ligands $\mathbf{p z} \mathbf{z}^{\mathbf{R}} \mathbf{L}$ ut did not tune the electronic structure of the cobalt ion in $\left[\mathbf{t p}^{\mathbf{R}}-\mathbf{F e} / \mathbf{p z}^{\mathbf{R}}{ }_{4} \mathbf{L u t}-\mathbf{C o}\right]$ for a thermal-driven CTIST (Chapter 2.4), the carbon connected derivative $\mathbf{L}^{\mathbf{p z}}$ (Scheme 2.15) reported by Siewert et al. ${ }^{129}$ was investigated. For the synthesis of the linear PBAs, the ligand $\mathbf{L}^{\mathbf{p z}}$ was provided by $\mathbf{M}$. Wilken of the Siewert group. The synthetic method already shown in Section 2.4.2 has been used to isolate the dinuclear PBA. After addition of $\mathrm{Bu} 4 \mathrm{~N}\left[\mathrm{tp}^{*} \mathrm{Fe}(\mathrm{CN})_{3}\right]$ to the in situ produced $\mathbf{L}^{\mathbf{p z}}$-Co species, the resulting reaction mixture was crystallized similar to the $\mathbf{p z}^{\mathbf{R}}{ }_{4} \mathbf{P y}$ system, which resulted in crystal growth after two days at room temperature. The ESI-MS spectrum (Appendix), shows a molecular ion peak $(920.25 \mathrm{~m} / \mathrm{z})$ with a isotopic pattern characteristic of $\left[\mathrm{tp}^{*} \mathrm{Fe}(\mathrm{CN})_{3} \mathrm{CoL}^{\mathbf{p z}}-\mathrm{H}^{\cdot}\right]^{+}$, indicating oxidation of the cobalt ion. The molecular structure of $\left[\mathbf{t p}^{*}-\mathbf{F e} / \mathbf{L}^{\mathbf{P z}} \mathbf{- C o}\right.$, shown in Figure 2.15, confirms, that the oxidation of the cobalt is coupled to a deprotonation of one of the pyrazole moieties, indicated by the short hydrogen bridge of two pyrazole moieties $\left(\mathrm{d}\left(\mathrm{HN}_{\mathrm{pz}}-\mathrm{N}_{\mathrm{pz}}\right)=2.7467(39)\right.$ and $\left.\angle\left(\mathrm{N}_{\mathrm{pz}}-\mathrm{H}-\mathrm{N}_{\mathrm{pz}}\right)=113.15(22)^{\circ}\right)$. In addition, the cobalt-ligand bond lengths appear to be shorter with $\mathrm{d}\left(\mathrm{Co}-\mathrm{N}_{\mathrm{Lut}}\right)=1.971(2)$ and $\mathrm{d}\left(\mathrm{Co}-\mathrm{N}_{\text {side }}\right)=1.891(2)-1.948(3) \AA$, compared to the already presented PBAs $\left(\mathrm{d}\left(\mathrm{Co}-\mathrm{N}_{\mathrm{Lut}}\right)=2.1152-2.1878\right.$ and $\mathrm{d}\left(\mathrm{Co}-\mathrm{N}_{\text {side }}\right)=2.083-$ $2.282 \AA$ ), which is consistent with an oxidation to $l s-\mathrm{Co}(\mathrm{III})$. The iron-ligand bond lengths $\left(\mathrm{d}\left(\mathrm{Fe}-\mathrm{N}_{\mathrm{pz}}\right)=1.980(2)-2.019(3) \AA\right)$ are similar to those of the earlier discussed PBAs $(\mathrm{d}(\mathrm{Fe}-$ $\mathrm{N}_{\mathrm{pz}}$ ) $=1.957-2.0186 \AA$ ). While this is not necessarily indicative a $l s-\mathrm{Fe}(\mathrm{III})$, because of the small structural changes observed with the IVCT for [FeCo], ${ }^{57} \mathrm{Fe}$ Mößbauer spectroscopy gives less ambiguous information. Selected bond lengths and angles of [tp*$\left.\mathbf{F e} / \mathbf{L}^{\mathbf{p z}}-\mathbf{C o}\right]$ are presented in Table 2.4 in comparison to one earlier discussed dinuclear PBA $\left[\mathrm{tp} *-\mathrm{Fe} / \mathrm{pz} *{ }_{4} \mathrm{Lut}-\mathrm{Co}\right]$. 


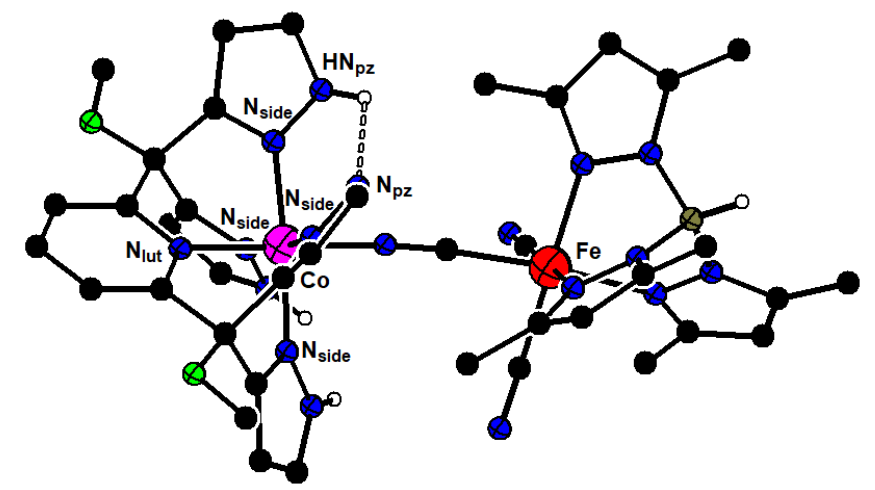

Figure 2.15 Molecular structure of $\left[\mathrm{tp}^{*} / \mathrm{L}^{\mathrm{pz}}\right]$, hydrogen atoms, triflate/hexafluorophosphate anions and solvent molecules are omitted for clarity.
Table 2.4 Selected bond lengths and angles of $\left[\mathbf{t p}^{*} / \mathrm{L}^{\mathrm{pz}}\right]$ and $\left[\mathrm{tp}^{*} / \mathrm{pz}^{*}{ }^{*} \mathrm{Lut}\right]$.

\begin{tabular}{|c|c|c|}
\hline $\begin{array}{l}d[\AA] / \\
\angle\left[^{\circ}\right]\end{array}$ & {$\left[\mathrm{tp} * / \mathrm{L}^{\mathrm{pz}}\right]$} & {$\left[\mathrm{tp} * / p z^{*}{ }_{4} \mathrm{Lut}\right]$} \\
\hline Co- $\mathrm{N}_{\text {side }}$ & $\begin{array}{r}1.891(2)- \\
1.948(3)\end{array}$ & $\begin{array}{r}2.219(2) \\
-2.245(2)\end{array}$ \\
\hline Co-N $N_{\text {Lut }}$ & $1.971(2)$ & $2.1152(19)$ \\
\hline Co-NC & $1.898(3)$ & $2.032(2)$ \\
\hline$H N_{p z}-N_{p z}$ & $2.7467(39)$ & - \\
\hline $\mathrm{Fe}-\mathrm{N}_{\mathrm{pz}}$ & $\begin{array}{r}1.980(2)- \\
2.019(3)\end{array}$ & $\begin{array}{r}1.996(2) \\
-2.004(2)\end{array}$ \\
\hline $\begin{array}{l}\mathrm{N}_{\text {Lut }}-\mathrm{Co}- \\
\mathrm{NC} \\
\end{array}$ & $177.30(10)$ & $170.66(8)$ \\
\hline
\end{tabular}

In the ${ }^{57} \mathrm{Fe}$ Mößbauer spectrum recorded at $80 \mathrm{~K}$, shown in Figure 2.16, one iron species with an isomer shift of $\delta=0.07 \mathrm{~mm} / \mathrm{s}$ and a quadrupole splitting of $\left|\Delta E_{Q}\right|=1.70 \mathrm{~mm} \cdot \mathrm{s}^{-1}$ is observed. In comparison to the data of the other dinuclear PBAs, the assignment of $l s$ $\mathrm{Fe}(\mathrm{III})$ with a low isomer shift and a high quadrupole splitting is reasonable

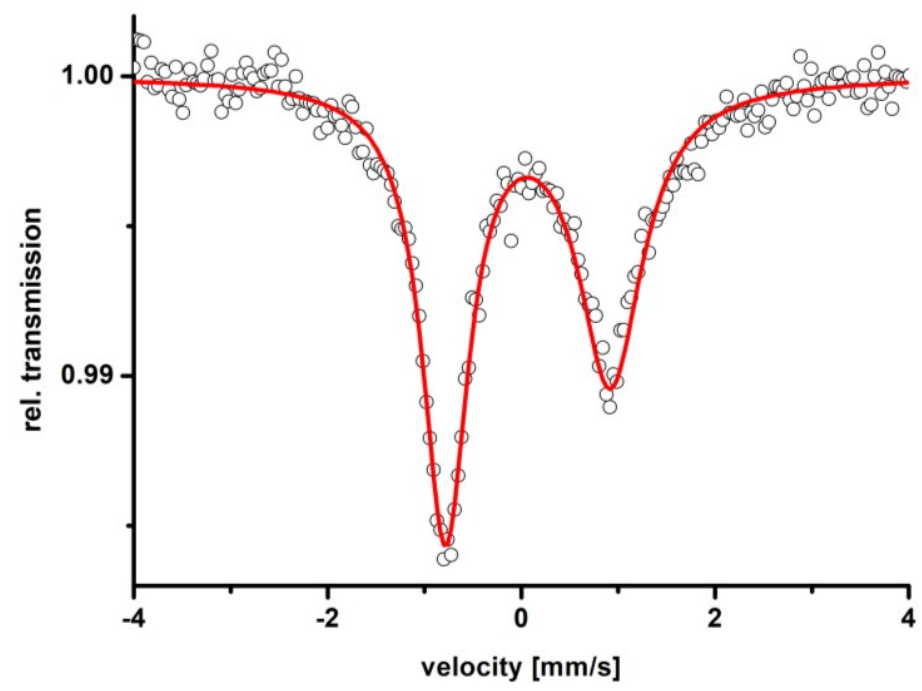

Figure 2.16 ${ }^{57} \mathrm{Fe}$ Mößbauer spectrum of solid [tp*-Fe/L $\left.{ }^{\mathrm{pz}}-\mathrm{Co}\right]$ recorded at $80 \mathrm{~K}$; dots represent the experimental data; red line shows the simulated fit (see text).

Due to the oxidation of the cobalt ion, no electron transfer is expected. To inhibit the deprotonation by adding a weak acid with various acids ([LutH]OTf, [DMF-H]OTf) resulted in oxidation as well. Therefore, the project was not further pursued. 


\subsubsection{Linear PBAs with pentadentate N4Py Capping Ligand}
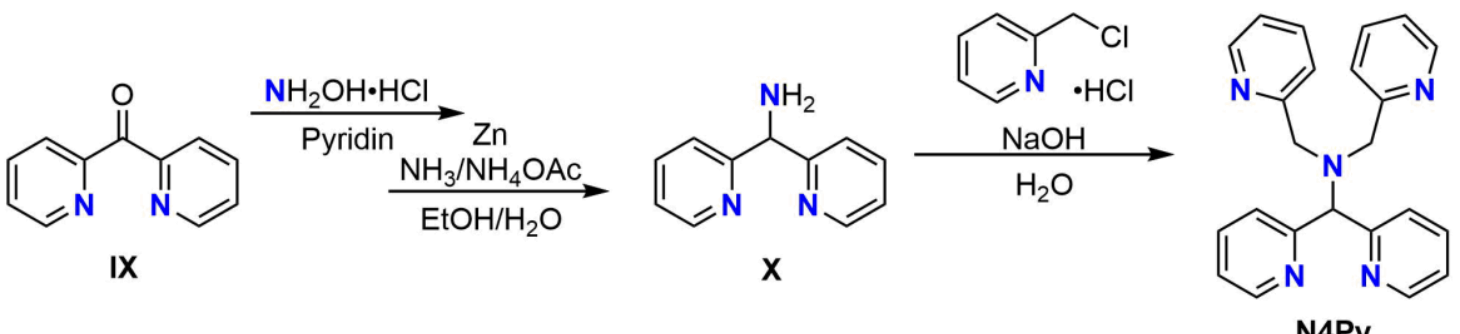

Scheme 2.16 Three step synthesis of N4Py according to Feringa et al. ${ }^{139}$

The ligand N4Py (Scheme 2.16) was chosen as it bears pyridine and amine N-donors to produce a PBA with a thermally driven CTIST. N4Py was developed by Feringa et al. ${ }^{139}$ as a pentadentate version of the well-known tris(2-pyridyl)amine ligand (tpa) and was applied in multiple fields of chemistry, mostly biomimetic or bioinspired inorganic chemistry, coordinated to iron, cobalt and manganese ions. ${ }^{139-147} \mathbf{N 4 P y}$ is synthesized according to the literature protocol starting with di(2-pyridyl) ketone IX. ${ }^{139}$ After condensation reaction with hydroxylamine and reduction with zinc, the amine $\mathbf{X}$ was used in a nucleophilic substitution with pycolyl chloride giving $\mathbf{N 4 P y}$ in moderate yields (Scheme 2.16).

With N4Py in hand, three dinuclear Fe/Co PBAs were synthesized under the same reaction conditions; they differ by the $\mathbf{t p}^{\mathbf{R}}$ ligands on Fe. Interestingly, right after the addition of the iron precursor the color of the solution changed, dependent on the trispyrazolyl borate $\mathbf{t p}^{\mathbf{R}}$ used. For the non-methylated [tp-Fe/N4Py-Co] the reaction mixture changed to green, the solution of [tp $\left.{ }^{\mathbf{M e}}-\mathbf{F e} / \mathbf{N} 4 \mathbf{P y}-\mathbf{C o}\right]$ changed to an orange-green and the one of [tp*-Fe/N4Py-Co] had a dark red color. This resulted also in different colored precipitates, when cobalt perchlorate was used instead of triflate. Multiple crystallization attempts under air or inert atmosphere resulted only in precipitation instead of single crystals. While this was unfortunate, the compounds gained interest, due to the different colors of the three samples, since similar observations were also found for other Fe/Co PBAs. ${ }^{116}$ Therefore, further characterization methods were applied to identify the electronic structure of the compounds.

Although, the ${ }^{1} \mathrm{H}-\mathrm{NMR}$ spectra of the species $\left[\mathbf{t p}^{\mathrm{Me}}-\mathbf{F e} / \mathbf{N} 4 \mathbf{P y}-\mathrm{Co}\right]$ and $\left[\mathbf{t p}{ }^{*}-\mathbf{F e} / \mathbf{N} 4 \mathbf{P y}-\mathbf{C o}\right]$ showed broad signals attributed to paramagnetism, for [tp-Fe/N4Py-Co] discrete signals were observed (Appendix). Additionally, the ${ }^{57} \mathrm{Fe}-\mathrm{Mößbauer}$ spectrum of [tp-Fe/N4Py-Co], presented in Figure 2.17 (left), shows only one new species with an isomer shift of $\delta=0.19 \mathrm{~mm} \cdot \mathrm{s}^{-1}$ and a quadrupole splitting of $\left|\Delta E_{Q}\right|=0.56 \mathrm{~mm} \cdot \mathrm{s}^{-1}$. The isomer shift is bigger compared to the complexes described in Section 2.4.3 $\left(\delta=0.05-0.11 \mathrm{~mm} \cdot \mathrm{s}^{-1}\right)$ and at the same time a smaller quadrupole splitting is observed $\left(\left|\Delta E_{Q}\right|=1.16-1.58 \mathrm{~mm} \cdot \mathrm{s}^{-1}\right)$. While the asymmetric intensities of the peak would rather hint at a $\mathrm{Fe}(\mathrm{III})$ center due to magnetic relaxation, the similarity with the Mößbauer 
parameters for the diamagnetic $[l s-\mathrm{Fe}(\mathrm{II}) / l s-\mathrm{Co}(\mathrm{III})]$ state of $\left[\mathbf{F e}_{2} \mathbf{C o}_{2}\right]$ is obvious. Additionally, the small quadrupole splitting indicates a symmetric charge distribution around the $\mathrm{Fe}$ nucleus, indicative for $l s-\mathrm{Fe}(\mathrm{II})$. Instead of magnetic relaxation, the asymmetry could be caused by multiple species with very similar Mößbauer parameters.
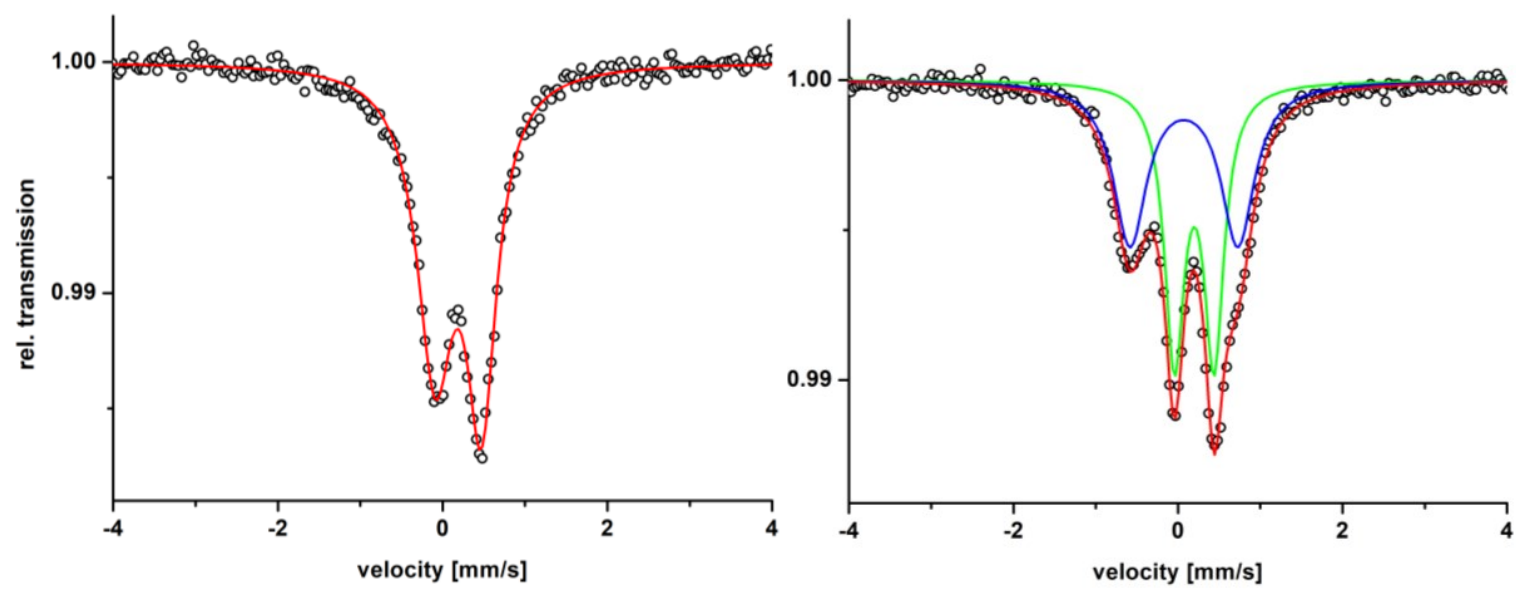

Figure $2.17{ }^{57} \mathrm{Fe}$ Mößbauer spectra of [tp-Fe/N4Py-Co] (left) and [tp*-Fe/N4Py-Co] (right) recorded at $80 \mathrm{~K}$, dots show experimental data, while the red, green and blue lines are the fits.

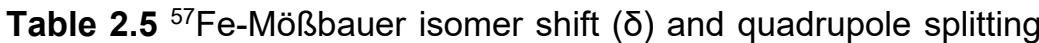
$\left(\left|\Delta \mathrm{E}_{\mathrm{Q}}\right|\right)$ for the presented PBAs with $\mathbf{N} 4 \mathrm{Py}$ recorded at $80 \mathrm{~K}$; for comparison the data from $\left[\mathrm{CO}_{2} \mathrm{Fe}_{2}\right]$ is included.

\begin{tabular}{|c|c|c|c|c|}
\hline \multicolumn{2}{|c|}{ Fe/Co PBAs } & $\begin{array}{c}\text { rel. } \\
\text { intensity }\end{array}$ & $\delta\left[\mathrm{mm} \cdot \mathrm{s}^{-1}\right]$ & $\left|\Delta E_{Q}\right|\left[\mathrm{mm} \cdot \mathrm{s}^{-1}\right]$ \\
\hline Tp-Fe & N4Py-Co & $100 \%$ & 0.19 & 0.56 \\
\hline \multirow{2}{*}{ tp $^{\mathrm{Me}}-\mathrm{Fe}$} & \multirow{2}{*}{ N4Py-Co } & $25 \%$ & 0.05 & 1.46 \\
\hline & & $75 \%$ & 0.22 & 0.49 \\
\hline \multirow{2}{*}{ tp*-Fe } & \multirow{2}{*}{ N4Py-Co } & $50 \%$ & 0.07 & 1.31 \\
\hline & & $50 \%$ & 0.20 & 0.48 \\
\hline \multirow{2}{*}[\mathrm{Fe}_{2}\mathrm{CO}_{2}]{} & para. ${ }^{[a]}$ & $100 \%$ & 0.00 & 0.91 \\
\hline & dia. ${ }^{[b]}$ & $100 \%$ & 0.22 & 0.43 \\
\hline
\end{tabular}

[a] recorded at $330 \mathrm{~K}$; $[\mathrm{b}]$ recorded at $20 \mathrm{~K}$

The Mößbauer spectra of [tp $\left.{ }^{\mathbf{M e}}-\mathbf{F e} / \mathbf{N} 4 \mathbf{P y}-\mathbf{C o}\right]$ and [tp*-Fe/N4Py-Co] (Figure 2.17, right), two sets of doublets with isomer shifts $\delta_{1}=0.05 / 0.07 \mathrm{~mm} \cdot \mathrm{s}^{-1}$ and $\delta_{2}=0.22 / 0.20 \mathrm{~mm} \cdot \mathrm{s}^{-1}$ are observed. The quadrupole splitting also differs greatly for the set of signals with $\left|\Delta E_{Q}\right|_{1}=1.46 / 1.31 \mathrm{~mm} \cdot \mathrm{s}^{-1}$ and $\left|\Delta E_{Q}\right|_{2}=0.48 / 0.49 \mathrm{~mm} \cdot \mathrm{s}^{-1}$. The parameters for all compounds are presented in Table 2.5. While the low isomer shift and high quadrupole splitting are indicative for $l s-\mathrm{Fe}(\mathrm{III})$, the other set of signals with higher isomer shift but lower quadrupole splitting show similar Mößbauer parameters to [tp/N4Py]. The different 
ratios between the two signals could indicate a partial thermally driven CTIST at $80 \mathrm{~K}$, but to evaluate this behavior, more evidence must be provided.

To further evaluate the electronic structure of the $\left[\mathbf{t p}^{\mathbf{R}}-\mathbf{F e} / \mathbf{N} 4 \mathbf{P y}-\mathbf{C o}\right]$ complexes at room temperature, crude product absorption spectra of the crude product were measured and are presented in Figure 2.18. The spectrum of [tp-Fe/N4Py-Co] shows a broad feature at 730 $\mathrm{nm}$, indicative of a Fe(II) $\rightarrow \mathrm{Co}$ (III) IVCT $^{116}$, next to the expected MLCT and LMCT bands at $400 \mathrm{~nm}$. For the spectra of $\left[\mathbf{t p}^{\mathrm{Me}}-\mathbf{F e} / \mathbf{N} 4 \mathbf{P y}-\mathbf{C o}\right]$ and $[\mathbf{t p} *-\mathbf{F e} / \mathbf{N} 4 \mathbf{P y}-\mathbf{C o}]$ the broad feature $>700 \mathrm{~nm}$ is not as pronounced and the MLCT and LMCT features are shifted to higher wavelengths 410 and $440 \mathrm{~nm}$, respectively. The presence of a weak band at $780 \mathrm{~nm}$ for the latter two systems could be an impurity, due to the presence in both spectra. Comparison to the photophysical features of the two magnetic states of $\left[\mathbf{F e}_{2} \mathbf{C o}_{2}\right]$, indicates a diamagnetic $\left[l s-\mathrm{Fe}(\mathrm{II}) / l_{s}-\mathrm{Co}\right.$ (III)] state for [tp-Fe/N4Py-Co] at room temperature, while for

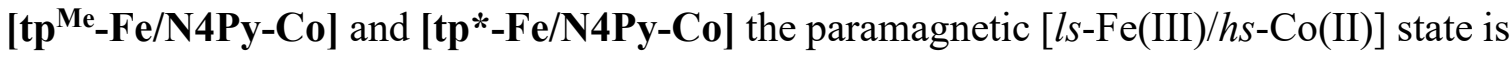
the ground state at room temperature.

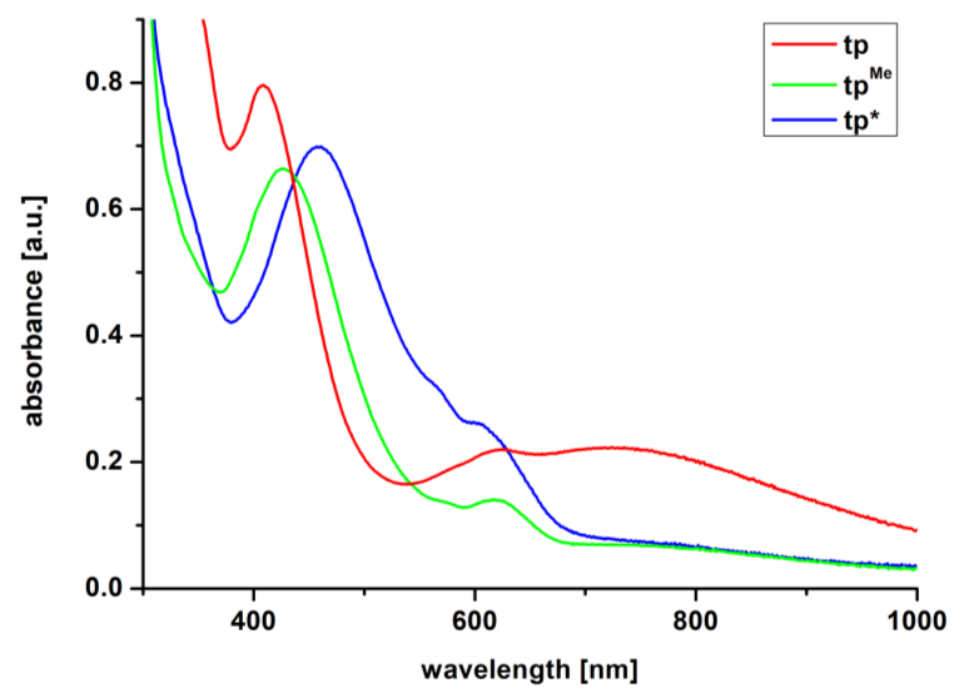

Figure 2.18 UV/vis spectra of [tp $\left.{ }^{R}-\mathrm{Fe} / \mathrm{N} 4 \mathrm{Py}-\mathrm{Co}\right]$ with $\mathrm{tp}^{\mathrm{R}}=$ tp, tp $\mathrm{p}^{\mathrm{Me}}$ and $\mathbf{t p}^{*}$.

In addition, a magnetic susceptibility measurement over the temperature range of $2-400$ $\mathrm{K}$ was conducted on solid material of [tp-Fe/N4Py-Co] (Figure 2.19). For comparison the fit for $[l s-\mathrm{Fe}(\mathrm{III}) / h s-\mathrm{Co}(\mathrm{II})]$ in $\left[\mathbf{t p}{ }^{*}-\mathbf{F e} / \mathbf{p z}^{*}{ }_{4} \mathbf{L u t}-\mathbf{C o}\right]$ is presented.

The variable temperature magnetic susceptibility measurement of [tp-Fe/N4Py-Co] shows a maximum value of $\chi_{m} T=0.2 \mathrm{~cm}^{3} \cdot \mathrm{K} \cdot \mathrm{mol}^{-1}$ (Spin-only value: $S=1 / 2 \rightarrow \chi_{m} T \approx 0.37$ $\mathrm{cm}^{3} \cdot \mathrm{K} \cdot \mathrm{mol}^{-1}$ ) at $300 \mathrm{~K}$, indicating a presence of an impurity in the sample. A decent fit was accomplished, by assuming a $10 \%$ impurity of a $h s-\mathrm{Co}$ (II) compound, most reasonable an unreacted cobalt(II) salt. The magnetic susceptibility measurements are therefore consistent with the UV/vis and Mößbauer data and suggest that [tp-Fe/N4Py-Co] is a diamagnetic species; however, the product obtained contained some paramagnetic impurity. Magnetic susceptibility measurement for the other complexes $\left[\mathbf{t p}^{\mathbf{M e}}-\mathbf{F e} / \mathbf{N} 4 \mathbf{P y}-\mathbf{C o}\right]$ and $[\mathbf{t p} * /-\mathbf{F e}$ 
N4Py-Co] were not pursued due to the challenging interpretation in case of possible paramagnetic impurities.

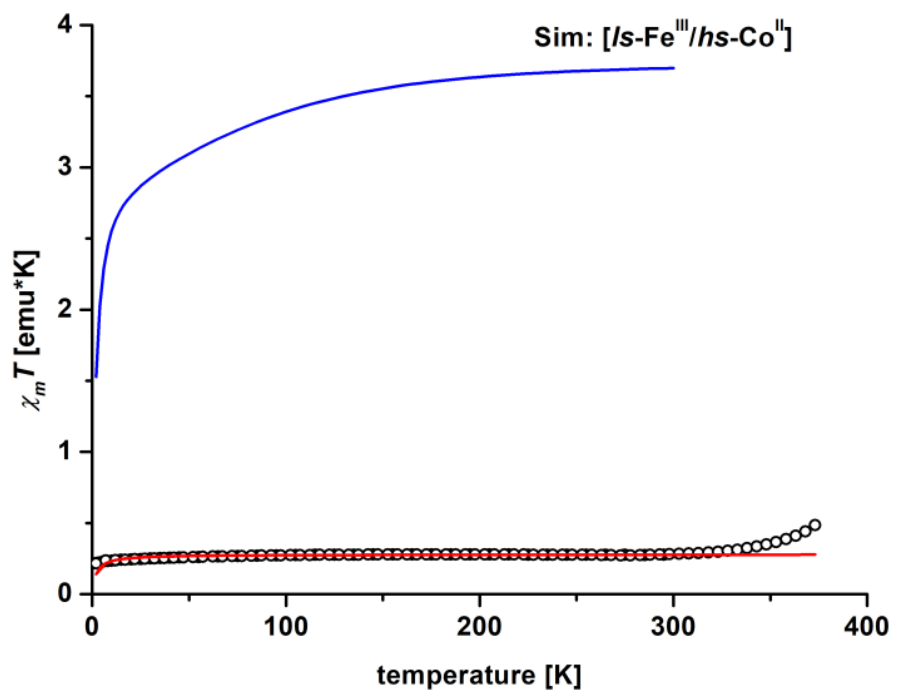

Figure $2.19 \chi_{m} T$ vs. T for crystalline [tp-Fe/N4Py-Co]; Dots representing experimental data and the solid red line simulation curve using a Curie-Weiss fit with a $10 \%$ impurity of $h s-\mathrm{Co}(\mathrm{II})$; the solid blue line is the simulated fit for [tp*-Fe/pz* ${ }_{4}$ Lut-Co] (Section 2.4.3).

In summary, the systems utilizing N4Py show different coloring of the compounds depending on the $\mathbf{t p}^{\mathbf{R}}$ derivative. Differences in electronic structures were also found for the iron center based on the ${ }^{57} \mathrm{Fe}$ Mößbauer data showing two different sets of signals. Interestingly, for [tp-Fe/N4Py-Co] a higher isomer shift and smaller quadrupole splitting is observed, indicative for $l s-\mathrm{Fe}$ (II) rather than $l s-\mathrm{Fe}(\mathrm{III})$. This assumption is consistent with the UV/vis measurements at room temperature. For [tp-Fe/N4Py-Co] a broad feature at $740 \mathrm{~nm}$ is found, which could be interpreted as a Fe(II) $\rightarrow$ Co(III) IVCT band. In contrast, the spectra of [tp $\left.{ }^{\mathrm{Me}}-\mathbf{F e} / \mathbf{N} 4 \mathbf{P y}-\mathbf{C o}\right]$ and $\left[\mathbf{t p}{ }^{*}-\mathbf{F e} / \mathbf{N} 4 \mathbf{P y}-\mathbf{C o}\right]$ do not provide such a red shifted feature. Finally, the observed diamagnetic state of [tp-Fe/N4Py-Co] is observed in the presented characterization methods. While there is a small paramagnetic contribution this could be mostly due to a $h s-\mathrm{Co}(\mathrm{II})$ impurity. 


\subsection{Ultrafast Time-Resolved Spectroscopy of the Photo-Induced Electron Transfer in Di- and Tetranuclear Fe/Co PBAs}

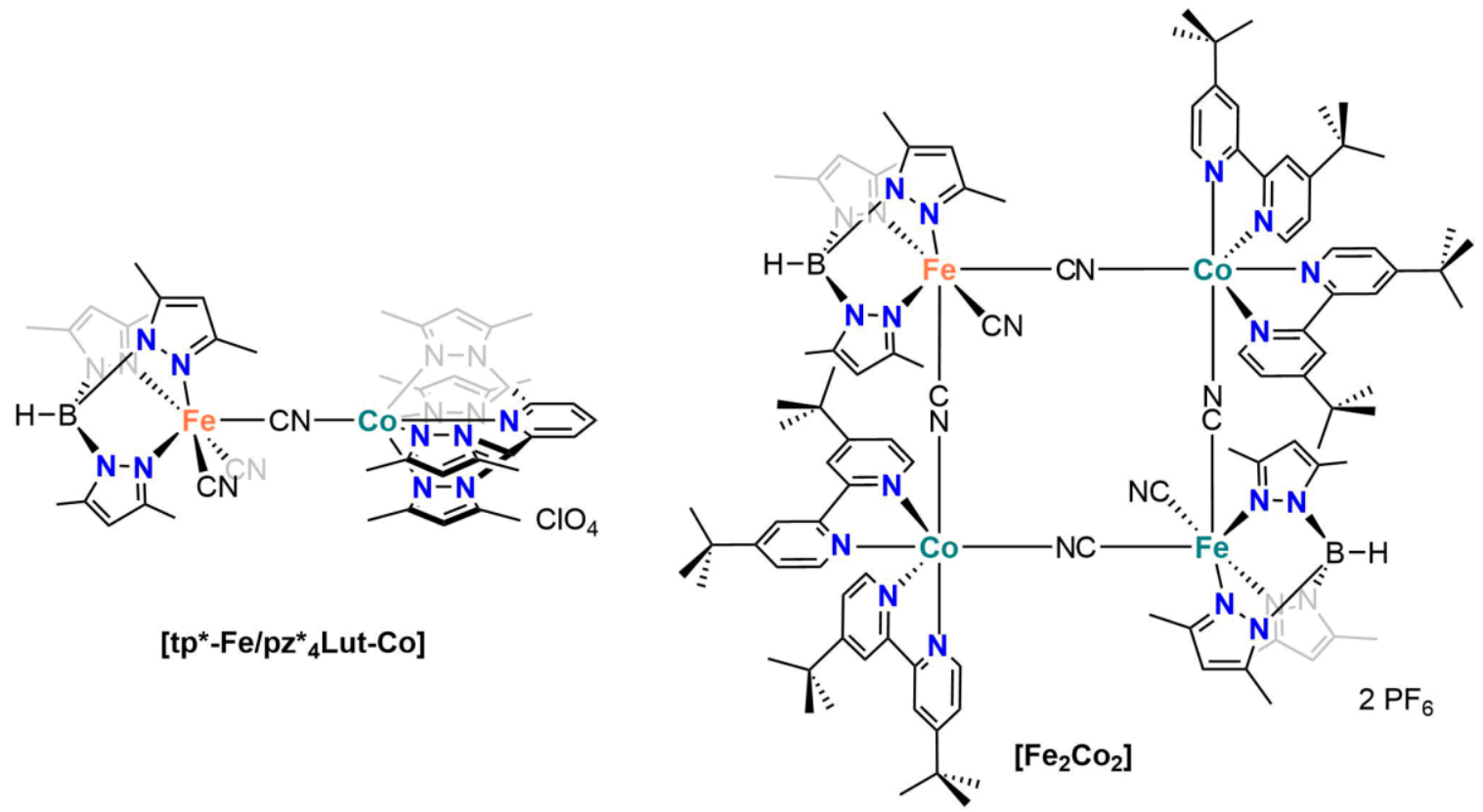

Scheme 2.17 Chosen PBAs [tp*-Fe/pz $\left.{ }^{*}{ }_{4} \mathrm{Lut}-\mathrm{Co}\right]$ and $\left[\mathrm{Fe}_{2} \mathrm{Co}_{2}\right]$ for pump probe spectroscopy.

In Chapter 2.3 and 2.4 the dinuclear Fe/Co PBAs containing pyrazole based [tp ${ }^{\mathbf{R}} / \mathbf{p z}^{\mathbf{R}}{ }_{4} \mathbf{P y}$ ] pentadentate ligands and the tetranuclear $\left[\mathbf{F e}_{2} \mathbf{C o}_{2}\right]$ were presented and their properties discussed. This section will now focus on the investigation of the CTIST via ultrafast pump probe spectroscopy. The two systems will be the tetranuclear system $\left[\mathrm{Fe}_{2} \mathbf{C o}_{2}\right]$ as a benchmark system and the dinuclear PBA [tp*/pz* $\left.{ }_{4} \mathbf{L u t}\right]$ (Scheme 2.17). While $\left[\mathbf{t p *} / \mathbf{p z}^{*}{ }_{4} \mathbf{L u t}\right]$ is found in the paramagnetic state over the whole temperature range, $\left[\mathrm{Fe}_{2} \mathrm{Co}_{2}\right]$ can be switched in solution to the diamagnetic state with decreasing temperature. Consequently, the differences between dinuclear and tetranuclear PBAs concerning their electronic structure changes through the CTIST mechanism may be studied. In addition, depending on the magnetic ground state, the possibility of two different pathways of the CTIST may occur and the relaxation pathway may be investigated. All time dependent measurements were carried out and analyzed by Dr. Ahrens and Dr. Schwarzer from the MPI für biophysikalische Chemie, Göttingen in the context of the SFB 1073. 


\subsubsection{Femtosecond UV/vis-Pump UV/vis-Probe Spectroscopy on [tp*-Fe/pz ${ }^{*}{ }_{4}$ Lut-Co] and [ $\left.\mathrm{Fe}_{2} \mathrm{Co}_{2}\right]$}

After the full characterization of both Fe/Co systems, we first investigated the femtosecond timescale changes of the PBAs through UV/vis spectroscopy. For this, pump pulses with two different excitation wavelengths $\left(\lambda_{\text {exc }}=385\right.$ and $775 \mathrm{~nm}$ ) were applied for the separate excitation of the diamagnetic and the paramagnetic state, respectively. All measurements were conducted in acetonitrile solution.

Starting with the dinuclear Fe/Co PBA [tp*-Fe/pz* $\left.{ }^{*} \mathbf{L u t}-\mathbf{C o}\right]$ the fs-pump-probe measurements were carried out at $22^{\circ} \mathrm{C}$ with an excitation wavelength of $\lambda_{\mathrm{ex}}=385 \mathrm{~nm}$, to excite the paramagnetic state present at that temperature. The time dependent difference spectra combined with the stationary UV/vis spectrum of [tp*-Fe/pz* $\left.{ }_{4} \mathbf{L u t}-\mathbf{C o}\right]$ and a time trace analysis are shown in Figure 2.20.

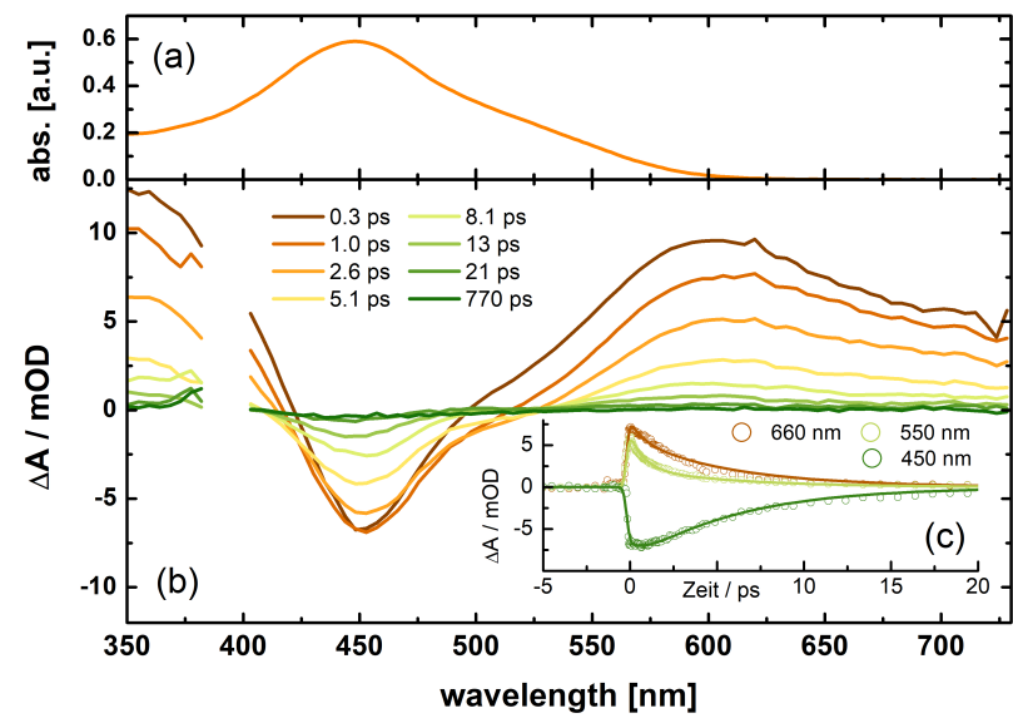

Figure 2.20 (a) Stationary UV/vis absorption spectrum of [tp*-Fe/pz ${ }^{*}{ }_{4}$ Lut-Co] recorded at $22^{\circ} \mathrm{C}$; (b) UV/vis transient difference spectra recorded at $22^{\circ} \mathrm{C}$ with $\lambda_{\text {exc }}=385 \mathrm{~nm}$. (c) Time traces at 450, 550 and $660 \mathrm{~nm}$. Measurements are done in $0.8 \mathrm{mM}$ acetonitrile solution using a quartz cuvette with (a) $1 \mathrm{~mm}$ and (b) $0.6 \mathrm{~mm}$ path length.

Right after excitation, a bleaching of the band at $450 \mathrm{~nm}$ is observed, which contains the $\mathrm{Co}(\mathrm{II}) \rightarrow \mathrm{Fe}(\mathrm{III})$ IVCT band next to the LMCT and MLCT bands. At the same time, a broad band with a maximum at $\sim 600 \mathrm{~nm}$ is observed which can be assigned to the backelectron transition (Fe(II) $\rightarrow \mathrm{Co}(\mathrm{III})$ IVCT band). Two lifetimes, $\tau_{1}=2.2 \pm 0.2 \mathrm{ps}$ and $\tau_{2}=$ $5.6 \pm 1.5 \mathrm{ps}$, were extracted by fitting the data at 600,550 and $440 \mathrm{~nm}$ to a biexponential function. The first time constant was assigned to the relaxation of a vibrationally hot state after electron transfer, which decays back through back electron donation to the ground state, similar to measurements in the literature. ${ }^{122}$ No long-living species undergoing 
intersystem crossing is observed, which normally decays on a time scale of 0.1 to $1 \mathrm{~ns}$ due to the spin forbidden nature of the transition. ${ }^{122}$

Although the excitation of [tp*-Fe/pz* ${ }_{4}$ Lut-Co] shows already an IVCT driven transition, a comparison to $\left[\mathbf{F e}_{2} \mathbf{C o}_{2}\right]$ is necessary. For the tetranuclear PBA both magnetic states can be excited, due to the thermal-driven CTIST which allows the diamagnetic state to be accessible for excitation. Therefore, [ $\left.\mathrm{Fe}_{2} \mathrm{Co}_{2}\right]$ was measured with a pump wavelength of $\lambda_{\mathrm{exc}}=385 \mathrm{~nm}$ at $22^{\circ} \mathrm{C}$, similar to the dinuclear PBA and with $\lambda_{\mathrm{exc}}=775 \mathrm{~nm}$ at $-30^{\circ} \mathrm{C}$ for the selective excitation of the diamagnetic state. The results are shown in Figure 2.21 with the stationary spectra at 30 and $-30^{\circ} \mathrm{C}$ and a time trace analysis.

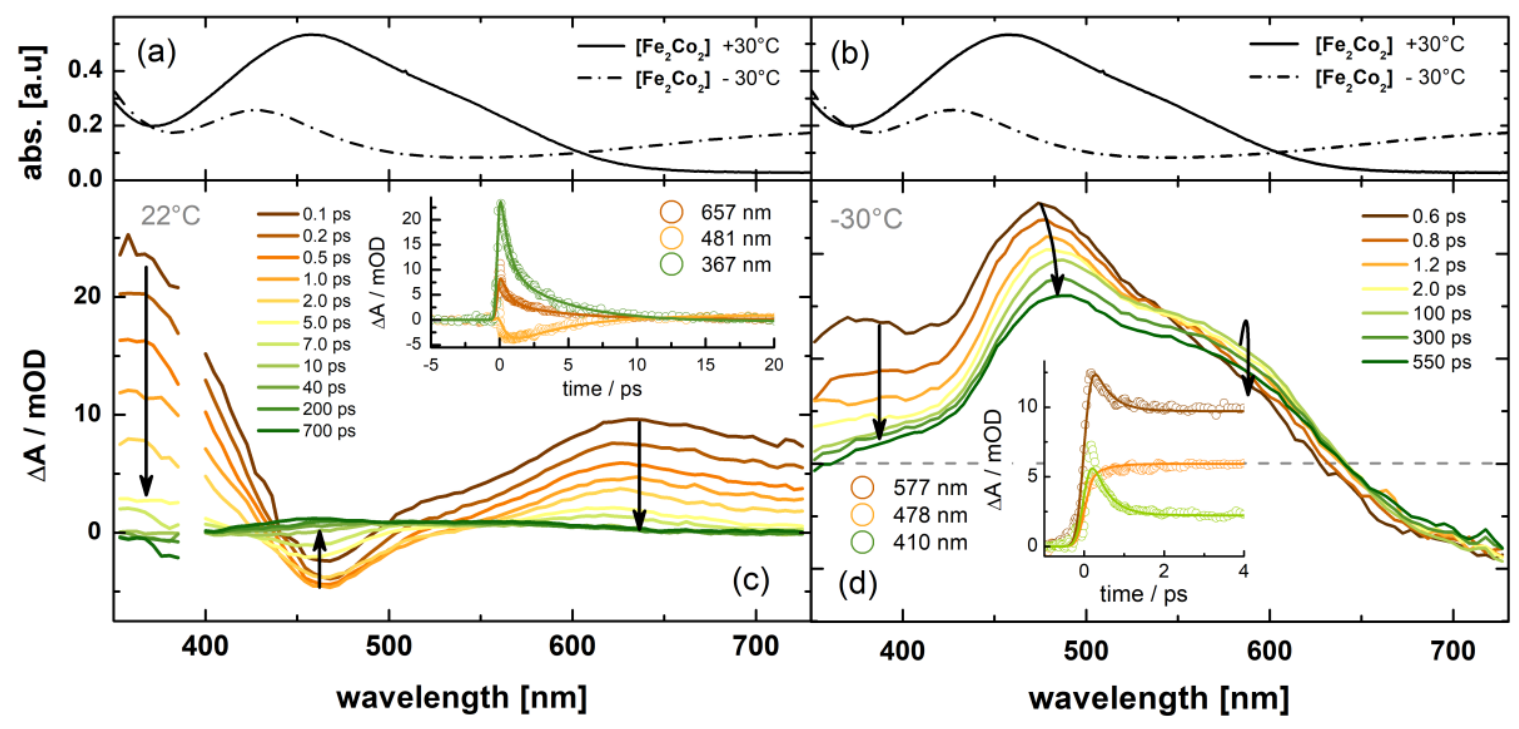

Figure 2.21 UV/vis transient difference spectra of [ $\left.\mathrm{Fe}_{2} \mathrm{Co}_{2}\right]$ (c) at $22^{\circ} \mathrm{C}$ (paramagnetic state) with $\lambda_{\mathrm{exc}}=385 \mathrm{~nm}$ and in (d) at $-30^{\circ} \mathrm{C}$ (diamagnetic state) with $\lambda_{\mathrm{exc}}=775 \mathrm{~nm}$. Both spectra were measured in $0.8 \mathrm{mM}$ acetonitrile solution under ambient conditions using a quartz cuvette with $0.6 \mathrm{~mm}$ path length. For comparison UV/vis absorption spectra at $30^{\circ} \mathrm{C}$ and at $-30^{\circ} \mathrm{C}$ in (a) and (b) are shown.

The transient absorption spectrum at room temperature is similar to the one measured with [tp*/pz* ${ }_{4}$ Lut] with a bleaching at $\sim 460 \mathrm{~nm}$ and two new bands at $385 \mathrm{~nm}$ and $\sim 660 \mathrm{~nm}$. The same excitation of the $\mathrm{Co}$ (II) $\rightarrow \mathrm{Fe}$ (III) IVCT is therefore reasonable. Those bands decay with two-time constants $\tau_{1}=0.6 \pm 0.15 \mathrm{ps}$ and $\tau_{2}=4.1 \pm 0.2 \mathrm{ps}$. Interestingly, a third long lifetime ( $\tau_{3}>>1 \mathrm{~ns}$ ) is observed, exceeding the timescale of the setup. This is due to a small percentage of the diamagnetic species present at $22^{\circ} \mathrm{C}$ which is excited because of the broad feature of the Fe(II) $\rightarrow \mathrm{Co}$ (III) IVCT band. This indicates that the two species have two different photophysical properties. Therefore, $\left[\mathbf{F e}_{2} \mathbf{C o}_{2}\right]$ was measured at $-30^{\circ} \mathrm{C}$ with an excitation wavelength of $\lambda_{\mathrm{exc}}=775 \mathrm{~nm}$, selectively exciting the diamagnetic state. Here, the broad band of the Fe(II) $\rightarrow \mathrm{Co}$ (III) IVCT is quenched at $\sim 650-750 \mathrm{~nm}$, and three bands at 410, 478 and $577 \mathrm{~nm}$ emerge. The bands at 478 and $577 \mathrm{~nm}$ are characteristic for the paramagnetic state when compared to the stationary spectrum. These bands decay again with two observable time constants, a short $\tau_{1}=0.35 \pm 0.15 \mathrm{ps}$ and a long living species with $\tau_{2}=2.5 \pm 0.1 \mathrm{~ns}$. Interestingly a third time constant for the intersystem crossing (ISC) 
between the short and long living species is not observe in contrast to the IR measurements (vide infra). In contrast to the low-temperature measurement, the room temperature UV/vis measurement does not show a long living species. To confirm excitation of the diamagnetic state at room temperature, the measurement was repeated but with an excitation at $\lambda_{\text {exc }}=775 \mathrm{~nm}$. The relaxation pathway of only the diamagnetic state was observed. Consistency of the data may be given through similar changes in the IR measurements, discussed in the next section.

\subsubsection{Femtosecond UV/vis-Pump Mid-IR-Probe Spectroscopy on [tp*-Fe/pz ${ }^{*}$ Lut-Co] and $\left[\mathrm{Fe}_{2} \mathrm{Co}_{2}\right]$}

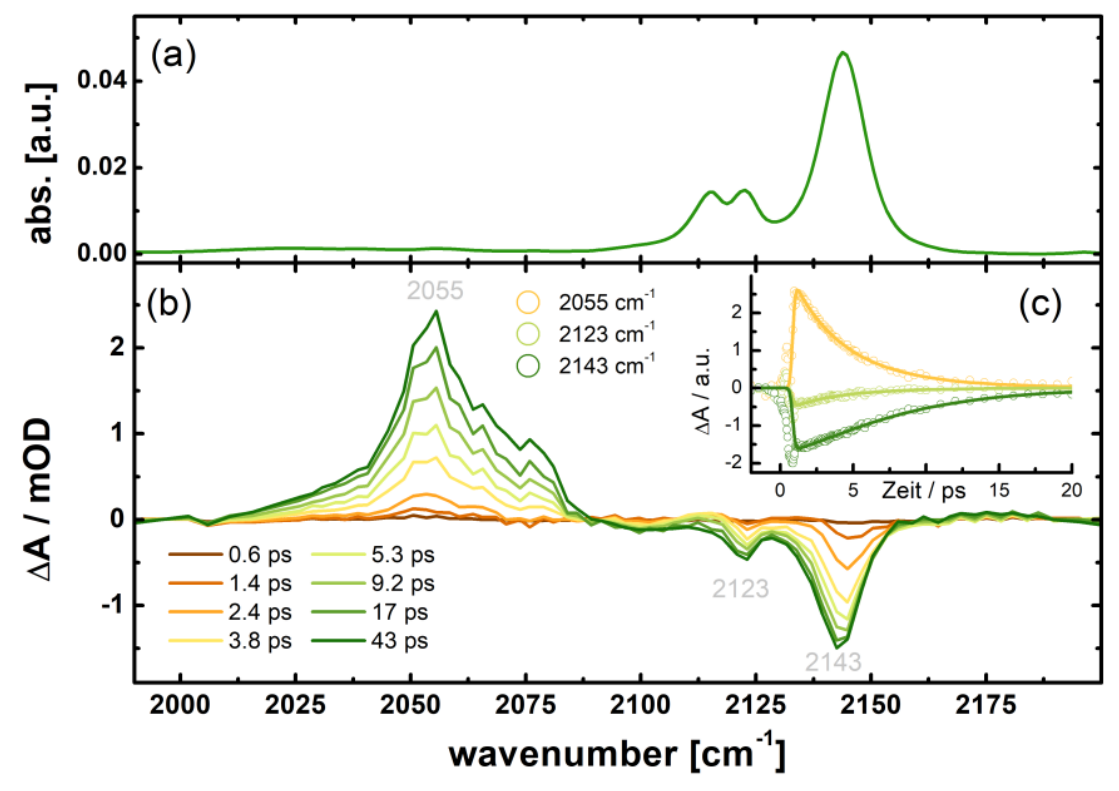

Figure 2.22 (a) FTIR spectrum of [tp*-Fe/pz ${ }^{*}{ }_{4}$ Lut-Co] in acetonitrile solution; (b) UV/vis-pump mid-IR-probe difference spectra at $+22^{\circ} \mathrm{C}$ (paramagnetic phase) with $\lambda_{\text {exc }}=385 \mathrm{~nm}$; (c) Time traces for bands at 2055, 2123 and $2143 \mathrm{~cm}^{-1}$. Measurements were performed in $0.8 \mathrm{mM}$ acetonitrile solution using a quartz cuvette with $0.6 \mathrm{~mm}$ path length.

The oxidation state sensitive $\mathrm{C}-\mathrm{N}$ stretching frequencies allow observation of the thermally driven CTIST for [ $\left.\mathbf{F e}_{2} \mathbf{C o}_{2}\right]$ via IR spectroscopy (Chapter 2.3). The changes of the bands corresponding to those vibrations can give information on any intermediates during the CTIST and their lifetimes.

Similar conditions as described in the previous chapter were used also for the UV/vis-pump IR-probe measurements of [tp*-Fe/pz* $\left.{ }_{4} \mathbf{L u t}-\mathbf{C o}\right]$. The results including stationary spectra and lifetime determinations are shown in Figure 2.22. In comparison to the stationary spectrum, the bleached bands at 2123 and $2145 \mathrm{~cm}^{-1}$ fit the characteristic bands for the 
paramagnetic state. While this is expected, interestingly, the bleach of the third band at $2113 \mathrm{~cm}^{-1}$ is not observed. This could be due to an energetic coupling of the two vibrational states, where only one excitation is observed. As expected from the stationary low temperature spectrum of $\left[\mathbf{F e}_{2} \mathbf{C o}_{2}\right]$, a band with a shoulder at lower wavenumbers is observed after excitation. These bands were assigned in the stationary spectrum for the diamagnetic state but based on the UV/vis data this assumption for the excitation of [tp*/pz* $\left.{ }_{4} \mathbf{L u t}\right]$ is questionable. The IR data confirms this hypothesis, showing again only two short lifetimes, $\tau_{1}=2.1 \pm 0.2 \mathrm{ps}$ and $\tau_{2}=9.9 \pm 1.5 \mathrm{ps}$. Considering the error of the measurement, those lifetimes are consistent with the data derived from the time-resolved $\mathrm{UV} / \mathrm{vis}$ measurements. Therefore, the same excitation pathway is reasonable, in which the electron transfer does not result in a spin transition and after electron back-donation a relaxation of a vibrationally hot ground state is observed.

Similar photophysical features are observed for $\left[\mathbf{F e}_{2} \mathbf{C o}_{2}\right]$ at room temperature after excitation at $385 \mathrm{~nm}$. Additionally, the selective excitation of the diamagnetic state was done at $-30^{\circ} \mathrm{C}$ with a pump laser of $\lambda_{\mathrm{exc}}=775 \mathrm{~nm}$. Both measurements are shown in Figure 2.23 .

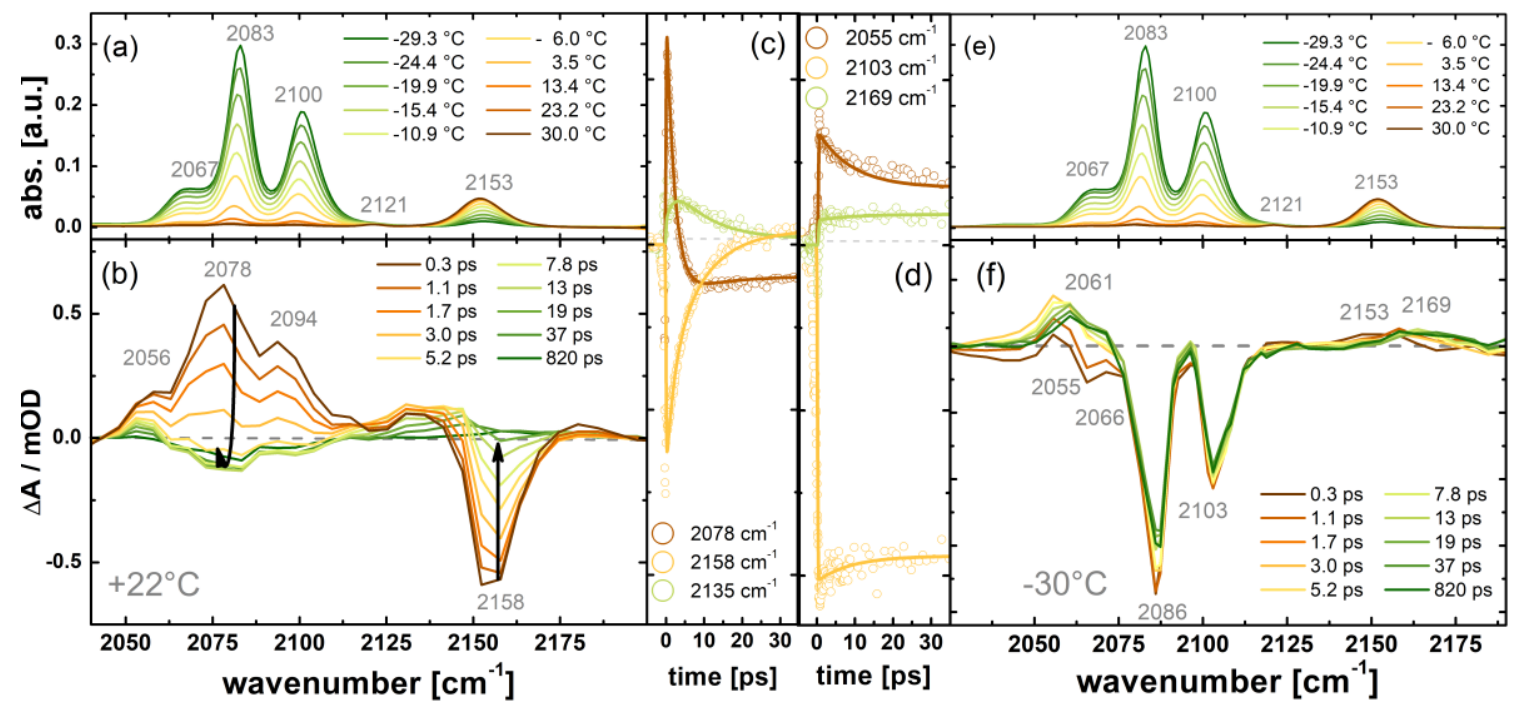

Figure 2.23 Temperature-dependent FTIR spectra of $\left[\mathrm{Fe}_{2} \mathrm{Co}_{2}\right]$ are shown in (a) and (e); IR transient difference spectra (b) at $+22^{\circ} \mathrm{C}$ (paramagnetic state) with $\lambda_{\operatorname{exc}}=385 \mathrm{~nm}$ and (f) at $-30^{\circ} \mathrm{C}$ (diamagnetic state) with $\lambda_{\mathrm{exc}}=775 \mathrm{~nm}$; time traces (c) for $22^{\circ} \mathrm{C}$ at 2078,2135 and $2158 \mathrm{~cm}^{-1}$ and (d) for $-30^{\circ} \mathrm{C}$ at 2055,2103 and $2169 \mathrm{~cm}^{-1}$; both measurements were performed in $0.8 \mathrm{mM}$ acetonitrile solution using a quartz cuvette with $0.6 \mathrm{~mm}$ path length.

The room temperature measurement appears to be similar to the measurement for [tp*-Fe/pz* $\left.{ }_{4} \mathbf{L u t}-\mathbf{C o}\right]$. In contrast to $\left[\mathbf{t p} *-\mathbf{F e} / \mathbf{p z}^{*}{ }_{4} \mathbf{L u t}-\mathbf{C o}\right.$ ] the spectrum shows a long-lived species, also observed in the time-resolved UV/vis measurement, indicating the presence of the diamagnetic species at $22^{\circ} \mathrm{C}$. The time constants with $\tau_{1}=2.1 \pm 0.5 \mathrm{ps}$ and $\tau_{1}=9.0 \pm 2.0$ ps showing the same behavior as $\left[\mathbf{t p}{ }^{*}-\mathbf{F e} / \mathbf{p z}^{*}{ }_{4} \mathbf{L u t}-\mathbf{C o}\right]$, where no spin transition is observed. The measurement at $-30^{\circ} \mathrm{C}$ and excitation at $775 \mathrm{~nm}$ shows a different behavior. Here, a bleach of the bands at 2066, 2086 and $2103 \mathrm{~cm}^{-1}$ is observed, corresponding to the diamagnetic $[l s-\mathrm{Fe}(\mathrm{II}) / l s-\mathrm{Co}(\mathrm{III})]$ state. Simultaneously, a higher energy band at $2153 \mathrm{~cm}^{-1}$ 
arises. In addition, the bleach of the $2066 \mathrm{~cm}^{-1}$ band slowly changes to a superimposed band at $2061 \mathrm{~cm}^{-1}$ with a very short time constant of $\tau_{1}=0.2 \pm 0.2 \mathrm{ps}$. This shift is coupled with another one of the bands at $2153 \mathrm{~cm}^{-1}$ to $2169 \mathrm{~cm}^{-1}$. Two additional lifetimes are extracted, $\tau_{2}=8.8 \pm 2.0 \mathrm{ps}$ and $\tau_{3}>>1 \mathrm{~ns}$. This substantiates the hypothesis, that only in the excitation of the diamagnetic state a CTIST is observed and in the case of the paramagnetic state only a electron transfer without spin transition is assumed. Only for the first case a long living species should observed, due to the spin-forbidden direct relaxation.

In conclusion, [tp*-Fe/pz $\left.{ }_{4}{ }_{4} \mathbf{L u t}-\mathbf{C o}\right]$ and $\left[\mathbf{F e}_{2} \mathbf{C o}_{2}\right]$ have been investigated by time-resolved UV/Vis and IR spectroscopy. While [tp*-Fe/pz* $\left.{ }_{4} \mathbf{L u t}-\mathbf{C o}\right]$ was only measured at room temperature, due to the absence of a thermal-driven CTIST, [Fe $\left.\mathbf{F e}_{2} \mathbf{C o}_{2}\right]$ was measured at room temperature and $-30^{\circ} \mathrm{C}$ with two different excitation wavelengths $\left(\lambda_{\mathrm{exc}}=385\right.$ and $785 \mathrm{~nm}$ ). The obtained lifetimes are summarized in Table 2.6. For the room temperature measurements both compounds show similar photophysical properties in UV/vis and IR with two short lifetimes. The presence of a long-lived species in case of $\left[\mathrm{Fe}_{2} \mathbf{C o}_{2}\right]$ is based on small amounts of the diamagnetic $\left[l s-\mathrm{Fe}(\mathrm{II}) / l_{s-\mathrm{Co}}\right.$ (III)] state at room temperature. In contrast to the measurements at $22^{\circ} \mathrm{C}$, excitation of $\left[\mathbf{F e}_{2} \mathbf{C o}_{2}\right]$ at $-30^{\circ} \mathrm{C}$ and $\lambda_{\mathrm{exc}}=775$ shows three different steps of the decay, the last one being longer than the limitation of the method ( $\tau>1 \mathrm{~ns}$ ). The presented measurements clearly show a dependency of the electron transfer pathway dependent on the excitation of the para- $[l s-\mathrm{Fe}(\mathrm{III}) / h s-\mathrm{Co}(\mathrm{II})]$ or diamagnetic $\left[l s-\mathrm{Fe}(\mathrm{II}) / l_{s}-\mathrm{Co}(\mathrm{III})\right]$ state. This hypothesis is supported by the similarity of the room temperature measurements of both compounds and the differences compared to the lowtemperature measurement of $\left[\mathbf{F e}_{2} \mathbf{C o}_{2}\right]$ in the diamagnetic state.

Table 2.6 Time trace analysis of the time-resolved measurements using UV/vis and mid-IR probe lasers for [ $\left.\mathrm{Fe}_{2} \mathrm{Co}_{2}\right]$ and [tp*-Fe/pz $\left.{ }_{4}{ }_{4} \mathrm{Lut}-\mathrm{Co}\right]$.

\begin{tabular}{|c|c|c|c|c|c|}
\hline & & & & & Vis \\
\hline PBA & $\mathrm{T}\left[{ }^{\circ} \mathrm{C}\right]$ & $385 \mathrm{~nm}$ & $775 \mathrm{~nm}$ & $385 \mathrm{~nm}$ & $775 \mathrm{~nm}$ \\
\hline & & & $0.2 \mathrm{ps}$ & $0.39 \mathrm{ps}$ & $0.35 \mathrm{ps}$ \\
\hline & -30 & & $8,8 \mathrm{ps}$ & $5.0 \mathrm{ps}$ & 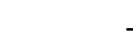 \\
\hline$\left[\mathrm{Fe}_{2} \mathrm{CO}_{2}\right]$ & & & $\gg 1 \mathrm{~ns}$ & $>1 \mathrm{~ns}$ & $\gg 1 \mathrm{~ns}$ \\
\hline & & $2.1 \mathrm{ps}$ & & $0.6 \mathrm{ps}$ & \\
\hline & 22 & $9.0 \mathrm{ps}$ & & 4.1ps & \\
\hline & & $>1 \mathrm{~ns}$ & & $\gg 1 \mathrm{~ns}$ & \\
\hline It & 25 & $2.1 \mathrm{ps}$ & & $2.2 \mathrm{ps}$ & \\
\hline & & $9.9 \mathrm{ps}$ & & $5.6 \mathrm{ps}$ & \\
\hline
\end{tabular}




\subsection{Summary and Outlook}

In this work six new dinuclear Fe/Co PBAs $\left[\mathbf{t p}^{\mathbf{R}}-\mathbf{F e} / \mathbf{p z}^{\mathbf{R}} \mathbf{4}_{\mathbf{L}} \mathbf{L u t}-\mathrm{Co}\right.$ ] bearing pentadentate tetrapyrazolyl lutidine ligands $\mathbf{p z}^{\mathbf{R}}{ }_{4}$ Lut were synthesized and characterized (Scheme 2.18).
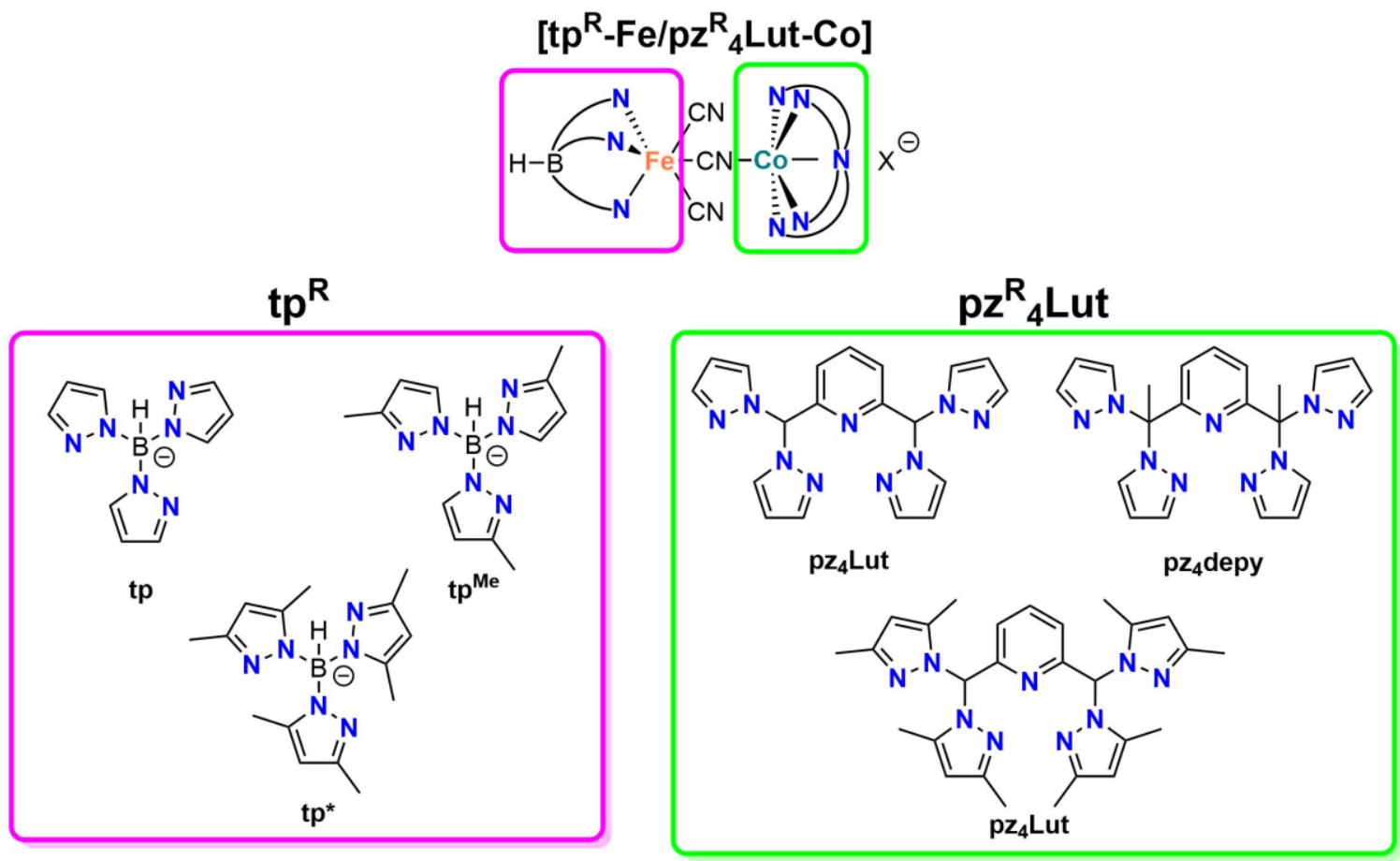

Synthesized Combinations in $\left[\operatorname{tp}^{\mathrm{R}}-\mathrm{Fe} / \mathrm{pz}^{\mathrm{R}}{ }_{4} \mathrm{Lut}-\mathrm{Co}\right]$
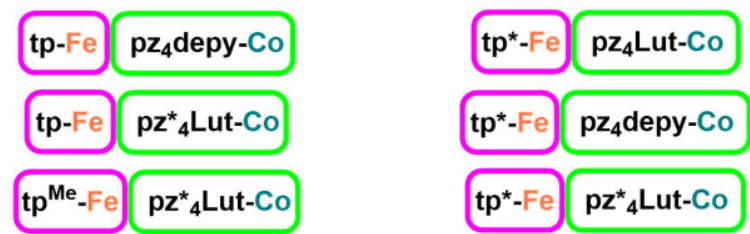

Scheme 2.18 Ligands $\operatorname{tp}^{R}$ and $p z^{R}{ }_{4}$ Lut used in the synthesis of six dinuclear Fe/Co PBAs $\left[\mathrm{tp}^{\mathrm{R}}-\mathrm{Fe} / \mathrm{pz}^{\mathrm{R}}{ }_{4}\right.$ Lut-Co].

In particular, the molecular and electronic structure of the complexes were investigated by UV/vis-, IR-, Mößbauer spectroscopy, X-ray diffraction analysis and magnetic susceptibility measurements towards a thermally driven CTIST between paramagnetic $\left[l_{s-}\right.$ $\mathrm{Fe}(\mathrm{III}) / h s-\mathrm{Co}(\mathrm{II})]$ and diamagnetic $[l s-\mathrm{Fe}(\mathrm{II}) / l s-\mathrm{Co}(\mathrm{III})]$ (Figure 2.24$)$. Despite the substitution pattern on the capping ligands, all of the synthesized complexes $\left[\mathbf{t p}{ }^{\mathbf{R}}-\mathbf{F e} / \mathbf{p z}^{\mathbf{R}}{ }_{4} \mathbf{L u t}-\mathbf{C o}\right]$ are found in the paramagnetic $[l s-\mathrm{Fe}(\mathrm{III}) / h s-\mathrm{Co}(\mathrm{II})]$ state from $2 \mathrm{~K}$ to $300 \mathrm{~K}$, consistent with variable temperature UV/vis and magnetic susceptibility measurements. The characteristic Fe(II) $\rightarrow \mathrm{Co}$ (III) IVCT band for the diamagnetic state is not present in the UV/vis spectra of any of the synthesized complexes (Figure 2.24, left). Additionally, magnetic susceptibility measurements of the complexes showed the magnetic 
behavior for antiferromagnetically coupled $[l s-\mathrm{Fe}(\mathrm{III}) / h s-\mathrm{Co}(\mathrm{II})]$ systems (Figure 2.24 , right).
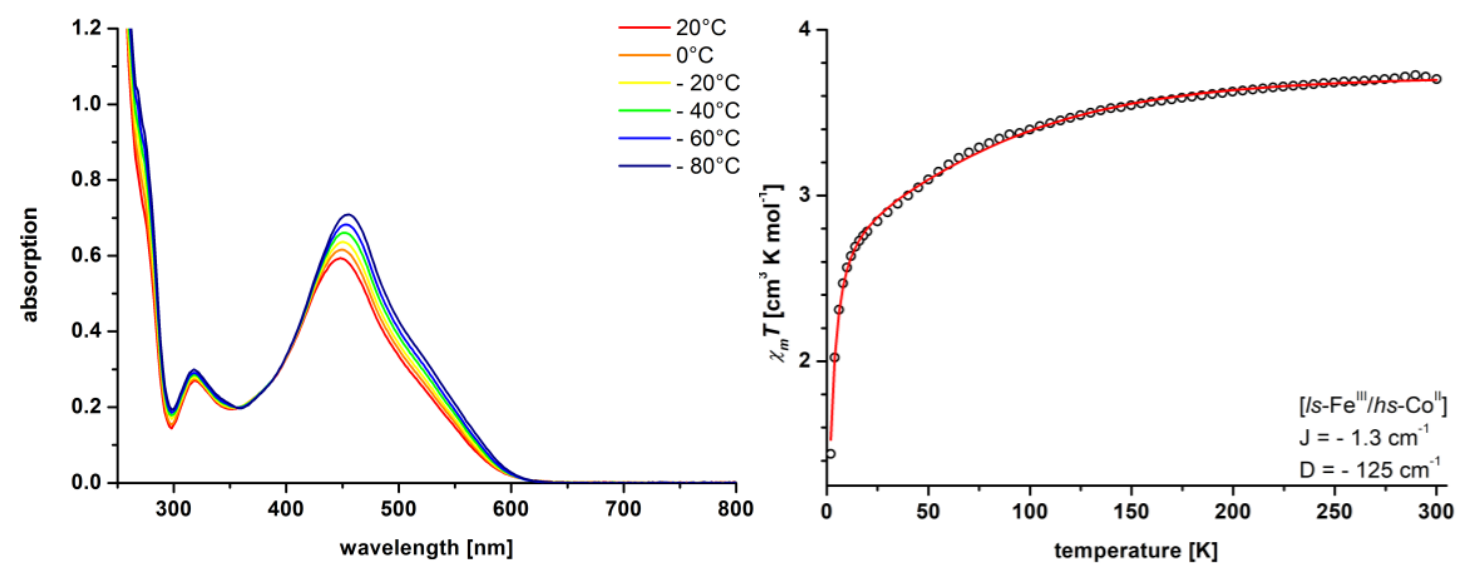

Figure 2.24 Variable temperature measurements of [tp*-Fe/pz* ${ }_{4}$ Lut-Co] give evidence for a paramagnetic state over the whole temperature range; left: Temperature-dependent UV/vis spectra in acetonitrile; right: $\chi_{m} T$ vs. $T$ of crystalline material with simulation representing a $[/ s-\mathrm{Fe}(\mathrm{III}) / h s-\mathrm{Co}(\mathrm{II})]$ spin state.

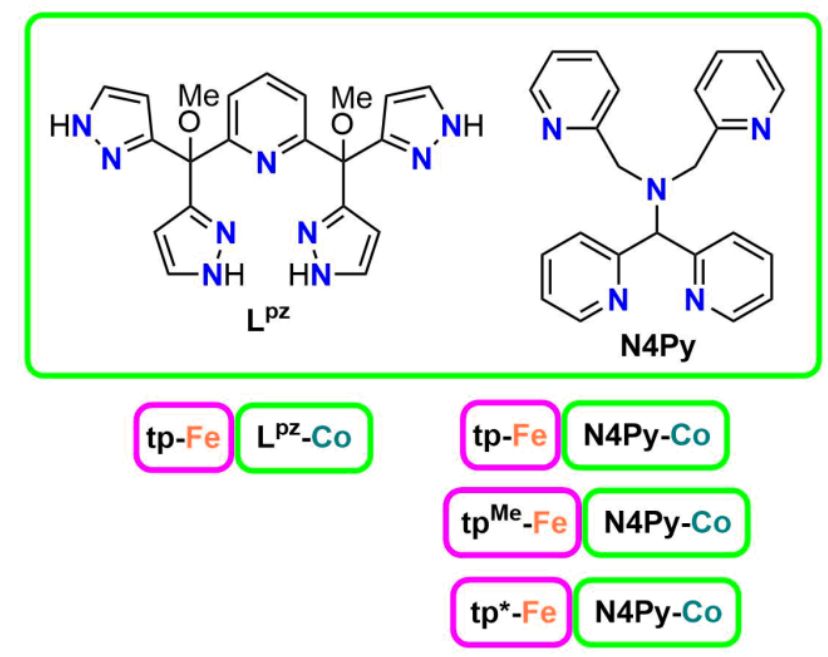

Scheme 2.19 Additional pentadentate $\mathrm{N}$-donor ligands, $\mathrm{L}^{\mathrm{pz}}$ and $\mathbf{N} \mathbf{4 P y}$ along with the investigated complexes.

In addition to the $\mathbf{p z} \mathbf{z}^{\mathbf{R}} \mathbf{L} \mathbf{u t}$ ligands, other pentadentate N-donor capping ligands $\mathbf{L}^{\mathbf{p z}}$ and N4Py were investigated (Scheme 2.19). The synthesized complex [tp*-Fe/ $\left.\mathbf{L}^{\mathbf{p z}}-\mathbf{C o}\right]$ exhibits oxidation coupled with the deprotonation of one pyrazole moiety of $\mathbf{L}^{\mathbf{p z}}$ to a $[l s-\mathrm{Fe}(\mathrm{III}) / l s$ Co(III)] species. In contrast to the discussed lutidine-based ligands, the complexes [tp ${ }^{\mathbf{R}_{-}}$ $\mathbf{F e} / \mathbf{N} 4 \mathbf{P y}-\mathbf{C o}]$ show different properties dependent on the substitution pattern of the $\mathbf{t p}^{\mathbf{R}}$ ligand. At room temperature, the complexes $\left[\mathbf{t p}^{\mathrm{Me}}-\mathbf{F e} / \mathbf{N} 4 \mathbf{P y}-\mathbf{C o}\right]$ and $[\mathbf{t p} * \mathbf{F e} / \mathbf{N} 4 \mathbf{P y}-\mathbf{C o}]$ were found also in the paramagnetic state. The complex [tp-Fe/N4Py-Co] was observed to be diamagnetic $[l s-\mathrm{Fe}(\mathrm{II}) / l s-\mathrm{Co}(\mathrm{III})]$, supported by UV/vis and Mößbauer spectroscopy. 
Variable temperature magnetic susceptibility measurements showed the diamagnetic state for [tp-Fe/N4Py-Co] in the temperature range of $2-400 \mathrm{~K}$ with a small paramagnetic impurity.

As a benchmark system, the literature-known tetranuclear $\mathrm{Fe} / \mathrm{Co} \mathrm{PBA}$ [ $\mathrm{Fe}_{2} \mathrm{Co}_{2}$ ] was synthesized and characterized. ${ }^{116}$ Complex [ $\left.\mathbf{F e}_{2} \mathbf{C o}_{2}\right]$ shows a thermally driven CTIST with $\mathrm{T}_{1 / 2}=275 \mathrm{~K}$ in acetonitrile solution and therefore allows for the excitation of the diamagnetic $[l s-\mathrm{Fe}(\mathrm{II}) / l s-\mathrm{Co}(\mathrm{III})]$ state and the paramagnetic $[l s-\mathrm{Fe}(\mathrm{III}) / h s-\mathrm{Co}(\mathrm{II})]$ state individually (Figure 2.25).
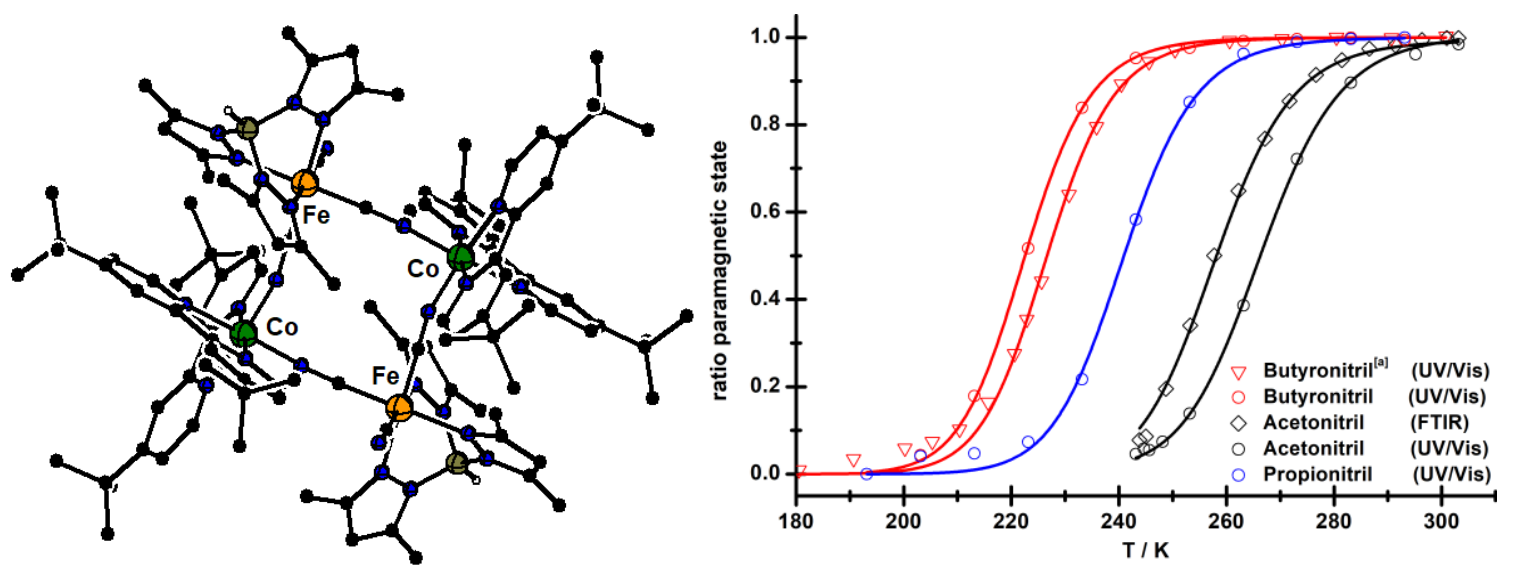

Figure 2.25 Left: molecular structure of [ $\left.\mathrm{Fe}_{2} \mathrm{Co}_{2}\right], \mathrm{PF}_{6}$ anions and hydrogen atoms are omitted for clarity; right: solvent dependency of the switching temperature of $\left[\mathrm{Fe}_{2} \mathrm{CO}_{2}\right]$ in different nitrile-based solvents.

Table 2.7 Time trace analysis of the time-resolved measurements using UV/vis and IR probe lasers for $\left[\mathrm{Fe}_{2} \mathrm{Co}_{2}\right]$ and $\left[\mathrm{tp}^{*}-\mathrm{Fe} / \mathrm{pz}^{*}{ }_{4} \mathrm{Lut}-\mathrm{Co}\right]$.

\begin{tabular}{|c|c|c|c|c|c|}
\hline \multirow{2}{*}{ Fe/Co PBAs } & \multirow{2}{*}{$\mathrm{T}\left[{ }^{\circ} \mathrm{C}\right]$} & \multicolumn{2}{|c|}{ IR } & \multicolumn{2}{|c|}{ UV/Vis } \\
\hline & & $385 \mathrm{~nm}$ & $775 \mathrm{~nm}$ & $385 \mathrm{~nm}$ & $775 \mathrm{~nm}$ \\
\hline \multirow{6}{*}[\mathrm{Fe}_{2}\mathrm{CO}_{2}]{} & \multirow{3}{*}{-30} & & $0.2 \mathrm{ps}$ & $0.39 \mathrm{ps}$ & $0.35 \mathrm{ps}$ \\
\hline & & & $8,8 \mathrm{ps}$ & $5.0 \mathrm{ps}$ & - \\
\hline & & & $>1 \mathrm{~ns}$ & $\gg 1 \mathrm{~ns}$ & $\gg 1 \mathrm{~ns}$ \\
\hline & \multirow{3}{*}{22} & $2.1 \mathrm{ps}$ & & $0.6 \mathrm{ps}$ & \\
\hline & & $9.0 \mathrm{ps}$ & & $4.1 \mathrm{ps}$ & \\
\hline & & > $1 \mathrm{~ns}$ & & $>1$ ns & \\
\hline \multirow{2}{*}{$\mathrm{tp} *-\mathrm{Fe} / \mathrm{pz}{ }_{4}{ }_{4}$ Lut-Co] } & \multirow{2}{*}{22} & $2.1 \mathrm{ps}$ & & $2.2 \mathrm{ps}$ & \\
\hline & & $9.9 \mathrm{ps}$ & & $5.6 \mathrm{ps}$ & \\
\hline
\end{tabular}


To investigate the mechanism of the CTIST, the dinuclear [tp*-Fe/pz* $\left.{ }_{4} \mathbf{L u t}-\mathbf{C o}\right]$ and tetranuclear [ $\left.\mathbf{F e}_{2} \mathbf{C o}_{2}\right]$ were chosen for UV/vis-pump UV/vis/mid-IR-probe spectroscopy. The excitation of the paramagnetic $[l s-\mathrm{Fe}(\mathrm{III}) / h s-\mathrm{Co}(\mathrm{II})]$ state $\left(\lambda_{\mathrm{exc}}=385 \mathrm{~nm}\right)$ at room temperature showed a two-step relaxation on the picosecond timescale for both compounds. Cooling down the solution of $\left[\mathbf{F e}_{2} \mathbf{C o}_{2}\right]$ results in a higher population of the diamagnetic $[l s-\mathrm{Fe}(\mathrm{II}) / l s-\mathrm{Co}(\mathrm{III})]$ state $\left(\lambda_{\mathrm{exc}}=775 \mathrm{~nm}\right)$ and when excited, a three-step relaxation with the last step having a long lifetime (Table 2.7). Based on the time trace analysis, two different relaxation pathways were developed dependent on the excitation of the para- or diamagnetic state (Scheme 2.20). Only in the case of the excitation of the diamagnetic state is a spin transition observed.
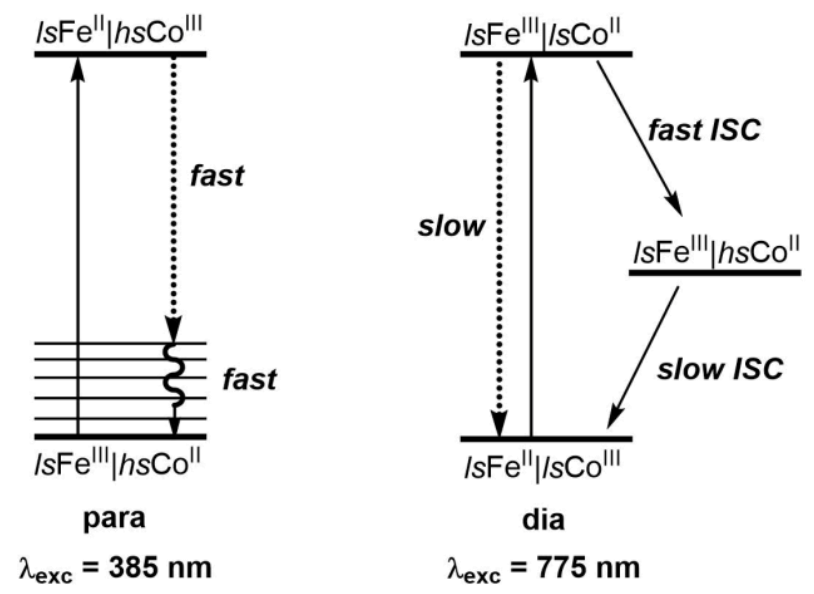

Scheme 2.20 Proposed mechanism for the excitation with (right) or without (left) CTIST dependent on ground state.

In conclusion, the identification of two different pathways dependent on the excitation of the magnetic ground state was, until this study, unknown. In contrast to the work of Bernhard et al., ${ }^{122}$ these pathways are not just found to be dependent on the laser pulse. The reason for the two pathways is still unknown, and theoretical calculations would give further insight. Additionally, the photophysical properties of [tp-Fe/N4PY-Co] should be further investigated via time-resolved spectroscopy, due to the possible diamagnetic ground state at room temperature. This could provide the missing information that is needed to fully elucidate the CTIST light harvesting process of Fe/Co PBAs. 


\section{Experimental Section}

\subsection{General Considerations}

All manipulations, if not stated otherwise, were performed under an anaerobic and anhydrous atmosphere of dried (phosphorous pentoxide on solid support [Sicapent, Merck]) argon by using standard Schlenk techniques or in a glove box $\left(\mathrm{O}_{2}<0.1 \mathrm{ppm}, \mathrm{H}_{2} \mathrm{O}\right.$ $<0.1 \mathrm{ppm}$ ) with $\mathrm{N}_{2}$ atmosphere. Hydrogen gas was purchased from Messer and deuterium gas from Sigma-Aldrich. Solvents and chemicals used were either present in the working group, were purchased from commercial sources or their synthesis is described below. $1 \mathrm{H}$ pyrazole-3,5-dicarboxamide (I) ${ }^{32}$, diphenyl aniline (IV $)^{63}, \mathrm{~d}_{1}$-benzaldehyde ${ }^{74}$, $\mathrm{Co}(\mathrm{OTf})_{2} \cdot 6 \mathrm{H}_{2} \mathrm{O}^{148}, 2,6$-pyridine dicarboxaldehyde ${ }^{133}$, di(pyridin-2-yl)methanone oxime ${ }^{149}$, di(pyridine-2-yl)methanamine ${ }^{149}, \mathbf{P y} 4 \mathbf{N}^{139}, \mathrm{Ktp}^{136}, \mathrm{Ktp}^{\mathrm{Me137}}, \mathrm{Ktp}^{* 138}, n \mathrm{Bu} 4 \mathrm{~N}\left[\mathrm{RFe}(\mathrm{CN})_{3}\right]$ $\left(\mathrm{R}=\mathbf{t p}, \mathbf{t p}^{\mathrm{Me}}, \mathbf{t p}^{*}\right)^{121}\left[\mathrm{Co}_{2} \mathrm{Fe}_{2}(\mathrm{CN})_{6}\left(\mathrm{tp}^{*}\right)_{2}\left(4,4^{\prime}-\mathrm{dtbbpy}\right)_{4}\right]\left(\mathrm{PF}_{6}\right)_{2} \cdot 2 \mathrm{MeOH}\left[\mathbf{C o}_{2} \mathbf{F e}_{2}\right]^{116}$ were synthesized through or similar to literature procedures. Glassware was dried at $120^{\circ} \mathrm{C}$. THF, diethyl ether, pentane and hexane were dried over sodium or sodium/potassium in the presence of benzophenone; DCM and Triethylamine was dried over Calcium hydride. $\mathrm{THF}-\mathrm{d} 8$ was also dried over sodium/potassium in the presence of benzophenone and cryodistilled, then stored over $3 \AA$ molecular sieves. Na and $\mathrm{K}$ were purchased as dispersions in mineral oil; they were washed repetitively with hexane and dried prior to use. NMR samples for $\mathrm{H}_{2}$ experiments were prepared under an inert atmosphere. Reactants were dissolved in THF- $\mathrm{d}^{8}$ and transferred into a J. Young NMR tube. The solution was degassed by means of the pump-freeze-thaw procedure with liquid nitrogen. The process was repeated three times. Because all compounds derived from $\left[\mathrm{L}^{\mathrm{Ph}} \mathrm{Ni}_{2}(\mu-\mathrm{Br})\right] \mathbf{1}$ are extremely sensitive to moisture (ultimately giving the hydroxide-bridged dinickel(II) complex 1-OH $\left.\left[\mathrm{L}^{\mathrm{Ph}} \mathrm{Ni}(\mathrm{II})(\mu-\mathrm{OH})\right]\right)$ gases such as $\mathrm{H}_{2}$ and $\mathrm{D}_{2}$ were dried with a cooling trap, cooled with liquid dinitrogen, or stirred above concentrated sulfuric acid before use. ${ }^{1} \mathrm{H}$ and ${ }^{13} \mathrm{C} \mathrm{NMR}$ spectra were recorded on Bruker Avance 300, 400 or $500 \mathrm{MHz}$ spectrometers. Chemical shifts are reported in parts per million relative to residual proton and carbon signals of the solvent. ${ }^{150}$ FTIR spectrometer was in Dial Path Technology with solid samples and analysed by FTIR MicroLab software. Elemental analyses were performed by the analytical laboratory of the Institute of Inorganic Chemistry at Georg-August-University using an Elementar Vario EL III instrument. Column chromatographic purifications (63-200 $\mu \mathrm{m}$ particle size) were performed on silica. TLC was performed on silica gel (Macherey-Nagel, Polygram SIL G/UV254). 


\subsection{X-Ray Data}

Crystal data and details of the data collections are given in Table 5.2 - 5.6 and molecular structures are shown in Figures 6.55-6.67. X-ray data were collected on a STOE IPDS II diffractometer (graphite monochromated Mo-K $\alpha$ radiation, $\lambda=0.71073 \AA$ ) by use of $\omega$ scans at $-140{ }^{\circ} \mathrm{C}$. The structures were solved with SHELXT and refined on $F^{2}$ using all reflections with SHELXL-2014/17/18. ${ }^{151,152}$ Non-hydrogen atoms were refined anisotropically. Most hydrogen atoms were placed in calculated positions and assigned to an isotropic displacement parameter of 1.5/1.2 $U_{\mathrm{eq}}(\mathrm{C})$. The metal hydrogen bond lengths must be considered with caution because of inherent ambiguities of $\mathrm{H}$ atom positions derived from X-ray crystallography. In case of disorder numerous restraints and constraints have been applied to model the disordered parts (see Table 3.1 for details). Face-indexed absorption corrections were performed numerically with the program X-RED. ${ }^{153}$ PLATON SQUEEZE $^{154}$ for strongly disordered lattice solvent molecules has been used in case of hs12120, hs281b, hs127, and hs127b. The contribution to the structure factors was calculated with PLATON SQUEEZE ${ }^{154}$ and the resulting fab file was processed with SHELXL using the ABIN instruction. The empirical formula and derived values are in accordance with the calculated cell content.

The nickel bound hydrogen atoms were refined freely in case of 3-K(crypt). The metal hydrogen bond lengths have to be considered with caution because of inherent ambiguities of $\mathrm{H}$ atom positions derived from X-ray crystallography. A potassium bound THF was found to be disordered about a center of inversion in $\mathbf{4 - K}$ and was refined at $1 / 2$ occupancy. Additionally, disordered lattice solvent THF molecules with occupancy factors of $0.523(15) / 0.477(15)$ in case of 3-K(crypt) and 0.512(7) / 0.488(7) in case of 4-K were observed. SAME, DELU and RIGU restraints were used to model the disordered parts. In case of 5-Na the sodium atoms together with the coordinated THF molecules are disordered over several positions as well as the two-fold axes of the $P 2 / c$ space group. In case of one sodium atom position the disorder could be modeled by using SAME restraints. The $\left[\mathrm{Na}(\mathrm{THF})_{3}\right]^{+}$cation was refined at $1 / 2$ occupancy. In case of a second sodium site no satisfactory model for a disorder could be found. The contribution to the structure factors was calculated with PLATON SQUEEZE ${ }^{154}$ and the resulting .fab file was processed with SHELXL using the ABIN instruction. The empirical formula and derived values are in accordance with the calculated cell content. Unfortunately, the charge is therefore not balanced in the present crystal structure. Several crystals from several crystallization batches have been tried but all of them showed the above described disorder. The anionic part, however, was not affected by the disorder. 
Table 3.1 Summary of disordered parts occupancy factors, and applied restraints and constraints.

\begin{tabular}{|c|c|c|c|c|}
\hline compound & disordered part & $\begin{array}{l}\text { occupancy } \\
\text { factors }\end{array}$ & restraints $\&$ constraints & remark \\
\hline \multirow{3}{*}{ [tp/pz $\left.z_{4} d e p y\right]$} & $\mathrm{CF}_{3} \mathrm{SO}_{3}^{-}$ & $\begin{array}{l}0.54(2) / \\
0.46(2)\end{array}$ & SAME, RIGU & 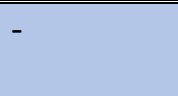 \\
\hline & $\mathrm{MeOH}$ & $\begin{array}{l}0.746(11) / \\
0.254(11)\end{array}$ & $\begin{array}{l}\operatorname{DFIX}(\mathrm{d}(\mathrm{C}-\mathrm{O})=1.41 \AA)) \text {; } \\
\operatorname{EADP}\end{array}$ & - \\
\hline & $\mathrm{MeOH}$ & $\begin{array}{l}0.655(7) / \\
0.345(7)\end{array}$ & $\operatorname{DFIX}(\mathrm{d}(\mathrm{C}-\mathrm{O})=1.41 \AA))$ & - \\
\hline$\left[\mathrm{tp} / \mathrm{pz} *{ }_{4}\right.$ Lut $]$ & $\mathrm{CF}_{3} \mathrm{SO}_{3}^{-}$ & $\begin{array}{l}0.730(5) / \\
0.270(5)\end{array}$ & SAME; RIGU; EADP & - \\
\hline \multirow[t]{2}{*}{$\mathrm{tp}^{\mathrm{Me}} / p z_{4}$ Lut] } & $\mathrm{MeOH}$ & 0.5 & $\begin{array}{l}\operatorname{DFIX}(d(C-O)=1.41 \AA)) \text {; } \\
\operatorname{RIGU}\end{array}$ & $\begin{array}{l}\text { disordered } \\
\text { about a } \\
\text { center of } \\
\text { inversion }\end{array}$ \\
\hline & $\mathrm{ClO}_{4}^{-}$ & $\begin{array}{l}0.875(9) / \\
0.125(9)\end{array}$ & $\begin{array}{l}\text { SADI }(d(C l-O), d(O \cdots O)) ; \\
\text { EADP }\end{array}$ & - \\
\hline \multirow{2}{*}[tp*/pz_{4}Lut]{} & $\mathrm{CF}_{3} \mathrm{SO}_{3}^{-}$ & $\begin{array}{l}0.760(4) / \\
0.240(4)\end{array}$ & SAME; RIGU & - \\
\hline & DMF & $\begin{array}{l}0.906(5) / \\
0.094(5)\end{array}$ & SIMU; RIGU & - \\
\hline [tp*/pz $z_{4}$ depy] & $\mathrm{CF}_{3} \mathrm{SO}_{3}^{-}$ & $\begin{array}{l}0.51(4) / \\
0.49(4)\end{array}$ & SAME; RIGU & - \\
\hline$\left[\mathrm{tp} * / p z *{ }_{4} L \mathrm{ut}\right]$ & $\mathrm{ClO}_{4}^{-}$ & $\begin{array}{l}0.892(5) / \\
0.108(5)\end{array}$ & SAME; RIGU & - \\
\hline \multirow[t]{2}{*}[\mathrm{tp}*/\mathrm{L}^{\mathrm{pz}}]{} & $\mathrm{PF}_{6}^{-} / \mathrm{CF}_{3} \mathrm{SO}_{3}^{-}$ & $0.5 / 0.5$ & $\begin{array}{l}\text { SADI }(d(P-F), d(F \cdots F), \\
d(O \cdots O)) ; \\
\text { DFIX }(d(S-O)=1.43 \AA \text {, } \\
d(S-C)=1.80 \AA, d(C-F)= \\
1.33 \AA)) ; R I G U\end{array}$ & $\begin{array}{l}\text { fixed } \\
\text { occupancy } \\
\text { factors }\end{array}$ \\
\hline & $\begin{array}{l}\mathrm{MeOH} / \mathrm{H}_{2} \mathrm{O} / \\
\mathrm{MeOH}\end{array}$ & $\begin{array}{l}0.5 / 0.25 / \\
0.25\end{array}$ & $\operatorname{DFIX}(\mathrm{d}(\mathrm{C}-\mathrm{O})=1.42 \AA))$ & $\begin{array}{l}\text { fixed } \\
\text { occupancy } \\
\text { factors }\end{array}$ \\
\hline
\end{tabular}




\subsection{Investigations on a Reversible C-H Activation Through a $\mathrm{Ni}(\mathrm{I}) \mathrm{Ni}(\mathrm{I})$ Intermediate and Investigations Towards Small Molecule Activation}

\subsubsection{Ligand Synthesis of $\mathbf{H}_{3} \mathrm{~L}^{\mathrm{Ph}}$}<smiles>NC(=O)c1cc(C(N)=O)[nH]n1</smiles><smiles>CC(C)C[C@H]1CCCCO1</smiles><smiles>N#Cc1cc(C#N)[nH]n1</smiles>

II

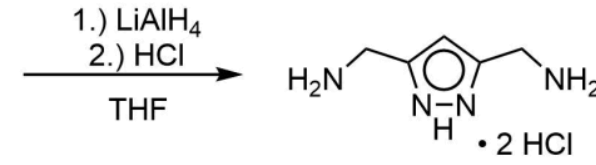

1.) $\left[\mathrm{Et}_{3} \mathrm{O}\right] \mathrm{BF}_{4}$<smiles>CC(=O)CC(C)(C)O</smiles>

IV<smiles>[CH]N(/C(C)=C\C(C)=O)c1c(P)cccc1-c1ccccc1</smiles>

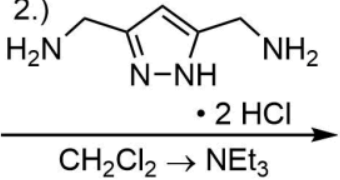

III

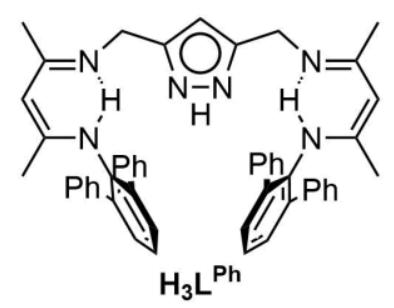

Scheme 3.1 Synthetic route for the Ligand $H_{3} L$.<smiles></smiles>

II

II was synthesized by a modified literature procedure ${ }^{32}$.

A suspension of $\mathbf{I}\left(20 \mathrm{~g}, 129.8 \mathrm{mmol}, 1 \mathrm{eq}\right.$.) in acetonitrile $(500 \mathrm{~mL})$ was cooled to $0^{\circ} \mathrm{C}$. One third of phosphoryl chloride $(20.2 \mathrm{~mL}, 214.0 \mathrm{mmol}, 1 \mathrm{eq}$.) was added dropwise. The resulting mixture was stirred for 5 hours at $75^{\circ} \mathrm{C}$. The brownish suspension was then cooled down again to $0^{\circ} \mathrm{C}$ and a second portion of phosphoryl chloride $(20.2 \mathrm{~mL}, 214.0 \mathrm{mmol}, 1$ eq.) was added before heating back to $75^{\circ} \mathrm{C}$. This procedure was then repeated with a third portion of phosphoryl chloride $(20.2 \mathrm{~mL}, 214.0 \mathrm{mmol}, 1$ eq.) after 18 hours of stirring at $75^{\circ} \mathrm{C}$. After the last addition and stirring for another 20 hours at $75^{\circ} \mathrm{C}$ the black suspension was poured carefully on ice water $(1 \mathrm{~L})$ and stirred for 18 hours at room temperature. The resulting dark purple solution was extracted with ethyl acetate $(3 \times 400 \mathrm{~mL})$, dried over magnesium sulphate and filtered. After removing of the solvent under vacuum the product was purified by column chromatography $\left(\mathrm{SiO}_{2}\right.$, Hexane / Ethyl acetate 7:3) as the second fraction. 1H-Pyrazole-dicarbonitrile II (9.71 g, $82.2 \mathrm{mmol}, 64 \%)$ was afforded as a slightly yellow solid.

${ }^{1} \mathrm{H}$ NMR $\left(\mathrm{DMSO}-\mathrm{d}_{6}, 300 \mathrm{MHz}\right) \delta=7.88\left(\mathrm{~s}, 1 \mathrm{H}, \mathrm{CH}^{\mathrm{pz}}\right) \mathrm{ppm}$. 


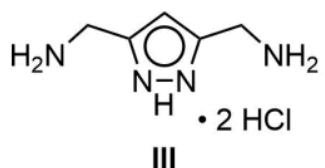

III was synthesized by a modified literature procedure ${ }^{32}$.

To a solution of II $(2.00 \mathrm{~g}, 16.8 \mathrm{mmol}, 1$ eq. $)$ in THF $(200 \mathrm{~mL})$ at $0^{\circ} \mathrm{C} \mathrm{LiAlH}_{4}$ in THF $(2.5$ M, $34 \mathrm{~mL}, 85.0 \mathrm{mmol}, 5$ eq.) was added. After warming up to $60^{\circ} \mathrm{C}$ a colorless precipitate formed. After stirring the suspension at $60^{\circ} \mathrm{C}$ for 18 hours, the suspension was cooled down to $0^{\circ} \mathrm{C}$ and water $(50 \mathrm{~mL})$ was carefully added. Solvents were removed under vacuum and the remaining solid was extracted with hot acetonitrile using a Sauxlett Apparatus for 3 days. After removal of the solvent the resulting yellow oil was dissolved in ethanol $(60 \mathrm{~mL})$ and $\mathrm{HCl}$ in $\mathrm{Et}_{2} \mathrm{O}(1.65 \mathrm{M}, 25 \mathrm{~mL}, 41.3 \mathrm{mmol}, 2.5$ eq.) was added to precipitate III as a white solid (3.06 g, $15.5 \mathrm{mmol}, 92 \%)$.

${ }^{1} \mathrm{H}$ NMR (DMSO-d $\left.6,300 \mathrm{MHz}\right) \delta=3.79\left(\mathrm{~d}, 4 \mathrm{H}, \mathrm{CH}_{2}\right), 6.26\left(\mathrm{~s}, 1 \mathrm{H}, \mathrm{CH}^{\mathrm{pz}}\right) \mathrm{ppm}$.

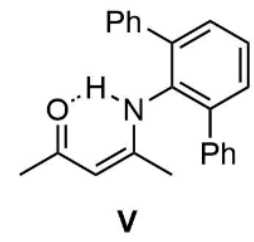

III was synthesized by a modified literature procedure. ${ }^{57}$

Diphenylaniline IV (15.00 g, $61.1 \mathrm{mmol}, 1$ eq.) was mixed with acetylacetone $(6.9 \mathrm{~mL}$, $6.73 \mathrm{~g}, 67.3 \mathrm{mmol}, 1.1 \mathrm{eq}$.$) and p$-Toluenesulfonic acid monohydrate $(0.43 \mathrm{~g}, 2.26 \mathrm{mmol}$, $3.7 \mathrm{~mol} \%)$ and dissolved in toluene $(250 \mathrm{~mL})$. The slightly yellow solution was stirred reflux for 14 hours using a Dean-Stark Apparatus. After cooling to room temperature, the solvent was removed and diethylether $(200 \mathrm{~mL})$ was added. The organic phase was then washed with water $(100 \mathrm{~mL})$ and the water phase was extracted with diethylether $(2 \times 50$ $\mathrm{mL}$ ). The combined organic phases were dried over sodium sulfate, filtered and the solvent was removed in vacuo. The crude product was recrystallized two times out of hexane and V was afforded as a beige crystalline solid (13.20 g, $40.4 \mathrm{mmol}, 66 \%)$.

${ }^{1} \mathrm{H}-\mathrm{NMR}\left(\mathrm{CDCl}_{3}, 400 \mathrm{MHz}\right) \delta=12.29(\mathrm{~s}, 1 \mathrm{H}, \mathrm{OH}), 7.45-7.38(\mathrm{~m}, 11 \mathrm{H}, \mathrm{Ar}), 7.34-7.30$ (m, 2H, Ar), $4.80(\mathrm{~s}, 1 \mathrm{H}, \mathrm{OCCHCN}), 1.94\left(\mathrm{~s}, 3 \mathrm{H}, \mathrm{HOCCH}_{3}\right), 1.32$ (s, 3H, NCCH$)$ ppm.

${ }^{13} \mathrm{C}$-NMR $\left(\mathrm{CDCl}_{3}, 101 \mathrm{MHz}\right) \delta=195.34(\mathrm{COH}), 161.63(\mathrm{CNR}), 140.56\left(\mathrm{C}^{\mathrm{Ar}}\right), 139.00$ $\left(\mathrm{C}^{\mathrm{Ar}}\right), 133.49\left(\mathrm{C}^{\mathrm{Ar}}\right), 129.99\left(\mathrm{C}^{\mathrm{Ar}}\right), 129.19\left(\mathrm{C}^{\mathrm{Ar}}\right), 128.26\left(\mathrm{C}^{\mathrm{Ar}}\right), 127.70\left(\mathrm{C}^{\mathrm{Ar}}\right), 127.76\left(\mathrm{C}^{\mathrm{Ar}}\right)$, $96.15(\mathrm{OCCHCN}), 28.74\left(\mathrm{HOCCH}_{3}\right), 19.24\left(\mathrm{NCCH}_{3}\right) \mathrm{ppm}$. 


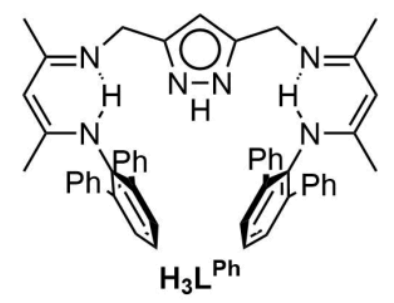

2-[(2,6-Diphenylphenyl)imido]-pent-2-en-4-one $\mathbf{V}(3.31 \mathrm{~g}, 10.10 \mathrm{mmol}, 2$ eq.) was dissolved in dry dichloromethane $(30 \mathrm{~mL})$ to give a slight yellow solution. To this solution triethyloxonium tetrafluoroborate $(3.07 \mathrm{~g}, 16.16 \mathrm{mmol}, 3.2 \mathrm{eq}$.) was added and stirred at room temperature for $19 \mathrm{~h}$. The pale-yellow solution was then dropwise added to a suspension of bis(3,5-aminomethyl)pyrazole hydrochloride III (1 g, $5.05 \mathrm{mmol}, 1.0$ eq.) in triethylamine $(10 \mathrm{~mL})$. The resulting suspension was stirred for $19 \mathrm{~h}$ at $40^{\circ} \mathrm{C}$. After removal of the solvent under reduced pressure, the crude solid was extracted multiple times with toluene $(2 \times 30 \mathrm{~mL})$. After another removal of the solvent the crude material was washed with Ethanol, cooled down to $-20^{\circ} \mathrm{C}$ and filtered. After multiple washings with pentane, $\mathbf{H}_{3} \mathbf{L}^{\mathbf{P h}}$ was obtained as a pale yellow solid (2.07 g, $\left.2.78 \mathrm{mmol}, 59 \%\right)$.

${ }^{1} \mathrm{H}-\mathrm{NMR}(400 \mathrm{MHz}, \mathrm{THF}-\mathrm{d} 8) \delta=10.79\left(\mathrm{~s}, 1 \mathrm{H}, \mathrm{NH}^{\text {nacnac }}\right), 7.38\left(\mathrm{~d}, J_{\mathrm{H}-\mathrm{H}}=8 \mathrm{~Hz}, 8 \mathrm{H}, \mathrm{Ph}\right)$, $7.24\left(\mathrm{~d}, J_{\mathrm{H}-\mathrm{H}}=8 \mathrm{~Hz}, 4 \mathrm{H}, \mathrm{Ph}\right), 7.19\left(\mathrm{~d}, J_{\mathrm{H}-\mathrm{H}}=8 \mathrm{~Hz}, 8 \mathrm{H}, \mathrm{Ph}\right), 7.12-7.07$ (m, 6H, Ph), 5.85 $\left(\mathrm{s}, 1 \mathrm{H}, \mathrm{CH}^{\mathrm{pz}}\right), 4.33\left(\mathrm{~s}, 2 \mathrm{H}, \mathrm{CHCNCH}_{3}\right), 4.22\left(\mathrm{~s}, 4 \mathrm{H}, \mathrm{CH}_{2} \mathrm{Pz}\right), 1.81\left(\mathrm{~s}, 6 \mathrm{H}, \mathrm{CH}_{2} \mathrm{~N}=\mathrm{CMe}\right)$. $1.23(\mathrm{~s}, 6 \mathrm{H}, \mathrm{ArN}=\mathrm{CMe}) \mathrm{ppm}$.

${ }^{13} \mathrm{C}-\mathrm{NMR}(101 \mathrm{MHz}, \mathrm{THF}) \delta=166.41(\mathrm{ArN}=\mathbf{C}), 156.41\left(\mathrm{CH}_{2} \mathrm{~N}=\mathbf{C}\right), 148.13\left(\mathrm{C}^{\mathrm{Ar}}\right), 147.70$ (3,5-Pz), $140.20\left(\mathrm{CH}^{\mathrm{Ar}}\right), 132.66\left(\mathrm{CH}^{\mathrm{Ar}}\right), 128.22\left(\mathrm{CH}^{\mathrm{Ar}}\right), 126.67\left(\mathrm{CH}^{\mathrm{Ar}}\right), 125.21\left(\mathrm{CH}^{\mathrm{Ar}}\right)$, $121.82\left(\mathrm{CH}^{\mathrm{Ar}}\right), 99.96\left(\mathrm{CH}^{\mathrm{Pz}}\right), 93.70\left(\mathrm{CHCNCH}_{3}\right), 38.84\left(\mathrm{CH}_{2}\right), 21.36\left(\mathrm{CH}_{3}\right), 18.07\left(\mathrm{CH}_{3}\right)$ ppm.

$\operatorname{MS}(\operatorname{ESI}(+), \mathrm{MeCN}) m / z$ : calcd. $[\mathrm{M}+\mathrm{H}]^{+} 745.40$ exp. $[\mathrm{M}+\mathrm{H}]^{+} 745.93$.

Anal. Calcd. for $\mathrm{C}_{51} \mathrm{H}_{48} \mathrm{~N}_{6}(744.39 \mathrm{~g} / \mathrm{mol})=\mathrm{C} 82.25, \mathrm{H} 6.57, \mathrm{~N} 11.17$; Found C 82.21, H 6.50, N 11.28. 


\subsubsection{Synthesis of Nickel(II) Complexes}

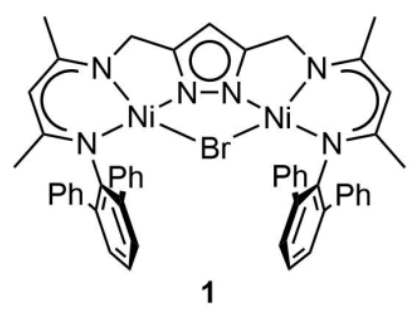

$\mathbf{H}_{3} \mathbf{L}^{\mathbf{P h}}$ (745 mg, $1.00 \mathrm{mmol}, 1.0$ eq.) was dissolved in THF (4 mL) and the clear yellow solution cooled to $-78^{\circ} \mathrm{C}$. $n$-Butyllithium $(2.6 \mathrm{M}$ in hexane, $1.2 \mathrm{~mL}, 3.2 \mathrm{mmol}, 3.2 \mathrm{eq}$.) was added slowly via a syringe to give a clear orange solution. Under stirring the reaction mixture was warmed to room temperature over 15 minutes to yield a dark red solution. (dme) $\mathrm{NiBr}_{2}$ (512 mg, $1.66 \mathrm{mmol}, 2.0$ eq.) was then added. The resulting dark-brown mixture was stirred overnight at $50^{\circ} \mathrm{C}$. The suspension was separated by a centrifuge and the THF phase discarded. The solid material was washed several times with acetone until the solvent remained colorless. After removing residual solvent under reduced pressure, 1 was obtained as a green fine powder. (525 mg, $0.56 \mathrm{mmol}, 56 \%$ ). Slow evaporation of a concentrated dichloromethane solution yielded cube-shaped crystals of $\mathbf{1}$, suitable for XRD analysis.

${ }^{1} \mathrm{H}-\mathrm{NMR}\left(400 \mathrm{MHz}, \mathrm{CD}_{2} \mathrm{Cl}_{2}\right) \delta=7.92\left(\mathrm{~d}, J_{\mathrm{H}-\mathrm{H}}=8 \mathrm{~Hz}, 8 \mathrm{H}, \mathrm{Ph}\right), 7.45-7.37(\mathrm{~m}, 12 \mathrm{H}, \mathrm{Ph})$, 7.09 (s, 6H, Ph), ), 5.46 (s, 1H, CH $\left.{ }^{\mathrm{pz}}\right), 4.49$ (s, 2H, $\left.\mathrm{CHCNCH}_{3}\right), 3.93$ (s, 4H, CH2 $\left.\mathrm{Pz}\right), 1.84$ $\left(\mathrm{s}, 6 \mathrm{H}, \mathrm{CH}_{2} \mathrm{~N}=\mathrm{CMe}\right) .1 .36(\mathrm{~s}, 6 \mathrm{H}, \mathrm{ArN}=\mathrm{CMe}) \mathrm{ppm}$.

${ }^{13} \mathrm{C}-\mathrm{NMR}(101 \mathrm{MHz}, \mathrm{THF}) \delta=160.82\left(\mathrm{CH}_{2} \mathrm{~N}=\mathbf{C}\right), 159.30(\mathrm{ArN}=\mathbf{C}), 154.02\left(\mathrm{C}^{\mathrm{pz}}\right), 148.58$ $\left(\mathrm{C}^{\mathrm{Ar}}\right), 141.73\left(\mathrm{C}^{\mathrm{Ar}}\right), 137.92\left(\mathrm{C}^{\mathrm{Ar}}\right), 131.02\left(\mathrm{CH}^{\mathrm{Ar}}\right), 130.51\left(\mathrm{CH}^{\mathrm{Ar}}\right), 128.16\left(\mathrm{CH}^{\mathrm{Ar}}\right), 127.16$ $\left(\mathrm{CH}^{\mathrm{Ar}}\right), 126.98\left(\mathrm{CH}^{\mathrm{Ar}}\right), 97.50\left(\mathrm{CHCNCH}_{3}\right), 92.39\left(\mathrm{CH}^{\mathrm{Pz}}\right), 54.99\left(\mathrm{CH}_{2}\right), 24.22\left(\mathrm{CH}_{3}{ }^{\mathrm{pz}}\right)$, $21.68\left(\mathrm{CH}_{3}{ }^{\mathrm{Ar}}\right)$ ppm..

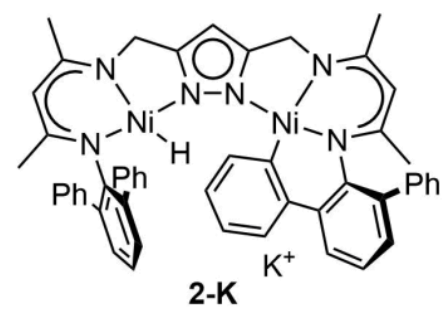

Method A: The green suspension of 1 (100 mg, $0.11 \mathrm{mmol}, 1$ eq.) in THF (2 mL) was treated with potassium triethyl borohydride in THF ( $1 \mathrm{M}, 0.3 \mathrm{~mL}, 0.3 \mathrm{mmol}, 3$ eq.). After stirring for 1 hour the suspension changed to a dark red color. The solution was filtered, and the solvent was removed under reduced pressure. The remaining solid was washed several times with hexane $(3 \times 10 \mathrm{~mL})$ and the remaining solvent was removed. Red tabular crystals of 2-K (69 mg, $0.07 \mathrm{mmol}, 64 \%$ ) were obtained by vapor diffusion of pentane into a prepared THF solution. 
Method B: The green suspension of 1 (100 mg, $0.11 \mathrm{mmol}, 1$ eq.) in THF (2 mL) was treated with potassium graphite $(32 \mathrm{mg}, 0.24 \mathrm{mmol}, 2.2 \mathrm{eq}$.). The reaction mixture was stirred for 6 hours at room temperature. After removal of the solvent, the crude product was washed with hexane ( $3 \times 4 \mathrm{~mL})$ and dried in vacuo. Crystallization by layering a THF solution with hexane, resulted in red tabular crystals of 2-K (71 mg, $0.08 \mathrm{mmol}, 72 \%)$.

${ }^{1} \mathrm{H}-\mathrm{NMR}\left(400 \mathrm{MHz}, \mathrm{THF}-\mathrm{d}_{8}\right) \delta=8.55\left(\mathrm{~d}, 2 \mathrm{H}, J_{\mathrm{H}-\mathrm{H}}=8 \mathrm{~Hz}, \mathrm{Ar}\right), 7.75\left(\mathrm{~d}, 2 \mathrm{H}, J_{\mathrm{H}-\mathrm{H}}=8 \mathrm{~Hz}\right.$, $\operatorname{Ar}), 7.67\left(\mathrm{~d}, 1 \mathrm{H}, J_{\mathrm{H}-\mathrm{H}}=8 \mathrm{~Hz}, \operatorname{Ar}\right), 7.47\left(\mathrm{~d}, 2 \mathrm{H}, J_{\mathrm{H}-\mathrm{H}}=8 \mathrm{~Hz}, \mathrm{Ar}\right), 7.32-6.96(\mathrm{~m}, 16 \mathrm{H}, \mathrm{Ar})$, $6.37\left(\mathrm{t}, 1 \mathrm{H}, J_{\mathrm{H}-\mathrm{H}}=8 \mathrm{~Hz}, \mathrm{Ar}\right), 6.29\left(\mathrm{t}, 2 \mathrm{H}, J_{\mathrm{H}-\mathrm{H}}=8 \mathrm{~Hz}, \mathrm{Ar}\right), 5.70\left(\mathrm{~s}, 1 \mathrm{H}, \mathrm{CH}^{\mathrm{pz}}\right), 4.48(\mathrm{~s}, 1 \mathrm{H}$, $\left.\mathrm{CHCNCH}_{3}\right), 4.43\left(\mathrm{~d}, J_{\mathrm{H}-\mathrm{H}}=24 \mathrm{~Hz}, 1 \mathrm{H}, \mathrm{CH}_{2} \mathrm{Pz}\right), 4.24\left(\mathrm{~s}, 1 \mathrm{H}, \mathrm{CHCNCH}_{3}\right), 4.21\left(\mathrm{~d}, J_{\mathrm{H}-\mathrm{H}}=\right.$ $24 \mathrm{~Hz}, 1 \mathrm{H}, \mathrm{CH}_{2} \mathrm{Pz}$ ), $4.06\left(\mathrm{~d}, J_{\mathrm{H}-\mathrm{H}}=24 \mathrm{~Hz}, 1 \mathrm{H}, \mathrm{CH}_{2} \mathrm{Pz}\right), 3.99$ (d, , $\left.J_{\mathrm{H}-\mathrm{H}}=24 \mathrm{~Hz}, 1 \mathrm{H}, \mathrm{CH}_{2} \mathrm{Pz}\right)$, 1.92 (s, 3H, Me), 1.05 (s, 6H, 2xllMe), -26.12 (s, 1H, Ni-H) ppm.

${ }^{13} \mathrm{C}-\mathrm{NMR}\left(101 \mathrm{MHz}, \mathrm{THF}-\mathrm{d}_{8}\right) \delta=160.72\left(\mathrm{C}^{\mathrm{q}}-\mathrm{Me}\right), 159.57\left(\mathrm{C}^{\mathrm{pz}}\right) 159.34\left(\mathrm{C}^{\mathrm{q}}-\mathrm{Me}\right), 158.90$ $\left(\mathrm{C}^{\mathrm{q}}-\mathrm{Me}\right), 157.14\left(\mathrm{C}^{\mathrm{q}}-\mathrm{Me}\right), 157.04\left(\mathrm{C}^{\mathrm{pz}}\right), 156.46\left(\mathrm{C}^{\mathrm{Ar}}\right), 154.69\left(\mathrm{C}^{\mathrm{Ar}}\right), 143.26\left(\mathrm{C}^{\mathrm{Ar}}\right), 143.21$ $\left(\mathrm{C}^{\mathrm{Ar}}\right), 142.97\left(\mathrm{C}^{\mathrm{Ar}}\right), 142.20\left(\mathrm{C}^{\mathrm{Ar}}\right), 141.75\left(\mathrm{C}^{\mathrm{Ar}}\right), 140.07\left(\mathrm{C}^{\mathrm{Ar}}\right), 137.31\left(\mathrm{C}^{\mathrm{Ar}}\right), 136.85\left(\mathrm{C}^{\mathrm{Ar}}\right)$, $135.87\left(\mathrm{C}^{\mathrm{Ar}}\right), 132.46\left(\mathrm{C}^{\mathrm{Ar}}\right), 131.19\left(\mathrm{C}^{\mathrm{Ar}}\right), 131.05\left(\mathrm{C}^{\mathrm{Ar}}\right), 130.14\left(\mathrm{C}^{\mathrm{Ar}}\right), 130.09\left(\mathrm{C}^{\mathrm{Ar}}\right), 129.88$ $\left(\mathrm{C}^{\mathrm{Ar}}\right), 128.80\left(\mathrm{C}^{\mathrm{Ar}}\right), 128.58\left(\mathrm{C}^{\mathrm{Ar}}\right), 128.38\left(\mathrm{C}^{\mathrm{Ar}}\right), 128.26\left(\mathrm{C}^{\mathrm{Ar}}\right), 127.78\left(\mathrm{C}^{\mathrm{Ar}}\right), 126.84\left(\mathrm{C}^{\mathrm{Ar}}\right)$, $126.74\left(\mathrm{C}^{\mathrm{Ar}}\right), 126.43\left(\mathrm{C}^{\mathrm{Ar}}\right), 126.29\left(\mathrm{C}^{\mathrm{Ar}}\right), 125.70\left(\mathrm{C}^{\mathrm{Ar}}\right), 125.37\left(\mathrm{C}^{\mathrm{Ar}}\right), 124.33\left(\mathrm{C}^{\mathrm{Ar}}\right), 123.45$ $\left(\mathrm{C}^{\mathrm{Ar}}\right), 123.02\left(\mathrm{C}^{\mathrm{Ar}}\right), 120.88\left(\mathrm{C}^{\mathrm{Ar}}\right), 103.80\left(\mathbf{C H C N C H}_{3}\right), 97.74\left(\mathbf{C H C N C H}_{3}\right), 92.97\left(\mathrm{CH}^{\mathrm{pz}}\right)$, $52.65\left(\mathrm{CH}_{2}\right), 52.22\left(\mathrm{CH}_{2}\right), 23.49(\mathrm{Me}), 23.19(\mathrm{Me}), 20.98(\mathrm{Me}), 20.21\left(\mathrm{CH}_{3}\right) \mathrm{ppm}$.

IR (ATR) $v / \mathrm{cm}^{-1}=3051(\mathrm{w}), 3023(\mathrm{w}), 2984(\mathrm{w}), 2908(\mathrm{w}), 2858(\mathrm{w}), 2821(\mathrm{w}), 1561(\mathrm{~m})$, 1511 (s), 1422 (s), 1387 (vs), 1306 (m), 1267 (s), 1248 (s), 1204 (s), 1156 (m), 1091 (m), 1068 (m), 1022 (s), 996 (m), 906 (m), 853 (m), 799 (m), 745 (vs), 692 (vs), 605 (s), 541 (s)

EA analysis: Anal. Calcd. for 2-K $\cdot$ THF $\mathrm{C}_{55} \mathrm{H}_{53} \mathrm{KN}_{6} \mathrm{Ni}_{2} \mathrm{O}(970.50 \mathrm{~g} / \mathrm{mol})=\mathrm{C} 68.06$, H 5.50, N 8.66; Found C 67.97, H 5.47, N 8.69.

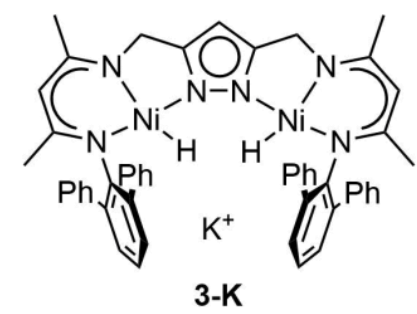

2-K was dissolved in minimal amounts of THF. After degassing the dark red solution three times, an atmosphere of dry dihydrogen was applied ( $\sim 1 \mathrm{~atm}$.). Crystallization attempts of $\mathbf{3 - K}$ even in a dihydrogen saturated solution always yielded a mixture of $\mathbf{2}-\mathbf{K}$ and $\mathbf{3}-\mathbf{K}$.

${ }^{1} \mathrm{H}-\mathrm{NMR}(400 \mathrm{MHz}, \mathrm{THF}-\mathrm{d} 8) \delta=7.78\left(\mathrm{~d}, 8 \mathrm{H}, J_{\mathrm{H}-\mathrm{H}}=8 \mathrm{~Hz}, \mathrm{Ar}\right), 7.20-7.19$ (m, 14H, Ar), $7.07\left(\mathrm{t}, 4 \mathrm{H}, J_{\mathrm{H}-\mathrm{H}}=8 \mathrm{~Hz}, \mathrm{Ar}\right), 5.42\left(\mathrm{~s}, 1 \mathrm{H}, \mathrm{CH}^{\mathrm{pz}}\right), 4.24\left(\mathrm{~s}, 2 \mathrm{H}, \mathrm{CHCNCH}_{3}\right), 4.04(\mathrm{~s}, 4 \mathrm{H}$, $\mathrm{CH}_{2} \mathrm{Pz}$ ), 1.65 (s, 6H, Me), 1.30 (s, 6H, Me), -23.80 (s, 2H, Ni-H) ppm. 
${ }^{13} \mathrm{C}-\mathrm{NMR}(101 \mathrm{MHz}, \mathrm{THF}-\mathrm{d} 8) \delta=158.86\left(\mathrm{C}^{\mathrm{q}}-\mathrm{Me}\right), 157.18\left(\mathrm{C}^{\mathrm{Ar}}\right), 156.98\left(\mathrm{C}^{\mathrm{q}}-\mathrm{Me}\right), 156.64$ $\left(\mathrm{C}^{\mathrm{pz}}\right), 143.79\left(\mathrm{C}^{\mathrm{Ar}}\right), 136.71\left(\mathrm{C}^{\mathrm{Ar}}\right), 131.42\left(\mathrm{C}^{\mathrm{Ar}}\right), 130.99\left(\mathrm{C}^{\mathrm{Ar}}\right), 130.15\left(\mathrm{C}^{\mathrm{Ar}}\right), 128.26\left(\mathrm{C}^{\mathrm{Ar}}\right)$, $128.21\left(\mathrm{C}^{\mathrm{Ar}}\right), 126.31\left(\mathrm{C}^{\mathrm{Ar}}\right), 125.71\left(\mathrm{C}^{\mathrm{Ar}}\right), 124.12\left(\mathrm{C}^{\mathrm{Ar}}\right), 97.82\left(\mathrm{CHCNCH}_{3}\right),, 92.31\left(\mathrm{CH}^{\mathrm{Pz}}\right)$, $53.42\left(\mathrm{CH}_{2}\right), 23.54\left(\mathrm{CH}_{3}\right), 20.61\left(\mathrm{CH}_{3}\right) \mathrm{ppm}$.

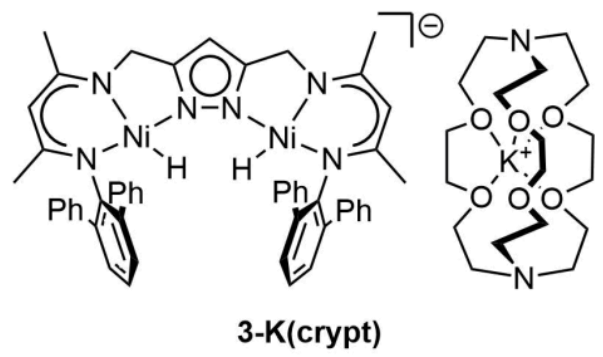

2-K (5.0 mg, $5.6 \mu \mathrm{mol}, 1$ eq.) was dissolved in minimal amounts of THF-d8 $(0.5 \mathrm{~mL})$. [2.2.2]cryptand $(2.1 \mathrm{mg}, 5.6 \mu \mathrm{mol}, 1$ eq.) was added as a solid and the resulting solution was stirred for 5 mins. After degassing the dark red solution three times, an atmosphere of dry dihydrogen was applied ( $\sim 1 \mathrm{~atm}$.). The complex 3-K(crypt) was used in situ for further NMR investigations, due to the instability in solution over a longer period of time. Crystallization was facilitated through cooling of the solution to $-30^{\circ} \mathrm{C}$.
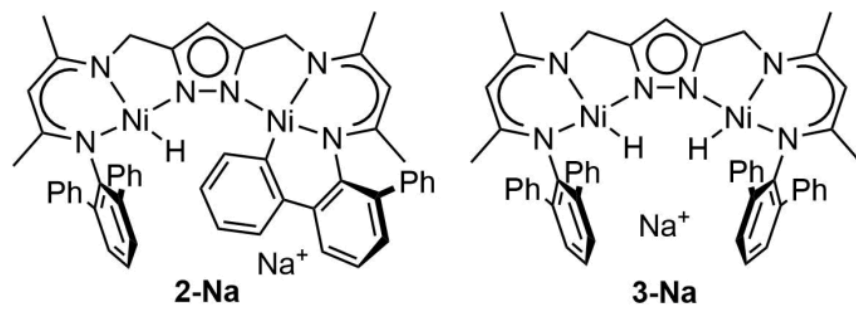

The green suspension of 1 (100 mg, $0.11 \mathrm{mmol}, 1$ eq.) in THF $(2 \mathrm{~mL})$ was treated with sodium triethylborohydride in THF ( $1 \mathrm{M}, 0.3 \mathrm{~mL}, 0.3 \mathrm{mmol}, 3$ eq.). After stirring for 1 hour the suspension changed to a dark red color. The solution was filtered, and the solvent was removed under reduced pressure. The remaining solid was washed several times with hexane $(3 \times 10 \mathrm{~mL})$ and the residual solvent was removed. Red tabular crystal mixture of 2-Na and 3-Na were obtained by vapor diffusion of pentane into a THF solution.

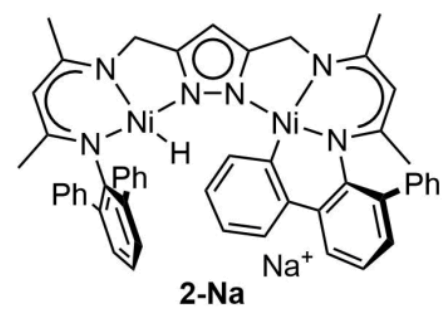

Alternative Synthesis for 2-Na:

The green suspension of 1 (200 mg, $0.21 \mathrm{mmol}, 1$ eq.) in THF $(4 \mathrm{~mL})$ was treated with a freshly prepared $1 \mathrm{M}$ solution of sodium naphtalide in THF $(0.5 \mathrm{~mL}, 0.50 \mathrm{mmol}, 2.4$ eq. $)$. 
After 2 hours, the red solution was filtered, and the solvent was evaporated. After washing with hexane (3 x $5 \mathrm{~mL})$ 2-Na was isolated as a red solid (181 mg, $0.20 \mathrm{mmol}, 95 \%)$.

${ }^{1} \mathrm{H}-\mathrm{NMR}(400 \mathrm{MHz}, \mathrm{THF}-\mathrm{d} 8) \delta=8.58\left(\mathrm{~d},, J_{\mathrm{H}-\mathrm{H}}=8 \mathrm{~Hz}, 2 \mathrm{H}, \mathrm{Ar}\right), 7.85\left(\mathrm{~d}, J_{\mathrm{H}-\mathrm{H}}=8 \mathrm{~Hz} 1 \mathrm{H}\right.$, $\operatorname{Ar}), 7.49\left(\mathrm{~d}, J_{\mathrm{H}-\mathrm{H}}=8 \mathrm{~Hz}, 2 \mathrm{H}, \mathrm{Ar}\right), 7.43\left(\mathrm{~d}, J_{\mathrm{H}-\mathrm{H}}=8 \mathrm{~Hz}, 1 \mathrm{H}, \mathrm{Ar}\right), 7.16-7.07$ (m, 17H, Ar), $6.38\left(\mathrm{t},, J_{\mathrm{H}-\mathrm{H}}=8 \mathrm{~Hz}, 1 \mathrm{H}, \mathrm{Ar}\right), 6.27\left(\mathrm{t}, J_{\mathrm{H}-\mathrm{H}}=8 \mathrm{~Hz}, 1 \mathrm{H}, \mathrm{Ar}\right), 5.69\left(\mathrm{~s}, 1 \mathrm{H}, \mathrm{CH}^{\mathrm{pz}}\right), 4.49(\mathrm{~s}$, $\left.1 \mathrm{H}, \mathrm{CHCNCH}_{3}\right), 4.41\left(\mathrm{~d}, J_{\mathrm{H}-\mathrm{H}}=18 \mathrm{~Hz}, 1 \mathrm{H}, \mathrm{CH}_{2} \mathrm{Pz}\right), 4.32\left(\mathrm{~s}, 1 \mathrm{H}, \mathrm{CHCNCH}_{3}\right), 4.18-3.94$ (m, 3H, $\mathrm{CH}_{2} \mathrm{Pz}$ ), 1.92 (s, 3H, Me), 1.74 (s, 3H, Me), 1.23 (s, 3H, Me), 1.06 (s, 3H, Me), 26.12 (s, 1H, Ni-H) ppm.

${ }^{13} \mathrm{C}-\mathrm{NMR}\left(75 \mathrm{MHz}, \mathrm{THF}-\mathrm{d}_{8}\right) \delta=160.59$ (C $\left.\mathrm{C}^{\mathrm{q}}-\mathrm{Me}\right), 160.16\left(\mathrm{C}^{\mathrm{q}}-\mathrm{Me}\right) 159.43\left(\mathrm{C}^{\mathrm{q}}-\mathrm{Me}\right), 158.89$ $\left(\mathrm{C}^{\mathrm{q}}-\mathrm{Me}\right), 157.96\left(\mathrm{C}^{\mathrm{Ar}}\right), 157.72\left(\mathrm{C}^{\mathrm{Ar}}\right), 157.71\left(\mathrm{C}^{\mathrm{Ar}}\right), 155.84\left(\mathrm{C}^{\mathrm{Ar}}\right), 155.82\left(\mathrm{C}^{\mathrm{Ar}}\right), 151.30\left(\mathrm{C}^{\mathrm{Ar}}\right)$, $144.17\left(\mathrm{C}^{\mathrm{Ar}}\right), 143.56\left(\mathrm{C}^{\mathrm{Ar}}\right), 143.53\left(\mathrm{C}^{\mathrm{Ar}}\right), 142.91\left(\mathrm{C}^{\mathrm{Ar}}\right), 142.61\left(\mathrm{C}^{\mathrm{Ar}}\right), 139.20\left(\mathrm{C}^{\mathrm{Ar}}\right), 137.77$ $\left(\mathrm{C}^{\mathrm{Ar}}\right), 137.02\left(\mathrm{C}^{\mathrm{Ar}}\right), 136.66\left(\mathrm{C}^{\mathrm{Ar}}\right), 134.04\left(\mathrm{C}^{\mathrm{Ar}}\right), 131.83\left(\mathrm{C}^{\mathrm{Ar}}\right), 130.81\left(\mathrm{C}^{\mathrm{Ar}}\right), 130.24\left(\mathrm{C}^{\mathrm{Ar}}\right)$, $130.15\left(\mathrm{C}^{\mathrm{Ar}}\right), 128.93\left(\mathrm{C}^{\mathrm{Ar}}\right), 128.58\left(\mathrm{C}^{\mathrm{Ar}}\right), 128.30\left(\mathrm{C}^{\mathrm{Ar}}\right), 128.01\left(\mathrm{C}^{\mathrm{Ar}}\right), 127.42\left(\mathrm{C}^{\mathrm{Ar}}\right), 126.58$ $\left(\mathrm{C}^{\mathrm{Ar}}\right), 126.54\left(\mathrm{C}^{\mathrm{Ar}}\right), 126.33\left(\mathrm{C}^{\mathrm{Ar}}\right), 125.02\left(\mathrm{C}^{\mathrm{Ar}}\right), 124.39\left(\mathrm{C}^{\mathrm{Ar}}\right), 124.29\left(\mathrm{C}^{\mathrm{Ar}}\right), 123.32\left(\mathrm{C}^{\mathrm{Ar}}\right)$, $120.85\left(\mathrm{C}^{\mathrm{Ar}}\right), 103.90\left(\mathrm{CHCNCH}_{3}\right), 98.01\left(\mathrm{CHCNCH}_{3}\right), 93.55\left(\mathrm{CH}^{\mathrm{pz}}\right), 52.41\left(\mathrm{CH}_{2}\right), 52.07$ $\left(\mathrm{CH}_{2}\right), 23.72(\mathrm{Me}), 23.10(\mathrm{Me}), 21.02(\mathrm{Me}), 20.03(\mathrm{Me}) \mathrm{ppm}$.

IR (ATR) $v / \mathrm{cm}^{-1}=3049(\mathrm{w}), 2952(\mathrm{w}), 2918(\mathrm{w}), 2858(\mathrm{w}), 1897(\mathrm{w}), 1557(\mathrm{~m}), 1519(\mathrm{~s})$, 1496 (m), 1450 (m), 1425 (s), 1386 (vs), 1355 (m), 1314 (m), 1274 (s), 1250 (m), 1208 (m), $1181(\mathrm{w}), 1156(\mathrm{w}), 1107$ (w), $1090(\mathrm{w}), 1073(\mathrm{w}), 1046(\mathrm{w}), 1021(\mathrm{~m}), 965(\mathrm{w}), 916$ (w), $869(\mathrm{w}), 803(\mathrm{w})$.

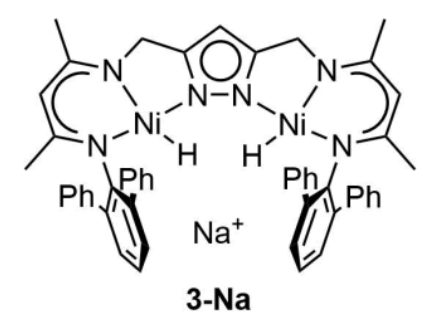

The mixture of 2-Na and 3-Na after washing with hexane was dissolved in a minimal amount of THF. The red solution was degassed three times before dry dihydrogen gas ( $\sim 1 \mathrm{~atm}$.) was added. Needle-shaped crystals of 3-Na for XRD analysis were obtained after layering of diethyl ether on top of the dihydrogen saturated THF solution. Crystals of 3-Na were only stable under $\mathrm{a}_{2}$ atmosphere over a long period of time.

${ }^{1} \mathrm{H}-\mathrm{NMR}\left(400 \mathrm{MHz}, \mathrm{THF}-\mathrm{d}_{8}\right) \delta=7.71-7.69\left(\mathrm{~d}, 8 \mathrm{H}, J_{\mathrm{H}-\mathrm{H}}=8 \mathrm{~Hz}, \mathrm{Ar}\right), 7.23-7.19(\mathrm{~m}, 14 \mathrm{H}$, Ar), $7.11\left(\mathrm{t}, 4 \mathrm{H}, J_{\mathrm{H}-\mathrm{H}}=8 \mathrm{~Hz}, \mathrm{Ar}\right), 5.46\left(\mathrm{~s}, 1 \mathrm{H}, \mathrm{CH}^{\mathrm{pz}}\right), 4.29\left(\mathrm{~s}, 2 \mathrm{H}, \mathrm{CHCNCH}_{3}\right), 4.06(\mathrm{~s}, 4 \mathrm{H}$, $\mathrm{CH}_{2} \mathrm{Pz}$ ), 1.68 (s, 6H, Me). 1.34 (s, 6H, Me), -23.61 (s, 2H, Ni-H) ppm.

${ }^{13} \mathrm{C}-\mathrm{NMR}\left(101 \mathrm{MHz}, \mathrm{THF}-\mathrm{d}_{8}\right) \delta=159.25\left(\mathrm{C}^{\mathrm{q}}-\mathrm{Me}\right), 157.38\left(\mathrm{C}^{\mathrm{pz}}\right), 157.35\left(\mathrm{C}^{\mathrm{q}}-\mathrm{Me}\right), 157.20$ $\left(\mathrm{C}^{\mathrm{Ar}}\right), 143.69\left(\mathrm{C}^{\mathrm{Ar}}\right), 131.42\left(\mathrm{C}^{\mathrm{Ar}}\right), 131.38\left(\mathrm{C}^{\mathrm{Ar}}\right), 131.04\left(\mathrm{C}^{\mathrm{Ar}}\right), 128.41\left(\mathrm{C}^{\mathrm{Ar}}\right), 128.18\left(\mathrm{C}^{\mathrm{Ar}}\right)$, 
$126.63\left(\mathrm{C}^{\mathrm{Ar}}\right), 124.37\left(\mathrm{C}^{\mathrm{Ar}}\right), 98.04\left(\mathrm{CHCNCH}_{3}\right), 92.94\left(\mathrm{CH}^{\mathrm{Pz}}\right), 53.48\left(\mathrm{CH}_{2}\right), 23.51\left(\mathrm{CH}_{3}\right)$, $20.64\left(\mathrm{CH}_{3}\right) \mathrm{ppm}$.

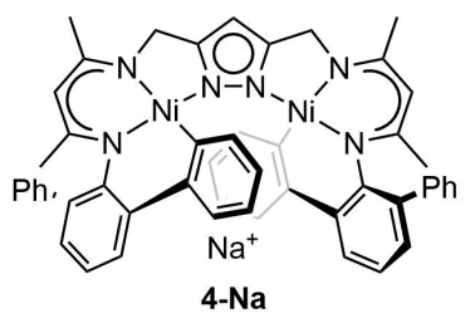

The mixture of 2-Na and 3-Na was dissolved in minimal amount of THF. The red solution was heated for at least three days at $60^{\circ} \mathrm{C}$ in a closed vessel. After cooling down, dark red needles of 4-Na were obtained by layering hexane on top of the THF solution.

${ }^{1} \mathrm{H}-\mathrm{NMR}(400 \mathrm{MHz}, \mathrm{THF}-\mathrm{d} 8) \delta=7.76\left(\mathrm{~d}, 4 \mathrm{H}, J_{\mathrm{H}-\mathrm{H}}=8 \mathrm{~Hz} \mathrm{Ar}\right), 7.51\left(\mathrm{~d}, 2 \mathrm{H}, J_{\mathrm{H}-\mathrm{H}}=8 \mathrm{~Hz}\right.$, Ar), $7.30\left(\mathrm{~d}, 2 \mathrm{H}, J_{\mathrm{H}-\mathrm{H}}=8 \mathrm{~Hz}, \mathrm{Ar}\right), 7.09\left(\mathrm{t}, 2 \mathrm{H}, J_{\mathrm{H}-\mathrm{H}}=8 \mathrm{~Hz}, \mathrm{Ar}\right), 6.97-6.87(\mathrm{~m}, 4 \mathrm{H}, \mathrm{Ar})$, $6.69\left(\mathrm{~d}, 2 \mathrm{H}, J_{\mathrm{H}-\mathrm{H}}=8 \mathrm{~Hz}, \mathrm{Ar}\right), 6.29\left(\mathrm{t}, 2 \mathrm{H}, J_{\mathrm{H}-\mathrm{H}}=8 \mathrm{~Hz}, \mathrm{Ar}\right), 5.94\left(\mathrm{t}, 2 \mathrm{H}, J_{\mathrm{H}-\mathrm{H}}=8 \mathrm{~Hz}, \mathrm{Ar}\right)$, $5.70\left(\mathrm{~s}, 1 \mathrm{H}, \mathrm{CH}^{\mathrm{pz}}\right), 4.30\left(\mathrm{~d}, 2 \mathrm{H}, J_{\mathrm{H}-\mathrm{H}}=16 \mathrm{~Hz} \mathrm{CH}_{2} \mathrm{Pz}\right), 4.23\left(\mathrm{~s}, 2 \mathrm{H}, \mathrm{CHCNCH}_{3}\right), 4.12$ (d, $2 \mathrm{H}, J_{\mathrm{H}-\mathrm{H}}=16 \mathrm{~Hz} \mathrm{CH} 2 \mathrm{Pz}$ ), 1.81 (s, 6H, Me). 1.02 (s, 6H, Me) ppm.

${ }^{13} \mathrm{C}-\mathrm{NMR}(101 \mathrm{MHz}, \mathrm{THF}-\mathrm{d} 8) \delta=158.69\left(\mathrm{C}^{\mathrm{q}}-\mathrm{Me}\right), 158.39\left(\mathrm{C}^{\mathrm{q}}-\mathrm{Me}\right), 156.54\left(\mathrm{C}^{\mathrm{Ar}}\right), 156.27$ $\left(\mathrm{C}^{\mathrm{pz}}\right), 144.94\left(\mathrm{C}^{\mathrm{Ar}}\right), 143.55\left(\mathrm{C}^{\mathrm{Ar}}\right), 140.00\left(\mathrm{C}^{\mathrm{Ar}}\right), 139.07\left(\mathrm{C}^{\mathrm{Ar}}\right), 137.47\left(\mathrm{Ni}-\mathrm{C}^{\mathrm{Ar}}\right), 135.39\left(\mathrm{C}^{\mathrm{Ar}}\right)$, $130.49\left(\mathrm{C}^{\mathrm{Ar}}\right), 127.81\left(\mathrm{C}^{\mathrm{Ar}}\right), 126.65\left(\mathrm{C}^{\mathrm{Ar}}\right), 126.58\left(\mathrm{C}^{\mathrm{Ar}}\right), 125.04\left(\mathrm{C}^{\mathrm{Ar}}\right), 122.68\left(\mathrm{C}^{\mathrm{Ar}}\right), 121.66$ $\left(\mathrm{C}^{\mathrm{Ar}}\right), 120.85\left(\mathrm{C}^{\mathrm{Ar}}\right), 120.38\left(\mathrm{C}^{\mathrm{Ar}}\right), 101.84\left(\mathrm{CHCNCH}_{3}\right), 91.05\left(\mathrm{CH}^{\mathrm{Pz}}\right), 52.69\left(\mathrm{CH}_{2}\right), 123.72$ $\left(\mathrm{CH}_{3}\right), 24.14\left(\mathrm{CH}_{3}\right), 20.56\left(\mathrm{CH}_{3}\right) \mathrm{ppm}$.

IR (ATR) $v / \mathrm{cm}^{-1}=3050(\mathrm{vw}), 3025(\mathrm{vw}) 2973(\mathrm{vw}), 2912(\mathrm{vw}), 2863(\mathrm{vw}), 1560(\mathrm{~m}), 1519$ (s), 1493 (m), 1446 (m), 1426 (s), 1386 (vs), 1313 (m), 1274 (s), 1212 (m), 1091 (m), 1045 (m), 1023 (s), $915(\mathrm{w}), 893(\mathrm{w}), 834(\mathrm{w}), 802(\mathrm{w}), 741(\mathrm{vs}), 721$ (s), $698(\mathrm{vs}), 639(\mathrm{~m}), 601$ (s), $542(\mathrm{~m}), 480(\mathrm{~m}), 457(\mathrm{~m}), 417(\mathrm{w})$.

\subsubsection{Substrate Activations of 2-M}

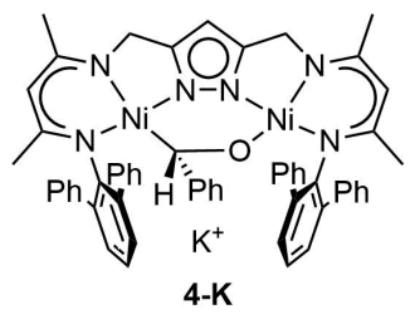

2-K (5.7 $\mu \mathrm{mol}, 1$ eq. $)$ was dissolved in THF $(0.5 \mathrm{~mL})$ and benzaldehyde $(0.59 \mu \mathrm{L}, 0.61 \mathrm{mg}$, $5.7 \mu \mathrm{mol}, 1$ eq.) was added. After stirring for $10 \mathrm{mins}$ at room temperature the brown solution was filtered. Crystals suitable for X-Ray diffraction analysis of 4-K (0.5 mg, $0.5 \mu \mathrm{mol}, 9 \%$ ) were produced by slow diffusion of pentane into a THF-solution. 


\section{4-K:}

${ }^{1} \mathrm{H}-\mathrm{NMR}\left(400 \mathrm{MHz}, \mathrm{C}_{6} \mathrm{D}_{6}\right) \delta=8.83\left(\mathrm{~d}, 2 \mathrm{H}, J_{\mathrm{H}-\mathrm{H}}=8 \mathrm{~Hz} \mathrm{Ar}\right), 8.03\left(\mathrm{~d}, 2 \mathrm{H}, J_{\mathrm{H}-\mathrm{H}}=8 \mathrm{~Hz}, \mathrm{Ar}\right)$, $7.71\left(\mathrm{~d}, 2 \mathrm{H}, J_{\mathrm{H}-\mathrm{H}}=4 \mathrm{~Hz}, \mathrm{Ar}\right), 7.54\left(\mathrm{~d}, 2 \mathrm{H}, J_{\mathrm{H}-\mathrm{H}}=8 \mathrm{~Hz}, \mathrm{Ar}\right), 7.50\left(\mathrm{~d}, 1 \mathrm{H}, J_{\mathrm{H}-\mathrm{H}}=8 \mathrm{~Hz}, \mathrm{Ar}\right)$ $7.31-7.21(\mathrm{~m}, 6 \mathrm{H}, \mathrm{Ar}), 7.13-7.05(\mathrm{~m}, 6 \mathrm{H}, \mathrm{Ar}) 6.97-6.87$ (m, 6H, Ar), $6.78-6.69(\mathrm{~m}$, $4 \mathrm{H}, \mathrm{Ar}), 5.83\left(\mathrm{~s}, 1 \mathrm{H}, \mathrm{CH}^{\mathrm{pz}}\right), 4.61\left(\mathrm{~s}, 1 \mathrm{H}, \mathrm{CHCNCH}_{3}\right), 4.36\left(\mathrm{~s}, 1 \mathrm{H}, \mathrm{CHCNCH}_{3}\right), 4.13(\mathrm{~d}$, $\left.1 \mathrm{H}, J_{\mathrm{H}-\mathrm{H}}=16 \mathrm{~Hz}, \mathrm{CH}_{2} \mathrm{Pz}\right), 4.03\left(\mathrm{~d}, 1 \mathrm{H}, J_{\mathrm{H}-\mathrm{H}}=16 \mathrm{~Hz}, \mathrm{CH}_{2} \mathrm{Pz}\right), 3.68\left(\mathrm{~s}, 2 \mathrm{H}, \mathrm{CH}_{2} \mathrm{Pz}\right), 2.03(\mathrm{~s}$, 1H, PhCHO), 1.61 (s, 3H, Me), $1.56(\mathrm{~s}, 3 \mathrm{H}, \mathrm{Me}), 1.36$ (s, 3H, Me), 1.22 (s, 3H, Me) ppm.

${ }^{13} \mathrm{C}-\mathrm{NMR}\left(101 \mathrm{MHz}, \mathrm{C}_{6} \mathrm{D}_{6}\right) \delta=159.92\left(\mathrm{C}^{\mathrm{q}}-\mathrm{Me}\right), 159.62\left(\mathrm{C}^{\mathrm{q}}-\mathrm{Me}\right), 158.68\left(\mathrm{C}^{\mathrm{q}}-\mathrm{Me}\right), 157.93$ $\left(\mathrm{C}^{\mathrm{q}}-\mathrm{Me}\right), 156.76\left(\mathrm{C}^{\mathrm{pz}}\right), 153.62\left(\mathrm{C}^{\mathrm{pz}}\right), 150.38\left(\mathrm{C}^{\mathrm{Ar}}\right), 148.28\left(\mathrm{C}^{\mathrm{Ar}}\right), 148.14\left(\mathrm{C}^{\mathrm{Ar}}\right), 146.64\left(\mathrm{C}^{\mathrm{Ar}}\right)$, $143.88\left(\mathrm{C}^{\mathrm{Ar}}\right), 142.92\left(\mathrm{C}^{\mathrm{Ar}}\right), 140.72\left(\mathrm{C}^{\mathrm{Ar}}\right), 140.68\left(\mathrm{C}^{\mathrm{Ar}}\right), 140.44\left(\mathrm{C}^{\mathrm{Ar}}\right), 138.52\left(\mathrm{C}^{\mathrm{Ar}}\right), 135.24$ $\left(\mathrm{C}^{\mathrm{Ar}}\right), 134.34\left(\mathrm{C}^{\mathrm{Ar}}\right), 132.07\left(\mathrm{C}^{\mathrm{Ar}}\right), 131.23\left(\mathrm{C}^{\mathrm{Ar}}\right), 131.03\left(\mathrm{C}^{\mathrm{Ar}}\right), 130.86\left(\mathrm{C}^{\mathrm{Ar}}\right), 130.73\left(\mathrm{C}^{\mathrm{Ar}}\right)$, $130.03\left(\mathrm{C}^{\mathrm{Ar}}\right), 129.52\left(\mathrm{C}^{\mathrm{Ar}}\right), 129.21\left(\mathrm{C}_{\mathbf{P h}} \mathrm{CHO}\right), 127.47\left(\mathrm{C}^{\mathrm{Ar}}\right), 127.22\left(\mathrm{C}^{\mathrm{Ar}}\right), 126.35\left(\mathrm{C}^{\mathrm{Ar}}\right)$, $125.95\left(\mathrm{C}^{\mathrm{Ar}}\right), 125.59\left(\mathrm{C}^{\mathrm{Ar}}\right), 125.33\left(\mathrm{C}^{\mathrm{Ar}}\right), 125.20\left(\mathrm{C}^{\mathrm{Ar}}\right), 124.39\left(\mathrm{C}^{\mathrm{Ar}}\right), 124.04\left(\mathrm{C}^{\mathrm{Ar}}\right), 97.63$ $\left(\mathrm{CHCNCH}_{3}\right), 97.27\left(\mathrm{CHCNCH}_{3}\right) 93.15\left(\mathrm{CH}^{\mathrm{pz}}\right), 86.70(\mathrm{CHO}), 51.97\left(\mathrm{CH}_{2}\right), 50.09\left(\mathrm{CH}_{2}\right)$, $27.98\left(\mathrm{CH}_{3}\right), 24.41\left(\mathrm{CH}_{3}\right), 22.12\left(\mathrm{CH}_{3}\right), 21.40\left(\mathrm{CH}_{3}\right) \mathrm{ppm}$.

IR (ATR) $v / \mathrm{cm}^{-1}=3050(\mathrm{vw}), 3025(\mathrm{vw}) 2973(\mathrm{vw}), 2912(\mathrm{vw}), 2863(\mathrm{vw}), 1560(\mathrm{~m}), 1519$ (s), 1493 (m), 1446 (m), 1426 (s), 1386 (vs), 1313 (m), 1274 (s), 1212 (m), 1091 (m), 1045 (m), 1023 (s), 915 (w), 893 (w), $834(\mathrm{w}), 802$ (w), 741 (vs), 721 (s), 698 (vs), 639 (m), 601 (s), $542(\mathrm{~m}), 480(\mathrm{~m}), 457(\mathrm{~m}), 417(\mathrm{w})$.

EA analysis: Anal. calcd. for 4-K 2 THF $\mathrm{C}_{66} \mathrm{H}_{67} \mathrm{KN}_{6} \mathrm{Ni}_{2} \mathrm{O}_{3}(1148.79 \mathrm{~g} / \mathrm{mol})=\mathrm{C} 69.01$, H 5.88, N 7.32; Found C 68.61, H 5.64, N 7.37.
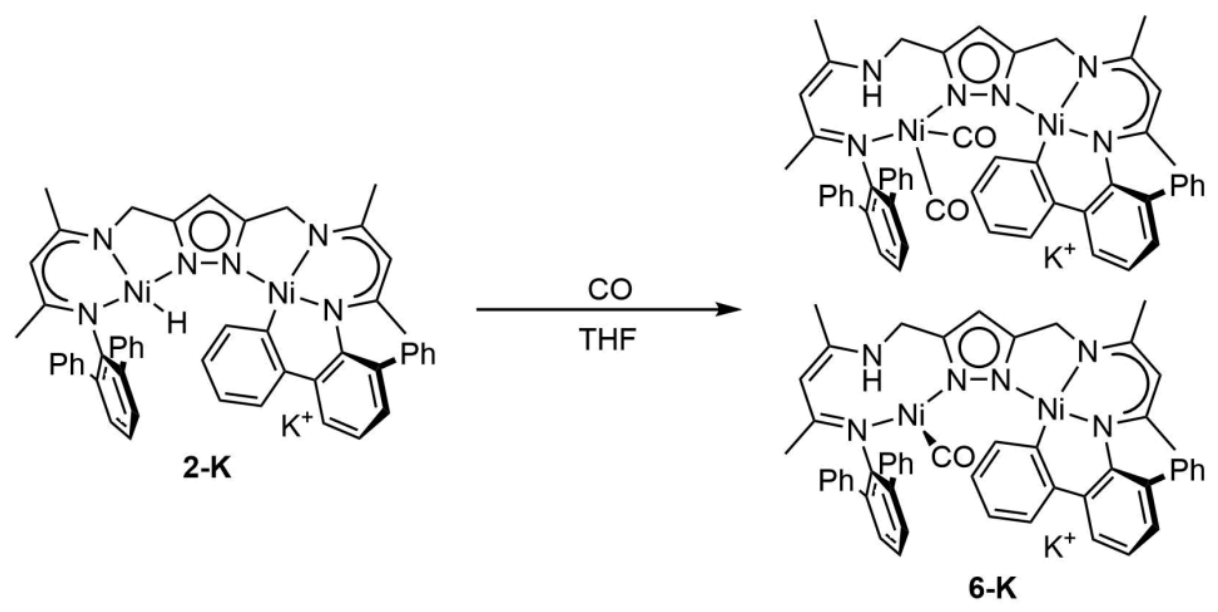

2-M (typical $5 \mu \mathrm{mol})$ was dissolved in THF $(0.5 \mathrm{~mL})$ and degassed via pump-freeze-thaw method. CO pressure of around $1 \mathrm{~atm}$. was applied to the reaction vessel and the light red solution was stirred for 48 hours. Evaporation of the solvent resulted in a light red powder of 6-K. 
${ }^{1} \mathrm{H}-\mathrm{NMR}(400 \mathrm{MHz}, \mathrm{THF}-\mathrm{d} 8) \delta=10.58\left(\mathrm{t}, J_{\mathrm{H}-\mathrm{H}}=6.0 \mathrm{~Hz}, 1 \mathrm{H}\right), 5.62\left(\mathrm{~s}, 1 \mathrm{H}, \mathrm{CH}^{\mathrm{pz}}\right), 4.51(\mathrm{~s}$, $\left.1 \mathrm{H}, \mathrm{CHCNCH}_{3}\right), 4.42\left(\mathrm{~d}, J_{\mathrm{H}-\mathrm{H}}=20 \mathrm{~Hz}, 1 \mathrm{H}, \mathrm{CH}_{2}\right), 4.31\left(\mathrm{~s}, 1 \mathrm{H}, \mathrm{CH}_{2}\right), 4.30\left(\mathrm{~d}, J_{\mathrm{H}-\mathrm{H}}=20 \mathrm{~Hz}\right.$ $\left.2 \mathrm{H}, \mathrm{CH}_{2}\right), 4.15\left(\mathrm{~s}, 1 \mathrm{H}, \mathrm{CHCNCH}_{3}\right), 4.12\left(\mathrm{~d}, \mathrm{~J}_{\mathrm{H}-\mathrm{H}}=20 \mathrm{~Hz}, 1 \mathrm{H}, \mathrm{CH} 2\right), 1.96$ (s, 3H,CH3), 1.86 (s, 3H, CH3), 1.20 (s, 3H, CH3), 1.17 (s, 3H, CH3) ppm.

${ }^{13} \mathrm{C}-\mathrm{NMR}\left(101 \mathrm{MHz}, \mathrm{THF}-\mathrm{d}_{8}\right) \delta=158.69\left(\mathrm{C}^{\mathrm{q}}-\mathrm{Me}\right), 158.39\left(\mathrm{C}^{\mathrm{q}}-\mathrm{Me}\right), 156.54\left(\mathrm{C}^{\mathrm{Ar}}\right), 156.27$ $\left(\mathrm{C}^{\mathrm{pz}}\right), 144.94\left(\mathrm{C}^{\mathrm{Ar}}\right), 143.55\left(\mathrm{C}^{\mathrm{Ar}}\right), 140.00\left(\mathrm{C}^{\mathrm{Ar}}\right), 139.07\left(\mathrm{C}^{\mathrm{Ar}}\right), 137.47\left(\mathrm{Ni}-\mathrm{C}^{\mathrm{Ar}}\right), 135.39\left(\mathrm{C}^{\mathrm{Ar}}\right)$, $130.49\left(\mathrm{C}^{\mathrm{Ar}}\right), 127.81\left(\mathrm{C}^{\mathrm{Ar}}\right), 126.65\left(\mathrm{C}^{\mathrm{Ar}}\right), 126.58\left(\mathrm{C}^{\mathrm{Ar}}\right), 125.04\left(\mathrm{C}^{\mathrm{Ar}}\right), 122.68\left(\mathrm{C}^{\mathrm{Ar}}\right), 121.66$ $\left(\mathrm{C}^{\mathrm{Ar}}\right), 120.85\left(\mathrm{C}^{\mathrm{Ar}}\right), 120.38\left(\mathrm{C}^{\mathrm{Ar}}\right), 101.84\left(\mathrm{CHCNCH}_{3}\right), 91.05\left(\mathrm{CH}^{\mathrm{Pz}}\right), 52.69\left(\mathrm{CH}_{2}\right), 123.72$ $\left(\mathrm{CH}_{3}\right), 24.14\left(\mathrm{CH}_{3}\right), 20.56\left(\mathrm{CH}_{3}\right) \mathrm{ppm}$.

IR (ATR) v/cm ${ }^{-1}=3050(\mathrm{vw}), 3025(\mathrm{vw}) 2973(\mathrm{vw}), 2912(\mathrm{vw}), 2863(\mathrm{vw}), 1560(\mathrm{~m}), 1519$ (s), 1493 (m), 1446 (m), 1426 (s), 1386 (vs), 1313 (m), 1274 (s), 1212 (m), 1091 (m), 1045 (m), 1023 (s), 915 (w), 893 (w), 834 (w), 802 (w), 741 (vs), 721 (s), 698 (vs), 639 (m), 601 (s), $542(\mathrm{~m}), 480(\mathrm{~m}), 457(\mathrm{~m}), 417(\mathrm{w})$. 


\subsection{Mechanistic Studies on the CTIST of Di- and Tetranuclear Co/Fe Prussian Blue Analogues using Time-Resolved Spectroscopy}

\subsubsection{Physical Measurements}

Electrospray ionization (ESI) mass spectra were collected on a Bruker HCTultra instrument. Elemental analyses were carried out with an Elementar 4.1 vario EL 3 instrument. IR spectra of solid samples were measured with a Cary 630 FTIR spectrometer equipped with a DialPath and Diamond ATR accessory (Agilent) placed. UV-vis spectra were recorded on an Agilent Cary 60 equipped with an Unisoku Cryostat (CoolSpek) and magnetic stirrer using quartz cuvettes with an attached tube and a screw cap with a septum.Temperature-dependent magnetic susceptibilities were measured using a QuantumDesign MPMS XL-5 SQUID magnetometer equipped. Mössbauer data were collected with a ${ }^{57}$ Co source embedded in a Rh matrix using an alternating constant acceleration Wissel Mössbauer spectrometer operated in the transmission mode and equipped with a Janis closed-cycle helium cryostat. Isomershifts are given relative to iron metal at ambient temperature. Simulation of the experimental data was performed with the Mfit program. ${ }^{39}$

\subsubsection{Laser Systems}

Near UV-pump-IR-probe experiments were performed with a laser system based on a 1 $\mathrm{kHz}$ Ti:sapphire oscillator/regenerative amplifier producing $100 \mathrm{fs}$ pulses at $800 \mathrm{~nm}$ with pulse energies of $0.75 \mathrm{~mJ}$. The excitation wavelength of $400 \mathrm{~nm}$ was produced by second harmonic generation of a small portion of the $800 \mathrm{~nm}$ regenerative amplifier output. Pump pulse energies entering the sample were about $400 \mathrm{~nJ}$ and focused to a diameter of $200 \mathrm{~mm}$. Tunable mid-infrared probe pulses were produced by difference frequency mixing of signal and idler pulses from a second OPA operated with the other half of the regenerative amplifier energy. The IR pulses were split into a probe and a reference beam. The latter was superimposed on the pump beam and both were focused into the sample cell. The spot size of the probe radiation was slightly below the pump diameter, i.e. $180 \mathrm{~mm}$. The relative plane of polarization was set to 54.71. Behind the sample the reference and probe beam were directed to a polychromator and their spectra were imaged on a liquid-nitrogen cooled $\mathrm{HgCdTe}$ detector of 2-32 pixels. To avoid spectral and temporal distortion of the IR pulses by $\mathrm{CO}_{2}$ and water absorptions in air, the whole pump-probe setup was purged with dry nitrogen. The overall time resolution of the pump-probe setup was about $200 \mathrm{fs}$. Temperature-depending UV/Vis and IR pump-probe measurements in a range of $-30^{\circ} \mathrm{C}$ to $+30^{\circ} \mathrm{C}$ were performed with an ethanol cooled flow cell equipped with $\mathrm{CaF}_{2}$ windows of 1 $\mathrm{mm}$ thickness. The optical path length inside the flow cell was $0.6 \mathrm{~mm}$. The Ethanol was tempered via an external cooler (Julabo Laboratory GmbH, Seelbach, type: F40-HC). 


\subsubsection{Magnetic Measurements}

Temperature-dependent magnetic susceptibility measurements were carried out with a Quantum-Design MPMS-XL-5 SQUID magnetometer equipped with a 5 Tesla magnet in the range from 295 to $2.0 \mathrm{~K}$ at a magnetic field of $0.5 \mathrm{~T}$. Data for pristine 2 were collected on freshly crystallized material. Each raw data file for the measured S3 magnetic moment was corrected for the diamagnetic contribution of the sample holder and the sample.

Experimental data were modelled with the julX program[1] using a fitting procedure to the spin Hamiltonian ${ }^{[2]}$ :

$$
\hat{H}=-2 J \hat{S}_{F e} \hat{S}_{C o}+g \mu_{B} \rho S_{F e}+\mu_{B}\left(S_{x(C o)} g_{x(C o)} B_{x}+S_{y(C o)} g_{y(C o)} B_{y}+S_{z(C o)} g_{z(C o)} B_{z}\right)+D_{C o}\left[\hat{S}_{z(C o)}^{2}-\frac{1}{3} S_{C o}\left(S_{C o}+1\right)\right]
$$

Temperature-independent paramagnetism (TIP) and paramagnetic impurities (PI) were included according to $\chi_{\text {calc }}=(1-\mathrm{PI}) \cdot \chi+\mathrm{PI} \cdot \chi_{\text {mono }}+\mathrm{TIP}$.

\subsubsection{Ligand Syntheses}
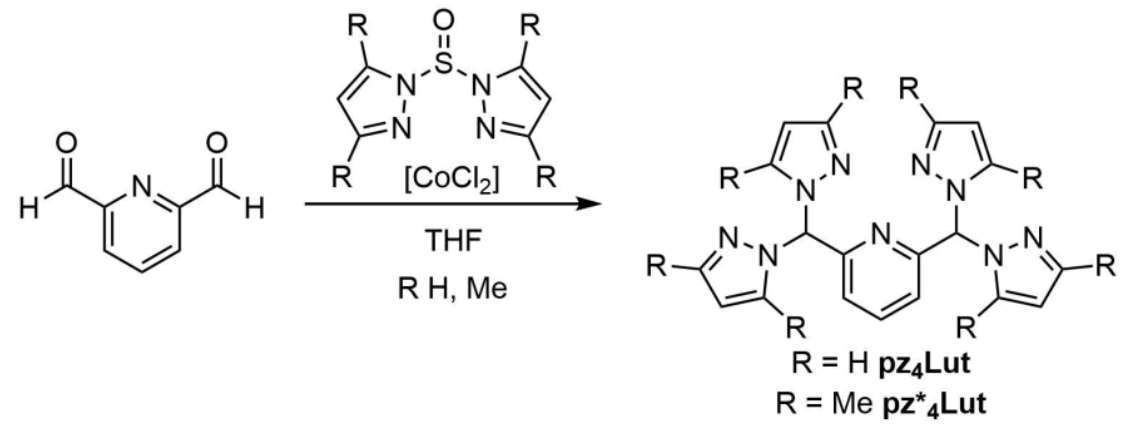

$\mathbf{p z}_{4}$ Lut and $\mathbf{p z}^{*}{ }_{4}$ Lut were synthesized according to slightly modified literature procedures. ${ }^{134,135}$

Pyrazole (4 eq.) were added to a suspension of sodium hydride (4 eq.) in THF. After the gas evolution ceased, thionyl chloride (2 eq.) was added slowly, while the mixture was kept at $0^{\circ} \mathrm{C}$. To the resulting suspension pyridine dicarbaldehyde (1 eq.) and cobalt(II) chloride (30 mol\%) was added. After stirring at $90^{\circ} \mathrm{C}$ for $21 \mathrm{~h}$ and cooling to room temperature the blue suspension was quenched carefully with water. The organic phase was separated, and the remaining water phase was extracted with dichloromethane. After drying the combined organic phases over magnesium sulfate the crude product was further purified by column chromatography on neutral aluminium oxide using diethylether as an eluent.

pz4Lut (Yield: 44\%): 
${ }^{1} \mathrm{H}-\mathrm{NMR}\left(300 \mathrm{MHz}, \mathrm{CDCl}_{3}\right) \delta=7.79\left(\mathrm{t}, 1 \mathrm{H}, J_{\mathrm{H}-\mathrm{H}}=8 \mathrm{~Hz}, p-\mathrm{H}_{\mathrm{py}}\right), 7.71\left(\mathrm{~s}, 2 \mathrm{H}, \mathrm{CHpz}_{2}\right), 7.60(\mathrm{dd}, 4 \mathrm{H}$, $\left.J_{\mathrm{H}-\mathrm{H}}=3 \mathrm{~Hz}, 3-\mathrm{H}_{\mathrm{pz}}\right), 7.54\left(\mathrm{dd}, 4 \mathrm{H}, J_{\mathrm{H}-\mathrm{H}}=3 \mathrm{~Hz}, 5-\mathrm{H}_{\mathrm{pz}}\right), 7.16\left(\mathrm{~d}, 2 \mathrm{H}, J_{\mathrm{H}-\mathrm{H}}=8 \mathrm{~Hz}, m-\mathrm{H}_{\mathrm{py}}\right), 6.31(\mathrm{dd}$, $\left.4 \mathrm{H}, J_{\mathrm{H}-\mathrm{H}}=3 \mathrm{~Hz}, 4-\mathrm{H}_{\mathrm{pz}}\right) \mathrm{ppm}$.

MS (ESI(+), MeCN) m/z: calcd. $[\mathrm{M}+\mathrm{H}]^{+} 372.17$ exp. $[\mathrm{M}+\mathrm{H}]^{+}$372.17, calcd. $[\mathrm{M}+\mathrm{Na}]^{+}$ $394.15 \exp [\mathrm{M}+\mathrm{Na}]^{+} 394.15$.

pz*$_{4}$ Lut (Yield: $85 \%$ ):

${ }^{1} \mathrm{H}-\mathrm{NMR}\left(300 \mathrm{MHz}, \mathrm{CDCl}_{3}\right) \delta=7.67\left(\mathrm{t}, 1 \mathrm{H}, J_{\mathrm{H}-\mathrm{H}}=8 \mathrm{~Hz}, p-\mathrm{H}_{\mathrm{py}}\right), 7.43$ (s, 2H, CHpz $), 7.16$ $\left(\mathrm{d}, 2 \mathrm{H}, J_{\mathrm{H}-\mathrm{H}}=8 \mathrm{~Hz}, m-\mathrm{H}_{\mathrm{py}}\right), 5.79\left(\mathrm{~s}, 4 \mathrm{H}, 4-\mathrm{H}_{\mathrm{pz}}{ }^{*}\right), 2.16(\mathrm{~s}, 12 \mathrm{H}, \mathrm{Me}), 2.06$ (s, 12H, Me) ppm.

MS (ESI(+), MeCN) $m / z$ : calcd. $[\mathrm{M}+\mathrm{H}]^{+} 484.29$ exp. $[\mathrm{M}+\mathrm{H}]^{+}$484.29, calcd. $[\mathrm{M}+\mathrm{Na}]^{+}$ $506.28 \exp [\mathrm{M}+\mathrm{Na}]^{+}$506.28.<smiles>c1ccc(C(n2cccn2)n2cccn2)nc1</smiles>

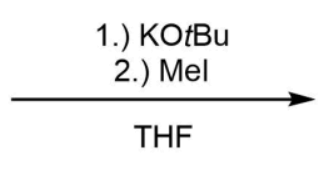

$\mathrm{pz}_{4}$ Lut

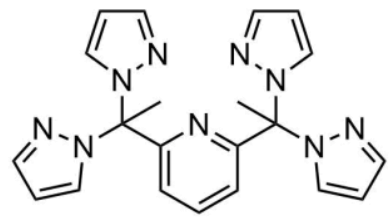

$\mathrm{pz}_{4}$ depy

$\mathbf{P z} \mathbf{z}_{\mathbf{4}}$ depy was synthesized according to a literature procedure. ${ }^{130}$

PzyLut (0.40 g, $1.08 \mathrm{mmol}, 1$ eq.) was dissolved in THF (15 mL) and potassium tertbutoxide ( $0.25 \mathrm{~g}, 2.23 \mathrm{mmol}, 2.1$ eq.) was added. After stirring the solution at room temperature for 30 mins, methyl iodide $(0.14 \mathrm{~mL}, 0.32 \mathrm{~g}, 2.25 \mathrm{mmol}, 2.1$ eq. $)$ was added dropwise. The solution was stirred for another $1.5 \mathrm{~h}$ and then quenched with water $(25 \mathrm{~mL})$. The organic phase was separated, and the water phase extracted with diethyl ether $(3 \mathrm{x}$ $25 \mathrm{~mL}$ ). The combined organic phases were dried over magnesium sulfate. The crude product was washed multiple times with diethyl ether to get pz4depy $(310 \mathrm{mg}, 0.77 \mathrm{mmol}$, $72 \%)$ as a white solid.

${ }^{1} \mathrm{H}-\mathrm{NMR}\left(300 \mathrm{MHz}, \mathrm{CDCl}_{3}\right) \delta=7.64\left(\mathrm{t}, 1 \mathrm{H}, J_{\mathrm{H}-\mathrm{H}}=8 \mathrm{~Hz}, p-\mathrm{H}_{\mathrm{py}}\right), 7.61\left(\mathrm{dd}, 4 \mathrm{H}, J_{\mathrm{H}-\mathrm{H}}=3 \mathrm{~Hz}\right.$, $\left.3-\mathrm{H}_{\mathrm{pz}}\right), 7.14\left(\mathrm{dd}, 4 \mathrm{H}, J_{\mathrm{H}-\mathrm{H}}=3 \mathrm{~Hz}, 5-\mathrm{H}_{\mathrm{pz}}\right), 6.46\left(\mathrm{~d}, 2 \mathrm{H}, J_{\mathrm{H}-\mathrm{H}}=8 \mathrm{~Hz}, m-\mathrm{H}_{\mathrm{py}}\right) 6.27(\mathrm{dd}, 4 \mathrm{H}$, $\left.J_{\mathrm{H}-\mathrm{H}}=3 \mathrm{~Hz}, 4-\mathrm{H}_{\mathrm{pz}}\right) \mathrm{ppm}$.

MS (ESI(+), MeCN) m/z: calcd. [M+Na $]^{+} 422.18$ exp. $[\mathrm{M}+\mathrm{Na}]^{+} 422.18$. 


\subsubsection{General Procedure for the Synthesis of Dinuclear Co/Fe PBAs Containing} Hydrotrispyrazolylborate and Tetrapyrazolyl Lutidine ligands

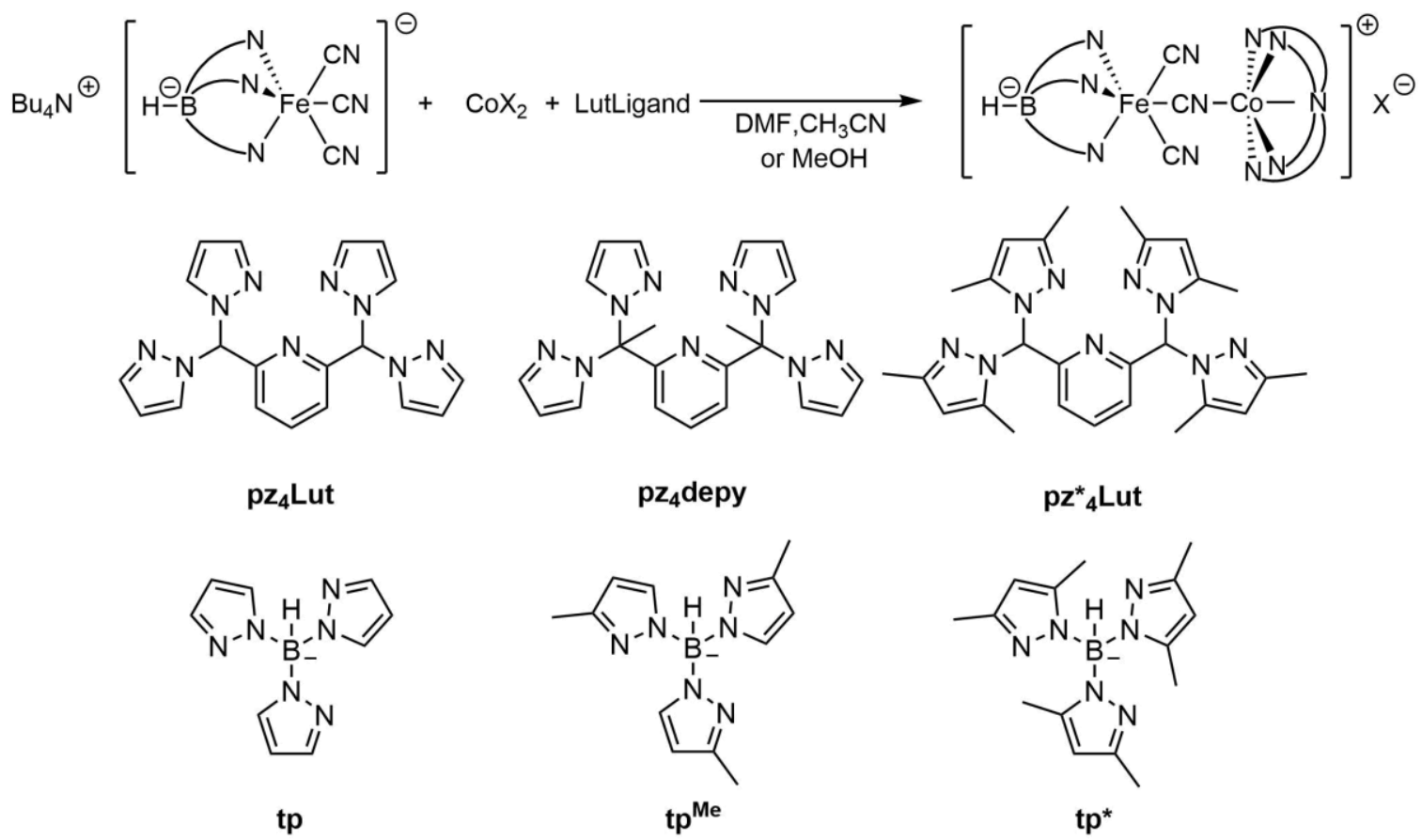

$\mathrm{CoX}_{2} \cdot \mathrm{xH}_{2} \mathrm{O}$ (1 eq.) and lutidine based ligand (1 eq.) was dissolved in solvent to produce a pale-yellow solution after 5 mins. $n \mathrm{Bu} 4 \mathrm{~N}\left[\mathrm{RFe}(\mathrm{CN})_{3}\right]$ (1 eq.) in solvent was added dropwise. The then dark red solution was stirred for 2 hours. For some ligand combinations a precipitate formed which was then used as it is after filtration. For the other ligand combinations crystallization was facilitated by slow diffusion of diethyl ether in the filtrate after the reaction.

$\left[\left(p z_{4} \mathrm{Lut}\right) \mathrm{CoFe}(\mathrm{CN})_{3}(\mathrm{tp})\right] \mathrm{OTf}[\mathbf{t p}-\mathbf{F e} / \mathbf{p z} \mathbf{4} \mathbf{L u t}-\mathbf{C o}]:$

Mößbauer spectroscopy $(80 \mathrm{~K}): \delta=0.04 \mathrm{~mm} \cdot \mathrm{s}^{-1}, \Delta \mathrm{E}_{\mathrm{Q}}=1.34 \mathrm{~mm} \cdot \mathrm{s}^{-1}$.

MS (ESI(+), MeCN) m/z: calcd. [M-OTf] 777.14 exp. [M-OTf] ${ }^{+} 777.14$.

$\left[\left(p_{4} d e p y\right) \mathrm{CoFe}(\mathrm{CN})_{3}(t p)\right] \mathrm{OTf}[\mathbf{t p}-\mathbf{F e} / \mathbf{p z} \mathbf{4} \mathbf{d e p y}-\mathbf{C o}]:$

Mößbauer spectroscopy $(80 \mathrm{~K}): \mathrm{A}(54 \%) \delta=0.02 \mathrm{~mm} \cdot \mathrm{s}^{-1}, \Delta \mathrm{EQ}_{\mathrm{Q}}=1.30 \mathrm{~mm} \cdot \mathrm{s}^{-1} ; \mathrm{B}(46 \%) \delta=$ $0.08 \mathrm{~mm} \cdot \mathrm{s}^{-1}, \Delta \mathrm{E}_{\mathrm{Q}}=1.74 \mathrm{~mm} \cdot \mathrm{s}^{-1}$.

$\operatorname{MS}(\operatorname{ESI}(+), \mathrm{MeCN}) m / z$ : calcd. [M-OTf] ${ }^{+} 805.17$ exp. $[\mathrm{M}-\mathrm{OTf}]^{+}$805.17.

IR $($ ATR $) v / \mathrm{cm}^{-1}=2512\left(v_{\mathrm{BH}}\right), 2160\left(v_{\mathrm{CN}}\right), 2149\left(v_{\mathrm{CN}}\right), 2136\left(v_{\mathrm{CN}}\right), 2123\left(v_{\mathrm{CN}}\right)$. 
$\left[\left(\mathrm{pz}^{*}{ }_{4} \mathrm{Lut}\right) \mathrm{CoFe}(\mathrm{CN})_{3}(\mathrm{tp})\right] \mathrm{OTf}\left[\mathbf{t p}-\mathbf{F e} / \mathbf{p z}{ }^{*} \mathbf{L u t}-\mathbf{C o}\right]:$

Mößbauer spectroscopy $(80 \mathrm{~K}): \delta=0.05 \mathrm{~mm} \cdot \mathrm{s}^{-1}, \Delta \mathrm{EQ}=1.16 \mathrm{~mm} \cdot \mathrm{s}^{-1}$

MS (ESI(+), MeCN) m/z: calcd. [M-OTf] 889.27 exp. [M-OTf] ${ }^{+} 889.27$.

$\operatorname{ATR}-I R\left(\mathrm{~cm}^{-1}\right): 2492\left(v_{\mathrm{BH}}\right), 2150\left(v_{\mathrm{CN}}\right), 2120\left(v_{\mathrm{CN}}\right)$.

$\left[\left(\mathrm{pz}_{4}{ }_{4} \mathrm{Lut}\right) \mathrm{CoFe}(\mathrm{CN})_{3}\left(\mathrm{tp}^{\mathrm{Me}}\right)\right] \mathrm{OTf}\left[\mathbf{t p} \mathbf{M e}_{-} \mathbf{F e} / \mathbf{p z}{ }_{4}{ }_{4} \mathbf{L u t}-\mathbf{C o}\right]:$

Mößbauer spectroscopy $(80 \mathrm{~K}): \delta=0.08 \mathrm{~mm} \cdot \mathrm{s}^{-1}, \Delta \mathrm{EQ}=1.58 \mathrm{~mm} \cdot \mathrm{s}^{-1}$

MS (ESI(+), MeCN) m/z: calcd. [M-OTf] 931.32 exp. [M-OTf] ${ }^{+}$931.32.

$\operatorname{ATR}-I R\left(\mathrm{~cm}^{-1}\right): 2519\left(v_{\mathrm{BH}}\right), 2139\left(v_{\mathrm{CN}}\right), 2122\left(v_{\mathrm{CN}}\right)$.

$\left[\left(p_{4} L u t\right) \operatorname{CoFe}(C N)_{3}\left(t p^{*}\right)\right] O T f\left[\mathbf{t p}^{*}\right.$-Fe/pz 4 Lut-Co]:

Mößbauer spectroscopy $(80 \mathrm{~K}): \delta=0.08 \mathrm{~mm} \cdot \mathrm{s}^{-1}, \Delta \mathrm{EQ}_{\mathrm{Q}}=1.48 \mathrm{~mm} \cdot \mathrm{s}^{-1}$.

MS (ESI(+), MeCN) m/z: calcd. [M-OTf] 861.24 exp. [M-OTf] ${ }^{+}$861.24.

IR $($ ATR $) v / \mathrm{cm}^{-1}=2538\left(v_{\mathrm{BH}}\right), 2134\left(v_{\mathrm{CN}}\right), 2115\left(v_{\mathrm{CN}}\right)$.

$\left[\left(p z{ }_{4} d e p y\right) \operatorname{CoFe}(C N)_{3}\left(t p^{*}\right)\right]$ OTf $\left[\mathbf{t p} * / \mathbf{p z}_{\mathbf{4}} \mathbf{d e p y}-\mathbf{C o}\right]:$

Mößbauer spectroscopy $(80 \mathrm{~K}): \delta=0.11 \mathrm{~mm} \cdot \mathrm{s}^{-1}, \Delta \mathrm{E}_{\mathrm{Q}}=1.33 \mathrm{~mm} \cdot \mathrm{s}^{-1}$.

MS (ESI(+), MeCN) m/z: calcd. [M-OTf] ${ }^{+} 889.27$ exp. [M-OTf] $]^{+} 889.27$.

IR $($ ATR $) v / \mathrm{cm}^{-1}=2546\left(v_{\mathrm{BH}}\right), 2139\left(v_{\mathrm{CN}}\right), 2119\left(v_{\mathrm{CN}}\right)$.

$\left[\left(\mathrm{pz}^{*}{ }_{4} \mathrm{Lut}\right) \mathrm{CoFe}(\mathrm{CN})_{3}\left(\mathrm{tp}{ }^{*}\right)\right] \mathrm{ClO}_{4}\left[\mathbf{t p}{ }^{*}-\mathbf{F e} / \mathbf{p z}^{*}{ }_{4} \mathbf{L u t}-\mathbf{C o}\right]:$

Mößbauer spectroscopy $(80 \mathrm{~K}): \delta=0.09 \mathrm{~mm} \cdot \mathrm{s}^{-1}, \Delta \mathrm{E}_{\mathrm{Q}}=1.18 \mathrm{~mm} \cdot \mathrm{s}^{-1}$

MS (ESI(+), MeCN) m/z: calcd. [M-ClO4] $]^{+} 973.36$ exp. [M-ClO4] $]^{+} 973.36$.

ATR-IR $\left(\mathrm{cm}^{-1}\right): 2547\left(v_{\mathrm{BH}}\right), 2138\left(v_{\mathrm{CN}}\right), 2120\left(v_{\mathrm{CN}}\right)$. 
Anal. Calcd. for $\mathrm{C}_{47} \mathrm{H}_{63} \mathrm{BClCoFeN} 18 \mathrm{O}_{6}$ : C, 49.64; H, 5.58; N, 22.17\%. Found: $\mathrm{C}, 49.30 ; \mathrm{H}$, $5.50 ; \mathrm{N}, 22.56$. 


\section{References}

1 S. Yao and M. Driess, Acc. Chem. Res., 2012, 45, 276-287.

2 M. Kreye, M. Freytag, P. G. Jones, P. G. Williard, W. H. Bernskoetter and M. D. Walter, Chem. Commun., 2015, 51, 2946-2949.

3 S. Pfirrmann, C. Limberg, C. Herwig, R. Stößer and B. Ziemer, Angew. Chem. Int. Ed., 2009, 48, 3357-3361.

4 B. S. Mandimutsira, J. L. Yamarik, T. C. Brunold, W. Gu, S. P. Cramer and C. G. Riordan, J. Am. Chem. Soc., 2001, 123, 9194-9195.

5 C. Yoo, S. Oh, J. Kim and Y. Lee, Chem. Sci., 2014, 5, 3853-3858.

6 F. Schneck, F. Schendzielorz, N. Hatami, M. Finger, C. Würtele and S. Schneider, Angew. Chem. Int. Ed., 2018, 57, 14482-14487.

7 J. A. Garduño, A. Arévalo and J. J. García, Dalton Trans., 2015, 44, 13419-13438.

8 P. Zimmermann and C. Limberg, J. Am. Chem. Soc., 2017, 139, 4233-4242.

9 C.-Y. Lin and P. P. Power, Chem. Soc. Rev., 2017, 46, 5347-5399.

10 N. A. Eberhardt and H. Guan, Chem. Rev., 2016, 116, 8373-8426.

11 G. Bai, P. Wei and D. W. Stephan, Organometallics, 2005, 24, 5901-5908.

12 J. I. van der Vlugt, Eur. J. Inorg. Chem., 2012, 2012, 363-375.

13 L. Bourget-Merle, M. F. Lappert and J. R. Severn, Chem. Rev., 2002, 102, 30313066.

14 C. Chen, S. M. Bellows and P. L. Holland, Dalton Trans., 2015, 44, 16654-16670.

15 D. J. Mindiola, Angew. Chem. Int. Ed., 2009, 48, 6198-6200.

16 C. Chen, S. M. Bellows and P. L. Holland, Dalton Trans., 2015, 44, 16654-16670.

17 C. Camp and J. Arnold, Dalton Trans., 2016, 45, 14462-14498.

18 R. L. Webster, Dalton Trans., 2017, 46, 4483-4498.

19 P. L. Holland, T. R. Cundari, L. L. Perez, N. A. Eckert and R. J. Lachicotte, J. Am. Chem. Soc., 2002, 124, 14416-14424.

20 S. C. Puiu and T. H. Warren, Organometallics, 2003, 22, 3974-3976.

21 S. Yao, E. Bill, C. Milsmann, K. Wieghardt and M. Driess, Angew. Chem. Int. Ed., 2008, 47, 7110-7113.

22 S. Yao, Y. Xiong, M. Vogt, H. Grützmacher, C. Herwig, C. Limberg and M. Driess, Angew. Chem. Int. Ed., 2009, 48, 8107-8110. 
23 S. Yao, C. Herwig, Y. Xiong, A. Company, E. Bill, C. Limberg and M. Driess, Angew. Chem. Int. Ed., 2010, 49, 7054-7058.

24 S. Pfirrmann, S. Yao, B. Ziemer, R. Stösser, M. Driess and C. Limberg, Organometallics, 2009, 28, 6855-6860.

25 B. Horn, S. Pfirrmann, C. Limberg, C. Herwig, B. Braun, S. Mebs and R. Metzinger, Zeitschrift für Anorg. und Allg. Chemie, 2011, 637, 1169-1174.

26 B. Horn, C. Limberg, C. Herwig and S. Mebs, Angew. Chem. Int. Ed., 2011, 50, 12621-12625.

27 B. Horn, C. Limberg, C. Herwig and B. Braun, Chem. Commun., 2013, 49, 10923.

28 S. Raugei, L. C. Seefeldt and B. M. Hoffman, Proc. Natl. Acad. Sci., 2018, 115, E10521-E10530.

29 D. Schilter, J. M. Camara, M. T. Huynh, S. Hammes-Schiffer and T. B. Rauchfuss, Chem. Rev., 2016, 116, 8693-8749.

30 S. Ogo, Coord. Chem. Rev., 2017, 334, 43-53.

31 D. E. Berning, B. C. Noll and D. L. DuBois, J. Am. Chem. Soc., 1999, 121, 1143211447.

32 D.-H. Manz, P.-C. Duan, S. Dechert, S. Demeshko, R. Oswald, M. John, R. A. Mata and F. Meyer, J. Am. Chem. Soc., 2017, 139, 16720-16731.

33 S. Pfirrmann, C. Limberg and B. Ziemer, Dalton Trans., 2008, 912, 6689.

34 K. Jonas and G. Wilke, Angew. Chem. Int. Ed., 1969, 8, 519-520.

35 A. Pietrzykowski, P. Buchalski and L. B. Jerzykiewicz, J. Organomet. Chem., 2000, 597, 133-138.

36 N. D. Clement, K. J. Cavell, C. Jones and C. J. Elsevier, Angew. Chem. Int. Ed., 2004, 43, 1277-1279.

37 A. Kempter, C. Gemel, T. Cadenbach and R. A. Fischer, Organometallics, 2007, 26, 4257-4264.

38 T. Steinke, B. K. Shaw, H. Jong, B. O. Patrick and M. D. Fryzuk, Organometallics, 2009, 28, 2830-2836.

39 J. A. Hatnean, R. Beck, J. D. Borrelli and S. A. Johnson, Organometallics, 2010, 29, 6077-6091.

40 P. Gandeepan, T. Müller, D. Zell, G. Cera, S. Warratz and L. Ackermann, Chem. Rev., 2019, 119, 2192-2452.

41 Q. Zhao, G. Meng, S. P. Nolan and M. Szostak, Chem. Rev., 2020, acs.chemrev.9b00634.

42 A. L. Keen and S. A. Johnson, J. Am. Chem. Soc., 2006, 128, 1806-1807.

43 A. L. Keen, M. Doster and S. A. Johnson, J. Am. Chem. Soc., 2007, 129, 810-819. 
44 A. Najafian and T. R. Cundari, Organometallics, 2018, 37, 3111-3121.

45 H. Yang, C. Zarate, W. N. Palmer, N. Rivera, D. Hesk and P. J. Chirik, ACS Catal., 2018, 8, 10210-10218.

46 C. Zarate, H. Yang, M. J. Bezdek, D. Hesk and P. J. Chirik, J. Am. Chem. Soc., 2019, 141, 5034-5044.

47 I. Pappas, S. Treacy and P. J. Chirik, ACS Catal., 2016, 6, 4105-4109.

48 N. G. Léonard and P. J. Chirik, ACS Catal., 2018, 8, 342-348.

49 J. Atzrodt, V. Derdau, W. J. Kerr and M. Reid, Angew. Chem. Int. Ed., 2018, 57, 3022-3047.

50 D. V. Vitanova, F. Hampel and K. C. Hultzsch, Dalton Trans., 2005, 559, 15651566.

51 D. F. J. Piesik, S. Range and S. Harder, Organometallics, 2008, 27, 6178-6187.

52 M. F. Pilz, C. Limberg, B. B. Lazarov, K. C. Hultzsch and B. Ziemer, Organometallics, 2007, 26, 3668-3676.

53 D. F.-J. Piesik, R. Stadler, S. Range and S. Harder, Eur. J. Inorg. Chem., 2009, 2009, 3569-3576.

54 N. Schuth, H. Gehring, B. Horn, P. Holze, R. Kositzki, P. Schrapers, C. Limberg and M. Haumann, J. Phys. Conf. Ser., 2016, 712, 012134.

55 H. Gehring, R. Metzinger, C. Herwig, J. Intemann, S. Harder and C. Limberg, Chem. Eur. J., 2013, 19, 1629-1636.

56 D.-H. Manz, Georg-August Universität Göttingen, 2016.

57 P.-C. Duan, Georg-August Universität Göttingen, 2017.

58 P.-C. Duan, D.-H. Manz, S. Dechert, S. Demeshko and F. Meyer, J. Am. Chem. Soc., 2018, 140, 4929-4939.

59 E. Ferretti, S. Dechert, S. Demeshko, M. C. Holthausen and F. Meyer, Angew. Chem. Int. Ed., 2019, 58, 1705-1709.

60 S. Kundu, S. C. E. Stieber, M. G. Ferrier, S. A. Kozimor, J. A. Bertke and T. H. Warren, Angew. Chem. Int. Ed., 2016, 55, 10321-10325.

61 E. Ferretti, S. Dechert and F. Meyer, Inorg. Chem., 2019, 58, 5154-5162.

62 B. M. Hoffman, D. Lukoyanov, Z.-Y. Yang, D. R. Dean and L. C. Seefeldt, Chem. Rev., 2014, 114, 4041-4062.

63 Š. Lachmanová, G. Dupeyre, J. Tarábek, P. Ochsenbein, C. Perruchot, I. Ciofini, M. Hromadová, L. Pospíšil and P. P. Lainé, J. Am. Chem. Soc., 2015, 137, 1134911364.

64 T. Steiner, Angew. Chem. Int. Ed., 2002, 41, 48-76. 
65 H. Maeda, Y. Ito, Y. Kusunose and T. Nakanishi, Chem. Commun., 2007, $1136-$ 1138 .

66 J. J. Scepaniak, C. G. Margarit, R. P. Bontchev and J. M. Smith, Acta Crystallogr. C., 2013, 69, 968-971.

67 P. Zanello, Inorganic Electrochemistry, Royal Society of Chemistry, Cambridge, 2007.

68 Q. Chen, J. Yu and J. Huang, Organometallics, 2007, 26, 617-625.

69 Z.-X. Wang and Z.-Y. Chai, Eur. J. Inorg. Chem., 2007, 2007, 4492-4499.

70 E. M. Matson, G. Espinosa Martinez, A. D. Ibrahim, B. J. Jackson, J. A. Bertke and A. R. Fout, Organometallics, 2015, 34, 399-407.

71 D. Britton, J. Chem. Crystallogr., 1998, 28, 601-604.

72 S. K. Nayak, R. Sathishkumar and T. N. G. Row, CrystEngComm, 2010, 12, 3112.

73 Z. Chai, T.-T. Zeng, Q. Li, L.-Q. Lu, W.-J. Xiao and D. Xu, J. Am. Chem. Soc., 2016, 138, 10128-10131.

74 T. Li, B.-H. Xu, D.-P. Zhu, Y.-F. Wang and S.-J. Zhang, Org. Chem. Front., 2018, 5, 1933-1939.

75 R. Cano, M. Yus and D. J. Ramón, Tetrahedron, 2011, 67, 8079-8085.

76 E. Dinjus, I. Gorski, H. Matschiner, E. Uhlig and D. Walther, Zeitschrift für Anorg. und Allg. Chemie, 1977, 436, 39-46.

77 S. D. Ittel, Inorg. Chem., 1977, 16, 2589-2597.

78 T. T. Tsou, J. C. Huffman and J. K. Kochi, Inorg. Chem., 1979, 18, 2311-2317.

79 Y.-J. Kim, K. Osakada and A. Yamamoto, Bull. Chem. Soc. Jpn., 1989, 62, 964966.

80 S. Ogoshi, H. Kamada and H. Kurosawa, Tetrahedron, 2006, 62, 7583-7588.

81 S. Ogoshi, Y. Hoshimoto and M. Ohashi, Chem. Commun., 2010, 46, 3354.

82 H. Yu and Y. Fu, Chem. Eur. J., 2012, 18, 16765-16773.

83 A. N. Desnoyer, W. He, S. Behyan, W. Chiu, J. A. Love and P. Kennepohl, Chem. - A Eur. J., 2019, 25, 5259-5268.

84 N. G. Connelly and W. E. Geiger, Chem. Rev., 1996, 96, 877-910.

85 H. Klein and H. H. Karsch, Chem. Ber., 1976, 109, 2524-2532.

86 F. Schneck, J. Ahrens, M. Finger, A. C. Stückl, C. Würtele, D. Schwarzer and S. Schneider, Nat. Commun., 2018, 9, 1161.

87 R. N. Perutz and B. Procacci, Chem. Rev., 2016, 116, 8506-8544.

88 M. Brookhart, M. L. H. Green and G. Parkin, Proc. Natl. Acad. Sci., 2007, 104, 
6908-6914.

89 M. Emmelius, G. Pawlowski and H. W. Vollmann, Angew. Chem. Int. Ed., 1989, 28, 1445-1471.

90 P. Gütlich, Eur. J. Inorg. Chem., 2013, 2013, 581-591.

91 D. N. Woodruff, R. E. P. Winpenny and R. A. Layfield, Chem. Rev., 2013, 113, $5110-5148$.

92 G. A. Craig and M. Murrie, Chem. Soc. Rev., 2015, 44, 2135-2147.

93 S. Decurtins, P. Gütlich, C. P. Köhler, H. Spiering and A. Hauser, Chem. Phys. Lett., 1984, 105, 1-4.

94 D. M. Adams, B. Li, J. D. Simon and D. N. Hendrickson, Angew. Chem. Int. Ed., 1995, 34, 1481-1483.

95 P. Gütlich, A. Hauser and H. Spiering, Angew. Chem. Int. Ed., 1994, 33, 2024 2054.

96 J.-F. Létard, J. Mater. Chem., 2006, 16, 2550-2559.

97 P. Gütlich, A. B. Gaspar and Y. Garcia, Beilstein J. Org. Chem., 2013, 9, 342-391.

98 T. Tezgerevska, K. G. Alley and C. Boskovic, Coord. Chem. Rev., 2014, 268, $23-$ 40 .

99 S. Hayami, S. M. Holmes and M. A. Halcrow, J. Mater. Chem. C, 2015, 3, 77757778.

100 D. Aguilà, Y. Prado, E. S. Koumousi, C. Mathonière and R. Clérac, Chem. Soc. Rev., 2016, 45, 203-224.

101 M. B. Robin, Inorg. Chem., 1962, 1, 337-342.

102 O. Sato, T. Iyoda, A. Fujishima and K. Hashimoto, Science, 1996, 272, 704-705.

103 S. Juszczyk, C. Johansson, M. Hanson, A. Ratuszna and G. Malecki, J. Phys. Condens. Matter, 1994, 6, 5697-5706.

104 M. Verdaguer, Science, 1996, 272, 698-699.

105 O. Sato, Y. Einaga, A. Fujishima and K. Hashimoto, Inorg. Chem., 1999, 38, 4405-4412.

106 A. Bleuzen, C. Lomenech, V. Escax, F. Villain, F. Varret, C. Cartier dit Moulin and M. Verdaguer, J. Am. Chem. Soc., 2000, 122, 6648-6652.

107 A. Vogler and H. Kunkely, Berichte der Bunsengesellschaft für Phys. Chemie, 1975, 79, 301-307.

108 R. E. Hester and E. M. Nour, J. Chem. Soc. Dalt. Trans., 1981, 939.

109 P. V. Bernhardt and M. Martinez, Inorg. Chem., 1999, 38, 424-425.

110 P. V. Bernhardt, B. P. Macpherson and M. Martinez, J. Chem. Soc. Dalt. Trans., 
$2002,1435$.

111 P. V. Bernhardt, F. Bozoglian, B. P. Macpherson, M. Martínez, G. González and

B. Sienra, Eur. J. Inorg. Chem., 2003, 2003, 2512-2518.

112 C. P. Berlinguette, A. Dragulescu-Andrasi, A. Sieber, J. R. Galán-Mascarós, H.-U. Güdel, C. Achim and K. R. Dunbar, J. Am. Chem. Soc., 2004, 126, 6222-6223.

113 C. P. Berlinguette, A. Dragulescu-Andrasi, A. Sieber, H.-U. Güdel, C. Achim and K. R. Dunbar, J. Am. Chem. Soc., 2005, 127, 6766-6779.

114 K. E. Funck, A. V. Prosvirin, C. Mathonière, R. Clérac and K. R. Dunbar, Inorg. Chem., 2011, 50, 2782-2789.

115 D. Li, R. Clérac, O. Roubeau, E. Harté, C. Mathonière, R. Le Bris and S. M. Holmes, J. Am. Chem. Soc., 2008, 130, 252-258.

116 M. Nihei, Y. Sekine, N. Suganami, K. Nakazawa, A. Nakao, H. Nakao, Y. Murakami and H. Oshio, J. Am. Chem. Soc., 2011, 133, 3592-3600.

117 Y. Sekine, M. Nihei, R. Kumai, H. Nakao, Y. Murakami and H. Oshio, Chem. Commun., 2014, 50, 4050-4052.

118 Y. Kitagawa, M. Asaoka, K. Miyagi, T. Matsui, M. Nihei, H. Oshio, M. Okumura and M. Nakano, Inorg. Chem. Front., 2015, 2, 771-779.

119 I.-R. Jeon, S. Calancea, A. Panja, D. M. Piñero Cruz, E. S. Koumousi, P. Dechambenoit, C. Coulon, A. Wattiaux, P. Rosa, C. Mathonière and R. Clérac, Chem. Sci., 2013, 4, 2463.

120 S. F. Jafri, E. S. Koumousi, M.-A. Arrio, A. Juhin, D. Mitcov, M. Rouzières, P. Dechambenoit, D. Li, E. Otero, F. Wilhelm, A. Rogalev, L. Joly, J.-P. Kappler, C. Cartier dit Moulin, C. Mathonière, R. Clérac and P. Sainctavit, J. Am. Chem. Soc., 2019, 141, 3470-3479.

121 E. S. Koumousi, I. Jeon, Q. Gao, P. Dechambenoit, D. N. Woodruff, P. Merzeau, L. Buisson, X. Jia, D. Li, F. Volatron, C. Mathonière and R. Clérac, J. Am. Chem. Soc., 2014, 136, 15461-15464.

122 B. P. Macpherson, P. V. Bernhardt, A. Hauser, S. Pagès and E. Vauthey, Inorg. Chem., 2005, 44, 5530-5536.

123 R. Berera, R. van Grondelle and J. T. M. Kennis, Photosynth. Res., 2009, 101, $105-118$.

124 A. Cannizzo, C. J. Milne, C. Consani, W. Gawelda, C. Bressler, F. van Mourik and M. Chergui, Coord. Chem. Rev., 2010, 254, 2677-2686.

125 J. Ahrens, Georg-August Universität Göttingen, 2017.

126 C. Bressler and M. Chergui, Chem. Rev., 2004, 104, 1781-1812.

127 P. V. Bernhardt, B. P. Macpherson and M. Martinez, Inorg. Chem., 2000, 39, 5203-5208.

128 P. V. Bernhardt, F. Bozoglian, B. P. Macpherson and M. Martinez, Dalton Trans., 
2004, 2582-2587.

129 M. Wilken, C. Würtele, M. Kügler, F. Chrobak and I. Siewert, Eur. J. Inorg. Chem., 2018, 2018, 2339-2344.

130 D. J. Wasylenko, H. M. Tatlock, L. S. Bhandari, J. R. Gardinier and C. P. Berlinguette, Chem. Sci., 2013, 4, 734-738.

131 N. Dehury, S. K. Tripathy, A. Sahoo, N. Maity and S. Patra, Dalton Trans., 2014, 43, 16597-16600.

132 A. Hoffmann, U. Flörke, M. Schürmann and S. Herres-Pawlis, European J. Org. Chem., 2010, 2010, 4136-4144.

133 F. Hamon, E. Largy, A. Guédin-Beaurepaire, M. Rouchon-Dagois, A. Sidibe, D. Monchaud, J. Mergny, J. Riou, C. Nguyen and M.-P. Teulade-Fichou, Angew. Chem. Int. Ed., 2011, 50, 8745-8749.

134 T. J. Morin, B. Bennett, S. V. Lindeman and J. R. Gardinier, Inorg. Chem., 2008, 47, 7468-7470.

135 T. J. Morin, A. Merkel, S. V. Lindeman and J. R. Gardinier, Inorg. Chem., 2010, 49, 7992-8002.

136 S. Trofimenko, J. R. Long, T. Nappier and S. G. Shore, in Inorganic Syntheses, 2007, vol. XII, pp. 99-109.

137 K. Niedenzu, P. M. Niedenzu and K. R. Warner, Inorg. Chem., 1985, 24, 1604 1606.

138 D. Coucouvanis, Inorganic Syntheses, John Wiley \& Sons, Inc., New York, USA, Inorganic., 2002, vol. 33.

139 M. Lubben, A. Meetsma, E. C. Wilkinson, B. Feringa and L. Que, Angew. Chem. Int. Ed., 1995, 34, 1512-1514.

140 M. R. Bukowski, P. Comba, A. Lienke, C. Limberg, C. Lopez de Laorden, R. MasBallesté, M. Merz and L. Que, Angew. Chem. Int. Ed., 2006, 45, 3446-3449.

141 A. A. Campanali, T. D. Kwiecien, L. Hryhorczuk and J. J. Kodanko, Inorg. Chem., 2010, 49, 4759-4761.

142 A. C. McQuilken, Y. Ha, K. D. Sutherlin, M. A. Siegler, K. O. Hodgson, B. Hedman, E. I. Solomon, G. N. L. Jameson and D. P. Goldberg, J. Am. Chem. Soc., 2013, 135, 14024-14027.

143 H. Yoon, Y.-M. Lee, X. Wu, K.-B. Cho, R. Sarangi, W. Nam and S. Fukuzumi, J. Am. Chem. Soc., 2013, 135, 9186-9194.

144 M. Makino, T. Ishizuka, S. Ohzu, J. Hua, H. Kotani and T. Kojima, Inorg. Chem., 2013, 52, 5507-5514.

145 J. Chen, Y.-M. Lee, K. M. Davis, X. Wu, M. S. Seo, K.-B. Cho, H. Yoon, Y. J. Park, S. Fukuzumi, Y. N. Pushkar and W. Nam, J. Am. Chem. Soc., 2013, 135, 6388-6391. 
146 S. D. Hicks, D. Kim, S. Xiong, G. A. Medvedev, J. Caruthers, S. Hong, W. Nam and M. M. Abu-Omar, J. Am. Chem. Soc., 2014, 136, 3680-3686.

147 E. Andris, R. Navrátil, J. Jašík, M. Srnec, M. Rodríguez, M. Costas and J. Roithová, Angew. Chem. Int. Ed., 2019, 58, 9619-9624.

148 J. S. Haynes, J. R. Sams and R. C. Thompson, Can. J. Chem., 1981, 59, 669-678.

149 D. Unjaroen, M. Swart and W. R. Browne, Inorg. Chem., 2017, 56, 470-479.

150 G. R. Fulmer, A. J. M. Miller, N. H. Sherden, H. E. Gottlieb, A. Nudelman, B. M. Stoltz, J. E. Bercaw and K. I. Goldberg, Organometallics, 2010, 29, 2176-2179.

151 G. M. Sheldrick, Acta Crystallogr. Sect. C Struct. Chem., 2015, 71, 3-8.

152 G. M. Sheldrick, Acta Crystallogr. Sect. A Found. Adv., 2015, 71, 3-8.

153 STOE \& CIE GmbH, 2002.

154 A. L. Spek, Acta Crystallogr. Sect. C Struct. Chem., 2015, 71, 9-18. 


\section{Appendix}

\subsection{NMR Spectra}

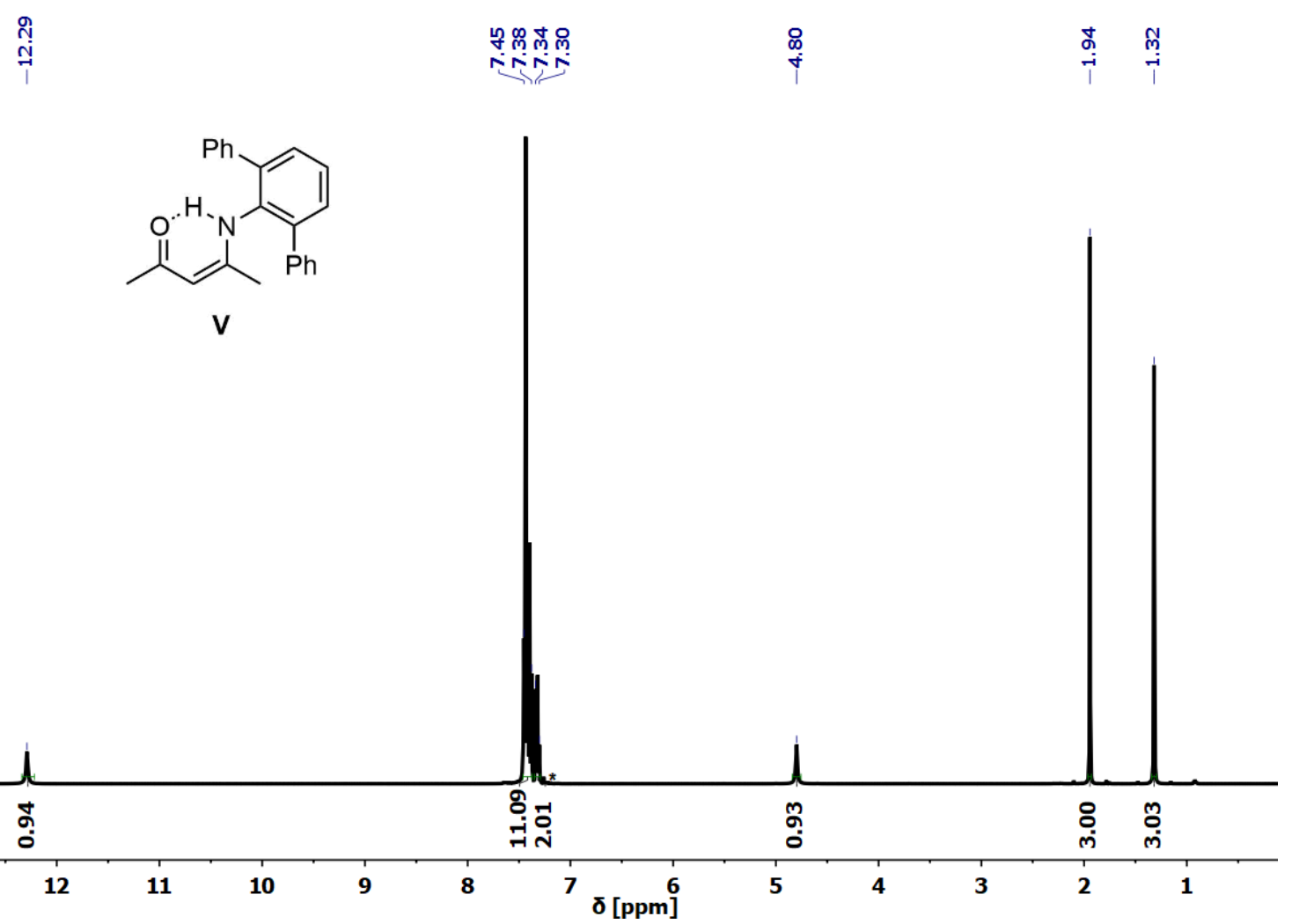

Figure $5.1{ }^{1} \mathrm{H}$ NMR $(300 \mathrm{MHz})$ spectrum of $\mathbf{V}$ in $\mathrm{CDCl}_{3}\left({ }^{*}\right)$.

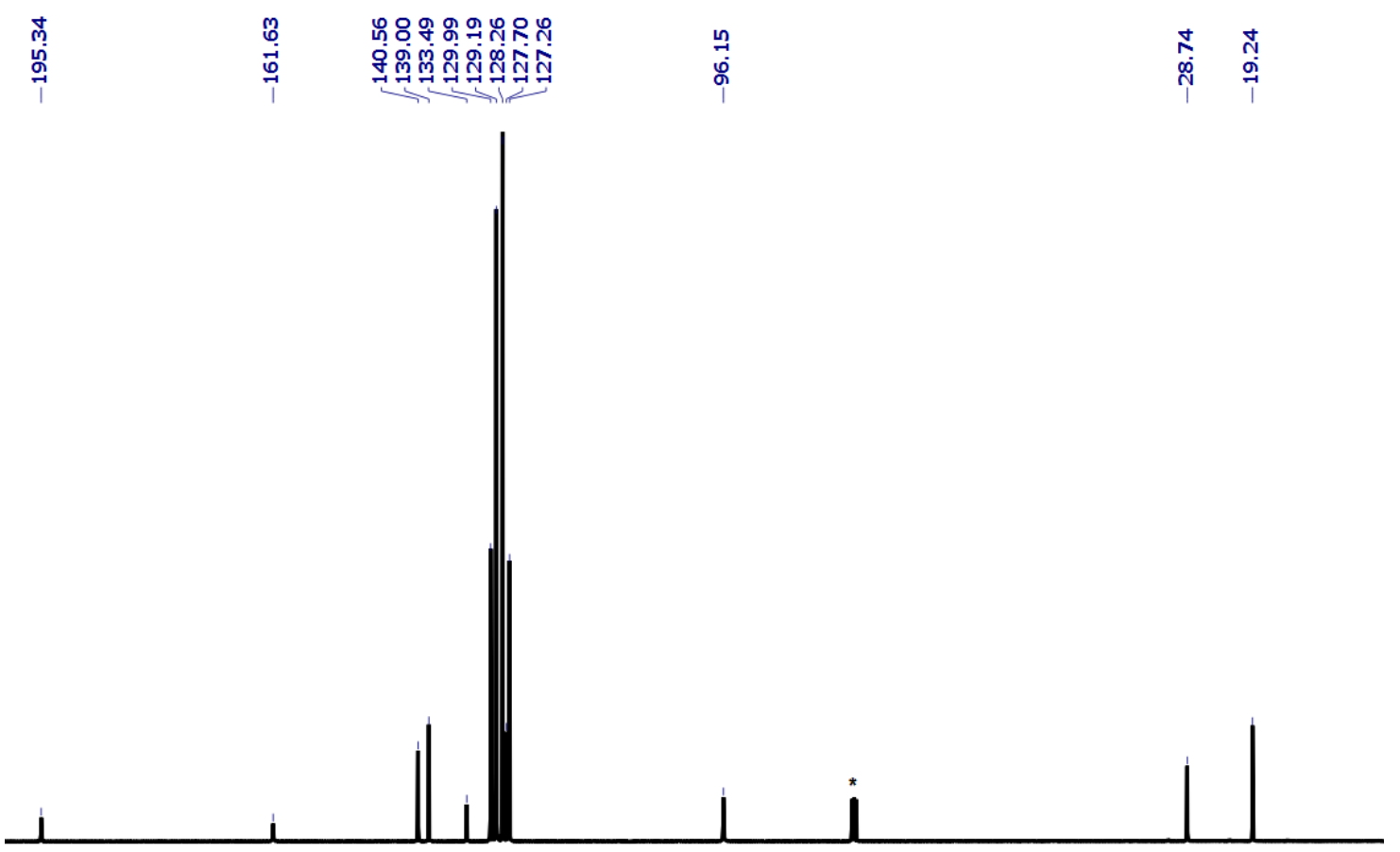

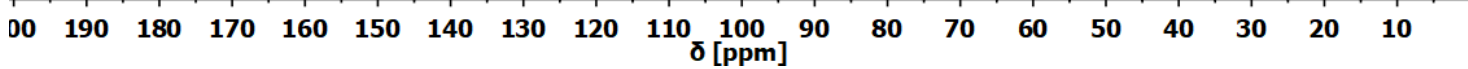

Figure $5.2{ }^{13} \mathrm{C}$ NMR $(101 \mathrm{MHz})$ spectrum of $\mathbf{V}$ in $\mathrm{CDCl}_{3}\left(^{*}\right)$. 

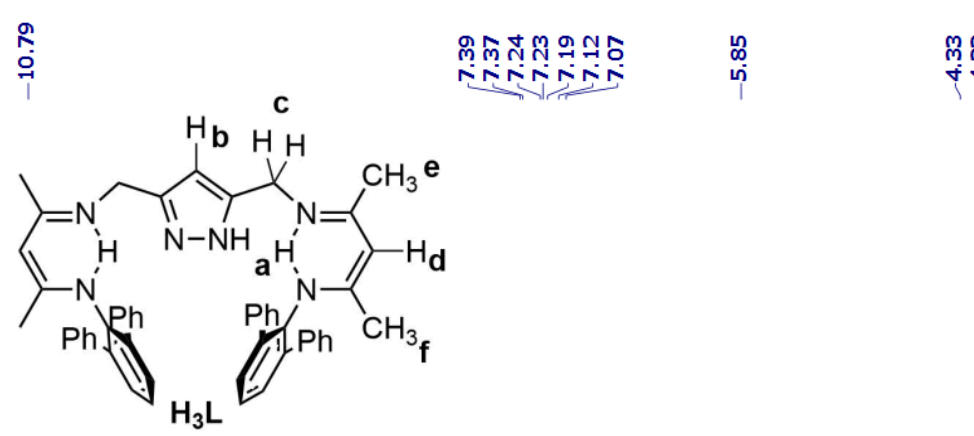

$\operatorname{mind~}$

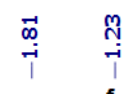

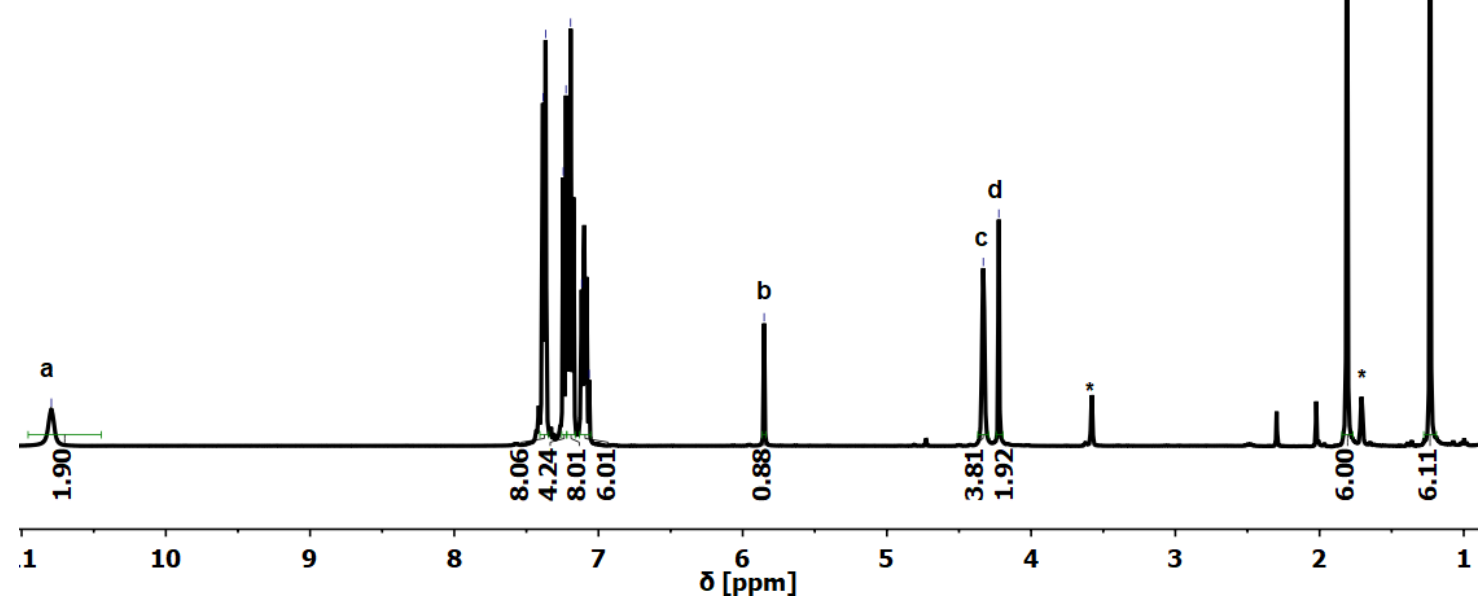

Figure $5.3{ }^{1} \mathrm{H}$ NMR $(400 \mathrm{MHz})$ spectrum of $\mathbf{H}_{3} \mathrm{~L}^{\mathrm{Ph}}$ in THF-d $\left(^{*}\right)$.
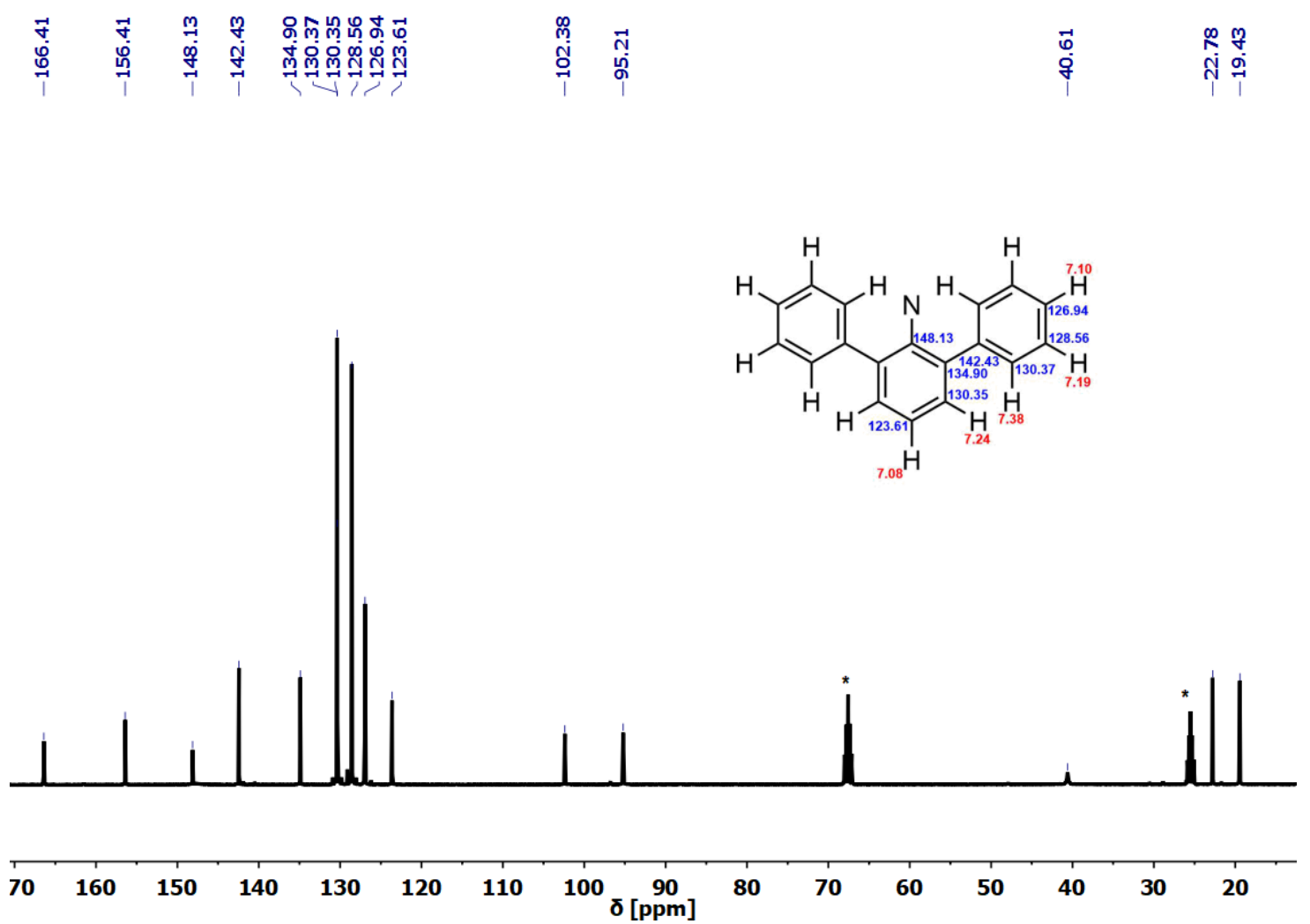

Figure $5.4{ }^{13} \mathrm{C}$ NMR (101 MHz) spectrum of $\mathbf{H}_{3} \mathrm{~L}^{\mathrm{Ph}}$ in THF-d $\left.8{ }^{*}\right)$. 


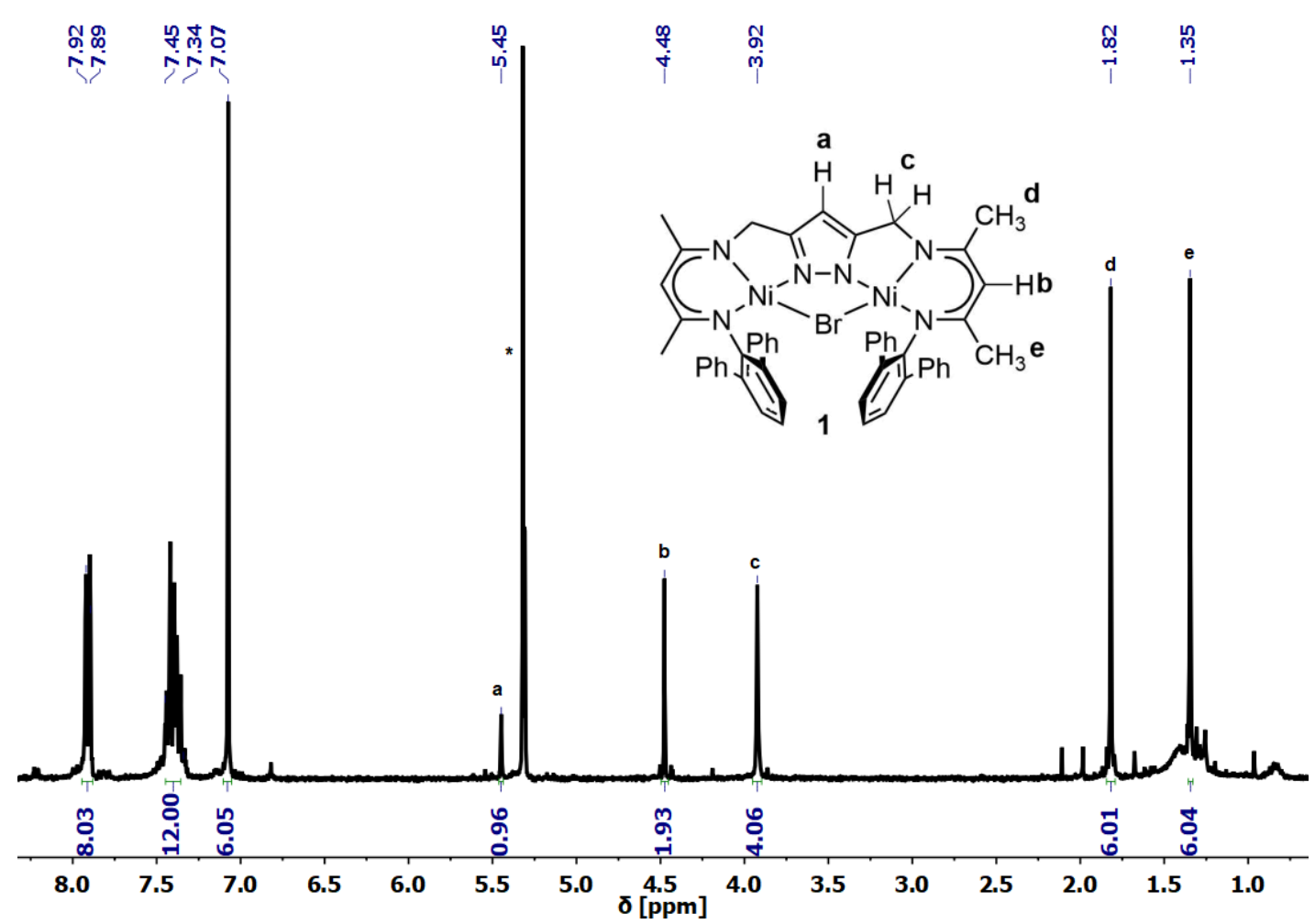

Figure $5.5^{1} \mathrm{H}$ NMR (400 MHz) spectrum of complex 1 in $\mathrm{CD}_{2} \mathrm{Cl}_{2}{ }^{*}$ ).

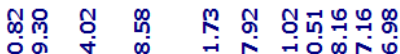

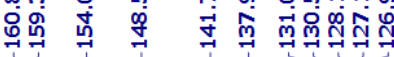

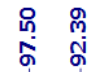

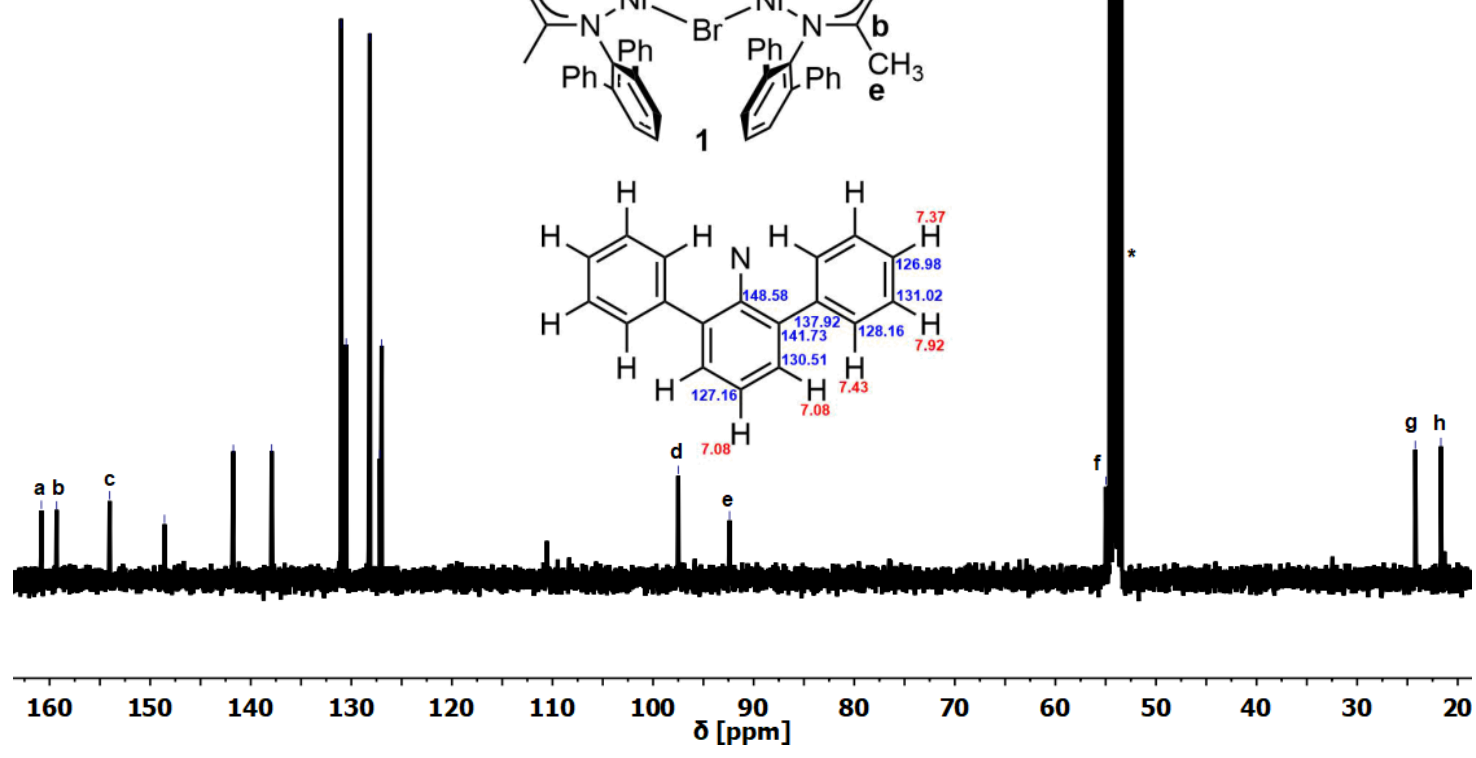

Figure $5.6{ }^{13} \mathrm{C}$ NMR $(101 \mathrm{MHz})$ spectrum of complex 1 in $\mathrm{CD}_{2} \mathrm{Cl}_{2}\left(^{*}\right)$. 


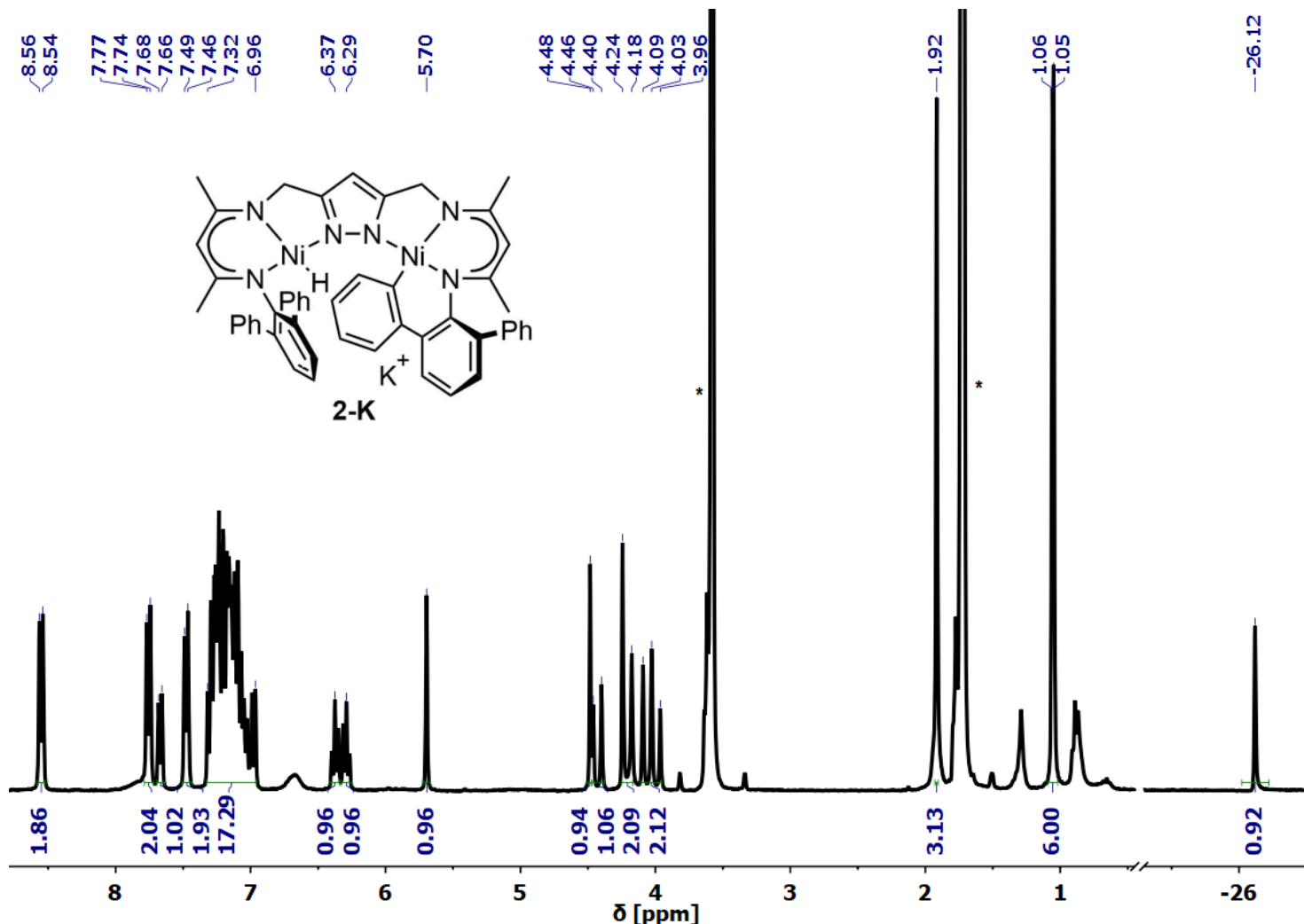

Figure $5.7{ }^{1} \mathrm{H}$ NMR $(400 \mathrm{MHz})$ spectrum of complex 2-K in THF-d $8\left(^{*}\right)$.

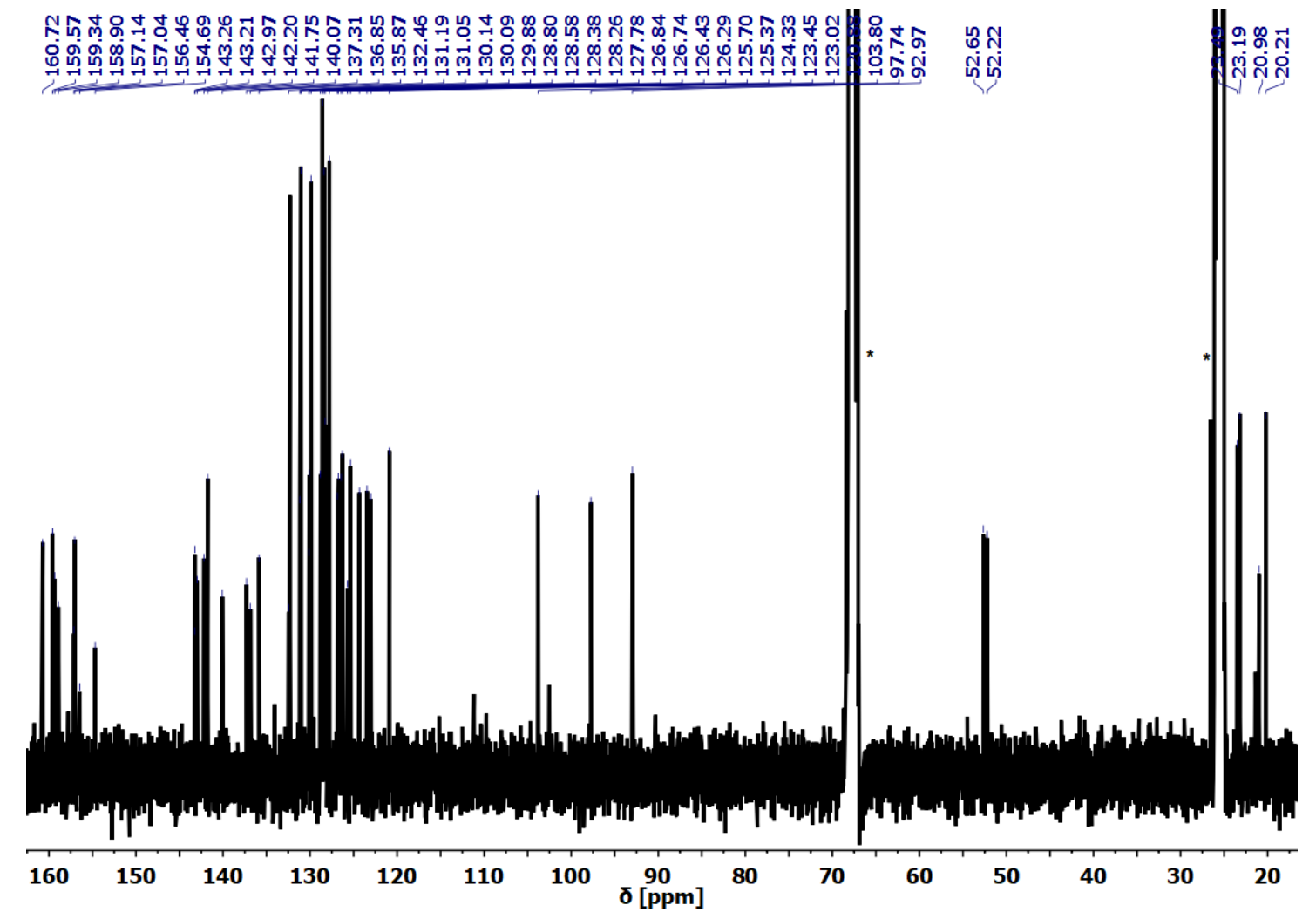

Figure $5.8{ }^{13} \mathrm{C}$ NMR $(101 \mathrm{MHz})$ spectrum of complex 2-K in THF-d $8\left(^{*}\right)$. 


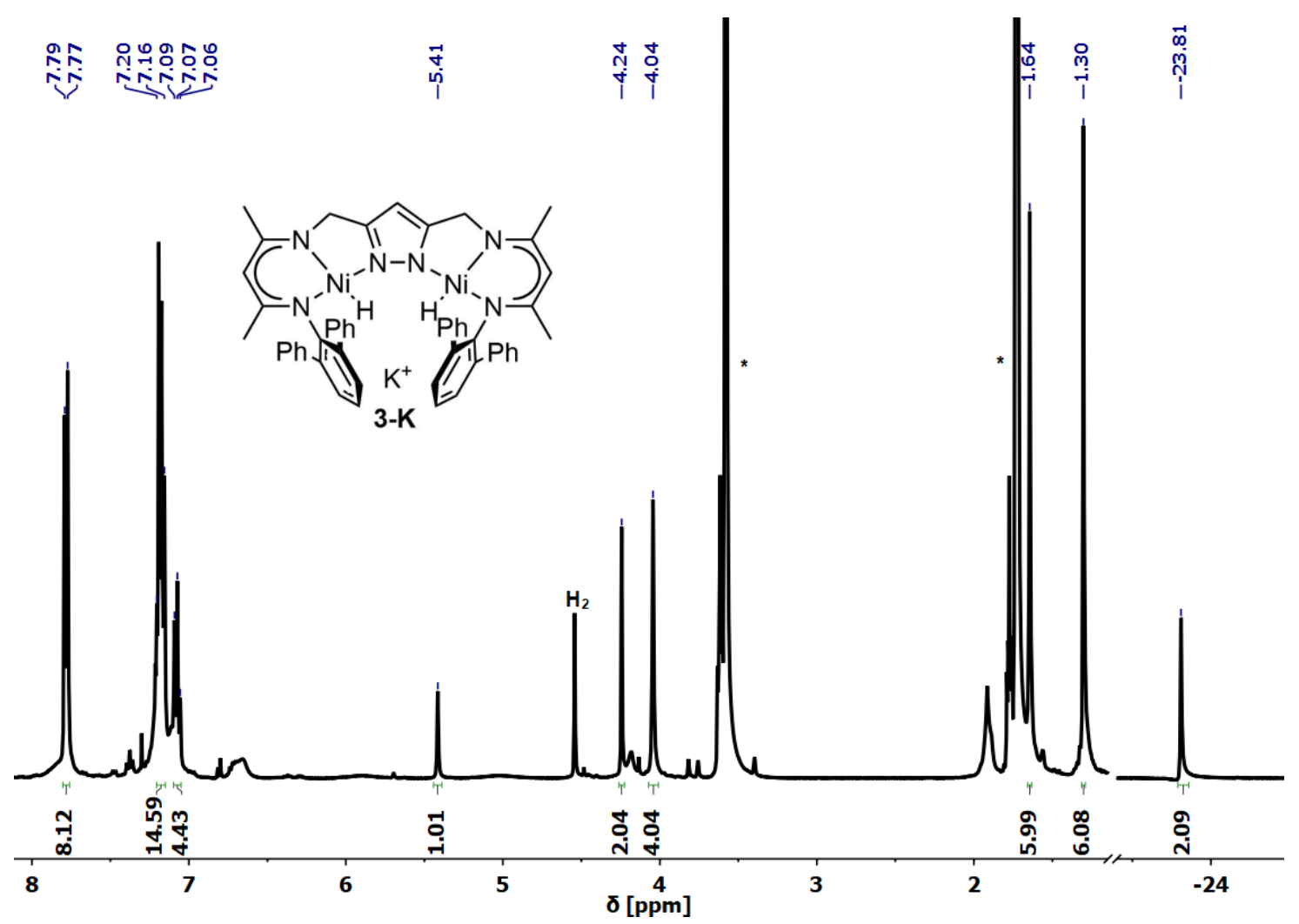

Figure $5.9{ }^{1} \mathrm{H}-\mathrm{NMR}(400 \mathrm{MHz})$ spectrum of $3-\mathrm{K}$ in THF-d $\left.8{ }^{*}\right)$ under $\mathrm{H}_{2}(1 \mathrm{~atm}$.$) .$

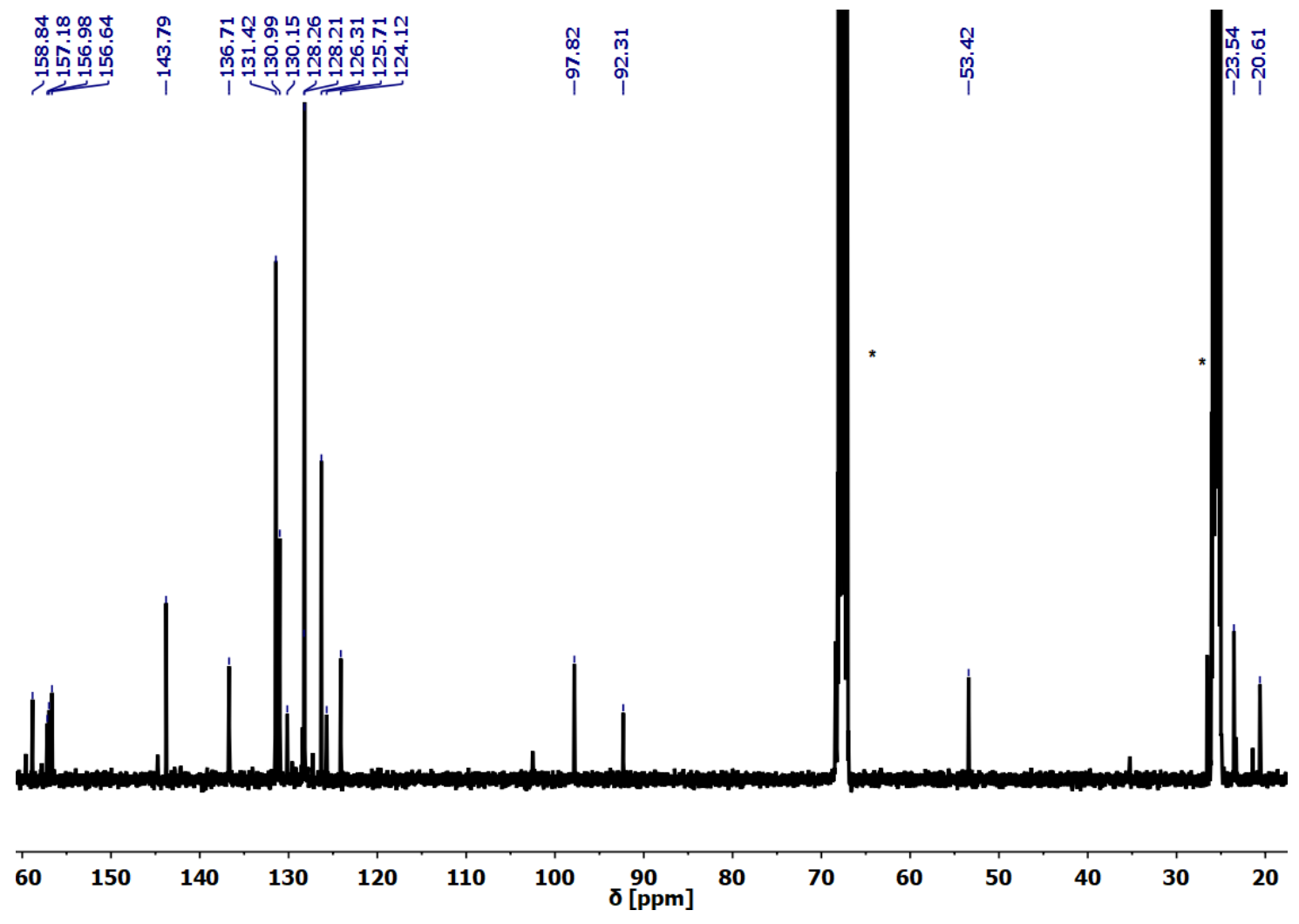

Figure $5.10{ }^{13} \mathrm{C}-\mathrm{NMR}(101 \mathrm{MHz})$ spectrum of 3-K in THF-d $8\left({ }^{*}\right)$ under $\mathrm{H}_{2}$ (1 atm.). 


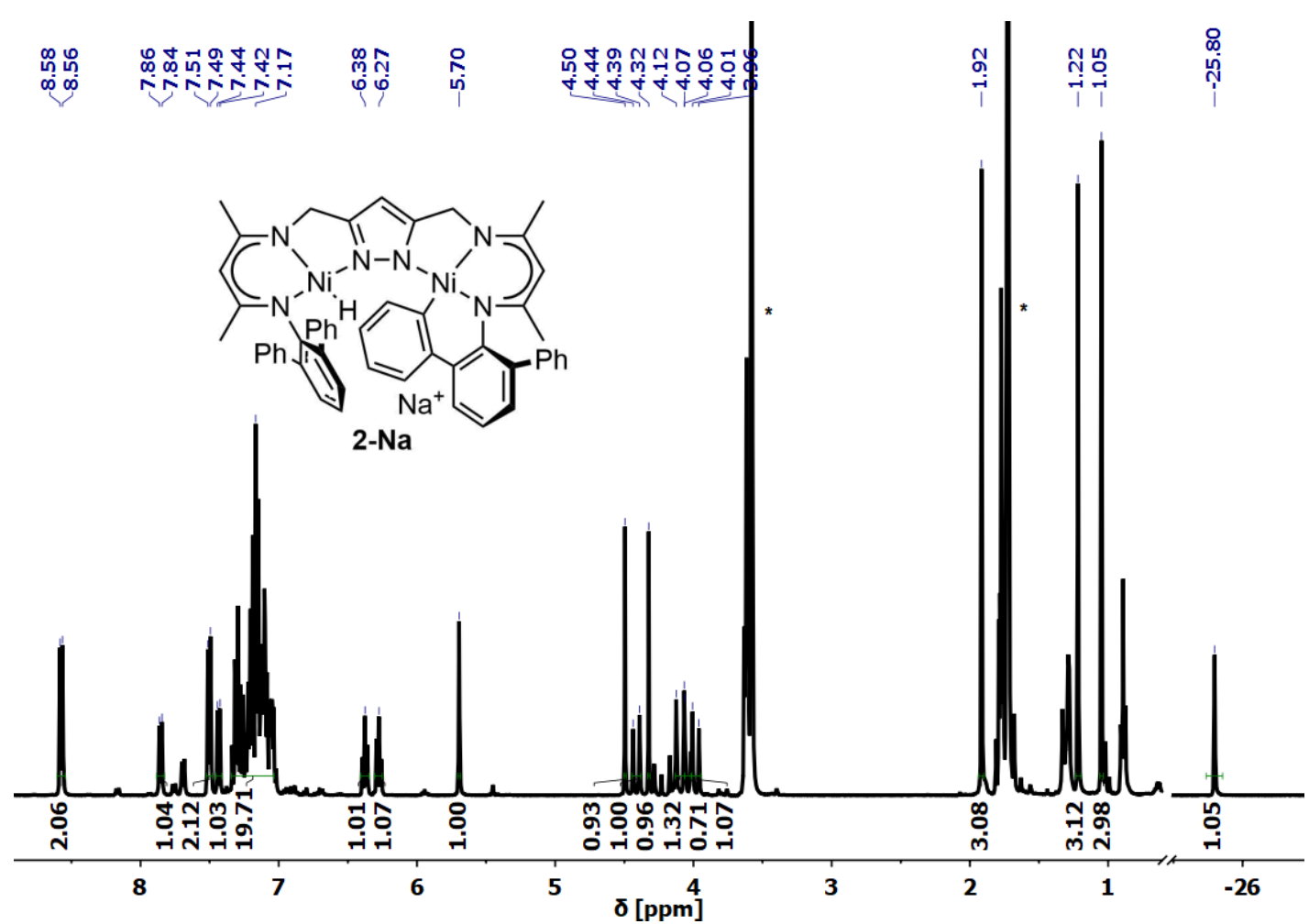

Figure $5.11{ }^{1} \mathrm{H}-\mathrm{NMR}(400 \mathrm{MHz})$ spectrum of $2-\mathrm{Na}$ in THF- $\mathrm{d}_{8}\left({ }^{*}\right)$.

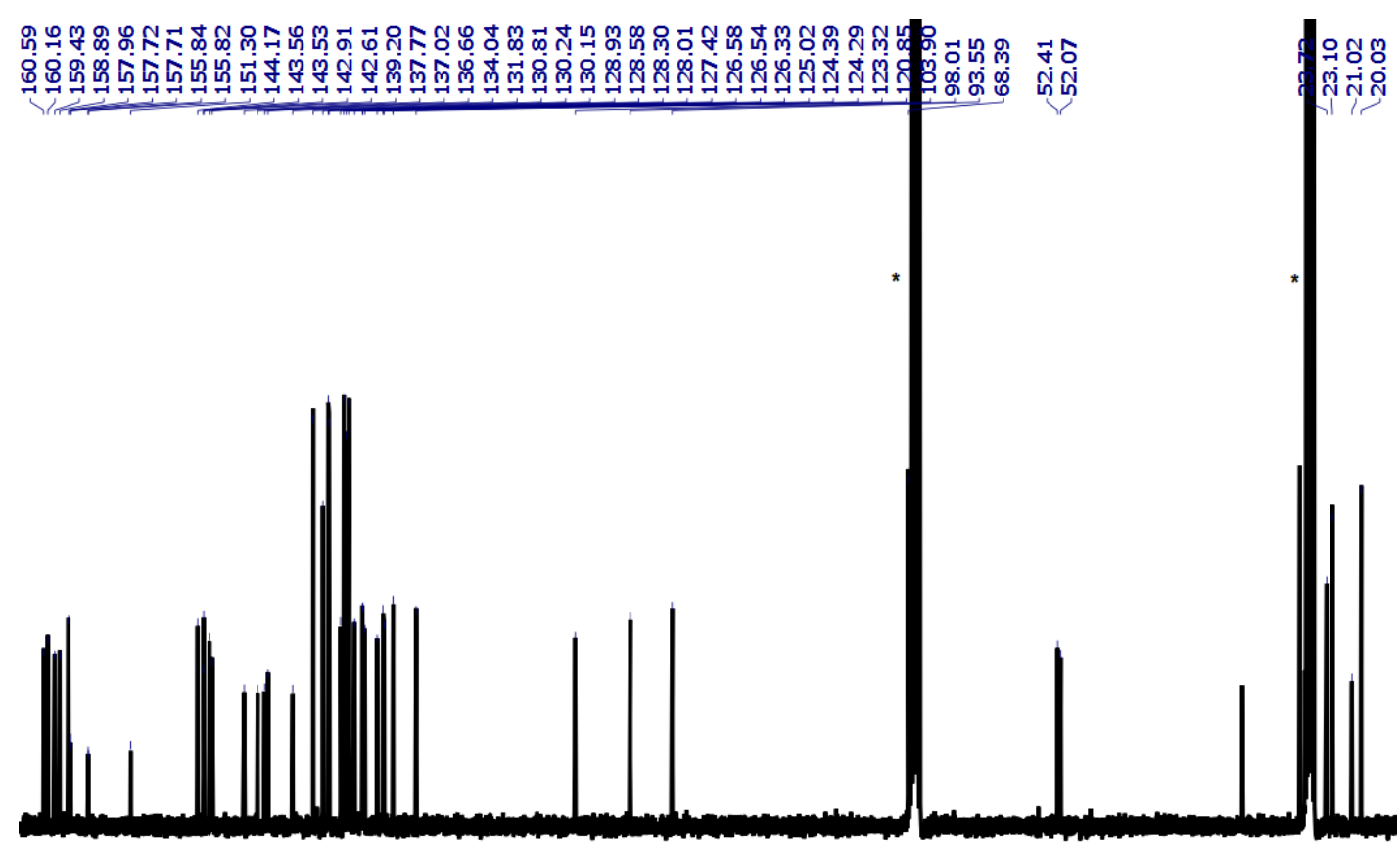

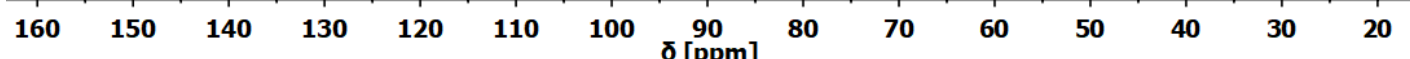

Figure $5.12{ }^{13} \mathrm{C}-\mathrm{NMR}(101 \mathrm{MHz})$ spectrum of $2-\mathrm{Na}$ in THF- $\mathrm{d}_{8}\left({ }^{*}\right)$. 


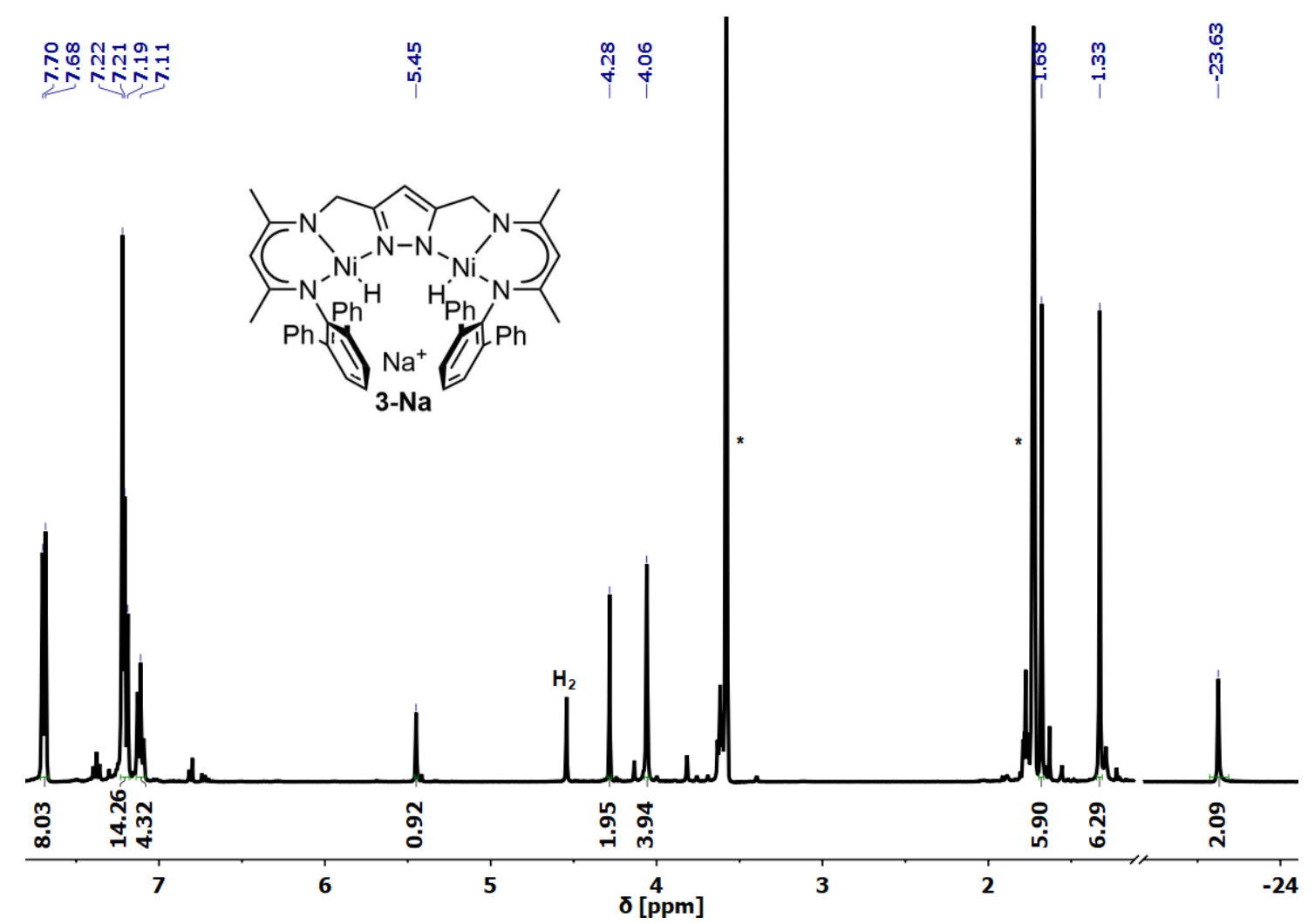

Figure $5.13{ }^{1} \mathrm{H}$ NMR spectrum $(400 \mathrm{MHz})$ of 3-Na in THF-d $8\left({ }^{*}\right)$ under $\mathrm{H}_{2}(1 \mathrm{~atm}$.$) .$

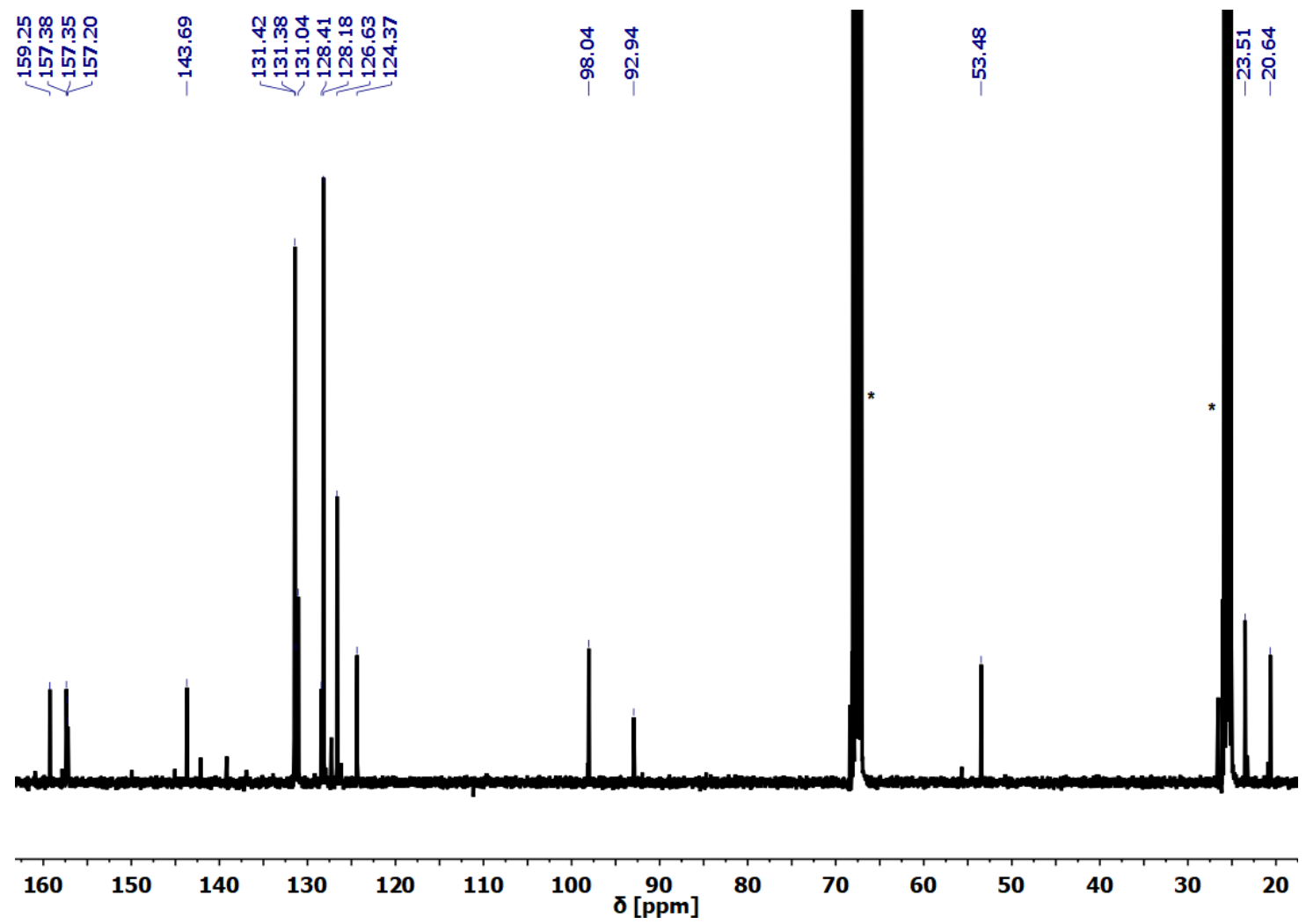

Figure $5.14{ }^{13} \mathrm{C}-\mathrm{NMR}(101 \mathrm{MHz})$ spectrum of 3-Na in THF- $\mathrm{d}_{8}\left({ }^{*}\right)$ under $\mathrm{H}_{2}(1 \mathrm{~atm}$.$) .$ 


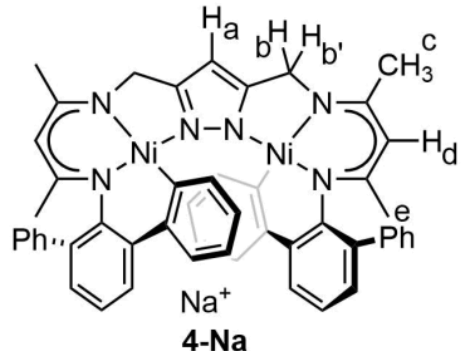
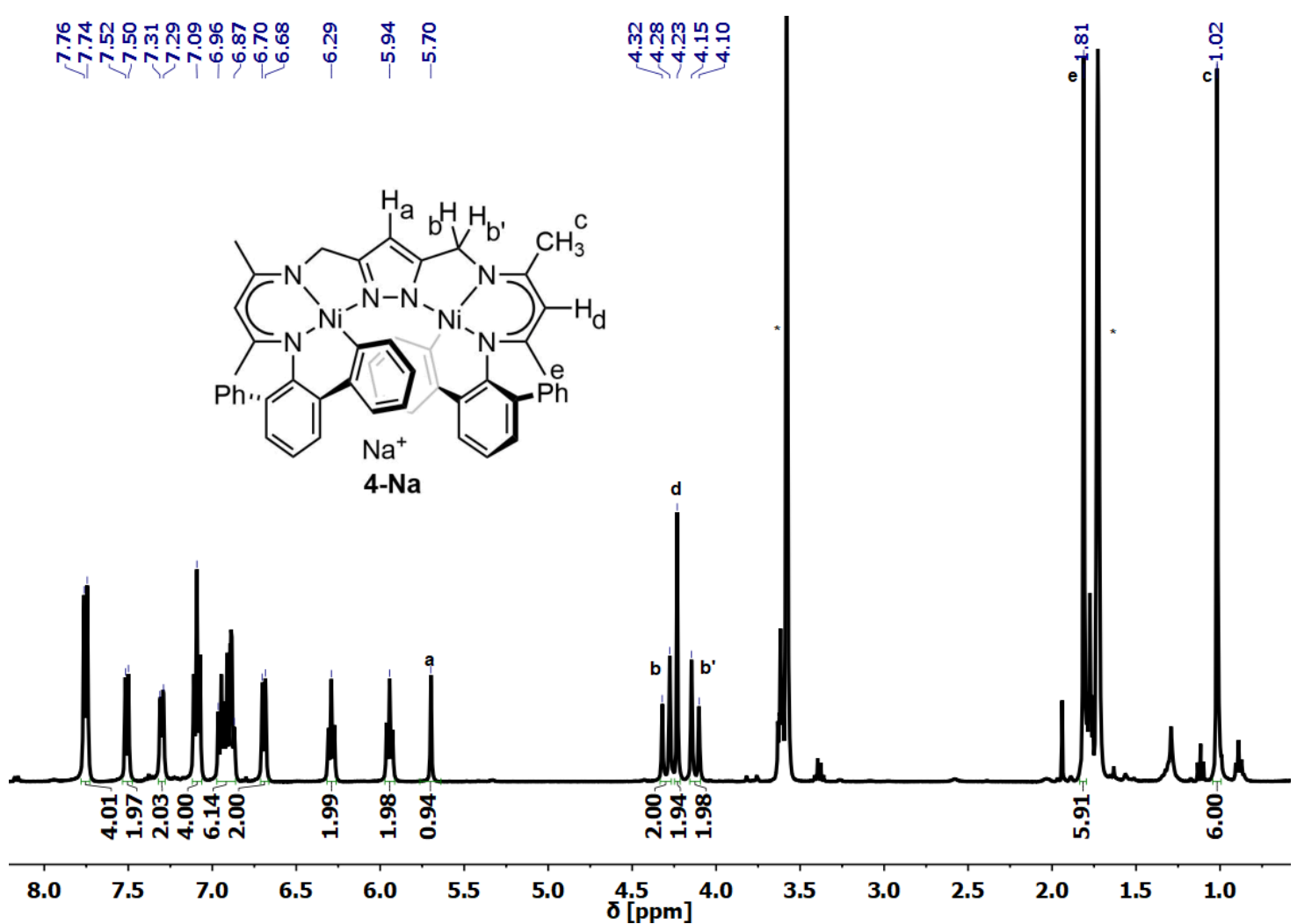

Figure $5.15^{1} \mathrm{H}$ NMR spectrum $(400 \mathrm{MHz})$ of complex 4-Na in THF-d $\left.8{ }^{*}\right)$.

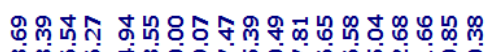

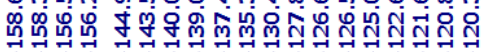
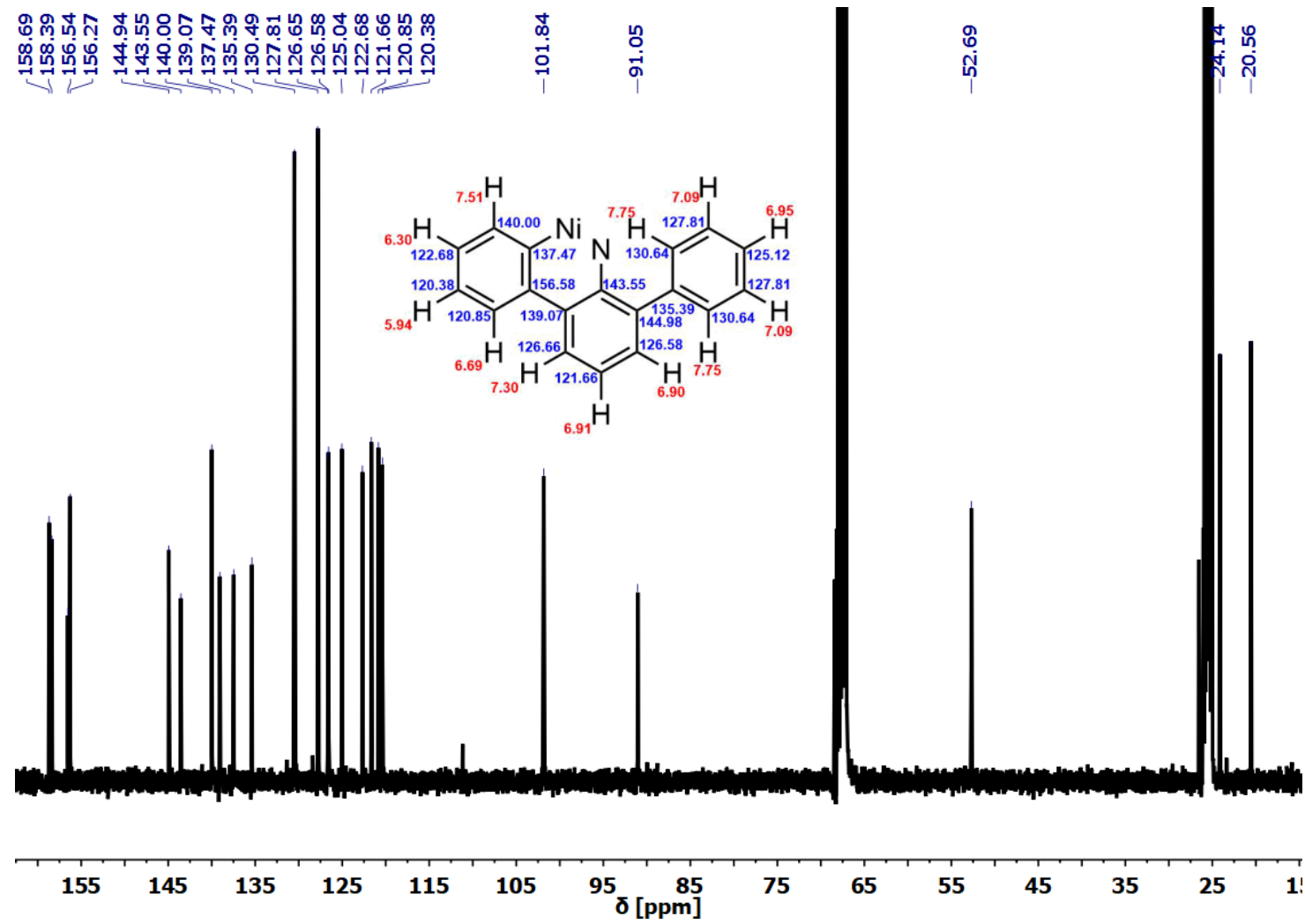

Figure $5.16{ }^{13} \mathrm{C}-\mathrm{NMR}(101 \mathrm{MHz})$ spectrum of 4-Na in THF- $\mathrm{d}_{8}\left({ }^{*}\right)$. 


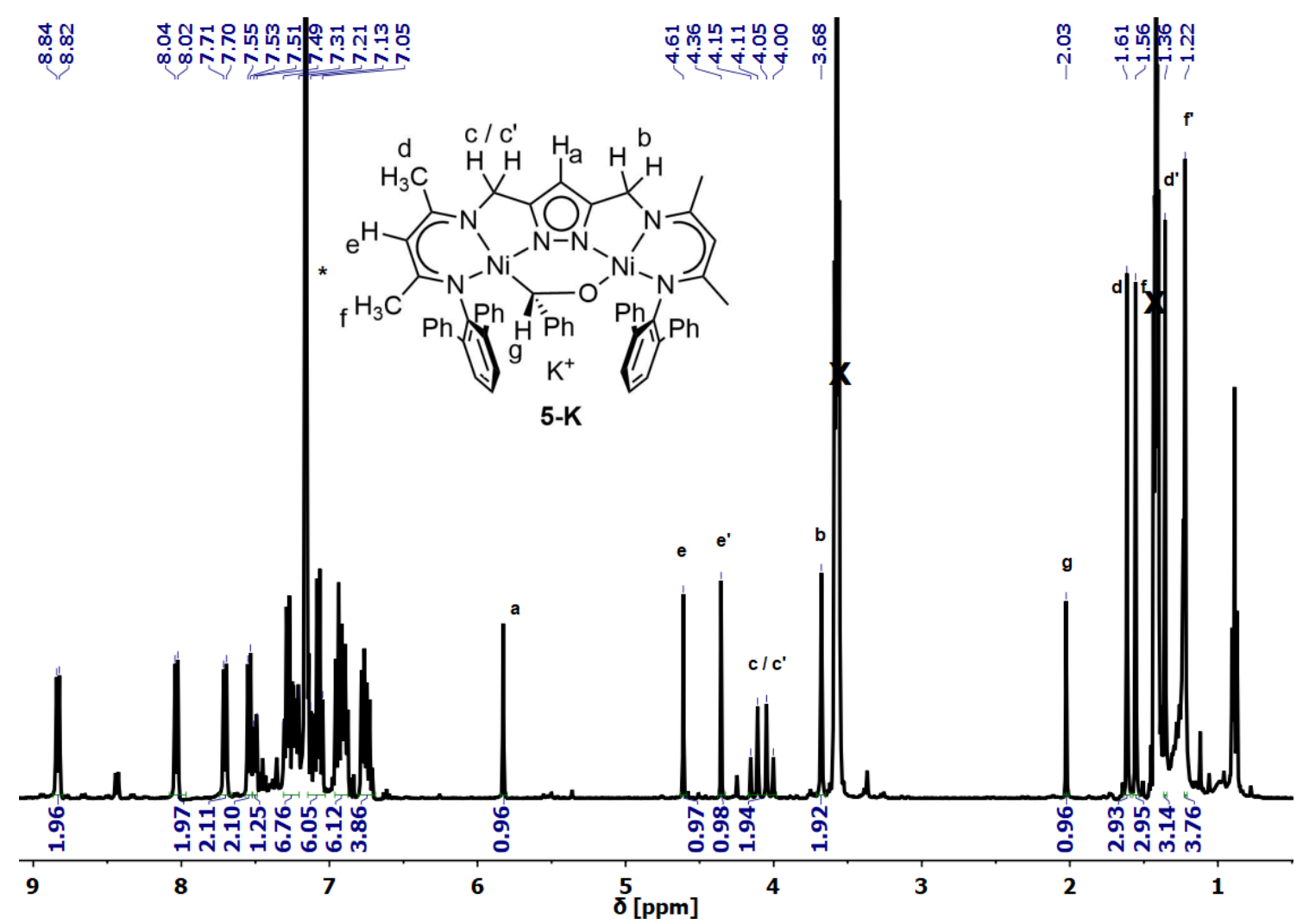

Figure $5.17{ }^{1} \mathrm{H}$ NMR spectrum $(400 \mathrm{MHz})$ of complex $5-\mathrm{K}$ in $\mathrm{C}_{6} \mathrm{D}_{6}\left({ }^{*}\right)$.

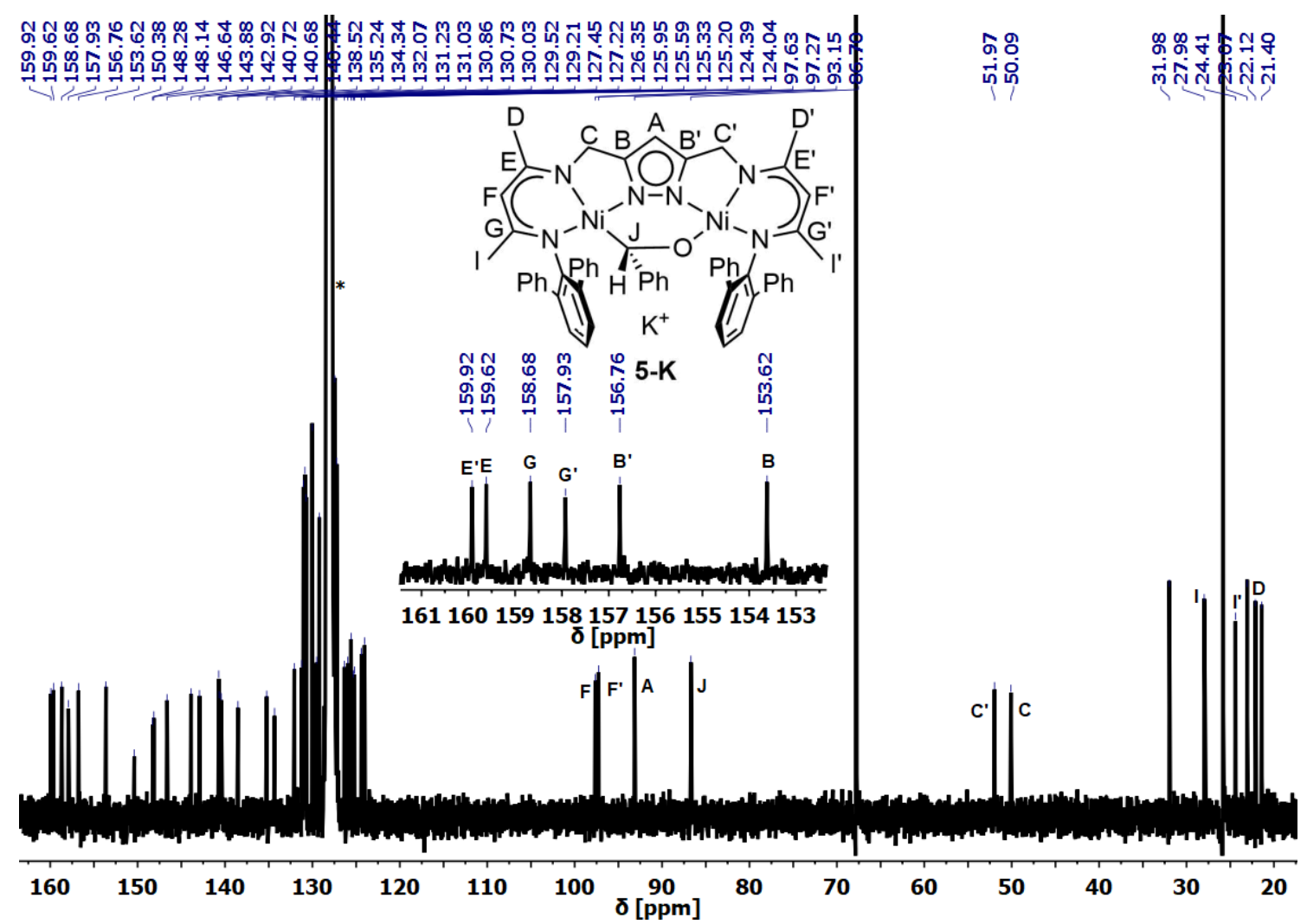

Figure $5.18{ }^{13} \mathrm{C}\left\{{ }^{1} \mathrm{H}\right\}-N M R$ spectrum $(101 \mathrm{MHz})$ of complex $5-\mathrm{K}$ in $\mathrm{C}_{6} \mathrm{D}_{6}\left({ }^{*}\right)$. 


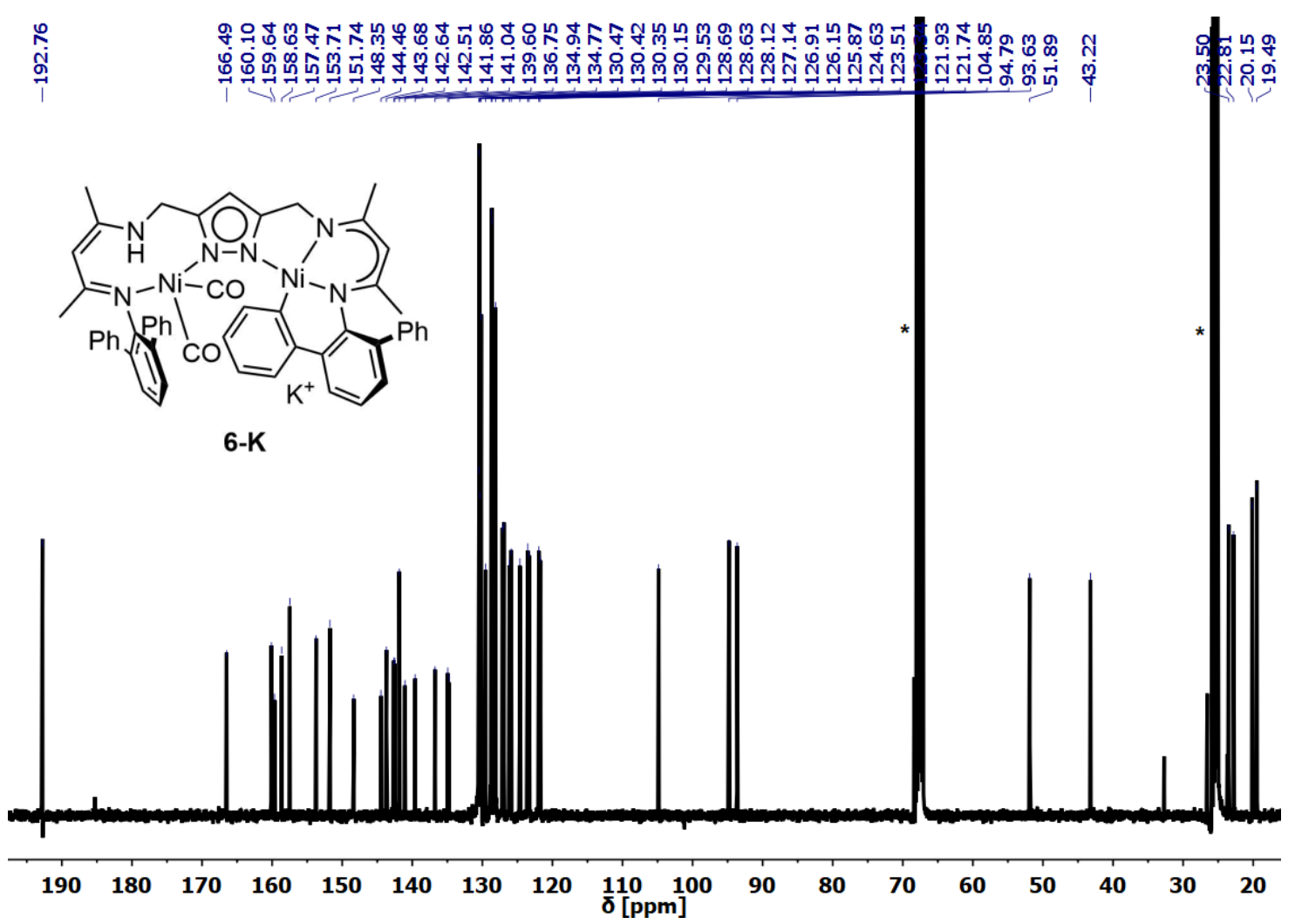

Figure $5.19{ }^{13} \mathrm{C}\left\{{ }^{1} \mathrm{H}\right\}-\mathrm{NMR}$ spectrum $(101 \mathrm{MHz})$ of complex 6-K in THF-d $\left.8{ }^{*}\right)$.

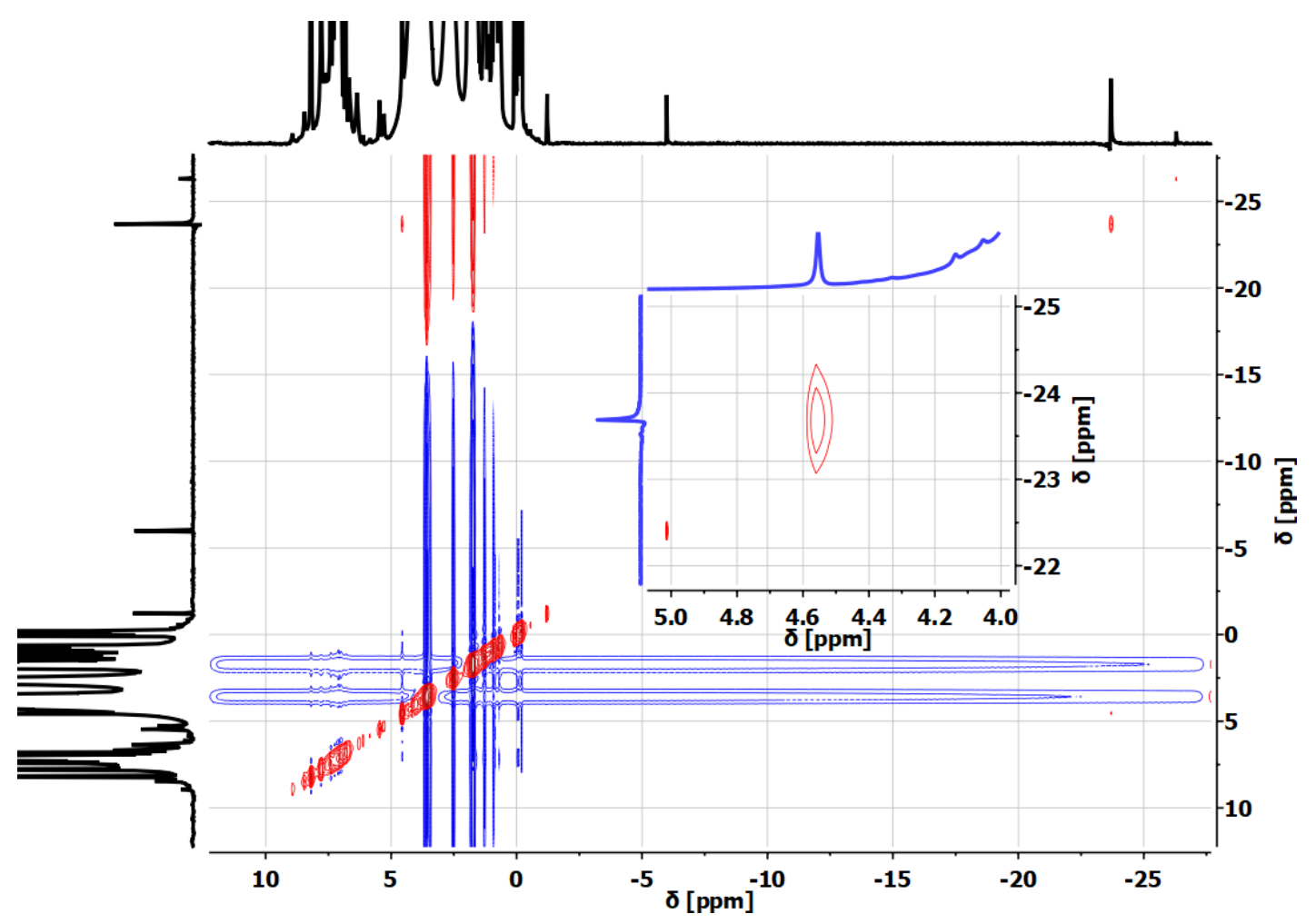

Figure $5.20{ }^{1} \mathrm{H}-{ }^{1} \mathrm{H}$ EXSY (500 MHz) spectrum of 3-K(crypt) under $\mathrm{H}_{2}(1 \mathrm{~atm}$.) in THF-d8 at $-30^{\circ} \mathrm{C}$. 


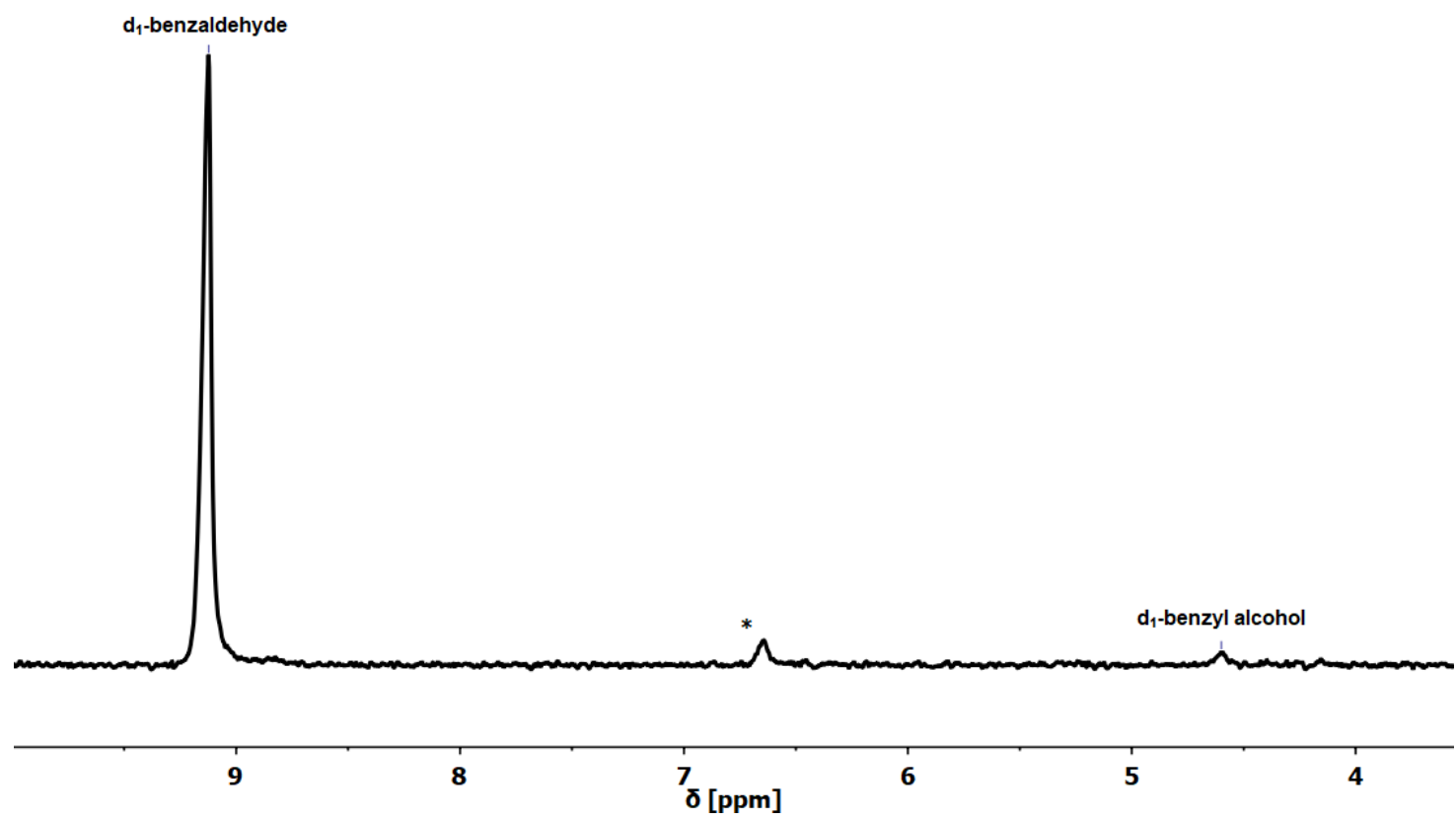

Figure $5.21{ }^{2} \mathrm{H}-\mathrm{NMR}(46 \mathrm{MHz})$ spectra of the reaction of $\mathrm{d}_{1}$-benzaldehyde with $2-\mathrm{K}$ in THF- $\mathrm{d}_{8}\left({ }^{*}\right)$.

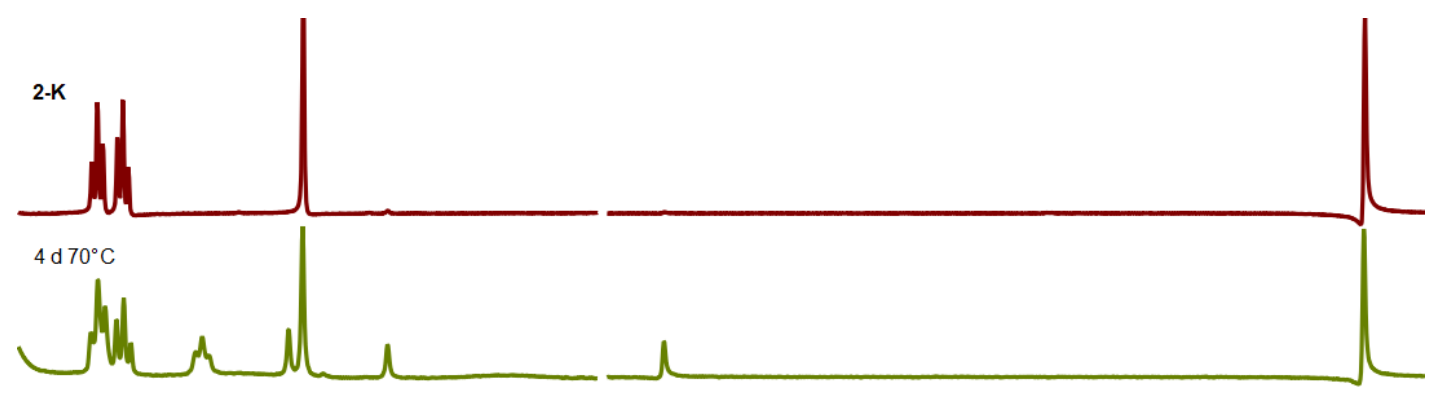

$\mathrm{H}_{2} / \mathrm{N}_{2}$ Exchange $6 \mathrm{~d} 70^{\circ} \mathrm{C}$

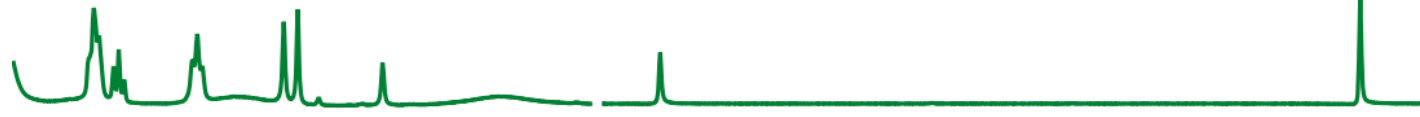

Pump-Freeze after $8 \mathrm{~d} 70^{\circ} \mathrm{C}$
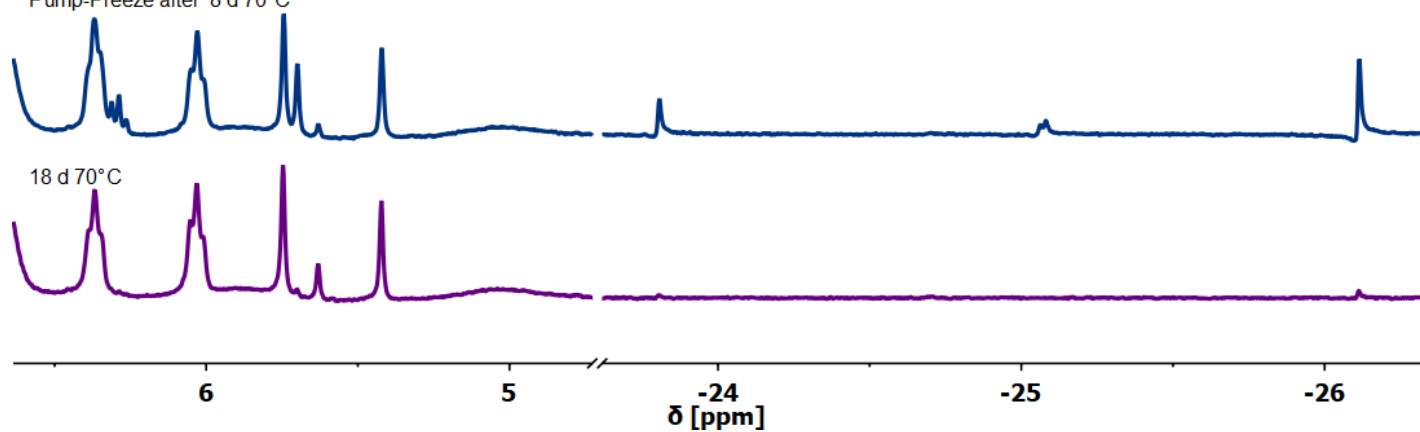

Figure 5.22 Time dependent ${ }^{1} \mathrm{H}-\mathrm{NMR}(300 \mathrm{MHz})$ spectra of 2-K at $70^{\circ} \mathrm{C}$ in THF-d8 over time. 


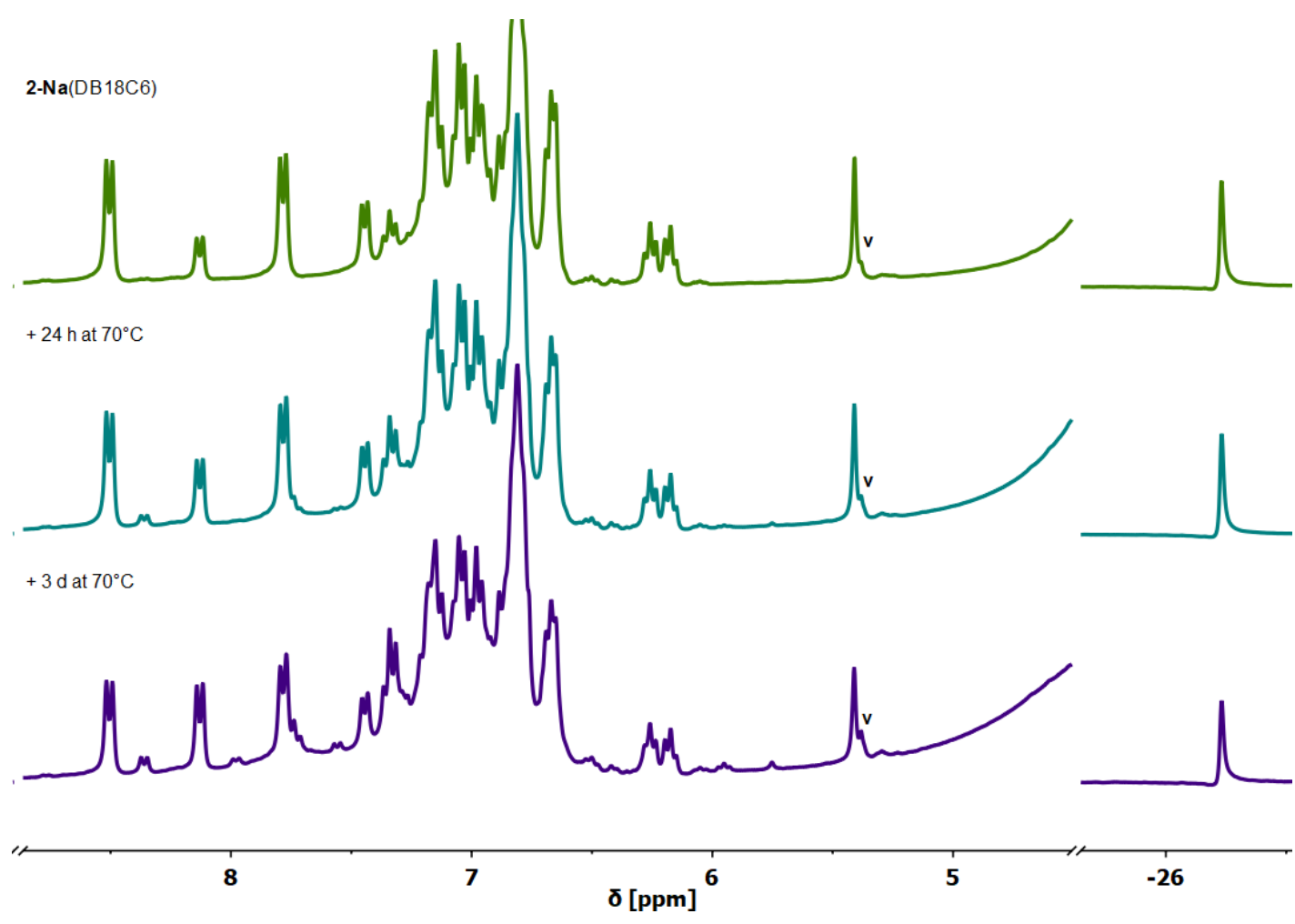

Figure 5.23 Time dependent ${ }^{1} \mathrm{H}-\mathrm{NMR}(300 \mathrm{MHz})$ spectra of $2-\mathrm{Na}(\mathrm{DB} 18 \mathrm{C} 6)$ at $70^{\circ} \mathrm{C}$ in THF-d 8 over time; marked $(\mathbf{V})$ is $1-\mathbf{O H}$.

$+\mathrm{H}_{2}(3 \mathrm{~atm})$

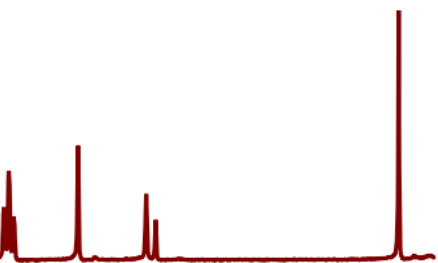

$41 \%\left[\mathrm{Ni}_{2}-\mathrm{H}_{2}\right]$
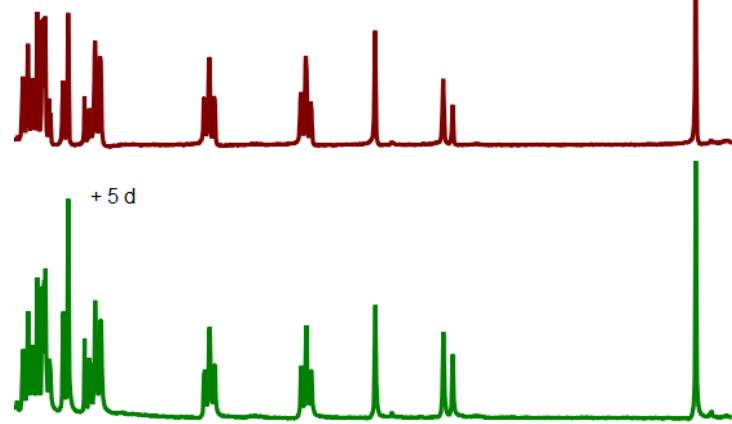

$45 \%\left[\mathrm{Ni}_{2}-\mathrm{H}_{2}\right]$

$+18 d$

$61 \%\left[\mathrm{Ni}_{2}-\mathrm{H}_{2}\right]$
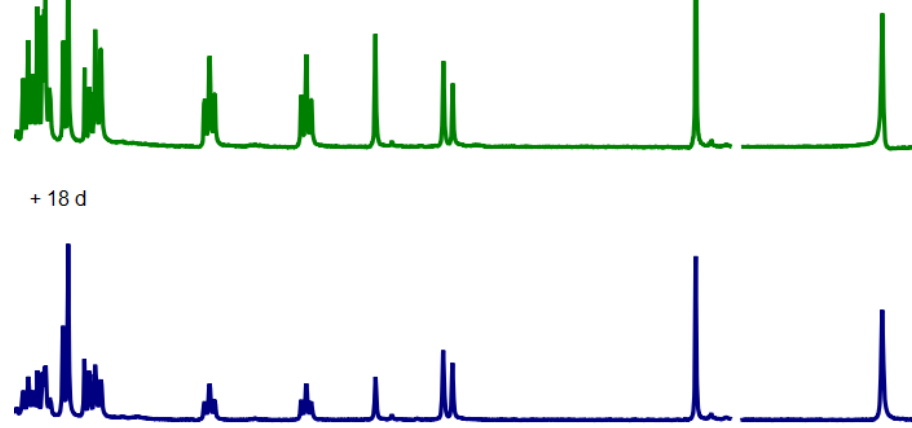

6

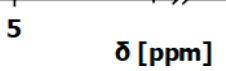

$-24$

$-25$

Figure 5.24 Time dependent ${ }^{1} \mathrm{H}-\mathrm{NMR}(300 \mathrm{MHz})$ spectra of $\mathbf{5}-\mathrm{Na}$ under $\mathrm{H}_{2}(3 \mathrm{~atm}$.) in THF-d8. 


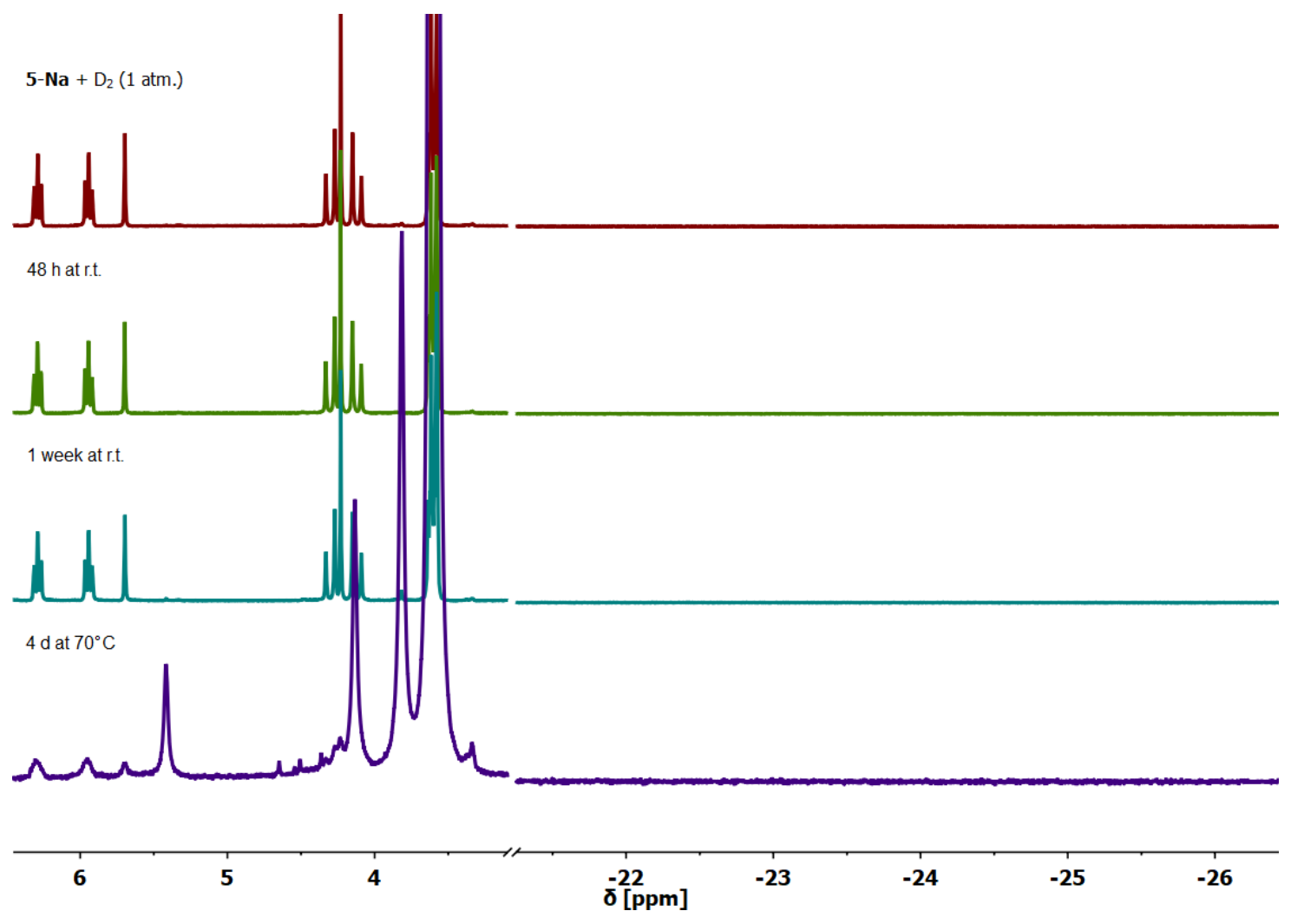

Figure 5.25 Time dependent ${ }^{1} \mathrm{H}-\mathrm{NMR}\left(300 \mathrm{MHz}\right.$ ) spectra of $5-\mathrm{Na}$ under $\mathrm{D}_{2}(1 \mathrm{~atm}$.$) in THF-d8.$

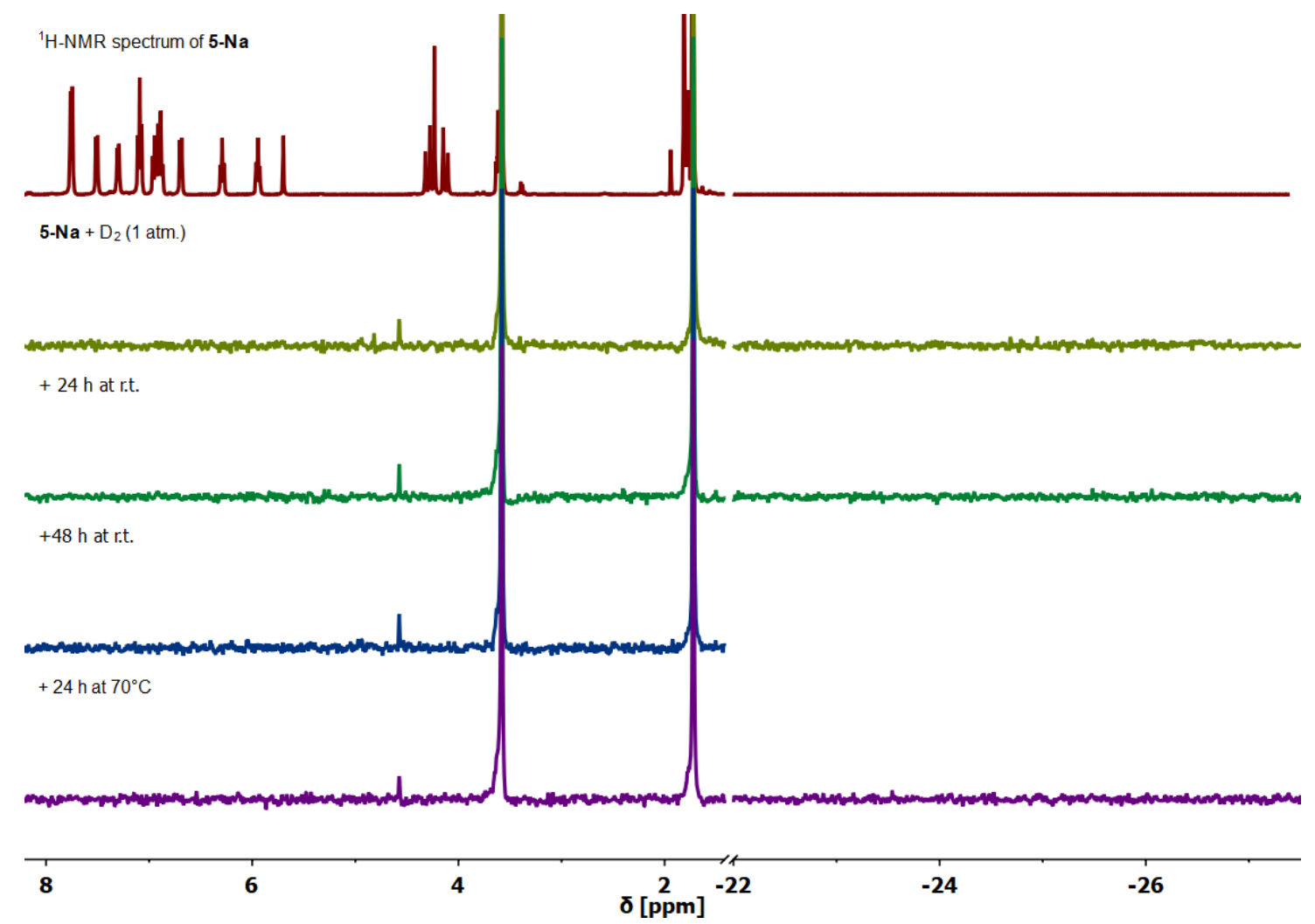

Figure 5.26 Time dependent ${ }^{2} \mathrm{H}-\mathrm{NMR}(46 \mathrm{MHz})$ spectra of 5-Na under $\mathrm{D}_{2}(1 \mathrm{~atm}$.$) in THF-d8.$ 


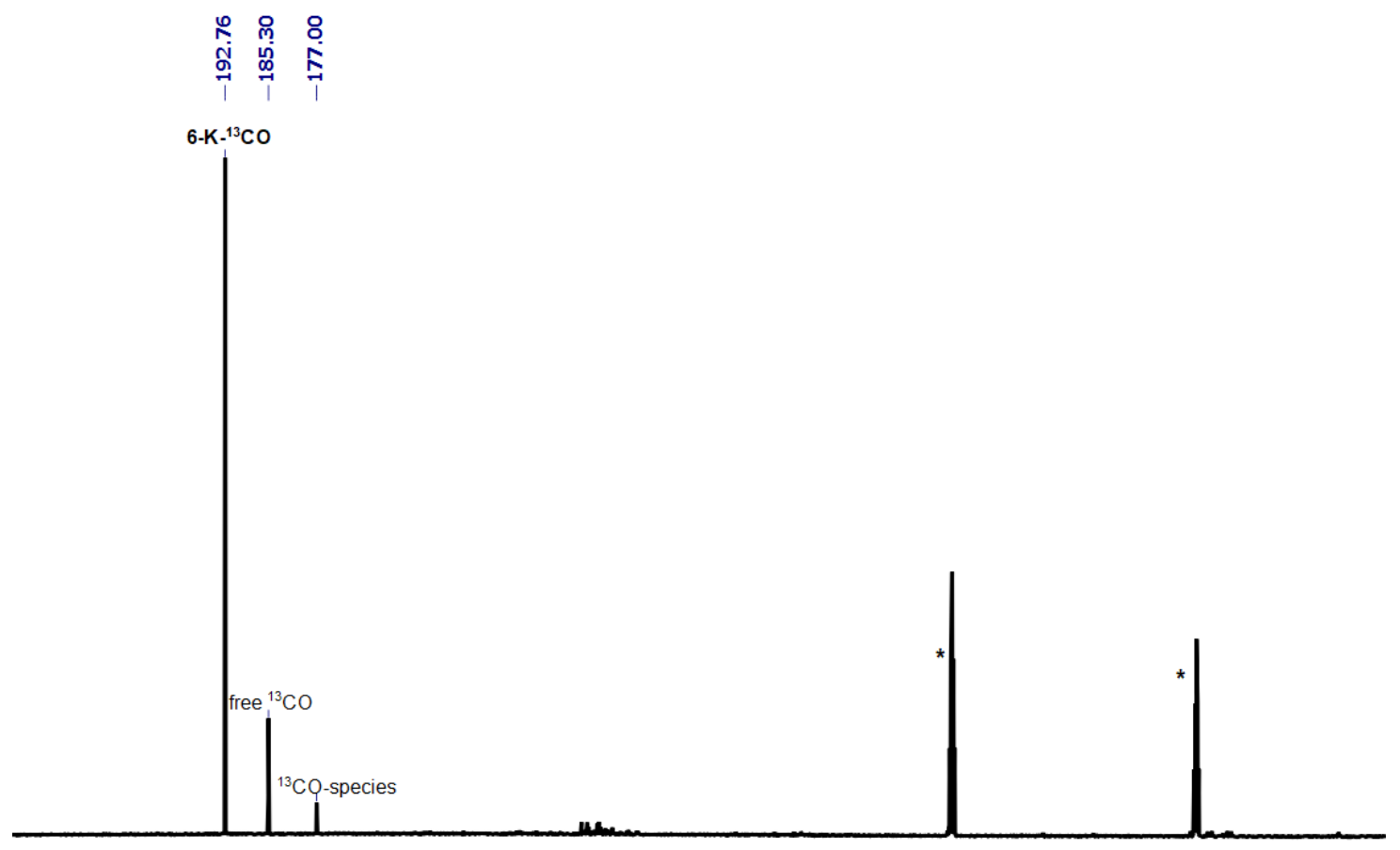

$\begin{array}{llllllllllllllllllllllllllll}220 & 210 & 200 & 190 & 180 & 170 & 160 & 150 & 140 & 130 & 120 & 110 & 100 & 90 & 80 & 70 & 60 & 50 & 40 & 30 & 20 & 10 & 0\end{array}$

Figure $5.27{ }^{13} \mathrm{C}-\mathrm{NMR}(101 \mathrm{MHz})$ spectrum of the reaction of $2-\mathrm{K}$ with ${ }^{13} \mathrm{CO}(1 \mathrm{~atm})$ to $6-\mathrm{K}-{ }^{13} \mathrm{CO}$ in THF-d $8\left(^{*}\right)$.

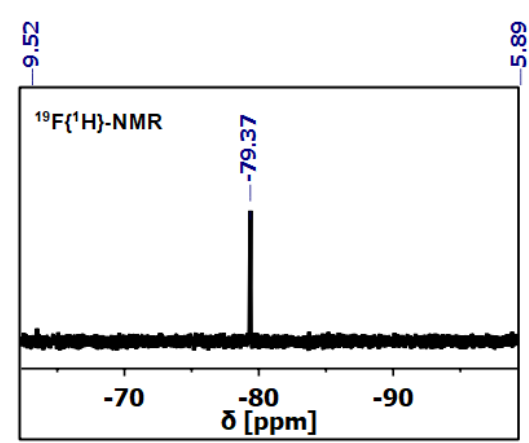

$\infty$
$\infty$
மீ

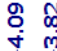

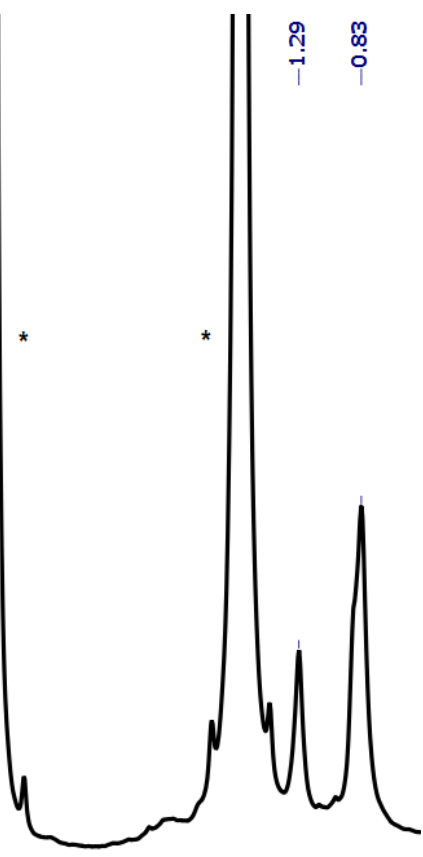

10

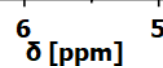

$5 \quad 4 \quad 3$

21

Figure 5.28 ${ }^{1} \mathrm{H}-\mathrm{NMR}$ spectrum of the crude product of the reaction of 2-K with lutidinium triflate in THF-d 8 at $\mathrm{rt}$; inset shows the ${ }^{19} \mathrm{~F}-\mathrm{NMR}$ spectrum of the crude product. 


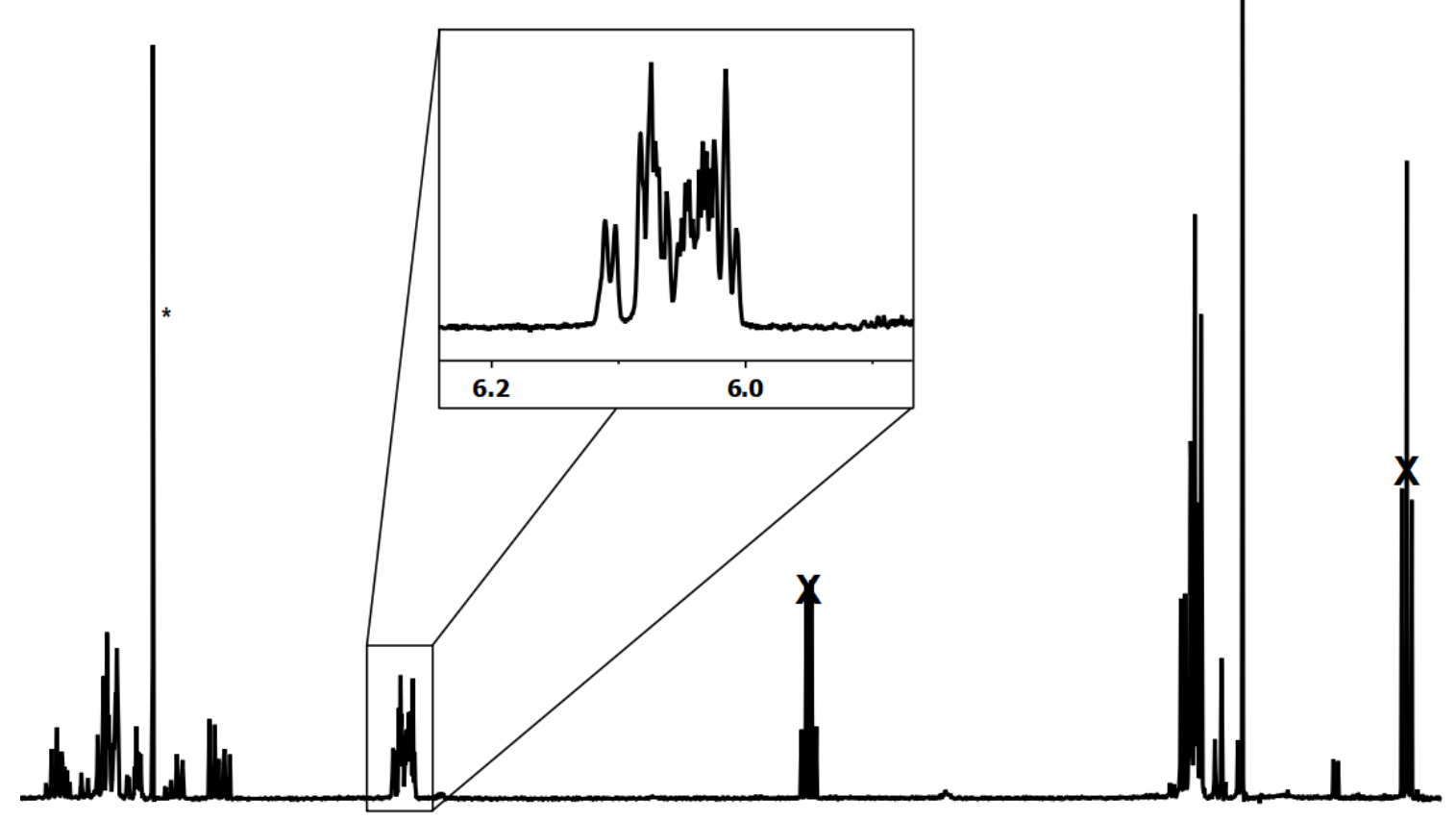

7 6

$5 \quad \delta[p p m]$

3

2

Figure $5.29{ }^{1} \mathrm{H}-\mathrm{NMR}(300 \mathrm{MHz})$ spectrum of the regio-isomers of the synthesis of $\mathbf{p z}^{\mathrm{Me}}{ }_{4} \mathrm{Lut}$ in $\mathrm{CDCl}_{3}\left({ }^{*}\right)$.

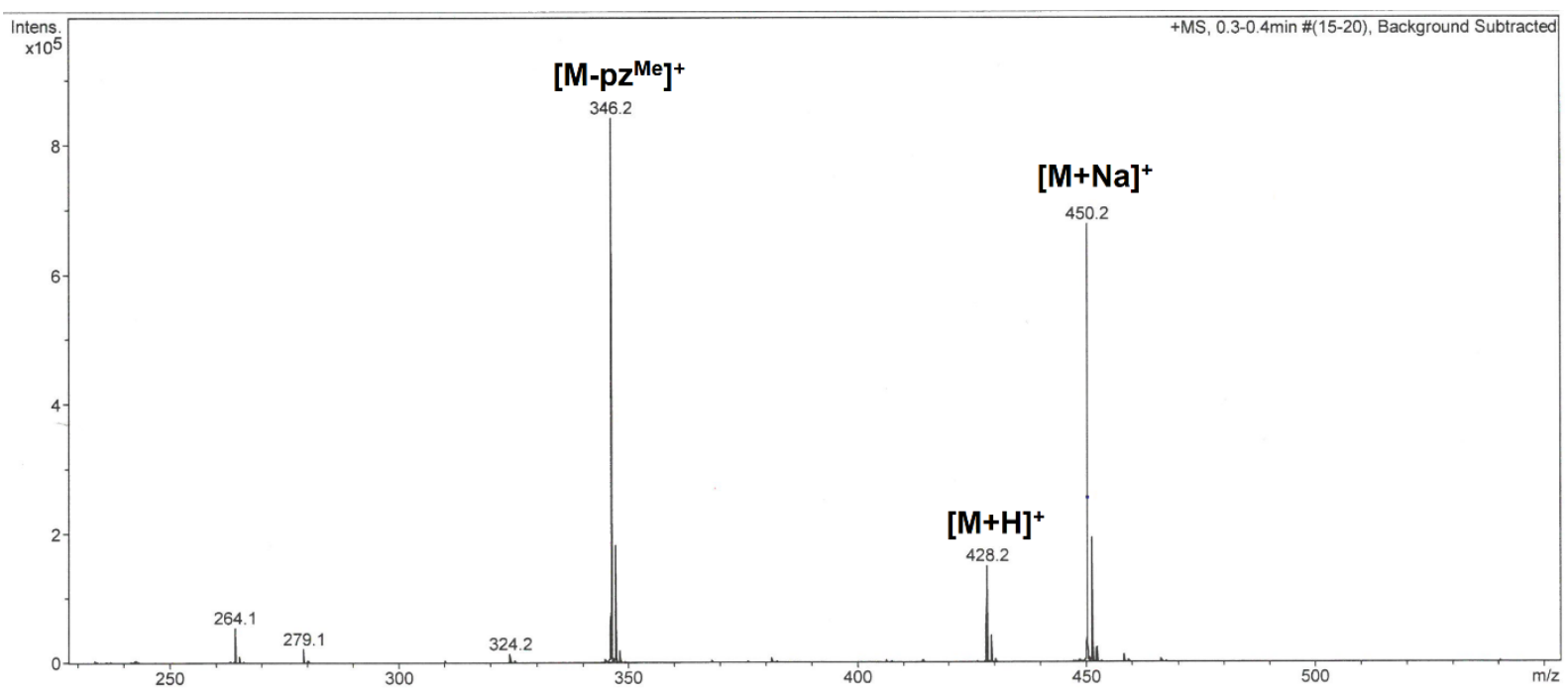

Figure 5.30 ESI-MS(+) spectrum of the synthesis of $\mathbf{p z}^{\mathrm{Me}}{ }_{4} \mathrm{Lut}$ in methanol. 


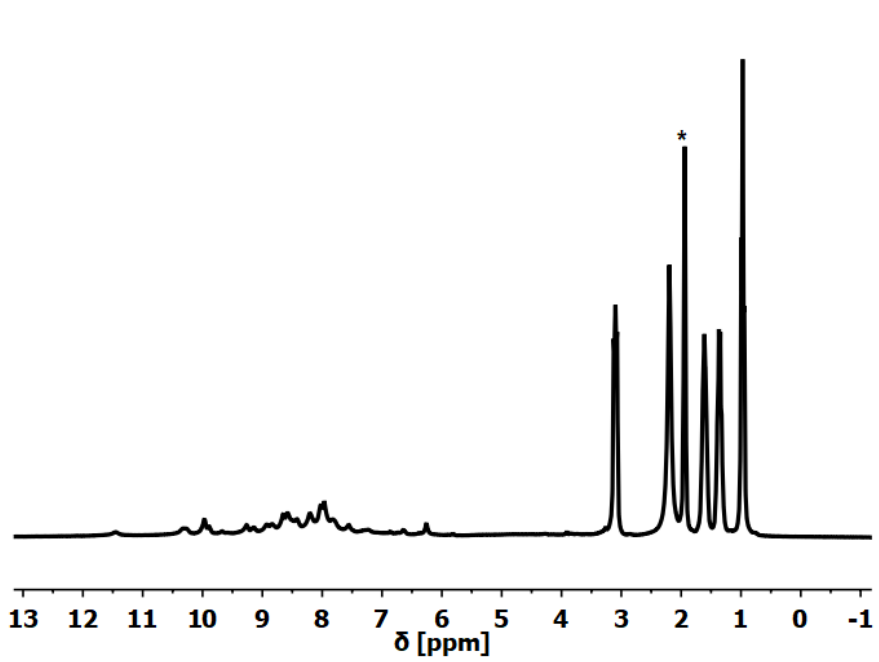

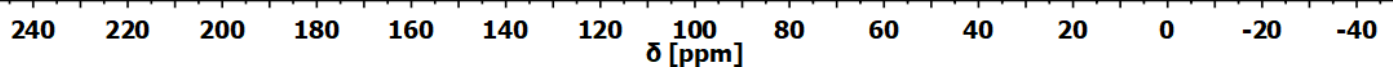

Figure $5.31{ }^{1} \mathrm{H}-\mathrm{NMR}(300 \mathrm{MHz})$ spectrum of the crude product of [tp/N4Py] in $\mathrm{CD}_{3} \mathrm{CN}\left({ }^{*}\right)$.

\subsection{IR Spectra}

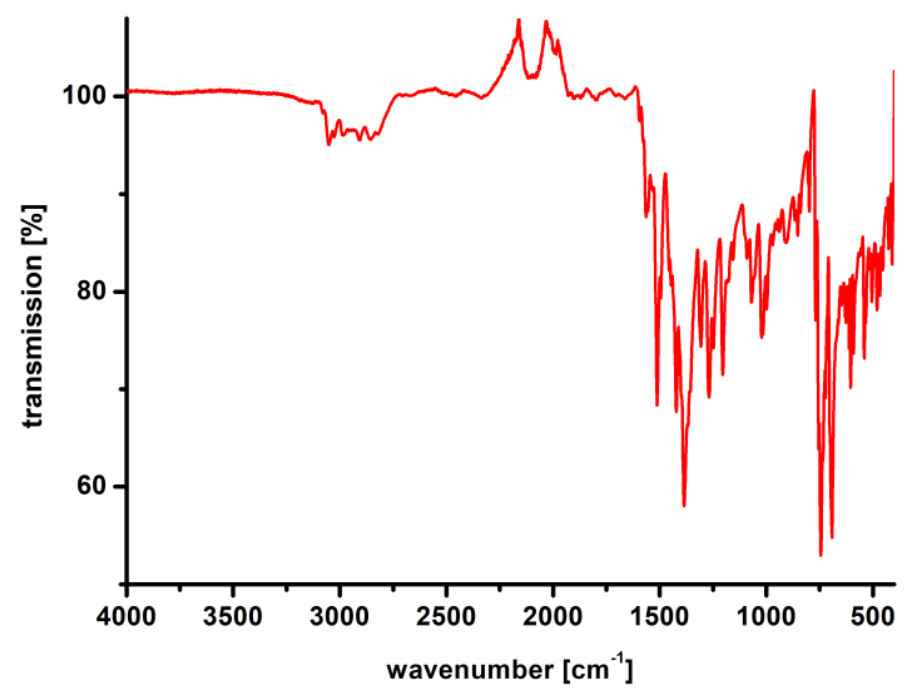

Figure 5.32 ATR-IR spectrum of crystalline 2-K at r.t. 


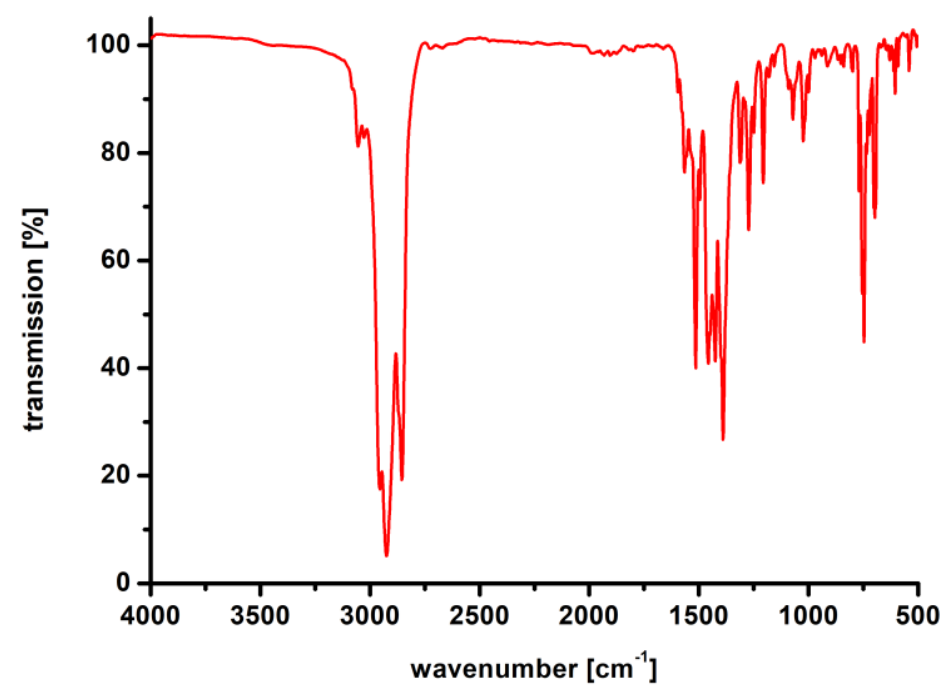

Figure 5.33 IR spectrum of crystalline 2-K in nujol at r.t.

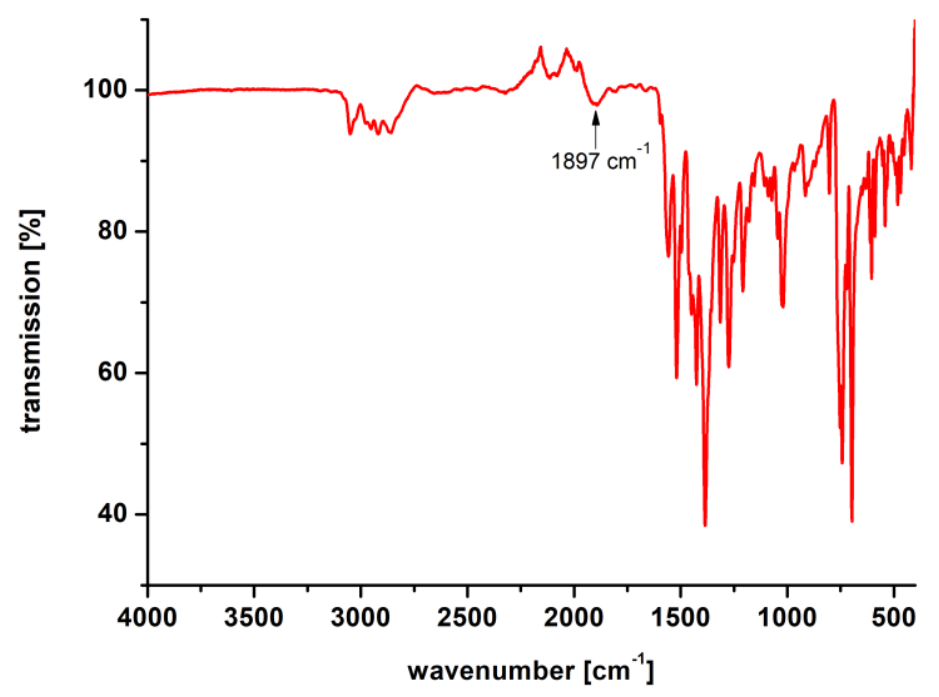

Figure 5.34 ATR-IR spectrum of crystalline 2-Na at r.t. 


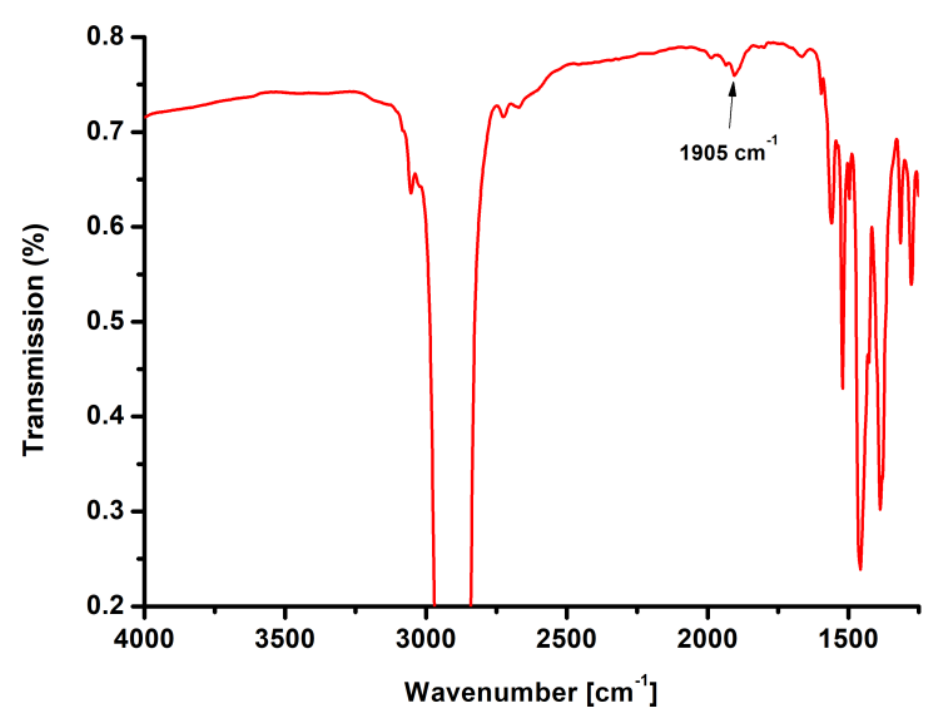

Figure 5.35 IR spectrum of crystalline 2-Na in nojul at r.t.

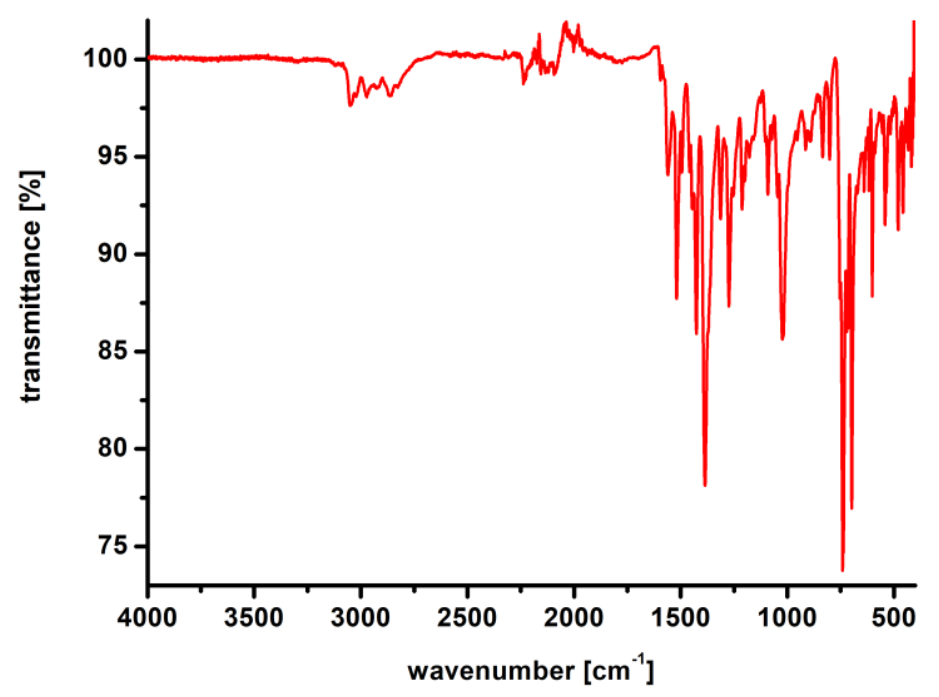

Figure 5.36 ATR-IR spectrum of crystalline 5-Na at r.t. 


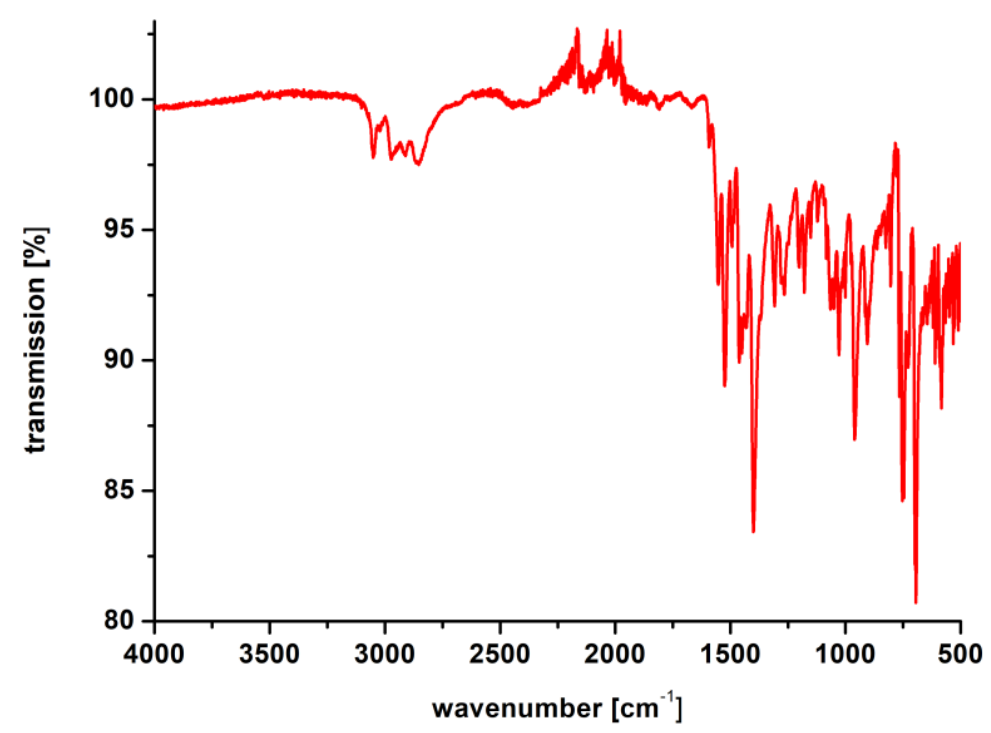

Figure 5.37 ATR-IR spectrum of crystalline 4-K at r.t.

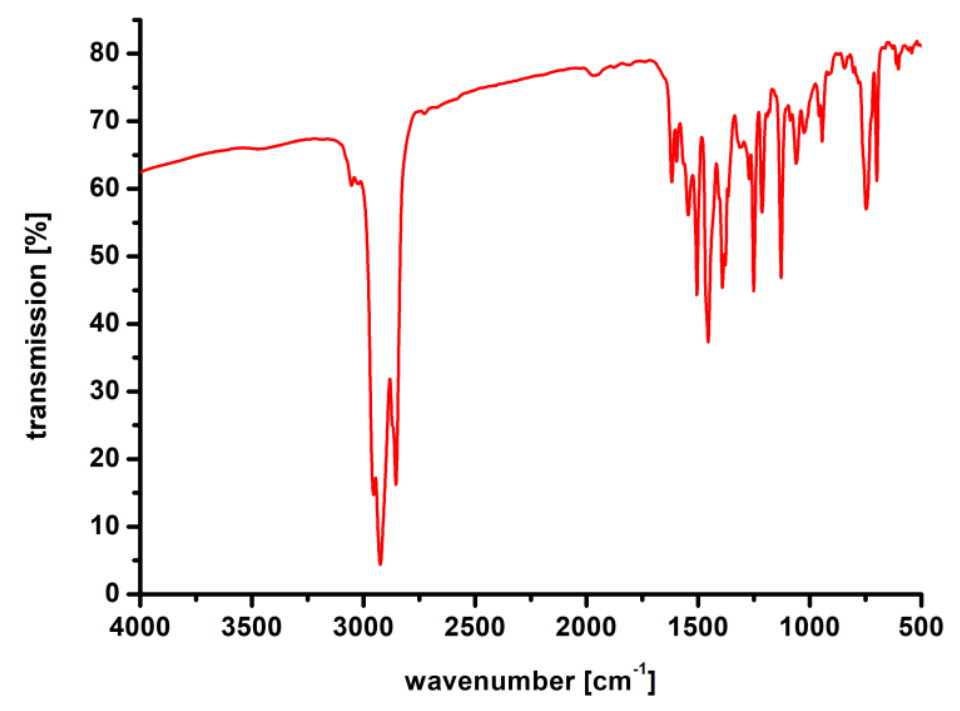

Figure 5.38 IR spectrum of 6-K in nujol at r.t. 


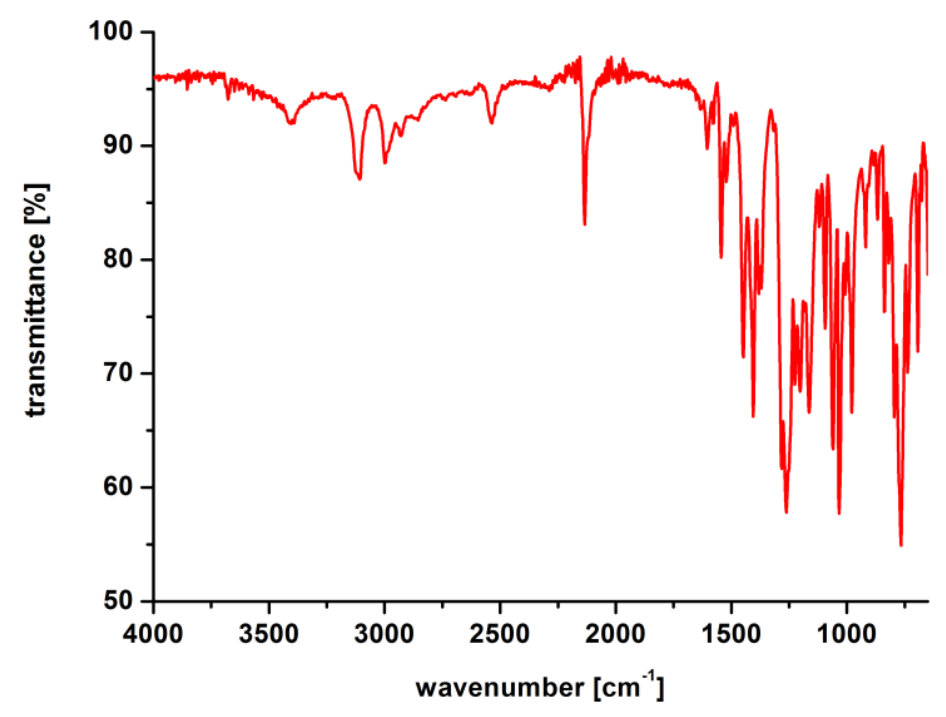

Figure 5.39 ATR-IR spectrum of crystalline [tp*-Fe/pz4Lut-Co] at r.t.

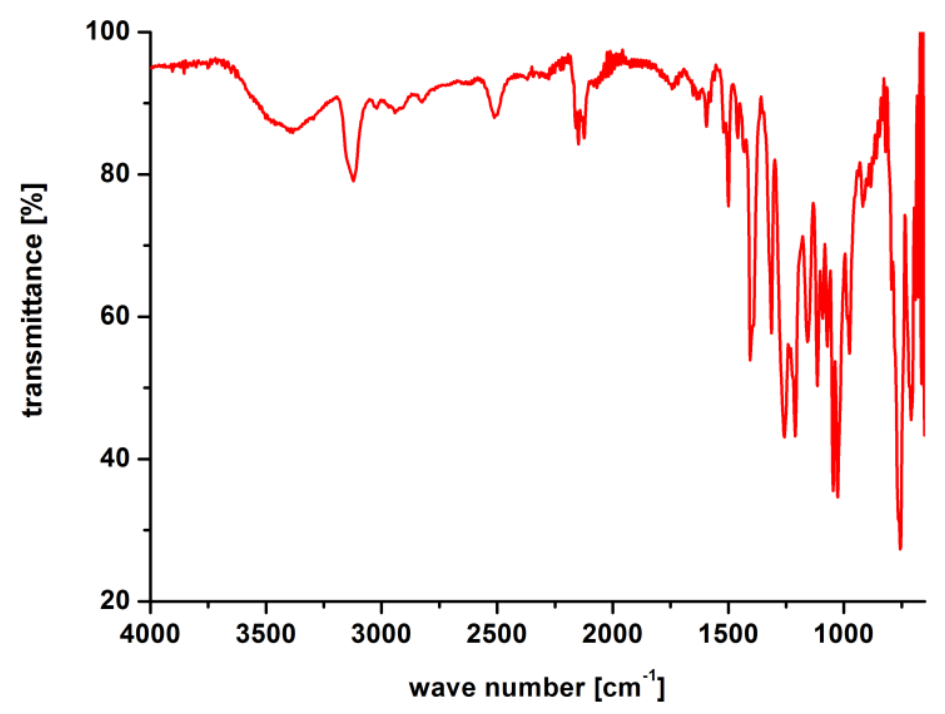

Figure 5.40 ATR-IR spectrum of crystalline [tp-Fe/pzudepy-Co] at r.t. 


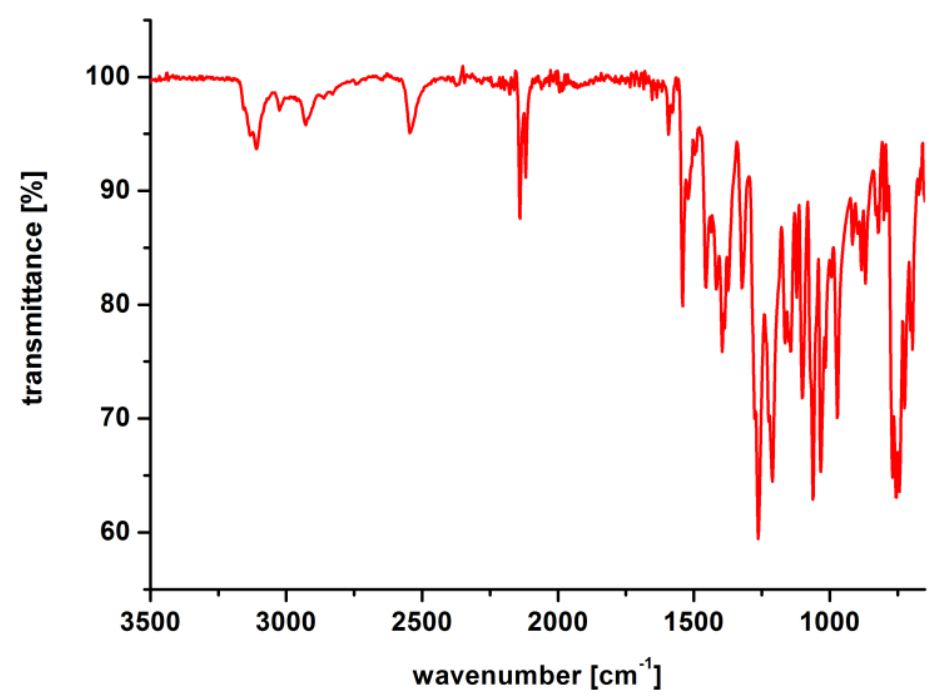

Figure 5.41 ATR-IR spectrum of crystalline [tp*-Fe/pz 4 depy-Co] at r.t.

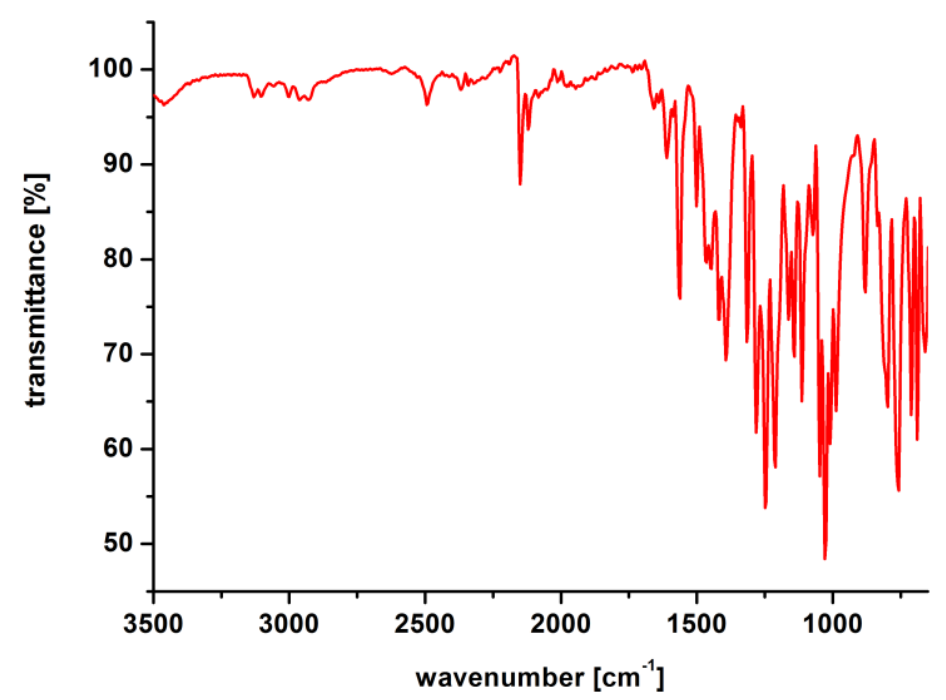

Figure 5.42 ATR-IR spectrum of crystalline [tp-Fe/pz ${ }^{*}{ }_{4}$ Lut-Co] at r.t. 


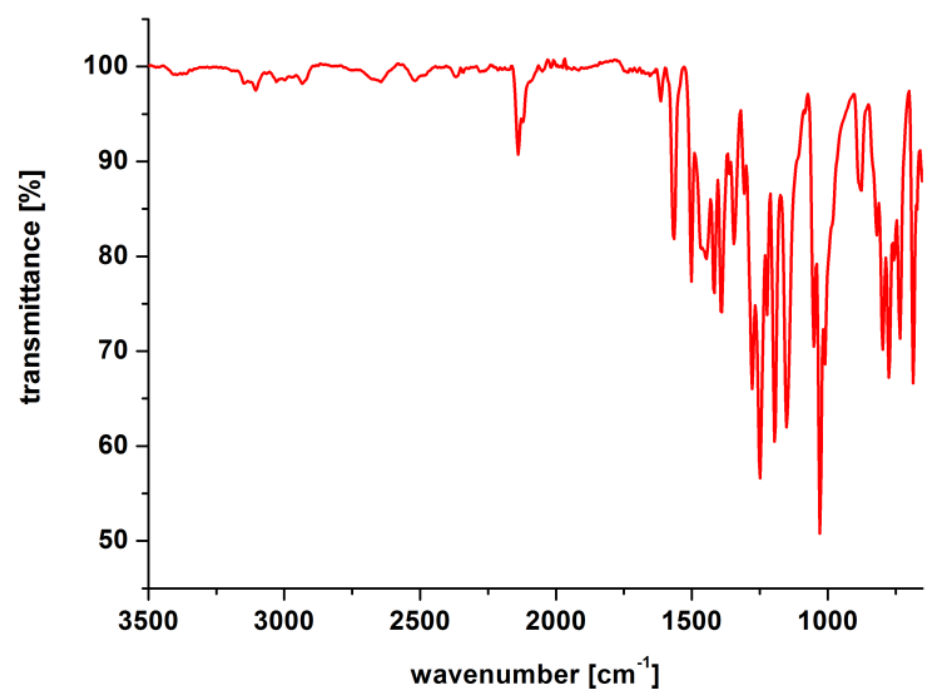

Figure 5.43 ATR-IR spectrum of crystalline $\left[\mathrm{tp}^{\mathrm{Me}}{ }_{-} \mathrm{Fe} / \mathrm{pz}^{*}{ }_{4}{ }_{\mathrm{Lut}}\right.$-Co] at r.t.

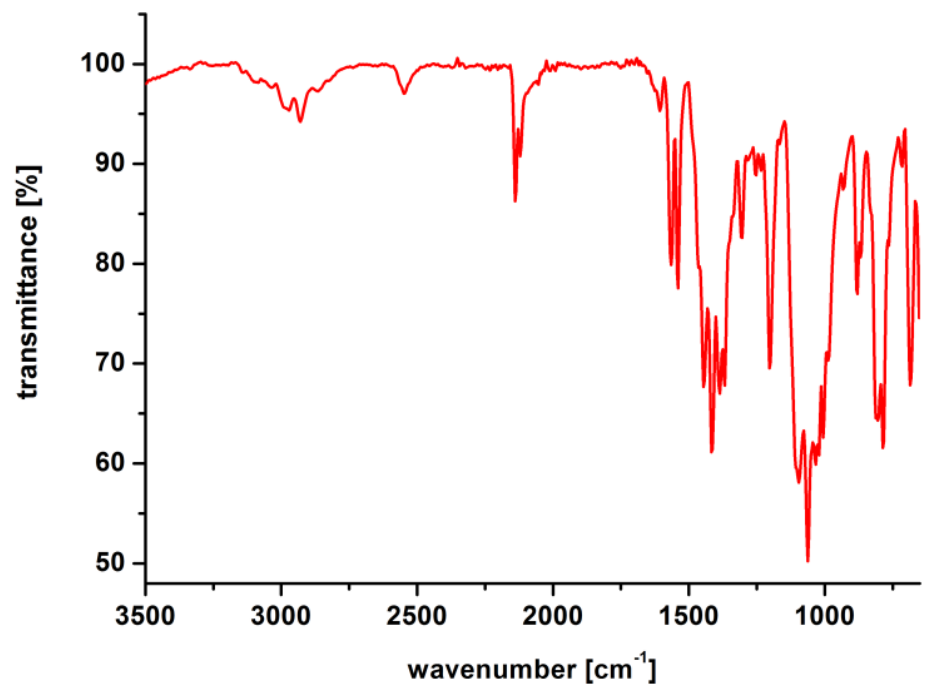

Figure 5.44 ATR-IR spectrum of crystalline [tp* $\left.{ }^{*}-\mathrm{Fe} / \mathrm{pz}^{*}{ }_{4} \mathrm{Lut}-\mathrm{Co}\right]$ at r.t. 


\subsection{UV/vis Spectra}

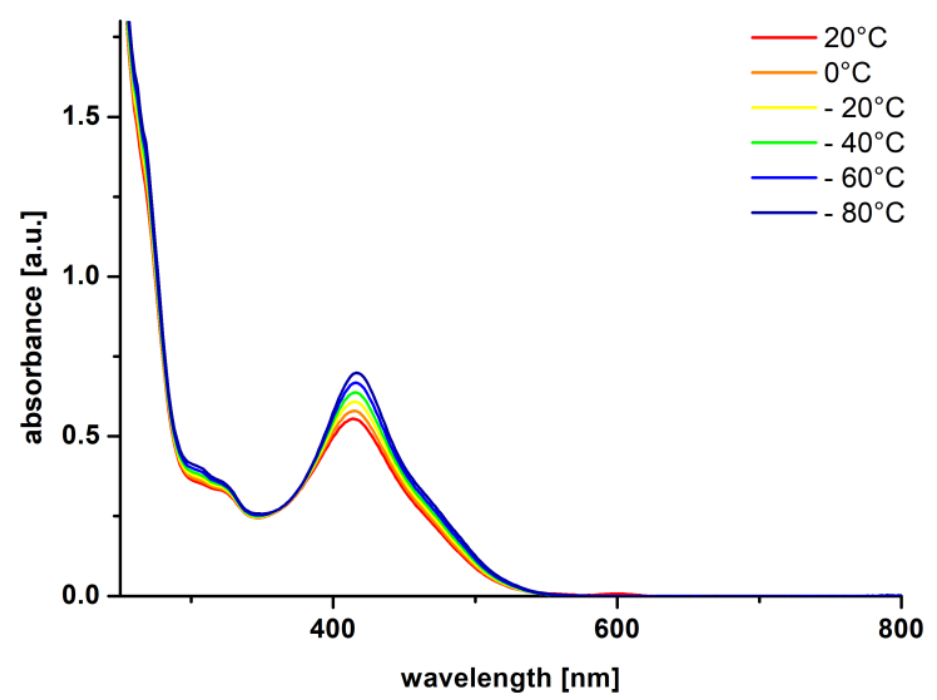

Figure 5.45 Temperature dependent UV/vis spectra of [tp-Fe/pz4depy-Co] in propionitrile.

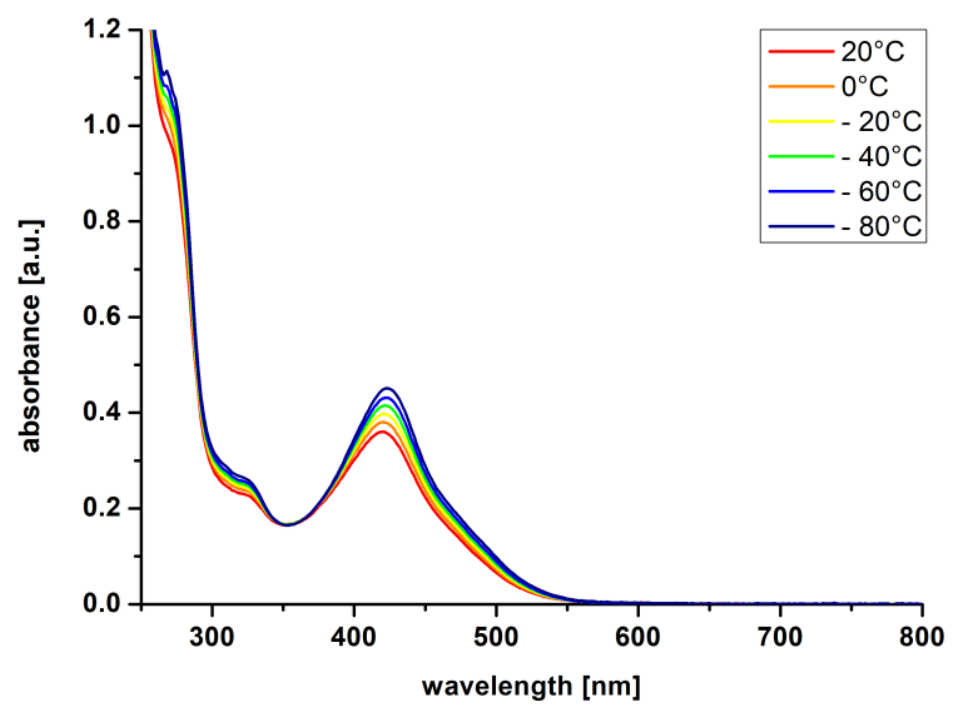

Figure 5.46 Temperature dependent UV/vis spectra of [tp-Fe/pz ${ }^{*}{ }_{4}$ Lut-Co] in propionitrile. 


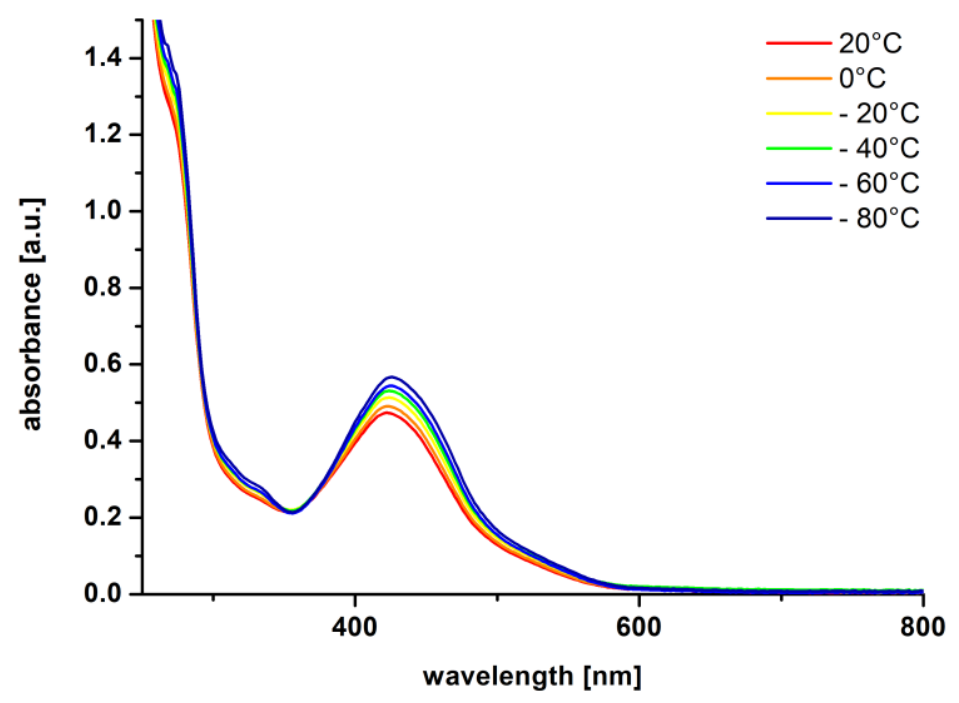

Figure 5.47 Temperature dependent UV/vis spectra of [tp $\left.{ }^{\mathrm{Me}}-\mathrm{Fe} / \mathrm{pz}^{*}{ }_{4} \mathrm{Lut}_{\mathrm{C}} \mathrm{Co}\right]$ in propionitrile.

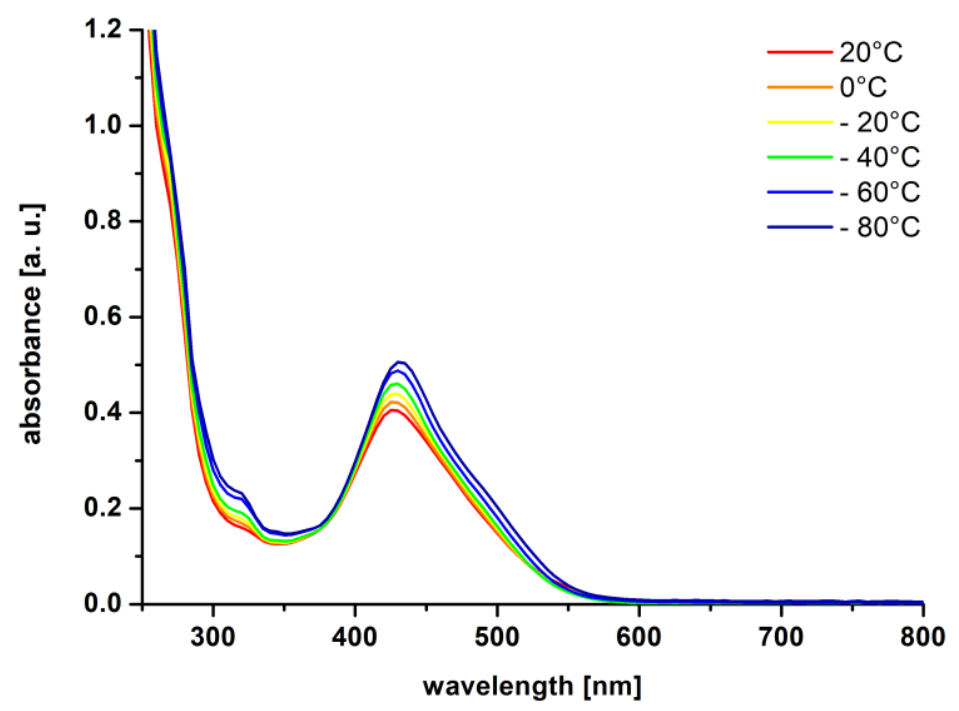

Figure 5.48 Temperature dependent UV/vis spectra of [tp*-Fe/pz 4 Lut-Co] in propionitrile. 


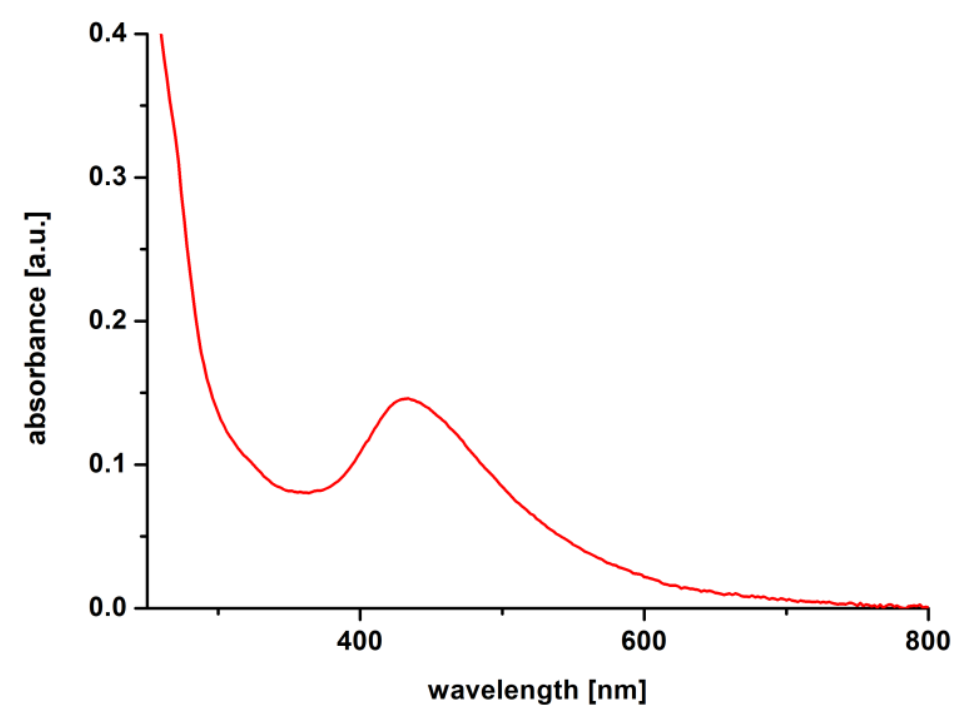

Figure 5.49 UV/vis spectrum of $\left[\mathrm{tp}^{*}-\mathrm{Fe} / \mathrm{pz}^{*}{ }_{4} \mathrm{depy}-\mathrm{Co}\right]$ in propionitrile at room temperature.

\subsection{Magnetic Susceptibility Measurements}

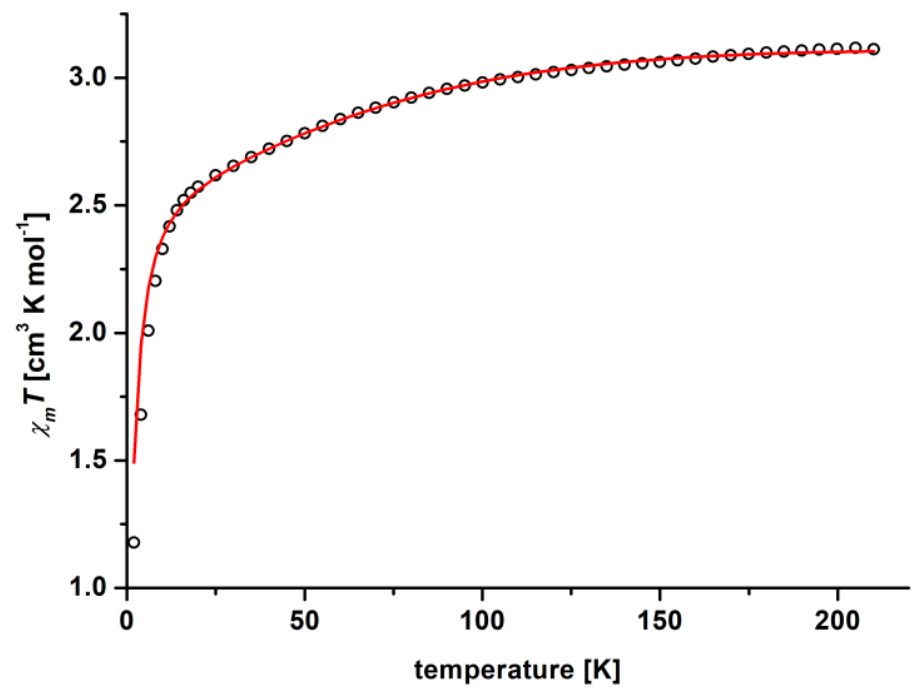

Figure $5.50 \chi_{m} T$ vs. T for crystalline [tp-Fe/pzudepy-Co]; Dots represent experimental data and the solid red line represent fits to the data. 


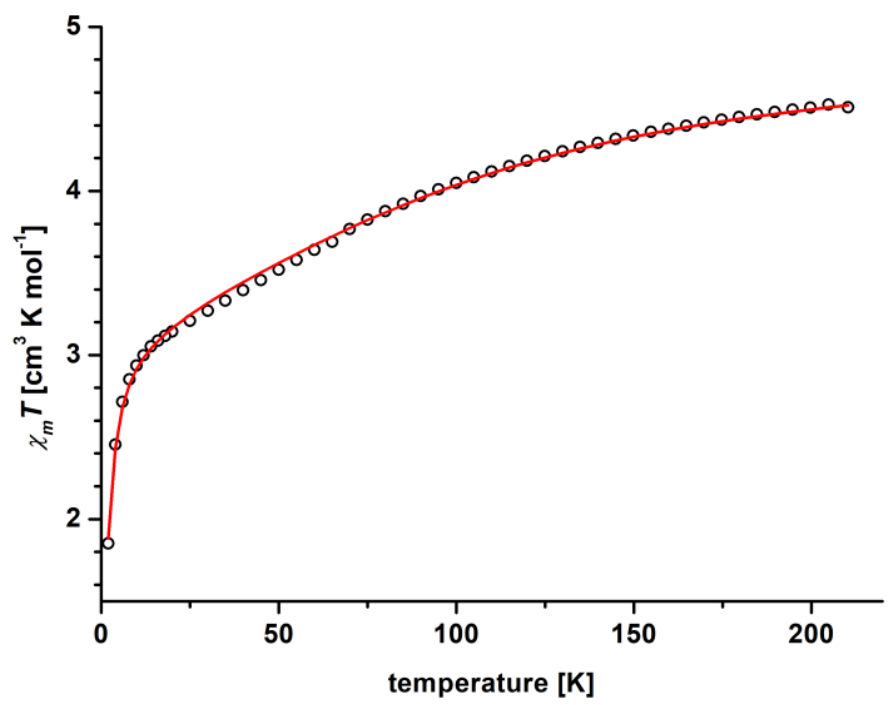

Figure 5.51 $\chi_{m} T$ vs. T for crystalline [tp-Fe/pz ${ }_{4}{ }_{4}$ Lut-Co]; Dots represent experimental data and the solid red line represent fits to the data.

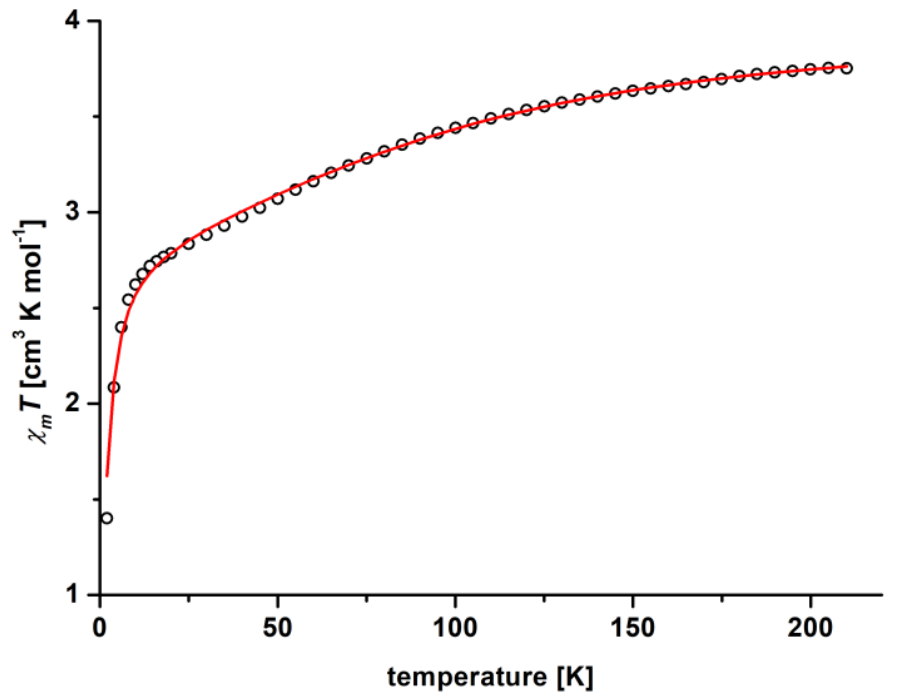

Figure $\quad 5.52 \quad \chi_{m} T$ vs. $\mathrm{T}$ for crystalline [tp $^{\mathrm{Me}}-\mathrm{Fe} / \mathrm{pz}^{*}{ }_{4}$ Lut-Co]; Dots represent experimental data and the solid red line represent fits to the data. 


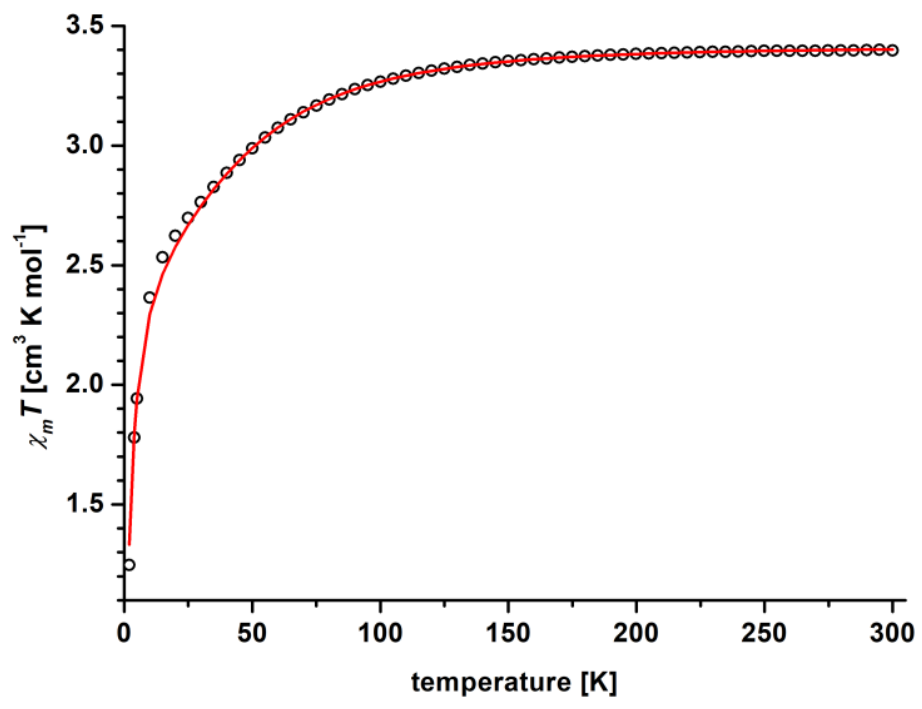

Figure $5.53 \chi_{m} T$ vs. T for crystalline [tp*-Fe/pz_Lut-Co]; Dots represent experimental data and the solid red line represent fits to the data.

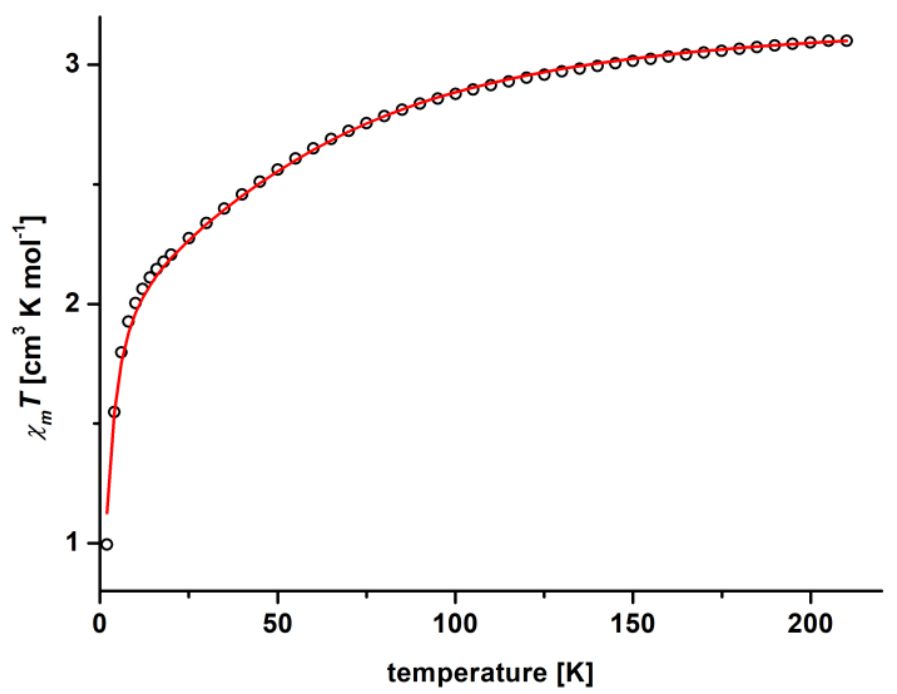

Figure 5.54 $\chi_{m} T$ vs. T for crystalline [tp*-Fe/pz 4 depy-Co]; Dots represent experimental data and the solid red line represent fits to the data. 
Table 5.1 Extracted data from the magnetic susceptibility measurements of the linear PBAs.

\begin{tabular}{|c|c|c|c|}
\hline \multicolumn{2}{|c|}{ Magnetic Susceptibility } & $\mathrm{D}\left[\mathrm{cm}^{-1}\right]$ & $2 J\left[\mathrm{~cm}^{-1}\right]$ \\
\hline \multirow{2}{*}{$\mathrm{Tp}-\mathrm{Fe}$} & pz ${ }_{4}$ depy-Co & -114.14 & -1.00 \\
\hline & pz ${ }_{4}{ }_{4}$ ut-Co & -114.70 & -0.94 \\
\hline tp $p^{\mathrm{Me}}-\mathrm{Fe}$ & pz ${ }_{4}{ }_{4}$ Lut-Co & -118.77 & -1.00 \\
\hline \multirow{3}{*}{ tp*-Fe } & pz $z_{4}$ Lut-Co & -60.90 & -1.19 \\
\hline & pz ${ }_{4}$ depy-Co & -74.581 & -1.09 \\
\hline & $\mathrm{pz}^{*}{ }_{4} \mathrm{Lut}-\mathrm{Co}$ & -125.367 & -1.31 \\
\hline
\end{tabular}

\subsection{X-Ray Structures}

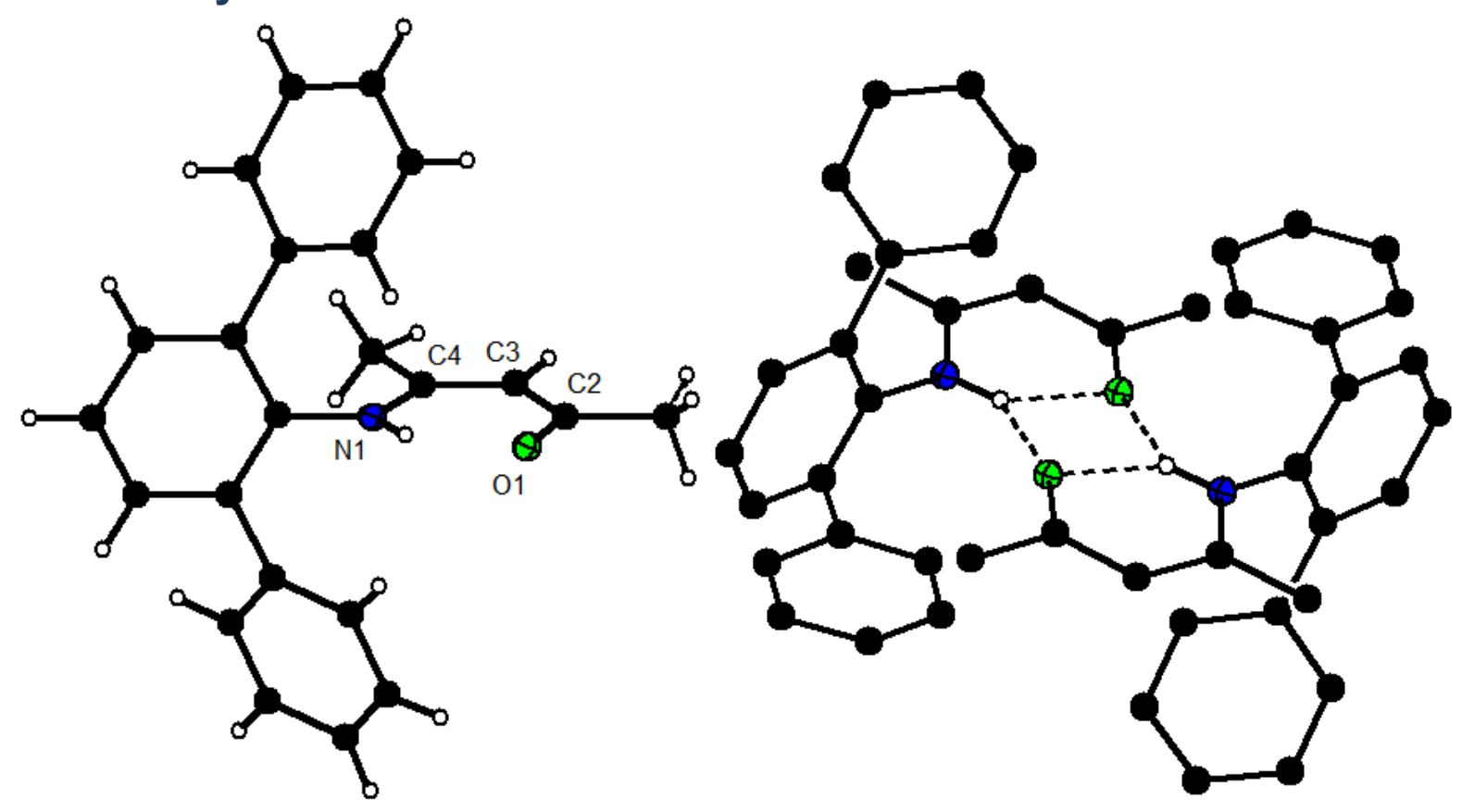

Figure 5.55 Molecular structure of $\mathbf{V}$; selected bond lengths $[\AA]$ and angles $\left[^{\circ}\right]$ : O1-C2 1.2461(13),C2C3 1.4247(15), C3-C4 1.3813(15), C4-N1 1.3368(13); O1-C2-C3 122.86(10), N1-C4-C3 120.61(9). 

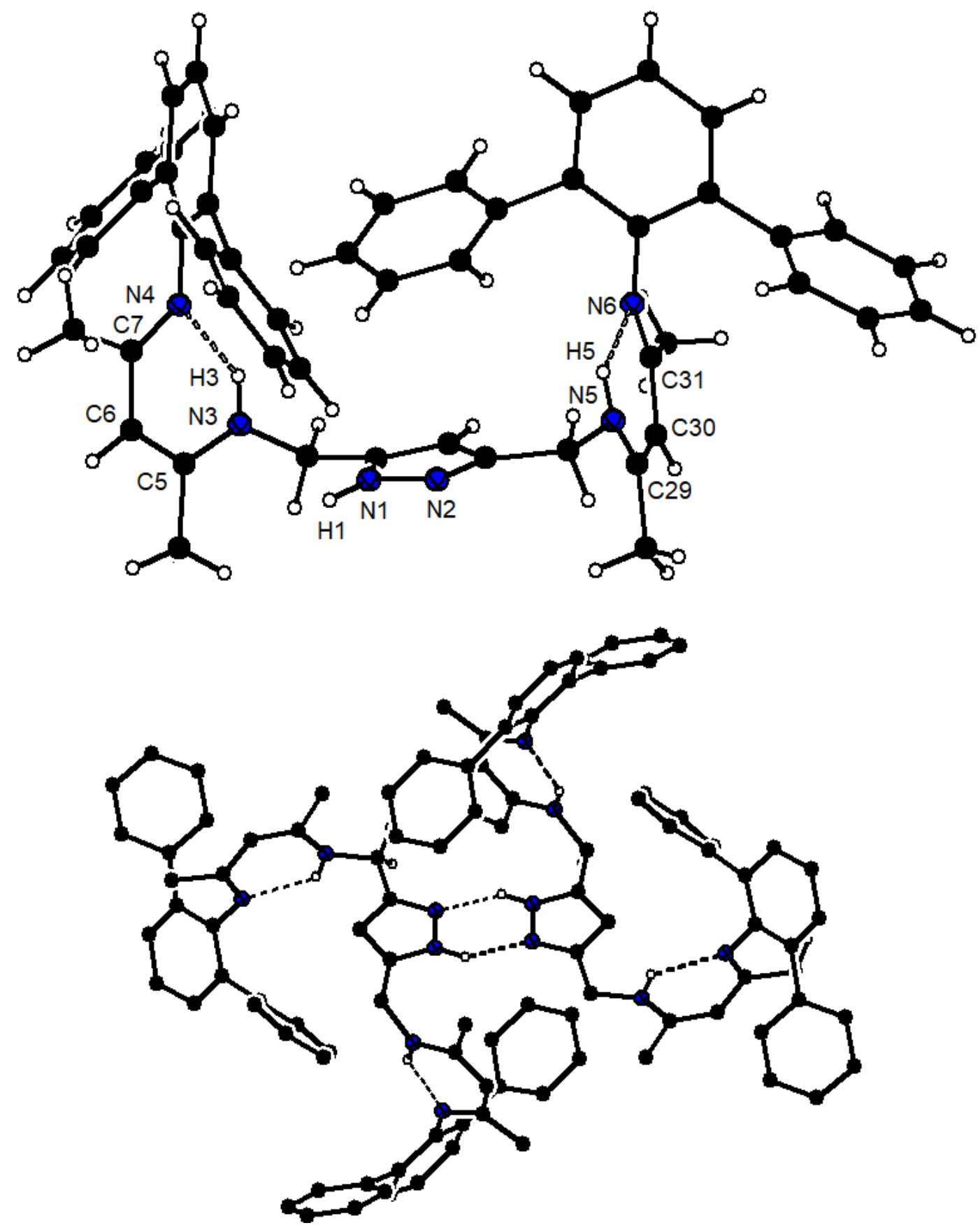

Figure 5.56 Molecular structure of $\mathbf{H}_{3} \mathbf{L}^{\mathrm{Ph}}$; Selected bond lengths [ $\AA \AA$ ] and angles [ $\left.{ }^{\circ}\right]$ : N1-N2 1.379(4), N3C5 1.341(4), N4-C7 1.305(3), N5-C29 1.346(4), N6-C31 1.313(3), C5-C6 1.378(4), C6-C7 1.434(4), C29-C30 1.338(4), C30-C31 1.429(4); N3-C5-C6 122.1(2), N4-C7-C6 119.7(2), N5-C29-C30 121.3(3), N6-C31-C30 121.8(3). 


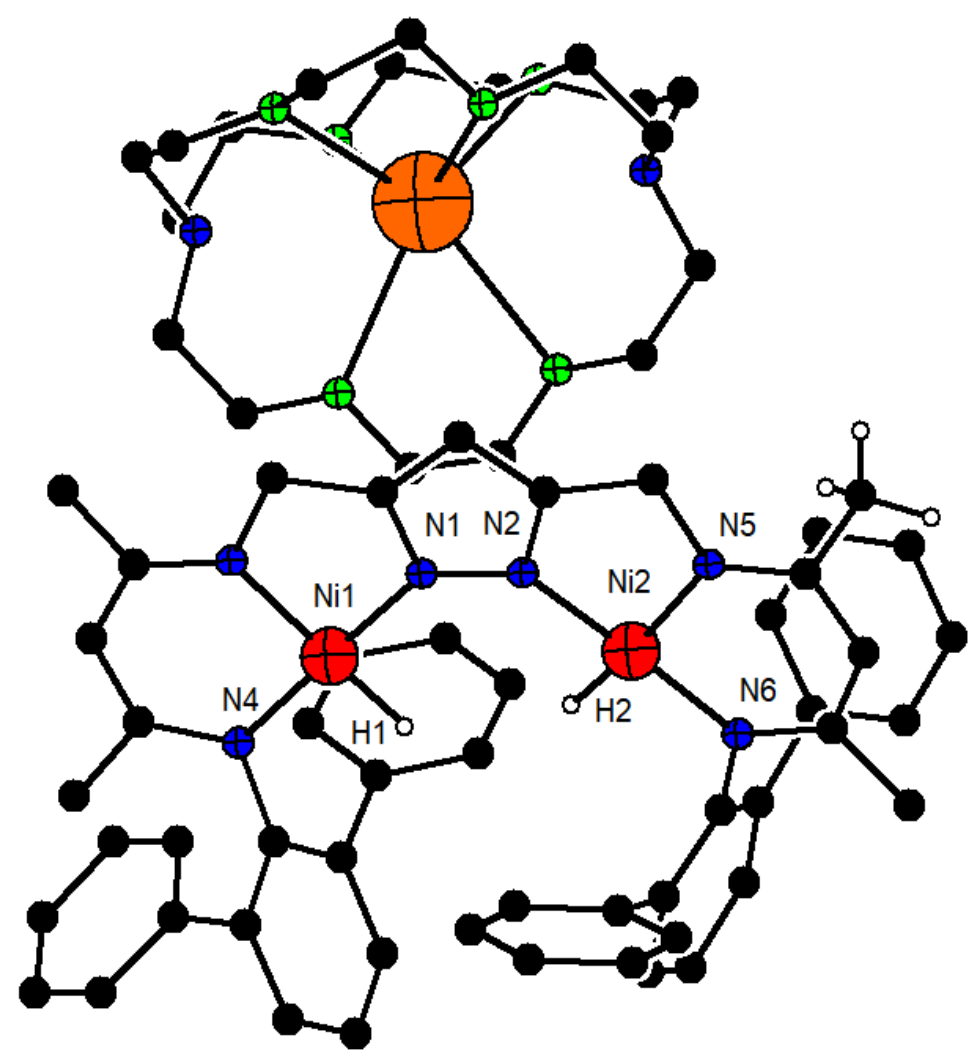

Figure 5.57 Molecular structure of 3-K(crypt); Selected bond lengths $[\AA]$ and angles [ $\left.{ }^{\circ}\right]$ : Ni1-H1 1.39(2), Ni1-N1 1.8651(16), Ni1-N4 1.8709(16), Ni1-N3 1.9154(17), Ni2-H2 1.42(2), Ni2-N6 1.8645(17), Ni2-N2 1.8672(17), Ni2-N5 1.9179(17); H1-Ni1-N1 91.3(9), H1-Ni1-N4 88.0(9), N1-Ni1-N4 178.71(7), H1-Ni1N3 174.1(9), N1-Ni1-N3 84.56(7), N4-Ni1-N3 96.09(7), H2-Ni2-N6 87.1(9), H2-Ni2-N2 92.8(9), N6-Ni2N2 177.67(7), H2-Ni2-N5 172.9(9), N6-Ni2-N5 96.41(7), N2-Ni2-N5 83.98(7), C2-N1-Ni1 116.51(13), N2-N1-Ni1 136.12(13), C3-N2-Ni2 116.76(13), N1-N2-Ni2 135.37(12), C5-N3-Ni1 125.93(14), C4-N3Ni1 114.90(13), C7-N4-Ni1 124.48(14), C10-N4-Ni1 116.42(12), C29-N5-Ni2 125.43(15), C28-N5-Ni2 115.16(13), C31-N6-Ni2 124.39(14), C34-N6-Ni2 118.95(13). 


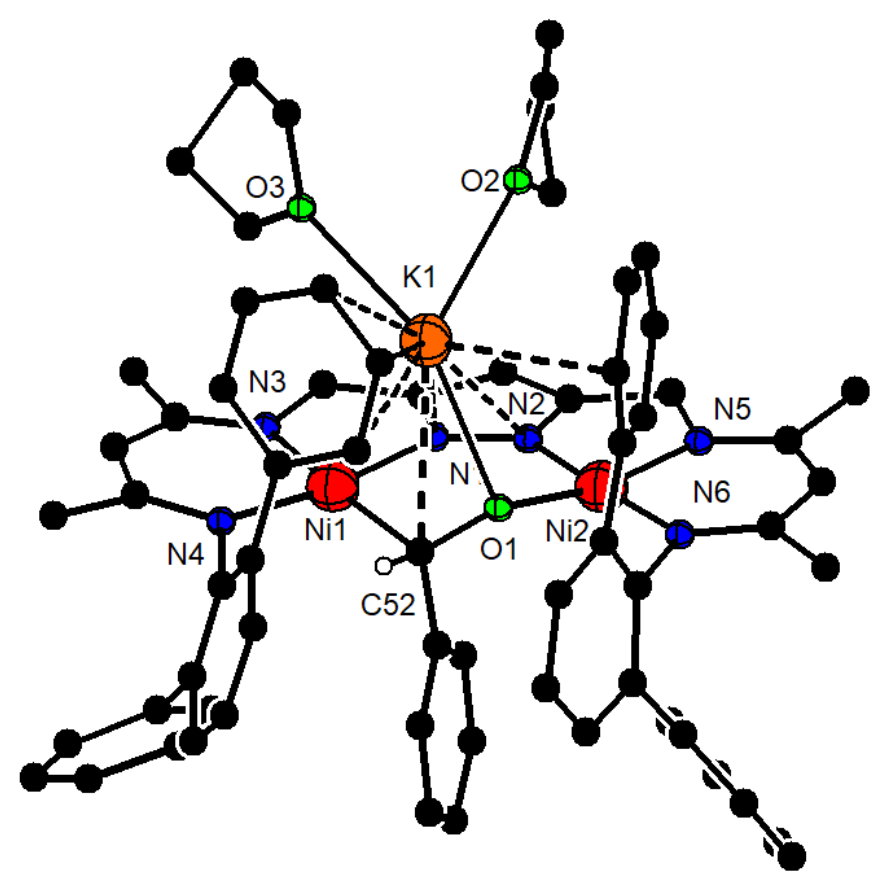

Figure 5.58 Molecular structure of 4-K; selected bond lengths $[\AA]$ and angles [ $\left.{ }^{\circ}\right]$ : Ni1-N1 1.8919(18), Ni1-N4 1.9336(18), Ni1-N3 1.9709(18), Ni1-C52 2.015(2), Ni1-K1 3.1133(6), Ni2-O1 1.8299(16), Ni2N2 1.8526(18), Ni2-N5 1.8813(18), Ni2-N6 1.9088(18), Ni2-K1 3.5297(6); N1-Ni1-N4 172.90(8), N1-Ni1N3 81.70(8), N4-Ni1-N3 93.87(8), N1-Ni1-C52 89.06(8), N4-Ni1-C52 95.97(9), N3-Ni1-C52 168.43(9), N1-Ni1-K1 71.36(6), N4-Ni1-K1 105.40(6), N3-Ni1-K1 111.05(6), C52-Ni1-K1 72.07(7), O1-Ni2-N2 90.02(7), O1-Ni2-N5 168.50(8), N2-Ni2-N5 83.74(8), O1-Ni2-N6 91.53(7), N2-Ni2-N6 178.36(8), N5Ni2-N6 94.64(8), O1-Ni2-K1 47.42(5), N2-Ni2-K1 66.82(6), N5-Ni2-K1 121.07(6), N6-Ni2-K1 113.96(5), O1-K1-Ni1 60.70(4), O3-K1-Ni1 91.68(10), O2-K1-Ni1 131.73(5), N1-K1-Ni1 35.55(3), Ni1-K1-C52 37.56(4), Ni1-K1-C18 114.08(6), Ni1-K1-C17 90.23(5), Ni1-K1-C19 117.55(6), Ni1-K1-N2 56.26(3), Ni1K1-C41 125.63(5), Ni1-K1-C20 97.20(5), C52-O1-Ni2 126.15(14), Ni2-O1-K1 102.12(7), C2-N1-Ni1 118.58(15), N2-N1-Ni1 134.65(14), Ni1-N1-K1 73.09(6), C3-N2-Ni2 118.35(15), N1-N2-Ni2 132.78(14), Ni2-N2-K1 81.87(6), C5-N3-Ni1 127.27(16), C4-N3-Ni1 115.78(14), C7-N4-Ni1 123.86(16), C10-N4-Ni1 122.31(14), C29-N5-Ni2 126.79(16), C28-N5-Ni2 115.99(14), C31-N6-Ni2 125.61(16), C34-N6-Ni2 117.86(14), O1-C52-Ni1 118.50(16), C53-C52-Ni1 104.61(14), Ni1-C52-K1 70.37(7). 


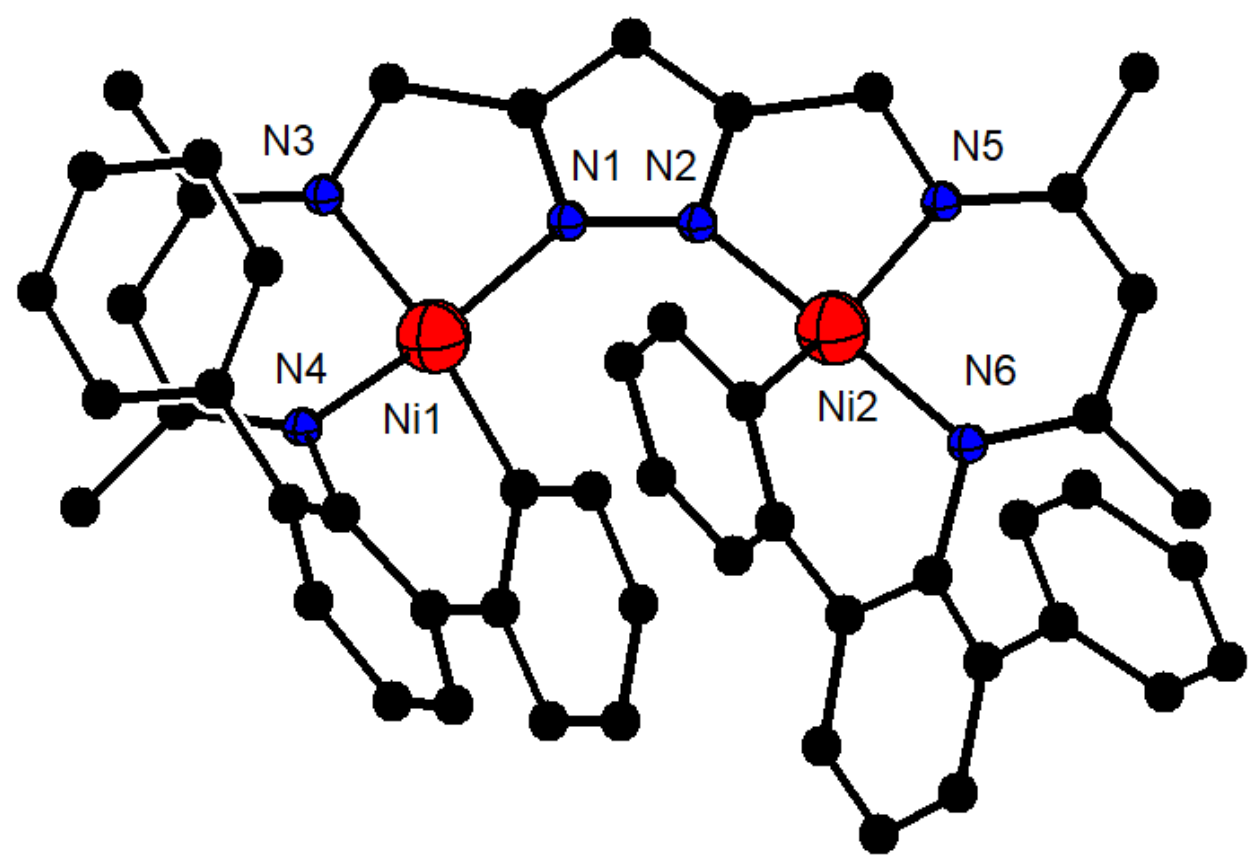

Figure 5.59 Molecular structure of 5-Na; selected bond lengths $[\AA]$ and angles [ $\left.{ }^{\circ}\right]$ : Ni1-N4 1.8646(15), Ni1-C21 1.8854(18), Ni1-N1 1.9046(16), Ni1-N3 1.9205(16), Ni2-N6 1.8630(18), Ni2-N2 1.8963(18), Ni2-C41 1.900(2), Ni2-N5 1.9310(18); N4-Ni1-C21 90.35(7), N4-Ni1-N1 166.55(6), C21-Ni1-N1 97.90(7), N4-Ni1-N3 93.62(6), C21-Ni1-N3 153.14(7), N1-Ni1-N3 83.83(7), N6-Ni2-N2 166.14(7), N6Ni2-C41 90.92(9), N2-Ni2-C41 98.02(9), N6-Ni2-N5 92.65(8), N2-Ni2-N5 84.08(8), C41-Ni2-N5 153.50(8), C2-N1-Ni1 115.10(14), N2-N1-Ni1 137.20(13), C3-N2-Ni2 115.49(15), N1-N2-Ni2 137.54(13), C5-N3-Ni1 125.64(13), C4-N3-Ni1 115.14(13), C7-N4-Ni1 124.93(12), C10-N4-Ni1 116.56(11), C29-N5-Ni2 125.27(18), C28-N5-Ni2 115.03(14), C31-N6-Ni2 125.38(15), C34-N6-Ni2 116.33(13), C20-C21-Ni1 116.54(14), C16-C21-Ni1 125.59(14), C40-C41-Ni2 123.90(17), C42-C41-Ni2 118.32(19). 


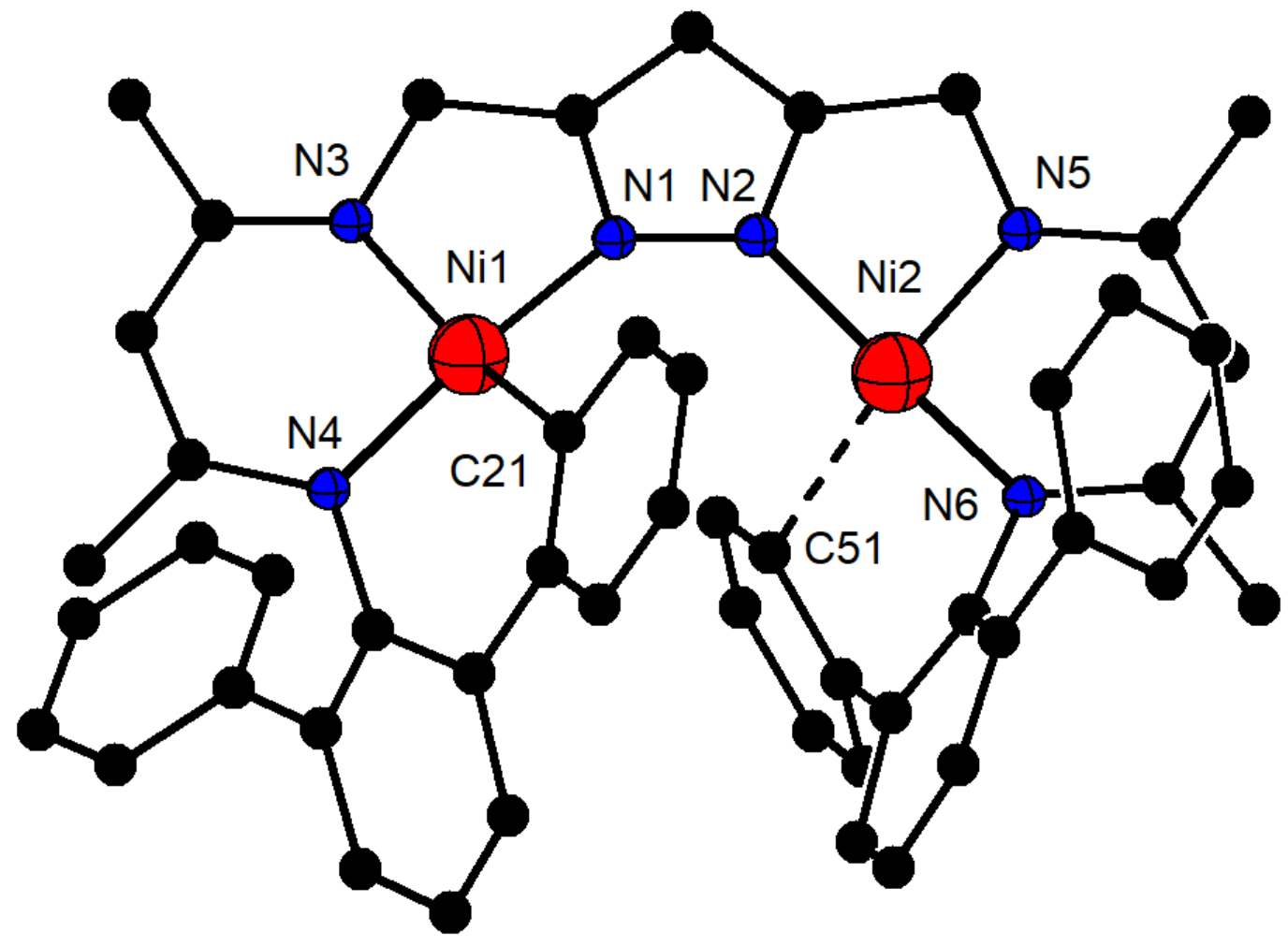

Figure 5.60 Molecular structure of 8; selected bond lengths $[\AA]$ and angles [ $\left.{ }^{\circ}\right]$ :Ni1-N4 1.852(3), Ni1-N1 1.879(3), Ni1-C21 1.899(4), Ni1-N3 1.917(3), Ni2-N6 1.845(3), Ni2-N5 1.860(3), Ni2-N2 1.878(3), Ni2-C51 2.275(3); N4-Ni1-N1 165.27(12), N4-Ni1-C21 90.35(13), N1-Ni1-C21 97.67(13), N4-Ni1-N3 94.75(12), N1-Ni1-N3 83.24(12), C21-Ni1-N3 154.82(13), N6-Ni2-N5 95.40(12), N6Ni2-N2 171.55(12), N5-Ni2-N2 84.23(12), N6-Ni2-C51 90.28(13), N5-Ni2-C51 144.41(13), N2-Ni2C51 94.92(12), C2-N1-Ni1 117.3(2), N2-N1-Ni1 135.5(2), C3-N2-Ni2 116.5(2), N1-N2-Ni2 133.9(2), C5-N3-Ni1 125.0(3), C4-N3-Ni1 116.1(2), C7-N4-Ni1 124.2(2), C10-N4-Ni1 116.3(2), C29-N5-Ni2 126.1(2), C28-N5-Ni2 116.1(2), C31-N6-Ni2 126.1(2), C34-N6-Ni2 115.8(2), C20-C21-Ni1 117.3(3), C16-C21-Ni1 125.6(3), C50-C51-Ni2 111.2(2), C46-C51-Ni2 109.9(3). 


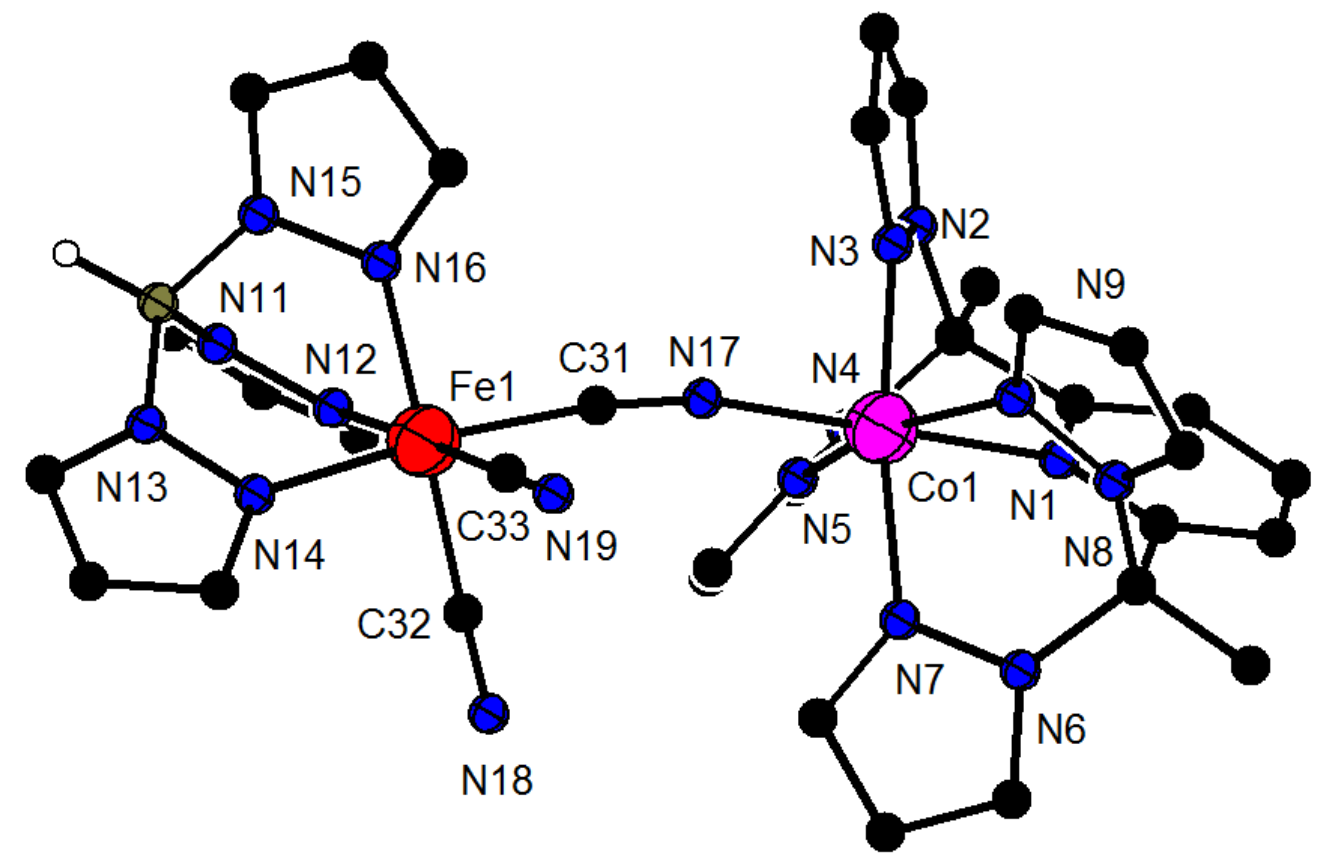

Figure 5.61 Molecular structure of [tp-Fe/pz depy-Co]; Co1-N17 2.056(5), Co1-N3 2.083(4), Co1-N9 2.085(4), Co1-N5 2.093(4), Co1-N7 2.105(5), Co1-N1 2.154(5); N17-Co1-N3 96.64(17), N17-Co1-N9 95.06(18), N3-Co1-N9 94.77(16), N17-Co1-N5 95.37(19), N3-Co1-N5 83.97(17), N9-Co1-N5 169.6(2), N17-Co1-N7 95.1(2), N3-Co1-N7 168.2(2), N9-Co1-N7 82.95(17), N5-Co1-N7 96.17(18), N17-Co1-N1 178.91(17), N3-Co1-N1 83.93(17), N9-Co1-N1 85.81(18), N5-Co1-N1 83.76(19), N7-Co1-N1 84.3(2), C5-N1-Co1 120.3(4), C1-N1-Co1 119.9(4), C10-N3-Co1 134.7(4), N2-N3-Co1 120.2(3), C13-N5-Co1 133.9(5), N4-N5-Co1 120.0(3), C18-N7-Co1 135.1(4), N6-N7-Co1 119.0(4), C21-N9-Co1 134.4(4), N8N9-Co1 119.4(3), C31-N17-Co1 159.9(5). 


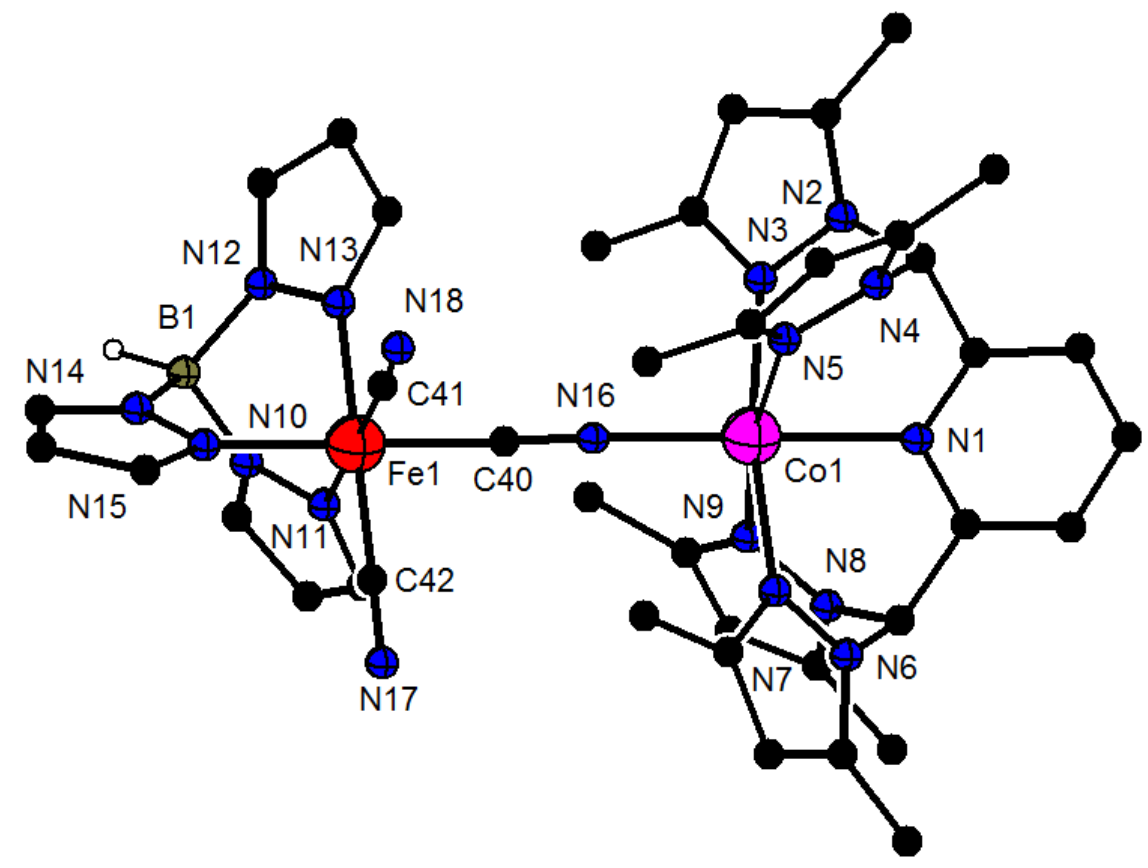

Figure 5.62 Molecular structure of [tp-Fe/pz $\left.{ }^{*}{ }_{4} \mathrm{Lut}-\mathrm{Co}\right]$; selected bond lengths $[\AA]$ and angles $\left[^{\circ}\right]$ : Co1N16 2.067(3), Co1-N1 2.148(3), Co1-N7 2.200(3), Co1-N3 2.218(3), Co1-N5 2.235(3), Co1-N9 2.269(3), Co2-N36 2.059(4), Co2-N21 2.155(3), Co2-N27 2.199(3), Co2-N25 2.217(4), Co2-N23 2.234(3), Co2-N29 2.253(3); N16-Co1-N1 177.95(12), N16-Co1-N7 94.43(13), N1-Co1-N7 84.78(13), N16-Co1-N3 96.26(13), N1-Co1-N3 84.59(13), N7-Co1-N3 169.12(13), N16-Co1-N5 95.71(12), N1Co1-N5 86.28(12), N7-Co1-N5 99.00(12), N3-Co1-N5 77.97(12), N16-Co1-N9 93.95(13), N1-Co1-N9 84.04(13), N7-Co1-N9 79.49(13), N3-Co1-N9 101.72(13), N5-Co1-N9 170.31(13), C5-N1-Co1 121.3(3), C1-N1-Co1 121.1(3), C9-N3-Co1 136.7(3), N2-N3-Co1 116.3(2), C14-N5-Co1 140.0(3), N4-N5-Co1 115.0(2), C20-N7-Co1 138.0(3), N6-N7-Co1 116.7(2), C25-N9-Co1 139.2(4), N8-N9-Co1 116.0(3), C40-N16-Co1 177.9(4), N36-Co2-N21 176.91(13), N36-Co2-N27 96.55(13), N21-Co2-N27 85.84(13), N36-Co2-N25 93.54(13), N21-Co2-N25 84.07(13), N27-Co2-N25 169.91(13), N36-Co2-N23 92.62(13), N21-Co2-N23 85.05(12), N27-Co2-N23 99.16(12), N25-Co2-N23 80.11(12), N36-Co2-N29 96.48(13), N21-Co2-N29 85.94(12), N27-Co2-N29 77.28(12), N25-Co2-N29 101.83(13), N23-Co2-N29 170.54(13), C55-N21-Co2 120.8(3), C51-N21-Co2 120.7(3), C59-N23-Co2 140.5(3), N22-N23-Co2 114.8(2), C64-N25-Co2 138.9(3), N24-N25-Co2 116.1(2), C70-N27-Co2 139.5(3), N26-N27-Co2 115.5(2), C75-N29-Co2 139.4(3), N28-N29-Co2 115.8(2), C90-N36-Co2 174.8(3). 


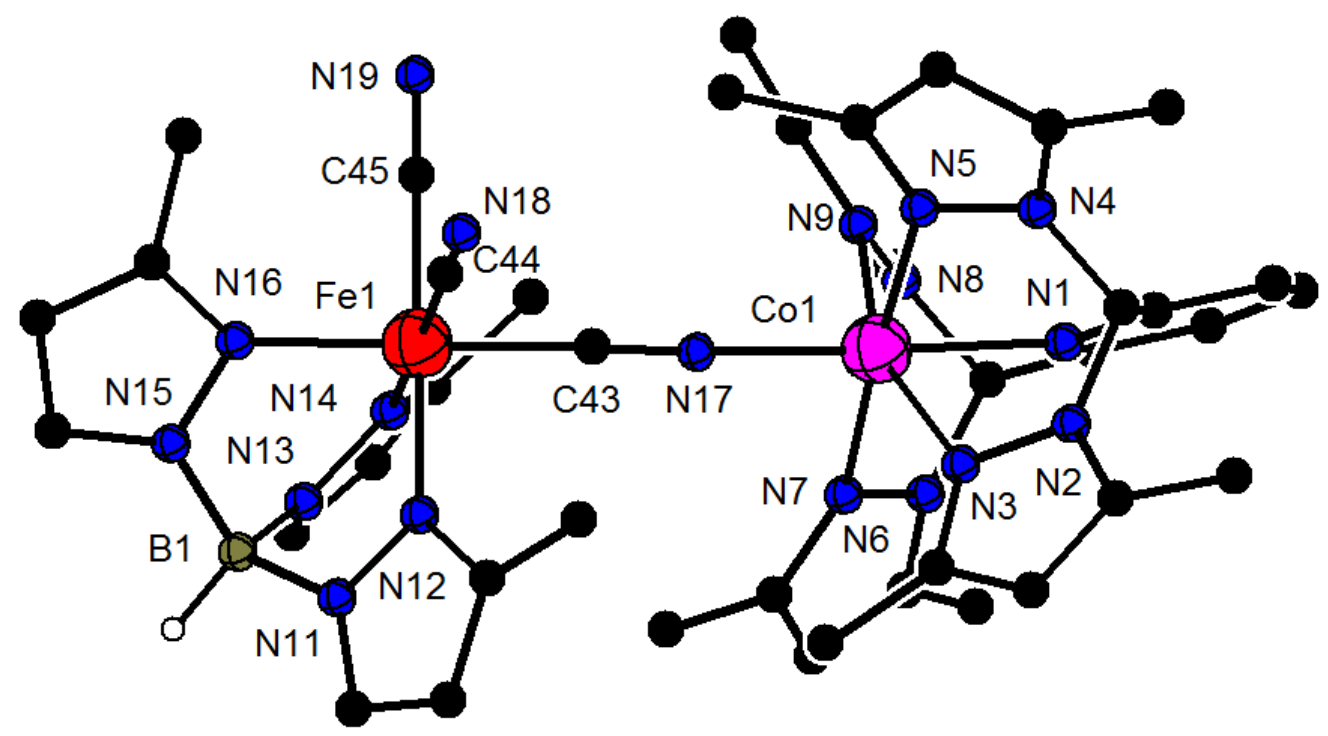

Figure 5.63 Molecular structure of [tp $\left.{ }^{\mathrm{Me}}-\mathrm{Fe} / \mathrm{pz}^{*}{ }_{4} \mathrm{Lut}-\mathrm{Co}\right]$; selected bond lengths $[\AA]$ and angles $\left[^{\circ}\right]$ : Co1N17 2.040(2), Co1-N1 2.137(2), Co1-N5 2.188(2), Co1-N7 2.221(2), Co1-N3 2.237(2), Co1-N9 2.282(2); N17-Co1-N1 177.82(9), N17-Co1-N5 91.65(8), N1-Co1-N5 88.58(8), N17-Co1-N7 91.36(8), N1-Co1-N7 88.46(8), N5-Co1-N7 176.74(8), N17-Co1-N3 100.86(9), N1-Co1-N3 81.31(8), N5-Co1-N3 79.56(8), N7-Co1-N3 98.64(8), N17-Co1-N9 98.42(9), N1-Co1-N9 79.41(8), N5-Co1-N9 98.55(8), N7Co1-N9 82.24(8), N3-Co1-N9 160.68(8), C5-N1-Co1 120.78(16), C1-N1-Co1 120.68(16), C9-N3-Co1 139.5(2), N2-N3-Co1 116.09(16), C14-N5-Co1 137.77(17), N4-N5-Co1 116.79(15), C20-N7-Co1 140.86(19), N6-N7-Co1 114.50(15), C25-N9-Co1 140.24(19), N8-N9-Co1 112.43(15), C43-N17-Co1 $172.9(2)$. 


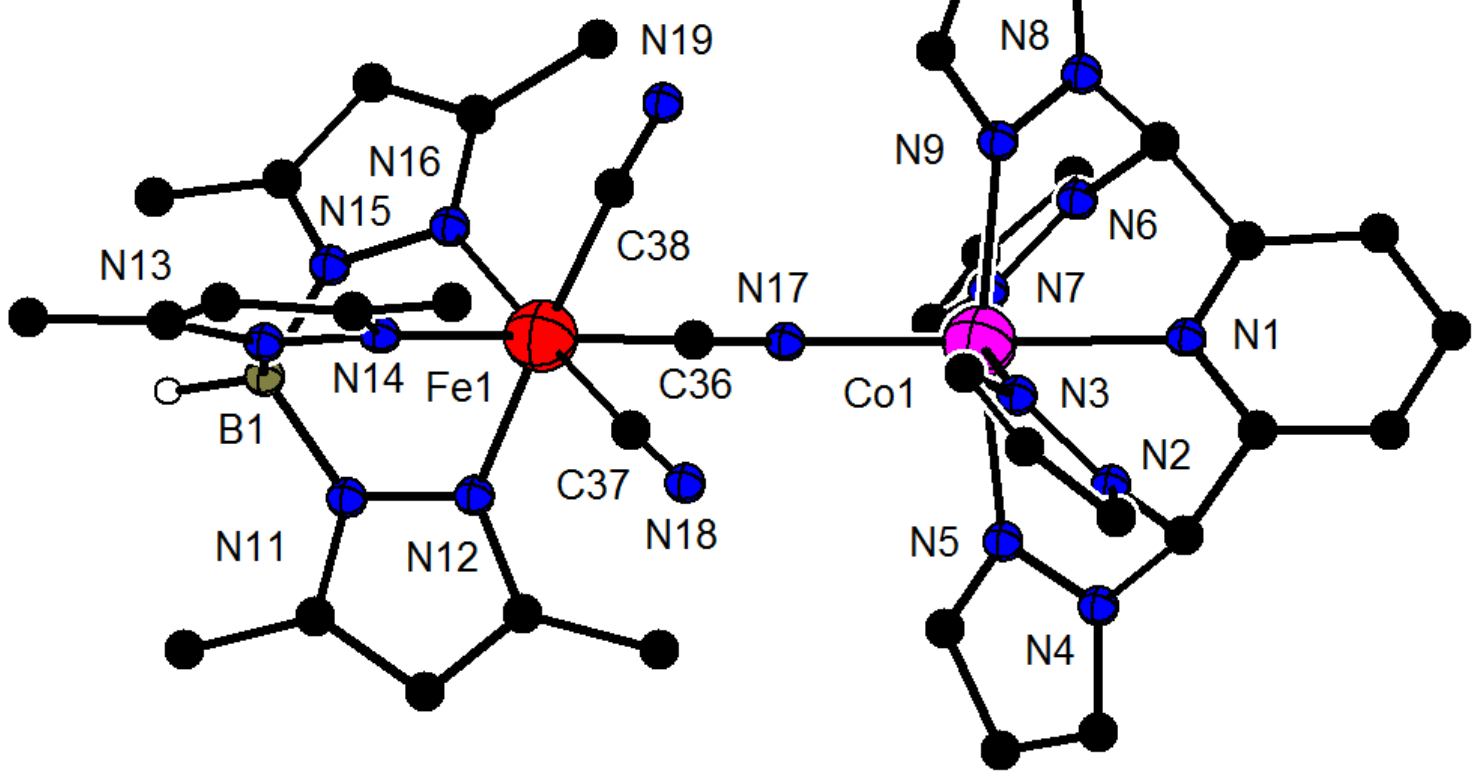

Figure 5.64 Molecular structure of [tp*-Fe/pz 4 Lut-Co]; selected bond lengths $[\AA]$ and angles $\left[^{\circ}\right]$ : Co1N17 2.0482(15), Co1-N9 2.1280(16), Co1-N5 2.1340(16), Co1-N3 2.1357(16), Co1-N7 2.1463(16), Co1-N1 2.1527(15); N17-Co1-N9 93.51(6), N17-Co1-N5 98.49(6), N9-Co1-N5 168.00(6), N17-Co1-N3 90.91(6), N9-Co1-N3 95.57(6), N5-Co1-N3 84.41(6), N17-Co1-N7 102.19(6), N9-Co1-N7 84.15(6), N5Co1-N7 93.16(6), N3-Co1-N7 166.90(6), N17-Co1-N1 173.81(6), N9-Co1-N1 84.28(6), N5-Co1-N1 83.79(6), N3-Co1-N1 83.57(6), N7-Co1-N1 83.37(6), C5-N1-Co1 120.21(12), C1-N1-Co1 120.54(12), C9-N3-Co1 136.73(13), N2-N3-Co1 118.07(12), C12-N5-Co1 136.43(13), N4-N5-Co1 118.12(11), C16N7-Co1 137.76(13), N6-N7-Co1 117.54(11), C19-N9-Co1 136.15(13), N8-N9-Co1 118.16(12), C36N17-Co1 156.94(14). 


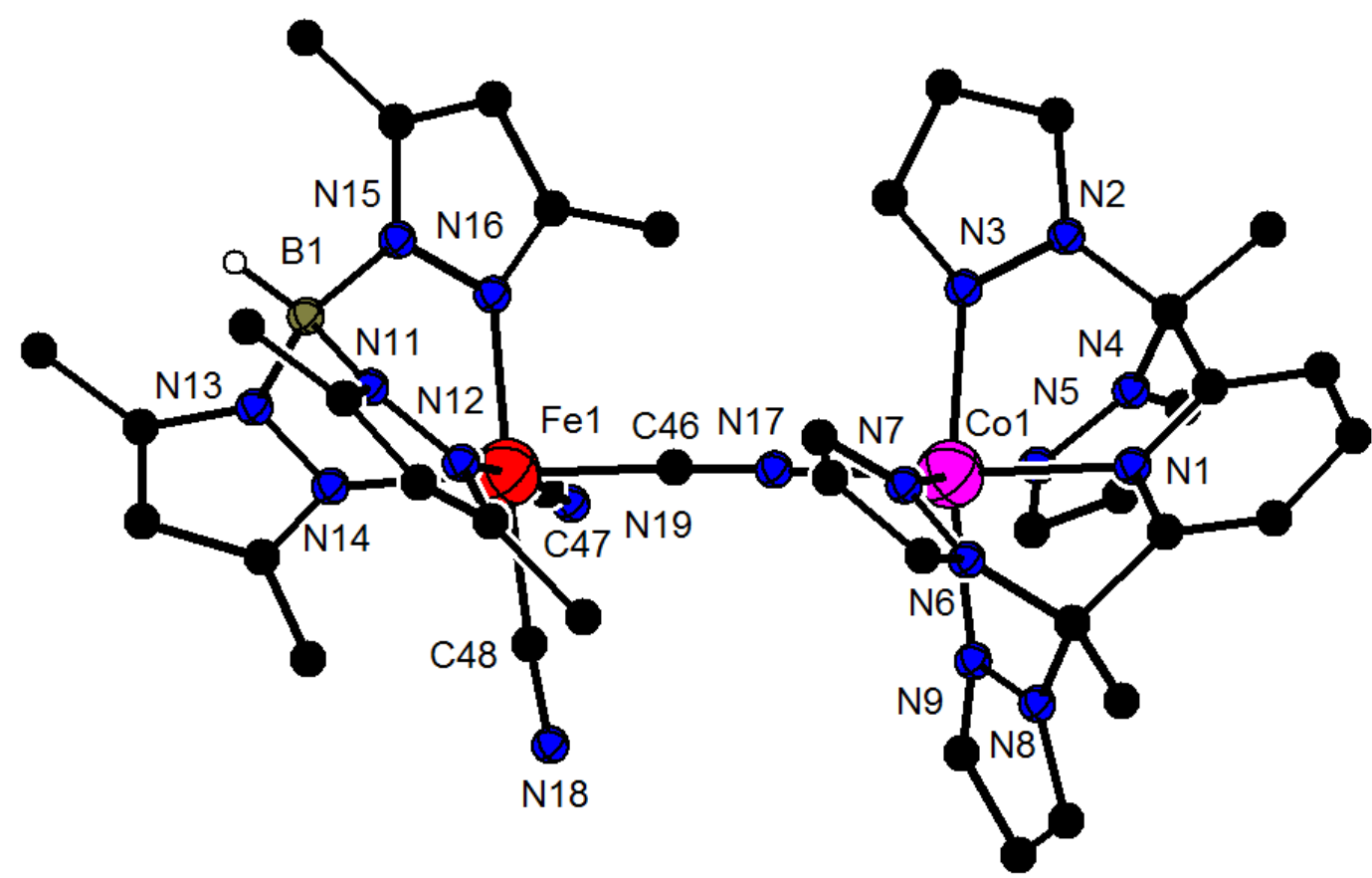

Figure 5.65 Molecular Structure of [tp*-Fe/pz 4 depy-Co]; selected bond lengths $[\AA \AA]$ and angles [ $\left.{ }^{\circ}\right]$ : Co1N17 2.0559(16), Co1-N5 2.0876(14), Co1-N3 2.1010(15), Co1-N7 2.1040(15), Co1-N9 2.1095(14), Co1-N1 2.1878(14); N17-Co1-N5 100.84(6), N17-Co1-N3 92.05(6), N5-Co1-N3 83.43(6), N17-Co1-N7 93.16(6), N5-Co1-N7 166.01(6), N3-Co1-N7 96.36(6), N17-Co1-N9 99.85(6), N5-Co1-N9 94.10(6), N3Co1-N9 168.10(6), N7-Co1-N9 83.23(6), N17-Co1-N1 174.67(6), N5-Co1-N1 82.63(5), N3-Co1-N1 84.29(5), N7-Co1-N1 83.43(5), N9-Co1-N1 83.85(5), C1-N1-Co1 120.30(11), C5-N1-Co1 120.30(11), C10-N3-Co1 134.22(12), N2-N3-Co1 120.08(11), C13-N5-Co1 133.70(12), N4-N5-Co1 120.43(10), C18-N7-Co1 135.04(13), N6-N7-Co1 119.60(11), C21-N9-Co1 133.96(12), N8-N9-Co1 119.05(10), C46-N17-Co1 174.73(15). 


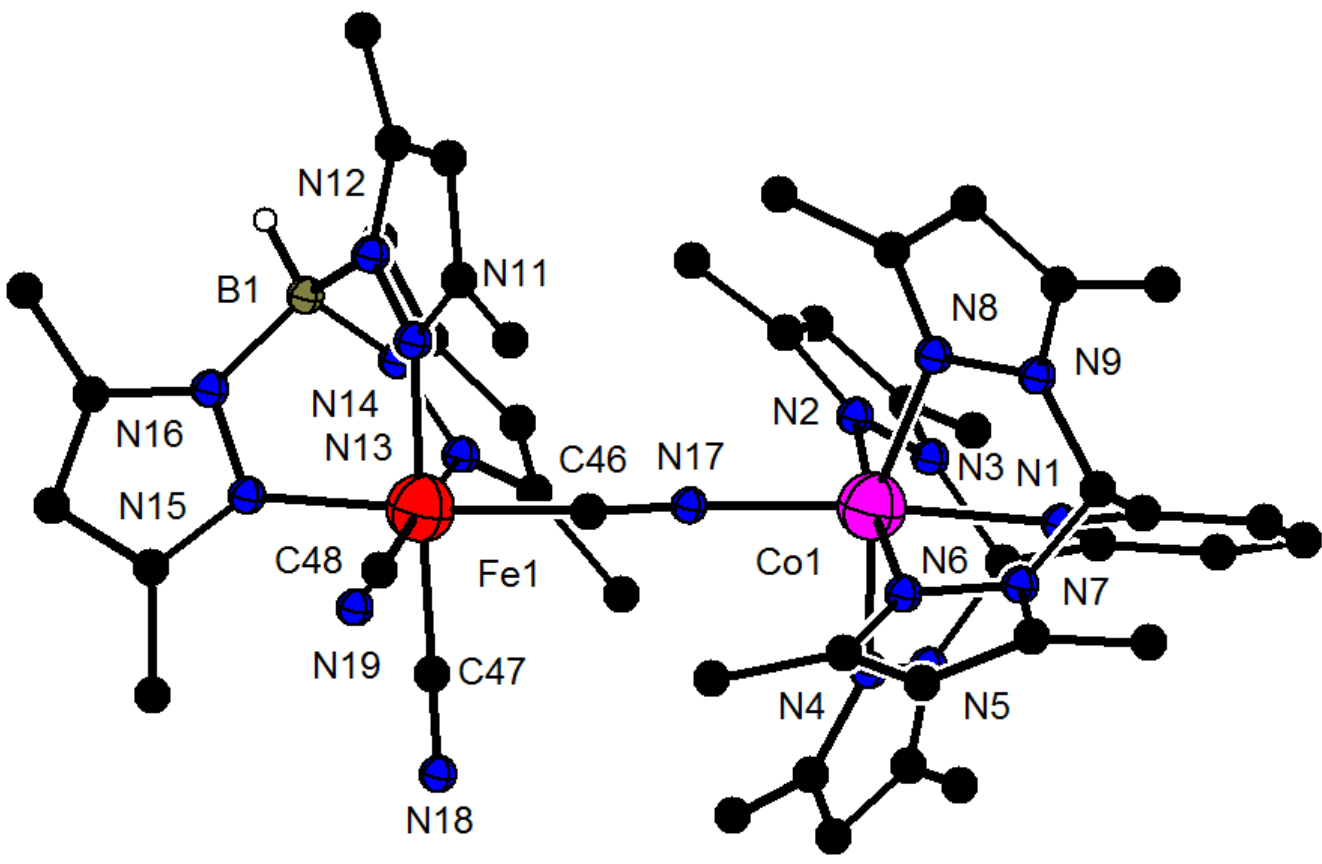

Figure 5.66 Molecular structure of [tp*-Fe/pz* ${ }_{4}$ Lut-Co]; selected bond lengths $[\AA]$ and angles [ $\left.{ }^{\circ}\right]$ : Co1N17 2.0510(15), Co1-N1 2.1496(14), Co1-N4 2.1979(15), Co1-N6 2.2118(15), Co1-N8 2.2297(15), Co1-N2 2.2349(15); N17-Co1-N1 171.79(6), N17-Co1-N4 92.25(6), N1-Co1-N4 80.16(5), N17-Co1-N6 94.55(6), N1-Co1-N6 89.59(5), N4-Co1-N6 97.65(5), N17-Co1-N8 104.52(6), N1-Co1-N8 83.29(6), N4Co1-N8 162.78(6), N6-Co1-N8 77.41(5), N17-Co1-N2 89.89(6), N1-Co1-N2 86.14(5), N4-Co1-N2 83.26(5), N6-Co1-N2 175.42(6), N8-Co1-N2 100.41(5), C5-N1-Co1 121.21(12), C1-N1-Co1 120.91(12), C7-N2-Co1 139.28(12), N3-N2-Co1 115.99(10), C12-N4-Co1 137.76(12), N5-N4-Co1 116.80(11), C18N6-Co1 140.45(13), N7-N6-Co1 114.75(10), C23-N8-Co1 139.05(13), N9-N8-Co1 115.51(10), C46N17-Co1 163.77(14). 


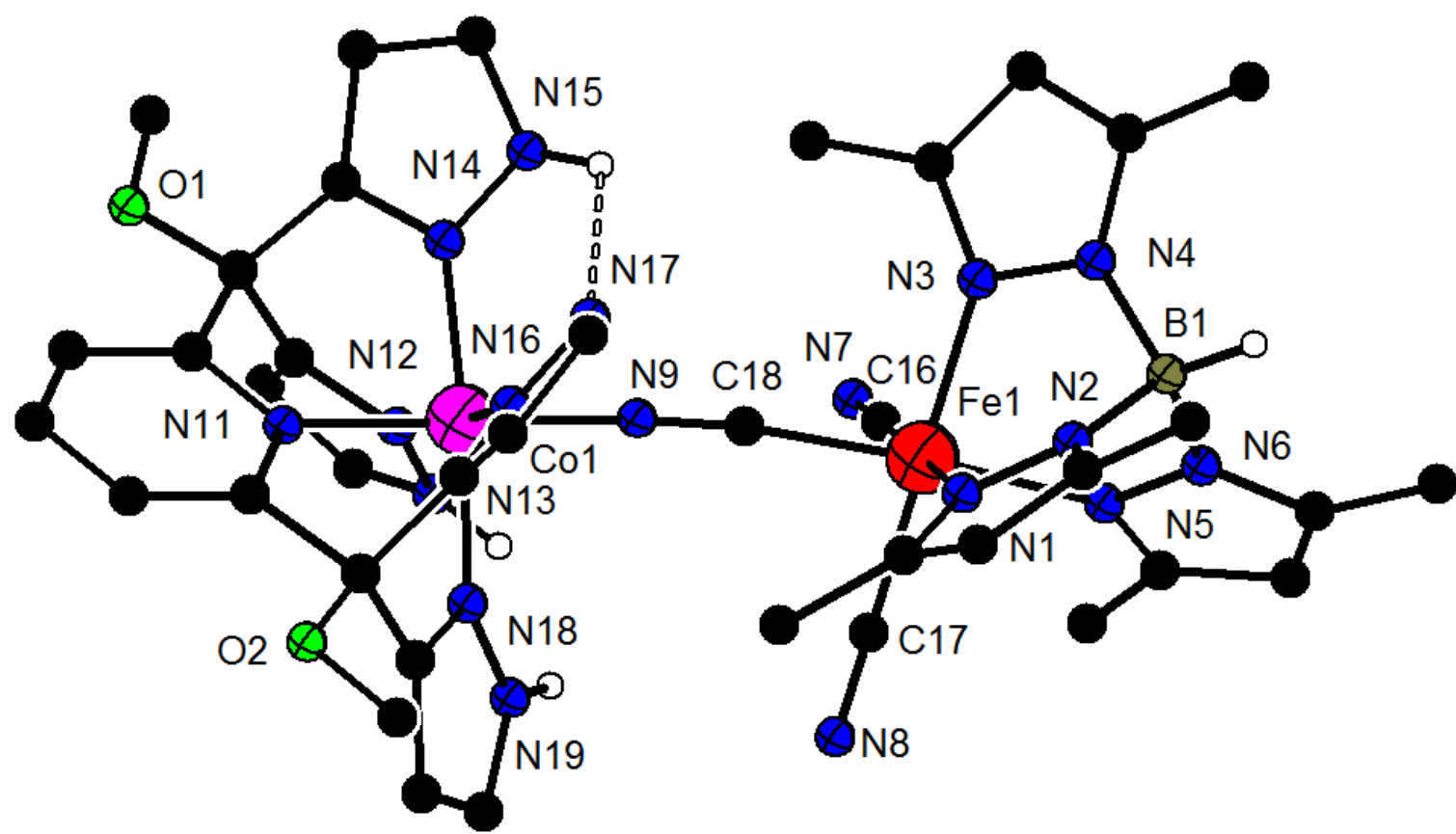

Figure 5.67 Molecular structure [tp ${ }^{*}-\mathrm{Fe} \mathrm{L}^{\mathrm{pz}}-\mathrm{Co}$ ]; selected bond lengths $[\AA]$ and angles $\left[^{\circ}\right]$ : $\mathrm{Co1}-\mathrm{N} 16$ 1.891(2), Co1-N14 1.894(3), Co1-N9 1.898(3), Co1-N18 1.932(3), Co1-N12 1.948(3), Co1-N11 1.971(2); N16-Co1-N14 90.09(11), N16-Co1-N9 90.66(11), N14-Co1-N9 93.97(11), N16-Co1-N18 87.72(11), N14-Co1-N18 174.68(11), N9-Co1-N18 90.90(11), N16-Co1-N12 174.31(11), N14-Co1-N12 86.79(11), N9-Co1-N12 94.29(11), N18-Co1-N12 94.99(11), N16-Co1-N11 86.91(10), N14-Co1-N11 87.22(10), N9-Co1-N11 177.30(10), N18-Co1-N11 87.82(11), N12-Co1-N11 88.19(11), C18-N9-Co1 168.7(3), C21-N11-Co1 120.49(19), C25-N11-Co1 120.5(2), C28-N12-Co1 120.8(2), N13-N12-Co1 132.3(2), C31-N14-Co1 123.5(2), N15-N14-Co1 127.2(2), C36-N16-Co1 123.21(19), N17-N16-Co1 127.00(19), C39-N18-Co1 121.7(2), N19-N18-Co1 131.7(2). 
Table 5.2 Crystal data and refinement details for ligand synthesis $\mathbf{V}$ and $\mathbf{H}_{3} \mathrm{~L}^{\mathrm{Ph}}$.

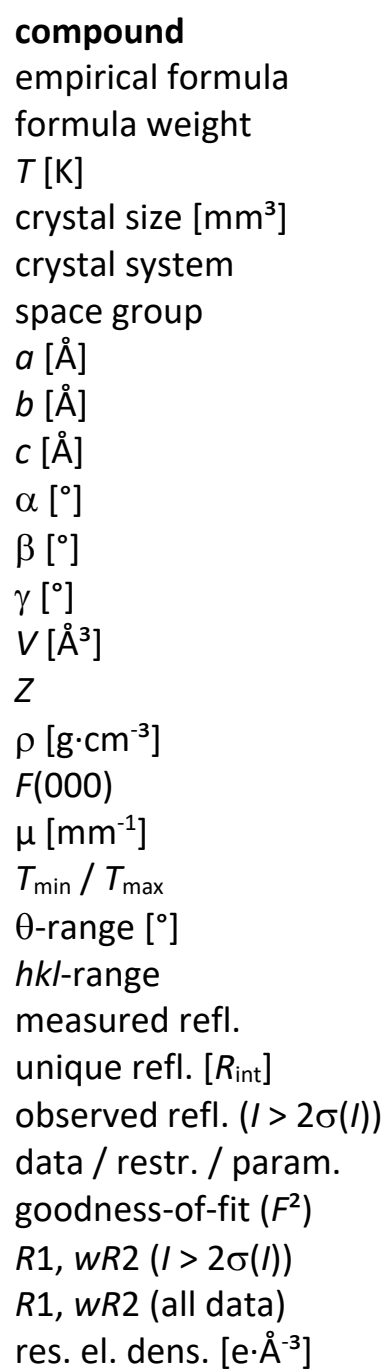

V

$\mathrm{C}_{23} \mathrm{H}_{21} \mathrm{NO}$

327.41

$133(2)$

$0.500 \times 0.490 \times 0.480$

monoclinic

$P 2_{1} / n$ (No. 14)

11.5527(4)

$8.0529(2)$

19.4888(8)

90

99.104(3)

90

$1790.26(11)$

4

1.215

696

0.074

$0.7961 / 0.9578$

$1.926-26.803$

-13 to $14, \pm 10, \pm 24$

24936

3792 [0.0233]

3340

3792 / 0 / 232

1.034

$0.0365 / 0.0940$

$0.0415 / 0.0975$

$-0.185 / 0.208$
$\mathrm{H}_{3} \mathrm{~L}^{\mathrm{Ph}}$

$\mathrm{C}_{51} \mathrm{H}_{48} \mathrm{~N}_{6}$

744.95

133(2)

$0.500 \times 0.290 \times 0.180$

triclinic

P-1 (No. 2)

7.7157(2)

14.6429(4)

$36.4144(9)$

$78.883(2)$

$89.123(2)$

$83.292(2)$

4009.16(18)

4

1.234

1584

0.073

0.8062 / 0.9791

$1.140-25.745$

$\pm 9, \pm 17, \pm 44$

50853

15101 [0.0710]

11436

15101 / 6 / 1059

1.075

0.0578 / 0.1443

$0.0793 / 0.1558$

$-0.244 / 0.303$ 
Table 5.3 Crystal data and refinement details for 3-K(crypt), 4-K, 5-Na, and 8.

\begin{tabular}{|c|c|c|c|c|}
\hline compound & 3-K(crypt) & $4-K$ & $5-\mathrm{Na}$ & 8 \\
\hline empirical formula & $\mathrm{C}_{77} \mathrm{H}_{99} \mathrm{KN}_{8} \mathrm{Ni}_{2} \mathrm{O}_{8}$ & $\mathrm{C}_{68} \mathrm{H}_{71} \mathrm{KN}_{6} \mathrm{Ni}_{2} \mathrm{O}_{3.5}$ & $\mathrm{C}_{57} \mathrm{H}_{55} \mathrm{~N}_{6} \mathrm{Na}_{0.50} \mathrm{Ni}_{2} \mathrm{O}_{1.5}$ & $\mathrm{C}_{51} \mathrm{H}_{44} \mathrm{~N}_{6} \mathrm{Ni}_{2}$ \\
\hline formula weight & 1421.16 & 1184.82 & 976.98 & 858.34 \\
\hline$T[\mathrm{~K}]$ & $133(2)$ & $133(2)$ & $133(2)$ & $133(2)$ \\
\hline crystal size $\left[\mathrm{mm}^{3}\right]$ & $\begin{array}{l}0.430 \times 0.320 \times \\
0.250\end{array}$ & $\begin{array}{l}0.500 \times 0.480 \times \\
0.420\end{array}$ & $0.500 \times 0.250 \times 0.240$ & $0.220 \times 0.200 \times 0.110$ \\
\hline crystal system & monoclinic & monoclinic & monoclinic & monoclinic \\
\hline space group & $P 2_{1} / n$ (No. 14) & $C 2 / c$ (No. 15) & $P 2 / c$ (No. 13) & $P 2_{1} / n$ (No. 14) \\
\hline$a[\AA]$ & $15.3579(3)$ & $30.8579(5)$ & $27.7413(5)$ & $10.4345(4)$ \\
\hline$b[\AA]$ & $17.4833(2)$ & $14.7943(3)$ & $10.6411(2)$ & $11.4682(3)$ \\
\hline$c[\AA]$ & $27.2615(5)$ & $25.3281(4)$ & $20.9606(4)$ & $34.8892(12)$ \\
\hline$\alpha\left[^{\circ}\right]$ & 90 & 90 & 90 & 90 \\
\hline$\beta\left[^{\circ}\right]$ & $95.2380(10)$ & $95.1870(10)$ & $108.0510(10)$ & $97.907(3)$ \\
\hline$\gamma\left[^{\circ}\right]$ & 90 & 90 & 90 & 90 \\
\hline$V\left[\AA^{3}\right]$ & $7289.3(2)$ & $11515.5(4)$ & $5882.98(19)$ & $4135.3(2)$ \\
\hline$Z$ & 4 & 8 & 4 & 4 \\
\hline$\rho\left[\mathrm{g} \cdot \mathrm{cm}^{-3}\right]$ & 1.295 & 1.367 & 1.103 & 1.379 \\
\hline$F(000)$ & 3024 & 4992 & 2050 & 1792 \\
\hline$\mu\left[\mathrm{mm}^{-1}\right]$ & 0.634 & 0.781 & 0.684 & 0.955 \\
\hline$T_{\min } / T_{\max }$ & 0.7545 / 0.8880 & 0.6210 / 0.7248 & 0.6769 / 0.9215 & 0.8683 / 0.9630 \\
\hline$\theta$-range $\left[{ }^{\circ}\right]$ & $1.385-25.741$ & $1.325-25.782$ & $1.544-25.786$ & $1.178-25.752$ \\
\hline$h k l$-range & $\begin{array}{l} \pm 18,-20 \text { to } 21 \\
\pm 33\end{array}$ & $\pm 37, \pm 17, \pm 30$ & $\pm 33, \pm 12,-25$ to 23 & $\pm 12, \pm 13,-42$ to 41 \\
\hline measured refl. & 80822 & 71528 & 71859 & 32752 \\
\hline unique refl. $\left[R_{\text {int }}\right]$ & $13774[0.0447]$ & 10855 [0.0396] & $11100[0.0510]$ & 7797 [0.0649] \\
\hline $\begin{array}{l}\text { observed refl. (I> } \\
2 \sigma(I))\end{array}$ & 10916 & 9203 & 8846 & 5071 \\
\hline $\begin{array}{l}\text { data / restr. / } \\
\text { param. }\end{array}$ & $\begin{array}{l}13774 \text { / } 110 \text { / } \\
923\end{array}$ & $\begin{array}{l}10855 \text { / } 220 \text { / } \\
798\end{array}$ & 11100 / 60 / 680 & 7797 / 0 / 536 \\
\hline goodness-of-fit $\left(F^{2}\right)$ & 1.051 & 1.063 & 1.031 & 1.015 \\
\hline$R 1, w R 2(I>2 \sigma(I))$ & 0.0340 / 0.0828 & 0.0366 / 0.0954 & 0.0336 / 0.0827 & 0.0429 / 0.0980 \\
\hline$R 1, w R 2$ (all data) & 0.0495 / 0.0906 & 0.0460 / 0.1014 & 0.0462 / 0.0881 & 0.0830 / 0.1189 \\
\hline res. el. dens. $\left[\mathrm{e} \cdot \AA^{-3}\right]$ & $-0.336 / 0.292$ & $-0.402 / 0.979$ & $-0.351 / 0.441$ & -0.273 / 0.936 \\
\hline
\end{tabular}


Table 5.4 Crystal data and refinement details for linear PBAs [tp/pz 4 depy], [tp/pz $\left.{ }^{*}{ }_{4} L u t\right]$ and [tp ${ }^{\mathrm{Me}} / \mathrm{pz} \mathbf{z}^{*}$ Lut].

\section{compound}

empirical formula

formula weight

$T[\mathrm{~K}]$

crystal size $\left[\mathrm{mm}^{3}\right]$

crystal system

space group

$a[\AA ̊]$

$b[\AA]$

$c[\AA ̊]$

$\alpha\left[^{\circ}\right]$

$\beta\left[^{\circ}\right]$

$\gamma\left[{ }^{\circ}\right]$

$V\left[\AA^{3}\right]$

$Z$

$\rho\left[\mathrm{g} \cdot \mathrm{cm}^{-3}\right]$

$F(000)$

$\mu\left[\mathrm{mm}^{-1}\right]$

$T_{\text {min }} / T_{\text {max }}$

$\theta$-range $\left[{ }^{\circ}\right]$

hkl-range

measured refl.

unique refl. [ $\left.R_{\text {int }}\right]$

observed refl. (I >

$2 \sigma(I))$

data / restr. / param.

goodness-of-fit $\left(F^{2}\right)$

$R 1, w R 2(I>2 \sigma(I))$

$R 1, w R 2$ (all data)

res. el. dens. $\left[\mathrm{e} \cdot \AA^{-3}\right]$

[tp/pz ${ }_{4}$ depy]
$\mathrm{C}_{36} \mathrm{H}_{39} \mathrm{BCoF} \mathrm{BeN}_{18} \mathrm{O}_{5} \mathrm{~S}$
1018.50
$133(2)$
$0.500 \times 0.260 \times 0.240$
triclinic
$P-1$ (No. 2)
$12.2105(5)$
$12.7513(6)$
$16.1599(8)$
$74.406(4)$
$72.699(4)$
$72.971(3)$
$2250.96(19)$
2
1.503
1044
0.813
$0.7106 / 0.8120$
$1.347-26.955$
-14 to $15, \pm 16, \pm 20$
29385
$9582[0.0349]$
7069
9582 / 137 / 715
1.128
$0.0700 / 0.1741$
$0.0969 / 0.1886$
$-1.348 / 0.903$

[tp/pz ${ }_{4}$ Lut]
$\mathrm{C}_{93} \mathrm{H}_{117} \mathrm{~B}_{2} \mathrm{Co}_{2} \mathrm{~F}_{6} \mathrm{Fe}_{2} \mathrm{~N}_{39} \mathrm{O}_{10} \mathrm{~S}_{2}$
2370.55
$133(2)$
$0.500 \times 0.230 \times 0.170$
triclinic
$P-1$ (No. 2)
$18.8816(6)$
$19.0593(7)$
$19.9679(7)$
$112.418(3)$
$95.261(3)$
$119.122(2)$
$5450.2(4)$
2
1.444
2460
0.683
0.6574 / 0.9154
$1.267-25.798$
$\pm 22, \pm 23,-23$ to 24
59778
20551 [0.0497]
16246
$20551 / 171 / 1504$
1.048
$0.0671 / 0.1767$
$0.0868 / 0.1978$
$-1.161 / 1.139$

[tp ${ }^{\mathrm{Me}} / \mathbf{p z}_{4}{ }_{4}$ Lut]
$\mathrm{C}_{44.50} \mathrm{H}_{59} \mathrm{BCICoFeN}{ }_{18} \mathrm{O}_{6.50}$
1111.13
$133(2)$
$0.500 \times 0.250 \times 0.150$
triclinic
$P-1$ (No. 2$)$
$11.2890(4)$
$14.5660(5)$
$19.0159(6)$
$67.689(3)$
$84.024(3)$
$71.401(3)$
$2741.21(18)$
2
1.346
1158
0.678
$0.8275 / 0.9215$
$1.583-26.861$
$\pm 14, \pm 18, \pm 24$
38165
$11631[0.0288]$
8936
$11631 / 62$ / 699
1.021
$0.0429 / 0.1161$
$0.0623 / 0.1295$
$-0.754 / 0.754$


Table 5.5 Crystal data and refinement details for [tp*/pz $\left.{ }_{4} L u t\right],\left[t p * / p z_{4} d e p y\right]$ and [tp*/pz* $\left.{ }_{4} L u t\right]$ Mod A.

\begin{tabular}{|c|c|c|c|}
\hline compound & {$\left[t p * / p z_{4}\right.$ Lut] } & [tp*/pz depy] & {$\left[t p * / p z *{ }_{4}\right.$ Lut $]$ Mod A } \\
\hline empirical formula & $\mathrm{C}_{44} \mathrm{H}_{53} \mathrm{BCoF}_{3} \mathrm{FeN}_{20} \mathrm{O}_{5} \mathrm{~S}$ & $\mathrm{C}_{40} \mathrm{H}_{43} \mathrm{BCoF}_{3} \mathrm{FeN}_{18} \mathrm{O}_{3} \mathrm{~S}$ & $\mathrm{C}_{47} \mathrm{H}_{63} \mathrm{BClCoFeN}{ }_{18} \mathrm{O}_{6}$ \\
\hline formula weight & 1156.71 & 1038.57 & 1137.19 \\
\hline$T[\mathrm{~K}]$ & $133(2)$ & $133(2)$ & $133(2)$ \\
\hline crystal size $\left[\mathrm{mm}^{3}\right]$ & $0.500 \times 0.240 \times 0.120$ & $0.500 \times 0.490 \times 0.160$ & $0.500 \times 0.440 \times 0.180$ \\
\hline crystal system & triclinic & monoclinic & triclinic \\
\hline space group & $P-1$ (No. 2) & $P 2_{1} / n$ (No. 14) & $P-1$ (No. 2) \\
\hline$a[\AA]$ & $9.5647(3)$ & $11.9219(3)$ & $12.2092(5)$ \\
\hline$b[\AA]$ & $15.2000(6)$ & $14.9496(2)$ & $14.8770(6)$ \\
\hline$c[\AA]$ & $17.8410(6)$ & $25.1164(6)$ & $16.8549(7)$ \\
\hline$\alpha\left[^{\circ}\right]$ & $84.210(3)$ & 90 & $97.597(3)$ \\
\hline$\beta\left[^{\circ}\right]$ & $82.657(3)$ & $96.937(2)$ & $104.474(3)$ \\
\hline$\gamma\left[^{\circ}\right]$ & $89.154(3)$ & 90 & $96.817(3)$ \\
\hline$V\left[\AA^{3}\right]$ & 2559.39(16) & $4443.67(17)$ & $2901.3(2)$ \\
\hline$Z$ & 2 & 4 & 2 \\
\hline$\rho\left[\mathrm{g} \cdot \mathrm{cm}^{-3}\right]$ & 1.501 & 1.552 & 1.302 \\
\hline$F(000)$ & 1196 & 2136 & 1188 \\
\hline$\mu\left[\mathrm{mm}^{-1}\right]$ & 0.726 & 0.822 & 0.642 \\
\hline$T_{\min } / T_{\max }$ & $0.7191 / 0.8905$ & $0.4616 / 0.9563$ & 0.6157 / 0.9172 \\
\hline$\theta$-range $\left[{ }^{\circ}\right]$ & $1.685-26.751$ & $1.588-26.912$ & $1.398-26.948$ \\
\hline hkl-range & -11 to $12, \pm 19, \pm 22$ & $\pm 15,-16$ to $19, \pm 31$ & $\pm 15, \pm 18,-21$ to 19 \\
\hline measured refl. & 33570 & 51351 & 38664 \\
\hline unique refl. $\left[R_{\text {int }}\right]$ & $10833[0.0522]$ & 9420 [0.0289] & 12295 [0.0194] \\
\hline $\begin{array}{l}\text { observed refl. (I> } \\
2 \sigma(I))\end{array}$ & 8943 & 8290 & 10540 \\
\hline $\begin{array}{l}\text { data / restr. / } \\
\text { param. }\end{array}$ & 10833 / 229 / 820 & 9420 / 133 / 698 & 12295 / 49 / 720 \\
\hline goodness-of-fit $\left(F^{2}\right)$ & 1.027 & 1.049 & 1.023 \\
\hline$R 1, w R 2(I>2 \sigma(I))$ & $0.0350 / 0.0860$ & 0.0317 / 0.0867 & $0.0331 / 0.0848$ \\
\hline$R 1, w R 2$ (all data) & 0.0465 / 0.0899 & 0.0377 / 0.0914 & $0.0421 / 0.0912$ \\
\hline res. el. dens. $\left[e \cdot \AA^{-3}\right]$ & $-0.359 / 0.524$ & -0.494 / 0.868 & -0.844 / 0.701 \\
\hline
\end{tabular}


Table 5.6 Crystal data and refinement details for $\left[\operatorname{tp}^{*} / \mathrm{pz}^{*}{ }_{4} L u t\right]$ Mod $B$ and $\left[\operatorname{tp}^{*} / L^{\mathrm{pz}}\right]$.

\begin{tabular}{|c|c|c|}
\hline compound & {$\left[\operatorname{tp} * / p z *{ }_{4}\right.$ Lut] Mod B } & {$\left[\operatorname{tp}^{*} / L^{\mathrm{pz}}\right]$} \\
\hline empirical formula & $\mathrm{C}_{47} \mathrm{H}_{63} \mathrm{BClCoFeN}{ }_{18} \mathrm{O}_{6}$ & $\mathrm{C}_{40.25} \mathrm{H}_{45} \mathrm{BCoF}_{4.50} \mathrm{FeN}_{18} \mathrm{O}_{4.50} \mathrm{P}_{0.50} \mathrm{~S}_{0.50}$ \\
\hline formula weight & 1137.19 & 1095.54 \\
\hline$T[\mathrm{~K}]$ & $133(2)$ & $133(2)$ \\
\hline crystal size $\left[\mathrm{mm}^{3}\right]$ & $0.500 \times 0.310 \times 0.240$ & $0.500 \times 0.370 \times 0.080$ \\
\hline crystal system & monoclinic & triclinic \\
\hline space group & $P 2_{1} / n$ (No. 14 ) & $P-1$ (No. 2) \\
\hline$a[\AA]$ & $15.9582(4)$ & $12.7063(5)$ \\
\hline$b[\AA]$ & $22.6779(7)$ & $14.3141(6)$ \\
\hline$c[\AA]$ & $16.5178(4)$ & $14.3864(5)$ \\
\hline$\alpha\left[^{\circ}\right]$ & 90 & $94.905(3)$ \\
\hline$\beta\left[^{\circ}\right]$ & $92.170(2)$ & $106.612(3)$ \\
\hline$\gamma\left[{ }^{\circ}\right]$ & 90 & $105.554(3)$ \\
\hline$V\left[\AA^{3}\right]$ & $5973.5(3)$ & 2377.99(17) \\
\hline$Z$ & 4 & 2 \\
\hline$\rho\left[\mathrm{g} \cdot \mathrm{cm}^{-3}\right]$ & 1.264 & 1.530 \\
\hline$F(000)$ & 2376 & 1125 \\
\hline$\mu\left[\mathrm{mm}^{-1}\right]$ & 0.624 & 0.774 \\
\hline$T_{\min } / T_{\max }$ & $0.7090 / 0.8768$ & $0.5539 / 0.8559$ \\
\hline$\theta$-range $\left[{ }^{\circ}\right]$ & $1.526-26.929$ & $1.500-26.723$ \\
\hline$h k l$-range & $\pm 20, \pm 28, \pm 20$ & $\pm 16, \pm 18, \pm 18$ \\
\hline measured refl. & 57482 & 31512 \\
\hline unique refl. $\left[R_{\text {int }}\right]$ & 12710 [0.0493] & 10058 [0.0523] \\
\hline observed refl. $(I>2 \sigma(I))$ & 9954 & 7765 \\
\hline data / restr. / param. & 12710 / 192 / 795 & 10058 / $183 / 753$ \\
\hline goodness-of-fit $\left(F^{2}\right)$ & 1.050 & 1.010 \\
\hline$R 1, w R 2(I>2 \sigma(I))$ & 0.0445 / 0.0998 & $0.0541 / 0.1536$ \\
\hline$R 1, w R 2$ (all data) & 0.0632 / 0.1092 & $0.0701 / 0.1630$ \\
\hline res. el. dens. $\left[\mathrm{e} \cdot \AA^{-3}\right]$ & $-0.474 / 0.306$ & $-0.537 / 0.995$ \\
\hline
\end{tabular}

